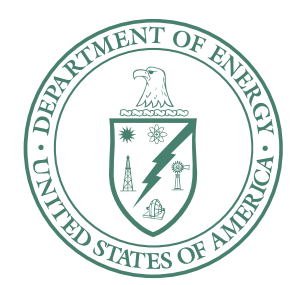

U.S. Department of Energy

Idaho Operations Office

\title{
Waste Area Group 10, Operable Unit 10-08, Remedial Investigation/Feasibility Study Annual Status Report for Fiscal Year 2006
}

May 2007 


\section{Waste Area Group 10, Operable Unit 10-08, Remedial Investigation/Feasibility Study Annual Status Report for Fiscal Year 2006}

May 2007 



\section{ABSTRACT}

This report provides a status of the progress made in Fiscal Year 2006 on tasks identified in the Waste Area Group 10, Operable Unit 10-08, Remedial Investigation/Feasibility Study Work Plan. Major accomplishments include:

(1) groundwater sampling and review of the groundwater monitoring data, (2) installation of a Sitewide groundwater-level monitoring network, (3) update of the Groundwater Monitoring and Field Sampling Plan of Operable Unit 10-08, (4) re-evaluation of the risk at Site TSF-08, (5) progress on the Operable Unit 10-08 Sitewide Groundwater Model. 


\section{CONTENTS}

ABSTRACT. iii

ACRONYMS vii

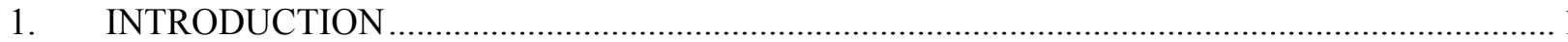

2. OPERABLE UNIT 10-08 DATA COLLECTION AND OTHER ACTIVITIES PERFORMED

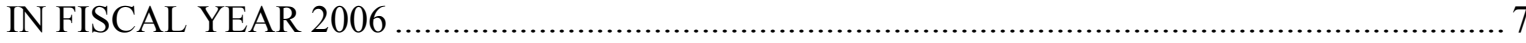

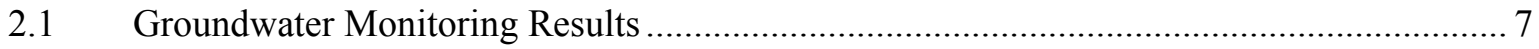

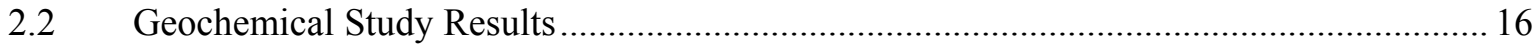

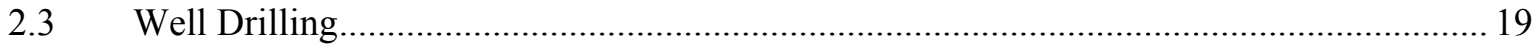

2.4 Sitewide Water-Level Measurements ............................................................................ 19

2.4.1 Selection of Well Locations and Installation for Automated Sitewide Water-Level Network................................................................................. 20

2.4.2 Evaluation of Vertical Gradients in Westbay Wells .....................................23

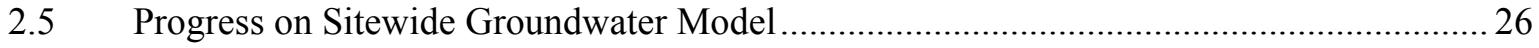

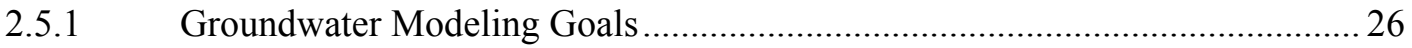

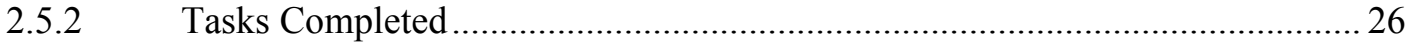

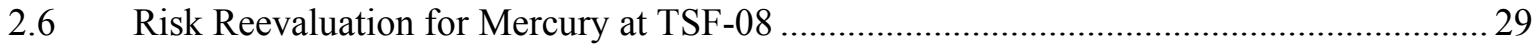

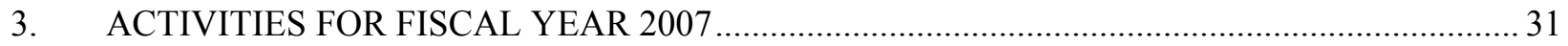

3.1 Recurring Tasks and Tasks Carried Over from Previous Annual Reports ........................ 31

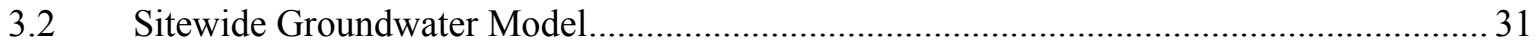

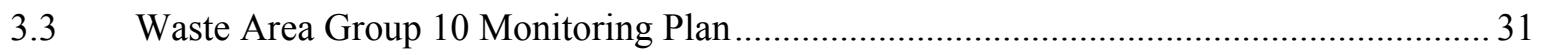

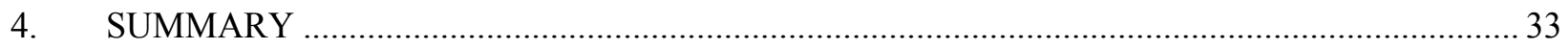

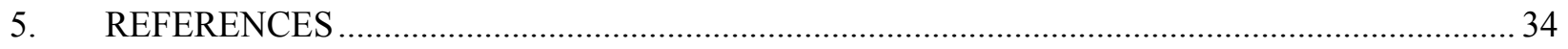

Appendix A-Analytical Results from Fiscal Year 2006 Groundwater Sampling ................................ A-1

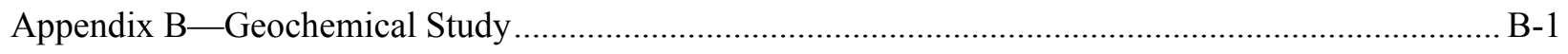

Appendix C—Summary of Operable Unit 10-08 Two-Dimensional Transport Model .......................... C-1 


\section{FIGURES}

1. Idaho National Laboratory site map showing waste area group locations .................................... 2

2. Location of boundary, guard, distal, and Westbay wells sampled in June and July 2006 .............. 10

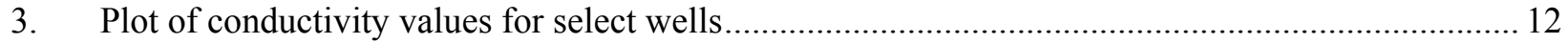

4. Tritium concentration trends for USGS-104 and USGS-106................................................ 14

5. Zinc concentrations at select wells that have galvanized riser pipes......................................... 19

6. Well locations for the automated Sitewide water-level monitoring network ...............................2

7. MIDDLE-2051 pressure heads plotted as water-level elevation versus depth

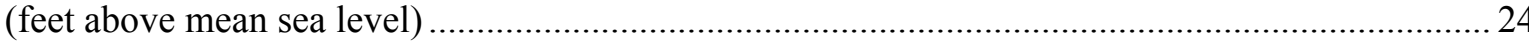

8. MIDDLE-2050A pressure heads plotted as water-level elevation versus depth (feet above mean sea level)

\section{TABLES}

1. Status of tasks identified in the Operable Unit 10-08 Remedial Investigation/Feasibility

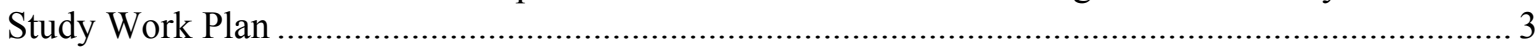

2. Well construction summary for Waste Area Group 10 wells ....................................................... 8

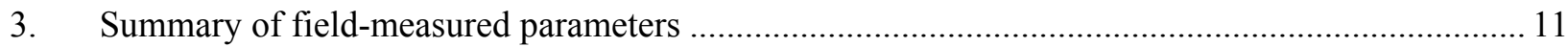

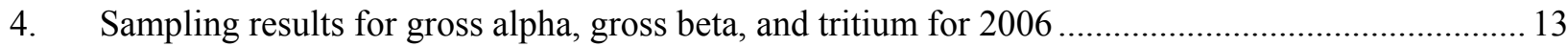

5. Sampling results for tritium, gross alpha, gross beta, uranium, and uranium isotopes for

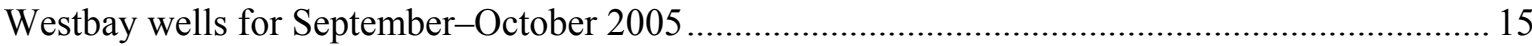

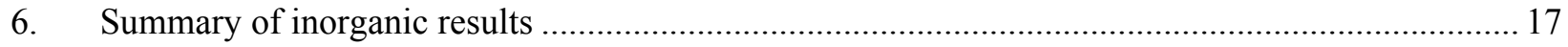

7. Proposed wells that were not used, with the corresponding replacement well or explanation for non-use 20

8. Wells used for automated Sitewide water-level monitoring network 


\section{ACRONYMS}

AMS accelerator mass spectrometry

bgs below ground surface

BLR Big Lost River

BRA baseline risk assessment

CERCLA Comprehensive Environmental Response, Compensation, and Liability Act

CFA Central Facilities Area

CFC chlorofluorocarbon

DEQ [Idaho] Department of Environmental Quality

DOE Department of Energy

DOE-ID Department of Energy Idaho Operations Office

EDW Environmental Data Warehouse

EPA Environmental Protection Agency

ESRP Eastern Snake River Plain Aquifer

FFA/CO Federal Facility Agreement and Consent Order

FY fiscal year

GMS groundwater model system

INL Idaho National Laboratory

INTEC Idaho Nuclear Technology and Engineering Center

MCL maximum contaminant level

MDA minimum detectable activity

OU operable unit

PCE tetrachloroethene

PRIME Purdue Rare Isotope Measurement (laboratory)

RI/FS remedial investigation/feasibility study

ROD Record of Decision 


$\begin{array}{ll}\text { RSM } & \text { Response Surface Model } \\ \text { RTC } & \text { Reactor Technology Complex } \\ \text { RWMC } & \text { Radioactive Waste Management Complex } \\ \text { SRPA } & \text { Snake River Plain Aquifer } \\ \text { SWGM } & \text { Sitewide groundwater-flow and contaminant-transport numerical model } \\ \text { TAN } & \text { Test Area North } \\ \text { TCE } & \text { trichloroethene } \\ \text { TSF } & \text { Technical Support Facility } \\ \text { USC } & \text { United States Code } \\ \text { USGS } & \text { United States Geological Survey } \\ \text { VOC } & \text { volatile organic compound } \\ \text { WAG } & \text { waste area group }\end{array}$




\section{Waste Area Group 10, Operable Unit 10-08, Remedial Investigation/Feasibility Study Annual Status Report for Fiscal Year 2006}

\section{INTRODUCTION}

The purpose of Waste Area Group (WAG) 10, Operable Unit (OU) 10-08, is the comprehensive evaluation of impacts to groundwater from operations at the Idaho National Laboratory (INL) Site. These operations have introduced radioactive and hazardous contaminants into the environment, and a number of these contaminants have been found in the Snake River Plain Aquifer (SRPA). The potential impacts to the groundwater from INL Site activities are being thoroughly investigated as part of the OU 10-08 remedial investigation/feasibility study (RI/FS). The requirement for this annual report was established in the Waste Area Group 10, Operable Unit 10-08, Remedial Investigation/Feasibility Study Work Plan (FINAL) (DOE-ID 2002a).

Waste Area Group 10 was created in accordance with the Federal Facility Agreement and Consent Order for the Idaho National Engineering Laboratory (DOE-ID 1991) and the Comprehensive Environmental Response, Compensation, and Liability Act (CERCLA) (42 USC $§ 9601$ et seq.), as amended by the Superfund Amendments and Reauthorization Act (Public Law 99-499). The OU 10-08 RI/FS will not follow the normal timeframe allocated under the Federal Facility Agreement and Consent Order (FFA/CO). The WAG 10 OU 10-08 RI/FS Work Plan (DOE-ID 2002a) describes the enforceable milestone schedule for OU 10-08 and the final deliverable date for the OU 10-08 Record of Decision (ROD). The OU 10-08 ROD is expected to be the last ROD completed at the INL Site, and the deliverable date depends on when the other WAGs' RODs are signed.

Waste Area Group 10 encompasses miscellaneous surface contamination sites and liquid disposal areas that are located throughout the INL Site but outside its nine major facility areas (Figure 1). These facility areas comprise the other nine INL Site WAGs. Operable Unit 10-08 addresses INL-related issues that are associated with the SRPA but are not addressed under the purview of the other WAGs. In addition, OU 10-08 will include the evaluation of new sites that are passed to WAG 10 by other WAGs, and sites discovered after the OU 10-04 work plan was signed. As of the last INL CERCLA 5-year review, over 76 new sites have been included for final disposition under OU 10-08 (DOE-ID 2006a). Additional new sites may be included (closure date to be established) in the OU 10-08 risk assessment depending on the date of discovery and the complexity of the site. New sites identified after the completion of the OU 10-08 comprehensive risk assessment will be evaluated based on criteria established in the OU 10-08 ROD. The 5-year review process will document decisions on those sites identified after the OU 10-08 ROD.

The work scope of the OU 10-08 RI/FS is based on completing tasks originally identified in the OU 10-08 RI/FS Work Plan (DOE-ID 2002a). The tasks identified in the OU 10-08 RI/FS Work Plan and the progress made toward their completion in Fiscal Year (FY) 2006 are summarized in Table 1. The activities in the work scope are necessary to characterize and assess INL Sitewide groundwater risks and ultimately will be used in support of the OU 10-08 ROD. It is important to note that many of the tasks performed under the OU 10-08 RI/FS also support individual WAGs. In addition, there will be a risk assessment of commingling plumes between the Idaho Nuclear Technology and Engineering Center (INTEC), Reactor Technology Complex (RTC), and the Radioactive Waste Management Complex (RWMC). 


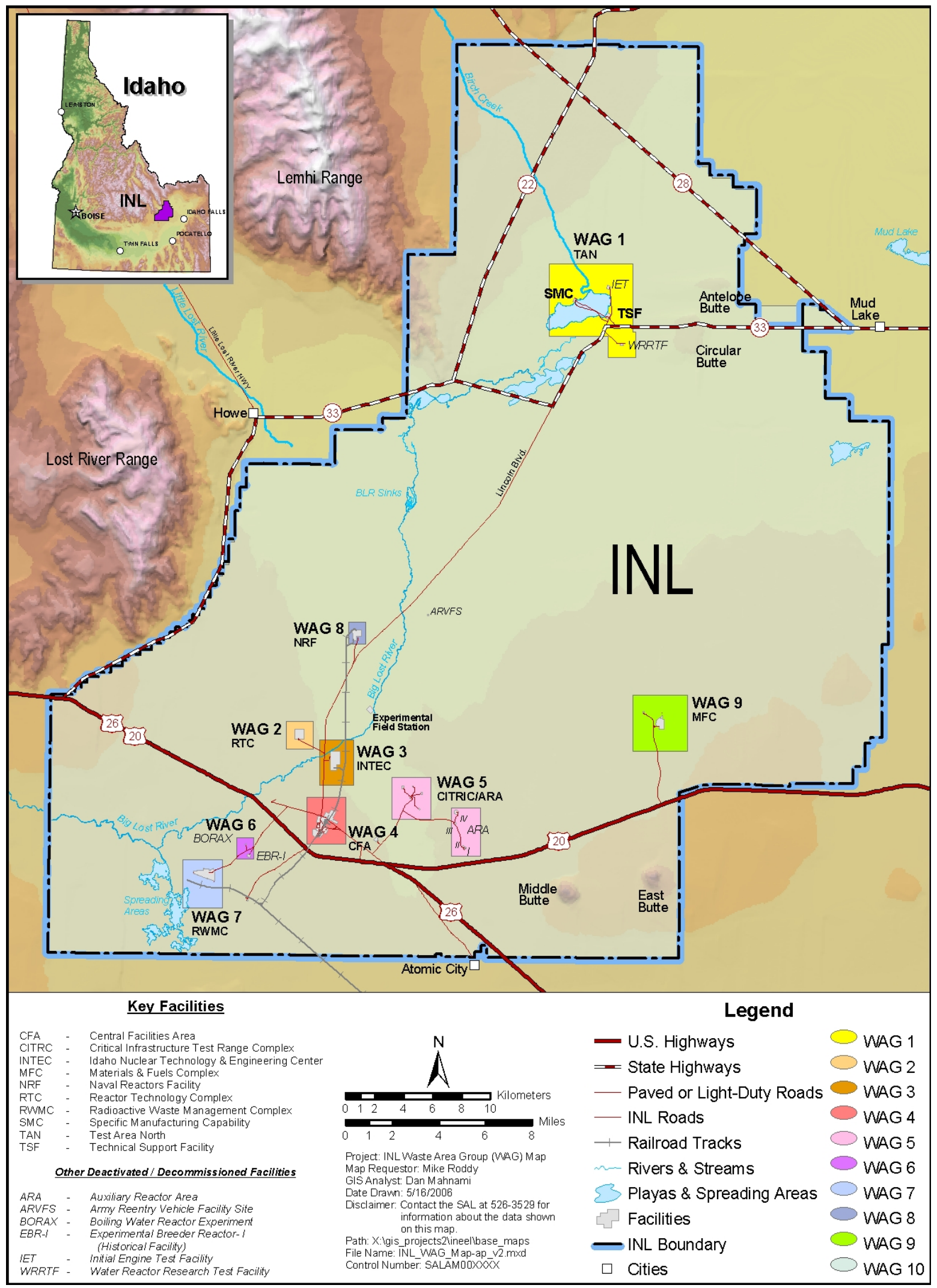

Figure 1. Idaho National Laboratory site map showing waste area group locations. 
Table 1. Status of tasks identified in the Operable Unit 10-08 Remedial Investigation/Feasibility Study Work Plan (DOE-ID 2002a).

\begin{tabular}{|c|c|c|}
\hline Description & Status & Comments \\
\hline \multicolumn{3}{|c|}{ Task 1: Development of a Comprehensive Groundwater Sample Results Database } \\
\hline $\begin{array}{l}\text { Develop a comprehensive database of groundwater } \\
\text { samples. }\end{array}$ & Completed in FY 2004. & $\begin{array}{l}\text { With a few exceptions, all data from the } \\
\text { Environmental Restoration Information System have } \\
\text { been entered into the Environmental Data Warehouse } \\
\text { (EDW). Data that were not put into the previous } \\
\text { database are occasionally discovered and entered into } \\
\text { EDW. All data collected in the future will be entered } \\
\text { into EDW. }\end{array}$ \\
\hline \multicolumn{3}{|c|}{ Task 2: Evaluate Groundwater Chemistry Data } \\
\hline $\begin{array}{l}\text { Evaluate the groundwater data from the WAG } 10 \\
\text { monitoring network to demonstrate compliance with } \\
\text { maximum contaminant levels (MCLs) or other } \\
\text { risk-based concentrations with the information } \\
\text { incorporated into the annual OU } 10-08 \text { RI/FS report. }\end{array}$ & Ongoing & $\begin{array}{l}\text { Monitoring data have been reviewed for FY } 2006 \text { in } \\
\text { this report. This task will continue on an annual basis. }\end{array}$ \\
\hline $\begin{array}{l}\text { Evaluate groundwater quality records to select wells } \\
\text { with suitable analytical histories for groundwater } \\
\text { model calibration. Trends from the selected wells } \\
\text { will be updated with new data (as data become } \\
\text { available) and compared to current groundwater } \\
\text { model predictions. }\end{array}$ & $\begin{array}{l}\text { Planned for FY } 2006 \text { through } \\
\text { FY } 2007 .\end{array}$ & $\begin{array}{l}\text { White paper has been prepared selecting wells for } \\
\text { model calibration. This task was begun in FY } 2006 \\
\text { and will continue in parallel with model development } \\
\text { for the RI/FS. }\end{array}$ \\
\hline $\begin{array}{l}\text { Revise the Groundwater Monitoring and } \\
\text { Field Sampling Plan of Operable Unit 10-08 } \\
\text { (DOE-ID 2005) to incorporate sampling in support } \\
\text { of the new deep core holes that will be converted } \\
\text { to monitoring wells. }\end{array}$ & $\begin{array}{l}\text { Revisions of the Groundwater } \\
\text { Monitoring and Field } \\
\text { Sampling Plan of Operable } \\
\text { Unit 10-08 to incorporate new } \\
\text { Westbay wells is ongoing. }\end{array}$ & $\begin{array}{l}\text { The subject document was revised in FY } 2006 \\
\text { to incorporate sampling of the Westbay wells } \\
\text { MIDDLE-2050A and MIDDLE-2051 } \\
\text { (DOE-ID 2006b). } \\
\text { Groundwater monitoring plans will be prepared } \\
\text { (or amended, as appropriate) upon additional well } \\
\text { installation and changing sampling needs. }\end{array}$ \\
\hline $\begin{array}{l}\text { Evaluate alternative groundwater well sampling and } \\
\text { purging methodology. }\end{array}$ & Completed in FY 2003. & $\begin{array}{l}\text { See the Waste Area Group 10, Operable Unit 10-08, } \\
\text { Remedial Investigation/Feasibility Study Annual } \\
\text { Report for Fiscal Year } 2003 \text { (DOE-ID 2004a). }\end{array}$ \\
\hline
\end{tabular}


Table 1. (continued).

\begin{tabular}{|c|c|c|}
\hline Description & Status & Comments \\
\hline \multicolumn{3}{|c|}{ Task 4: Evaluate Potentially Commingled Plumes } \\
\hline $\begin{array}{l}\text { Evaluate potentially commingled plumes, } \\
\text { incorporating annual data. }\end{array}$ & $\begin{array}{l}\text { Ongoing. Sampling for } \\
\text { geochemical study to address } \\
\text { issue of commingling plumes } \\
\text { performed in FY } 2005 \text {. } \\
\text { Additional samples collected } \\
\text { in FY } 2006 .\end{array}$ & $\begin{array}{l}\text { Work necessary to complete this task is discussed in } \\
\text { the Idaho National Engineering and Environmental } \\
\text { Laboratory Operable Unit 10-08 Sitewide } \\
\text { Groundwater Model Work Plan (DOE-ID 2004b). } \\
\text { Evaluation of commingling plumes will be completed } \\
\text { after the Sitewide numerical model is completed. }\end{array}$ \\
\hline \multicolumn{3}{|c|}{ Task 5: Evaluate Groundwater Quality for Current Compliance with Maximum Contaminant Levels or Other Risk-Based Concentrations } \\
\hline $\begin{array}{l}\text { Evaluate downgradient guard and boundary wells to } \\
\text { assess compliance with groundwater MCLs or } \\
\text { risk-based concentrations on a Sitewide basis. }\end{array}$ & $\begin{array}{l}\text { Ongoing. Sampling results for } \\
2006 \text { presented in this report. }\end{array}$ & $\begin{array}{l}\text { WAG } 10 \text { 's responsibility is to interact with other } \\
\text { WAGs to monitor the success of the individual } \\
\text { WAG's remedial action. Currently, WAG } 10 \\
\text { personnel review the applicable documents (e.g., } \\
\text { annual monitoring reports and 5-year reviews) to } \\
\text { remain familiar with conditions at these WAGs. }\end{array}$ \\
\hline \multicolumn{3}{|c|}{ Task 6: Methods to Incorporate New Sites into WAG 10, OU 10-08 } \\
\hline $\begin{array}{l}\text { Review the procedures for incorporating new sites } \\
\text { into WAG } 10 .\end{array}$ & Completed in FY 2004. & $\begin{array}{l}\text { The latest revision of "Inclusion of New Sites under } \\
\text { the Federal Facility Agreement and Consent Order" } \\
\text { (MCP-3448) was completed in FY } 2005 . \\
\text { Documents or procedures used to incorporate new } \\
\text { sites into WAG } 10 \text { will be reviewed and updated } \\
\text { when requirements change or when otherwise } \\
\text { determined to be necessary. }\end{array}$ \\
\hline \multicolumn{3}{|c|}{ Task 7: Evaluation of Phytoremediation of Mercury in Soils at Site TSF-08 } \\
\hline $\begin{array}{l}\text { Evaluate the applicability of phytoremediation at this } \\
\text { site. Reevaluate the risk from Hg at this site using } \\
\text { updated fate and transport information now available } \\
\text { from the U.S. Environmental Protection Agency } \\
\text { (EPA). Also, include all applicable sampling data in } \\
\text { this reassessment. }\end{array}$ & In progress & See Section 2.6 of this report. \\
\hline
\end{tabular}


Table 1. (continued).

\begin{tabular}{|c|c|c|}
\hline Description & Status & Comments \\
\hline \multicolumn{3}{|c|}{ Task 8: Revise Sitewide Groundwater Model } \\
\hline Revise the Sitewide groundwater model. & In progress & $\begin{array}{l}\text { This task is addressed in the Idaho National } \\
\text { Engineering and Environmental Laboratory Operable } \\
\text { Unit 10-08 Sitewide Groundwater Model Work Plan } \\
\text { (DOE-ID 2004b). }\end{array}$ \\
\hline \multicolumn{3}{|c|}{ Task 9: Institutional Controls } \\
\hline $\begin{array}{l}\text { The development of comprehensive Sitewide } \\
\text { institutional controls resides within the Remedial } \\
\text { Design/Remedial Action Work Plan for Operable } \\
\text { Units 6-05 and 10-04, Phase I (DOE-ID 2004c), as } \\
\text { directed by the Record of Decision Experimental } \\
\text { Breeder Reactor-I/Boiling Water Reactor Experiment } \\
\text { Area and Miscellaneous Sites (DOE-ID 2002b). }\end{array}$ & $\begin{array}{l}\text { INEEL Sitewide Institutional } \\
\text { Controls Plan was completed } \\
\text { in FY 2004. An annual report } \\
\text { summarizing institutional } \\
\text { controls assessment for } \\
\text { FY 2006 also was completed } \\
\text { (DOE-ID 2006c). }\end{array}$ & $\begin{array}{l}\text { The INL Sitewide Institutional Controls Plan } \\
\text { (DOE-ID 2006d) was completed as part of the } \\
\text { Remedial Design/Remedial Action Work Plan for } \\
\text { Operable Units 6-05 and 10-04, Phase I } \\
\text { (DOE-ID 2004c). }\end{array}$ \\
\hline \multicolumn{3}{|c|}{ Task 10: Risk Evaluation for Groundwater } \\
\hline $\begin{array}{l}\text { This task will be among the last completed during the } \\
\text { OU } 10-08 \mathrm{RI} / \mathrm{FS} \text {. The risk assessment will be } \\
\text { completed to determine risk on the WAG } 10 \text { scale, } \\
\text { incorporating up-to-date numerical modeling } \\
\text { efforts and analytical data. }\end{array}$ & $\begin{array}{l}\text { Ongoing. An INL-scale } \\
\text { evaluation is planned for } \\
\text { future years. } \\
\text { The facility-scale evaluation } \\
\text { is ongoing. }\end{array}$ & $\begin{array}{l}\text { Currently, WAG } 10 \text { is interfacing with individual } \\
\text { WAGs in order to incorporate the work and findings } \\
\text { of individual WAGs into the Sitewide groundwater } \\
\text { model used for the risk assessment. } \\
\text { The Sitewide work is to take place after individual } \\
\text { WAGs have transferred monitoring responsibilities to } \\
\text { WAG } 10 \text {. } \\
\text { Evaluations of contaminants of concern in the } \\
\text { perched-water zones and aquifers at various facilities } \\
\text { were reviewed to establish familiarity with the } \\
\text { conditions at these facilities and to maintain } \\
\text { institutional knowledge. }\end{array}$ \\
\hline
\end{tabular}


Table 1. (continued).

\begin{tabular}{|l|l|l|}
\hline \multicolumn{1}{|c|}{ Description } & \multicolumn{1}{|c|}{ Status } & Comments \\
\hline \multicolumn{3}{|c|}{ Task 11: Verification of Water Level Measuring Points } \\
\hline $\begin{array}{l}\text { The correct water-level measuring points, casing } \\
\text { stick-ups, and well surveys for all wells used for } \\
\text { Sitewide groundwater monitoring will be verified. }\end{array}$ & Ongoing & $\begin{array}{l}\text { During FY 2006, dedicated insitu water-level } \\
\text { data loggers were installed in 52 wells across the INL } \\
\text { (see Section 2.4). }\end{array}$ \\
\hline EDW $=$ Environmental Data Warehouse. \\
FY $=$ fiscal year. \\
INEEL $=$ Idaho National Engineering and Environmental Laboratory. \\
INL = Idaho National Laboratory. \\
MCL = maximum contaminant level. \\
MCP = management control procedure. \\
OU = operable unit. \\
RI/FS $=$ remedial investigation/feasibility study. \\
ROD $=$ Record of Decision. \\
TSF $=$ Technical Support Facility. \\
WAG $=$ waste area group.
\end{tabular}




\section{OPERABLE UNIT 10-08 DATA COLLECTION AND OTHER ACTIVITIES PERFORMED IN FISCAL YEAR 2006}

The WAG 10 field sampling activities are planned and approved by the Agencies (i.e., U.S. Department of Energy [DOE], U.S. Environmental Protection Agency [EPA], and Idaho Department of Environmental Quality [DEQ]) through the preparation of formal work planning documents, e.g., the Groundwater Monitoring and Field Sampling Plan (DOE-ID 2006b) and Idaho National Engineering and Environmental Laboratory OU 10-08 Sitewide Groundwater Model Work Plan (DOE-ID 2004b). Progress under the controlling documents is tracked during semimonthly conference calls with the Agencies. As required to meet program objectives, the controlling documents are modified in revisions. The results of the groundwater monitoring effort for FY 2006 and progress on the groundwater flow model in FY 2006 are discussed in the following sections. In addition, the re-evaluation of mercury risk at TSF-08 is presented.

\subsection{Groundwater Monitoring Results}

The groundwater monitoring task in FY 2006 included gathering, reviewing, and organizing available water quality and construction data for wells that are currently being monitored under the purview of OU 10-08. The wells sampled are divided into five groups: boundary, guard, baseline, distal, and Westbay. In accordance with the field sampling plan, the baseline wells were not sampled in FY 2006 but will be sampled in FY 2007. The downgradient boundary wells and guard wells are considered the most important to fill data gaps and are sampled annually. The baseline wells are utilized for evaluating potential impacts of off-Site sources migrating on-Site and are now sampled every other year. The distal wells are intended to evaluate off-Site downgradient impacts and are sampled every other year. The Westbay wells are multi-level wells that are sampled to evaluate the vertical distribution of contaminants and to evaluate vertical groundwater gradients. The collected data are being used to evaluate groundwater quality at the scale of the INL Site for the OU 10-08 RI/FS and for preparation of parameter trends for model calibration and compliance monitoring. In FY 2006, well construction information was reviewed and compiled as part of a revision to the Groundwater Monitoring and Field Sampling Plan (DOE-ID 2006b) (Table 2). The well locations sampled in 2006 are shown on Figure 2.

Sampling was conducted in accordance with the Groundwater Monitoring and Field Sampling Plan (DOE-ID 2006b). Wells were sampled for the analytes listed on the sampling and analysis plan tables in Appendix A of that plan. Eighteen wells and two Westbay wells with five sampling intervals in each were sampled for volatile organic compounds (VOCs) (Contract Laboratory Program target analyte list), metals (filtered), anions, and radionuclides (I-129, tritium, Tc-99, gross alpha, gross beta, and Sr-90) during June and July 2006. Full analytical results for each well are provided in Appendix A of this report. Well USGS-103 could not be sampled because of pump problems. That well is presently being deepened with the intention that a Westbay system will be installed in FY 2007. In addition to the wells sampled as part of the regular WAG 10 sampling, two wells, M7S and the RWMC production well, were sampled for the geochemical study as called for in the Groundwater Monitoring and Field Sampling Plan (DOE-ID 2006b). The analytical results for I-129 from the geochemical study sampling conducted in 2005 are presented and discussed in Appendix B. Also in Appendix B, the major ion data from the 2005 and 2006 Westbay and packer well sampling are discussed in relation to groundwater flow paths.

The data for field-measured parameters (including temperature, $\mathrm{pH}$, and conductivity) and approximate water level at the time of sampling are summarized in Table 3 . The dissolved oxygen probe was not working during the sampling event. Data for field parameters are included because abnormal $\mathrm{pH}$ (high or low) and high-conductivity values can be used as indicators of contamination. The conductivity

trends over time for select wells are shown in Figure 3. The recent rise in conductivity values for most wells could be related to the drop in water levels. 
Table 2. Well construction summary for Waste Area Group 10 wells.

\begin{tabular}{|c|c|c|c|c|}
\hline $\begin{array}{c}\text { Well } \\
\text { Identifier }^{\mathrm{a}} \\
\end{array}$ & Well Name & $\begin{array}{c}\text { Screened or } \\
\text { Open Hole } \\
(\mathrm{ft})\end{array}$ & $\begin{array}{l}\text { Pump Depth } \\
(\mathrm{ft})^{\mathrm{b}}\end{array}$ & $\begin{array}{c}\text { Approximate } \\
\text { Depth to Water } \\
\text { (ft) }\end{array}$ \\
\hline \multicolumn{5}{|c|}{ Boundary Wells } \\
\hline 450 & USGS-001 & 600 to 630 perforated & 612 & 588 \\
\hline 458 & USGS-009 & 620 to 650 perforated & 635 & 607 \\
\hline 535 & USGS-086 & 48 to 691 open hole & 678 & 649 \\
\hline 550 & USGS-101 & 750 to 865 perforated & 790 & 771 \\
\hline 552 & USGS-103 & 575 to 760 open hole & 700 & 583 \\
\hline 554 & USGS-105 & 400 to 800 open hole & 700 & 670 \\
\hline 557 & USGS-108 & 400 to 760 open hole & 637 & 609 \\
\hline 558 & USGS-109 & 600 to 800 open hole & 656 & 621 \\
\hline 559 & USGS-110 & 580 to 780 open hole & 612 & 566 \\
\hline \multicolumn{5}{|c|}{ Guard Wells } \\
\hline 184 & Highway 3 & 680 to 750 perforated & 567 & 538 \\
\hline 451 & USGS-002 & 675 to 696 perforated & 683 & 659 \\
\hline 549 & USGS-100 & 662 to 750 open hole & 703 & 686 \\
\hline 553 & USGS-104 & 550 to 700 open hole & 592 & 555 \\
\hline 555 & USGS-106 & 605 to 760 open hole & 609 & 584 \\
\hline 556 & USGS-107 & 270 to 690 open hole & 531 & 477 \\
\hline \multicolumn{5}{|c|}{ Baseline Wells } \\
\hline 453 & USGS-004 & $\begin{array}{l}285 \text { to } 315 \text { perforated } \\
322 \text { to } 553 \text { open hole }\end{array}$ & 303 & 251 \\
\hline 457 & USGS-008 & 782 to 812 perforated & 801 & 766 \\
\hline 468 & USGS-019 & 289 to 305 perforated & 323 & 276 \\
\hline 475 & USGS-026 & 232 to 266.5 perforated & 255 & 212 \\
\hline 476 & USGS-027 & $\begin{array}{l}250 \text { to } 260 \text { perforated } \\
298 \text { to } 308 \text { perforated }\end{array}$ & 262 & 228 \\
\hline 1346 & USGS-126B & 400 to 452 open hole & 420 & 408 \\
\hline 147 & DH-1B & 380 to 400 open hole & 320 & 268 \\
\hline 250 & $\mathrm{P} \& \mathrm{~W}-3$ & 322 to 401 perforated & 340 & 304 \\
\hline \multicolumn{5}{|c|}{ Distal Wells } \\
\hline 460 & USGS-011 & 672.5 to 703.8 perforated & 687 & $658^{\mathrm{c}}$ \\
\hline 463 & USGS-014 & 720 to 746 perforated & 739 & $720^{\mathrm{c}}$ \\
\hline 987 & USGS-124 & 750 to 800 slotted & Not available & $-^{\mathrm{c}}$ \\
\hline 988 & USGS-125 & 620 to 774 slotted & 700 & $634^{\mathrm{c}}$ \\
\hline
\end{tabular}


Table 2. (continued).

\begin{tabular}{|c|c|c|c|c|}
\hline $\begin{array}{c}\text { Well } \\
\text { Identifier }^{\mathrm{a}} \\
\end{array}$ & Well Name & $\begin{array}{c}\text { Screened or } \\
\text { Open Hole } \\
(\mathrm{ft})\end{array}$ & $\begin{array}{l}\text { Pump Depth } \\
(\mathrm{ft})^{\mathrm{b}}\end{array}$ & $\begin{array}{c}\text { Approximate } \\
\text { Depth to Water } \\
\text { (ft) }\end{array}$ \\
\hline \multicolumn{5}{|c|}{ Westbay Wells } \\
\hline 2050 & MIDDLE-2050A & Sampling port -474 & Not applicable & Not applicable \\
\hline 2050 & MIDDLE-2050A & Sampling port -647 & Not applicable & Not applicable \\
\hline 2050 & MIDDLE-2050A & Sampling port -790 & Not applicable & Not applicable \\
\hline 2050 & MIDDLE-2050A & Sampling port -1005 & Not applicable & Not applicable \\
\hline 2050 & MIDDLE-2050A & Sampling port -1212 & Not applicable & Not applicable \\
\hline 2051 & MIDDLE-2051 & Sampling port -608 & Not applicable & Not applicable \\
\hline 2051 & MIDDLE-2051 & Sampling port -748 & Not applicable & Not applicable \\
\hline 2051 & MIDDLE-2051 & Sampling port -834 & Not applicable & Not applicable \\
\hline 2051 & MIDDLE-2051 & Sampling port -1098 & Not applicable & Not applicable \\
\hline 2051 & MIDDLE-2051 & Sampling port -1148 & Not applicable & Not applicable \\
\hline
\end{tabular}

The primary radiological analytes detected in the boundary, guard, and distal wells included gross alpha, gross beta, and tritium (Table 4). These analytes were below their respective maximum contaminant levels (MCLs). The concentrations of gross alpha, gross beta, and uranium in the WAG 10 wells were similar to background, based on background values from Knobel, Orr, and Cecil (1992). Tritium was detected in two wells, USGS-104 and USGS-106, and both of these wells have a history of tritium detections (Figure 4). Over the past 20 years, both wells exhibit a downward trend in tritium concentration (Figure 4). The tritium concentrations in these wells currently are less than $1,100 \mathrm{pCi} / \mathrm{L}$ and considerably less than the MCL of $20,000 \mathrm{pCi} / \mathrm{L}$ (Table 4).

In the Westbay wells, tritium, gross alpha and gross beta were also the primary radiological analytes detected. Gross alpha and gross beta were at background concentrations (Tables 4 and 5). Tritium was detected in two intervals, 748 and $834 \mathrm{ft} \mathrm{bgs,} \mathrm{from} \mathrm{MIDDLE-2051} \mathrm{in} \mathrm{2005.} \mathrm{In} \mathrm{addition} \mathrm{to}$ being detected in the 748- and 834-ft intervals, tritium was detected in the 1,098- and 1,148-ft depth samples in the 2006 samples from MIDDLE-2051. Cross contamination due to the addition of drilling water should not be an issue since the water used for the Westbay wells was from the fire station well located upgradient of INTEC and RTC. Contaminants of interest in the fire station well are below detection limits or at background levels. Both Westbay wells were also sampled for uranium isotopes in the 2005 sampling event (Table 5). Uranium isotope concentrations were within the range for background. The $2005 \mathrm{Sr}-90$ detection in the deepest sample (1,148 ft) from MIDDLE-2051 was below the minimum detectable activity (MDA) and Sr-90 was not detected in the 2006 sample from this interval. Similarly, Sr-90 was detected in 2006 in the two deepest intervals from MIDDLE-2050A, but the reported Sr-90 concentrations were below the MDA. The occurrence of Sr-90 in these samples below its MDA is questionable. 


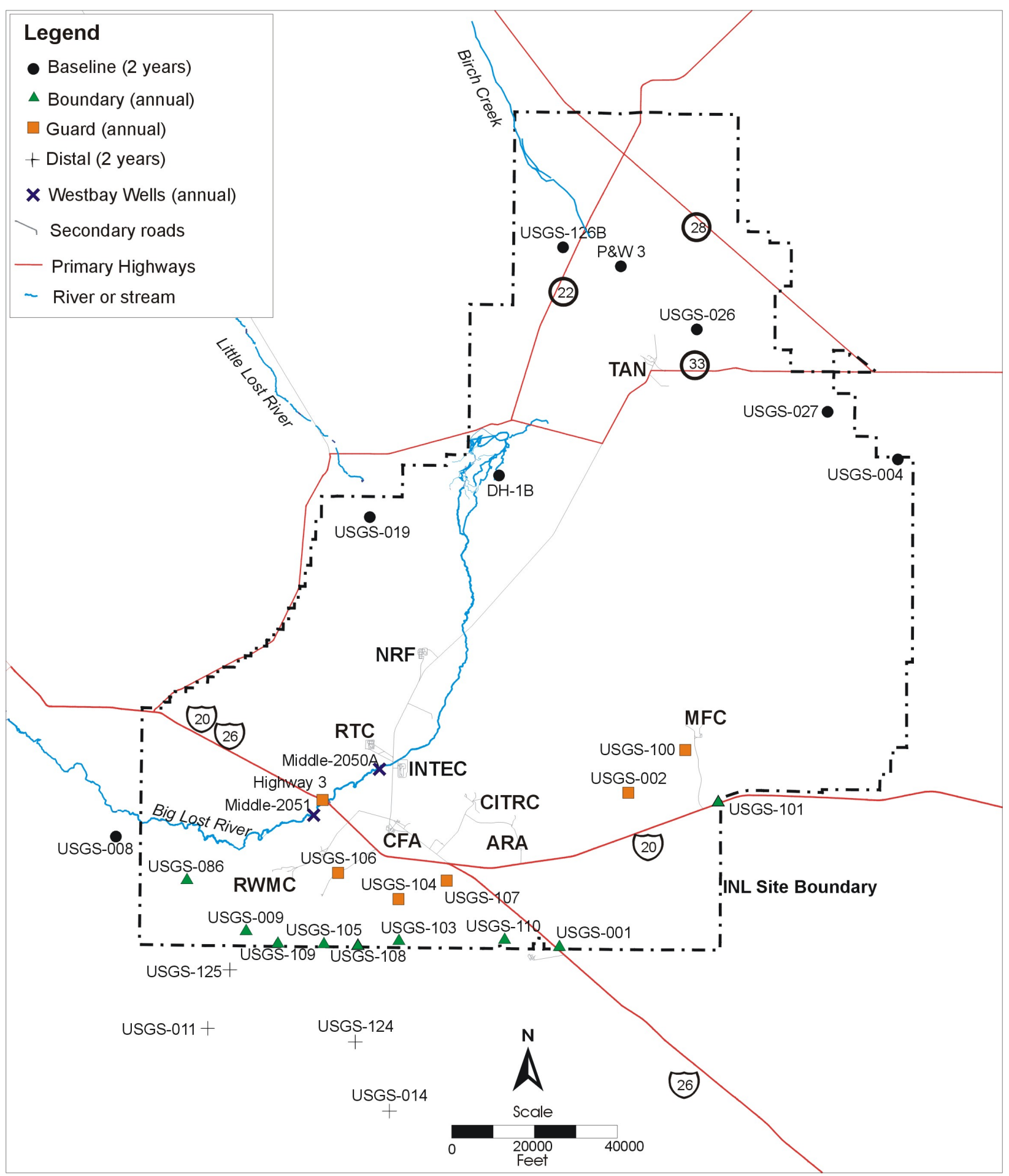

Figure 2. Location of boundary, guard, distal, and Westbay wells sampled in June and July 2006.

(NOTE: Additional wells were sampled for the geochemical study described in Appendix B. The baseline wells were not sampled in FY 2006, but will be sampled in FY 2007. The distal wells were sampled in FY 2006, but will not be sampled in FY 2007.) 
Table 3. Summary of field-measured parameters.

\begin{tabular}{|c|c|c|c|c|c|c|c|}
\hline Well Name & $\begin{array}{l}\text { Water } \\
\text { Level } \\
(\mathrm{ft} \mathrm{bgs})^{\mathrm{a}}\end{array}$ & $\begin{array}{c}\text { Date } \\
\text { Sampled } \\
\end{array}$ & $\begin{array}{c}\text { Time } \\
\text { Sampled } \\
\end{array}$ & $\begin{array}{l}\text { Temp. } \\
\left({ }^{\circ} \mathrm{C}\right)^{\mathrm{b}}\end{array}$ & $\mathrm{pH}$ & $\begin{array}{c}\text { Specific } \\
\text { Conductivity } \\
\text { (mmhos/cm) }\end{array}$ & $\begin{array}{c}\text { Dissolved } \\
\text { Oxygen } \\
(\mathrm{mg} / \mathrm{L})^{\mathrm{c}}\end{array}$ \\
\hline \multicolumn{8}{|l|}{ Boundary Wells } \\
\hline USGS-001 & 594.55 & $6 / 13 / 2006$ & 950 & 14.8 & $\mathrm{NF}^{\mathrm{d}}$ & 0.346 & $\mathrm{NF}$ \\
\hline USGS-009 & 608.3 & $6 / 20 / 2006$ & 1159 & 11.55 & 7.86 & 0.39 & $\mathrm{NF}$ \\
\hline USGS-086 & 656.05 & $6 / 27 / 2006$ & 1157 & 15.02 & 8.00 & 0.345 & $\mathrm{NF}$ \\
\hline USGS-101 & 778.49 & $6 / 15 / 2006$ & 922 & 13.84 & NF & 0.308 & NF \\
\hline USGS-103 & NS & NS & NS & NS & NS & NS & NS \\
\hline USGS-105 & 676 & $7 / 6 / 2006$ & 950 & NR & NR & NR & NR \\
\hline USGS-108 & 614.67 & $7 / 11 / 2006$ & 910 & 13.9 & 7.88 & 0.379 & $\mathrm{NF}$ \\
\hline USGS-109 & 626.79 & $6 / 20 / 2006$ & 1103 & 13.76 & NF & 0.403 & $\mathrm{NF}$ \\
\hline USGS-110 & 569.99 & $6 / 13 / 2006$ & 1222 & 16.73 & NF & 0.396 & $\mathrm{NF}$ \\
\hline \multicolumn{8}{|l|}{ Guard Wells } \\
\hline USGS-100 & 686.4 & $6 / 14 / 2006$ & 1020 & 13.44 & NF & 0.401 & $\mathrm{NF}$ \\
\hline Highway 3 & NA & $6 / 19 / 2006$ & 825 & 12.12 & NF & 0.369 & NF \\
\hline USGS-002 & 668.8 & $6 / 12 / 2006$ & 1317 & 13.6 & NF & 0.386 & $\mathrm{NF}$ \\
\hline USGS-104 & 562.06 & $6 / 19 / 2006$ & 1355 & 13.3 & NF & 0.351 & NF \\
\hline USGS-106 & 592.68 & $6 / 21 / 2006$ & 1216 & 14.49 & NF & 0.427 & $\mathrm{NF}$ \\
\hline USGS-107 & 486.32 & $6 / 21 / 2006$ & 956 & 15.32 & NF & 0.44 & NF \\
\hline \multicolumn{8}{|l|}{ Distal Wells } \\
\hline USGS-011 & 658.27 & $6 / 20 / 2006$ & 942 & 13.98 & NF & 0.371 & $\mathrm{NF}$ \\
\hline USGS-014 & 721.76 & $6 / 26 / 2006$ & 1246 & 15.77 & 8.14 & 0.415 & $\mathrm{NF}$ \\
\hline USGS-124 & 687.78 & $6 / 26 / 2006$ & 1330 & 14.48 & NF & 0.397 & $\mathrm{NF}$ \\
\hline USGS-125 & 633.19 & $6 / 27 / 2006$ & 938 & 13.02 & NF & 0.389 & $\mathrm{NF}$ \\
\hline \multicolumn{8}{|l|}{ Packer Samples } \\
\hline USGS-105 -769 & 764 & $7 / 5 / 2006$ & 914 & 13.61 & 6.78 & 0.393 & $\mathrm{NF}$ \\
\hline USGS-105 -676 & 676 & $7 / 5 / 2006$ & 1502 & 14.15 & 7.46 & 0.411 & $\mathrm{NF}$ \\
\hline \multicolumn{8}{|c|}{ No readings from USGS-108 } \\
\hline \multicolumn{8}{|c|}{$\begin{array}{l}\text { bgs }=\text { below ground surface } \\
\mathrm{NA}=\text { not available. } \\
\mathrm{NF}=\text { not functioning. } \\
\mathrm{NR}=\text { no readings. } \\
\mathrm{NS}=\text { not sampled. } \\
\text { USGS = United States Geological Survey. }\end{array}$} \\
\hline
\end{tabular}




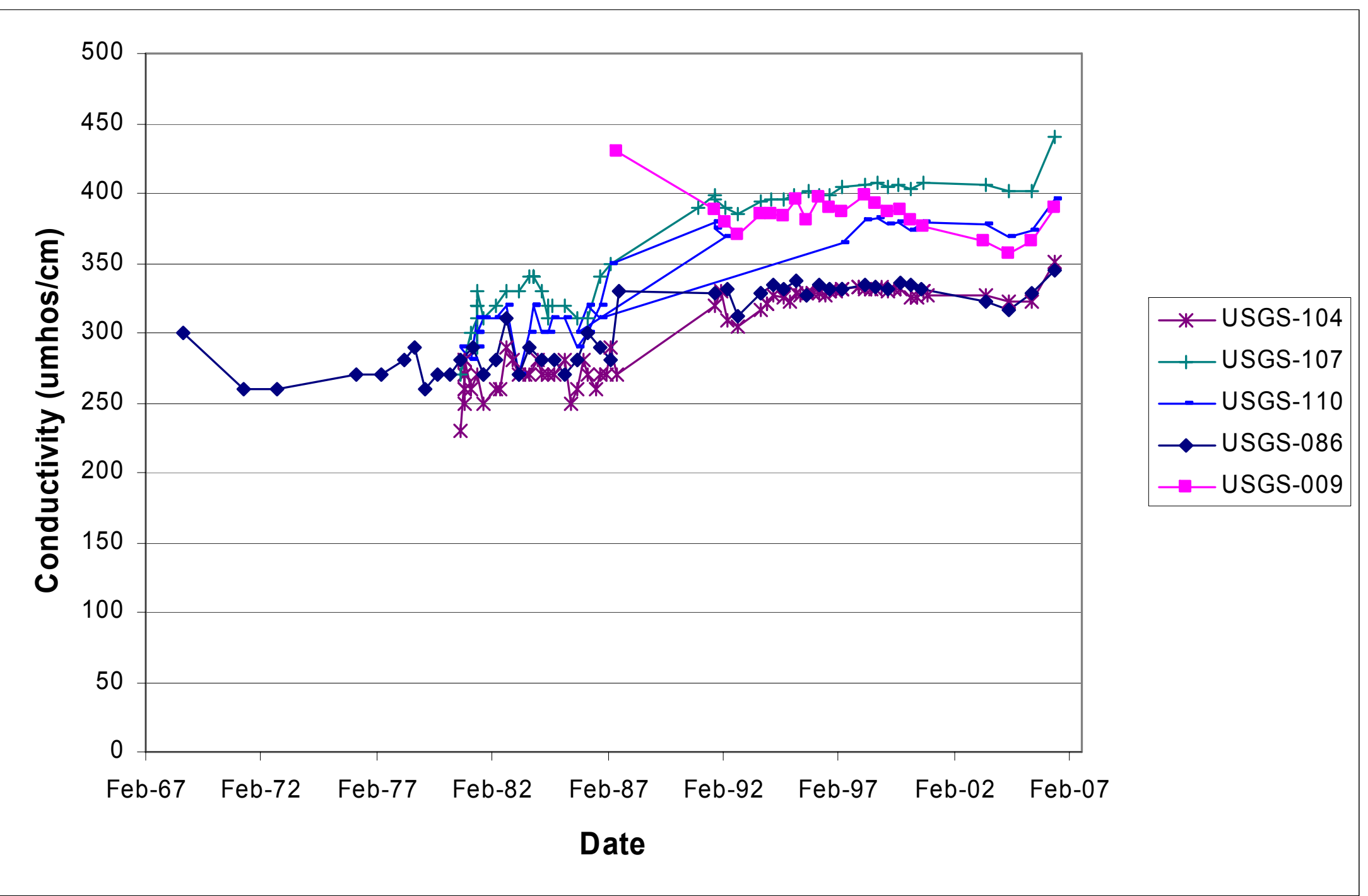

Figure 3. Plot of conductivity values for select wells. 
Table 4. Sampling results for gross alpha, gross beta, and tritium for 2006.

\begin{tabular}{|c|c|c|c|c|c|c|c|c|c|}
\hline \multirow[b]{2}{*}{ Well } & \multicolumn{3}{|c|}{$\begin{array}{c}\text { Gross Alpha } \\
(\mathrm{MCL}=15 \mathrm{pCi} / \mathrm{L})\end{array}$} & \multicolumn{3}{|c|}{$\begin{array}{c}\text { Gross Beta } \\
(\mathrm{MCL}=5 \mathrm{mrem} / \mathrm{yr})^{\mathrm{a}}\end{array}$} & \multicolumn{3}{|c|}{$\begin{array}{c}\text { Tritium } \\
(\mathrm{MCL}=20,000 \mathrm{pCi} / \mathrm{L})\end{array}$} \\
\hline & $\mathrm{pCi} / \mathrm{L}$ & $+/-$ & VF & $\mathrm{pCi} / \mathrm{L}$ & $+1-$ & VF & $\mathrm{pCi} / \mathrm{L}$ & $+/-$ & VF \\
\hline \multicolumn{10}{|l|}{ Distal Wells } \\
\hline USGS-011 & 2.31 & 0.463 & & 2.11 & 0.499 & & -83.2 & 118 & $\mathrm{U}$ \\
\hline USGS-014 & 2.32 & 0.618 & & 3.52 & 0.774 & & 121 & 108 & $\mathrm{U}$ \\
\hline USGS-124 & 2.86 & 0.665 & & 2.31 & 0.802 & UJ & 171 & 110 & $\mathrm{U}$ \\
\hline USGS-125 & 2.76 & 0.732 & & 1.84 & 0.78 & UJ & 99.2 & 108 & $\mathrm{U}$ \\
\hline \multicolumn{10}{|l|}{ Guard Wells } \\
\hline HIGHWAY 3 & 1.03 & 0.429 & UJ & 2.14 & 0.583 & & -85.2 & 121 & $\mathrm{U}$ \\
\hline USGS-002 & 2.81 & 0.725 & & 4.2 & 0.842 & & -43.6 & 125 & $\mathrm{U}$ \\
\hline USGS-100 & 1.93 & 0.584 & & 5.21 & 0.835 & & 0 & 113 & $\mathrm{U}$ \\
\hline USGS-104 & 1.11 & 0.312 & & 2.55 & 0.489 & & 1080 & 162 & \\
\hline USGS-106 & 1.76 & 0.438 & & 4.03 & 0.594 & & 580 & 141 & \\
\hline USGS-107 & 2.85 & 0.482 & & 3.4 & 0.58 & & -124 & 115 & $\mathrm{U}$ \\
\hline \multicolumn{10}{|l|}{ INL Boundary Wells } \\
\hline USGS-001 dup & 2.26 & 0.437 & & 3.51 & 0.505 & & $-261^{b}$ & 116 & $\mathrm{U}$ \\
\hline USGS-001 & 2.13 & 0.379 & & 3.71 & 0.559 & & -80.6 & 114 & $\mathrm{U}$ \\
\hline USGS-009 & 2.13 & 0.373 & & 2.59 & 0.54 & & -166 & 114 & $\mathrm{U}$ \\
\hline USGS-086 & 1.25 & 0.529 & UJ & 2.41 & 0.807 & UJ & 49.6 & 106 & $\mathrm{U}$ \\
\hline USGS-101 & 2.33 & 0.616 & & 1.97 & 0.799 & UJ & -216 & 117 & $\mathrm{U}$ \\
\hline USGS-105 & 2.23 & 0.678 & & 3.4 & 0.88 & & 320 & 115 & UJ \\
\hline USGS-108 & 1.62 & 0.507 & & 4.2 & 0.782 & & 262 & 113 & UJ \\
\hline USGS-109 & 1.54 & 0.303 & & 3.17 & 0.502 & & 41.8 & 123 & $\mathrm{U}$ \\
\hline USGS-110 & 2.21 & 0.375 & & 3.39 & 0.535 & & -43.4 & 125 & $\mathrm{U}$ \\
\hline \multicolumn{10}{|l|}{ Westbay Wells } \\
\hline \multicolumn{10}{|l|}{ MIDDLE-2050A } \\
\hline 474 & 1.83 & 0.333 & & 3.42 & 0.397 & & -140 & 112 & $\mathrm{U}$ \\
\hline 647 & 1.22 & 0.541 & UJ & 3.44 & 0.836 & & 18 & 117 & $\mathrm{U}$ \\
\hline 790 & 1.77 & 0.302 & & 5.5 & 0.423 & & -74.1 & 112 & $\mathrm{U}$ \\
\hline 1005 & 1.84 & 0.318 & & 3.91 & 0.397 & $\mathrm{~J}$ & -35.3 & 113 & $\mathrm{U}$ \\
\hline 1212 & 0.837 & 0.247 & $\mathrm{~J}$ & 5.72 & 0.429 & & -162 & 112 & $\mathrm{U}$ \\
\hline \multicolumn{10}{|l|}{ MIDDLE-2051 } \\
\hline 608 & 1.84 & 0.623 & UJ & 4.67 & 0.875 & & 7.72 & 112 & $\mathrm{U}$ \\
\hline 748 & 2.08 & 0.678 & & 2.22 & 0.833 & UJ & 449 & 123 & \\
\hline 834 & 1.92 & 0.688 & UJ & 3.03 & 0.904 & & 565 & 128 & \\
\hline 834 & 1.14 & 0.554 & UJ & 3.62 & 1.04 & & 576 & 126 & \\
\hline 1098 & 1.33 & 0.652 & UJ & 3.04 & 0.829 & & 428 & 125 & \\
\hline 1148 & 1.56 & 0.64 & UJ & 4.19 & 0.888 & & 587 & 123 & \\
\hline
\end{tabular}


Table 4. (continued).

\begin{tabular}{|c|c|c|c|c|c|c|c|c|c|c|}
\hline \multirow[b]{2}{*}{ Well } & & \multicolumn{3}{|c|}{$\begin{array}{c}\text { Gross Alpha } \\
(\mathrm{MCL}=15 \mathrm{pCi} / \mathrm{L})\end{array}$} & \multicolumn{3}{|c|}{$\begin{array}{c}\text { Gross Beta } \\
(\mathrm{MCL}=5 \mathrm{mrem} / \mathrm{yr})^{\mathrm{a}}\end{array}$} & \multicolumn{3}{|c|}{$\begin{array}{c}\text { Tritium } \\
(\mathrm{MCL}=20,000 \mathrm{pCi} / \mathrm{L})\end{array}$} \\
\hline & & $\mathrm{pCi} / \mathrm{L}$ & $+/-$ & VF & $\mathrm{pCi} / \mathrm{L}$ & $+/-$ & VF & $\mathrm{pCi} / \mathrm{L}$ & $+/-$ & $\mathrm{VF}$ \\
\hline \multicolumn{11}{|l|}{ Packer Sampling } \\
\hline \multicolumn{11}{|l|}{ USGS-105 } \\
\hline 704 & & 2.14 & 0.717 & UJ & 5.27 & 1.01 & & 329 & 116 & UJ \\
\hline 769 & & 1.5 & 0.568 & UJ & 4.68 & 0.868 & & 206 & 111 & $\mathrm{U}$ \\
\hline 769 & dup & 2.57 & 0.713 & & 6.61 & 0.986 & & 163 & 109 & $\mathrm{U}$ \\
\hline \multicolumn{11}{|l|}{ USGS-108 } \\
\hline 627 & & 2.3 & 0.273 & & 2.64 & 0.372 & & 0 & 104 & $\mathrm{U}$ \\
\hline $657-760$ & & 1.38 & 0.546 & UJ & 3.45 & 0.852 & & 7.52 & 106 & $\mathrm{U}$ \\
\hline
\end{tabular}

a. The MCL for gross beta is based on an exposure criterion of $5 \mathrm{mrem} / \mathrm{yr}$.

b. The tritium result is an outlier for the analytical method. If the lab gets negative numbers on a regular basis, then the lab is required to investigate and determine a new zero.

Dup $=$ duplicate sample.

MCL = maximum contaminant level.

USGS $=$ United States Geological Survey

$\mathrm{VF}=$ validation flag. See Appendix A for data flag definitions.

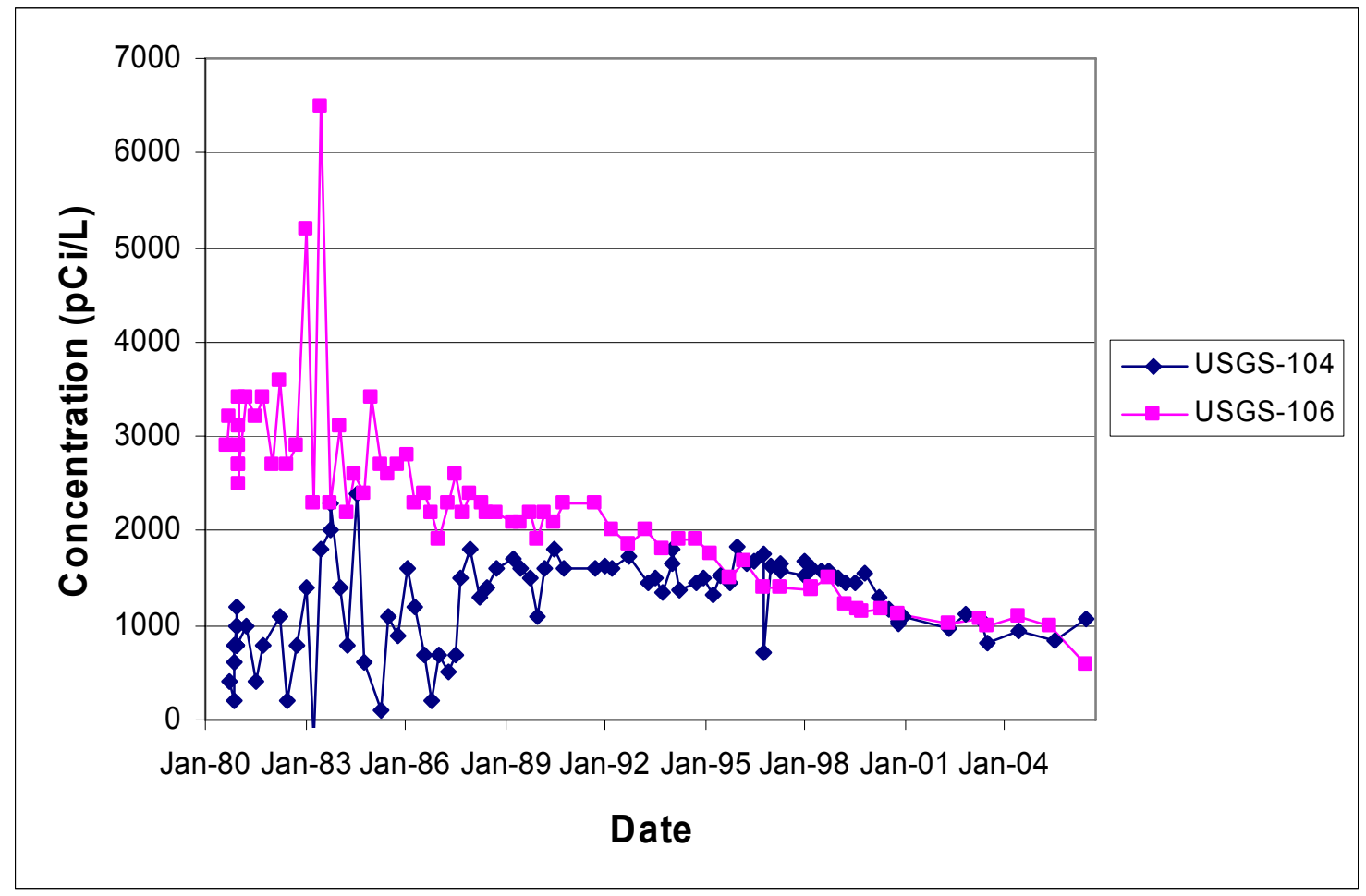

Figure 4. Tritium concentration trends for USGS-104 and USGS-106. 
Table 5. Sampling results for tritium, gross alpha, gross beta, uranium, and uranium isotopes for Westbay wells for September-October 2005.

\begin{tabular}{|c|c|c|c|c|c|c|c|c|c|c|c|c|c|}
\hline \multirow[b]{2}{*}{ Location } & \multirow[b]{2}{*}{ Depth } & \multicolumn{3}{|c|}{ Gross Alpha } & \multicolumn{3}{|c|}{ Gross Beta } & \multicolumn{3}{|c|}{ Tritium } & & & \\
\hline & & $\mathrm{pCi} / \mathrm{L}$ & $+/-$ & VF & $\mathrm{pCi} / \mathrm{L}$ & $+/-$ & VF & $\mathrm{pCi} / \mathrm{L}$ & $+/-$ & $\mathrm{VF}$ & & & \\
\hline MIDDLE-2050A & 474 & 0.402 & 0.6 & $\mathrm{U}$ & 3.4 & 0.953 & & 310 & 104 & UJ & & & \\
\hline MIDDLE-2050A & 647 & 3.17 & 1.06 & UJ & 2.13 & 1.06 & UJ & 230 & 86.3 & UJ & & & \\
\hline MIDDLE-2050A & 790 & 1.91 & 0.533 & & 3 & 0.797 & & 223 & 84.8 & UJ & & & \\
\hline MIDDLE-2050A & 1005 & 0.772 & 0.781 & $\mathrm{U}$ & -0.732 & 0.93 & $\mathrm{U}$ & -8.57 & 114 & $\mathrm{U}$ & & & \\
\hline MIDDLE-2050A & 1005 & 1.89 & 0.586 & & 1.7 & 0.846 & UJ & -86.6 & 111 & $\mathrm{U}$ & & & \\
\hline MIDDLE-2050A & 1212 & 2.1 & 0.726 & UJ & 5.12 & 1.04 & & -27.8 & 78.5 & $\mathrm{U}$ & & & \\
\hline MIDDLE-2051 & 608 & 1.75 & 0.651 & $\mathrm{~J}$ & 3.37 & 1.06 & $\mathrm{~J}$ & 68 & 116 & $\mathrm{U}$ & & & \\
\hline MIDDLE-2051 & 748 & 1.41 & 0.603 & UJ & 0.562 & 0.947 & $\mathrm{U}$ & 632 & 136 & & & & \\
\hline MIDDLE-2051 & 834 & 3.01 & 0.81 & & 2.41 & 0.986 & UJ & 745 & 141 & & & & \\
\hline MIDDLE-2051 & 1098 & 0.503 & 0.593 & $\mathrm{U}$ & 2.41 & 0.938 & UJ & 123 & 116 & $\mathrm{U}$ & & & \\
\hline MIDDLE-2051 & 1148 & 1.21 & 0.612 & $\mathrm{U}$ & 3.9 & 0.995 & & 172 & 120 & $\mathrm{U}$ & & & \\
\hline & & Ura & $m-233 / 2$ & & Ura & iium-235 & & & ium-238 & & & iium & \\
\hline Location & Depth & $\mathrm{pCi} / \mathrm{L}$ & $+/-$ & $\mathrm{VF}$ & $\mathrm{pCi} / \mathrm{L}$ & $+/-$ & VF & $\mathrm{pCi} / \mathrm{L}$ & $+/-$ & $\mathrm{VF}$ & $\mathrm{ug} / \mathrm{L}$ & LF & VF \\
\hline MIDDLE-2050A & 474 & 1.25 & 0.141 & & 0.131 & 0.0424 & & 0.644 & 0.0931 & & 1.8 & $\mathrm{~B}$ & \\
\hline MIDDLE-2050A & 647 & 2.04 & 0.252 & & 0.136 & 0.0566 & $\mathrm{~J}$ & 0.946 & 0.152 & & 2.3 & $\mathrm{~B}$ & \\
\hline MIDDLE-2050A & 790 & 1.41 & 0.163 & & 0.106 & 0.0439 & UJ & 0.682 & 0.103 & & 1.4 & $\mathrm{~B}$ & \\
\hline MIDDLE-2050A & 1005 & 1.17 & 0.111 & & 0.0402 & 0.0207 & $\mathrm{U}$ & 0.447 & 0.0605 & & 2 & $\mathrm{~B}$ & $\mathrm{~J}$ \\
\hline MIDDLE-2050A & 1005 & 1.36 & 0.115 & & 0.0904 & 0.0256 & & 0.515 & 0.0613 & & 1.8 & $\mathrm{~B}$ & $\mathrm{~J}$ \\
\hline MIDDLE-2050A & 1212 & 0.96 & 0.13 & & 0.113 & 0.0433 & $\mathrm{~J}$ & 0.43 & 0.0818 & & 2.1 & $\mathrm{~B}$ & \\
\hline MIDDLE-2051 & 608 & 0.964 & 0.0816 & & 0.0397 & 0.0167 & $\mathrm{UJ}$ & 0.572 & 0.0583 & & 2.3 & $\mathrm{~B}$ & \\
\hline MIDDLE-2051 & 748 & 1.41 & 0.161 & & 0.0952 & 0.0395 & $\mathrm{~J}$ & 0.638 & 0.0969 & & 2 & $\mathrm{~B}$ & \\
\hline MIDDLE-2051 & 834 & 1.49 & 0.15 & & 0.0426 & 0.023 & $\mathrm{U}$ & 0.566 & 0.0803 & & 1.8 & $\mathrm{~B}$ & $\mathrm{~J}$ \\
\hline MIDDLE-2051 & 1098 & 1.27 & 0.159 & & 0.00466 & 0.018 & $\mathrm{U}$ & 0.508 & 0.0904 & & 2.1 & $\mathrm{~B}$ & $\mathrm{~J}$ \\
\hline MIDDLE-2051 & 1148 & 1.66 & 0.172 & & 0.0525 & 0.0265 & & 0.473 & 0.0777 & $\mathrm{U}$ & 2.1 & $\mathrm{~B}$ & $\mathrm{~J}$ \\
\hline
\end{tabular}


Four VOCs, acetone, chloromethane, toluene, and carbon tetrachloride, were detected at concentrations well below MCLs. Toluene was detected in samples from two wells at concentrations of $2.9 \mu \mathrm{g} / \mathrm{L}$ (USGS-108) and $4.8 \mu \mathrm{g} / \mathrm{L}$ (USGS-105). Toluene was also detected in packer samples from USGS-108 (627 ft, $0.25 \mu \mathrm{g} / \mathrm{L})$ and USGS-105 (769 ft, $2 \mu \mathrm{g} / \mathrm{L})$. All the toluene detections were below the MCL for toluene of $1,000 \mu \mathrm{g} / \mathrm{L}$. The source of the toluene is uncertain, but the lack of other hydrocarbons at the locations with the toluene detections is not consistent with fuel migration. Toluene is a common laboratory contaminant and that source cannot be ruled out. Carbon tetrachloride was detected at 0.15 $\mu \mathrm{g} / \mathrm{L}$ in USGS-109, located directly south of the RWMC on the INL boundary. The carbon tetrachloride concentration in USGS-109 is an estimated value or J flagged and is close to the method detection limit. A carbon tetrachloride plume originates at the RWMC and this carbon tetrachloride detection could represent migration from the RWMC. Chloromethane was detected in the deepest sample from MIDDLE-2051, but the concentration was near a detection limit. Acetone was detected in three samples at very low concentrations (less than $1.5 \mu \mathrm{g} / \mathrm{L}$ ) from the 2005 Westbay sampling, but it was not detected in the 2006 sampling event. Like toluene, acetone is a common laboratory contaminant.

The data for anions, common cations, and metals are summarized in Table 6 and are compared to MCLs or secondary MCLs. Data are also compared to U.S. Geological Survey background values for the INL Site (USGS 1999). Review of the WAG 10 boundary, guard, and distal wells indicates that no inorganics are above their respective MCLs or secondary MCLs.

In the Westbay wells, only manganese was above its secondary MCL of $50 \mu \mathrm{g} / \mathrm{L}$. The elevated manganese concentrations occurred in three samples with a maximum concentration of $424 \mu \mathrm{g} / \mathrm{L}$ in the deepest sample from MIDDLE-2051. However, the elevated manganese detections did not occur at the same sample depths in the 2005 and 2006 sampling events. The inconsistent manganese detections above the secondary MCL make the occurrences suspect and they are not traceable back to any known source at INL.

Although not above its secondary MCL, zinc concentrations in the groundwater samples from USGS-011, USGS-086, USGS-100, USGS-103, USGS-104, USGS-106, USGS-108, USGS-109, and the Highway 3 well were elevated (Figure 5). The elevated zinc concentrations in these groundwater monitoring wells are probably the result of corroding galvanized discharge/riser pipe used in their construction. Elevated zinc concentrations in groundwater have been correlated to galvanized riser pipes for other wells at the INL Site (INEEL 2003; ICP 2004).

\subsection{Geochemical Study Results}

Although the geochemical study was conducted primarily in FY 2005, the I-129 data are incorporated into the FY 2006 annual monitoring report because it was not received in time to be incorporated into the FY 2005 report. Sampling for the geochemical study described in the OU 10-08 Groundwater Monitoring and Field Sampling Plan (DOE-ID 2005) was completed in April-July 2005. In May-June 2006, two additional I-129 samples were collected to fill in data gaps. The I-129 samples were collected at the RWMC production well and M7S. The goals of the geochemical study were to resolve the source of the tritium in the aquifer at the RWMC, identify flow paths of contaminants from INTEC and the RTC, determine the source of the anion anomaly south of the RWMC, and identify flow paths and evaluate contaminant influence south of the southern INL Site boundary. Identification of groundwater flow paths is essential for development and calibration of the OU 10-08 (i.e., Sitewide) groundwater model. In addition, data from the geochemical study are used to evaluate the potential for commingled plumes, which might elevate the cumulative risk above levels calculated for each plume individually. 
Table 6. Summary of inorganic results.

\begin{tabular}{|c|c|c|c|c|c|c|c|c|c|c|c|c|c|}
\hline \multirow[b]{2}{*}{ Compound } & \multirow[b]{2}{*}{$\begin{array}{c}\text { Sample } \\
\text { Units }\end{array}$} & \multicolumn{2}{|c|}{ Guard } & \multicolumn{2}{|c|}{ Boundary $^{\mathrm{a}}$} & \multicolumn{2}{|c|}{ Distal } & \multicolumn{2}{|c|}{ Westbay $^{\mathrm{b}}$} & \multirow[b]{2}{*}{$\begin{array}{l}\text { MCL or } \\
\text { SMCL }^{c}\end{array}$} & \multirow[b]{2}{*}{ Background $^{\mathrm{d}}$} & \multirow{2}{*}{$\begin{array}{l}\text { Detections } \\
\text { above } \\
\text { Background }\end{array}$} & \multirow{2}{*}{$\begin{array}{c}\text { Detections } \\
\text { above } \\
\text { MCL or } \\
\text { SMCL }\end{array}$} \\
\hline & & $\operatorname{Max}$ & Min & $\operatorname{Max}$ & Min & Max & Min & Max & Min & & & & \\
\hline \multicolumn{14}{|l|}{ Anions } \\
\hline Alkalinity & $\mathrm{mg} / \mathrm{L}$ & 158 & 122 & 204 & 104 & 144 & 136 & 182 & 140 & None & $169-174$ & Yes & NA \\
\hline Chloride & $\mathrm{mg} / \mathrm{L}$ & 23.7 & 7.4 & 19.2 & 10.7 & 23.1 & 10.2 & 17.1 & 6.7 & 250 & $16-27$ & No & 0 \\
\hline Fluoride & $\mathrm{mg} / \mathrm{L}$ & 0.66 & 0.17 & 0.86 & 0.17 & 0.92 & 0.25 & 0.23 & 0.11 & 2 & $0.3-0.5$ & Yes & 0 \\
\hline Nitrate/Nitrite as $\mathrm{N}$ & $\mathrm{mg} / \mathrm{L}$ & 1,880 & 937 & 1,570 & 671 & 1,180 & 584 & 1.1 & $\mathrm{U}$ & 10 & 1 to 2 & No & 0 \\
\hline Sulfate & $\mathrm{mg} / \mathrm{L}$ & 25.7 & 16.1 & 28 & 9.3 & 24.6 & 20.7 & 27.7 & 17.4 & 250 & $24-31$ & No & 0 \\
\hline \multicolumn{14}{|l|}{ Common Cations } \\
\hline Calcium & $\mu \mathrm{g} / \mathrm{L}$ & 42,900 & 31,600 & 37,500 & 27,600 & 38,400 & 33,400 & 57,300 & 33,700 & None & $43,000-46,000$ & Yes & NA \\
\hline Magnesium & $\mu \mathrm{g} / \mathrm{L}$ & 15,800 & 11,000 & 15,000 & 8,820 & 13,800 & 12,900 & 19,600 & 11,200 & None & 15,000 & Yes & NA \\
\hline Potassium & $\mu \mathrm{g} / \mathrm{L}$ & 3,210 & 2,170 & 3,120 & 2,300 & 2,450 & 2,100 & 3,050 & 1,630 & None & $3,100-3,500$ & No & NA \\
\hline Sodium & $\mu \mathrm{g} / \mathrm{L}$ & 16,700 & 5,630 & 18,000 & 10,000 & 15,400 & 7,800 & 13,100 & 5,820 & None & $14,000-17,000$ & Yes & NA \\
\hline \multicolumn{14}{|l|}{ Metals } \\
\hline Aluminum & $\mu \mathrm{g} / \mathrm{L}$ & 11 & $\mathrm{U}$ & 39.3 & $\mathrm{U}$ & $\mathrm{U}$ & $\mathrm{U}$ & 27.6 & $\mathrm{U}$ & 50 to 200 & $10-13$ & Yes & 0 \\
\hline Antimony & $\mu \mathrm{g} / \mathrm{L}$ & 0.54 & $\mathrm{U}$ & 0.66 & U & U & $\mathrm{U}$ & 0.59 & U & 6 & - & - & 0 \\
\hline Arsenic & $\mu \mathrm{g} / \mathrm{L}$ & 2.1 & $\mathrm{U}$ & 2.8 & $\mathrm{U}$ & $\mathrm{U}$ & $\mathrm{U}$ & $\mathrm{U}$ & $\mathrm{U}$ & 10 & 2 to 3 & No & 0 \\
\hline Barium & $\mu \mathrm{g} / \mathrm{L}$ & 58.1 & 32.3 & 39.7 & 16.7 & 53.3 & 19.9 & 116 & 35.3 & 2,000 & 50 to 70 & Yes & 0 \\
\hline Beryllium & $\mu \mathrm{g} / \mathrm{L}$ & $\mathrm{U}$ & $\mathrm{U}$ & $\mathrm{U}$ & $\mathrm{U}$ & $\mathrm{U}$ & $\mathrm{U}$ & $\mathrm{U}$ & $\mathrm{U}$ & 4 & $\mathrm{~N}$ & No & 0 \\
\hline Cadmium & $\mu \mathrm{g} / \mathrm{L}$ & 0.43 & $\mathrm{U}$ & 0.13 & $\mathrm{U}$ & $\mathrm{U}$ & $\mathrm{U}$ & 1.4 & $\mathrm{U}$ & 5 & $<1$ & No & 0 \\
\hline Chromium & $\mu \mathrm{g} / \mathrm{L}$ & 8 & $\mathrm{U}$ & 13.4 & $\mathrm{U}$ & 5.9 & 3.8 & 40 & U & 100 & 2 to 3 & Yes & 0 \\
\hline Cobalt & $\mu \mathrm{g} / \mathrm{L}$ & $\mathrm{U}$ & $\mathrm{U}$ & $\mathrm{U}$ & $\mathrm{U}$ & $\mathrm{U}$ & $\mathrm{U}$ & 4.3 & $\mathrm{U}$ & None & 3 & No & NA \\
\hline Copper & $\mu \mathrm{g} / \mathrm{L}$ & 3.5 & 0.42 & 2 & 0.34 & 0.9 & 0.33 & 3.8 & 0.38 & $1,300 / 1,000$ & $<1$ & No & 0 \\
\hline Iron & $\mu \mathrm{g} / \mathrm{L}$ & 9.4 & $\mathrm{U}$ & 105 & $\mathrm{U}$ & 9.4 & $\mathrm{U}$ & 209 & $\mathrm{U}$ & 300 & $16-25$ & Yes & 0 \\
\hline Lead & $\mu \mathrm{g} / \mathrm{L}$ & 12.2 & $\mathrm{U}$ & 2.2 & $\mathrm{U}$ & 0.9 & $\mathrm{U}$ & $\mathrm{U}$ & $\mathrm{U}$ & $15^{\mathrm{e}}$ & 1 to 5 & Yes & 0 \\
\hline Manganese & $\mu \mathrm{g} / \mathrm{L}$ & 1.6 & $\mathrm{U}$ & 9 & $\mathrm{U}$ & 7.9 & 0.9 & 424 & U & 50 & 7 & Yes & $3^{\mathrm{f}}$ \\
\hline Mercury & $\mu \mathrm{g} / \mathrm{L}$ & $\mathrm{U}$ & $\mathrm{U}$ & 0.17 & $\mathrm{U}$ & U & $\mathrm{U}$ & 0.14 & U & 2 & $\mathrm{~N}$ & No & 0 \\
\hline
\end{tabular}


Table 6. (continued).

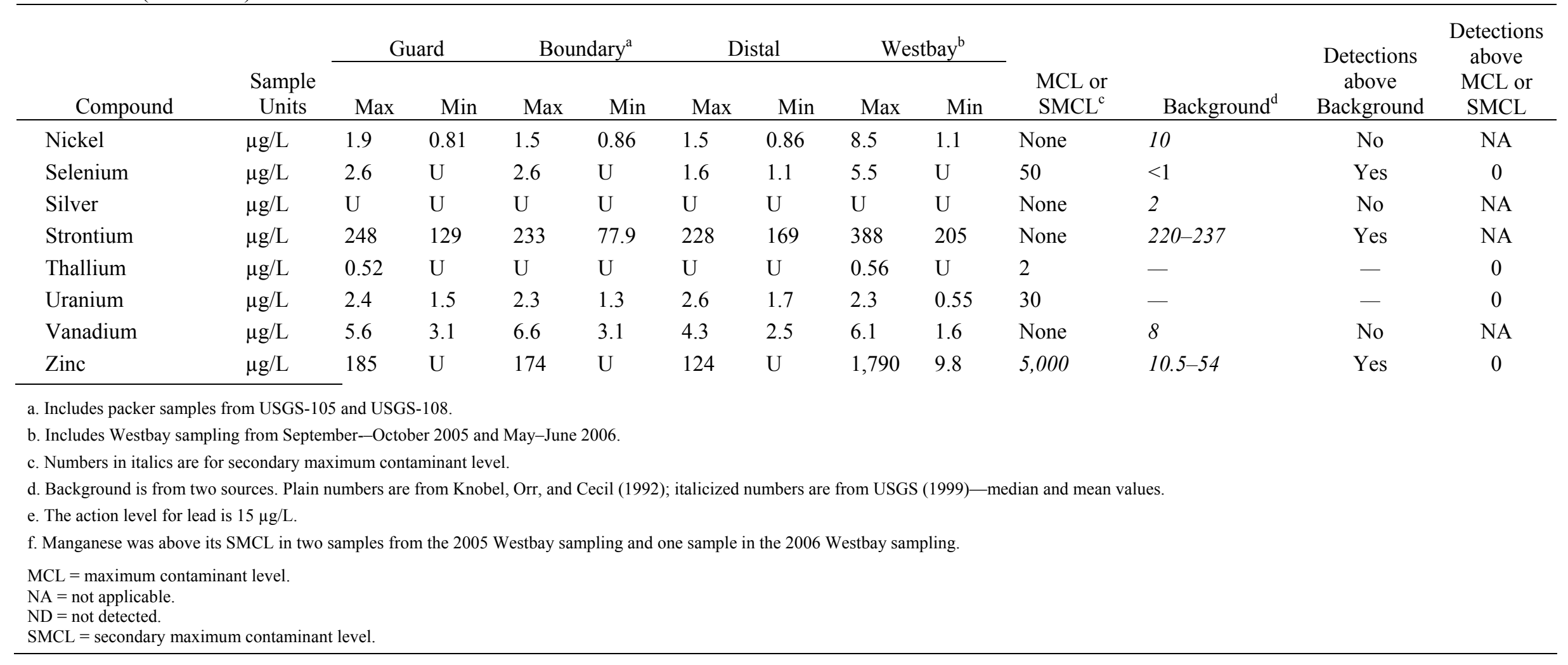




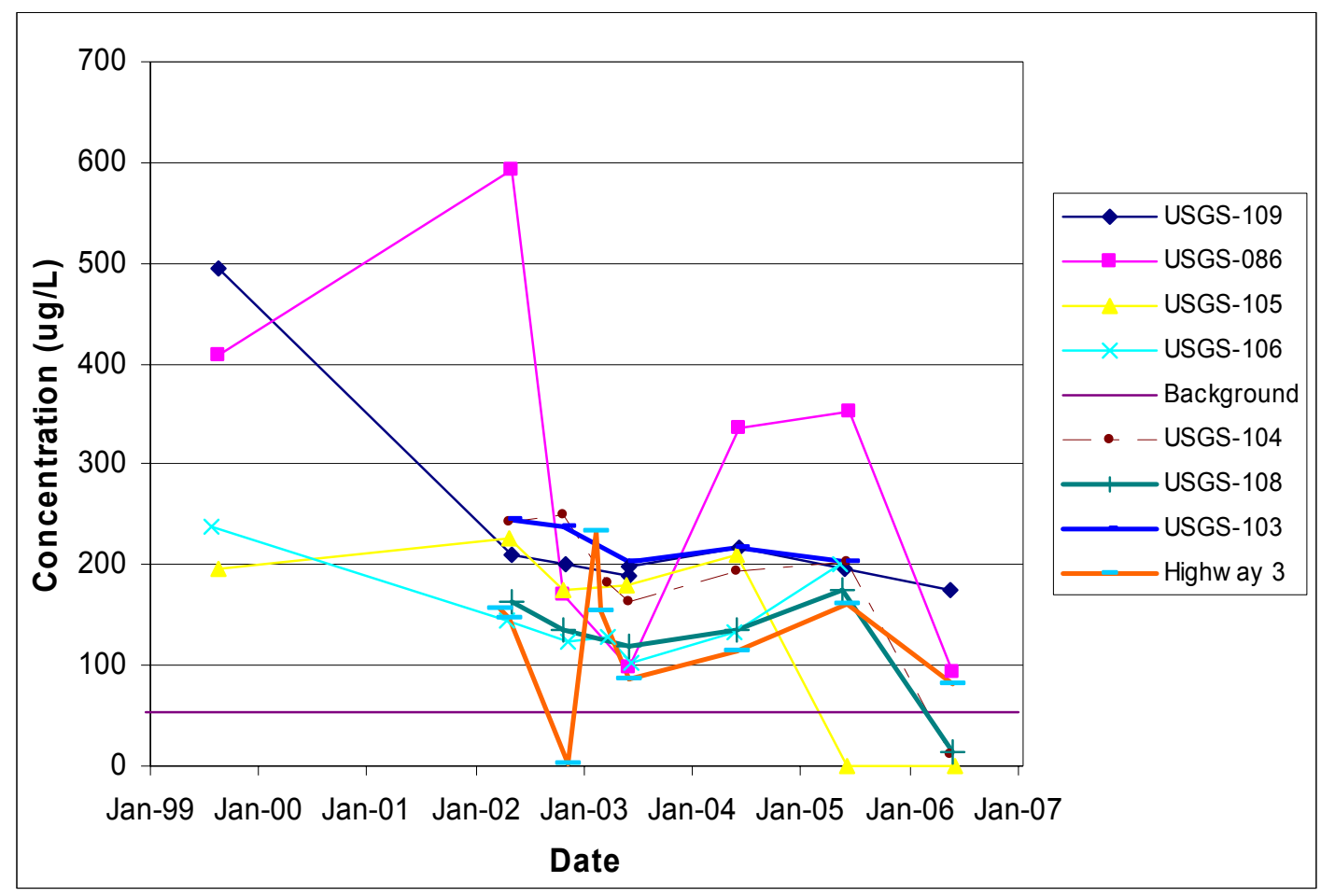

Figure 5. Zinc concentrations at select wells that have galvanized riser pipes.

The data collected for geochemical study and discussion of groundwater flow paths are presented in Appendix B.

\subsection{Well Drilling}

The following tasks were completed in FY 2006:

- $\quad$ Prepared an end-of-well report for the deep core holes and Westbay installations (RPT-178 [ICP 2006]).

- $\quad$ Purchased Westbay multilevel groundwater monitoring systems for USGS-132 and USGS-134. The Westbay systems were installed by the USGS in both wells.

\subsection{Sitewide Water-Level Measurements}

Water levels are one of the most frequently used calibration targets for groundwater models and a critical data need is accurate water table maps. In April and May 2006, an INL Sitewide automated water-level measurement network was installed. The water-level measurement data from the automated network will be presented in the FY 2007 annual report. The water-level monitoring network may be supplemented by water-level measurements from individual WAGs and the USGS's National Water Information Storage (NWIS) database (USGS 2006). Water levels, as determined from pressure readings, were measured for the newly installed Westbay wells and are discussed below. 


\subsubsection{Selection of Well Locations and Installation for Automated Sitewide Water-Level Network}

Wells were selected for a Sitewide automated water-level monitoring network to provide data to evaluate water-level changes across the entire INL and to monitor the water-level response from flow in the Big Lost River (BLR). Table A-2 in the OU 10-08 Groundwater Monitoring and Field Sampling Plan (DOE-ID 2005) was used as a starting point for well selection. This table identified 62 wells that were prioritized for water-level measurements based upon their location and completion characteristics. This list of wells was compared with wells either currently being continuously monitored or planned for continuous monitoring by other organizations and agencies (e.g., WAG 3 and the USGS). This list also was compared with wells identified by the groundwater modeling team as being important for model calibration. The density of priority wells was reduced in areas where a significant number of monitoring wells have been identified in the OU 10-08 Groundwater Monitoring Plan and/or other organizations or agencies that are collecting data. Wells were added in areas where coverage was deemed to be sparse or where a particular well may be needed to control contour extrapolation of water table maps.

Some of the locations proposed in the Groundwater Monitoring Plan (DOE-ID 2006b) could not be used because of well construction or access issues (Table 7). The well locations used for the Sitewide network are shown on Figure 6 and listed in Table 8. Most of the wells selected are "skimmer" wells, or wells that are completed with a small open interval across the water table. Several wells with deeper completions were also selected to evaluate vertical gradients for the three-dimensional (3-D) evaluation of the aquifer response.

The data logger selected for the Sitewide measurement system was the Solinist Levelogger model 3001. The sampling interval was preprogrammed to collect data every 15 minutes for the first 180 days, and then once an hour for the next 100 weeks. The leveloggers were placed inside the water-level access, suspended with a 1/16-in. stainless steel cable. Field installation of leveloggers in 49 wells was performed from April 21, 2006, to May 9, 2006, with three more installed in June. In addition to leveloggers, three wells, ANP-06, USGS-008, and USGS-101, were equipped with barologgers to monitor barometric pressure changes.

Table 7. Proposed wells that were not used, with the corresponding replacement well or explanation for non-use.

\begin{tabular}{ll}
\hline Original Well Not Instrumented & \multicolumn{1}{c}{ Replacement Well/Explanation } \\
\hline CFA-MON-A-001 & CFA-MON-A-002 \\
LF2-08 & CFA-1931 \\
USGS-006 & Water-level access pipe diameter \\
USGS-083 & USGS-104 \\
USGS-084 & Water-level access pipe diameter \\
USGS-089 & Water-level access line shorter than length \\
USGS-097 & Water-level access pipe diameter \\
USGS-098 & USGS-079 \\
USGS-103 & Well to be deepened by the USGS \\
USGS-105 & USGS-109 \\
USGS-117 & Logger stuck above set elevation \\
USGS-122 & Bottomed out. Line is capped or dry. \\
\hline USGS = United States Geological Survey. & \\
\hline
\end{tabular}




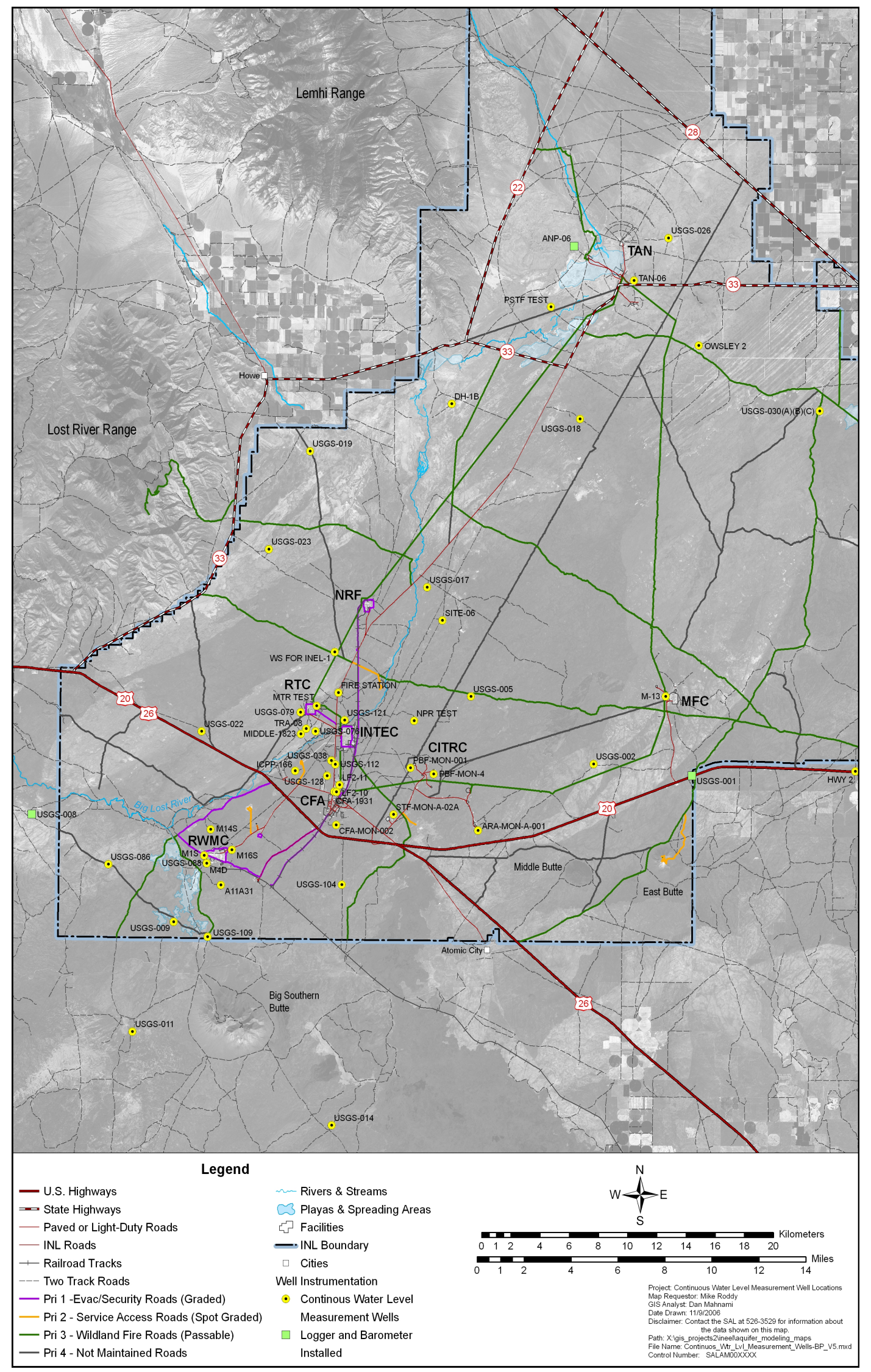

Figure 6. Well locations for the automated Sitewide water-level monitoring network. 
Table 8. Wells used for automated Sitewide water-level monitoring network.

\begin{tabular}{|c|c|c|c|c|c|}
\hline Well Name & Well ID & $\begin{array}{c}\text { Corrected } \\
\text { June } 2005 \\
\text { Depth to Water } \\
\text { (ft bgs) }\end{array}$ & $\begin{array}{c}\text { Land Surface } \\
\text { Datum } \\
(\mathrm{ft} \mathrm{msl})\end{array}$ & $\begin{array}{c}\text { Water Table } \\
\text { Elevation } \\
(\mathrm{ft} \mathrm{msl})^{\mathrm{a}}\end{array}$ & $\begin{array}{l}\text { Completion } \\
\text { Depth }\end{array}$ \\
\hline ANL-MON-A-013 & 1075 & 646.26 & 5120.37 & 4474.11 & 662 \\
\hline ANP-6 & 74 & 225.95 & 4797.49 & 4571.54 & 305 \\
\hline ARA-MON-A-001 & 1003 & 596.00 & 5034.30 & 4438.30 & 640 \\
\hline CFA-MON-A-002 & 1078 & 491.78 & 4932.24 & 4440.46 & 518 \\
\hline CFA-1931 & 1931 & 485.47 & 4931.25 & 4445.78 & 520 \\
\hline DH1B & 147 & 284.24 & 4792.33 & 4508.09 & 380 \\
\hline FIRE STATION WELL & 158 & 438.50 & 4901.13 & 4462.63 & 516 \\
\hline HWY-2 & 183 & 734.95 & 5216.55 & 4481.60 & 786 \\
\hline ICPP-MON-A-166 & 1352 & 509.52 & 4956.00 & 4446.48 & 527 \\
\hline LF 2-11 & 199 & 481.96 & 4928.36 & 4446.40 & 499 \\
\hline LF2-10 & 198 & 488.04 & 4932.48 & 4444.44 & 766 \\
\hline M1S & 765 & 590.58 & 5011.09 & 4420.51 & 638 \\
\hline M4D & 767 & 600.85 & 5022.53 & 4421.68 & 838 \\
\hline Middle-1823 & 1823 & 492.62 & 4939.36 & 4446.74 & 720 \\
\hline MTR-TEST & 231 & 469.40 & 4917.15 & 4447.75 & 588 \\
\hline NPR-TEST & 239 & 472.68 & 4933.15 & 4460.46 & 600 \\
\hline OWSLEY-2 & 247 & 234.30 & 4786.87 & 4552.57 & 310 \\
\hline PBF-MON-A-001 & 1085 & 451.72 & 4906.15 & 4454.43 & 489 \\
\hline PBF-MON-A-004 & 1094 & 500.29 & 4939.66 & 4439.36 & $522-542$ \\
\hline PSTF-TEST & 256 & 219.15 & 4786.37 & 4567.22 & 319 \\
\hline $\begin{array}{l}\text { RWMC-MON-A-013 } \\
\text { (A11A31) }\end{array}$ & 906 & 646.67 & 5065.40 & 4418.73 & 675 \\
\hline SITE-06 & 274 & 369.69 & 4836.17 & 4466.48 & 523 \\
\hline $\begin{array}{l}\text { SOUTH-MON-A-004 } \\
\text { (M14S) }\end{array}$ & 1215 & 610.15 & 5032.46 & 4422.31 & 645 \\
\hline $\begin{array}{l}\text { SOUTH-MON-A-010 } \\
(\mathrm{M} 16 \mathrm{~S})\end{array}$ & 1339 & 582.94 & 5004.34 & 4421.40 & 578 \\
\hline STF-MON-A-02A & 999 & 502.39 & 4937.30 & 4434.91 & 530 \\
\hline TAN-06 & 746 & 218.60 & 4786.87 & 4568.27 & 261 \\
\hline TRA-08 & 732 & 488.15 & 4934.93 & 4446.78 & 502 \\
\hline USGS-002 & 451 & 668.96 & 5125.99 & 4457.03 & 704 \\
\hline USGS-005 & 454 & 477.45 & 4937.79 & 4460.34 & 453 \\
\hline USGS-008 & 457 & 774.00 & 5195.57 & 4421.57 & 812 \\
\hline USGS-009 & 458 & 615.26 & 5031.86 & 4416.61 & 654 \\
\hline USGS-011 & 460 & 658.79 & 5067.22 & 4408.43 & 704 \\
\hline USGS-014 & 463 & 721.38 & 5133.08 & 4411.70 & 752 \\
\hline USGS-017 & 466 & 366.53 & 4834.01 & 4467.48 & 498 \\
\hline USGS-018 & 467 & 282.34 & 4804.82 & 4522.47 & 329 \\
\hline USGS-019 & 468 & 281.46 & 4800.62 & 4519.16 & 399 \\
\hline USGS-022 & 471 & 618.57 & 5048.74 & 4430.17 & 657 \\
\hline
\end{tabular}


Table 8. (continued).

\begin{tabular}{lccccc}
\hline \multicolumn{1}{c}{ Well Name } & Well ID & $\begin{array}{c}\text { Corrected } \\
\text { June 2005 } \\
\text { Depth to Water } \\
(\mathrm{ft} \text { bgs })\end{array}$ & $\begin{array}{c}\text { Land Surface } \\
\text { Datum } \\
(\mathrm{ft} \mathrm{msl)}\end{array}$ & $\begin{array}{c}\text { Water Table } \\
\text { Elevation } \\
(\mathrm{ft} \mathrm{msl})\end{array}$ & $\begin{array}{c}\text { Completion } \\
\text { Depth }\end{array}$ \\
\hline USGS-023 & 472 & 413.16 & 4884.67 & 4471.51 & 463 \\
USGS-026 & 475 & 220.70 & 4789.53 & 4568.83 & 267 \\
USGS-030A & 479 & 269.39 & 4794.84 & 4525.45 & 300 \\
USGS-038 & 487 & 482.79 & 4929.63 & 4446.84 & 724 \\
USGS-076 & 525 & 482.87 & 4929.70 & 4446.83 & 718 \\
USGS-079 & 528 & 483.91 & 4931.08 & 4447.18 & 281 \\
USGS-086 & 535 & 656.39 & 5077.04 & 4420.65 & 691 \\
USGS-088 & 537 & 599.65 & 5021.26 & 4421.61 & 663 \\
USGS-101 & 550 & 780.07 & 5251.60 & 4471.52 & 865 \\
USGS-104 & 553 & 562.57 & 4988.65 & 4426.08 & 550 \\
USGS-109 & 558 & 627.16 & 5043.61 & 4416.45 & 600 \\
USGS-112 & 561 & 480.92 & 4927.84 & 4446.91 & 563 \\
USGS-121 & 570 & 461.66 & 4909.65 & 4447.99 & 485 \\
USGS-128 & 1413 & 488.37 & 4934.92 & 4446.55 & 615 \\
WS-INEL1 & 595 & 408.25 & 4872.83 & 4464.58 & 507 \\
\hline a. A water-level contour map for the 2005 water-level data is shown in the annual report for FY 2005 (DOE-ID 2006e). & \\
bgs = below ground surface. & & & & & \\
msl= mean sea level. & & & &
\end{tabular}

\subsubsection{Evaluation of Vertical Gradients in Westbay Wells}

Water levels for the Westbay wells were determined from pressure readings measured in September 2005 and May 2006. The hydraulic head as a function of elevation from the Westbay systems showed that both wells MIDDLE-2050A and MIDDLE-2051 display shifts in gradient direction. The vertical direction of groundwater flow is determined from the difference in head (water levels) at two depths with water flowing in the direction of the lower head. Gradient analysis is only a partial indicator of water movement, and knowledge of or estimates of formation permeability are also necessary to evaluate water movement.

In the September 2005 measurements for MIDDLE-2051, initially there is a downward gradient from the water table ( $\sim 563 \mathrm{ft}$ bgs) to a depth of approximately $900 \mathrm{ft}$ below ground surface (Figure 7). Calculated water levels below $900 \mathrm{ft}$ are higher than at approximately $900 \mathrm{ft}$ and would indicate an upward gradient below $900 \mathrm{ft}$ bgs. This gradient pattern is also apparent in the May 2006 data, but fewer data points were collected in May (Figure 7).

The September 2005 data for MIDDLE-2050A show a distinct decrease in head, indicating downward vertical gradient, with increasing depth from the water table to about $800 \mathrm{ft}$ below ground surface or approximately $320 \mathrm{ft}$ below the water table ( $480 \mathrm{ft} \mathrm{bgs}$ ) (Figure 8 ). The gradient reverses sharply just below $900 \mathrm{ft}$, to an upward gradient. Below $900 \mathrm{ft}$, the vertical groundwater gradient is slightly downward. 
2051, Septem ber 23, 2005

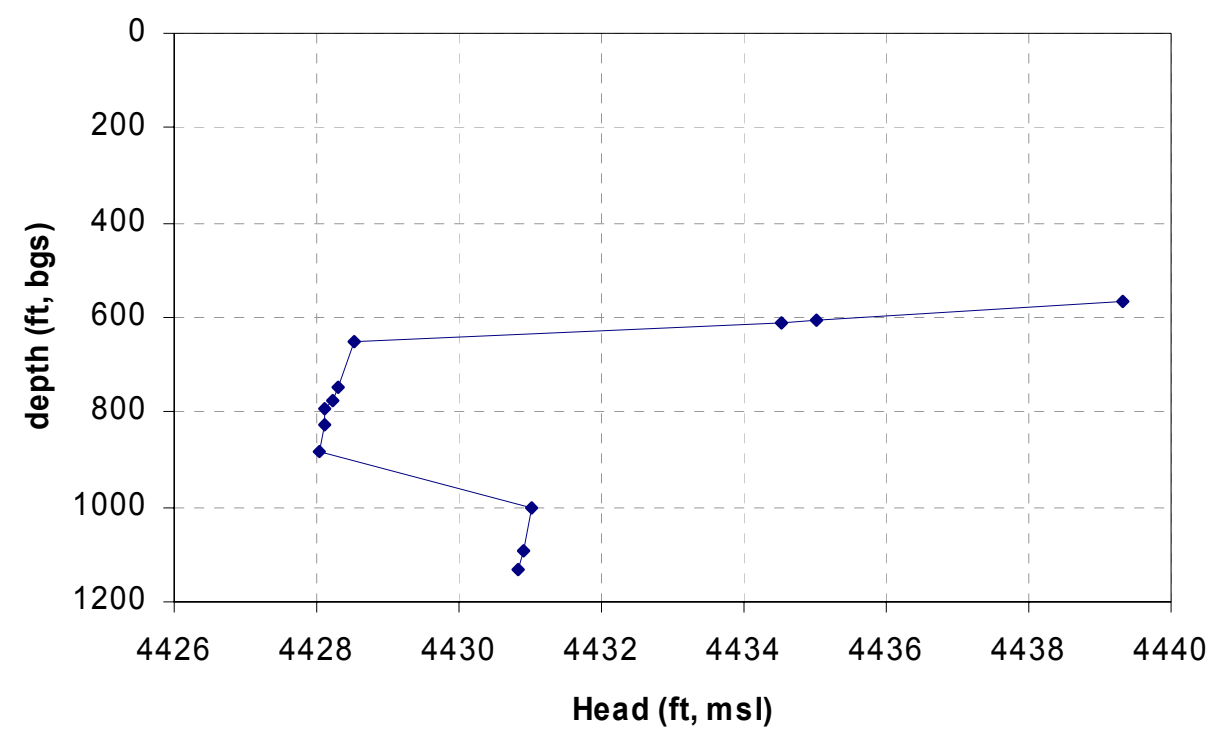

2051, May 5, 2006

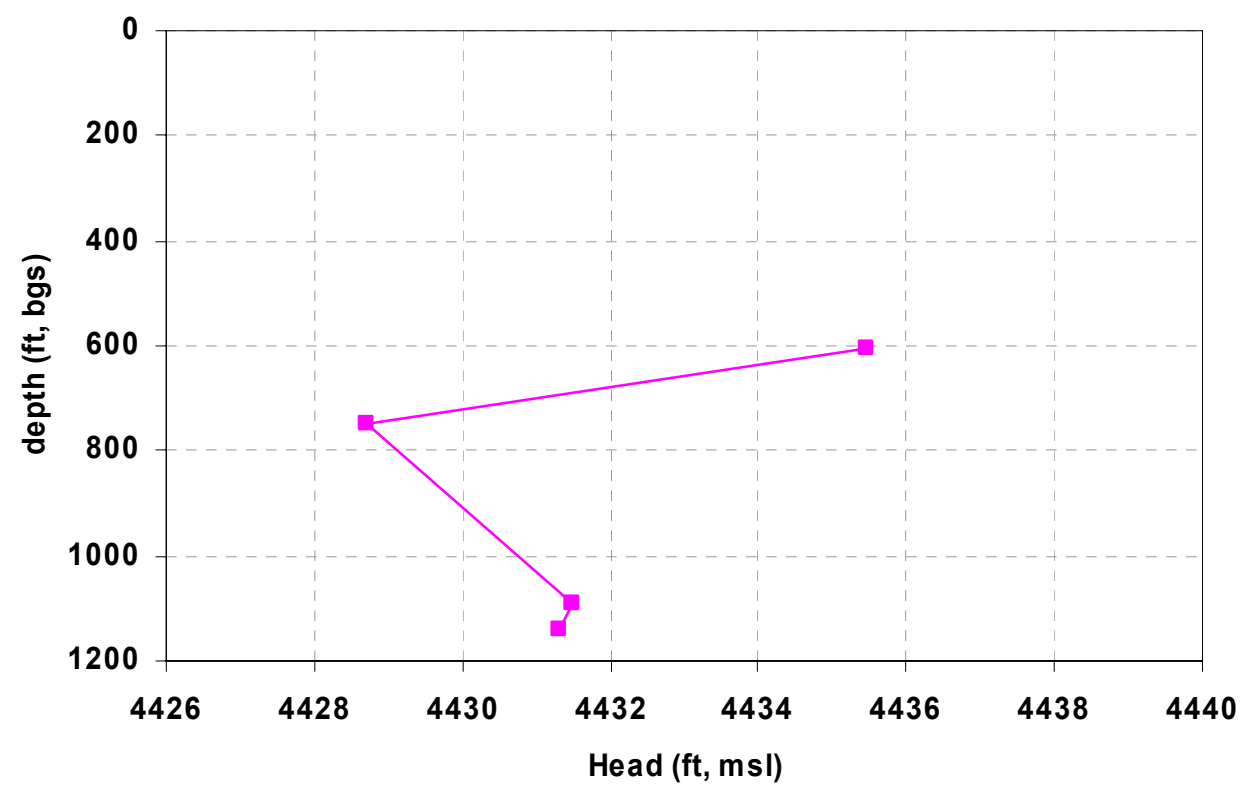

Figure 7. MIDDLE-2051 pressure heads plotted as water-level elevation versus depth (feet above mean sea level). 
2050A, Septe mber 23, 2005
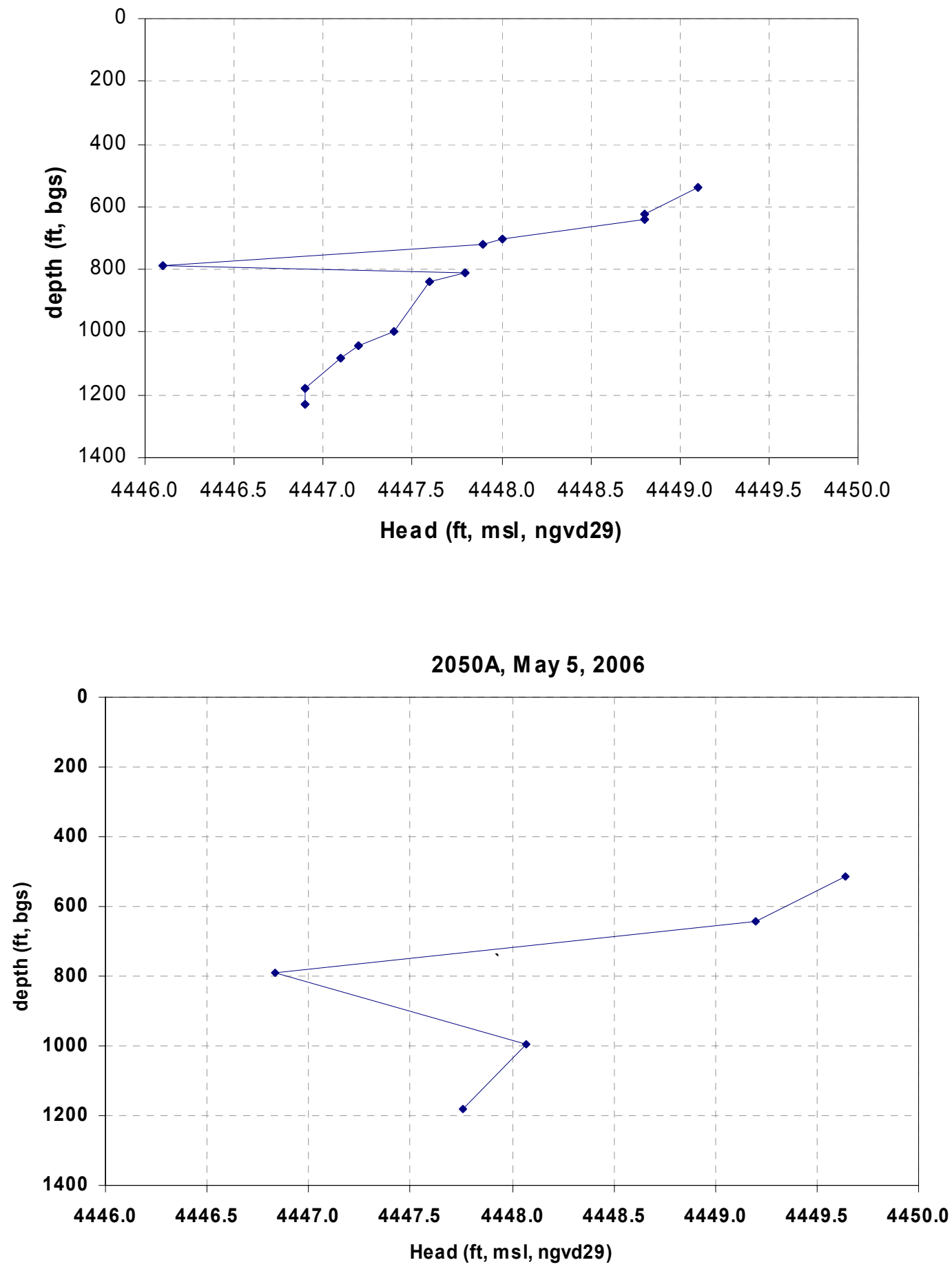

Figure 8. MIDDLE-2050A pressure heads plotted as water-level elevation versus depth (feet above mean sea level). 


\subsection{Progress on Sitewide Groundwater Model}

A major component of the OU 10-08 RI/FS effort is the preparation of a Sitewide groundwater flow and transport model. The results of the OU 10-08 Sitewide groundwater flow model will be used as input into the regulatory decisions made under OU 10-08. The Sitewide groundwater flow model is updated with recently collected subsurface information and is consistent with groundwater models prepared under CERCLA by individual WAGs. Previous modeling performed by the individual WAGs was summarized in the 2005 Annual Report (DOE-ID 2006e). The complete description of the plan for the OU 10-08 groundwater model activities is presented in the Idaho National Engineering and Environmental Laboratory Operable Unit 10-08 Sitewide Groundwater Model Work Plan (DOE-ID 2004b).

\subsubsection{Groundwater Modeling Goals}

The overall goal of the Sitewide Groundwater Model Project is based on concurrence reached among representatives from the DOE, state and EPA regulators, and INL Site contractors. The goal is given as follows (DOE-ID 2004b):

Develop a Sitewide flow and transport model of the active flow portion of the SRPA that can be used to evaluate OU 10-08 remedial action alternatives and to ensure all remedies remain protective of the SRPA. The model will provide credible estimates of contaminant concentrations from sources at the INL over relevant future timeframes.

\subsubsection{Tasks Completed}

Tasks to develop the Sitewide groundwater model were identified in the OU 10-08 Sitewide Groundwater Model Work Plan (DOE-ID 2004b). These tasks were identified as necessary to meet the goals and scope identified above. Tasks completed in activities through FY 2005 were described in the 2005 Annual Report (DOE-ID 2006e). Tasks completed in FY 2006 toward developing the Sitewide groundwater model are discussed in this section. Although there is overlap, these tasks fall into two primary divisions: conceptual modeling and numerical modeling.

2.5.2.1 Conceptual Model. The comprehensive analysis of the geological, hydrological, chemical, and geophysical data sets to define the 3-D hydrostratigraphic conceptual model was completed. This analysis was summarized in draft form and will be finalized in FY 2007.

The methodology was developed and implemented to define 3-D regions corresponding to hydrogeologic units defined by the hydrostratigraphic conceptual model. Bounds on hydrologic properties for the 3-D regions were estimated. A description of the methodology and implementation was completed in draft form and will be finalized in FY 2007.

Distribution of water influx across the northeast model domain boundary was conceptualized in FY 2006 for the two-dimensional (2-D) transport model using results from previous modeling. This horizontal distribution was further partitioned vertically for the 3-D flow model. Distribution of water from the tributary basins also was revisited for the conceptual model. Rather than simply entering the aquifer on the side of the model domain, a more realistic interpretation was envisioned where the local aquifers in the tributary basin are disconnected from the regional aquifer. Recharge water from tributary basins enters the regional aquifer at the water table within an areal transition zone at the mouths of tributary valleys. These changes to the conceptual model were described in draft form and will be finalized in FY 2007. 
Velocities were estimated from chemical disequilibrium measurements along interpreted preferential pathways. These velocities averaged $4 \mathrm{~m} /$ day along the central pathway from Little Lost River southward and $2.6 \mathrm{~m} /$ day along an eastern pathway from Birch Creek southward. Velocities, or more correctly specific discharge, also was estimated using vertical temperature profiles and horizontal water table gradients. These specific discharge estimates are used to estimate velocities when adjusted for porosity. The velocity estimates will serve as calibration points for joint inversion on water levels and water velocities.

An analysis conducted on transient water-level data determined that steady-state conditions were generally appropriate for representing external boundaries. Although water levels change with time throughout the model domain, they generally rise and fall similarly so that flow directions (overall) do not significantly change. The obvious exception to this is observed in the transient influence of the BLR locally where it flows across portions of the INL. Water levels from calendar year 2004 were compiled for use in calibration. This analysis also determined vertical gradients wherever they could be calculated across the INL for use in constraining the 3-D flow model. These analyses were written in draft form and will be finalized in FY 2007.

2.5.2.2 Numerical Model. The 2-D transport model, constructed primarily to assist in development of a method of contaminant loading that was consistent with facility-scale models, was completed and presented at the June agency summit. A decision was made to not calibrate the 2-D transport model. This decision was based on the fact that the 2-D model averages contaminant concentrations vertically over the entire effective thickness of the aquifer, and these simulated concentrations are diluted. Attempting calibration using these diluted concentrations would not be meaningful. Contaminant sources for Test Area North (TAN), INTEC, and RWMC are now implemented with assurance that the mass loading is correct. A description of the 2-D transport modeling activity is included as Appendix C.

A draft discussion was prepared describing contaminant monitoring results at facilities on the INL and identifying aquifer monitoring well locations for use in calibrating the 3-D Sitewide aquifer transport model. Contaminants for model calibration were selected for each facility based on the list of contaminants present in the aquifer beneath each facility. The wells and contaminants selected in this draft discussion may be modified as needed depending on preliminary model results. Ranges of contaminant transport parameters used in previous facility-specific aquifer models were described for use in determining likely bounds on these parameters for the 3-D transport model.

A primary activity for FY 2006 was the development of the 3-D flow model. The horizontal domain was that defined by the 2-D flow model. Horizontal discretization of this domain was accomplished with a maximum grid block size of $750 \mathrm{~m}$ and included refinement at each of the principal INL facilities. The facilities with grid refinement are TAN, Naval Reactors Facility, RTC, INTEC, Materials and Fuels Complex, Central Facilities Area (CFA), Boiling Water Reactor Experiment, and RWMC. A grid block with dimensions of $150 \times 150 \mathrm{~m}$ is located at the approximate center of each facility. The factor for increasing grid dimension away from this central grid block was 1.1, which keeps the grid dimensions less than $200 \mathrm{~m}$ up to approximately $700 \mathrm{~m}$ around each facility. Vertical discretization of the model domain was accomplished through an iterative process that balanced adequate representation of the 3-D volumes from the conceptual model and computational burden. Eventually, the vertical domain was discretized into six layers. These discretizations were accomplished for both the thick and thin aquifer scenarios.

The estimated distribution of water flux along the northeast boundary was distributed vertically along this same boundary for the 3-D flow model. Likewise, the revised interpretation of tributary basin water fluxes entering onto the top of the regional aquifer over a transition zone extending out over the aquifer was implemented in the 3-D flow model. This revision had a benefit of removing a problem from 
the 2-D flow model where unrealistically elevated water levels resulted where the tributary fluxes were applied to the side of the aquifer model.

Two methods for calibration of the 3-D steady-state flow model were used. The first method was a pure pilot point approach where pilot points were distributed in each model layer and the model was run in an inverse mode to estimate hydraulic conductivities that minimized the difference between observed and simulated water levels. The second method used the 3-D volumes from the conceptual model to constrain hydraulic conductivities within the model regions representing the $3-\mathrm{D}$ volumes. This was performed primarily for sediment regions and with all the basalt regions combined. The assigned bounds on the hydraulic properties for the basalt regions were not sufficiently defined to allow distinction between them. The preliminary results for both these approaches on both the thick and thin aquifer scenarios were included in a draft discussion that will be finalized in FY 2007.

An overall objective in the development of a representative 3-D transport model is to simultaneously use multiple data sources in the calibration. These data sources are water levels, anthropogenic contaminant monitoring histories, velocities estimated from geochemical studies, and temperature data. The interface within the groundwater model system (GMS) shell was modified in FY 2006 to allow PEST to simultaneously interact with MODFLOW and MT3-DMS to allow joint inversion. Joint inversion to water levels and velocities was initiated in FY 2006 and will be completed in FY 2007. This joint inversion also will include the contaminant transport and prediction(s) of contaminant concentrations.

Another model task accomplished in FY 2006 was development of a 3-D regional aquifer thermal transport model. This model was developed to serve as a constraint on the transport modeling, given the similarities between contaminant and thermal transport. The HST3D simulation code was used to implement the 3-D regional aquifer model. This model utilized the horizontal domain, discretization, and hydrologic property distribution from the updated Idaho Water Resources Research Institute model (Cosgrove et al. 2006). The vertical domain was the same for the base of the aquifer but required extension upwards to land surface to allow surface temperatures to be assigned for the upper boundary. The model was parameterized to allow vertical energy transport in the vadose zone portion of the domain but no horizontal advection of water. Results of the 3-D heat flow modeling for the regional Eastern Snake River Plain aquifer demonstrate that adequate information is available to constrain a heat flow model, even for an area much larger than is the focus of the OU 10-08 study. Efforts in FY 2007 will include use of this regional model to provide boundary conditions for a local-scale model that only considers the portion of the aquifer of interest to the OU 10-08 project. This modeling effort provides a secondary constraint for inverse modeling to estimate hydraulic conductivity for the WAG 10 subregional flow and transport model.

The last numerical modeling activity involved continued development of the Response Surface Model (RSM). This model eventually will serve as the risk assessment tool for OU 10-08. The RSM model utilizes results from the 3-D transport model and then-using response surface functionsmathematically represents the results of the 3-D transport model more simply and efficiently. It does not replace development of the 3-D transport model; it mimics the 3-D model once it is created and also performs the linear risk calculations. During FY 2006, the RSM was implemented into an Access database structure. The unit response functions for INTEC and RWMC sources were updated using the final 2-D transport model. The RSM was then validated by running the RSM with these response functions for Tc-99 and I-129 but with a tritium source term for INTEC and RWMC. Running the RSM model in this way provided an estimate of tritium transport that would have been derived using the completed 2-D transport model. The tritium source term also was run with the 2-D transport model to provide a comparison result to the RSM model. The results compared favorably with these two methods 
indicating the RSM model adequately mimicked the more complicated 2-D model. This validation process was presented at the June agency meeting.

2.5.2.3 Other Activities. One of the objectives outlined in the OU 10-08 Groundwater Modeling Work Plan (DOE-ID 2004b) was to develop methods to convey information to both the regional technical community and the general public. The Great Rift Science Symposium was held at the Idaho State University campus in October 2005, thus providing an opportunity to meet this objective. Most members of the OU 10-08 project presented results of either conceptual or numerical model development activities. Peer-reviewed papers covering the material in the presentations are expected to be released in 2007.

\subsection{Risk Reevaluation for Mercury at TSF-08}

The TSF-08 Mercury Spill Site Area 13B was evaluated for phytoremediation as discussed in the Final Record of Decision for Test Area North, Operable Unit 1-10 (DOE-ID 1999), the Explanation of Significant Differences for the Record of Decision for the Test Area North Operable Unit 1-10 (DOE-ID 2003), and the OU 10-08 RI/FS Work Plan (DOE-ID 2002a). As several studies have reported (Jonasson 1970; Shcherbakov et al. 1970; and Landa 1978), and as summarized in Trace Elements in Soils and Plants (Kabata-Pendias 2001), Hg is likely to be strongly bound when added to soils as elemental $\mathrm{Hg}$. However, these studies also found that this behavior in soils is highly dependent on its form, which is a function of several soil parameters, and of the initial $\mathrm{Hg}$ concentration in the soil solution, as well as of other ions (e.g., Cl) (Kabata-Pendias 2001).

Spills of elemental mercury occurred onto the railroad bed that carried the Heat Transfer Reactor Experiment No. 3 (HTRE-III), which used Hg for radiation protection and was tested in 1959 and 1960. The HTRE-III was drained in 1960 and stored at the TSF-08 site before decontamination and decommissioning in 1987. The 1994 remediation at this site confirmed that the mercury that had not volatilized remained in the elemental form. This was primarily due to the fact that the spill site was on compacted railroad bed with a clay substrate. During the 1994 remediation activities, all the observed mercury was collected, but it was observed that elemental mercury was beading in cracks in clay and was not present at depth (INEL 1995).

This finding is consistent with the findings of a study on spilled elemental $\mathrm{Hg}$ (Harju et al. 1995). The Harju study found that spilled elemental $\mathrm{Hg}$ usually breaks into small beads or droplets in the immediate vicinity of the spill and, generally, infiltration is limited to 0.5 to $1.0 \mathrm{~m}$ (Harju et al. 1995). Deeper migration, however, may occur if fracture or macropores are present (Henke et al. 1993). As found during the remediation, the spilled elemental mercury (and the associated remediation) followed the cracks in the clay. Thus, it is determined that this would not be a productive site to attempt phytoremediation.

Finally, the EPA Study Report to Congress (Volume III) on the Fate and Transport of Mercury (EPA 1997a) found that $\operatorname{Hg}(0)$ spilled onto the soil will diffuse through the soil and re-enter the atmosphere. In the soil, any of the $\mathrm{Hg}(0)$ that is converted to $\mathrm{Hg}(\mathrm{II})$ and methylmercury complexes would be available theoretically for plant uptake and translocation, potentially resulting in transfer through the terrestrial food chain (EPA 1997a). However, EPA (1997a) found that in reality plant uptake from ordinary soils, especially to above-ground parts of plants, appears to be insignificant (Schuster 1991; Lindqvist et al. 1991, Chapter 9). Therefore, it has been determined that phytoremediation is not a viable option at this site, based on the form of the mercury present (elemental) and the other site characteristics.

During this evaluation and review of the site remediation, it became apparent that additional information as well as sampling results were available that were not included in the OU 1-10 risk assessment (DOE-ID 1997). The OU 1-10 risk assessment had used the maximum concentration detected 
in post remediation sampling as representative of the mercury concentration across the entire Site, thereby greatly overestimating the risk. In addition, since the performance of the OU 1-10 risk assessment, new information concerning mercury has become available from EPA sources, such as updated toxicity data (EPA 2001). For human health, the EPA Mercury Report to Congress (EPA 1997a) provides updated fate and transport information (EPA 1997a). This is critical to the risk characterization of this site since the conservative uptake factors used in the OU 1-10 risk assessment contributed to the risk for the resident from homegrown produce. The EPA mercury report provides a more realistic estimation of the amount of mercury available through the homegrown produce pathway (EPA 1997a). For ecological receptors, more accurate modeling as well as additional toxicity, fate, and transport information has become available (EPA 1997b, 1999). The OU 10-08 Work Plan (DOE-ID 2002a) directs that the site should be reassessed after phytoremediation. Since the phytoremediation was deemed to be inappropriate, and due to the new information available for assessment, this site will be reevaluated. 


\section{ACTIVITIES FOR FISCAL YEAR 2007}

Several tasks are scheduled to support the OU 10-08 RI/FS in FY 2007. These tasks have been identified in the OU 10-08 RI/FS Work Plan (DOE-ID 2002a), in previous annual reports, and in the WAG 10 monitoring and field sampling plan (DOE-ID 2006b). Several tasks for FY 2007 are identified below, but additional tasks may be performed as the need arises and as negotiated with the Agencies and funded by DOE.

\subsection{Recurring Tasks and Tasks Carried Over from Previous Annual Reports}

The following tasks have been identified in previous annual reports or in the OU 10-08 RI/FS Work Plan (DOE-ID 2002a). Several tasks have been completed in previous years and will continue to be performed annually, while other tasks have yet to be completed but will be performed in future years. These tasks include the following:

- $\quad$ Evaluate potentially commingled plumes, incorporating annual data from all OU 10-08 monitored wells and individual WAGs as appropriate. This task is currently being evaluated as part of the geochemical study (see Appendix B). An analysis of commingling plumes also will be conducted as part of the updated Sitewide groundwater model.

- Evaluate groundwater data collected from the OU 10-08 monitoring network, particularly focusing on trends and determining baselines for long-term monitoring. This information will be incorporated into the annual OU 10-08 RI/FS report, along with recommendations for future sampling.

- $\quad$ Prepare the FY 2006 annual OU 10-08 RI/FS report (this report) and submit it to the Department of Energy Idaho Operations Office (DOE-ID) for transmittal to the regulatory Agencies.

\subsection{Sitewide Groundwater Model}

A major effort is underway to create a 3-D Sitewide groundwater model that will include contaminant transport. Many detailed tasks have been identified and are described in the Sitewide Groundwater Model Work Plan (DOE-ID 2004b). A high-level overview of the tasks is as follows:

Fiscal Year 2007

Tasks for FY 2007 include:

- $\quad$ Deliverables include a 3-D flow modeling report to be finalized in mid-FY 2007

- $\quad$ Prepare a comprehensive, 3-D, subregional conceptual model

- $\quad$ Prepare a subregional-scale, 3-D, aquifer-flow numerical model

- $\quad$ Prepare a 3-D flow and transport model.

\subsection{Waste Area Group 10 Monitoring Plan}

The Groundwater Monitoring and Field Sampling Plan for WAG 10 will be updated during FY 2007 to include sampling intervals from the new Westbay Well USGS-132 completed in FY 2006 
and to propose a sampling plan for MIDDLE-2050A and MIDDLE-2051. The plan will guide WAG 10 groundwater monitoring activities through the completion of the OU 10-08 ROD or until otherwise updated or modified. 


\section{SUMMARY}

Several activities were completed in FY 2006 in support of the OU 10-08 RI/FS. Most notably, a groundwater sampling round was completed on the OU 10-08 monitoring well network, a comprehensive Sitewide water-level measurement system was installed, two new multilevel Westbay wells were installed by the USGS, the Groundwater Monitoring and Field Sampling Plan for OU 10-08 was updated, and the summary report for the 2-D groundwater model was produced (Appendix C).

No contaminant exceeded an MCL in a well along the southern boundary of the INL Site or downgradient of the Site in the FY 2006 groundwater monitoring. 


\section{REFERENCES}

42 USC § 9601 et seq., 1980, "Comprehensive Environmental Response, Compensation, and Liability Act of 1980 (CERCLA/Superfund)," United States Code, December 11, 1980.

Cosgrove, D. M., B. A. Contor, G. S. Johnson, 2006, Enhanced Snake River Plain Aquifer Model Final Report, Idaho Water Resources Research Institute Technical Report 06-002, Idaho Water Resources Research Institute, University of Idaho, July 2006.

DOE-ID, 1991, Federal Facility Agreement and Consent Order for the Idaho National Engineering Laboratory, Administrative Docket No. 1088-06-29-120, U.S. Department of Energy Idaho Operations Office; U.S. Environmental Protection Agency, Region 10; Idaho Department of Health and Welfare, December 4, 1991.

DOE-ID, 1997, Comprehensive Remedial Investigation/Feasibility Study for the Test Area North Operable Unit 1-10 at the Idaho National Engineering and Environmental Laboratory, DOE/ID-10557, Rev. 0, U.S. Department of Energy Idaho Operations Office, November 1997.

DOE-ID, 1999, Final Record of Decision for Test Area North, Operable Unit 1-10, DOE/ID-10682, Rev. 0, U.S. Department of Energy Idaho Operations Office; U.S. Environmental Protection Agency, Region 10; and Idaho Department of Health and Welfare, October 1999.

DOE-ID, 2002a, Waste Area Group 10, Operable Unit 10-08, Remedial Investigation/Feasibility Study Work Plan (FINAL), DOE/ID-10902, Rev. 0, U.S. Department of Energy Idaho Operations Office, August 2002.

DOE-ID, 2002b, Record of Decision Experimental Breeder Reactor-I/Boiling Water Reactor Experiment Area and Miscellaneous Sites, DOE/ID-10980, Rev. 0, U.S. Department of Energy Idaho Operations Office, November 2002.

DOE-ID, 2003, Explanation of Significant Differences for the Record of Decision for the Test Area North Operable Unit 1-10, DOE-ID-11050, Rev. 0, U.S. Department of Energy Idaho Operations Office; U.S. Environmental Protection Agency, Region 10; and Idaho Department of Health and Welfare, April 2003.

DOE-ID, 2004a, Waste Area Group 10, Operable Unit 10-08, Remedial Investigation/Feasibility Study Annual Report for Fiscal Year 2003, DOE/ID-11116, Rev. 0, U.S. Department of Energy Idaho Operations Office, April 2004.

DOE-ID, 2004b, Idaho National Engineering and Environmental Laboratory Operable Unit 10-08 Sitewide Groundwater Model Work Plan, DOE/NE-ID-11188, Rev. 0, U.S. Department of Energy Idaho Operations Office, December 2004.

DOE-ID, 2004c, Remedial Design/Remedial Action Work Plan for Operable Units 6-05 and 10-04, Phase I, DOE/ID-11101, Rev. 0, U.S. Department of Energy Idaho Operations Office, February 2004.

DOE-ID, 2005, Groundwater Monitoring and Field Sampling Plan of Operable Unit 10-08, DOE/NE-ID-11210, Rev. 0, U.S. Department of Energy Idaho Operations Office, March 2005. 
DOE-ID, 2006a, Five-Year Review of CERCLA Response Actions at the Idaho National Laboratory, DOE/ID-11201, Rev. 1, U.S. Department of Energy Idaho Operations Office, June 2006.

DOE-ID, 2006b, Groundwater Monitoring and Field Sampling Plan of Operable Unit 10-08, DOE/NE-ID-11210, Rev. 1, U.S. Department of Energy Idaho Operations Office, May 2006.

DOE-ID, 2006c, INL Sitewide Institutional Controls Annual Report-FY 2006, DOE/ID-11289, Rev. 0, U.S. Department of Energy Idaho Operations Office, August 2006.

DOE-ID, 2006d, INL Sitewide Institutional Controls Plan, DOE/ID-11042, Rev. 3, U.S. Department of Energy Idaho Operations Office, July 2006.

DOE-ID, 2006e, Waste Area Group 10, Operable Unit 10-08, Remedial Investigation/Feasibility Study Annual Status Report for Fiscal Year 2005, DOE/NE-ID-11274, Rev. 1, U.S. Department of Energy Idaho Operations Office, May 2006.

EPA, 1997a, Mercury Study Report to Congress, Volume III, Fate and Transport of Mercury in the Environment, EPA-452/R-97-003, United States Environmental Protection Agency Office of Air Quality Planning and Standards and Office of Research and Development, December 1997.

EPA, 1997b, Ecological Risk Assessment Guidance for Superfund: Process for Designing and Conducting Ecological Risk Assessments - Interim Final, EPA-540-R-97-006, U.S. Environmental Protection Agency, Office of Solid Waste and Emergency Response, June 1997.

EPA, 1999, Screening Level Ecological Risk Assessment Protocol for Hazardous Waste Combustion Facilities, Peer Review Draft, EPA/530-D-99-001A, U.S. Environmental Protection Agency, Office of Solid Waste and Emergency Response, Washington, DC., EPA, 1999.

EPA, 2001, Integrated Risk Information System (IRIS), Duluth, MN. (Note: Methyl mercury toxicity values were updated $7 / 21 / 01$ ).

Harju, J. A., V. Kühnel, D. S. Charlton, and J. M. Evans, 1995, "Field-Based Research on Elemental Mercury Spills,” Water, Air, \& Soil Poll., Vol. 80, Numbers 1-4, pp. 1191-1197, February 1995.

Henke, K. R., V. Kühnel, D. J. Stepan, R. H. Fraley, C. M. Robinson, D. S. Charlton, H. M. Gust, and N. S. Bloom, 1993, Critical Review of Mercury Contamination Issues Relevant to Manometers at Natural Gas Industry Sites, GRI-93/0117, Gas Research Institute, 92 pp.

ICP, 2004, Annual Groundwater Monitoring Status Report for Waste Area Group 5 for Fiscal Year 2004, ICP/EXT-04-00369, Rev. 1, Idaho National Engineering and Environmental Laboratory, Idaho Completion Project, November 2004.

ICP, 2006, The Drilling, Coring, and Installation of Two Deep Monitoring Wells (MIDDLE-2051 and MIDDLE-2050A) in Fiscal Year 2005, RPT-178, Rev. 0, Idaho National Laboratory, Idaho Cleanup Project, August 2006.

INEEL, 2003, Central Facilities Area Landfills I, II, and III Annual Monitoring Report (2002), INEEL/EXT-03-00024, Rev. 0, Idaho National Engineering and Environmental Laboratory, September 2003.

INEL, 1995, Preliminary Scoping Track 2 Summary Report for Operable Unit 1-08, INEL-94/0170, Rev. 0, Lockheed Martin Idaho Technologies Company, September 1995. 
Jonasson, I. R., 1970, "Mercury in the Natural Environment: A Review of Recent Work, Geol. Surv. Can., Vol. 70, Issue 57, p. 37.

Kabata-Pendias, A., 2001, Trace Elements in Soils and Plants, Third Edition, Boca Raton, Florida, CRC Press.

Knobel, L. L., B. R. Orr, and L. D. Cecil, 1992, "Summary of Concentrations of Selected Radiochemical and Chemical Constituents in Groundwater from the Snake River Plain Aquifer, Idaho: Estimated from an Analysis of Previously Published Data," Journal of Idaho Academy of Science, Vol. 28, No. 1, pp. 48-61, June 1992.

Landa, E. R., 1978, “The Retention of Metallic Mercury Vapor by Soils," Geochim. Cosmochim. Acta, Vol. 42, p. 1407.

Lindqvist, O., K. Johansson, M. Aastrup, A. Anderson, L. Bringmark, G. Hovsenius, L. Hakanson, A. Iverfeldt, M. Meili, and B. Timm, 1991, "Mercury in the Swedish Environment - Recent Research on Causes, Consequences, and Corrective Methods," Water, Air, \& Soil Poll., Vol. 55, all chapters.

MCP-3448, 2005, "Inclusion of New Sites under the Federal Facility Agreement and Consent Order," Rev. 7, Idaho National Engineering and Environmental Laboratory, Idaho Completion Project, June 2005.

Public Law 99-499, 1986, "Superfund Amendments and Reauthorization Act of 1986 (SARA)," 100 Statutes 1728, Public Law, October 17, 1986.

Schuster, E., 1991, "The Behavior of Mercury in the Soil, with Special Emphasis on Complexation and Adsorption Processes-A Review of the Literature," Water, Air, and Soil Poll., Vol. 56, pp. 667-680.

Shcherbakov, V. P., Dvornikov, A. T., and Zakrenichnya, G. L., 1970, "New Data on Occurrence and Forms of Mercury in the Donbas Coals, Dopov. ANUR, 2b, 126, Uk.

USGS, 1999, Chemical Constituents in Ground Water from 39 Selected Sites with an Evaluation of Associated Quality Assurance Data, Idaho National Engineering and Environmental Laboratory and Vicinity, Idaho, USGS Open File Report 99-246, DOE/ID-22159, United States Geological Survey.

USGS, 2006, Ground-Water Data for Idaho, http://waterdata.usgs.gov/id/nwis/gw, U.S. Geological Survey, Web page visited November 15, 2006. 


\section{Appendix A}

\section{Analytical Results from Fiscal Year 2006 Groundwater Sampling}




$$
\text { A-2 }
$$




\section{Appendix A}

\section{Analytical Results from Fiscal Year 2006 Groundwater Sampling}

\section{A-1. INTRODUCTION}

This appendix presents the groundwater analytical results from Fiscal Year 2006 for Waste Area Group (WAG) 10. The analytes and analytical methods are summarized in Table A-1. The data for WAG 10 (boundary, guard, and distal wells), packer sampling, and Westbay wells are contained in Tables A-2, A-3, and A-4, respectively. The data for the Westbay sampling in September-October 2005 also are included since they have not been previously included in an annual report (Table A-5). The complete groundwater data set (in Excel) is provided on the compact disc attached to the inside back cover of this report. The low detection limit accelerator mass spectrometry (AMS) I-129 data are presented in Appendix B, but the analytical methods are summarized in this appendix following the definition of data flags.

Note that alkalinity data are reported in units of $\mathrm{mg} / \mathrm{L}$ as calcium carbonate. In Tables A-2 through A-5 and on the compact disc, sample and duplicate samples are designated as 01 and 02 before the two-character analysis code. For example, GWM32102RH is the duplicate for GWM32101RH.

Table A-1. Analytes and method codes.

\begin{tabular}{|c|c|c|}
\hline Analyte & $\begin{array}{l}\text { Method } \\
\text { Code }\end{array}$ & Method Description \\
\hline VOCs & SW8260B & VOCs by GC/MS \\
\hline Sulfate, chloride, fluoride & E300 & Inorganic anions by ion chromatography \\
\hline Alkalinity, total as $\mathrm{CaCO}_{3}$ & E310.1 & Alkalinity, total (as carbonate) \\
\hline Nitrogen, nitrate/nitrite & E353.1 & Nitrogen, nitrate-nitrite \\
\hline Tritium & LSC & Liquid scintillation \\
\hline Metals, except mercury & SW6020 & Inductively coupled plasma - mass spectrometry \\
\hline Mercury & SW7470A & Mercury in liquid waste (manual cold-vapor technique) \\
\hline Sr-90 & GFP & Gas flow proportional counter \\
\hline Tc-99 & LSC & Liquid scintillation \\
\hline I-129 & GMS & Gamma spectroscopy \\
\hline Gross alpha & GAB & Gross alpha/beta \\
\hline Gross beta & GAB & Gross alpha/beta \\
\hline
\end{tabular}


Table A-2. Waste Area Group 10 groundwater monitoring data.

\begin{tabular}{|c|c|c|c|c|c|c|c|c|c|c|c|}
\hline $\begin{array}{c}\text { Field Sample } \\
\text { Number }\end{array}$ & Location & Depth & Compound & $\begin{array}{c}\text { Sample } \\
\text { Result }\end{array}$ & $\begin{array}{c}\text { Sample } \\
\text { Error }\end{array}$ & $\begin{array}{c}\text { Result } \\
\text { Qualifier }\end{array}$ & $\begin{array}{c}\text { Validation } \\
\text { Flag }\end{array}$ & $\begin{array}{c}\text { Sample } \\
\text { Units }\end{array}$ & $\begin{array}{c}\text { Date Sample } \\
\text { Collected }\end{array}$ & MDA & $\begin{array}{c}\text { L\&V Report } \\
\text { Number }\end{array}$ \\
\hline GWM442012X & HIGHWAY 3 & 567 & Aluminum & 7.9 & & $U$ & & UG/L & $06 / 19 / 2006$ & & DNT-330-06 \\
\hline GWM442012X & HIGHWAY 3 & 567 & Antimony & 0.5 & & $\mathrm{U}$ & & UG/L & $06 / 19 / 2006$ & & DNT-330-06 \\
\hline GWM442012X & HIGHWAY 3 & 567 & Arsenic & 2 & & U & & UG/L & $06 / 19 / 2006$ & & DNT-330-06 \\
\hline GWM442012X & HIGHWAY 3 & 567 & Beryllium & 0.088 & & $U$ & & UG/L & $06 / 19 / 2006$ & & DNT-330-06 \\
\hline GWM442012X & HIGHWAY 3 & 567 & Cadmium & 0.057 & & U & & UG/L & $06 / 19 / 2006$ & & DNT-330-06 \\
\hline GWM442012X & HIGHWAY 3 & 567 & Calcium & 42900 & & & & UG/L & $06 / 19 / 2006$ & & DNT-330-06 \\
\hline GWM442012X & HIGHWAY 3 & 567 & Chromium & 2.8 & & U & & UG/L & $06 / 19 / 2006$ & & DNT-330-06 \\
\hline GWM442012X & HIGHWAY 3 & 567 & Cobalt & 0.31 & & U & & UG/L & $06 / 19 / 2006$ & & DNT-330-06 \\
\hline GWM442012X & HIGHWAY 3 & 567 & Copper & 3.5 & & & & UG/L & $06 / 19 / 2006$ & & DNT-330-06 \\
\hline GWM442012X & HIGHWAY 3 & 567 & Iron & 9.4 & & U & & UG/L & $06 / 19 / 2006$ & & DNT-330-06 \\
\hline GWM442012X & HIGHWAY 3 & 567 & Lead & 1.6 & & B & & UG/L & $06 / 19 / 2006$ & & DNT-330-06 \\
\hline GWM442012X & HIGHWAY 3 & 567 & Magnesium & 11000 & & $\mathrm{E}$ & $\mathrm{J}$ & UG/L & $06 / 19 / 2006$ & & DNT-330-06 \\
\hline GWM442012X & HIGHWAY 3 & 567 & Manganese & 0.42 & & B & & UG/L & $06 / 19 / 2006$ & & DNT-330-06 \\
\hline GWM442012X & HIGHWAY 3 & 567 & Nickel & 1 & & B & & UG/L & $06 / 19 / 2006$ & & DNT-330-06 \\
\hline GWM442012X & HIGHWAY 3 & 567 & Potassium & 2270 & & E & $\mathrm{J}$ & UG/L & $06 / 19 / 2006$ & & DNT-330-06 \\
\hline GWM442012X & HIGHWAY 3 & 567 & Selenium & 1 & & $\mathrm{U}$ & & UG/L & $06 / 19 / 2006$ & & DNT-330-06 \\
\hline GWM442012X & HIGHWAY 3 & 567 & Silver & 0.2 & & U & & UG/L & $06 / 19 / 2006$ & & DNT-330-06 \\
\hline GWM442012X & HIGHWAY 3 & 567 & Sodium & 5630 & & & & UG/L & $06 / 19 / 2006$ & & DNT-330-06 \\
\hline GWM442012X & HIGHWAY 3 & 567 & Uranium & 2 & & & & UG/L & $06 / 19 / 2006$ & & DNT-330-06 \\
\hline GWM442012X & HIGHWAY 3 & 567 & Vanadium & 5.3 & & B & & UG/L & $06 / 19 / 2006$ & & DNT-330-06 \\
\hline GWM442012X & HIGHWAY 3 & 567 & Zinc & 82.1 & & & & UG/L & $06 / 19 / 2006$ & & DNT-330-06 \\
\hline GWM442012X & HIGHWAY 3 & 567 & Mercury & 0.093 & & U & & UG/L & $06 / 19 / 2006$ & & DNT-330-06 \\
\hline GWM44201A1 & HIGHWAY 3 & 567 & Total Alkalinity & 148 & & & $\mathrm{~J}$ & $M G / L$ & $06 / 19 / 2006$ & & DNT-281-06 \\
\hline GWM44201AN & HIGHWAY 3 & 567 & Chloride & 7.4 & & $\mathrm{~J}$ & & $M G / L$ & 06/19/2006 & & DNT-281-06 \\
\hline GWM44201AN & HIGHWAY 3 & 567 & Fluoride & 0.34 & & & $\mathrm{~J}$ & $M G / L$ & $06 / 19 / 2006$ & & DNT-281-06 \\
\hline GWM44201AN & HIGHWAY 3 & 567 & Sulfate & 19.8 & & & & $M G / L$ & $06 / 19 / 2006$ & & DNT-281-06 \\
\hline GWM44201N2 & HIGHWAY 3 & 567 & Nitrate/Nitrite as $\mathrm{N}$ & 1790 & & & & UG/L & $06 / 19 / 2006$ & & DNT-281-06 \\
\hline GWM44201VL & HIGHWAY 3 & 567 & Acetone & 2 & & $U$ & $\mathrm{R}$ & UG/L & $06 / 19 / 2006$ & & JGJ-066-06 \\
\hline GWM44201VL & HIGHWAY 3 & 567 & Benzene & 1 & & $\mathrm{U}$ & & UG/L & $06 / 19 / 2006$ & & JGJ-066-06 \\
\hline GWM44201VL & HIGHWAY 3 & 567 & Bromodichloromethane & 1 & & $\mathrm{U}$ & & UG/L & $06 / 19 / 2006$ & & JGJ-066-06 \\
\hline GWM44201VL & HIGHWAY 3 & 567 & Bromoform & 1 & & $U$ & & UG/L & $06 / 19 / 2006$ & & JGJ-066-06 \\
\hline GWM44201VL & HIGHWAY 3 & 567 & Bromomethane & 2 & & U & & UG/L & $06 / 19 / 2006$ & & JGJ-066-06 \\
\hline GWM44201VL & HIGHWAY 3 & 567 & 2-Butanone & 5 & & $\mathrm{U}$ & $\mathrm{R}$ & UG/L & $06 / 19 / 2006$ & & JGJ-066-06 \\
\hline GWM44201VL & HIGHWAY 3 & 567 & Carbon disulfide & 1 & & U & & UG/L & $06 / 19 / 2006$ & & JGJ-066-06 \\
\hline GWM44201VL & HIGHWAY 3 & 567 & Carbon tetrachloride & 1 & & $\mathrm{U}$ & & UG/L & $06 / 19 / 2006$ & & JGJ-066-06 \\
\hline GWM44201VL & HIGHWAY 3 & 567 & Chlorobenzene & 1 & & $\mathrm{U}$ & & UG/L & $06 / 19 / 2006$ & & JGJ-066-06 \\
\hline GWM44201VL & HIGHWAY 3 & 567 & Dibromochloromethane & 1 & & U & & UG/L & $06 / 19 / 2006$ & & JGJ-066-06 \\
\hline GWM44201VL & HIGHWAY 3 & 567 & Chloromethane & 2 & & $U$ & & UG/L & $06 / 19 / 2006$ & & JGJ-066-06 \\
\hline GWM44201VL & HIGHWAY 3 & 567 & Cyclohexane & 1 & & $\mathrm{U}$ & & UG/L & $06 / 19 / 2006$ & & JGJ-066-06 \\
\hline GWM44201VL & HIGHWAY 3 & 567 & 1,2-Dibromoethane & 1 & & $\mathrm{U}$ & & UG/L & $06 / 19 / 2006$ & & JGJ-066-06 \\
\hline GWM44201VL & HIGHWAY 3 & 567 & 1,2-Dichlorobenzene & 1 & & U & & UG/L & 06/19/2006 & & JGJ-066-06 \\
\hline GWM44201VL & HIGHWAY 3 & 567 & 1,3-Dichlorobenzene & 1 & & $\mathrm{U}$ & & UG/L & $06 / 19 / 2006$ & & JGJ-066-06 \\
\hline GWM44201VL & HIGHWAY 3 & 567 & 1,4-Dichlorobenzene & 1 & & $U$ & & UG/L & $06 / 19 / 2006$ & & JGJ-066-06 \\
\hline GWM44201VL & HIGHWAY 3 & 567 & Dichlorodifluoromethane & 2 & & U & & UG/L & $06 / 19 / 2006$ & & JGJ-066-06 \\
\hline GWM44201VL & HIGHWAY 3 & 567 & 1,1-Dichloroethane & 1 & & U & & UG/L & $06 / 19 / 2006$ & & JGJ-066-06 \\
\hline GWM44201VL & HIGHWAY 3 & 567 & 1,2-Dichloroethane & 1 & & $\mathrm{U}$ & & UG/L & $06 / 19 / 2006$ & & JGJ-066-06 \\
\hline GWM44201VL & HIGHWAY 3 & 567 & 1,1-Dichloroethene & 1 & & $\mathrm{U}$ & & UG/L & $06 / 19 / 2006$ & & JGJ-066-06 \\
\hline GWM44201VL & HIGHWAY 3 & 567 & cis-1,2-Dichloroethene & 1 & & U & & UG/L & $06 / 19 / 2006$ & & JGJ-066-06 \\
\hline GWM44201VL & HIGHWAY 3 & 567 & trans-1,2-Dichloroethene & 1 & & $\mathrm{U}$ & & UG/L & $06 / 19 / 2006$ & & JGJ-066-06 \\
\hline GWM44201VL & HIGHWAY 3 & 567 & 1,2-Dichloropropane & 1 & & $\mathrm{U}$ & & UG/L & $06 / 19 / 2006$ & & JGJ-066-06 \\
\hline GWM44201VL & HIGHWAY 3 & 567 & cis-1,3-Dichloropropene & 1 & & U & & UG/L & $06 / 19 / 2006$ & & JGJ-066-06 \\
\hline GWM44201VL & HIGHWAY 3 & 567 & trans-1,3-Dichloropropene & 1 & & $\mathrm{U}$ & & UG/L & $06 / 19 / 2006$ & & JGJ-066-06 \\
\hline GWM44201VL & HIGHWAY 3 & 567 & Ethylbenzene & 1 & & $\mathrm{U}$ & & UG/L & $06 / 19 / 2006$ & & JGJ-066-06 \\
\hline GWM44201VL & HIGHWAY 3 & 567 & Trichlorofluoromethane & 1 & & U & & UG/L & $06 / 19 / 2006$ & & JGJ-066-06 \\
\hline GWM44201VL & HIGHWAY 3 & 567 & 2-Hexanone & 5 & & $\mathrm{U}$ & $\mathrm{R}$ & UG/L & $06 / 19 / 2006$ & & JGJ-066-06 \\
\hline GWM44201VL & HIGHWAY 3 & 567 & Isopropylbenzene & 1 & & $\mathrm{U}$ & & UG/L & $06 / 19 / 2006$ & & JGJ-066-06 \\
\hline GWM44201VL & HIGHWAY 3 & 567 & Methyl acetate & 5 & & U & $\mathrm{R}$ & UG/L & $06 / 19 / 2006$ & & JGJ-066-06 \\
\hline GWM44201VL & HIGHWAY 3 & 567 & Methyl cyclohexane & 4 & & $\mathrm{U}$ & & UG/L & $06 / 19 / 2006$ & & JGJ-066-06 \\
\hline GWM44201VL & HIGHWAY 3 & 567 & Methylene Chloride & 1 & & $\mathrm{U}$ & & UG/L & $06 / 19 / 2006$ & & JGJ-066-06 \\
\hline GWM44201VL & HIGHWAY 3 & 567 & 4-Methyl-2-pentanone & 5 & & U & & UG/L & $06 / 19 / 2006$ & & JGJ-066-06 \\
\hline GWM44201VL & HIGHWAY 3 & 567 & Styrene & 1 & & $U$ & & UG/L & $06 / 19 / 2006$ & & JGJ-066-06 \\
\hline GWM44201VL & HIGHWAY 3 & 567 & 1,1,2,2-Tetrachloroethane & 1 & & $U$ & & UG/L & $06 / 19 / 2006$ & & JGJ-066-06 \\
\hline GWM44201VL & HIGHWAY 3 & 567 & Tetrachloroethene & 1 & & $\mathrm{U}$ & & UG/L & $06 / 19 / 2006$ & & JGJ-066-06 \\
\hline GWM44201VL & HIGHWAY 3 & 567 & Toluene & 1 & & U & & UG/L & $06 / 19 / 2006$ & & JGJ-066-06 \\
\hline GWM44201VL & HIGHWAY 3 & 567 & 1,2,4-Trichlorobenzene & 1 & & $\mathrm{U}$ & & UG/L & $06 / 19 / 2006$ & & JGJ-066-06 \\
\hline GWM44201VL & HIGHWAY 3 & 567 & 1,1,1-Trichloroethane & 1 & & $U$ & & UG/L & $06 / 19 / 2006$ & & JGJ-066-06 \\
\hline GWM44201VL & HIGHWAY 3 & 567 & 1,1,2-Trichloroethane & 1 & & $\mathrm{U}$ & & UG/L & $06 / 19 / 2006$ & & JGJ-066-06 \\
\hline GWM44201VL & HIGHWAY 3 & 567 & Trichloroethene & 1 & & $\mathrm{U}$ & & UG/L & $06 / 19 / 2006$ & & JGJ-066-06 \\
\hline GWM44201VL & HIGHWAY 3 & 567 & 1,1,2-Trichloro-1,2,2-trifluoroethane & 1 & & $\mathrm{U}$ & & UG/L & $06 / 19 / 2006$ & & JGJ-066-06 \\
\hline GWM44201VL & HIGHWAY 3 & 567 & Vinyl Chloride & 2 & & U & & UG/L & $06 / 19 / 2006$ & & JGJ-066-06 \\
\hline GWM44201VL & HIGHWAY 3 & 567 & Xylene (Total) & 3 & & U & & UG/L & $06 / 19 / 2006$ & & JGJ-066-06 \\
\hline GWM44201VL & HIGHWAY 3 & 567 & Methyl t-butyl ether & 2 & & $\mathrm{U}$ & & UG/L & $06 / 19 / 2006$ & & JGJ-066-06 \\
\hline
\end{tabular}


Table A-2. (continued).

\begin{tabular}{|c|c|c|c|c|c|c|c|c|c|c|c|}
\hline $\begin{array}{c}\text { Field Sample } \\
\text { Number }\end{array}$ & Location & Depth & Compound & $\begin{array}{c}\text { Sample } \\
\text { Result }\end{array}$ & $\begin{array}{l}\text { Sample } \\
\text { Error }\end{array}$ & $\begin{array}{c}\text { Result } \\
\text { Qualifier }\end{array}$ & $\begin{array}{l}\text { Validation } \\
\text { Flag }\end{array}$ & $\begin{array}{c}\text { Sample } \\
\text { Units }\end{array}$ & $\begin{array}{l}\text { Date Sample } \\
\text { Collected }\end{array}$ & MDA & $\begin{array}{l}\text { L\&V Report } \\
\text { Number }\end{array}$ \\
\hline GWM44201R8 & HIGHWAY 3 & 567 & Tritium & $-8.52 \mathrm{E}+01$ & $1.21 \mathrm{E}+02$ & & $U$ & $\mathrm{PCl} / \mathrm{L}$ & $06 / 19 / 2006$ & $4.33 \mathrm{E}+02$ & SOS-TL199-06 \\
\hline GWM44201RH & HIGHWAY 3 & 567 & Gross Alpha & $1.03 E+00$ & 4.29E-01 & & UJ & $\mathrm{PCI} / \mathrm{L}$ & $06 / 19 / 2006$ & $1.48 \mathrm{E}+00$ & SOS-TL198-06 \\
\hline GWM44201RH & HIGHWAY 3 & 567 & Gross Beta & $2.14 \mathrm{E}+00$ & $5.83 \mathrm{E}-01$ & & & $\mathrm{PCl} / \mathrm{L}$ & $06 / 19 / 2006$ & $2.11 \mathrm{E}+00$ & SOS-TL198-06 \\
\hline GWM44201RH & HIGHWAY 3 & 567 & Strontium-90 & $-2.35 \mathrm{E}-01$ & 4.33E-02 & & $u$ & $\mathrm{PCI} / \mathrm{L}$ & $06 / 19 / 2006$ & 3.73E-01 & SOS-TL198-06 \\
\hline GWM44201RH & HIGHWAY 3 & 567 & Technetium-99 & $-7.03 E+00$ & $2.75 \mathrm{E}+00$ & & U & $\mathrm{PCl} / \mathrm{L}$ & $06 / 19 / 2006$ & $9.42 \mathrm{E}+00$ & SOS-TL198-06 \\
\hline GWM44201UX & HIGHWAY 3 & 567 & lodine-129 & $5.40 \mathrm{E}-02$ & $2.91 \mathrm{E}-02$ & & $\mathrm{u}$ & $\mathrm{PCI} / \mathrm{L}$ & $06 / 19 / 2006$ & 1.05E-01 & SOS-TL197-06 \\
\hline GWM452012X & FIELD BLANK & NA & Aluminum & 7.9 & & $U$ & & UG/L & $06 / 21 / 2006$ & & DNT-330-06 \\
\hline GWM452012X & FIELD BLANK & NA & Antimony & 0.5 & & $\mathrm{U}$ & & UG/L & $06 / 21 / 2006$ & & DNT-330-06 \\
\hline GWM452012X & FIELD BLANK & NA & Barium & 1.2 & & B & $\mathrm{U}$ & UG/L & $06 / 21 / 2006$ & & DNT-330-06 \\
\hline GWM452012X & FIELD BLANK & NA & Beryllium & 0.088 & & $U$ & & UG/L & $06 / 21 / 2006$ & & DNT-330-06 \\
\hline GWM452012X & FIELD BLANK & NA & Cadmium & 0.057 & & $\mathrm{U}$ & & UG/L & $06 / 21 / 2006$ & & DNT-330-06 \\
\hline GWM452012X & FIELD BLANK & NA & Calcium & 24.3 & & B & & UG/L & $06 / 21 / 2006$ & & DNT-330-06 \\
\hline GWM452012X & FIELD BLANK & NA & Chromium & 2.8 & & $U$ & & UG/L & $06 / 21 / 2006$ & & DNT-330-06 \\
\hline GWM452012X & FIELD BLANK & NA & Cobalt & 0.31 & & $\mathrm{U}$ & & UG/L & $06 / 21 / 2006$ & & DNT-330-06 \\
\hline GWM452012X & FIELD BLANK & NA & Copper & 0.75 & & B & & UG/L & $06 / 21 / 2006$ & & DNT-330-06 \\
\hline GWM452012X & FIELD BLANK & NA & Iron & 9.4 & & $U$ & & UG/L & $06 / 21 / 2006$ & & DNT-330-06 \\
\hline GWM452012X & FIELD BLANK & NA & Lead & 0.49 & & U & & UG/L & $06 / 21 / 2006$ & & DNT-330-06 \\
\hline GWM452012X & FIELD BLANK & NA & Magnesium & 6.4 & & UE & UJ & UG/L & $06 / 21 / 2006$ & & DNT-330-06 \\
\hline GWM452012X & FIELD BLANK & NA & Nickel & 0.52 & & $U$ & & UG/L & $06 / 21 / 2006$ & & DNT-330-06 \\
\hline GWM452012X & FIELD BLANK & NA & Potassium & 10 & & UE & UJ & UG/L & $06 / 21 / 2006$ & & DNT-330-06 \\
\hline GWM452012X & FIELD BLANK & NA & Selenium & 1 & & $U$ & & UG/L & $06 / 21 / 2006$ & & DNT-330-06 \\
\hline GWM452012X & FIELD BLANK & NA & Silver & 0.2 & & $\mathrm{U}$ & & UG/L & $06 / 21 / 2006$ & & DNT-330-06 \\
\hline GWM452012X & FIELD BLANK & NA & Sodium & 11 & & $\mathrm{U}$ & & UG/L & $06 / 21 / 2006$ & & DNT-330-06 \\
\hline GWM452012X & FIELD BLANK & NA & Strontium & 0.53 & & UE & UJ & UG/L & $06 / 21 / 2006$ & & DNT-330-06 \\
\hline GWM452012X & FIELD BLANK & NA & Thallium & 0.32 & & $\mathrm{U}$ & & UG/L & $06 / 21 / 2006$ & & DNT-330-06 \\
\hline GWM452012X & FIELD BLANK & NA & Uranium & 0.21 & & $\mathrm{U}$ & & UG/L & $06 / 21 / 2006$ & & DNT-330-06 \\
\hline GWM452012X & FIELD BLANK & NA & Vanadium & 1.6 & & $\mathrm{U}$ & & UG/L & $06 / 21 / 2006$ & & DNT-330-06 \\
\hline GWM452012X & FIELD BLANK & NA & Zinc & 2 & & B & $\mathrm{U}$ & UG/L & $06 / 21 / 2006$ & & DNT-330-06 \\
\hline GWM452012X & FIELD BLANK & NA & Mercury & 0.093 & & $\mathrm{U}$ & & UG/L & $06 / 21 / 2006$ & & DNT-330-06 \\
\hline GWM452022X & FIELD BLANK & NA & Aluminum & 7.9 & & $\mathrm{U}$ & & UG/L & $06 / 27 / 2006$ & & DNT-336-06 \\
\hline GWM452022X & FIELD BLANK & NA & Antimony & 0.5 & & U & & UG/L & $06 / 27 / 2006$ & & DNT-336-06 \\
\hline GWM452022X & FIELD BLANK & NA & Arsenic & 2 & & $\mathrm{U}$ & & UG/L & $06 / 27 / 2006$ & & DNT-336-06 \\
\hline GWM452022X & FIELD BLANK & NA & Barium & 0.25 & & $\mathrm{U}$ & & UG/L & $06 / 27 / 2006$ & & DNT-336-06 \\
\hline GWM452022X & FIELD BLANK & NA & Beryllium & 0.088 & & $U$ & & UG/L & $06 / 27 / 2006$ & & DNT-336-06 \\
\hline GWM452022X & FIELD BLANK & NA & Cadmium & 0.057 & & $\mathrm{U}$ & & UG/L & $06 / 27 / 2006$ & & DNT-336-06 \\
\hline GWM452022X & FIELD BLANK & NA & Calcium & 21 & & U & & UG/L & $06 / 27 / 2006$ & & DNT-336-06 \\
\hline GWM452022X & FIELD BLANK & NA & Chromium & 2.8 & & U & & UG/L & $06 / 27 / 2006$ & & DNT-336-06 \\
\hline GWM452022X & FIELD BLANK & NA & Cobalt & 0.31 & & $U$ & & UG/L & $06 / 27 / 2006$ & & DNT-336-06 \\
\hline GWM452022X & FIELD BLANK & NA & Magnesium & 6.4 & & U & & UG/L & $06 / 27 / 2006$ & & DNT-336-06 \\
\hline GWM452022X & FIELD BLANK & NA & Manganese & 0.34 & & U & & UG/L & $06 / 27 / 2006$ & & DNT-336-06 \\
\hline GWM452022X & FIELD BLANK & NA & Nickel & 0.52 & & U & & UG/L & $06 / 27 / 2006$ & & DNT-336-06 \\
\hline GWM452022X & FIELD BLANK & NA & Potassium & 10 & & $\mathrm{U}$ & & UG/L & $06 / 27 / 2006$ & & DNT-336-06 \\
\hline GWM452022X & FIELD BLANK & NA & Selenium & 1 & & $\mathrm{U}$ & & UG/L & $06 / 27 / 2006$ & & DNT-336-06 \\
\hline GWM452022X & FIELD BLANK & NA & Silver & 0.2 & & $U$ & & UG/L & $06 / 27 / 2006$ & & DNT-336-06 \\
\hline GWM452022X & FIELD BLANK & NA & Sodium & 11 & & $U$ & & UG/L & $06 / 27 / 2006$ & & DNT-336-06 \\
\hline GWM452022X & FIELD BLANK & NA & Strontium & 0.53 & & $\mathrm{U}$ & & UG/L & $06 / 27 / 2006$ & & DNT-336-06 \\
\hline GWM452022X & FIELD BLANK & NA & Thallium & 0.32 & & U & & UG/L & $06 / 27 / 2006$ & & DNT-336-06 \\
\hline GWM452022X & FIELD BLANK & NA & Uranium & 0.21 & & U & & UG/L & $06 / 27 / 2006$ & & DNT-336-06 \\
\hline GWM452022X & FIELD BLANK & NA & Vanadium & 1.6 & & $\mathrm{U}$ & & UG/L & $06 / 27 / 2006$ & & DNT-336-06 \\
\hline GWM452022X & FIELD BLANK & NA & Zinc & 2.8 & & $\mathrm{BE}$ & $\mathrm{U}$ & UG/L & $06 / 27 / 2006$ & & DNT-336-06 \\
\hline GWM452022X & FIELD BLANK & NA & Mercury & 0.14 & & B & & UG/L & $06 / 27 / 2006$ & & DNT-336-06 \\
\hline GWM45202A1 & FIELD BLANK & NA & Total Alkalinity & 5 & & $\mathrm{U}$ & & $M G / L$ & $06 / 27 / 2006$ & & DNT-324-06 \\
\hline GWM45202AN & FIELD BLANK & NA & Chloride & 2 & & $\mathrm{JU}$ & & $M G / L$ & $06 / 27 / 2006$ & & DNT-324-06 \\
\hline GWM45202AN & FIELD BLANK & NA & Fluoride & 0.1 & & $\mathrm{U}$ & & $M G / L$ & $06 / 27 / 2006$ & & DNT-324-06 \\
\hline GWM45202AN & FIELD BLANK & NA & Sulfate & 5 & & $\mathrm{U}$ & & $M G / L$ & $06 / 27 / 2006$ & & DNT-324-06 \\
\hline GWM45202N2 & FIELD BLANK & NA & Nitrate/Nitrite as $\mathrm{N}$ & 50 & & $\mathrm{U}$ & & UG/L & $06 / 27 / 2006$ & & DNT-324-06 \\
\hline GWM45201A1 & FIELD BLANK & NA & Total Alkalinity & 5 & & $U$ & & $M G / L$ & $06 / 21 / 2006$ & & DNT-282-06 \\
\hline GWM45201AN & FIELD BLANK & NA & Chloride & 0.2 & & $\mathrm{JU}$ & & $M G / L$ & $06 / 21 / 2006$ & & DNT-282-06 \\
\hline GWM45201AN & FIELD BLANK & NA & Fluoride & 0.1 & & $\mathrm{U}$ & & $M G / L$ & $06 / 21 / 2006$ & & DNT-282-06 \\
\hline GWM45201AN & FIELD BLANK & NA & Sulfate & 0.5 & & U & & $M G / L$ & $06 / 21 / 2006$ & & DNT-282-06 \\
\hline GWM45201N2 & FIELD BLANK & NA & Nitrate/Nitrite as $\mathrm{N}$ & 50 & & $\mathrm{U}$ & & UG/L & $06 / 21 / 2006$ & & DNT-282-06 \\
\hline GWM45301VL & TRIP BLANK & NA & Acetone & 2 & & $\mathrm{U}$ & & UG/L & $06 / 12 / 2006$ & & JGJ-062-06 \\
\hline GWM45301VL & TRIP BLANK & NA & Benzene & 1 & & $U$ & & UG/L & $06 / 12 / 2006$ & & JGJ-062-06 \\
\hline GWM45301VL & TRIP BLANK & NA & Bromodichloromethane & 1 & & $\mathrm{U}$ & & UG/L & $06 / 12 / 2006$ & & JGJ-062-06 \\
\hline GWM45301VL & TRIP BLANK & NA & Bromoform & 1 & & $\mathrm{U}$ & & UG/L & $06 / 12 / 2006$ & & JGJ-062-06 \\
\hline GWM45301VL & TRIP BLANK & NA & Bromomethane & 2 & & $\mathrm{U}$ & & UG/L & $06 / 12 / 2006$ & & JGJ-062-06 \\
\hline GWM45301VL & TRIP BLANK & NA & 2-Butanone & 5 & & $U$ & $\mathrm{R}$ & UG/L & $06 / 12 / 2006$ & & JGJ-062-06 \\
\hline GWM45301VL & TRIP BLANK & NA & Carbon disulfide & 1 & & $\mathrm{U}$ & & UG/L & $06 / 12 / 2006$ & & JGJ-062-06 \\
\hline GWM45301VL & TRIP BLANK & NA & Carbon tetrachloride & 1 & & $\mathrm{U}$ & & UG/L & $06 / 12 / 2006$ & & JGJ-062-06 \\
\hline GWM45301VL & TRIP BLANK & NA & Chlorobenzene & 1 & & U & & UG/L & $06 / 12 / 2006$ & & JGJ-062-06 \\
\hline GWM45301VL & TRIP BLANK & NA & Dibromochloromethane & 1 & & U & & UG/L & $06 / 12 / 2006$ & & JGJ-062-06 \\
\hline GWM45301VL & TRIP BLANK & NA & 1,2-Dibromo-3-chloropropane & 1 & & $\mathrm{U}$ & & UG/L & $06 / 12 / 2006$ & & JGJ-062-06 \\
\hline GWM45301VL & TRIP BLANK & NA & Chloroethane & 2 & & $\mathrm{U}$ & & UG/L & $06 / 12 / 2006$ & & JGJ-062-06 \\
\hline
\end{tabular}


Table A-2. (continued).

\begin{tabular}{|c|c|c|c|c|c|c|c|c|c|c|c|}
\hline $\begin{array}{l}\text { Field Sample } \\
\text { Number }\end{array}$ & Location & Depth & Compound & $\begin{array}{c}\text { Sample } \\
\text { Result }\end{array}$ & $\begin{array}{l}\text { Sample } \\
\text { Error }\end{array}$ & $\begin{array}{c}\text { Result } \\
\text { Qualifier }\end{array}$ & $\begin{array}{c}\text { Validation } \\
\text { Flag }\end{array}$ & $\begin{array}{c}\text { Sample } \\
\text { Units }\end{array}$ & $\begin{array}{c}\text { Date Sample } \\
\text { Collected }\end{array}$ & MDA & $\begin{array}{l}\text { L\&V Report } \\
\text { Number }\end{array}$ \\
\hline GWM45301VL & TRIP BLANK & NA & Chloroform & 1 & & $U$ & & UG/L & $06 / 12 / 2006$ & & JGJ-062-06 \\
\hline GWM45301VL & TRIP BLANK & NA & Chloromethane & 2 & & $\mathrm{U}$ & & UG/L & $06 / 12 / 2006$ & & JGJ-062-06 \\
\hline GWM45301VL & TRIP BLANK & NA & Cyclohexane & 1 & & $u$ & & UG/L & $06 / 12 / 2006$ & & JGJ-062-06 \\
\hline GWM45301VL & TRIP BLANK & NA & 1,2-Dichlorobenzene & 1 & & U & & UG/L & $06 / 12 / 2006$ & & JGJ-062-06 \\
\hline GWM45301VL & TRIP BLANK & NA & 1,3-Dichlorobenzene & 1 & & $\mathrm{U}$ & & UG/L & $06 / 12 / 2006$ & & JGJ-062-06 \\
\hline GWM45301VL & TRIP BLANK & NA & 1,4-Dichlorobenzene & 1 & & $u$ & & UG/L & $06 / 12 / 2006$ & & JGJ-062-06 \\
\hline GWM45301VL & TRIP BLANK & NA & Dichlorodifluoromethane & 2 & & $\mathrm{U}$ & & UG/L & $06 / 12 / 2006$ & & JGJ-062-06 \\
\hline GWM45301VL & TRIP BLANK & NA & 1,2-Dichloroethane & 1 & & U & & UG/L & $06 / 12 / 2006$ & & JGJ-062-06 \\
\hline GWM45301VL & TRIP BLANK & NA & 1,1-Dichloroethene & 1 & & u & & UG/L & $06 / 12 / 2006$ & & JGJ-062-06 \\
\hline GWM45301VL & TRIP BLANK & NA & cis-1,2-Dichloroethene & 1 & & $\mathrm{U}$ & & UG/L & $06 / 12 / 2006$ & & JGJ-062-06 \\
\hline GWM45301VL & TRIP BLANK & NA & trans-1,2-Dichloroethene & 1 & & u & & UG/L & $06 / 12 / 2006$ & & JGJ-062-06 \\
\hline GWM45301VL & TRIP BLANK & NA & 1,2-Dichloropropane & 1 & & $\mathrm{U}$ & & UG/L & $06 / 12 / 2006$ & & JGJ-062-06 \\
\hline GWM45301VL & TRIP BLANK & NA & cis-1,3-Dichloropropene & 1 & & $\mathrm{U}$ & & UG/L & $06 / 12 / 2006$ & & JGJ-062-06 \\
\hline GWM45301VL & TRIP BLANK & NA & trans-1,3-Dichloropropene & 1 & & U & & UG/L & $06 / 12 / 2006$ & & JGJ-062-06 \\
\hline GWM45301VL & TRIP BLANK & NA & Ethylbenzene & 1 & & $\mathrm{u}$ & & UG/L & $06 / 12 / 2006$ & & JGJ-062-06 \\
\hline GWM45301VL & TRIP BLANK & NA & Trichlorofluoromethane & 1 & & $u$ & & UG/L & $06 / 12 / 2006$ & & JGJ-062-06 \\
\hline GWM45301VL & TRIP BLANK & NA & 2-Hexanone & 5 & & $\mathrm{U}$ & & UG/L & $06 / 12 / 2006$ & & JGJ-062-06 \\
\hline GWM45301VL & TRIP BLANK & NA & Methyl cyclohexane & 4 & & $u$ & & UG/L & $06 / 12 / 2006$ & & JGJ-062-06 \\
\hline GWM45301VL & TRIP BLANK & NA & Methylene Chloride & 1 & & $\mathrm{U}$ & & UG/L & $06 / 12 / 2006$ & & JGJ-062-06 \\
\hline GWM45301VL & TRIP BLANK & NA & 4-Methyl-2-pentanone & 5 & & $\mathrm{U}$ & & UG/L & $06 / 12 / 2006$ & & JGJ-062-06 \\
\hline GWM45301VL & TRIP BLANK & NA & Styrene & 1 & & $\mathrm{U}$ & & UG/L & $06 / 12 / 2006$ & & JGJ-062-06 \\
\hline GWM45301VL & TRIP BLANK & NA & 1,1,2,2-Tetrachloroethane & 1 & & U & & UG/L & $06 / 12 / 2006$ & & JGJ-062-06 \\
\hline GWM45301VL & TRIP BLANK & NA & Tetrachloroethene & 1 & & $\mathrm{u}$ & & UG/L & $06 / 12 / 2006$ & & JGJ-062-06 \\
\hline GWM45301VL & TRIP BLANK & NA & Toluene & 1 & & $\mathrm{U}$ & & UG/L & $06 / 12 / 2006$ & & JGJ-062-06 \\
\hline GWM45301VL & TRIP BLANK & NA & 1,2,4-Trichlorobenzene & 1 & & $\mathrm{U}$ & & UG/L & $06 / 12 / 2006$ & & JGJ-062-06 \\
\hline GWM45301VL & TRIP BLANK & NA & 1,1,1-Trichloroethane & 1 & & $\mathrm{u}$ & & UG/L & $06 / 12 / 2006$ & & JGJ-062-06 \\
\hline GWM45301VL & TRIP BLANK & NA & 1,1,2-Trichloroethane & 1 & & U & & UG/L & $06 / 12 / 2006$ & & JGJ-062-06 \\
\hline GWM45301VL & TRIP BLANK & NA & Trichloroethene & 1 & & $\mathrm{U}$ & & UG/L & $06 / 12 / 2006$ & & JGJ-062-06 \\
\hline GWM45301VL & TRIP BLANK & NA & 1,1,2-Trichloro-1,2,2-trifluoroethane & 1 & & $\mathrm{u}$ & & UG/L & $06 / 12 / 2006$ & & JGJ-062-06 \\
\hline GWM45301VL & TRIP BLANK & NA & Vinyl Chloride & 2 & & $\mathrm{U}$ & & UG/L & $06 / 12 / 2006$ & & JGJ-062-06 \\
\hline GWM45301VL & TRIP BLANK & NA & Xylene (Total) & 3 & & U & & UG/L & $06 / 12 / 2006$ & & JGJ-062-06 \\
\hline GWM45301VL & TRIP BLANK & NA & Methyl t-butyl ether & 2 & & U & & UG/L & $06 / 12 / 2006$ & & JGJ-062-06 \\
\hline GWM45201VL & FIELD BLANK & NA & Acetone & 2 & & $\mathrm{U}$ & $\mathrm{R}$ & UG/L & $06 / 21 / 2006$ & & JGJ-066-06 \\
\hline GWM45201VL & FIELD BLANK & NA & Benzene & 1 & & $\mathrm{U}$ & & UG/L & $06 / 21 / 2006$ & & JGJ-066-06 \\
\hline GWM45201VL & FIELD BLANK & NA & Bromodichloromethane & 1 & & $\mathrm{u}$ & & UG/L & $06 / 21 / 2006$ & & JGJ-066-06 \\
\hline GWM45201VL & FIELD BLANK & NA & Bromoform & 1 & & $\mathrm{U}$ & & UG/L & $06 / 21 / 2006$ & & JGJ-066-06 \\
\hline GWM45201VL & FIELD BLANK & NA & Carbon tetrachloride & 1 & & $\mathrm{U}$ & & UG/L & $06 / 21 / 2006$ & & JGJ-066-06 \\
\hline GWM45201VL & FIELD BLANK & NA & Chlorobenzene & 1 & & $\mathrm{U}$ & & UG/L & $06 / 21 / 2006$ & & JGJ-066-06 \\
\hline GWM45201VL & FIELD BLANK & NA & Dibromochloromethane & 1 & & $\mathrm{u}$ & & UG/L & $06 / 21 / 2006$ & & JGJ-066-06 \\
\hline GWM45201VL & FIELD BLANK & NA & 1,2-Dibromo-3-chloropropane & 1 & & $\mathrm{U}$ & $\mathrm{R}$ & UG/L & $06 / 21 / 2006$ & & JGJ-066-06 \\
\hline GWM45201VL & FIELD BLANK & NA & Chloroethane & 2 & & $\mathrm{U}$ & & UG/L & $06 / 21 / 2006$ & & JGJ-066-06 \\
\hline GWM45201VL & FIELD BLANK & NA & Chloroform & 1 & & U & & UG/L & $06 / 21 / 2006$ & & JGJ-066-06 \\
\hline GWM45201VL & FIELD BLANK & NA & Chloromethane & 2 & & $\mathrm{U}$ & & UG/L & $06 / 21 / 2006$ & & JGJ-066-06 \\
\hline GWM45201VL & FIELD BLANK & NA & Cyclohexane & 1 & & $\mathrm{U}$ & & UG/L & $06 / 21 / 2006$ & & JGJ-066-06 \\
\hline GWM45201VL & FIELD BLANK & NA & 1,2-Dibromoethane & 1 & & U & & UG/L & $06 / 21 / 2006$ & & JGJ-066-06 \\
\hline GWM45201VL & FIELD BLANK & NA & 1,2-Dichlorobenzene & 1 & & $\mathrm{u}$ & & UG/L & $06 / 21 / 2006$ & & JGJ-066-06 \\
\hline GWM45201VL & FIELD BLANK & NA & 1,3-Dichlorobenzene & 1 & & U & & UG/L & $06 / 21 / 2006$ & & JGJ-066-06 \\
\hline GWM45201VL & FIELD BLANK & NA & 1,4-Dichlorobenzene & 1 & & $\mathrm{U}$ & & UG/L & $06 / 21 / 2006$ & & JGJ-066-06 \\
\hline GWM45201VL & FIELD BLANK & NA & Dichlorodifluoromethane & 2 & & $\mathrm{u}$ & & UG/L & $06 / 21 / 2006$ & & JGJ-066-06 \\
\hline GWM45201VL & FIELD BLANK & NA & 1,1-Dichloroethane & 1 & & $\mathrm{U}$ & & UG/L & $06 / 21 / 2006$ & & JGJ-066-06 \\
\hline GWM45201VL & FIELD BLANK & NA & 1,2-Dichloroethane & 1 & & U & & UG/L & $06 / 21 / 2006$ & & JGJ-066-06 \\
\hline GWM45201VL & FIELD BLANK & NA & 1,1-Dichloroethene & 1 & & $\mathrm{u}$ & & UG/L & $06 / 21 / 2006$ & & JGJ-066-06 \\
\hline GWM45201VL & FIELD BLANK & NA & cis-1,2-Dichloroethene & 1 & & $\mathrm{U}$ & & UG/L & $06 / 21 / 2006$ & & JGJ-066-06 \\
\hline GWM45201VL & FIELD BLANK & NA & trans-1,2-Dichloroethene & 1 & & $\mathrm{U}$ & & UG/L & $06 / 21 / 2006$ & & JGJ-066-06 \\
\hline GWM45201VL & FIELD BLANK & NA & 1,2-Dichloropropane & 1 & & U & & UG/L & $06 / 21 / 2006$ & & JGJ-066-06 \\
\hline GWM45201VL & FIELD BLANK & NA & cis-1,3-Dichloropropene & 1 & & $\mathrm{U}$ & & UG/L & $06 / 21 / 2006$ & & JGJ-066-06 \\
\hline GWM45201VL & FIELD BLANK & NA & trans-1,3-Dichloropropene & 1 & & $\mathrm{U}$ & & UG/L & $06 / 21 / 2006$ & & JGJ-066-06 \\
\hline GWM45201VL & FIELD BLANK & NA & Ethylbenzene & 1 & & $\mathrm{u}$ & & UG/L & $06 / 21 / 2006$ & & JGJ-066-06 \\
\hline GWM45201VL & FIELD BLANK & NA & Trichlorofluoromethane & 1 & & $\mathrm{U}$ & & UG/L & $06 / 21 / 2006$ & & JGJ-066-06 \\
\hline GWM45201VL & FIELD BLANK & NA & 2-Hexanone & 5 & & U & $\mathrm{R}$ & UG/L & $06 / 21 / 2006$ & & JGJ-066-06 \\
\hline GWM45201VL & FIELD BLANK & NA & Isopropylbenzene & 1 & & U & & UG/L & $06 / 21 / 2006$ & & JGJ-066-06 \\
\hline GWM45201VL & FIELD BLANK & NA & Methyl acetate & 5 & & $\mathrm{u}$ & $\mathrm{R}$ & UG/L & $06 / 21 / 2006$ & & JGJ-066-06 \\
\hline GWM45201VL & FIELD BLANK & NA & Methyl cyclohexane & 4 & & $\mathrm{U}$ & & UG/L & $06 / 21 / 2006$ & & JGJ-066-06 \\
\hline GWM45201VL & FIELD BLANK & NA & Methylene Chloride & 1 & & $U$ & & UG/L & $06 / 21 / 2006$ & & JGJ-066-06 \\
\hline GWM45201VL & FIELD BLANK & NA & 4-Methyl-2-pentanone & 5 & & $U$ & & UG/L & $06 / 21 / 2006$ & & JGJ-066-06 \\
\hline GWM45201VL & FIELD BLANK & NA & Styrene & 1 & & $\mathrm{U}$ & & UG/L & $06 / 21 / 2006$ & & JGJ-066-06 \\
\hline GWM45201VL & FIELD BLANK & NA & 1,1,2,2-Tetrachloroethane & 1 & & $\mathrm{U}$ & & UG/L & $06 / 21 / 2006$ & & JGJ-066-06 \\
\hline GWM45201VL & FIELD BLANK & NA & Tetrachloroethene & 1 & & U & & UG/L & $06 / 21 / 2006$ & & JGJ-066-06 \\
\hline GWM45201VL & FIELD BLANK & NA & Toluene & 1 & & $\mathrm{U}$ & & UG/L & $06 / 21 / 2006$ & & JGJ-066-06 \\
\hline GWM45201VL & FIELD BLANK & NA & 1,2,4-Trichlorobenzene & 1 & & $U$ & & UG/L & $06 / 21 / 2006$ & & JGJ-066-06 \\
\hline GWM45201VL & FIELD BLANK & NA & 1,1,1-Trichloroethane & 1 & & U & & UG/L & $06 / 21 / 2006$ & & JGJ-066-06 \\
\hline
\end{tabular}


Table A-2. (continued).

\begin{tabular}{|c|c|c|c|c|c|c|c|c|c|c|c|}
\hline $\begin{array}{l}\text { Field Sample } \\
\text { Number }\end{array}$ & Location & Depth & Compound & $\begin{array}{c}\text { Sample } \\
\text { Result }\end{array}$ & $\begin{array}{l}\text { Sample } \\
\text { Error }\end{array}$ & $\begin{array}{c}\text { Result } \\
\text { Qualifier }\end{array}$ & $\begin{array}{c}\text { Validation } \\
\text { Flag }\end{array}$ & $\begin{array}{c}\text { Sample } \\
\text { Units }\end{array}$ & $\begin{array}{c}\text { Date Sample } \\
\text { Collected }\end{array}$ & MDA & $\begin{array}{l}\text { L\&V Report } \\
\text { Number }\end{array}$ \\
\hline GWM45201VL & FIELD BLANK & NA & 1,1,2-Trichloroethane & 1 & & $U$ & & UG/L & $06 / 21 / 2006$ & & JGJ-066-06 \\
\hline GWM45201VL & FIELD BLANK & NA & Trichloroethene & 1 & & $\mathrm{U}$ & & UG/L & $06 / 21 / 2006$ & & JGJ-066-06 \\
\hline GWM45201VL & FIELD BLANK & NA & 1,1,2-Trichloro-1,2,2-trifluoroethane & 1 & & $u$ & & UG/L & $06 / 21 / 2006$ & & JGJ-066-06 \\
\hline GWM45201VL & FIELD BLANK & NA & Xylene (Total) & 3 & & U & & UG/L & $06 / 21 / 2006$ & & JGJ-066-06 \\
\hline GWM45201VL & FIELD BLANK & NA & Methyl t-butyl ether & 2 & & $\mathrm{U}$ & & UG/L & $06 / 21 / 2006$ & & JGJ-066-06 \\
\hline GWM45302VL & TRIP BLANK & NA & Acetone & 2 & & $\mathrm{u}$ & $\mathrm{R}$ & UG/L & $06 / 19 / 2006$ & & JGJ-066-06 \\
\hline GWM45302VL & TRIP BLANK & NA & Benzene & 1 & & $\mathrm{U}$ & & UG/L & $06 / 19 / 2006$ & & JGJ-066-06 \\
\hline GWM45302VL & TRIP BLANK & NA & Bromoform & 1 & & U & & UG/L & $06 / 19 / 2006$ & & JGJ-066-06 \\
\hline GWM45302VL & TRIP BLANK & NA & Bromomethane & 2 & & u & & UG/L & $06 / 19 / 2006$ & & JGJ-066-06 \\
\hline GWM45302VL & TRIP BLANK & NA & 2-Butanone & 5 & & $\mathrm{U}$ & $\mathrm{R}$ & UG/L & $06 / 19 / 2006$ & & JGJ-066-06 \\
\hline GWM45302VL & TRIP BLANK & NA & Carbon disulfide & 1 & & u & & UG/L & $06 / 19 / 2006$ & & JGJ-066-06 \\
\hline GWM45302VL & TRIP BLANK & NA & Carbon tetrachloride & 1 & & $\mathrm{U}$ & & UG/L & 06/19/2006 & & JGJ-066-06 \\
\hline GWM45302VL & TRIP BLANK & NA & Chlorobenzene & 1 & & $\mathrm{U}$ & & UG/L & 06/19/2006 & & JGJ-066-06 \\
\hline GWM45302VL & TRIP BLANK & NA & Dibromochloromethane & 1 & & $\mathrm{U}$ & & UG/L & $06 / 19 / 2006$ & & JGJ-066-06 \\
\hline GWM45302VL & TRIP BLANK & NA & 1,2-Dibromo-3-chloropropane & 1 & & $\mathrm{u}$ & $\mathrm{R}$ & UG/L & $06 / 19 / 2006$ & & JGJ-066-06 \\
\hline GWM45302VL & TRIP BLANK & NA & Chloroethane & 2 & & $u$ & & UG/L & $06 / 19 / 2006$ & & JGJ-066-06 \\
\hline GWM45302VL & TRIP BLANK & NA & Chloroform & 1 & & $\mathrm{U}$ & & UG/L & $06 / 19 / 2006$ & & JGJ-066-06 \\
\hline GWM45302VL & TRIP BLANK & NA & 1,2-Dibromoethane & 1 & & $u$ & & UG/L & $06 / 19 / 2006$ & & JGJ-066-06 \\
\hline GWM45302VL & TRIP BLANK & NA & 1,2-Dichlorobenzene & 1 & & $\mathrm{U}$ & & UG/L & $06 / 19 / 2006$ & & JGJ-066-06 \\
\hline GWM45302VL & TRIP BLANK & NA & 1,3-Dichlorobenzene & 1 & & $\mathrm{U}$ & & UG/L & $06 / 19 / 2006$ & & JGJ-066-06 \\
\hline GWM45302VL & TRIP BLANK & NA & 1,4-Dichlorobenzene & 1 & & $\mathrm{U}$ & & UG/L & $06 / 19 / 2006$ & & JGJ-066-06 \\
\hline GWM45302VL & TRIP BLANK & NA & Dichlorodifluoromethane & 2 & & $u$ & & UG/L & $06 / 19 / 2006$ & & JGJ-066-06 \\
\hline GWM45302VL & TRIP BLANK & NA & 1,1-Dichloroethane & 1 & & $\mathrm{U}$ & & UG/L & $06 / 19 / 2006$ & & JGJ-066-06 \\
\hline GWM45302VL & TRIP BLANK & NA & 1,2-Dichloroethane & 1 & & $\mathrm{U}$ & & UG/L & 06/19/2006 & & JGJ-066-06 \\
\hline GWM45302VL & TRIP BLANK & NA & 1,1-Dichloroethene & 1 & & U & & UG/L & 06/19/2006 & & JGJ-066-06 \\
\hline GWM45302VL & TRIP BLANK & NA & cis-1,2-Dichloroethene & 1 & & $\mathrm{u}$ & & UG/L & $06 / 19 / 2006$ & & JGJ-066-06 \\
\hline GWM45302VL & TRIP BLANK & NA & trans-1,2-Dichloroethene & 1 & & $\mathrm{U}$ & & UG/L & 06/19/2006 & & JGJ-066-06 \\
\hline GWM45302VL & TRIP BLANK & NA & 1,2-Dichloropropane & 1 & & $\mathrm{U}$ & & UG/L & $06 / 19 / 2006$ & & JGJ-066-06 \\
\hline GWM45302VL & TRIP BLANK & NA & cis-1,3-Dichloropropene & 1 & & $\mathrm{u}$ & & UG/L & $06 / 19 / 2006$ & & JGJ-066-06 \\
\hline GWM45302VL & TRIP BLANK & NA & trans-1,3-Dichloropropene & 1 & & $\mathrm{U}$ & & UG/L & 06/19/2006 & & JGJ-066-06 \\
\hline GWM45302VL & TRIP BLANK & NA & Ethylbenzene & 1 & & $u$ & & UG/L & 06/19/2006 & & JGJ-066-06 \\
\hline GWM45302VL & TRIP BLANK & NA & Trichlorofluoromethane & 1 & & $u$ & & UG/L & $06 / 19 / 2006$ & & JGJ-066-06 \\
\hline GWM45302VL & TRIP BLANK & NA & 2-Hexanone & 5 & & $\mathrm{U}$ & $\mathrm{R}$ & UG/L & $06 / 19 / 2006$ & & JGJ-066-06 \\
\hline GWM45302VL & TRIP BLANK & NA & Isopropylbenzene & 1 & & $\mathrm{U}$ & & UG/L & 06/19/2006 & & JGJ-066-06 \\
\hline GWM45302VL & TRIP BLANK & NA & Methyl acetate & 5 & & $\mathrm{U}$ & $\mathrm{R}$ & UG/L & $06 / 19 / 2006$ & & JGJ-066-06 \\
\hline GWM45302VL & TRIP BLANK & NA & Methyl cyclohexane & 4 & & $u$ & & UG/L & $06 / 19 / 2006$ & & JGJ-066-06 \\
\hline GWM45302VL & TRIP BLANK & NA & 1,1,2,2-Tetrachloroethane & 1 & & $\mathrm{U}$ & & UG/L & 06/19/2006 & & JGJ-066-06 \\
\hline GWM45302VL & TRIP BLANK & NA & Tetrachloroethene & 1 & & $\mathrm{U}$ & & UG/L & $06 / 19 / 2006$ & & JGJ-066-06 \\
\hline GWM45302VL & TRIP BLANK & NA & Toluene & 1 & & $\mathrm{u}$ & & UG/L & $06 / 19 / 2006$ & & JGJ-066-06 \\
\hline GWM45302VL & TRIP BLANK & NA & 1,2,4-Trichlorobenzene & 1 & & $\mathrm{U}$ & & UG/L & 06/19/2006 & & JGJ-066-06 \\
\hline GWM45302VL & TRIP BLANK & NA & 1,1,1-Trichloroethane & 1 & & $\mathrm{U}$ & & UG/L & $06 / 19 / 2006$ & & JGJ-066-06 \\
\hline GWM45302VL & TRIP BLANK & NA & 1,1,2-Trichloroethane & 1 & & $u$ & & UG/L & $06 / 19 / 2006$ & & JGJ-066-06 \\
\hline GWM45302VL & TRIP BLANK & NA & Trichloroethene & 1 & & $\mathrm{U}$ & & UG/L & $06 / 19 / 2006$ & & JGJ-066-06 \\
\hline GWM45302VL & TRIP BLANK & NA & 1,1,2-Trichloro-1,2,2-trifluoroethane & 1 & & $\mathrm{U}$ & & UG/L & $06 / 19 / 2006$ & & JGJ-066-06 \\
\hline GWM45302VL & TRIP BLANK & NA & Vinyl Chloride & 2 & & $u$ & & UG/L & $06 / 19 / 2006$ & & JGJ-066-06 \\
\hline GWM45302VL & TRIP BLANK & NA & Xylene (Total) & 3 & & $\mathrm{u}$ & & UG/L & 06/19/2006 & & JGJ-066-06 \\
\hline GWM45302VL & TRIP BLANK & NA & Methyl t-butyl ether & 2 & & $\mathrm{U}$ & & UG/L & $06 / 19 / 2006$ & & JGJ-066-06 \\
\hline GWM45202VL & FIELD BLANK & NA & Acetone & 2 & & $\mathrm{U}$ & $\mathrm{R}$ & UG/L & $06 / 27 / 2006$ & & JGJ-067-06 \\
\hline GWM45202VL & FIELD BLANK & NA & Benzene & 1 & & $\mathrm{u}$ & & UG/L & $06 / 27 / 2006$ & & JGJ-067-06 \\
\hline GWM45202VL & FIELD BLANK & NA & Bromodichloromethane & 1 & & $\mathrm{U}$ & & UG/L & $06 / 27 / 2006$ & & JGJ-067-06 \\
\hline GWM45202VL & FIELD BLANK & NA & Bromoform & 1 & & U & & UG/L & $06 / 27 / 2006$ & & JGJ-067-06 \\
\hline GWM45202VL & FIELD BLANK & NA & Bromomethane & 2 & & $\mathrm{u}$ & & UG/L & $06 / 27 / 2006$ & & JGJ-067-06 \\
\hline GWM45202VL & FIELD BLANK & NA & 2-Butanone & 5 & & $\mathrm{U}$ & $\mathrm{R}$ & UG/L & $06 / 27 / 2006$ & & JGJ-067-06 \\
\hline GWM45202VL & FIELD BLANK & NA & Carbon disulfide & 1 & & $\mathrm{U}$ & & UG/L & $06 / 27 / 2006$ & & JGJ-067-06 \\
\hline GWM45202VL & FIELD BLANK & NA & Carbon tetrachloride & 1 & & $\mathrm{u}$ & & UG/L & $06 / 27 / 2006$ & & JGJ-067-06 \\
\hline GWM45202VL & FIELD BLANK & NA & Chlorobenzene & 1 & & $\mathrm{U}$ & & UG/L & $06 / 27 / 2006$ & & JGJ-067-06 \\
\hline GWM45202VL & FIELD BLANK & NA & Dibromochloromethane & 1 & & $\mathrm{U}$ & & UG/L & $06 / 27 / 2006$ & & JGJ-067-06 \\
\hline GWM45202VL & FIELD BLANK & NA & 1,2-Dibromo-3-chloropropane & 1 & & $\mathrm{u}$ & & UG/L & $06 / 27 / 2006$ & & JGJ-067-06 \\
\hline GWM45202VL & FIELD BLANK & NA & Chloroethane & 2 & & $\mathrm{U}$ & & UG/L & $06 / 27 / 2006$ & & JGJ-067-06 \\
\hline GWM45202VL & FIELD BLANK & NA & Chloroform & 1 & & $\mathrm{U}$ & & UG/L & $06 / 27 / 2006$ & & JGJ-067-06 \\
\hline GWM45202VL & FIELD BLANK & NA & Chloromethane & 2 & & U & & UG/L & $06 / 27 / 2006$ & & JGJ-067-06 \\
\hline GWM45202VL & FIELD BLANK & NA & Cyclohexane & 1 & & $\mathrm{u}$ & & UG/L & $06 / 27 / 2006$ & & JGJ-067-06 \\
\hline GWM45202VL & FIELD BLANK & NA & 1,2-Dibromoethane & 1 & & $\mathrm{U}$ & & UG/L & $06 / 27 / 2006$ & & JGJ-067-06 \\
\hline GWM45202VL & FIELD BLANK & NA & 1,2-Dichlorobenzene & 1 & & U & & UG/L & $06 / 27 / 2006$ & & JGJ-067-06 \\
\hline GWM45202VL & FIELD BLANK & NA & 1,3-Dichlorobenzene & 1 & & $\mathrm{U}$ & & UG/L & $06 / 27 / 2006$ & & JGJ-067-06 \\
\hline GWM45202VL & FIELD BLANK & NA & 1,4-Dichlorobenzene & 1 & & $\mathrm{U}$ & & UG/L & $06 / 27 / 2006$ & & JGJ-067-06 \\
\hline GWM45202VL & FIELD BLANK & NA & Dichlorodifluoromethane & 2 & & $\mathrm{U}$ & & UG/L & $06 / 27 / 2006$ & & JGJ-067-06 \\
\hline GWM45202VL & FIELD BLANK & NA & 1,1-Dichloroethane & 1 & & U & & UG/L & $06 / 27 / 2006$ & & JGJ-067-06 \\
\hline GWM45202VL & FIELD BLANK & NA & 1,2-Dichloroethane & 1 & & $\mathrm{U}$ & & UG/L & 06/27/2006 & & JGJ-067-06 \\
\hline GWM45202VL & FIELD BLANK & NA & 1,1-Dichloroethene & 1 & & $U$ & & UG/L & $06 / 27 / 2006$ & & JGJ-067-06 \\
\hline GWM45202VL & FIELD BLANK & NA & cis-1,2-Dichloroethene & 1 & & $U$ & & UG/L & $06 / 27 / 2006$ & & JGJ-067-06 \\
\hline
\end{tabular}


Table A-2. (continued).

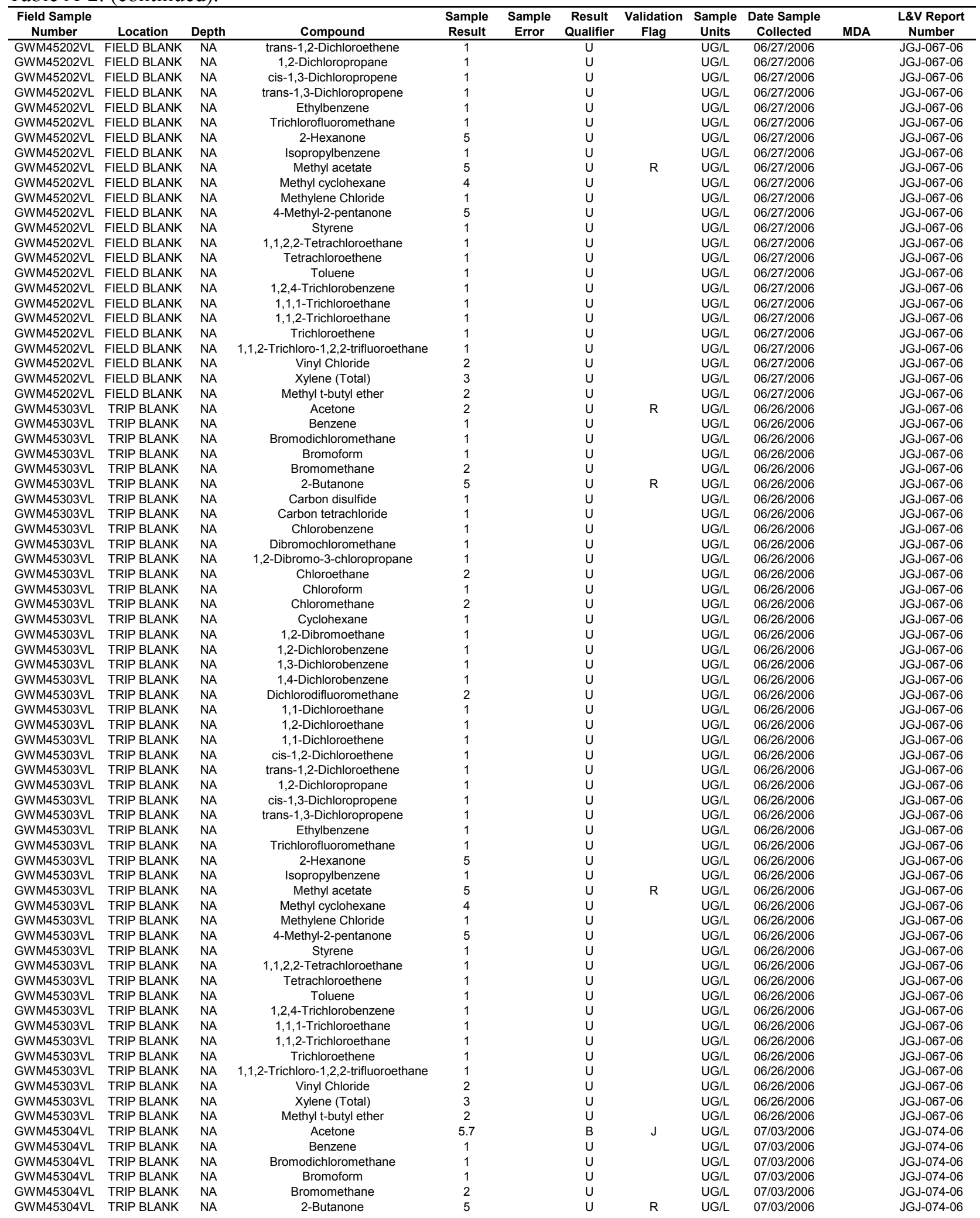


Table A-2. (continued).

\begin{tabular}{|c|c|c|c|c|c|c|c|c|c|c|c|}
\hline $\begin{array}{l}\text { Field Sample } \\
\text { Number }\end{array}$ & Location & Depth & Compound & $\begin{array}{c}\text { Sample } \\
\text { Result }\end{array}$ & $\begin{array}{l}\text { Sample } \\
\text { Error }\end{array}$ & $\begin{array}{c}\text { Result } \\
\text { Qualifier }\end{array}$ & $\begin{array}{c}\text { Validation } \\
\text { Flag }\end{array}$ & $\begin{array}{c}\text { Sample } \\
\text { Units }\end{array}$ & $\begin{array}{c}\text { Date Sample } \\
\text { Collected }\end{array}$ & MDA & $\begin{array}{l}\text { L\&V Report } \\
\text { Number }\end{array}$ \\
\hline GWM45304VL & TRIP BLANK & NA & Carbon disulfide & 1 & & $U$ & & UG/L & $07 / 03 / 2006$ & & JGJ-074-06 \\
\hline GWM45304VL & TRIP BLANK & NA & Carbon tetrachloride & 1 & & $\mathrm{U}$ & & UG/L & 07/03/2006 & & JGJ-074-06 \\
\hline GWM45304VL & TRIP BLANK & NA & Chlorobenzene & 1 & & $u$ & & UG/L & $07 / 03 / 2006$ & & JGJ-074-06 \\
\hline GWM45304VL & TRIP BLANK & NA & 1,2-Dibromo-3-chloropropane & 1 & & U & $\mathrm{R}$ & UG/L & $07 / 03 / 2006$ & & JGJ-074-06 \\
\hline GWM45304VL & TRIP BLANK & NA & Chloroethane & 2 & & $\mathrm{U}$ & & UG/L & $07 / 03 / 2006$ & & JGJ-074-06 \\
\hline GWM45304VL & TRIP BLANK & NA & Chloroform & 1 & & $u$ & & UG/L & $07 / 03 / 2006$ & & JGJ-074-06 \\
\hline GWM45304VL & TRIP BLANK & NA & Chloromethane & 2 & & $\mathrm{U}$ & & UG/L & 07/03/2006 & & JGJ-074-06 \\
\hline GWM45304VL & TRIP BLANK & NA & 1,2-Dibromoethane & 1 & & U & & UG/L & $07 / 03 / 2006$ & & JGJ-074-06 \\
\hline GWM45304VL & TRIP BLANK & NA & 1,2-Dichlorobenzene & 1 & & $\mathrm{U}$ & & UG/L & $07 / 03 / 2006$ & & JGJ-074-06 \\
\hline GWM45304VL & TRIP BLANK & NA & 1,3-Dichlorobenzene & 1 & & $\mathrm{U}$ & & UG/L & $07 / 03 / 2006$ & & JGJ-074-06 \\
\hline GWM45304VL & TRIP BLANK & NA & 1,4-Dichlorobenzene & 1 & & u & & UG/L & $07 / 03 / 2006$ & & JGJ-074-06 \\
\hline GWM45304VL & TRIP BLANK & NA & Dichlorodifluoromethane & 2 & & $\mathrm{U}$ & & UG/L & 07/03/2006 & & JGJ-074-06 \\
\hline GWM45304VL & TRIP BLANK & NA & 1,1-Dichloroethane & 1 & & $\mathrm{U}$ & & UG/L & 07/03/2006 & & JGJ-074-06 \\
\hline GWM45304VL & TRIP BLANK & NA & 1,2-Dichloroethane & 1 & & U & & UG/L & $07 / 03 / 2006$ & & JGJ-074-06 \\
\hline GWM45304VL & TRIP BLANK & NA & 1,1-Dichloroethene & 1 & & $\mathrm{u}$ & & UG/L & $07 / 03 / 2006$ & & JGJ-074-06 \\
\hline GWM45304VL & TRIP BLANK & NA & cis-1,2-Dichloroethene & 1 & & $\mathrm{U}$ & & UG/L & $07 / 03 / 2006$ & & JGJ-074-06 \\
\hline GWM45304VL & TRIP BLANK & NA & trans-1,2-Dichloroethene & 1 & & $\mathrm{U}$ & & UG/L & $07 / 03 / 2006$ & & JGJ-074-06 \\
\hline GWM45304VL & TRIP BLANK & NA & trans-1,3-Dichloropropene & 1 & & $u$ & & UG/L & 07/03/2006 & & JGJ-074-06 \\
\hline GWM45304VL & TRIP BLANK & NA & Ethylbenzene & 1 & & U & & UG/L & $07 / 03 / 2006$ & & JGJ-074-06 \\
\hline GWM45304VL & TRIP BLANK & NA & Trichlorofluoromethane & 1 & & $\mathrm{U}$ & & UG/L & $07 / 03 / 2006$ & & JGJ-074-06 \\
\hline GWM45304VL & TRIP BLANK & NA & 2-Hexanone & 5 & & $\mathrm{U}$ & & UG/L & $07 / 03 / 2006$ & & JGJ-074-06 \\
\hline GWM45304VL & TRIP BLANK & NA & Isopropylbenzene & 1 & & $u$ & & UG/L & $07 / 03 / 2006$ & & JGJ-074-06 \\
\hline GWM45304VL & TRIP BLANK & NA & Methyl acetate & 5 & & $\mathrm{U}$ & $\mathrm{R}$ & UG/L & $07 / 03 / 2006$ & & JGJ-074-06 \\
\hline GWM45304VL & TRIP BLANK & NA & Methyl cyclohexane & 4 & & $\mathrm{U}$ & & UG/L & 07/03/2006 & & JGJ-074-06 \\
\hline GWM45304VL & TRIP BLANK & NA & Methylene Chloride & 1 & & U & & UG/L & 07/03/2006 & & JGJ-074-06 \\
\hline GWM45304VL & TRIP BLANK & NA & 4-Methyl-2-pentanone & 5 & & $\mathrm{u}$ & & UG/L & $07 / 03 / 2006$ & & JGJ-074-06 \\
\hline GWM45304VL & TRIP BLANK & NA & Styrene & 1 & & $\mathrm{U}$ & & UG/L & 07/03/2006 & & JGJ-074-06 \\
\hline GWM45304VL & TRIP BLANK & NA & 1,1,2,2-Tetrachloroethane & 1 & & $\mathrm{U}$ & & UG/L & $07 / 03 / 2006$ & & JGJ-074-06 \\
\hline GWM45304VL & TRIP BLANK & NA & Tetrachloroethene & 1 & & $\mathrm{U}$ & & UG/L & $07 / 03 / 2006$ & & JGJ-074-06 \\
\hline GWM45304VL & TRIP BLANK & NA & Toluene & 1 & & $\mathrm{U}$ & & UG/L & 07/03/2006 & & JGJ-074-06 \\
\hline GWM45304VL & TRIP BLANK & NA & 1,2,4-Trichlorobenzene & 1 & & $u$ & & UG/L & $07 / 03 / 2006$ & & JGJ-074-06 \\
\hline GWM45304VL & TRIP BLANK & NA & 1,1,1-Trichloroethane & 1 & & U & & UG/L & $07 / 03 / 2006$ & & JGJ-074-06 \\
\hline GWM45304VL & TRIP BLANK & NA & 1,1,2-Trichloroethane & 1 & & $\mathrm{U}$ & & UG/L & $07 / 03 / 2006$ & & JGJ-074-06 \\
\hline GWM45304VL & TRIP BLANK & NA & Trichloroethene & 1 & & $\mathrm{U}$ & & UG/L & $07 / 03 / 2006$ & & JGJ-074-06 \\
\hline GWM45304VL & TRIP BLANK & NA & 1,1,2-Trichloro-1,2,2-trifluoroethane & 1 & & $\mathrm{U}$ & & UG/L & 07/03/2006 & & JGJ-074-06 \\
\hline GWM45304VL & TRIP BLANK & NA & Vinyl Chloride & 2 & & $\mathrm{U}$ & & UG/L & 07/03/2006 & & JGJ-074-06 \\
\hline GWM45305VL & TRIP BLANK & NA & Benzene & 1 & & $\mathrm{U}$ & & UG/L & 07/10/2006 & & JGJ-075-06 \\
\hline GWM45305VL & TRIP BLANK & NA & Bromodichloromethane & 1 & & $\mathrm{U}$ & & UG/L & 07/10/2006 & & JGJ-075-06 \\
\hline GWM45305VL & TRIP BLANK & NA & Bromoform & 1 & & $\mathrm{u}$ & UJ & UG/L & $07 / 10 / 2006$ & & JGJ-075-06 \\
\hline GWM45305VL & TRIP BLANK & NA & Bromomethane & 2 & & $\mathrm{U}$ & & UG/L & 07/10/2006 & & JGJ-075-06 \\
\hline GWM45305VL & TRIP BLANK & NA & 2-Butanone & 5 & & $\mathrm{U}$ & $\mathrm{R}$ & UG/L & 07/10/2006 & & JGJ-075-06 \\
\hline GWM45305VL & TRIP BLANK & NA & Carbon disulfide & 1 & & $u$ & & UG/L & $07 / 10 / 2006$ & & JGJ-075-06 \\
\hline GWM45305VL & TRIP BLANK & NA & Carbon tetrachloride & 1 & & $\mathrm{U}$ & & UG/L & 07/10/2006 & & JGJ-075-06 \\
\hline GWM45305VL & TRIP BLANK & NA & Chlorobenzene & 1 & & $\mathrm{U}$ & & UG/L & 07/10/2006 & & JGJ-075-06 \\
\hline GWM45305VL & TRIP BLANK & NA & Dibromochloromethane & 1 & & U & & UG/L & $07 / 10 / 2006$ & & JGJ-075-06 \\
\hline GWM45305VL & TRIP BLANK & NA & 1,2-Dibromo-3-chloropropane & 1 & & $\mathrm{u}$ & $\mathrm{R}$ & UG/L & $07 / 10 / 2006$ & & JGJ-075-06 \\
\hline GWM45305VL & TRIP BLANK & NA & Chloroethane & 2 & & U & & UG/L & $07 / 10 / 2006$ & & JGJ-075-06 \\
\hline GWM45305VL & TRIP BLANK & NA & Chloroform & 1 & & $\mathrm{U}$ & & UG/L & $07 / 10 / 2006$ & & JGJ-075-06 \\
\hline GWM45305VL & TRIP BLANK & NA & Chloromethane & 2 & & $\mathrm{u}$ & & UG/L & $07 / 10 / 2006$ & & JGJ-075-06 \\
\hline GWM45305VL & TRIP BLANK & NA & Cyclohexane & 1 & & $\mathrm{U}$ & & UG/L & 07/10/2006 & & JGJ-075-06 \\
\hline GWM45305VL & TRIP BLANK & NA & 1,2-Dibromoethane & 1 & & U & & UG/L & $07 / 10 / 2006$ & & JGJ-075-06 \\
\hline GWM45305VL & TRIP BLANK & NA & 1,2-Dichlorobenzene & 1 & & $\mathrm{u}$ & & UG/L & $07 / 10 / 2006$ & & JGJ-075-06 \\
\hline GWM45305VL & TRIP BLANK & NA & 1,3-Dichlorobenzene & 1 & & $\mathrm{U}$ & & UG/L & 07/10/2006 & & JGJ-075-06 \\
\hline GWM45305VL & TRIP BLANK & NA & 1,4-Dichlorobenzene & 1 & & $\mathrm{U}$ & & UG/L & $07 / 10 / 2006$ & & JGJ-075-06 \\
\hline GWM45305VL & TRIP BLANK & NA & Dichlorodifluoromethane & 2 & & $u$ & & UG/L & $07 / 10 / 2006$ & & JGJ-075-06 \\
\hline GWM45305VL & TRIP BLANK & NA & 1,1-Dichloroethane & 1 & & $\mathrm{U}$ & & UG/L & 07/10/2006 & & JGJ-075-06 \\
\hline GWM45305VL & TRIP BLANK & NA & 1,2-Dichloroethane & 1 & & $\mathrm{U}$ & & UG/L & 07/10/2006 & & JGJ-075-06 \\
\hline GWM45305VL & TRIP BLANK & NA & 1,1-Dichloroethene & 1 & & $\mathrm{u}$ & & UG/L & $07 / 10 / 2006$ & & JGJ-075-06 \\
\hline GWM45305VL & TRIP BLANK & NA & cis-1,2-Dichloroethene & 1 & & $\mathrm{U}$ & & UG/L & 07/10/2006 & & JGJ-075-06 \\
\hline GWM45305VL & TRIP BLANK & NA & trans-1,2-Dichloroethene & 1 & & U & & UG/L & 07/10/2006 & & JGJ-075-06 \\
\hline GWM45305VL & TRIP BLANK & NA & 1,2-Dichloropropane & 1 & & U & & UG/L & $07 / 10 / 2006$ & & JGJ-075-06 \\
\hline GWM45305VL & TRIP BLANK & NA & cis-1,3-Dichloropropene & 1 & & $\mathrm{u}$ & & UG/L & $07 / 10 / 2006$ & & JGJ-075-06 \\
\hline GWM45305VL & TRIP BLANK & NA & trans-1,3-Dichloropropene & 1 & & $\mathrm{U}$ & & UG/L & 07/10/2006 & & JGJ-075-06 \\
\hline GWM45305VL & TRIP BLANK & NA & Ethylbenzene & 1 & & U & & UG/L & $07 / 10 / 2006$ & & JGJ-075-06 \\
\hline GWM45305VL & TRIP BLANK & NA & Trichlorofluoromethane & 1 & & $U$ & & UG/L & $07 / 10 / 2006$ & & JGJ-075-06 \\
\hline GWM45305VL & TRIP BLANK & NA & 2-Hexanone & 5 & & $\mathrm{U}$ & & UG/L & 07/10/2006 & & JGJ-075-06 \\
\hline GWM45305VL & TRIP BLANK & NA & Isopropylbenzene & 1 & & $\mathrm{U}$ & & UG/L & $07 / 10 / 2006$ & & JGJ-075-06 \\
\hline GWM45305VL & TRIP BLANK & NA & Methyl acetate & 5 & & U & $\mathrm{R}$ & UG/L & $07 / 10 / 2006$ & & JGJ-075-06 \\
\hline GWM45305VL & TRIP BLANK & NA & Methyl cyclohexane & 4 & & $\mathrm{U}$ & & UG/L & 07/10/2006 & & JGJ-075-06 \\
\hline GWM45305VL & TRIP BLANK & NA & Methylene Chloride & 1 & & $U$ & & UG/L & $07 / 10 / 2006$ & & JGJ-075-06 \\
\hline GWM45305VL & TRIP BLANK & NA & 4-Methyl-2-pentanone & 5 & & $U$ & & UG/L & $07 / 10 / 2006$ & & JGJ-075-06 \\
\hline
\end{tabular}


Table A-2. (continued).

\begin{tabular}{|c|c|c|c|c|c|c|c|c|c|c|c|}
\hline $\begin{array}{l}\text { Field Sample } \\
\text { Number }\end{array}$ & Location & Depth & Compound & $\begin{array}{c}\text { Sample } \\
\text { Result }\end{array}$ & $\begin{array}{c}\text { Sample } \\
\text { Error }\end{array}$ & $\begin{array}{c}\text { Result } \\
\text { Qualifier }\end{array}$ & $\begin{array}{c}\text { Validation } \\
\text { Flag }\end{array}$ & $\begin{array}{c}\text { Sample } \\
\text { Units }\end{array}$ & $\begin{array}{c}\text { Date Sample } \\
\text { Collected }\end{array}$ & MDA & $\begin{array}{l}\text { L\&V Report } \\
\text { Number }\end{array}$ \\
\hline GWM45305VL & TRIP BLANK & NA & Styrene & 1 & & $\mathrm{U}$ & & UG/L & $07 / 10 / 2006$ & & JGJ-075-06 \\
\hline GWM45305VL & TRIP BLANK & NA & 1,1,2,2-Tetrachloroethane & 1 & & U & & UG/L & $07 / 10 / 2006$ & & JGJ-075-06 \\
\hline GWM45305VL & TRIP BLANK & NA & Tetrachloroethene & 1 & & $\mathrm{U}$ & UJ & UG/L & $07 / 10 / 2006$ & & JGJ-075-06 \\
\hline GWM45305VL & TRIP BLANK & NA & 1,2,4-Trichlorobenzene & 1 & & $\mathrm{U}$ & & UG/L & $07 / 10 / 2006$ & & JGJ-075-06 \\
\hline GWM45305VL & TRIP BLANK & NA & 1,1,1-Trichloroethane & 1 & & $\mathrm{U}$ & & UG/L & $07 / 10 / 2006$ & & JGJ-075-06 \\
\hline GWM45305VL & TRIP BLANK & NA & 1,1,2-Trichloroethane & 1 & & u & & UG/L & 07/10/2006 & & JGJ-075-06 \\
\hline GWM45305VL & TRIP BLANK & NA & Trichloroethene & 1 & & $\mathrm{U}$ & & UG/L & $07 / 10 / 2006$ & & JGJ-075-06 \\
\hline GWM45305VL & TRIP BLANK & NA & Vinyl Chloride & 2 & & $U$ & & UG/L & $07 / 10 / 2006$ & & JGJ-075-06 \\
\hline GWM45305VL & TRIP BLANK & NA & Xylene (Total) & 3 & & $\mathrm{U}$ & & UG/L & $07 / 10 / 2006$ & & JGJ-075-06 \\
\hline GWM45305VL & TRIP BLANK & NA & Methyl t-butyl ether & 2 & & $\mathrm{U}$ & & UG/L & $07 / 10 / 2006$ & & JGJ-075-06 \\
\hline GWM45201R8 & FIELD BLANK & NA & Tritium & $-4.10 \mathrm{E}+01$ & 1.17E+02 & & $U$ & $\mathrm{PCl} / \mathrm{L}$ & $06 / 21 / 2006$ & 4.16E+02 & SOS-TL199-06 \\
\hline GWM45202R8 & FIELD BLANK & NA & Tritium & $-9.90 E+01$ & $1.01 \mathrm{E}+02$ & & $\mathrm{U}$ & $\mathrm{PCl} / \mathrm{L}$ & $06 / 27 / 2006$ & $3.62 \mathrm{E}+02$ & SOS-TL228-06 \\
\hline GWM45202RH & FIELD BLANK & NA & Gross Alpha & $3.23 \mathrm{E}-01$ & $2.51 \mathrm{E}-01$ & & $\mathrm{U}$ & $\mathrm{PCl} / \mathrm{L}$ & $06 / 27 / 2006$ & $1.02 \mathrm{E}+00$ & SOS-TL227-06 \\
\hline GWM45202RH & FIELD BLANK & NA & Gross Beta & $7.50 \mathrm{E}-01$ & $5.73 \mathrm{E}-01$ & & $\mathrm{U}$ & $\mathrm{PCl} / \mathrm{L}$ & $06 / 27 / 2006$ & $2.44 E+00$ & SOS-TL227-06 \\
\hline GWM45202RH & FIELD BLANK & NA & Strontium-90 & 4.25E-01 & $1.23 \mathrm{E}-01$ & & $\mathrm{~J}$ & $\mathrm{PCl} / \mathrm{L}$ & $06 / 27 / 2006$ & 4.33E-01 & SOS-TL227-06 \\
\hline GWM45202RH & FIELD BLANK & NA & Technetium-99 & 4.93E-01 & $1.86 \mathrm{E}+00$ & & $\mathrm{U}$ & $\mathrm{PCl} / \mathrm{L}$ & $06 / 27 / 2006$ & $6.25 \mathrm{E}+00$ & SOS-TL227-06 \\
\hline GWM45202UX & FIELD BLANK & NA & lodine-129 & 4.12E-02 & $2.51 \mathrm{E}-02$ & & UJ & $\mathrm{PCl} / \mathrm{L}$ & $06 / 27 / 2006$ & $5.98 \mathrm{E}-02$ & SOS-TL226-06 \\
\hline GWM45201RH & FIELD BLANK & NA & Strontium-90 & $-9.83 E-02$ & $5.75 \mathrm{E}-02$ & & U & $\mathrm{PCl} / \mathrm{L}$ & $06 / 21 / 2006$ & $3.54 \mathrm{E}-01$ & SOS-TL198-06 \\
\hline GWM45201RH & FIELD BLANK & NA & Technetium-99 & $1.08 \mathrm{E}+00$ & $2.88 \mathrm{E}+00$ & & U & $\mathrm{PCl} / \mathrm{L}$ & $06 / 21 / 2006$ & $9.57 \mathrm{E}+00$ & SOS-TL198-06 \\
\hline GWM45201UX & FIELD BLANK & NA & lodine- 129 & $5.23 \mathrm{E}-03$ & $2.75 \mathrm{E}-02$ & & $U$ & $\mathrm{PCl} / \mathrm{L}$ & $06 / 21 / 2006$ & 8.37E-02 & SOS-TL197-06 \\
\hline GWM435022X & USGS-001 & 612 & Aluminum & 8.2 & & B & & UG/L & $06 / 13 / 2006$ & & DNT-275-06 \\
\hline GWM435022X & USGS-001 & 612 & Antimony & 0.5 & & $\mathrm{U}$ & & UG/L & $06 / 13 / 2006$ & & DNT-275-06 \\
\hline GWM435022X & USGS-001 & 612 & Arsenic & 2.4 & & B & & UG/L & $06 / 13 / 2006$ & & DNT-275-06 \\
\hline GWM435022X & USGS-001 & 612 & Barium & 29.1 & & & & UG/L & $06 / 13 / 2006$ & & DNT-275-06 \\
\hline GWM435022X & USGS-001 & 612 & Beryllium & 0.088 & & $U$ & & UG/L & $06 / 13 / 2006$ & & DNT-275-06 \\
\hline GWM435022X & USGS-001 & 612 & Cadmium & 0.057 & & U & & UG/L & $06 / 13 / 2006$ & & DNT-275-06 \\
\hline GWM435022X & USGS-001 & 612 & Calcium & 30500 & & & & UG/L & $06 / 13 / 2006$ & & DNT-275-06 \\
\hline GWM435022X & USGS-001 & 612 & Chromium & 3 & & B & & UG/L & $06 / 13 / 2006$ & & DNT-275-06 \\
\hline GWM435022X & USGS-001 & 612 & Cobalt & 0.31 & & $U$ & & UG/L & $06 / 13 / 2006$ & & DNT-275-06 \\
\hline GWM435022X & USGS-001 & 612 & Copper & 0.64 & & B & & UG/L & $06 / 13 / 2006$ & & DNT-275-06 \\
\hline GWM435022X & USGS-001 & 612 & Iron & 9.4 & & U & & UG/L & $06 / 13 / 2006$ & & DNT-275-06 \\
\hline GWM435022X & USGS-001 & 612 & Lead & 0.49 & & U & & UG/L & $06 / 13 / 2006$ & & DNT-275-06 \\
\hline GWM435022X & USGS-001 & 612 & Magnesium & 11600 & & & & UG/L & $06 / 13 / 2006$ & & DNT-275-06 \\
\hline GWM435022X & USGS-001 & 612 & Manganese & 1.4 & & B & & UG/L & $06 / 13 / 2006$ & & DNT-275-06 \\
\hline GWM435022X & USGS-001 & 612 & Nickel & 1 & & B & & UG/L & $06 / 13 / 2006$ & & DNT-275-06 \\
\hline GWM435022X & USGS-001 & 612 & Potassium & 2990 & & & & UG/L & $06 / 13 / 2006$ & & DNT-275-06 \\
\hline GWM435022X & USGS-001 & 612 & Strontium & 128 & & & & UG/L & $06 / 13 / 2006$ & & DNT-275-06 \\
\hline GWM435022X & USGS-001 & 612 & Thallium & 0.32 & & $\mathrm{U}$ & & UG/L & $06 / 13 / 2006$ & & DNT-275-06 \\
\hline GWM435022X & USGS-001 & 612 & Uranium & 1.9 & & & & UG/L & $06 / 13 / 2006$ & & DNT-275-06 \\
\hline GWM435022X & USGS-001 & 612 & Vanadium & 4.3 & & B & & UG/L & $06 / 13 / 2006$ & & DNT-275-06 \\
\hline GWM435022X & USGS-001 & 612 & Zinc & 4.9 & & B & $U$ & UG/L & $06 / 13 / 2006$ & & DNT-275-06 \\
\hline GWM435022X & USGS-001 & 612 & Mercury & 0.093 & & $\mathrm{U}$ & & UG/L & $06 / 13 / 2006$ & & DNT-275-06 \\
\hline GWM435012X & USGS-001 & 612 & Aluminum & 7.9 & & U & & UG/L & $06 / 13 / 2006$ & & DNT-275-06 \\
\hline GWM435012X & USGS-001 & 612 & Antimony & 0.66 & & B & & UG/L & $06 / 13 / 2006$ & & DNT-275-06 \\
\hline GWM435012X & USGS-001 & 612 & Arsenic & 2.8 & & B & & UG/L & $06 / 13 / 2006$ & & DNT-275-06 \\
\hline GWM435012X & USGS-001 & 612 & Barium & 27.6 & & & & UG/L & $06 / 13 / 2006$ & & DNT-275-06 \\
\hline GWM435012X & USGS-001 & 612 & Beryllium & 0.088 & & U & & UG/L & $06 / 13 / 2006$ & & DNT-275-06 \\
\hline GWM435012X & USGS-001 & 612 & Cadmium & 0.057 & & U & & UG/L & $06 / 13 / 2006$ & & DNT-275-06 \\
\hline GWM435012X & USGS-001 & 612 & Calcium & 30200 & & & & UG/L & $06 / 13 / 2006$ & & DNT-275-06 \\
\hline GWM435012X & USGS-001 & 612 & Chromium & 2.8 & & U & & UG/L & $06 / 13 / 2006$ & & DNT-275-06 \\
\hline GWM435012X & USGS-001 & 612 & Cobalt & 0.31 & & U & & UG/L & $06 / 13 / 2006$ & & DNT-275-06 \\
\hline GWM435012X & USGS-001 & 612 & Copper & 0.64 & & B & & UG/L & $06 / 13 / 2006$ & & DNT-275-06 \\
\hline GWM435012X & USGS-001 & 612 & Iron & 9.4 & & U & & UG/L & $06 / 13 / 2006$ & & DNT-275-06 \\
\hline GWM435012X & USGS-001 & 612 & Lead & 0.49 & & $\mathrm{U}$ & & UG/L & $06 / 13 / 2006$ & & DNT-275-06 \\
\hline GWM435012X & USGS-001 & 612 & Magnesium & 11300 & & & & UG/L & $06 / 13 / 2006$ & & DNT-275-06 \\
\hline GWM435012X & USGS-001 & 612 & Manganese & 1.5 & & B & & UG/L & $06 / 13 / 2006$ & & DNT-275-06 \\
\hline GWM435012X & USGS-001 & 612 & Nickel & 1.4 & & B & & UG/L & $06 / 13 / 2006$ & & DNT-275-06 \\
\hline GWM435012X & USGS-001 & 612 & Potassium & 2900 & & & & UG/L & $06 / 13 / 2006$ & & DNT-275-06 \\
\hline GWM435012X & USGS-001 & 612 & Selenium & 2.5 & & B & $\mathrm{U}$ & UG/L & $06 / 13 / 2006$ & & DNT-275-06 \\
\hline GWM435012X & USGS-001 & 612 & Silver & 0.2 & & $U$ & & UG/L & $06 / 13 / 2006$ & & DNT-275-06 \\
\hline GWM435012X & USGS-001 & 612 & Sodium & 14600 & & & & UG/L & $06 / 13 / 2006$ & & DNT-275-06 \\
\hline GWM435012X & USGS-001 & 612 & Strontium & 123 & & & & UG/L & $06 / 13 / 2006$ & & DNT-275-06 \\
\hline GWM435012X & USGS-001 & 612 & Thallium & 0.76 & & B & $\mathrm{U}$ & UG/L & $06 / 13 / 2006$ & & DNT-275-06 \\
\hline GWM435012X & USGS-001 & 612 & Uranium & 2 & & & & UG/L & $06 / 13 / 2006$ & & DNT-275-06 \\
\hline GWM435012X & USGS-001 & 612 & Vanadium & 4.9 & & B & & UG/L & $06 / 13 / 2006$ & & DNT-275-06 \\
\hline GWM435012X & USGS-001 & 612 & Zinc & 3.1 & & B & $\mathrm{U}$ & UG/L & $06 / 13 / 2006$ & & DNT-275-06 \\
\hline GWM435012X & USGS-001 & 612 & Mercury & 0.093 & & U & & UG/L & $06 / 13 / 2006$ & & DNT-275-06 \\
\hline GWM43501A1 & USGS-001 & 612 & Total Alkalinity & 126 & & & & $M G / L$ & $06 / 13 / 2006$ & & DNT-283-06 \\
\hline GWM43502A1 & USGS-001 & 612 & Total Alkalinity & 128 & & & & $M G / L$ & $06 / 13 / 2006$ & & DNT-283-06 \\
\hline GWM43501AN & USGS-001 & 612 & Chloride & 14.1 & & & $\mathrm{~J}$ & $M G / L$ & $06 / 13 / 2006$ & & DNT-283-06 \\
\hline GWM43501AN & USGS-001 & 612 & Fluoride & 0.65 & & & & $M G / L$ & $06 / 13 / 2006$ & & DNT-283-06 \\
\hline
\end{tabular}


Table A-2. (continued).

\begin{tabular}{|c|c|c|c|c|c|c|c|c|c|c|c|}
\hline $\begin{array}{c}\text { Field Sample } \\
\text { Number }\end{array}$ & Location & Depth & Compound & $\begin{array}{c}\text { Sample } \\
\text { Result }\end{array}$ & $\begin{array}{c}\text { Sample } \\
\text { Error }\end{array}$ & $\begin{array}{c}\text { Result } \\
\text { Qualifier }\end{array}$ & $\begin{array}{c}\text { Validation } \\
\text { Flag }\end{array}$ & $\begin{array}{c}\text { Sample } \\
\text { Units }\end{array}$ & $\begin{array}{c}\text { Date Sample } \\
\text { Collected }\end{array}$ & MDA & $\begin{array}{c}\text { L\&V Report } \\
\text { Number }\end{array}$ \\
\hline GWM43501AN & USGS-001 & 612 & Sulfate & 14.1 & & & $\mathrm{~J}$ & MG/L & $06 / 13 / 2006$ & & DNT-283-06 \\
\hline GWM43502AN & USGS-001 & 612 & Chloride & 14.8 & & & $\mathrm{~J}$ & MG/L & $06 / 13 / 2006$ & & DNT-283-06 \\
\hline GWM43502AN & USGS-001 & 612 & Fluoride & 0.69 & & & & MG/L & $06 / 13 / 2006$ & & DNT-283-06 \\
\hline GWM43501N2 & USGS-001 & 612 & Nitrate/Nitrite as $\mathrm{N}$ & 1170 & & & & UG/L & $06 / 13 / 2006$ & & DNT-283-06 \\
\hline GWM43502N2 & USGS-001 & 612 & Nitrate/Nitrite as $\mathrm{N}$ & 1190 & & & & UG/L & $06 / 13 / 2006$ & & DNT-283-06 \\
\hline GWM43501VL & USGS-001 & 612 & Acetone & 2 & & $\mathrm{U}$ & & UG/L & $06 / 13 / 2006$ & & JGJ-062-06 \\
\hline GWM43501VL & USGS-001 & 612 & Benzene & 1 & & $\mathrm{U}$ & & UG/L & $06 / 13 / 2006$ & & JGJ-062-06 \\
\hline GWM43501VL & USGS-001 & 612 & Bromodichloromethane & 1 & & $\mathrm{U}$ & & UG/L & $06 / 13 / 2006$ & & JGJ-062-06 \\
\hline GWM43501VL & USGS-001 & 612 & Bromoform & 1 & & U & & UG/L & $06 / 13 / 2006$ & & JGJ-062-06 \\
\hline GWM43501VL & USGS-001 & 612 & Bromomethane & 2 & & U & & UG/L & $06 / 13 / 2006$ & & JGJ-062-06 \\
\hline GWM43501VL & USGS-001 & 612 & 2-Butanone & 5 & & $u$ & $\mathrm{R}$ & UG/L & $06 / 13 / 2006$ & & JGJ-062-06 \\
\hline GWM43501VL & USGS-001 & 612 & Carbon disulfide & 1 & & $\mathrm{U}$ & & UG/L & $06 / 13 / 2006$ & & JGJ-062-06 \\
\hline GWM43501VL & USGS-001 & 612 & Carbon tetrachloride & 1 & & $\mathrm{U}$ & & UG/L & $06 / 13 / 2006$ & & JGJ-062-06 \\
\hline GWM43501VL & USGS-001 & 612 & Chlorobenzene & 1 & & $\mathrm{U}$ & & UG/L & $06 / 13 / 2006$ & & JGJ-062-06 \\
\hline GWM43501VL & USGS-001 & 612 & Dibromochloromethane & 1 & & U & & UG/L & $06 / 13 / 2006$ & & JGJ-062-06 \\
\hline GWM43501VL & USGS-001 & 612 & 1,2-Dibromo-3-chloropropane & 1 & & $\mathrm{U}$ & $\mathrm{R}$ & UG/L & $06 / 13 / 2006$ & & JGJ-062-06 \\
\hline GWM43501VL & USGS-001 & 612 & Chloroethane & 2 & & $\mathrm{U}$ & & UG/L & $06 / 13 / 2006$ & & JGJ-062-06 \\
\hline GWM43501VL & USGS-001 & 612 & Chloroform & 1 & & $\mathrm{u}$ & & UG/L & $06 / 13 / 2006$ & & JGJ-062-06 \\
\hline GWM43501VL & USGS-001 & 612 & 1,2-Dibromoethane & 1 & & $u$ & & UG/L & $06 / 13 / 2006$ & & JGJ-062-06 \\
\hline GWM43501VL & USGS-001 & 612 & 1,2-Dichlorobenzene & 1 & & $\mathrm{U}$ & & UG/L & $06 / 13 / 2006$ & & JGJ-062-06 \\
\hline GWM43501VL & USGS-001 & 612 & 1,3-Dichlorobenzene & 1 & & $\mathrm{U}$ & & UG/L & $06 / 13 / 2006$ & & JGJ-062-06 \\
\hline GWM43501VL & USGS-001 & 612 & 1,4-Dichlorobenzene & 1 & & $\mathrm{U}$ & & UG/L & $06 / 13 / 2006$ & & JGJ-062-06 \\
\hline GWM43501VL & USGS-001 & 612 & Dichlorodifluoromethane & 2 & & $\mathrm{U}$ & & UG/L & $06 / 13 / 2006$ & & JGJ-062-06 \\
\hline GWM43501VL & USGS-001 & 612 & 1,1-Dichloroethane & 1 & & $u$ & & UG/L & $06 / 13 / 2006$ & & JGJ-062-06 \\
\hline GWM43501VL & USGS-001 & 612 & 1,2-Dichloroethane & 1 & & $\mathrm{U}$ & & UG/L & $06 / 13 / 2006$ & & JGJ-062-06 \\
\hline GWM43501VL & USGS-001 & 612 & 1,1-Dichloroethene & 1 & & $\mathrm{U}$ & & UG/L & $06 / 13 / 2006$ & & JGJ-062-06 \\
\hline GWM43501VL & USGS-001 & 612 & cis-1,2-Dichloroethene & 1 & & $u$ & & UG/L & $06 / 13 / 2006$ & & JGJ-062-06 \\
\hline GWM43501VL & USGS-001 & 612 & trans-1,2-Dichloroethene & 1 & & $\mathrm{U}$ & & UG/L & $06 / 13 / 2006$ & & JGJ-062-06 \\
\hline GWM43501VL & USGS-001 & 612 & 1,2-Dichloropropane & 1 & & U & & UG/L & $06 / 13 / 2006$ & & JGJ-062-06 \\
\hline GWM43501VL & USGS-001 & 612 & cis-1,3-Dichloropropene & 1 & & $\mathrm{U}$ & & UG/L & $06 / 13 / 2006$ & & JGJ-062-06 \\
\hline GWM43501VL & USGS-001 & 612 & trans-1,3-Dichloropropene & 1 & & $\mathrm{U}$ & & UG/L & $06 / 13 / 2006$ & & JGJ-062-06 \\
\hline GWM43501VL & USGS-001 & 612 & Ethylbenzene & 1 & & $\mathrm{U}$ & & UG/L & $06 / 13 / 2006$ & & JGJ-062-06 \\
\hline GWM43501VL & USGS-001 & 612 & Trichlorofluoromethane & 1 & & $\mathrm{U}$ & & UG/L & $06 / 13 / 2006$ & & JGJ-062-06 \\
\hline GWM43501VL & USGS-001 & 612 & 2-Hexanone & 5 & & $\mathrm{U}$ & & UG/L & $06 / 13 / 2006$ & & JGJ-062-06 \\
\hline GWM43501VL & USGS-001 & 612 & Isopropylbenzene & 1 & & $\mathrm{U}$ & & UG/L & $06 / 13 / 2006$ & & JGJ-062-06 \\
\hline GWM43501VL & USGS-001 & 612 & Methyl acetate & 5 & & $\mathrm{U}$ & & UG/L & $06 / 13 / 2006$ & & JGJ-062-06 \\
\hline GWM43501VL & USGS-001 & 612 & Methyl cyclohexane & 4 & & U & & UG/L & $06 / 13 / 2006$ & & JGJ-062-06 \\
\hline GWM43501VL & USGS-001 & 612 & 1,1,2,2-Tetrachloroethane & 1 & & $\mathrm{U}$ & & UG/L & $06 / 13 / 2006$ & & JGJ-062-06 \\
\hline GWM43501VL & USGS-001 & 612 & Tetrachloroethene & 1 & & $\mathrm{U}$ & & UG/L & $06 / 13 / 2006$ & & JGJ-062-06 \\
\hline GWM43501VL & USGS-001 & 612 & Toluene & 1 & & $u$ & & UG/L & $06 / 13 / 2006$ & & JGJ-062-06 \\
\hline GWM43501VL & USGS-001 & 612 & 1,2,4-Trichlorobenzene & 1 & & U & & UG/L & 06/13/2006 & & JGJ-062-06 \\
\hline GWM43501VL & USGS-001 & 612 & 1,1,1-Trichloroethane & 1 & & $\mathrm{U}$ & & UG/L & $06 / 13 / 2006$ & & JGJ-062-06 \\
\hline GWM43501VL & USGS-001 & 612 & 1,1,2-Trichloroethane & 1 & & $u$ & & UG/L & $06 / 13 / 2006$ & & JGJ-062-06 \\
\hline GWM43501VL & USGS-001 & 612 & Trichloroethene & 1 & & $\mathrm{U}$ & & UG/L & $06 / 13 / 2006$ & & JGJ-062-06 \\
\hline GWM43501VL & USGS-001 & 612 & 1,1,2-Trichloro-1,2,2-trifluoroethane & 1 & & $\mathrm{U}$ & & UG/L & $06 / 13 / 2006$ & & JGJ-062-06 \\
\hline GWM43501VL & USGS-001 & 612 & Vinyl Chloride & 2 & & U & & UG/L & $06 / 13 / 2006$ & & JGJ-062-06 \\
\hline GWM43501VL & USGS-001 & 612 & Xylene (Total) & 3 & & $\mathrm{u}$ & & UG/L & $06 / 13 / 2006$ & & JGJ-062-06 \\
\hline GWM43501VL & USGS-001 & 612 & Methyl t-butyl ether & 2 & & $\mathrm{U}$ & & UG/L & $06 / 13 / 2006$ & & JGJ-062-06 \\
\hline GWM43502VL & USGS-001 & 612 & Acetone & 2 & & $\mathrm{U}$ & & UG/L & $06 / 13 / 2006$ & & JGJ-062-06 \\
\hline GWM43502VL & USGS-001 & 612 & Benzene & 1 & & $\mathrm{U}$ & & UG/L & $06 / 13 / 2006$ & & JGJ-062-06 \\
\hline GWM43502VL & USGS-001 & 612 & Bromodichloromethane & 1 & & $\mathrm{U}$ & & UG/L & $06 / 13 / 2006$ & & JGJ-062-06 \\
\hline GWM43502VL & USGS-001 & 612 & Bromoform & 1 & & $\mathrm{U}$ & & UG/L & 06/13/2006 & & JGJ-062-06 \\
\hline GWM43502VL & USGS-001 & 612 & Bromomethane & 2 & & $U$ & & UG/L & 06/13/2006 & & JGJ-062-06 \\
\hline GWM43502VL & USGS-001 & 612 & 2-Butanone & 5 & & $u$ & $\mathrm{R}$ & UG/L & $06 / 13 / 2006$ & & JGJ-062-06 \\
\hline GWM43502VL & USGS-001 & 612 & Carbon disulfide & 1 & & $\mathrm{U}$ & & UG/L & $06 / 13 / 2006$ & & JGJ-062-06 \\
\hline GWM43502VL & USGS-001 & 612 & Carbon tetrachloride & 1 & & $\mathrm{U}$ & & UG/L & $06 / 13 / 2006$ & & JGJ-062-06 \\
\hline GWM43502VL & USGS-001 & 612 & Chlorobenzene & 1 & & $\mathrm{U}$ & & UG/L & $06 / 13 / 2006$ & & JGJ-062-06 \\
\hline GWM43502VL & USGS-001 & 612 & Dibromochloromethane & 1 & & $\mathrm{U}$ & & UG/L & $06 / 13 / 2006$ & & JGJ-062-06 \\
\hline GWM43502VL & USGS-001 & 612 & 1,2-Dibromo-3-chloropropane & 1 & & U & $\mathrm{R}$ & UG/L & 06/13/2006 & & JGJ-062-06 \\
\hline GWM43502VL & USGS-001 & 612 & Chloroethane & 2 & & U & & UG/L & $06 / 13 / 2006$ & & JGJ-062-06 \\
\hline GWM43502VL & USGS-001 & 612 & Chloroform & 1 & & $\mathrm{U}$ & & UG/L & 06/13/2006 & & JGJ-062-06 \\
\hline GWM43502VL & USGS-001 & 612 & Chloromethane & 2 & & $U$ & & UG/L & 06/13/2006 & & JGJ-062-06 \\
\hline GWM43502VL & USGS-001 & 612 & Cyclohexane & 1 & & $\mathrm{U}$ & & UG/L & $06 / 13 / 2006$ & & JGJ-062-06 \\
\hline GWM43502VL & USGS-001 & 612 & 1,2-Dibromoethane & 1 & & U & & UG/L & $06 / 13 / 2006$ & & JGJ-062-06 \\
\hline GWM43502VL & USGS-001 & 612 & 1,2-Dichlorobenzene & 1 & & $\mathrm{U}$ & & UG/L & 06/13/2006 & & JGJ-062-06 \\
\hline GWM43502VL & USGS-001 & 612 & 1,3-Dichlorobenzene & 1 & & $\mathrm{U}$ & & UG/L & $06 / 13 / 2006$ & & JGJ-062-06 \\
\hline GWM43502VL & USGS-001 & 612 & 1,4-Dichlorobenzene & 1 & & $U$ & & UG/L & $06 / 13 / 2006$ & & JGJ-062-06 \\
\hline GWM43502VL & USGS-001 & 612 & Dichlorodifluoromethane & 2 & & $U$ & & UG/L & $06 / 13 / 2006$ & & JGJ-062-06 \\
\hline GWM43502VL & USGS-001 & 612 & 1,1-Dichloroethane & 1 & & U & & UG/L & $06 / 13 / 2006$ & & JGJ-062-06 \\
\hline GWM43502VL & USGS-001 & 612 & 1,2-Dichloroethane & 1 & & $\mathrm{U}$ & & UG/L & $06 / 13 / 2006$ & & JGJ-062-06 \\
\hline GWM43502VL & USGS-001 & 612 & 1,1-Dichloroethene & 1 & & $\mathrm{U}$ & & UG/L & 06/13/2006 & & JGJ-062-06 \\
\hline GWM43502VL & USGS-001 & 612 & cis-1,2-Dichloroethene & 1 & & U & & UG/L & 06/13/2006 & & JGJ-062-06 \\
\hline
\end{tabular}


Table A-2. (continued)

\begin{tabular}{|c|c|c|c|c|c|c|c|c|c|c|c|}
\hline $\begin{array}{c}\text { Field Sample } \\
\text { Number }\end{array}$ & Location & Depth & Compound & $\begin{array}{c}\text { Sample } \\
\text { Result }\end{array}$ & $\begin{array}{c}\text { Sample } \\
\text { Error }\end{array}$ & $\begin{array}{c}\text { Result } \\
\text { Qualifier }\end{array}$ & $\begin{array}{c}\text { Validation } \\
\text { Flag }\end{array}$ & $\begin{array}{c}\text { Sample } \\
\text { Units }\end{array}$ & $\begin{array}{c}\text { Date Sample } \\
\text { Collected }\end{array}$ & MDA & $\begin{array}{c}\text { L\&V Report } \\
\text { Number }\end{array}$ \\
\hline GWM43502VL & USGS-001 & 612 & trans-1,2-Dichloroethene & 1 & & $U$ & & UG/L & $06 / 13 / 2006$ & & JGJ-062-06 \\
\hline GWM43502VL & USGS-001 & 612 & 1,2-Dichloropropane & 1 & & $U$ & & UG/L & $06 / 13 / 2006$ & & JGJ-062-06 \\
\hline GWM43502VL & USGS-001 & 612 & cis-1,3-Dichloropropene & 1 & & $\mathrm{U}$ & & UG/L & $06 / 13 / 2006$ & & JGJ-062-06 \\
\hline GWM43502VL & USGS-001 & 612 & Ethylbenzene & 1 & & $\mathrm{U}$ & & UG/L & $06 / 13 / 2006$ & & JGJ-062-06 \\
\hline GWM43502VL & USGS-001 & 612 & Trichlorofluoromethane & 1 & & $\mathrm{U}$ & & UG/L & $06 / 13 / 2006$ & & JGJ-062-06 \\
\hline GWM43502VL & USGS-001 & 612 & 2-Hexanone & 5 & & $\mathrm{U}$ & & UG/L & $06 / 13 / 2006$ & & JGJ-062-06 \\
\hline GWM43502VL & USGS-001 & 612 & Isopropylbenzene & 1 & & $\mathrm{U}$ & & UG/L & $06 / 13 / 2006$ & & JGJ-062-06 \\
\hline GWM43502VL & USGS-001 & 612 & Methyl acetate & 5 & & $\mathrm{U}$ & & UG/L & $06 / 13 / 2006$ & & JGJ-062-06 \\
\hline GWM43502VL & USGS-001 & 612 & Methyl cyclohexane & 4 & & $\mathrm{U}$ & & UG/L & $06 / 13 / 2006$ & & JGJ-062-06 \\
\hline GWM43502VL & USGS-001 & 612 & Methylene Chloride & 1 & & U & & UG/L & $06 / 13 / 2006$ & & JGJ-062-06 \\
\hline GWM43502VL & USGS-001 & 612 & 4-Methyl-2-pentanone & 5 & & $\mathrm{U}$ & & UG/L & $06 / 13 / 2006$ & & JGJ-062-06 \\
\hline GWM43502VL & USGS-001 & 612 & Styrene & 1 & & $\mathrm{U}$ & & UG/L & $06 / 13 / 2006$ & & JGJ-062-06 \\
\hline GWM43502VL & USGS-001 & 612 & 1,1,2,2-Tetrachloroethane & 1 & & $\mathrm{U}$ & & UG/L & $06 / 13 / 2006$ & & JGJ-062-06 \\
\hline GWM43502VL & USGS-001 & 612 & Tetrachloroethene & 1 & & $\mathrm{U}$ & & UG/L & $06 / 13 / 2006$ & & JGJ-062-06 \\
\hline GWM43502VL & USGS-001 & 612 & Toluene & 1 & & $\mathrm{U}$ & & UG/L & $06 / 13 / 2006$ & & JGJ-062-06 \\
\hline GWM43502VL & USGS-001 & 612 & 1,2,4-Trichlorobenzene & 1 & & $\mathrm{U}$ & & UG/L & $06 / 13 / 2006$ & & JGJ-062-06 \\
\hline GWM43502VL & USGS-001 & 612 & 1,1,1-Trichloroethane & 1 & & $U$ & & UG/L & 06/13/2006 & & JGJ-062-06 \\
\hline GWM43502VL & USGS-001 & 612 & 1,1,2-Trichloroethane & 1 & & $\mathrm{U}$ & & UG/L & $06 / 13 / 2006$ & & JGJ-062-06 \\
\hline GWM43502VL & USGS-001 & 612 & Vinyl Chloride & 2 & & $\mathrm{U}$ & & UG/L & $06 / 13 / 2006$ & & JGJ-062-06 \\
\hline GWM43502VL & USGS-001 & 612 & Xylene (Total) & 3 & & $\mathrm{U}$ & & UG/L & 06/13/2006 & & JGJ-062-06 \\
\hline GWM43502VL & USGS-001 & 612 & Methyl t-butyl ether & 2 & & U & & UG/L & $06 / 13 / 2006$ & & JGJ-062-06 \\
\hline GWM43501R8 & USGS-001 & 612 & Tritium & $-8.06 E+01$ & $1.14 \mathrm{E}+02$ & & $\mathrm{U}$ & $\mathrm{PCI} / \mathrm{L}$ & $06 / 13 / 2006$ & $4.09 E+02$ & SOS-TL199-06 \\
\hline GWM43502R8 & USGS-001 & 612 & Tritium & $-2.61 \mathrm{E}+02$ & $1.16 \mathrm{E}+02$ & & $\mathrm{u}$ & $\mathrm{PCI} / \mathrm{L}$ & $06 / 13 / 2006$ & $4.41 \mathrm{E}+02$ & SOS-TL199-06 \\
\hline GWM43502RH & USGS-001 & 612 & Gross Alpha & $2.26 \mathrm{E}+00$ & 4.37E-01 & & & $\mathrm{PCI} / \mathrm{L}$ & $06 / 13 / 2006$ & $1.22 \mathrm{E}+00$ & SOS-TL198-06 \\
\hline GWM43502RH & USGS-001 & 612 & Gross Beta & $3.51 \mathrm{E}+00$ & 5.05E-01 & & & $\mathrm{PCI} / \mathrm{L}$ & 06/13/2006 & $1.61 \mathrm{E}+00$ & SOS-TL198-06 \\
\hline GWM43501RH & USGS-001 & 612 & Gross Alpha & $2.13 \mathrm{E}+00$ & 3.79E-01 & & & $\mathrm{PCI} / \mathrm{L}$ & $06 / 13 / 2006$ & 6.62E-01 & SOS-TL198-06 \\
\hline GWM43501RH & USGS-001 & 612 & Gross Beta & $3.71 \mathrm{E}+00$ & 5.59E-01 & & & $\mathrm{PCl} / \mathrm{L}$ & $06 / 13 / 2006$ & $1.56 \mathrm{E}+00$ & SOS-TL198-06 \\
\hline GWM43502RH & USGS-001 & 612 & Strontium-90 & $1.03 \mathrm{E}-01$ & $1.02 \mathrm{E}-01$ & & $U$ & $\mathrm{PCI} / \mathrm{L}$ & 06/13/2006 & 4.57E-01 & SOS-TL198-06 \\
\hline GWM43501RH & USGS-001 & 612 & Strontium-90 & $-5.77 \mathrm{E}-02$ & 7.26E-02 & & $u$ & $\mathrm{PCl} / \mathrm{L}$ & $06 / 13 / 2006$ & 3.94E-01 & SOS-TL198-06 \\
\hline GWM43502RH & USGS-001 & 612 & Technetium-99 & $3.75 \mathrm{E}+00$ & $2.89 \mathrm{E}+00$ & & $u$ & $\mathrm{PCl} / \mathrm{L}$ & $06 / 13 / 2006$ & $9.55 \mathrm{E}+00$ & SOS-TL198-06 \\
\hline GWM43501RH & USGS-001 & 612 & Technetium-99 & $-4.03 E+00$ & $2.79 \mathrm{E}+00$ & & $u$ & $\mathrm{PCl} / \mathrm{L}$ & $06 / 13 / 2006$ & $9.46 \mathrm{E}+00$ & SOS-TL198-06 \\
\hline GWM43502UX & USGS-001 & 612 & lodine-129 & $-1.71 \mathrm{E}-02$ & $1.81 \mathrm{E}-02$ & & $\mathrm{U}$ & $\mathrm{PCl} / \mathrm{L}$ & $06 / 13 / 2006$ & $6.26 \mathrm{E}-02$ & SOS-TL197-06 \\
\hline GWM43501UX & USGS-001 & 612 & lodine-129 & $-1.34 \mathrm{E}-02$ & 1.90E-02 & & $\mathrm{U}$ & $\mathrm{PCI} / \mathrm{L}$ & $06 / 13 / 2006$ & $6.69 \mathrm{E}-02$ & SOS-TL197-06 \\
\hline GWM443012X & USGS-002 & 683 & Aluminum & 8 & & B & & UG/L & $06 / 12 / 2006$ & & DNT-276-06 \\
\hline GWM443012X & USGS-002 & 683 & Antimony & 0.54 & & B & & UG/L & $06 / 12 / 2006$ & & DNT-276-06 \\
\hline GWM443012X & USGS-002 & 683 & Arsenic & 2 & & $\mathrm{U}$ & & UG/L & $06 / 12 / 2006$ & & DNT-276-06 \\
\hline GWM443012X & USGS-002 & 683 & Barium & 36.6 & & & & UG/L & $06 / 12 / 2006$ & & DNT-276-06 \\
\hline GWM443012X & USGS-002 & 683 & Chromium & 2.8 & & $\bar{U}$ & & UG/L & $06 / 12 / 2006$ & & DNT-276-06 \\
\hline GWM443012X & USGS-002 & 683 & Cobalt & 0.31 & & $\mathrm{U}$ & & UG/L & $06 / 12 / 2006$ & & DNT-276-06 \\
\hline GWM443012X & USGS-002 & 683 & Copper & 0.94 & & B & & UG/L & $06 / 12 / 2006$ & & DNT-276-06 \\
\hline GWM443012X & USGS-002 & 683 & Iron & 9.4 & & $U$ & & UG/L & $06 / 12 / 2006$ & & DNT-276-06 \\
\hline GWM443012X & USGS-002 & 683 & Lead & 0.49 & & $U$ & & UG/L & $06 / 12 / 2006$ & & DNT-276-06 \\
\hline GWM443012X & USGS-002 & 683 & Magnesium & 11200 & & $\mathrm{E}$ & $\mathrm{J}$ & UG/L & $06 / 12 / 2006$ & & DNT-276-06 \\
\hline GWM443012X & USGS-002 & 683 & Manganese & 1.6 & & B & & UG/L & $06 / 12 / 2006$ & & DNT-276-06 \\
\hline GWM443012X & USGS-002 & 683 & Nickel & 0.94 & & $\mathrm{~B}$ & & UG/L & $06 / 12 / 2006$ & & DNT-276-06 \\
\hline GWM443012X & USGS-002 & 683 & Potassium & 2940 & & $\mathrm{E}$ & $\mathrm{J}$ & UG/L & $06 / 12 / 2006$ & & DNT-276-06 \\
\hline GWM443012X & USGS-002 & 683 & Selenium & 1.7 & & B & $\mathrm{U}$ & UG/L & $06 / 12 / 2006$ & & DNT-276-06 \\
\hline GWM443012X & USGS-002 & 683 & Silver & 0.2 & & $U$ & & UG/L & $06 / 12 / 2006$ & & DNT-276-06 \\
\hline GWM443012X & USGS-002 & 683 & Sodium & 15800 & & $\mathrm{NE}$ & $\mathrm{J}$ & UG/L & $06 / 12 / 2006$ & & DNT-276-06 \\
\hline GWM443012X & USGS-002 & 683 & Strontium & 129 & & $\mathrm{E}$ & $\mathrm{J}$ & UG/L & $06 / 12 / 2006$ & & DNT-276-06 \\
\hline GWM443012X & USGS-002 & 683 & Thallium & 0.75 & & B & $\mathrm{U}$ & UG/L & $06 / 12 / 2006$ & & DNT-276-06 \\
\hline GWM443012X & USGS-002 & 683 & Uranium & 2.2 & & & & UG/L & $06 / 12 / 2006$ & & DNT-276-06 \\
\hline GWM443012X & USGS-002 & 683 & Vanadium & 4.1 & & B & & UG/L & $06 / 12 / 2006$ & & DNT-276-06 \\
\hline GWM443012X & USGS-002 & 683 & Zinc & 3.7 & & B & $u$ & UG/L & $06 / 12 / 2006$ & & DNT-276-06 \\
\hline GWM443012X & USGS-002 & 683 & Mercury & 0.093 & & U & UJ & UG/L & $06 / 12 / 2006$ & & DNT-276-06 \\
\hline GWM44301A1 & USGS-002 & 683 & Total Alkalinity & 132 & & & & $M G / L$ & $06 / 12 / 2006$ & & DNT-280-06 \\
\hline GWM44301AN & USGS-002 & 683 & Chloride & 17.6 & & $\mathrm{~J}$ & & $M G / L$ & $06 / 12 / 2006$ & & DNT-280-06 \\
\hline GWM44301AN & USGS-002 & 683 & Fluoride & 0.64 & & & & MG/L & $06 / 12 / 2006$ & & DNT-280-06 \\
\hline GWM44301AN & USGS-002 & 683 & Sulfate & 16.1 & & & $\mathrm{~J}$ & $M G / L$ & $06 / 12 / 2006$ & & DNT-280-06 \\
\hline GWM44301N2 & USGS-002 & 683 & Nitrate/Nitrite as $\mathrm{N}$ & 1740 & & & & UG/L & $06 / 12 / 2006$ & & DNT-280-06 \\
\hline GWM44301VL & USGS-002 & 683 & Acetone & 2 & & U & & UG/L & $06 / 12 / 2006$ & & JGJ-062-06 \\
\hline GWM44301VL & USGS-002 & 683 & Benzene & 1 & & $\mathrm{U}$ & & UG/L & $06 / 12 / 2006$ & & JGJ-062-06 \\
\hline GWM44301VL & USGS-002 & 683 & Bromodichloromethane & 1 & & $\mathrm{U}$ & & UG/L & $06 / 12 / 2006$ & & JGJ-062-06 \\
\hline GWM44301VL & USGS-002 & 683 & Bromoform & 1 & & $\mathrm{U}$ & & UG/L & $06 / 12 / 2006$ & & JGJ-062-06 \\
\hline GWM44301VL & USGS-002 & 683 & Bromomethane & 2 & & $\mathrm{U}$ & & UG/L & $06 / 12 / 2006$ & & JGJ-062-06 \\
\hline GWM44301VL & USGS-002 & 683 & 2-Butanone & 5 & & $\mathrm{U}$ & $\mathrm{R}$ & UG/L & $06 / 12 / 2006$ & & JGJ-062-06 \\
\hline GWM44301VL & USGS-002 & 683 & Carbon disulfide & 1 & & U & & UG/L & $06 / 12 / 2006$ & & JGJ-062-06 \\
\hline GWM44301VL & USGS-002 & 683 & Carbon tetrachloride & 1 & & $\mathrm{U}$ & & UG/L & $06 / 12 / 2006$ & & JGJ-062-06 \\
\hline GWM44301VL & USGS-002 & 683 & Chlorobenzene & 1 & & $\mathrm{U}$ & & UG/L & $06 / 12 / 2006$ & & JGJ-062-06 \\
\hline GWM44301VL & USGS-002 & 683 & Dibromochloromethane & 1 & & U & & UG/L & $06 / 12 / 2006$ & & JGJ-062-06 \\
\hline GWM44301VL & USGS-002 & 683 & 1,2-Dibromo-3-chloropropane & 1 & & $\mathrm{U}$ & $\mathrm{R}$ & UG/L & $06 / 12 / 2006$ & & JGJ-062-06 \\
\hline GWM44301VL & USGS-002 & 683 & Chloroethane & 2 & & $\mathrm{U}$ & & UG/L & $06 / 12 / 2006$ & & JGJ-062-06 \\
\hline
\end{tabular}


Table A-2. (continued).

\begin{tabular}{|c|c|c|c|c|c|c|c|c|c|c|c|}
\hline $\begin{array}{c}\text { Field Sample } \\
\text { Number }\end{array}$ & Location & Depth & Compound & $\begin{array}{c}\text { Sample } \\
\text { Result }\end{array}$ & $\begin{array}{c}\text { Sample } \\
\text { Error }\end{array}$ & $\begin{array}{c}\text { Result } \\
\text { Qualifier }\end{array}$ & $\begin{array}{c}\text { Validation } \\
\text { Flag }\end{array}$ & $\begin{array}{c}\text { Sample } \\
\text { Units }\end{array}$ & $\begin{array}{c}\text { Date Sample } \\
\text { Collected }\end{array}$ & MDA & $\begin{array}{c}\text { L\&V Report } \\
\text { Number }\end{array}$ \\
\hline GWM44301VL & USGS-002 & 683 & Chloroform & 1 & & $U$ & & UG/L & $06 / 12 / 2006$ & & JGJ-062-06 \\
\hline GWM44301VL & USGS-002 & 683 & Chloromethane & 2 & & U & & UG/L & $06 / 12 / 2006$ & & JGJ-062-06 \\
\hline GWM44301VL & USGS-002 & 683 & Cyclohexane & 1 & & $\mathrm{U}$ & & UG/L & $06 / 12 / 2006$ & & JGJ-062-06 \\
\hline GWM44301VL & USGS-002 & 683 & 1,2-Dichlorobenzene & 1 & & $\mathrm{U}$ & & UG/L & $06 / 12 / 2006$ & & JGJ-062-06 \\
\hline GWM44301VL & USGS-002 & 683 & 1,3-Dichlorobenzene & 1 & & $\mathrm{U}$ & & UG/L & $06 / 12 / 2006$ & & JGJ-062-06 \\
\hline GWM44301VL & USGS-002 & 683 & 1,4-Dichlorobenzene & 1 & & $\mathrm{U}$ & & UG/L & $06 / 12 / 2006$ & & JGJ-062-06 \\
\hline GWM44301VL & USGS-002 & 683 & Dichlorodifluoromethane & 2 & & U & & UG/L & $06 / 12 / 2006$ & & JGJ-062-06 \\
\hline GWM44301VL & USGS-002 & 683 & 1,1-Dichloroethane & 1 & & $\mathrm{U}$ & & UG/L & $06 / 12 / 2006$ & & JGJ-062-06 \\
\hline GWM44301VL & USGS-002 & 683 & 1,2-Dichloroethane & 1 & & $\mathrm{U}$ & & UG/L & $06 / 12 / 2006$ & & JGJ-062-06 \\
\hline GWM44301VL & USGS-002 & 683 & 1,1-Dichloroethene & 1 & & U & & UG/L & $06 / 12 / 2006$ & & JGJ-062-06 \\
\hline GWM44301VL & USGS-002 & 683 & cis-1,2-Dichloroethene & 1 & & U & & UG/L & $06 / 12 / 2006$ & & JGJ-062-06 \\
\hline GWM44301VL & USGS-002 & 683 & trans-1,2-Dichloroethene & 1 & & $\mathrm{U}$ & & UG/L & $06 / 12 / 2006$ & & JGJ-062-06 \\
\hline GWM44301VL & USGS-002 & 683 & 1,2-Dichloropropane & 1 & & U & & UG/L & $06 / 12 / 2006$ & & JGJ-062-06 \\
\hline GWM44301VL & USGS-002 & 683 & cis-1,3-Dichloropropene & 1 & & U & & UG/L & $06 / 12 / 2006$ & & JGJ-062-06 \\
\hline GWM44301VL & USGS-002 & 683 & trans-1,3-Dichloropropene & 1 & & $\mathrm{U}$ & & UG/L & $06 / 12 / 2006$ & & JGJ-062-06 \\
\hline GWM44301VL & USGS-002 & 683 & Ethylbenzene & 1 & & $\mathrm{U}$ & & UG/L & $06 / 12 / 2006$ & & JGJ-062-06 \\
\hline GWM44301VL & USGS-002 & 683 & Trichlorofluoromethane & 1 & & U & & UG/L & $06 / 12 / 2006$ & & JGJ-062-06 \\
\hline GWM44301VL & USGS-002 & 683 & 2-Hexanone & 5 & & $\mathrm{U}$ & & UG/L & $06 / 12 / 2006$ & & JGJ-062-06 \\
\hline GWM44301VL & USGS-002 & 683 & Methyl cyclohexane & 4 & & U & & UG/L & $06 / 12 / 2006$ & & JGJ-062-06 \\
\hline GWM44301VL & USGS-002 & 683 & Methylene Chloride & 1 & & U & & UG/L & $06 / 12 / 2006$ & & JGJ-062-06 \\
\hline GWM44301VL & USGS-002 & 683 & 4-Methyl-2-pentanone & 5 & & $U$ & & UG/L & $06 / 12 / 2006$ & & JGJ-062-06 \\
\hline GWM44301VL & USGS-002 & 683 & Styrene & 1 & & $\mathrm{U}$ & & UG/L & $06 / 12 / 2006$ & & JGJ-062-06 \\
\hline GWM44301VL & USGS-002 & 683 & 1,1,2,2-Tetrachloroethane & 1 & & U & & UG/L & $06 / 12 / 2006$ & & JGJ-062-06 \\
\hline GWM44301VL & USGS-002 & 683 & Tetrachloroethene & 1 & & $U$ & & UG/L & $06 / 12 / 2006$ & & JGJ-062-06 \\
\hline GWM44301VL & USGS-002 & 683 & Toluene & 1.9 & & B & & UG/L & $06 / 12 / 2006$ & & JGJ-062-06 \\
\hline GWM44301VL & USGS-002 & 683 & 1,2,4-Trichlorobenzene & 1 & & U & & UG/L & $06 / 12 / 2006$ & & JGJ-062-06 \\
\hline GWM44301VL & USGS-002 & 683 & 1,1,1-Trichloroethane & 1 & & $U$ & & UG/L & $06 / 12 / 2006$ & & JGJ-062-06 \\
\hline GWM44301VL & USGS-002 & 683 & 1,1,2-Trichloroethane & 1 & & U & & UG/L & $06 / 12 / 2006$ & & JGJ-062-06 \\
\hline GWM44301VL & USGS-002 & 683 & Trichloroethene & 1 & & $\mathrm{U}$ & & UG/L & $06 / 12 / 2006$ & & JGJ-062-06 \\
\hline GWM44301VL & USGS-002 & 683 & 1,1,2-Trichloro-1,2,2-trifluoroethane & 1 & & $\mathrm{U}$ & & UG/L & $06 / 12 / 2006$ & & JGJ-062-06 \\
\hline GWM44301VL & USGS-002 & 683 & Vinyl Chloride & 2 & & $\mathrm{U}$ & & UG/L & $06 / 12 / 2006$ & & JGJ-062-06 \\
\hline GWM44301VL & USGS-002 & 683 & Xylene (Total) & 3 & & $\mathrm{U}$ & & UG/L & $06 / 12 / 2006$ & & JGJ-062-06 \\
\hline GWM44301VL & USGS-002 & 683 & Methyl t-butyl ether & 2 & & U & & UG/L & $06 / 12 / 2006$ & & JGJ-062-06 \\
\hline GWM44301R8 & USGS-002 & 683 & Tritium & $-4.36 \mathrm{E}+01$ & $1.25 \mathrm{E}+02$ & & $\mathrm{U}$ & $\mathrm{PCl} / \mathrm{L}$ & $06 / 12 / 2006$ & $4.43 E+02$ & SOS-TL199-06 \\
\hline GWM44301RH & USGS-002 & 683 & Gross Alpha & $2.81 \mathrm{E}+00$ & 7.25E-01 & & & $\mathrm{PCl} / \mathrm{L}$ & $06 / 12 / 2006$ & $2.26 \mathrm{E}+00$ & SOS-TL198-06 \\
\hline GWM44301RH & USGS-002 & 683 & Gross Beta & $4.20 \mathrm{E}+00$ & $8.42 \mathrm{E}-01$ & & & $\mathrm{PCI} / \mathrm{L}$ & $06 / 12 / 2006$ & $3.09 \mathrm{E}+00$ & SOS-TL198-06 \\
\hline GWM44301RH & USGS-002 & 683 & Strontium-90 & $9.40 \mathrm{E}-02$ & 1.01E-01 & & $U$ & $\mathrm{PCl} / \mathrm{L}$ & $06 / 12 / 2006$ & 4.56E-01 & SOS-TL198-06 \\
\hline GWM436012X & USGS-009 & 656 & Antimony & 0.5 & & $\mathrm{U}$ & & UG/L & $06 / 20 / 2006$ & & DNT-330-06 \\
\hline GWM436012X & USGS-009 & 656 & Arsenic & 2 & & U & & UG/L & $06 / 20 / 2006$ & & DNT-330-06 \\
\hline GWM436012X & USGS-009 & 656 & Barium & 36.4 & & & & UG/L & $06 / 20 / 2006$ & & DNT-330-06 \\
\hline GWM436012X & USGS-009 & 656 & Beryllium & 0.088 & & $\mathrm{U}$ & & UG/L & $06 / 20 / 2006$ & & DNT-330-06 \\
\hline GWM436012X & USGS-009 & 656 & Cadmium & 0.057 & & U & & UG/L & $06 / 20 / 2006$ & & DNT-330-06 \\
\hline GWM436012X & USGS-009 & 656 & Calcium & 36700 & & & & UG/L & $06 / 20 / 2006$ & & DNT-330-06 \\
\hline GWM436012X & USGS-009 & 656 & Chromium & 3.4 & & B & & UG/L & $06 / 20 / 2006$ & & DNT-330-06 \\
\hline GWM436012X & USGS-009 & 656 & Cobalt & 0.31 & & $\mathrm{U}$ & & UG/L & $06 / 20 / 2006$ & & DNT-330-06 \\
\hline GWM436012X & USGS-009 & 656 & Copper & 0.42 & & B & & UG/L & $06 / 20 / 2006$ & & DNT-330-06 \\
\hline GWM436012X & USGS-009 & 656 & Iron & 36 & & B & & UG/L & $06 / 20 / 2006$ & & DNT-330-06 \\
\hline GWM436012X & USGS-009 & 656 & Lead & 0.49 & & U & & UG/L & $06 / 20 / 2006$ & & DNT-330-06 \\
\hline GWM436012X & USGS-009 & 656 & Magnesium & 14000 & & $\mathrm{E}$ & $\mathrm{J}$ & UG/L & $06 / 20 / 2006$ & & DNT-330-06 \\
\hline GWM436012X & USGS-009 & 656 & Manganese & 9 & & & & UG/L & $06 / 20 / 2006$ & & DNT-330-06 \\
\hline GWM436012X & USGS-009 & 656 & Nickel & 0.86 & & B & & UG/L & $06 / 20 / 2006$ & & DNT-330-06 \\
\hline GWM436012X & USGS-009 & 656 & Potassium & 2790 & & $\mathrm{E}$ & $\mathrm{J}$ & UG/L & $06 / 20 / 2006$ & & DNT-330-06 \\
\hline GWM436012X & USGS-009 & 656 & Selenium & 1.6 & & B & & UG/L & $06 / 20 / 2006$ & & DNT-330-06 \\
\hline GWM436012X & USGS-009 & 656 & Silver & 0.2 & & U & & UG/L & $06 / 20 / 2006$ & & DNT-330-06 \\
\hline GWM436012X & USGS-009 & 656 & Sodium & 11100 & & & & UG/L & $06 / 20 / 2006$ & & DNT-330-06 \\
\hline GWM436012X & USGS-009 & 656 & Strontium & 175 & & E & $\mathrm{J}$ & UG/L & $06 / 20 / 2006$ & & DNT-330-06 \\
\hline GWM436012X & USGS-009 & 656 & Thallium & 0.81 & & B & $\mathrm{U}$ & UG/L & $06 / 20 / 2006$ & & DNT-330-06 \\
\hline GWM436012X & USGS-009 & 656 & Uranium & 1.9 & & & & UG/L & $06 / 20 / 2006$ & & DNT-330-06 \\
\hline GWM436012X & USGS-009 & 656 & Vanadium & 3.9 & & B & & UG/L & $06 / 20 / 2006$ & & DNT-330-06 \\
\hline GWM436012X & USGS-009 & 656 & Zinc & 4.1 & & B & $\mathrm{U}$ & UG/L & $06 / 20 / 2006$ & & DNT-330-06 \\
\hline GWM436012X & USGS-009 & 656 & Mercury & 0.093 & & $U$ & & UG/L & $06 / 20 / 2006$ & & DNT-330-06 \\
\hline GWM43601A1 & USGS-009 & 656 & Total Alkalinity & 136 & & & $\mathrm{~J}$ & $M G / L$ & $06 / 20 / 2006$ & & DNT-321-06 \\
\hline GWM43601AN & USGS-009 & 656 & Chloride & 17.1 & & & & $M G / L$ & $06 / 20 / 2006$ & & DNT-321-06 \\
\hline GWM43601AN & USGS-009 & 656 & Fluoride & 0.26 & & & $\mathrm{~J}$ & $M G / L$ & $06 / 20 / 2006$ & & DNT-321-06 \\
\hline GWM43601AN & USGS-009 & 656 & Sulfate & 24.2 & & & & $M G / L$ & $06 / 20 / 2006$ & & DNT-321-06 \\
\hline GWM43601N2 & USGS-009 & 656 & Nitrate/Nitrite as $\mathrm{N}$ & 671 & & & & UG/L & $06 / 20 / 2006$ & & DNT-321-06 \\
\hline GWM43601VL & USGS-009 & 656 & Acetone & 2 & & U & $\mathrm{R}$ & UG/L & $06 / 20 / 2006$ & & JGJ-066-06 \\
\hline GWM43601VL & USGS-009 & 656 & Benzene & 1 & & $U$ & & UG/L & $06 / 20 / 2006$ & & JGJ-066-06 \\
\hline GWM43601VL & USGS-009 & 656 & Bromodichloromethane & 1 & & $\mathrm{U}$ & & UG/L & $06 / 20 / 2006$ & & JGJ-066-06 \\
\hline GWM43601VL & USGS-009 & 656 & Bromoform & 1 & & U & & UG/L & $06 / 20 / 2006$ & & JGJ-066-06 \\
\hline GWM43601VL & USGS-009 & 656 & Bromomethane & 2 & & U & & UG/L & $06 / 20 / 2006$ & & JGJ-066-06 \\
\hline GWM43601VL & USGS-009 & 656 & 2-Butanone & 5 & & $U$ & $\mathrm{R}$ & UG/L & $06 / 20 / 2006$ & & JGJ-066-06 \\
\hline
\end{tabular}


Table A-2. (continued).

\begin{tabular}{|c|c|c|c|c|c|c|c|c|c|c|c|}
\hline $\begin{array}{l}\text { Field Sample } \\
\text { Number }\end{array}$ & Location & Depth & Compound & $\begin{array}{c}\text { Sample } \\
\text { Result }\end{array}$ & $\begin{array}{l}\text { Sample } \\
\text { Error }\end{array}$ & $\begin{array}{c}\text { Result } \\
\text { Qualifier }\end{array}$ & $\begin{array}{c}\text { Validation } \\
\text { Flag }\end{array}$ & $\begin{array}{c}\text { Sample } \\
\text { Units }\end{array}$ & $\begin{array}{c}\text { Date Sample } \\
\text { Collected }\end{array}$ & MDA & $\begin{array}{l}\text { L\&V Report } \\
\text { Number }\end{array}$ \\
\hline GWM43601VL & USGS-009 & 656 & Carbon disulfide & 1 & & $\mathrm{U}$ & & UG/L & $06 / 20 / 2006$ & & JGJ-066-06 \\
\hline GWM43601VL & USGS-009 & 656 & Carbon tetrachloride & 1 & & U & & UG/L & 06/20/2006 & & JGJ-066-06 \\
\hline GWM43601VL & USGS-009 & 656 & Chlorobenzene & 1 & & $\mathrm{U}$ & & UG/L & $06 / 20 / 2006$ & & JGJ-066-06 \\
\hline GWM43601VL & USGS-009 & 656 & 1,2-Dibromo-3-chloropropane & 1 & & $\mathrm{U}$ & $\mathrm{R}$ & UG/L & $06 / 20 / 2006$ & & JGJ-066-06 \\
\hline GWM43601VL & USGS-009 & 656 & Chloroethane & 2 & & U & & UG/L & 06/20/2006 & & JGJ-066-06 \\
\hline GWM43601VL & USGS-009 & 656 & Chloroform & 1 & & U & & UG/L & $06 / 20 / 2006$ & & JGJ-066-06 \\
\hline GWM43601VL & USGS-009 & 656 & Chloromethane & 2 & & $U$ & & UG/L & $06 / 20 / 2006$ & & JGJ-066-06 \\
\hline GWM43601VL & USGS-009 & 656 & 1,2-Dibromoethane & 1 & & U & & UG/L & $06 / 20 / 2006$ & & JGJ-066-06 \\
\hline GWM43601VL & USGS-009 & 656 & 1,2-Dichlorobenzene & 1 & & $U$ & & UG/L & $06 / 20 / 2006$ & & JGJ-066-06 \\
\hline GWM43601VL & USGS-009 & 656 & 1,3-Dichlorobenzene & 1 & & U & & UG/L & $06 / 20 / 2006$ & & JGJ-066-06 \\
\hline GWM43601VL & USGS-009 & 656 & 1,4-Dichlorobenzene & 1 & & U & & UG/L & 06/20/2006 & & JGJ-066-06 \\
\hline GWM43601VL & USGS-009 & 656 & Dichlorodifluoromethane & 2 & & $\mathrm{u}$ & & UG/L & 06/20/2006 & & JGJ-066-06 \\
\hline GWM43601VL & USGS-009 & 656 & 1,1-Dichloroethane & 1 & & $\mathrm{U}$ & & UG/L & 06/20/2006 & & JGJ-066-06 \\
\hline GWM43601VL & USGS-009 & 656 & 1,2-Dichloroethane & 1 & & $\mathrm{U}$ & & UG/L & $06 / 20 / 2006$ & & JGJ-066-06 \\
\hline GWM43601VL & USGS-009 & 656 & 1,1-Dichloroethene & 1 & & $U$ & & UG/L & 06/20/2006 & & JGJ-066-06 \\
\hline GWM43601VL & USGS-009 & 656 & cis-1,2-Dichloroethene & 1 & & $\mathrm{U}$ & & UG/L & 06/20/2006 & & JGJ-066-06 \\
\hline GWM43601VL & USGS-009 & 656 & trans-1,2-Dichloroethene & 1 & & $\mathrm{U}$ & & UG/L & $06 / 20 / 2006$ & & JGJ-066-06 \\
\hline GWM43601VL & USGS-009 & 656 & cis-1,3-Dichloropropene & 1 & & U & & UG/L & $06 / 20 / 2006$ & & JGJ-066-06 \\
\hline GWM43601VL & USGS-009 & 656 & trans-1,3-Dichloropropene & 1 & & $\mathrm{U}$ & & UG/L & $06 / 20 / 2006$ & & JGJ-066-06 \\
\hline GWM43601VL & USGS-009 & 656 & Ethylbenzene & 1 & & $\mathrm{U}$ & & UG/L & 06/20/2006 & & JGJ-066-06 \\
\hline GWM43601VL & USGS-009 & 656 & Trichlorofluoromethane & 1 & & $U$ & & UG/L & $06 / 20 / 2006$ & & JGJ-066-06 \\
\hline GWM43601VL & USGS-009 & 656 & 2-Hexanone & 5 & & $\mathrm{U}$ & $\mathrm{R}$ & UG/L & 06/20/2006 & & JGJ-066-06 \\
\hline GWM43601VL & USGS-009 & 656 & Isopropylbenzene & 1 & & $\mathrm{U}$ & & UG/L & $06 / 20 / 2006$ & & JGJ-066-06 \\
\hline GWM43601VL & USGS-009 & 656 & Methyl acetate & 5 & & U & $\mathrm{R}$ & UG/L & $06 / 20 / 2006$ & & JGJ-066-06 \\
\hline GWM43601VL & USGS-009 & 656 & Methyl cyclohexane & 4 & & $\mathrm{U}$ & & UG/L & 06/20/2006 & & JGJ-066-06 \\
\hline GWM43601VL & USGS-009 & 656 & Methylene Chloride & 1 & & $\mathrm{U}$ & & UG/L & 06/20/2006 & & JGJ-066-06 \\
\hline GWM43601VL & USGS-009 & 656 & 4-Methyl-2-pentanone & 5 & & U & & UG/L & 06/20/2006 & & JGJ-066-06 \\
\hline GWM43601VL & USGS-009 & 656 & Styrene & 1 & & $\mathrm{U}$ & & UG/L & 06/20/2006 & & JGJ-066-06 \\
\hline GWM43601VL & USGS-009 & 656 & 1,1,2,2-Tetrachloroethane & 1 & & $\mathrm{u}$ & & UG/L & 06/20/2006 & & JGJ-066-06 \\
\hline GWM43601VL & USGS-009 & 656 & Tetrachloroethene & 1 & & U & & UG/L & 06/20/2006 & & JGJ-066-06 \\
\hline GWM43601VL & USGS-009 & 656 & Toluene & 1 & & $\mathrm{U}$ & & UG/L & 06/20/2006 & & JGJ-066-06 \\
\hline GWM43601VL & USGS-009 & 656 & 1,2,4-Trichlorobenzene & 1 & & $\mathrm{U}$ & & UG/L & $06 / 20 / 2006$ & & JGJ-066-06 \\
\hline GWM43601VL & USGS-009 & 656 & 1,1,1-Trichloroethane & 1 & & $\mathrm{U}$ & & UG/L & $06 / 20 / 2006$ & & JGJ-066-06 \\
\hline GWM43601VL & USGS-009 & 656 & 1,1,2-Trichloroethane & 1 & & $U$ & & UG/L & $06 / 20 / 2006$ & & JGJ-066-06 \\
\hline GWM43601VL & USGS-009 & 656 & Trichloroethene & 1 & & $\mathrm{U}$ & & UG/L & 06/20/2006 & & JGJ-066-06 \\
\hline GWM43601VL & USGS-009 & 656 & 1,1,2-Trichloro-1,2,2-trifluoroethane & 1 & & U & & UG/L & 06/20/2006 & & JGJ-066-06 \\
\hline GWM43601VL & USGS-009 & 656 & Vinyl Chloride & 2 & & U & & UG/L & 06/20/2006 & & JGJ-066-06 \\
\hline GWM43601RH & USGS-009 & 656 & Gross Alpha & $2.13 \mathrm{E}+00$ & 3.73E-01 & & & $\mathrm{PCl} / \mathrm{L}$ & $06 / 20 / 2006$ & 8.83E-01 & SOS-TL198-06 \\
\hline GWM43601RH & USGS-009 & 656 & Gross Beta & $2.59 \mathrm{E}+00$ & $5.40 \mathrm{E}-01$ & & & $\mathrm{PCI} / \mathrm{L}$ & $06 / 20 / 2006$ & $1.89 \mathrm{E}+00$ & SOS-TL198-06 \\
\hline GWM43601RH & USGS-009 & 656 & Strontium-90 & $-2.26 \mathrm{E}-02$ & 8.84E-02 & & $\mathrm{U}$ & $\mathrm{PCl} / \mathrm{L}$ & 06/20/2006 & 4.59E-01 & SOS-TL198-06 \\
\hline GWM43601RH & USGS-009 & 656 & Technetium-99 & $5.30 \mathrm{E}+00$ & $2.13 E+00$ & & UJ & $\mathrm{PCl} / \mathrm{L}$ & 06/20/2006 & $6.93 \mathrm{E}+00$ & SOS-TL198-06 \\
\hline GWM43601UX & USGS-009 & 656 & lodine-129 & $2.65 \mathrm{E}-03$ & $2.91 \mathrm{E}-02$ & & $\mathrm{U}$ & $\mathrm{PCl} / \mathrm{L}$ & 06/20/2006 & $9.66 \mathrm{E}-02$ & SOS-TL197-06 \\
\hline GWM448012X & USGS-011 & 687 & Aluminum & 7.9 & & $U$ & & UG/L & $06 / 20 / 2006$ & & DNT-330-06 \\
\hline GWM448012X & USGS-011 & 687 & Antimony & 0.5 & & $U$ & & UG/L & $06 / 20 / 2006$ & & DNT-330-06 \\
\hline GWM448012X & USGS-011 & 687 & Arsenic & 2 & & $\mathrm{U}$ & & UG/L & $06 / 20 / 2006$ & & DNT-330-06 \\
\hline GWM448012X & USGS-011 & 687 & Barium & 53.3 & & & & UG/L & 06/20/2006 & & DNT-330-06 \\
\hline GWM448012X & USGS-011 & 687 & Beryllium & 0.088 & & $U$ & & UG/L & 06/20/2006 & & DNT-330-06 \\
\hline GWM448012X & USGS-011 & 687 & Cadmium & 0.057 & & U & & UG/L & $06 / 20 / 2006$ & & DNT-330-06 \\
\hline GWM448012X & USGS-011 & 687 & Calcium & 38400 & & & & UG/L & 06/20/2006 & & DNT-330-06 \\
\hline GWM448012X & USGS-011 & 687 & Chromium & 3.8 & & B & & UG/L & 06/20/2006 & & DNT-330-06 \\
\hline GWM448012X & USGS-011 & 687 & Cobalt & 0.31 & & U & & UG/L & $06 / 20 / 2006$ & & DNT-330-06 \\
\hline GWM448012X & USGS-011 & 687 & Copper & 0.41 & & B & & UG/L & $06 / 20 / 2006$ & & DNT-330-06 \\
\hline GWM448012X & USGS-011 & 687 & Iron & 9.4 & & $U$ & & UG/L & 06/20/2006 & & DNT-330-06 \\
\hline GWM448012X & USGS-011 & 687 & Lead & 0.49 & & U & & UG/L & $06 / 20 / 2006$ & & DNT-330-06 \\
\hline GWM448012X & USGS-011 & 687 & Magnesium & 13300 & & $\mathrm{E}$ & $\mathrm{J}$ & UG/L & 06/20/2006 & & DNT-330-06 \\
\hline GWM448012X & USGS-011 & 687 & Manganese & 1.1 & & B & & UG/L & $06 / 20 / 2006$ & & DNT-330-06 \\
\hline GWM448012X & USGS-011 & 687 & Nickel & 0.86 & & B & & UG/L & $06 / 20 / 2006$ & & DNT-330-06 \\
\hline GWM448012X & USGS-011 & 687 & Potassium & 2100 & & $\mathrm{E}$ & $\mathrm{J}$ & UG/L & $06 / 20 / 2006$ & & DNT-330-06 \\
\hline GWM448012X & USGS-011 & 687 & Selenium & 1.1 & & B & & UG/L & 06/20/2006 & & DNT-330-06 \\
\hline GWM448012X & USGS-011 & 687 & Silver & 0.2 & & $U$ & & UG/L & $06 / 20 / 2006$ & & DNT-330-06 \\
\hline GWM448012X & USGS-011 & 687 & Sodium & 7800 & & & & UG/L & $06 / 20 / 2006$ & & DNT-330-06 \\
\hline GWM448012X & USGS-011 & 687 & Strontium & 228 & & $\mathrm{E}$ & $\mathrm{J}$ & UG/L & 06/20/2006 & & DNT-330-06 \\
\hline GWM448012X & USGS-011 & 687 & Thallium & 0.32 & & $U$ & & UG/L & 06/20/2006 & & DNT-330-06 \\
\hline GWM448012X & USGS-011 & 687 & Uranium & 1.8 & & & & UG/L & 06/20/2006 & & DNT-330-06 \\
\hline GWM448012X & USGS-011 & 687 & Vanadium & 3.6 & & B & & UG/L & 06/20/2006 & & DNT-330-06 \\
\hline GWM448012X & USGS-011 & 687 & Zinc & 124 & & & & UG/L & 06/20/2006 & & DNT-330-06 \\
\hline GWM448012X & USGS-011 & 687 & Mercury & 0.093 & & U & & UG/L & 06/20/2006 & & DNT-330-06 \\
\hline GWM44801A1 & USGS-011 & 687 & Total Alkalinity & 140 & & & $\mathrm{~J}$ & $M G / L$ & $06 / 20 / 2006$ & & DNT-321-06 \\
\hline GWM44801AN & USGS-011 & 687 & Chloride & 10.2 & & & & $M G / L$ & 06/20/2006 & & DNT-321-06 \\
\hline GWM44801AN & USGS-011 & 687 & Fluoride & 0.25 & & & $J$ & $M G / L$ & 06/20/2006 & & DNT-321-06 \\
\hline GWM44801AN & USGS-011 & 687 & Sulfate & 21.9 & & & & $M G / L$ & $06 / 20 / 2006$ & & DNT-321-06 \\
\hline GWM44801N2 & USGS-011 & 687 & Nitrate/Nitrite as $\mathrm{N}$ & 745 & & & & UG/L & 06/20/2006 & & DNT-321-06 \\
\hline
\end{tabular}


Table A-2. (continued).

\begin{tabular}{|c|c|c|c|c|c|c|c|c|c|c|c|}
\hline $\begin{array}{l}\text { Field Sample } \\
\text { Number }\end{array}$ & Location & Depth & Compound & $\begin{array}{c}\text { Sample } \\
\text { Result }\end{array}$ & $\begin{array}{l}\text { Sample } \\
\text { Error }\end{array}$ & $\begin{array}{c}\text { Result } \\
\text { Qualifier }\end{array}$ & $\begin{array}{c}\text { Validation } \\
\text { Flag }\end{array}$ & $\begin{array}{c}\text { Sample } \\
\text { Units }\end{array}$ & $\begin{array}{c}\text { Date Sample } \\
\text { Collected }\end{array}$ & MDA & $\begin{array}{l}\text { L\&V Report } \\
\text { Number }\end{array}$ \\
\hline GWM44801VL & USGS-011 & 687 & Acetone & 2 & & $\mathrm{U}$ & $\mathrm{R}$ & UG/L & $06 / 20 / 2006$ & & JGJ-066-06 \\
\hline GWM44801VL & USGS-011 & 687 & Benzene & 1 & & U & & UG/L & $06 / 20 / 2006$ & & JGJ-066-06 \\
\hline GWM44801VL & USGS-011 & 687 & Bromodichloromethane & 1 & & $\mathrm{U}$ & & UG/L & $06 / 20 / 2006$ & & JGJ-066-06 \\
\hline GWM44801VL & USGS-011 & 687 & Bromomethane & 2 & & $U$ & & UG/L & $06 / 20 / 2006$ & & JGJ-066-06 \\
\hline GWM44801VL & USGS-011 & 687 & 2-Butanone & 5 & & $\mathrm{U}$ & $\mathrm{R}$ & UG/L & $06 / 20 / 2006$ & & JGJ-066-06 \\
\hline GWM44801VL & USGS-011 & 687 & Carbon disulfide & 1 & & U & & UG/L & $06 / 20 / 2006$ & & JGJ-066-06 \\
\hline GWM44801VL & USGS-011 & 687 & Carbon tetrachloride & 1 & & $U$ & & UG/L & $06 / 20 / 2006$ & & JGJ-066-06 \\
\hline GWM44801VL & USGS-011 & 687 & Dibromochloromethane & 1 & & $\mathrm{U}$ & & UG/L & $06 / 20 / 2006$ & & JGJ-066-06 \\
\hline GWM44801VL & USGS-011 & 687 & 1,2-Dibromo-3-chloropropane & 1 & & $U$ & $\mathrm{R}$ & UG/L & $06 / 20 / 2006$ & & JGJ-066-06 \\
\hline GWM44801VL & USGS-011 & 687 & Chloroethane & 2 & & U & & UG/L & $06 / 20 / 2006$ & & JGJ-066-06 \\
\hline GWM44801VL & USGS-011 & 687 & Chloroform & 1 & & U & & UG/L & $06 / 20 / 2006$ & & JGJ-066-06 \\
\hline GWM44801VL & USGS-011 & 687 & Chloromethane & 2 & & $\mathrm{u}$ & & UG/L & $06 / 20 / 2006$ & & JGJ-066-06 \\
\hline GWM44801VL & USGS-011 & 687 & Cyclohexane & 1 & & $\mathrm{U}$ & & UG/L & $06 / 20 / 2006$ & & JGJ-066-06 \\
\hline GWM44801VL & USGS-011 & 687 & 1,2-Dibromoethane & 1 & & $\mathrm{U}$ & & UG/L & $06 / 20 / 2006$ & & JGJ-066-06 \\
\hline GWM44801VL & USGS-011 & 687 & 1,2-Dichlorobenzene & 1 & & $U$ & & UG/L & $06 / 20 / 2006$ & & JGJ-066-06 \\
\hline GWM44801VL & USGS-011 & 687 & 1,3-Dichlorobenzene & 1 & & $\mathrm{U}$ & & UG/L & $06 / 20 / 2006$ & & JGJ-066-06 \\
\hline GWM44801VL & USGS-011 & 687 & 1,4-Dichlorobenzene & 1 & & $\mathrm{U}$ & & UG/L & $06 / 20 / 2006$ & & JGJ-066-06 \\
\hline GWM44801VL & USGS-011 & 687 & 1,1-Dichloroethane & 1 & & U & & UG/L & $06 / 20 / 2006$ & & JGJ-066-06 \\
\hline GWM44801VL & USGS-011 & 687 & 1,2-Dichloroethane & 1 & & $\mathrm{U}$ & & UG/L & $06 / 20 / 2006$ & & JGJ-066-06 \\
\hline GWM44801VL & USGS-011 & 687 & 1,1-Dichloroethene & 1 & & $\mathrm{U}$ & & UG/L & 06/20/2006 & & JGJ-066-06 \\
\hline GWM44801VL & USGS-011 & 687 & cis-1,2-Dichloroethene & 1 & & 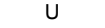 & & UG/L & $06 / 20 / 2006$ & & JGJ-066-06 \\
\hline GWM44801VL & USGS-011 & 687 & trans-1,2-Dichloroethene & 1 & & $\mathrm{U}$ & & UG/L & $06 / 20 / 2006$ & & JGJ-066-06 \\
\hline GWM44801VL & USGS-011 & 687 & 1,2-Dichloropropane & 1 & & $\mathrm{U}$ & & UG/L & $06 / 20 / 2006$ & & JGJ-066-06 \\
\hline GWM44801VL & USGS-011 & 687 & cis-1,3-Dichloropropene & 1 & & $U$ & & UG/L & $06 / 20 / 2006$ & & JGJ-066-06 \\
\hline GWM44801VL & USGS-011 & 687 & trans-1,3-Dichloropropene & 1 & & $\mathrm{U}$ & & UG/L & $06 / 20 / 2006$ & & JGJ-066-06 \\
\hline GWM44801VL & USGS-011 & 687 & Ethylbenzene & 1 & & $\mathrm{U}$ & & UG/L & $06 / 20 / 2006$ & & JGJ-066-06 \\
\hline GWM44801VL & USGS-011 & 687 & Trichlorofluoromethane & 1 & & U & & UG/L & $06 / 20 / 2006$ & & JGJ-066-06 \\
\hline GWM44801VL & USGS-011 & 687 & 2-Hexanone & 5 & & $\mathrm{U}$ & $\mathrm{R}$ & UG/L & $06 / 20 / 2006$ & & JGJ-066-06 \\
\hline GWM44801VL & USGS-011 & 687 & Isopropylbenzene & 1 & & $\mathrm{U}$ & & UG/L & $06 / 20 / 2006$ & & JGJ-066-06 \\
\hline GWM44801VL & USGS-011 & 687 & Methyl acetate & 5 & & U & $\mathrm{R}$ & UG/L & $06 / 20 / 2006$ & & JGJ-066-06 \\
\hline GWM44801VL & USGS-011 & 687 & Methyl cyclohexane & 4 & & $\mathrm{u}$ & & UG/L & $06 / 20 / 2006$ & & JGJ-066-06 \\
\hline GWM44801VL & USGS-011 & 687 & Methylene Chloride & 1 & & $\mathrm{U}$ & & UG/L & $06 / 20 / 2006$ & & JGJ-066-06 \\
\hline GWM44801VL & USGS-011 & 687 & 4-Methyl-2-pentanone & 5 & & $\mathrm{U}$ & & UG/L & $06 / 20 / 2006$ & & JGJ-066-06 \\
\hline GWM44801VL & USGS-011 & 687 & Styrene & 1 & & $U$ & & UG/L & $06 / 20 / 2006$ & & JGJ-066-06 \\
\hline GWM44801VL & USGS-011 & 687 & 1,1,2,2-Tetrachloroethane & 1 & & $\mathrm{U}$ & & UG/L & $06 / 20 / 2006$ & & JGJ-066-06 \\
\hline GWM44801VL & USGS-011 & 687 & Tetrachloroethene & 1 & & U & & UG/L & $06 / 20 / 2006$ & & JGJ-066-06 \\
\hline GWM44801VL & USGS-011 & 687 & Toluene & 1 & & $\mathrm{U}$ & & UG/L & $06 / 20 / 2006$ & & JGJ-066-06 \\
\hline GWM44801VL & USGS-011 & 687 & Trichloroethene & 1 & & U & & UG/L & $06 / 20 / 2006$ & & JGJ-066-06 \\
\hline GWM44801VL & USGS-011 & 687 & 1,1,2-Trichloro-1,2,2-trifluoroethane & 1 & & $\mathrm{U}$ & & UG/L & $06 / 20 / 2006$ & & JGJ-066-06 \\
\hline GWM44801VL & USGS-011 & 687 & Vinyl Chloride & 2 & & $\mathrm{U}$ & & UG/L & $06 / 20 / 2006$ & & JGJ-066-06 \\
\hline GWM44801VL & USGS-011 & 687 & Xylene (Total) & 3 & & $\mathrm{U}$ & & UG/L & $06 / 20 / 2006$ & & JGJ-066-06 \\
\hline GWM44801VL & USGS-011 & 687 & Methyl t-butyl ether & 2 & & $\mathrm{U}$ & & UG/L & $06 / 20 / 2006$ & & JGJ-066-06 \\
\hline GWM44801R8 & USGS-011 & 687 & Tritium & $-8.32 \mathrm{E}+01$ & $1.18 \mathrm{E}+02$ & & $\mathrm{U}$ & $\mathrm{PCI} / \mathrm{L}$ & $06 / 20 / 2006$ & $4.23 \mathrm{E}+02$ & SOS-TL199-06 \\
\hline GWM44801RH & USGS-011 & 687 & Gross Alpha & $2.31 \mathrm{E}+00$ & 4.63E-01 & & & $\mathrm{PCl} / \mathrm{L}$ & $06 / 20 / 2006$ & $1.39 \mathrm{E}+00$ & SOS-TL198-06 \\
\hline GWM44801RH & USGS-011 & 687 & Gross Beta & $2.11 \mathrm{E}+00$ & 4.99E-01 & & & $\mathrm{PCl} / \mathrm{L}$ & $06 / 20 / 2006$ & $1.76 \mathrm{E}+00$ & SOS-TL198-06 \\
\hline GWM44801RH & USGS-011 & 687 & Strontium-90 & 1.76E-01 & $1.09 \mathrm{E}-01$ & & U & $\mathrm{PCl} / \mathrm{L}$ & $06 / 20 / 2006$ & 4.59E-01 & SOS-TL198-06 \\
\hline GWM44801RH & USGS-011 & 687 & Technetium-99 & $-7.90 \mathrm{E}+00$ & $2.74 \mathrm{E}+00$ & & $U$ & $\mathrm{PCl} / \mathrm{L}$ & $06 / 20 / 2006$ & $9.39 \mathrm{E}+00$ & SOS-TL198-06 \\
\hline GWM44801UX & USGS-011 & 687 & lodine-129 & $-2.52 \mathrm{E}-02$ & $2.31 \mathrm{E}-02$ & & $\mathrm{U}$ & $\mathrm{PCl} / \mathrm{L}$ & $06 / 20 / 2006$ & $6.51 \mathrm{E}-02$ & SOS-TL197-06 \\
\hline GWM449012X & USGS-014 & 739 & Aluminum & 7.9 & & $U$ & & UG/L & $06 / 26 / 2006$ & & DNT-336-06 \\
\hline GWM449012X & USGS-014 & 739 & Antimony & 0.5 & & $U$ & & UG/L & $06 / 26 / 2006$ & & DNT-336-06 \\
\hline GWM449012X & USGS-014 & 739 & Arsenic & 2 & & U & & UG/L & $06 / 26 / 2006$ & & DNT-336-06 \\
\hline GWM449012X & USGS-014 & 739 & Barium & 19.9 & & & & UG/L & $06 / 26 / 2006$ & & DNT-336-06 \\
\hline GWM449012X & USGS-014 & 739 & Beryllium & 0.088 & & $U$ & & UG/L & $06 / 26 / 2006$ & & DNT-336-06 \\
\hline GWM449012X & USGS-014 & 739 & Cadmium & 0.057 & & U & & UG/L & $06 / 26 / 2006$ & & DNT-336-06 \\
\hline GWM449012X & USGS-014 & 739 & Calcium & 33400 & & & & UG/L & $06 / 26 / 2006$ & & DNT-336-06 \\
\hline GWM449012X & USGS-014 & 739 & Chromium & 3.8 & & B & & UG/L & $06 / 26 / 2006$ & & DNT-336-06 \\
\hline GWM449012X & USGS-014 & 739 & Cobalt & 0.31 & & U & & UG/L & $06 / 26 / 2006$ & & DNT-336-06 \\
\hline GWM449012X & USGS-014 & 739 & Copper & 0.33 & & B & & UG/L & $06 / 26 / 2006$ & & DNT-336-06 \\
\hline GWM449012X & USGS-014 & 739 & Iron & 9.4 & & $U$ & & UG/L & $06 / 26 / 2006$ & & DNT-336-06 \\
\hline GWM449012X & USGS-014 & 739 & Lead & 0.9 & & B & & UG/L & $06 / 26 / 2006$ & & DNT-336-06 \\
\hline GWM449012X & USGS-014 & 739 & Magnesium & 13300 & & & & UG/L & $06 / 26 / 2006$ & & DNT-336-06 \\
\hline GWM449012X & USGS-014 & 739 & Manganese & 0.9 & & B & & UG/L & $06 / 26 / 2006$ & & DNT-336-06 \\
\hline GWM449012X & USGS-014 & 739 & Nickel & 1.1 & & B & & UG/L & $06 / 26 / 2006$ & & DNT-336-06 \\
\hline GWM449012X & USGS-014 & 739 & Potassium & 2450 & & & & UG/L & $06 / 26 / 2006$ & & DNT-336-06 \\
\hline GWM449012X & USGS-014 & 739 & Selenium & 1.6 & & B & & UG/L & $06 / 26 / 2006$ & & DNT-336-06 \\
\hline GWM449012X & USGS-014 & 739 & Silver & 0.2 & & $U$ & & UG/L & $06 / 26 / 2006$ & & DNT-336-06 \\
\hline GWM449012X & USGS-014 & 739 & Sodium & 15400 & & & & UG/L & $06 / 26 / 2006$ & & DNT-336-06 \\
\hline GWM449012X & USGS-014 & 739 & Strontium & 169 & & & & UG/L & $06 / 26 / 2006$ & & DNT-336-06 \\
\hline GWM449012X & USGS-014 & 739 & Thallium & 0.68 & & B & $U$ & UG/L & $06 / 26 / 2006$ & & DNT-336-06 \\
\hline GWM449012X & USGS-014 & 739 & Uranium & 2.6 & & & & UG/L & $06 / 26 / 2006$ & & DNT-336-06 \\
\hline GWM449012X & USGS-014 & 739 & Vanadium & 4.3 & & B & & UG/L & $06 / 26 / 2006$ & & DNT-336-06 \\
\hline GWM449012X & USGS-014 & 739 & Zinc & 46.8 & & $\mathrm{E}$ & & UG/L & $06 / 26 / 2006$ & & DNT-336-06 \\
\hline
\end{tabular}


Table A-2. (continued).

\begin{tabular}{|c|c|c|c|c|c|c|c|c|c|c|c|}
\hline $\begin{array}{l}\text { Field Sample } \\
\text { Number }\end{array}$ & Location & Depth & Compound & $\begin{array}{c}\text { Sample } \\
\text { Result }\end{array}$ & $\begin{array}{l}\text { Sample } \\
\text { Error }\end{array}$ & $\begin{array}{c}\text { Result } \\
\text { Qualifier }\end{array}$ & $\begin{array}{c}\text { Validation } \\
\text { Flag }\end{array}$ & $\begin{array}{c}\text { Sample } \\
\text { Units }\end{array}$ & $\begin{array}{c}\text { Date Sample } \\
\text { Collected }\end{array}$ & MDA & $\begin{array}{l}\text { L\&V Report } \\
\text { Number }\end{array}$ \\
\hline GWM449012X & USGS-014 & 739 & Mercury & 0.093 & & $\mathrm{U}$ & & UG/L & $06 / 26 / 2006$ & & DNT-336-06 \\
\hline GWM44901A1 & USGS-014 & 739 & Total Alkalinity & 136 & & & & $M G / L$ & $06 / 26 / 2006$ & & DNT-322-06 \\
\hline GWM44901AN & USGS-014 & 739 & Chloride & 23.1 & & $\mathrm{~J}$ & $\mathrm{~J}$ & $\mathrm{MG} / \mathrm{L}$ & $06 / 26 / 2006$ & & DNT-322-06 \\
\hline GWM44901AN & USGS-014 & 739 & Sulfate & 20.7 & & & & $M G / L$ & $06 / 26 / 2006$ & & DNT-322-06 \\
\hline GWM44901N2 & USGS-014 & 739 & Nitrate/Nitrite as $\mathrm{N}$ & 1180 & & & & UG/L & 06/26/2006 & & DNT-322-06 \\
\hline GWM44901VL & USGS-014 & 739 & Acetone & 2 & & $\mathrm{u}$ & $\mathrm{R}$ & UG/L & $06 / 26 / 2006$ & & JGJ-067-06 \\
\hline GWM44901VL & USGS-014 & 739 & Benzene & 1 & & $\mathrm{U}$ & & UG/L & $06 / 26 / 2006$ & & JGJ-067-06 \\
\hline GWM44901VL & USGS-014 & 739 & Bromoform & 1 & & $\mathrm{U}$ & & UG/L & $06 / 26 / 2006$ & & JGJ-067-06 \\
\hline GWM44901VL & USGS-014 & 739 & Bromomethane & 2 & & $\mathrm{U}$ & & UG/L & $06 / 26 / 2006$ & & JGJ-067-06 \\
\hline GWM44901VL & USGS-014 & 739 & 2-Butanone & 5 & & $\mathrm{U}$ & $\mathrm{R}$ & UG/L & 06/26/2006 & & JGJ-067-06 \\
\hline GWM44901VL & USGS-014 & 739 & Carbon disulfide & 1 & & $U$ & & UG/L & $06 / 26 / 2006$ & & JGJ-067-06 \\
\hline GWM44901VL & USGS-014 & 739 & Carbon tetrachloride & 1 & & $\mathrm{U}$ & & UG/L & 06/26/2006 & & JGJ-067-06 \\
\hline GWM44901VL & USGS-014 & 739 & Chlorobenzene & 1 & & $\mathrm{U}$ & & UG/L & 06/26/2006 & & JGJ-067-06 \\
\hline GWM44901VL & USGS-014 & 739 & Dibromochloromethane & 1 & & $U$ & & UG/L & $06 / 26 / 2006$ & & JGJ-067-06 \\
\hline GWM44901VL & USGS-014 & 739 & 1,2-Dibromo-3-chloropropane & 1 & & $\mathrm{U}$ & & UG/L & 06/26/2006 & & JGJ-067-06 \\
\hline GWM44901VL & USGS-014 & 739 & Chloroethane & 2 & & $U$ & & UG/L & 06/26/2006 & & JGJ-067-06 \\
\hline GWM44901VL & USGS-014 & 739 & Chloroform & 1 & & $\mathrm{U}$ & & UG/L & $06 / 26 / 2006$ & & JGJ-067-06 \\
\hline GWM44901VL & USGS-014 & 739 & 1,2-Dibromoethane & 1 & & $\mathrm{U}$ & & UG/L & $06 / 26 / 2006$ & & JGJ-067-06 \\
\hline GWM44901VL & USGS-014 & 739 & 1,2-Dichlorobenzene & 1 & & $\mathrm{U}$ & & UG/L & 06/26/2006 & & JGJ-067-06 \\
\hline GWM44901VL & USGS-014 & 739 & 1,3-Dichlorobenzene & 1 & & $\mathrm{U}$ & & UG/L & $06 / 26 / 2006$ & & JGJ-067-06 \\
\hline GWM44901VL & USGS-014 & 739 & 1,4-Dichlorobenzene & 1 & & $\mathrm{U}$ & & UG/L & 06/26/2006 & & JGJ-067-06 \\
\hline GWM44901VL & USGS-014 & 739 & Dichlorodifluoromethane & 2 & & $\mathrm{U}$ & & UG/L & $06 / 26 / 2006$ & & JGJ-067-06 \\
\hline GWM44901VL & USGS-014 & 739 & 1,1-Dichloroethane & 1 & & $\mathrm{U}$ & & UG/L & $06 / 26 / 2006$ & & JGJ-067-06 \\
\hline GWM44901VL & USGS-014 & 739 & 1,2-Dichloroethane & 1 & & $\mathrm{U}$ & & UG/L & 06/26/2006 & & JGJ-067-06 \\
\hline GWM44901VL & USGS-014 & 739 & 1,1-Dichloroethene & 1 & & $U$ & & UG/L & $06 / 26 / 2006$ & & JGJ-067-06 \\
\hline GWM44901VL & USGS-014 & 739 & cis-1,2-Dichloroethene & 1 & & $U$ & & UG/L & $06 / 26 / 2006$ & & JGJ-067-06 \\
\hline GWM44901VL & USGS-014 & 739 & trans-1,2-Dichloroethene & 1 & & $\mathrm{U}$ & & UG/L & 06/26/2006 & & JGJ-067-06 \\
\hline GWM44901VL & USGS-014 & 739 & 1,2-Dichloropropane & 1 & & $\mathrm{U}$ & & UG/L & 06/26/2006 & & JGJ-067-06 \\
\hline GWM44901VL & USGS- 014 & 739 & cis-1,3-Dichloropropene & 1 & & u & & UG/L & $06 / 26 / 2006$ & & JGJ-067-06 \\
\hline GWM44901VL & USGS-014 & 739 & trans-1,3-Dichloropropene & 1 & & $\mathrm{U}$ & & UG/L & 06/26/2006 & & JGJ-067-06 \\
\hline GWM44901VL & USGS-014 & 739 & Ethylbenzene & 1 & & $U$ & & UG/L & $06 / 26 / 2006$ & & JGJ-067-06 \\
\hline GWM44901VL & USGS-014 & 739 & Trichlorofluoromethane & 1 & & $U$ & & UG/L & $06 / 26 / 2006$ & & JGJ-067-06 \\
\hline GWM44901VL & USGS-014 & 739 & 2-Hexanone & 5 & & $\mathrm{U}$ & & UG/L & $06 / 26 / 2006$ & & JGJ-067-06 \\
\hline GWM44901VL & USGS-014 & 739 & Isopropylbenzene & 1 & & $\mathrm{U}$ & & UG/L & 06/26/2006 & & JGJ-067-06 \\
\hline GWM44901VL & USGS-014 & 739 & Methyl acetate & 5 & & $\mathrm{u}$ & $\mathrm{R}$ & UG/L & $06 / 26 / 2006$ & & JGJ-067-06 \\
\hline GWM44901VL & USGS-014 & 739 & Methyl cyclohexane & 4 & & $\mathrm{U}$ & & UG/L & 06/26/2006 & & JGJ-067-06 \\
\hline GWM44901VL & USGS-014 & 739 & 1,1,2,2-Tetrachloroethane & 1 & & $\mathrm{U}$ & & UG/L & 06/26/2006 & & JGJ-067-06 \\
\hline GWM44901VL & USGS-014 & 739 & Tetrachloroethene & 1 & & $\mathrm{U}$ & & UG/L & 06/26/2006 & & JGJ-067-06 \\
\hline GWM44901VL & USGS-014 & 739 & Toluene & 1 & & $\mathrm{U}$ & & UG/L & 06/26/2006 & & JGJ-067-06 \\
\hline GWM44901VL & USGS-014 & 739 & 1,2,4-Trichlorobenzene & 1 & & $\mathrm{U}$ & & UG/L & 06/26/2006 & & JGJ-067-06 \\
\hline GWM44901VL & USGS-014 & 739 & 1,1,1-Trichloroethane & 1 & & $\mathrm{U}$ & & UG/L & $06 / 26 / 2006$ & & JGJ-067-06 \\
\hline GWM44901VL & USGS-014 & 739 & $1,1,2$-Trichloroethane & 1 & & $U$ & & UG/L & $06 / 26 / 2006$ & & JGJ-067-06 \\
\hline GWM44901VL & USGS-014 & 739 & Trichloroethene & 1 & & $\mathrm{U}$ & & UG/L & $06 / 26 / 2006$ & & JGJ-067-06 \\
\hline GWM44901VL & USGS-014 & 739 & 1,1,2-Trichloro-1,2,2-trifluoroethane & 1 & & $\mathrm{U}$ & & UG/L & 06/26/2006 & & JGJ-067-06 \\
\hline GWM44901VL & USGS-014 & 739 & Vinyl Chloride & 2 & & U & & UG/L & $06 / 26 / 2006$ & & JGJ-067-06 \\
\hline GWM44901VL & USGS-014 & 739 & Xylene (Total) & 3 & & U & & UG/L & $06 / 26 / 2006$ & & JGJ-067-06 \\
\hline GWM44901VL & USGS-014 & 739 & Methyl t-butyl ether & 2 & & $\mathrm{U}$ & & UG/L & 06/26/2006 & & JGJ-067-06 \\
\hline GWM44901R8 & USGS-014 & 739 & Tritium & $1.21 \mathrm{E}+02$ & $1.08 \mathrm{E}+02$ & & $\mathrm{U}$ & $\mathrm{PCI} / \mathrm{L}$ & 06/26/2006 & $3.62 \mathrm{E}+02$ & SOS-TL228-06 \\
\hline GWM44901RH & USGS-014 & 739 & Gross Alpha & $2.32 \mathrm{E}+00$ & $6.18 \mathrm{E}-01$ & & & $\mathrm{PCl} / \mathrm{L}$ & 06/26/2006 & $1.38 \mathrm{E}+00$ & SOS-TL227-06 \\
\hline GWM44901RH & USGS-014 & 739 & Gross Beta & $3.52 \mathrm{E}+00$ & 7.74E-01 & & & $\mathrm{PCl} / \mathrm{L}$ & $06 / 26 / 2006$ & $2.56 \mathrm{E}+00$ & SOS-TL227-06 \\
\hline GWM44901RH & USGS-014 & 739 & Strontium-90 & $1.72 \mathrm{E}-01$ & 1.02E-01 & & $\mathrm{U}$ & $\mathrm{PCI} / \mathrm{L}$ & 06/26/2006 & 4.25E-01 & SOS-TL227-06 \\
\hline GWM44901RH & USGS-014 & 739 & Technetium-99 & $-2.02 \mathrm{E}+00$ & $1.85 \mathrm{E}+00$ & & $U$ & $\mathrm{PCl} / \mathrm{L}$ & $06 / 26 / 2006$ & $6.38 \mathrm{E}+00$ & SOS-TL227-06 \\
\hline GWM44901UX & USGS-014 & 739 & lodine-129 & $-3.87 \mathrm{E}-02$ & $2.68 \mathrm{E}-02$ & & UJ & $\mathrm{PCI} / \mathrm{L}$ & 06/26/2006 & $8.67 \mathrm{E}-02$ & SOS-TL226-06 \\
\hline GWM437012X & USGS-086 & 678 & Aluminum & 7.9 & & $\mathrm{U}$ & & UG/L & $06 / 27 / 2006$ & & DNT-336-06 \\
\hline GWM437012X & USGS-086 & 678 & Antimony & 0.5 & & $\mathrm{U}$ & & UG/L & $06 / 27 / 2006$ & & DNT-336-06 \\
\hline GWM437012X & USGS-086 & 678 & Arsenic & 2 & & $\mathrm{U}$ & & UG/L & 06/27/2006 & & DNT-336-06 \\
\hline GWM437012X & USGS-086 & 678 & Barium & 16.7 & & & & UG/L & 06/27/2006 & & DNT-336-06 \\
\hline GWM437012X & USGS-086 & 678 & Beryllium & 0.088 & & $\mathrm{U}$ & & UG/L & 06/27/2006 & & DNT-336-06 \\
\hline GWM437012X & USGS-086 & 678 & Cadmium & 0.057 & & $\mathrm{U}$ & & UG/L & 06/27/2006 & & DNT-336-06 \\
\hline GWM437012X & USGS-086 & 678 & Calcium & 34800 & & & & UG/L & $06 / 27 / 2006$ & & DNT-336-06 \\
\hline GWM437012X & USGS-086 & 678 & Chromium & 13.4 & & & & UG/L & $06 / 27 / 2006$ & & DNT-336-06 \\
\hline GWM437012X & USGS-086 & 678 & Cobalt & 0.31 & & U & & UG/L & $06 / 27 / 2006$ & & DNT-336-06 \\
\hline GWM437012X & USGS-086 & 678 & Copper & 2 & & & & UG/L & 06/27/2006 & & DNT-336-06 \\
\hline GWM437012X & USGS-086 & 678 & Iron & 9.4 & & U & & UG/L & $06 / 27 / 2006$ & & DNT-336-06 \\
\hline GWM437012X & USGS-086 & 678 & Lead & 0.56 & & B & & UG/L & $06 / 27 / 2006$ & & DNT-336-06 \\
\hline GWM437012X & USGS-086 & 678 & Magnesium & 9310 & & & & UG/L & 06/27/2006 & & DNT-336-06 \\
\hline GWM437012X & USGS-086 & 678 & Manganese & 0.34 & & $U$ & & UG/L & $06 / 27 / 2006$ & & DNT-336-06 \\
\hline GWM437012X & USGS-086 & 678 & Nickel & 1.2 & & B & & UG/L & $06 / 27 / 2006$ & & DNT-336-06 \\
\hline GWM437012X & USGS-086 & 678 & Potassium & 2690 & & & & UG/L & $06 / 27 / 2006$ & & DNT-336-06 \\
\hline GWM437012X & USGS-086 & 678 & Selenium & 2.6 & & B & & UG/L & $06 / 27 / 2006$ & & DNT-336-06 \\
\hline GWM437012X & USGS-086 & 678 & Silver & 0.2 & & $U$ & & UG/L & $06 / 27 / 2006$ & & DNT-336-06 \\
\hline
\end{tabular}


Table A-2. (continued).

\begin{tabular}{|c|c|c|c|c|c|c|c|c|c|c|c|}
\hline $\begin{array}{c}\text { Field Sample } \\
\text { Number }\end{array}$ & Location & Depth & Compound & $\begin{array}{c}\text { Sample } \\
\text { Result }\end{array}$ & $\begin{array}{c}\text { Sample } \\
\text { Error }\end{array}$ & $\begin{array}{c}\text { Result } \\
\text { Qualifier }\end{array}$ & $\begin{array}{c}\text { Validation } \\
\text { Flag }\end{array}$ & $\begin{array}{c}\text { Sample } \\
\text { Units }\end{array}$ & $\begin{array}{c}\text { Date Sample } \\
\text { Collected }\end{array}$ & MDA & $\begin{array}{l}\text { L\&V Report } \\
\text { Number }\end{array}$ \\
\hline GWM437012X & USGS-086 & 678 & Sodium & 10400 & & & & UG/L & $06 / 27 / 2006$ & & DNT-336-06 \\
\hline GWM437012X & USGS-086 & 678 & Strontium & 144 & & & & UG/L & $06 / 27 / 2006$ & & DNT-336-06 \\
\hline GWM437012X & USGS-086 & 678 & Thallium & 0.94 & & B & $U$ & UG/L & $06 / 27 / 2006$ & & DNT-336-06 \\
\hline GWM437012X & USGS-086 & 678 & Vanadium & 5.7 & & B & & UG/L & $06 / 27 / 2006$ & & DNT-336-06 \\
\hline GWM437012X & USGS-086 & 678 & Zinc & 92.7 & & $\mathrm{E}$ & & UG/L & $06 / 27 / 2006$ & & DNT-336-06 \\
\hline GWM437012X & USGS-086 & 678 & Mercury & 0.093 & & $\bar{U}$ & & UG/L & $06 / 27 / 2006$ & & DNT-336-06 \\
\hline GWM43701A1 & USGS-086 & 678 & Total Alkalinity & 104 & & & & $M G / L$ & $06 / 27 / 2006$ & & DNT-324-06 \\
\hline GWM43701AN & USGS-086 & 678 & Fluoride & 0.17 & & & $\mathrm{~J}$ & $\mathrm{MG} / \mathrm{L}$ & $06 / 27 / 2006$ & & DNT-324-06 \\
\hline GWM43701AN & USGS-086 & 678 & Sulfate & 21.9 & & & & $M G / L$ & $06 / 27 / 2006$ & & DNT-324-06 \\
\hline GWM43701N2 & USGS-086 & 678 & Nitrate/Nitrite as $\mathrm{N}$ & 1570 & & & & UG/L & $06 / 27 / 2006$ & & DNT-324-06 \\
\hline GWM43701VL & USGS-086 & 678 & Acetone & 2 & & U & $\mathrm{R}$ & UG/L & $06 / 27 / 2006$ & & JGJ-067-06 \\
\hline GWM43701VL & USGS-086 & 678 & Benzene & 1 & & $\mathrm{U}$ & & UG/L & $06 / 27 / 2006$ & & JGJ-067-06 \\
\hline GWM43701VL & USGS-086 & 678 & Bromodichloromethane & 1 & & $\mathrm{U}$ & & UG/L & $06 / 27 / 2006$ & & JGJ-067-06 \\
\hline GWM43701VL & USGS-086 & 678 & Bromoform & 1 & & $u$ & & UG/L & $06 / 27 / 2006$ & & JGJ-067-06 \\
\hline GWM43701VL & USGS-086 & 678 & Bromomethane & 2 & & U & & UG/L & $06 / 27 / 2006$ & & JGJ-067-06 \\
\hline GWM43701VL & USGS-086 & 678 & 2-Butanone & 5 & & $\mathrm{U}$ & $\mathrm{R}$ & UG/L & $06 / 27 / 2006$ & & JGJ-067-06 \\
\hline GWM43701VL & USGS-086 & 678 & Carbon disulfide & 1 & & $\mathrm{U}$ & & UG/L & $06 / 27 / 2006$ & & JGJ-067-06 \\
\hline GWM43701VL & USGS-086 & 678 & Dibromochloromethane & 1 & & $\mathrm{U}$ & & UG/L & $06 / 27 / 2006$ & & JGJ-067-06 \\
\hline GWM43701VL & USGS-086 & 678 & 1,2-Dibromo-3-chloropropane & 1 & & $\mathrm{U}$ & & UG/L & $06 / 27 / 2006$ & & JGJ-067-06 \\
\hline GWM43701VL & USGS-086 & 678 & Chloroethane & 2 & & U & & UG/L & $06 / 27 / 2006$ & & JGJ-067-06 \\
\hline GWM43701VL & USGS-086 & 678 & Chloroform & 1 & & $\mathrm{U}$ & & UG/L & $06 / 27 / 2006$ & & JGJ-067-06 \\
\hline GWM43701VL & USGS-086 & 678 & Chloromethane & 2 & & $U$ & & UG/L & $06 / 27 / 2006$ & & JGJ-067-06 \\
\hline GWM43701VL & USGS-086 & 678 & Cyclohexane & 1 & & U & & UG/L & $06 / 27 / 2006$ & & JGJ-067-06 \\
\hline GWM43701VL & USGS-086 & 678 & 1,2-Dibromoethane & 1 & & $\mathrm{u}$ & & UG/L & $06 / 27 / 2006$ & & JGJ-067-06 \\
\hline GWM43701VL & USGS-086 & 678 & 1,2-Dichlorobenzene & 1 & & $\mathrm{U}$ & & UG/L & $06 / 27 / 2006$ & & JGJ-067-06 \\
\hline GWM43701VL & USGS-086 & 678 & 1,3-Dichlorobenzene & 1 & & U & & UG/L & $06 / 27 / 2006$ & & JGJ-067-06 \\
\hline GWM43701VL & USGS-086 & 678 & 1,4-Dichlorobenzene & 1 & & $U$ & & UG/L & $06 / 27 / 2006$ & & JGJ-067-06 \\
\hline GWM43701VL & USGS-086 & 678 & Dichlorodifluoromethane & 2 & & $\mathrm{U}$ & & UG/L & $06 / 27 / 2006$ & & JGJ-067-06 \\
\hline GWM43701VL & USGS-086 & 678 & 1,1-Dichloroethane & 1 & & U & & UG/L & $06 / 27 / 2006$ & & JGJ-067-06 \\
\hline GWM43701VL & USGS-086 & 678 & 1,2-Dichloroethane & 1 & & $\mathrm{U}$ & & UG/L & $06 / 27 / 2006$ & & JGJ-067-06 \\
\hline GWM43701VL & USGS-086 & 678 & 1,1-Dichloroethene & 1 & & $\mathrm{U}$ & & UG/L & $06 / 27 / 2006$ & & JGJ-067-06 \\
\hline GWM43701VL & USGS-086 & 678 & cis-1,2-Dichloroethene & 1 & & U & & UG/L & $06 / 27 / 2006$ & & JGJ-067-06 \\
\hline GWM43701VL & USGS-086 & 678 & trans-1,2-Dichloroethene & 1 & & U & & UG/L & $06 / 27 / 2006$ & & JGJ-067-06 \\
\hline GWM43701VL & USGS-086 & 678 & 1,2-Dichloropropane & 1 & & $\mathrm{U}$ & & UG/L & $06 / 27 / 2006$ & & JGJ-067-06 \\
\hline GWM43701VL & USGS-086 & 678 & cis-1,3-Dichloropropene & 1 & & U & & UG/L & $06 / 27 / 2006$ & & JGJ-067-06 \\
\hline GWM43701VL & USGS-086 & 678 & trans-1,3-Dichloropropene & 1 & & U & & UG/L & $06 / 27 / 2006$ & & JGJ-067-06 \\
\hline GWM43701VL & USGS-086 & 678 & Isopropylbenzene & 1 & & $\mathrm{U}$ & & UG/L & $06 / 27 / 2006$ & & JGJ-067-06 \\
\hline GWM43701VL & USGS-086 & 678 & Methyl acetate & 5 & & $\mathrm{U}$ & $\mathrm{R}$ & UG/L & $06 / 27 / 2006$ & & JGJ-067-06 \\
\hline GWM43701VL & USGS-086 & 678 & Methyl cyclohexane & 4 & & 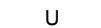 & & UG/L & $06 / 27 / 2006$ & & JGJ-067-06 \\
\hline GWM43701VL & USGS-086 & 678 & Methylene Chloride & 1 & & $\mathrm{U}$ & & UG/L & $06 / 27 / 2006$ & & JGJ-067-06 \\
\hline GWM43701VL & USGS-086 & 678 & 4-Methyl-2-pentanone & 5 & & $\mathrm{U}$ & & UG/L & $06 / 27 / 2006$ & & JGJ-067-06 \\
\hline GWM43701VL & USGS-086 & 678 & Styrene & 1 & & U & & UG/L & $06 / 27 / 2006$ & & JGJ-067-06 \\
\hline GWM43701VL & USGS-086 & 678 & 1,1,2,2-Tetrachloroethane & 1 & & $\mathrm{u}$ & & UG/L & $06 / 27 / 2006$ & & JGJ-067-06 \\
\hline GWM43701VL & USGS-086 & 678 & Tetrachloroethene & 1 & & $\mathrm{U}$ & & UG/L & $06 / 27 / 2006$ & & JGJ-067-06 \\
\hline GWM43701VL & USGS-086 & 678 & Toluene & 1 & & $\mathrm{U}$ & & UG/L & $06 / 27 / 2006$ & & JGJ-067-06 \\
\hline GWM43701VL & USGS-086 & 678 & 1,2,4-Trichlorobenzene & 1 & & U & & UG/L & $06 / 27 / 2006$ & & JGJ-067-06 \\
\hline GWM43701VL & USGS-086 & 678 & 1,1,1-Trichloroethane & 1 & & $\mathrm{U}$ & & UG/L & $06 / 27 / 2006$ & & JGJ-067-06 \\
\hline GWM43701VL & USGS-086 & 678 & 1,1,2-Trichloroethane & 1 & & $\mathrm{U}$ & & UG/L & $06 / 27 / 2006$ & & JGJ-067-06 \\
\hline GWM43701VL & USGS-086 & 678 & Trichloroethene & 1 & & U & & UG/L & $06 / 27 / 2006$ & & JGJ-067-06 \\
\hline GWM43701VL & USGS-086 & 678 & 1,1,2-Trichloro-1,2,2-trifluoroethane & 1 & & $\mathrm{U}$ & & UG/L & $06 / 27 / 2006$ & & JGJ-067-06 \\
\hline GWM43701VL & USGS-086 & 678 & Vinyl Chloride & 2 & & $\mathrm{U}$ & & UG/L & $06 / 27 / 2006$ & & JGJ-067-06 \\
\hline GWM43701VL & USGS-086 & 678 & Xylene (Total) & 3 & & U & & UG/L & $06 / 27 / 2006$ & & JGJ-067-06 \\
\hline GWM43701VL & USGS-086 & 678 & Methyl t-butyl ether & 2 & & $\mathrm{U}$ & & UG/L & $06 / 27 / 2006$ & & JGJ-067-06 \\
\hline GWM43701R8 & USGS-086 & 678 & Tritium & $4.96 \mathrm{E}+01$ & $1.06 \mathrm{E}+02$ & & $\mathrm{U}$ & $\mathrm{PCl} / \mathrm{L}$ & $06 / 27 / 2006$ & $3.63 E+02$ & SOS-TL228-06 \\
\hline GWM43701RH & USGS-086 & 678 & Gross Alpha & $1.25 \mathrm{E}+00$ & $5.29 \mathrm{E}-01$ & & UJ & $\mathrm{PCl} / \mathrm{L}$ & $06 / 27 / 2006$ & $1.72 E+00$ & SOS-TL227-06 \\
\hline GWM43701RH & USGS-086 & 678 & Gross Beta & $2.41 \mathrm{E}+00$ & 8.07E-01 & & UJ & $\mathrm{PCl} / \mathrm{L}$ & $06 / 27 / 2006$ & $3.16 \mathrm{E}+00$ & SOS-TL227-06 \\
\hline GWM43701RH & USGS-086 & 678 & Strontium-90 & $2.14 \mathrm{E}-02$ & 7.67E-02 & & $U$ & $\mathrm{PCl} / \mathrm{L}$ & $06 / 27 / 2006$ & $3.69 \mathrm{E}-01$ & SOS-TL227-06 \\
\hline GWM43701RH & USGS-086 & 678 & Technetium-99 & $-1.89 E+00$ & $1.84 \mathrm{E}+00$ & & $U$ & $\mathrm{PCl} / \mathrm{L}$ & $06 / 27 / 2006$ & $6.34 \mathrm{E}+00$ & SOS-TL227-06 \\
\hline GWM43701UX & USGS-086 & 678 & lodine-129 & $6.62 \mathrm{E}-02$ & 3.31E-02 & & UJ & $\mathrm{PCl} / \mathrm{L}$ & $06 / 27 / 2006$ & 1.17E-01 & SOS-TL226-06 \\
\hline GWM444012X & USGS-100 & 693 & Aluminum & 11 & & B & & UG/L & $06 / 14 / 2006$ & & DNT-275-06 \\
\hline GWM444012X & USGS-100 & 693 & Antimony & 0.5 & & U & & UG/L & $06 / 14 / 2006$ & & DNT-275-06 \\
\hline GWM444012X & USGS-100 & 693 & Arsenic & 2.1 & & B & & UG/L & $06 / 14 / 2006$ & & DNT-275-06 \\
\hline GWM444012X & USGS-100 & 693 & Barium & 39.1 & & & & UG/L & $06 / 14 / 2006$ & & DNT-275-06 \\
\hline GWM444012X & USGS-100 & 693 & Beryllium & 0.088 & & U & & UG/L & $06 / 14 / 2006$ & & DNT-275-06 \\
\hline GWM444012X & USGS-100 & 693 & Cadmium & 0.43 & & B & & UG/L & $06 / 14 / 2006$ & & DNT-275-06 \\
\hline GWM444012X & USGS-100 & 693 & Calcium & 36400 & & & & UG/L & $06 / 14 / 2006$ & & DNT-275-06 \\
\hline GWM444012X & USGS-100 & 693 & Chromium & 5 & & B & & UG/L & $06 / 14 / 2006$ & & DNT-275-06 \\
\hline GWM444012X & USGS-100 & 693 & Cobalt & 0.31 & & $\mathrm{U}$ & & UG/L & $06 / 14 / 2006$ & & DNT-275-06 \\
\hline GWM444012X & USGS-100 & 693 & Copper & 1 & & & & UG/L & $06 / 14 / 2006$ & & DNT-275-06 \\
\hline GWM444012X & USGS-100 & 693 & Iron & 9.4 & & $U$ & & UG/L & $06 / 14 / 2006$ & & DNT-275-06 \\
\hline GWM444012X & USGS-100 & 693 & Lead & 12.2 & & & & UG/L & $06 / 14 / 2006$ & & DNT-275-06 \\
\hline
\end{tabular}


Table A-2. (continued).

\begin{tabular}{|c|c|c|c|c|c|c|c|c|c|c|c|}
\hline $\begin{array}{c}\text { Field Sample } \\
\text { Number }\end{array}$ & Location & Depth & Compound & $\begin{array}{c}\text { Sample } \\
\text { Result }\end{array}$ & $\begin{array}{l}\text { Sample } \\
\text { Error }\end{array}$ & $\begin{array}{c}\text { Result } \\
\text { Qualifier }\end{array}$ & $\begin{array}{c}\text { Validation } \\
\text { Flag }\end{array}$ & $\begin{array}{c}\text { Sample } \\
\text { Units }\end{array}$ & $\begin{array}{c}\text { Date Sample } \\
\text { Collected }\end{array}$ & MDA & $\begin{array}{l}\text { L\&V Report } \\
\text { Number }\end{array}$ \\
\hline GWM444012X & USGS-100 & 693 & Magnesium & 11700 & & & & UG/L & $06 / 14 / 2006$ & & DNT-275-06 \\
\hline GWM444012X & USGS-100 & 693 & Manganese & 0.89 & & B & & UG/L & $06 / 14 / 2006$ & & DNT-275-06 \\
\hline GWM444012X & USGS-100 & 693 & Nickel & 1.9 & & B & & UG/L & $06 / 14 / 2006$ & & DNT-275-06 \\
\hline GWM444012X & USGS-100 & 693 & Selenium & 2.6 & & B & U & UG/L & $06 / 14 / 2006$ & & DNT-275-06 \\
\hline GWM444012X & USGS-100 & 693 & Silver & 0.2 & & U & & UG/L & $06 / 14 / 2006$ & & DNT-275-06 \\
\hline GWM444012X & USGS-100 & 693 & Sodium & 16400 & & & & UG/L & $06 / 14 / 2006$ & & DNT-275-06 \\
\hline GWM444012X & USGS-100 & 693 & Strontium & 131 & & & & UG/L & $06 / 14 / 2006$ & & DNT-275-06 \\
\hline GWM444012X & USGS-100 & 693 & Uranium & 1.5 & & & & UG/L & $06 / 14 / 2006$ & & DNT-275-06 \\
\hline GWM444012X & USGS-100 & 693 & Vanadium & 3.4 & & B & & UG/L & $06 / 14 / 2006$ & & DNT-275-06 \\
\hline GWM444012X & USGS-100 & 693 & Zinc & 185 & & & & UG/L & $06 / 14 / 2006$ & & DNT-275-06 \\
\hline GWM444012X & USGS-100 & 693 & Mercury & 0.093 & & $\mathrm{U}$ & & UG/L & $06 / 14 / 2006$ & & DNT-275-06 \\
\hline GWM44401A1 & USGS-100 & 693 & Total Alkalinity & 136 & & & & $M G / L$ & $06 / 14 / 2006$ & & DNT-287-06 \\
\hline GWM44401AN & USGS-100 & 693 & Chloride & 19.1 & & $\mathrm{~J}$ & & $M G / L$ & $06 / 14 / 2006$ & & DNT-287-06 \\
\hline GWM44401AN & USGS-100 & 693 & Fluoride & 0.66 & & & $\mathrm{~J}$ & $M G / L$ & $06 / 14 / 2006$ & & DNT-287-06 \\
\hline GWM44401AN & USGS-100 & 693 & Sulfate & 20.6 & & & $\mathrm{~J}$ & $M G / L$ & $06 / 14 / 2006$ & & DNT-287-06 \\
\hline GWM44401N2 & USGS-100 & 693 & Nitrate/Nitrite as $\mathrm{N}$ & 1880 & & & & UG/L & $06 / 14 / 2006$ & & DNT-287-06 \\
\hline GWM44401VL & USGS-100 & 693 & Acetone & 2 & & $\mathrm{U}$ & & UG/L & $06 / 14 / 2006$ & & JGJ-062-06 \\
\hline GWM44401VL & USGS-100 & 693 & Bromoform & 1 & & $\mathrm{U}$ & & UG/L & $06 / 14 / 2006$ & & JGJ-062-06 \\
\hline GWM44401VL & USGS-100 & 693 & Bromomethane & 2 & & U & & UG/L & $06 / 14 / 2006$ & & JGJ-062-06 \\
\hline GWM44401VL & USGS-100 & 693 & 2-Butanone & 5 & & U & $\mathrm{R}$ & UG/L & $06 / 14 / 2006$ & & JGJ-062-06 \\
\hline GWM44401VL & USGS-100 & 693 & Carbon disulfide & 1 & & U & & UG/L & $06 / 14 / 2006$ & & JGJ-062-06 \\
\hline GWM44401VL & USGS-100 & 693 & Carbon tetrachloride & 1 & & $U$ & & UG/L & $06 / 14 / 2006$ & & JGJ-062-06 \\
\hline GWM44401VL & USGS-100 & 693 & Chlorobenzene & 1 & & U & & UG/L & $06 / 14 / 2006$ & & JGJ-062-06 \\
\hline GWM44401VL & USGS-100 & 693 & Dibromochloromethane & 1 & & $\mathrm{u}$ & & UG/L & $06 / 14 / 2006$ & & JGJ-062-06 \\
\hline GWM44401VL & USGS-100 & 693 & 1,2-Dibromo-3-chloropropane & 1 & & $\mathrm{U}$ & $\mathrm{R}$ & UG/L & $06 / 14 / 2006$ & & JGJ-062-06 \\
\hline GWM44401VL & USGS-100 & 693 & Chloroethane & 2 & & U & & UG/L & $06 / 14 / 2006$ & & JGJ-062-06 \\
\hline GWM44401VL & USGS-100 & 693 & Chloroform & 1 & & $\mathrm{u}$ & & UG/L & $06 / 14 / 2006$ & & JGJ-062-06 \\
\hline GWM44401VL & USGS-100 & 693 & Chloromethane & 2 & & $\mathrm{U}$ & & UG/L & $06 / 14 / 2006$ & & JGJ-062-06 \\
\hline GWM44401VL & USGS-100 & 693 & Cyclohexane & 1 & & U & & UG/L & $06 / 14 / 2006$ & & JGJ-062-06 \\
\hline GWM44401VL & USGS-100 & 693 & 1,2-Dibromoethane & 1 & & $\mathrm{u}$ & & UG/L & $06 / 14 / 2006$ & & JGJ-062-06 \\
\hline GWM44401VL & USGS-100 & 693 & 1,2-Dichlorobenzene & 1 & & $\mathrm{U}$ & & UG/L & $06 / 14 / 2006$ & & JGJ-062-06 \\
\hline GWM44401VL & USGS-100 & 693 & 1,3-Dichlorobenzene & 1 & & U & & UG/L & $06 / 14 / 2006$ & & JGJ-062-06 \\
\hline GWM44401VL & USGS-100 & 693 & 1,4-Dichlorobenzene & 1 & & U & & UG/L & $06 / 14 / 2006$ & & JGJ-062-06 \\
\hline GWM44401VL & USGS-100 & 693 & Dichlorodifluoromethane & 2 & & $\mathrm{u}$ & & UG/L & $06 / 14 / 2006$ & & JGJ-062-06 \\
\hline GWM44401VL & USGS-100 & 693 & 1,1-Dichloroethane & 1 & & u & & UG/L & $06 / 14 / 2006$ & & JGJ-062-06 \\
\hline GWM44401VL & USGS-100 & 693 & 1,2-Dichloroethane & 1 & & U & & UG/L & $06 / 14 / 2006$ & & JGJ-062-06 \\
\hline GWM44401VL & USGS-100 & 693 & 1,2-Dichloropropane & 1 & & $\mathrm{U}$ & & UG/L & $06 / 14 / 2006$ & & JGJ-062-06 \\
\hline GWM44401VL & USGS-100 & 693 & cis-1,3-Dichloropropene & 1 & & $\mathrm{U}$ & & UG/L & $06 / 14 / 2006$ & & JGJ-062-06 \\
\hline GWM44401VL & USGS-100 & 693 & trans-1,3-Dichloropropene & 1 & & 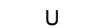 & & UG/L & $06 / 14 / 2006$ & & JGJ-062-06 \\
\hline GWM44401VL & USGS-100 & 693 & Ethylbenzene & 1 & & $\mathrm{U}$ & & UG/L & $06 / 14 / 2006$ & & JGJ-062-06 \\
\hline GWM44401VL & USGS-100 & 693 & Trichlorofluoromethane & 1 & & $\mathrm{U}$ & & UG/L & $06 / 14 / 2006$ & & JGJ-062-06 \\
\hline GWM44401VL & USGS-100 & 693 & 2-Hexanone & 5 & & U & & UG/L & $06 / 14 / 2006$ & & JGJ-062-06 \\
\hline GWM44401VL & USGS-100 & 693 & Isopropylbenzene & 1 & & $\mathrm{U}$ & & UG/L & $06 / 14 / 2006$ & & JGJ-062-06 \\
\hline GWM44401VL & USGS-100 & 693 & Methyl acetate & 5 & & $\mathrm{U}$ & & UG/L & $06 / 14 / 2006$ & & JGJ-062-06 \\
\hline GWM44401VL & USGS-100 & 693 & Methyl cyclohexane & 4 & & $U$ & & UG/L & $06 / 14 / 2006$ & & JGJ-062-06 \\
\hline GWM44401VL & USGS-100 & 693 & Methylene Chloride & 1 & & U & & UG/L & $06 / 14 / 2006$ & & JGJ-062-06 \\
\hline GWM44401VL & USGS-100 & 693 & 4-Methyl-2-pentanone & 5 & & $\mathrm{U}$ & & UG/L & $06 / 14 / 2006$ & & JGJ-062-06 \\
\hline GWM44401VL & USGS-100 & 693 & Styrene & 1 & & U & & UG/L & $06 / 14 / 2006$ & & JGJ-062-06 \\
\hline GWM44401VL & USGS-100 & 693 & 1,1,2,2-Tetrachloroethane & 1 & & U & & UG/L & $06 / 14 / 2006$ & & JGJ-062-06 \\
\hline GWM44401VL & USGS-100 & 693 & Tetrachloroethene & 1 & & $\mathrm{U}$ & & UG/L & $06 / 14 / 2006$ & & JGJ-062-06 \\
\hline GWM44401VL & USGS-100 & 693 & Toluene & 1 & & $\mathrm{U}$ & & UG/L & $06 / 14 / 2006$ & & JGJ-062-06 \\
\hline GWM44401VL & USGS-100 & 693 & 1,2,4-Trichlorobenzene & 1 & & U & & UG/L & $06 / 14 / 2006$ & & JGJ-062-06 \\
\hline GWM44401VL & USGS-100 & 693 & 1,1,1-Trichloroethane & 1 & & $\mathrm{U}$ & & UG/L & $06 / 14 / 2006$ & & JGJ-062-06 \\
\hline GWM44401VL & USGS-100 & 693 & 1,1,2-Trichloroethane & 1 & & $\mathrm{U}$ & & UG/L & $06 / 14 / 2006$ & & JGJ-062-06 \\
\hline GWM44401VL & USGS-100 & 693 & Trichloroethene & 1 & & $U$ & & UG/L & $06 / 14 / 2006$ & & JGJ-062-06 \\
\hline GWM44401VL & USGS-100 & 693 & 1,1,2-Trichloro-1,2,2-trifluoroethane & 1 & & $\mathrm{U}$ & & UG/L & $06 / 14 / 2006$ & & JGJ-062-06 \\
\hline GWM44401VL & USGS-100 & 693 & Vinyl Chloride & 2 & & $\mathrm{U}$ & & UG/L & $06 / 14 / 2006$ & & JGJ-062-06 \\
\hline GWM44401VL & USGS-100 & 693 & Xylene (Total) & 3 & & U & & UG/L & $06 / 14 / 2006$ & & JGJ-062-06 \\
\hline GWM44401VL & USGS-100 & 693 & Methyl t-butyl ether & 2 & & $\mathrm{U}$ & & UG/L & $06 / 14 / 2006$ & & JGJ-062-06 \\
\hline GWM44401R8 & USGS-100 & 693 & Tritium & $0.00 \mathrm{E}+00$ & $1.13 E+02$ & & $U$ & $\mathrm{PCI} / \mathrm{L}$ & $06 / 14 / 2006$ & $3.92 \mathrm{E}+02$ & SOS-TL199-06 \\
\hline GWM44401RH & USGS-100 & 693 & Gross Alpha & $1.93 E+00$ & $5.84 \mathrm{E}-01$ & & & $\mathrm{PCl} / \mathrm{L}$ & $06 / 14 / 2006$ & $1.30 E+00$ & SOS-TL198-06 \\
\hline GWM44401RH & USGS-100 & 693 & Gross Beta & $5.21 \mathrm{E}+00$ & 8.35E-01 & & & $\mathrm{PCI} / \mathrm{L}$ & $06 / 14 / 2006$ & $2.69 E+00$ & SOS-TL198-06 \\
\hline GWM44401RH & USGS-100 & 693 & Strontium-90 & $1.17 \mathrm{E}-02$ & $1.01 \mathrm{E}-01$ & & $\mathrm{U}$ & $\mathrm{PCl} / \mathrm{L}$ & $06 / 14 / 2006$ & 4.94E-01 & SOS-TL198-06 \\
\hline GWM44401RH & USGS-100 & 693 & Technetium-99 & $-3.11 E+00$ & $2.84 \mathrm{E}+00$ & & U & $\mathrm{PCl} / \mathrm{L}$ & $06 / 14 / 2006$ & $9.58 E+00$ & SOS-TL198-06 \\
\hline GWM44401UX & USGS-100 & 693 & lodine-129 & $3.87 \mathrm{E}-03$ & 3.01E-02 & & $U$ & $\mathrm{PCl} / \mathrm{L}$ & $06 / 14 / 2006$ & $1.13 \mathrm{E}-01$ & SOS-TL197-06 \\
\hline GWM438012X & USGS-101 & 790 & Aluminum & 9.2 & & B & & UG/L & $06 / 14 / 2006$ & & DNT-275-06 \\
\hline GWM438012X & USGS-101 & 790 & Antimony & 0.5 & & U & & UG/L & $06 / 14 / 2006$ & & DNT-275-06 \\
\hline GWM438012X & USGS-101 & 790 & Arsenic & 2 & & U & & UG/L & $06 / 14 / 2006$ & & DNT-275-06 \\
\hline GWM438012X & USGS-101 & 790 & Barium & 19.4 & & & & UG/L & $06 / 14 / 2006$ & & DNT-275-06 \\
\hline GWM438012X & USGS-101 & 790 & Beryllium & 0.088 & & U & & UG/L & $06 / 14 / 2006$ & & DNT-275-06 \\
\hline GWM438012X & USGS-101 & 790 & Cadmium & 0.057 & & $U$ & & UG/L & $06 / 14 / 2006$ & & DNT-275-06 \\
\hline
\end{tabular}


Table A-2. (continued).

\begin{tabular}{|c|c|c|c|c|c|c|c|c|c|c|c|}
\hline $\begin{array}{c}\text { Field Sample } \\
\text { Number }\end{array}$ & Location & Depth & Compound & $\begin{array}{c}\text { Sample } \\
\text { Result }\end{array}$ & $\begin{array}{l}\text { Sample } \\
\text { Error }\end{array}$ & $\begin{array}{c}\text { Result } \\
\text { Qualifier }\end{array}$ & $\begin{array}{c}\text { Validation } \\
\text { Flag }\end{array}$ & $\begin{array}{c}\text { Sample } \\
\text { Units }\end{array}$ & $\begin{array}{c}\text { Date Sample } \\
\text { Collected }\end{array}$ & MDA & $\begin{array}{l}\text { L\&V Report } \\
\text { Number }\end{array}$ \\
\hline GWM438012X & USGS-101 & 790 & Calcium & 27600 & & & & UG/L & $06 / 14 / 2006$ & & DNT-275-06 \\
\hline GWM438012X & USGS-101 & 790 & Chromium & 2.8 & & U & & UG/L & $06 / 14 / 2006$ & & DNT-275-06 \\
\hline GWM438012X & USGS-101 & 790 & Cobalt & 0.31 & & U & & UG/L & $06 / 14 / 2006$ & & DNT-275-06 \\
\hline GWM438012X & USGS-101 & 790 & Iron & 9.4 & & $U$ & & UG/L & $06 / 14 / 2006$ & & DNT-275-06 \\
\hline GWM438012X & USGS-101 & 790 & Lead & 0.49 & & 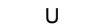 & & UG/L & $06 / 14 / 2006$ & & DNT-275-06 \\
\hline GWM438012X & USGS-101 & 790 & Magnesium & 8820 & & & & UG/L & $06 / 14 / 2006$ & & DNT-275-06 \\
\hline GWM438012X & USGS-101 & 790 & Manganese & 1.6 & & B & & UG/L & $06 / 14 / 2006$ & & DNT-275-06 \\
\hline GWM438012X & USGS-101 & 790 & Potassium & 2570 & & & & UG/L & $06 / 14 / 2006$ & & DNT-275-06 \\
\hline GWM438012X & USGS-101 & 790 & Selenium & 1 & & U & & UG/L & $06 / 14 / 2006$ & & DNT-275-06 \\
\hline GWM438012X & USGS-101 & 790 & Silver & 0.2 & & $U$ & & UG/L & $06 / 14 / 2006$ & & DNT-275-06 \\
\hline GWM438012X & USGS-101 & 790 & Sodium & 13700 & & & & UG/L & $06 / 14 / 2006$ & & DNT-275-06 \\
\hline GWM438012X & USGS-101 & 790 & Strontium & 77.9 & & & & UG/L & $06 / 14 / 2006$ & & DNT-275-06 \\
\hline GWM438012X & USGS-101 & 790 & Thallium & 0.32 & & U & & UG/L & $06 / 14 / 2006$ & & DNT-275-06 \\
\hline GWM438012X & USGS-101 & 790 & Uranium & 1.5 & & & & UG/L & $06 / 14 / 2006$ & & DNT-275-06 \\
\hline GWM438012X & USGS-101 & 790 & Vanadium & 3.9 & & B & & UG/L & $06 / 14 / 2006$ & & DNT-275-06 \\
\hline GWM438012X & USGS-101 & 790 & Zinc & 1.9 & & B & U & UG/L & $06 / 14 / 2006$ & & DNT-275-06 \\
\hline GWM438012X & USGS-101 & 790 & Mercury & 0.093 & & $\mathrm{U}$ & & UG/L & $06 / 14 / 2006$ & & DNT-275-06 \\
\hline GWM43801AN & USGS-101 & 790 & Fluoride & 0.86 & & & $\mathrm{~J}$ & $M G / L$ & $06 / 14 / 2006$ & & DNT-287-06 \\
\hline GWM43801AN & USGS-101 & 790 & Sulfate & 9.3 & & & $\mathrm{~J}$ & MG/L & $06 / 14 / 2006$ & & DNT-287-06 \\
\hline GWM43801N2 & USGS-101 & 790 & Nitrate/Nitrite as $\mathrm{N}$ & 1170 & & & & UG/L & $06 / 14 / 2006$ & & DNT-287-06 \\
\hline GWM43801VL & USGS-101 & 790 & Acetone & 2 & & U & & UG/L & $06 / 14 / 2006$ & & JGJ-062-06 \\
\hline GWM43801VL & USGS-101 & 790 & Benzene & 1 & & U & & UG/L & $06 / 14 / 2006$ & & JGJ-062-06 \\
\hline GWM43801VL & USGS-101 & 790 & Bromodichloromethane & 1 & & U & & UG/L & $06 / 14 / 2006$ & & JGJ-062-06 \\
\hline GWM43801VL & USGS-101 & 790 & Bromoform & 1 & & $\mathrm{U}$ & & UG/L & $06 / 14 / 2006$ & & JGJ-062-06 \\
\hline GWM43801VL & USGS-101 & 790 & Bromomethane & 2 & & $\mathrm{U}$ & & UG/L & $06 / 14 / 2006$ & & JGJ-062-06 \\
\hline GWM43801VL & USGS-101 & 790 & 2-Butanone & 5 & & U & $\mathrm{R}$ & UG/L & $06 / 14 / 2006$ & & JGJ-062-06 \\
\hline GWM43801VL & USGS-101 & 790 & Carbon disulfide & 1 & & $\mathrm{U}$ & & UG/L & $06 / 14 / 2006$ & & JGJ-062-06 \\
\hline GWM43801VL & USGS-101 & 790 & Carbon tetrachloride & 1 & & $\mathrm{u}$ & & UG/L & $06 / 14 / 2006$ & & JGJ-062-06 \\
\hline GWM43801VL & USGS-101 & 790 & Chlorobenzene & 1 & & U & & UG/L & $06 / 14 / 2006$ & & JGJ-062-06 \\
\hline GWM43801VL & USGS-101 & 790 & Dibromochloromethane & 1 & & $\mathrm{U}$ & & UG/L & $06 / 14 / 2006$ & & JGJ-062-06 \\
\hline GWM43801VL & USGS-101 & 790 & 1,2-Dibromo-3-chloropropane & 1 & & $\mathrm{U}$ & $\mathrm{R}$ & UG/L & $06 / 14 / 2006$ & & JGJ-062-06 \\
\hline GWM43801VL & USGS-101 & 790 & Chloroethane & 2 & & $U$ & & UG/L & $06 / 14 / 2006$ & & JGJ-062-06 \\
\hline GWM43801VL & USGS-101 & 790 & Chloroform & 1 & & $U$ & & UG/L & $06 / 14 / 2006$ & & JGJ-062-06 \\
\hline GWM43801VL & USGS-101 & 790 & Chloromethane & 2 & & $\mathrm{U}$ & & UG/L & $06 / 14 / 2006$ & & JGJ-062-06 \\
\hline GWM43801VL & USGS-101 & 790 & Cyclohexane & 1 & & U & & UG/L & $06 / 14 / 2006$ & & JGJ-062-06 \\
\hline GWM43801VL & USGS-101 & 790 & 1,2-Dibromoethane & 1 & & $U$ & & UG/L & $06 / 14 / 2006$ & & JGJ-062-06 \\
\hline GWM43801VL & USGS-101 & 790 & Dichlorodifluoromethane & 2 & & U & & UG/L & $06 / 14 / 2006$ & & JGJ-062-06 \\
\hline GWM43801VL & USGS-101 & 790 & 1,1-Dichloroethane & 1 & & $\mathrm{u}$ & & UG/L & $06 / 14 / 2006$ & & JGJ-062-06 \\
\hline GWM43801VL & USGS-101 & 790 & 1,2-Dichloroethane & 1 & & U & & UG/L & $06 / 14 / 2006$ & & JGJ-062-06 \\
\hline GWM43801VL & USGS-101 & 790 & 1,1-Dichloroethene & 1 & & $\mathrm{U}$ & & UG/L & $06 / 14 / 2006$ & & JGJ-062-06 \\
\hline GWM43801VL & USGS-101 & 790 & cis-1,2-Dichloroethene & 1 & & $\mathrm{U}$ & & UG/L & $06 / 14 / 2006$ & & JGJ-062-06 \\
\hline GWM43801VL & USGS-101 & 790 & trans-1,2-Dichloroethene & 1 & & U & & UG/L & $06 / 14 / 2006$ & & JGJ-062-06 \\
\hline GWM43801VL & USGS-101 & 790 & 1,2-Dichloropropane & 1 & & $U$ & & UG/L & $06 / 14 / 2006$ & & JGJ-062-06 \\
\hline GWM43801VL & USGS-101 & 790 & cis-1,3-Dichloropropene & 1 & & $\mathrm{U}$ & & UG/L & $06 / 14 / 2006$ & & JGJ-062-06 \\
\hline GWM43801VL & USGS-101 & 790 & trans-1,3-Dichloropropene & 1 & & U & & UG/L & $06 / 14 / 2006$ & & JGJ-062-06 \\
\hline GWM43801VL & USGS-101 & 790 & Ethylbenzene & 1 & & $U$ & & UG/L & $06 / 14 / 2006$ & & JGJ-062-06 \\
\hline GWM43801VL & USGS-101 & 790 & Trichlorofluoromethane & 1 & & $\mathrm{U}$ & & UG/L & $06 / 14 / 2006$ & & JGJ-062-06 \\
\hline GWM43801VL & USGS-101 & 790 & 2-Hexanone & 5 & & U & & UG/L & $06 / 14 / 2006$ & & JGJ-062-06 \\
\hline GWM43801VL & USGS-101 & 790 & Isopropylbenzene & 1 & & $U$ & & UG/L & $06 / 14 / 2006$ & & JGJ-062-06 \\
\hline GWM43801VL & USGS-101 & 790 & Methyl acetate & 5 & & U & & UG/L & $06 / 14 / 2006$ & & JGJ-062-06 \\
\hline GWM43801VL & USGS-101 & 790 & Methyl cyclohexane & 4 & & $\mathrm{U}$ & & UG/L & $06 / 14 / 2006$ & & JGJ-062-06 \\
\hline GWM43801VL & USGS-101 & 790 & Methylene Chloride & 1 & & $U$ & & UG/L & $06 / 14 / 2006$ & & JGJ-062-06 \\
\hline GWM43801VL & USGS-101 & 790 & 4-Methyl-2-pentanone & 5 & & $\mathrm{U}$ & & UG/L & $06 / 14 / 2006$ & & JGJ-062-06 \\
\hline GWM43801VL & USGS-101 & 790 & Styrene & 1 & & $\mathrm{U}$ & & UG/L & $06 / 14 / 2006$ & & JGJ-062-06 \\
\hline GWM43801VL & USGS-101 & 790 & 1,1,2,2-Tetrachloroethane & 1 & & $\mathrm{U}$ & & UG/L & $06 / 14 / 2006$ & & JGJ-062-06 \\
\hline GWM43801VL & USGS-101 & 790 & Tetrachloroethene & 1 & & U & & UG/L & $06 / 14 / 2006$ & & JGJ-062-06 \\
\hline GWM43801VL & USGS-101 & 790 & Toluene & 1 & & $\mathrm{U}$ & & UG/L & $06 / 14 / 2006$ & & JGJ-062-06 \\
\hline GWM43801VL & USGS-101 & 790 & 1,2,4-Trichlorobenzene & 1 & & $\mathrm{U}$ & & UG/L & $06 / 14 / 2006$ & & JGJ-062-06 \\
\hline GWM43801VL & USGS-101 & 790 & 1,1,1-Trichloroethane & 1 & & $\mathrm{U}$ & & UG/L & $06 / 14 / 2006$ & & JGJ-062-06 \\
\hline GWM43801VL & USGS-101 & 790 & 1,1,2-Trichloroethane & 1 & & $\mathrm{U}$ & & UG/L & $06 / 14 / 2006$ & & JGJ-062-06 \\
\hline GWM43801VL & USGS-101 & 790 & Trichloroethene & 1 & & U & & UG/L & $06 / 14 / 2006$ & & JGJ-062-06 \\
\hline GWM43801VL & USGS-101 & 790 & 1,1,2-Trichloro-1,2,2-trifluoroethane & 1 & & U & & UG/L & $06 / 14 / 2006$ & & JGJ-062-06 \\
\hline GWM43801VL & USGS-101 & 790 & Vinyl Chloride & 2 & & $U$ & & UG/L & $06 / 14 / 2006$ & & JGJ-062-06 \\
\hline GWM43801VL & USGS-101 & 790 & Xylene (Total) & 3 & & U & & UG/L & $06 / 14 / 2006$ & & JGJ-062-06 \\
\hline GWM43801VL & USGS-101 & 790 & Methyl t-butyl ether & 2 & & $U$ & & UG/L & $06 / 14 / 2006$ & & JGJ-062-06 \\
\hline GWM43801R8 & USGS-101 & 790 & Tritium & $-2.16 \mathrm{E}+02$ & $1.17 \mathrm{E}+02$ & & $\mathrm{U}$ & $\mathrm{PCl} / \mathrm{L}$ & $06 / 14 / 2006$ & $4.38 \mathrm{E}+02$ & SOS-TL199-06 \\
\hline GWM43801RH & USGS-101 & 790 & Gross Alpha & $2.33 E+00$ & $6.16 \mathrm{E}-01$ & & & $\mathrm{PCI} / \mathrm{L}$ & $06 / 14 / 2006$ & $1.90 \mathrm{E}+00$ & SOS-TL198-06 \\
\hline GWM43801RH & USGS-101 & 790 & Gross Beta & $1.97 \mathrm{E}+00$ & 7.99E-01 & & UJ & $\mathrm{PCl} / \mathrm{L}$ & $06 / 14 / 2006$ & $3.21 \mathrm{E}+00$ & SOS-TL198-06 \\
\hline GWM43801RH & USGS-101 & 790 & Strontium-90 & $-1.99 \mathrm{E}-02$ & 8.09E-02 & & $U$ & $\mathrm{PCl} / \mathrm{L}$ & $06 / 14 / 2006$ & 4.14E-01 & SOS-TL198-06 \\
\hline GWM43801RH & USGS-101 & 790 & Technetium-99 & $1.56 \mathrm{E}+00$ & $2.15 E+00$ & & $\mathrm{U}$ & $\mathrm{PCl} / \mathrm{L}$ & $06 / 14 / 2006$ & $7.16 \mathrm{E}+00$ & SOS-TL198-06 \\
\hline GWM43801UX & USGS-101 & 790 & lodine-129 & $-3.46 \mathrm{E}-02$ & $2.36 \mathrm{E}-02$ & & U & $\mathrm{PCI} / \mathrm{L}$ & $06 / 14 / 2006$ & 7.73E-02 & SOS-TL197-06 \\
\hline
\end{tabular}


Table A-2. (continued).

\begin{tabular}{|c|c|c|c|c|c|c|c|c|c|c|c|}
\hline $\begin{array}{l}\text { Field Sample } \\
\text { Number }\end{array}$ & Location & Depth & Compound & $\begin{array}{c}\text { Sample } \\
\text { Result }\end{array}$ & $\begin{array}{l}\text { Sample } \\
\text { Error }\end{array}$ & $\begin{array}{c}\text { Result } \\
\text { Qualifier }\end{array}$ & $\begin{array}{c}\text { Validation } \\
\text { Flag }\end{array}$ & $\begin{array}{c}\text { Sample } \\
\text { Units }\end{array}$ & $\begin{array}{c}\text { Date Sample } \\
\text { Collected }\end{array}$ & MDA & $\begin{array}{l}\text { L\&V Report } \\
\text { Number }\end{array}$ \\
\hline GWM445012X & USGS-104 & 592 & Aluminum & 7.9 & & $U$ & & UG/L & $06 / 19 / 2006$ & & DNT-330-06 \\
\hline GWM445012X & USGS-104 & 592 & Antimony & 0.5 & & U & & UG/L & $06 / 19 / 2006$ & & DNT-330-06 \\
\hline GWM445012X & USGS-104 & 592 & Arsenic & 2 & & $u$ & & UG/L & $06 / 19 / 2006$ & & DNT-330-06 \\
\hline GWM445012X & USGS-104 & 592 & Beryllium & 0.088 & & U & & UG/L & $06 / 19 / 2006$ & & DNT-330-06 \\
\hline GWM445012X & USGS-104 & 592 & Cadmium & 0.057 & & U & & UG/L & $06 / 19 / 2006$ & & DNT-330-06 \\
\hline GWM445012X & USGS-104 & 592 & Calcium & 31600 & & & & UG/L & $06 / 19 / 2006$ & & DNT-330-06 \\
\hline GWM445012X & USGS-104 & 592 & Chromium & 7.4 & & & & UG/L & $06 / 19 / 2006$ & & DNT-330-06 \\
\hline GWM445012X & USGS-104 & 592 & Copper & 0.42 & & B & & UG/L & $06 / 19 / 2006$ & & DNT-330-06 \\
\hline GWM445012X & USGS-104 & 592 & Iron & 9.4 & & u & & UG/L & $06 / 19 / 2006$ & & DNT-330-06 \\
\hline GWM445012X & USGS-104 & 592 & Lead & 0.49 & & U & & UG/L & 06/19/2006 & & DNT-330-06 \\
\hline GWM445012X & USGS-104 & 592 & Magnesium & 12300 & & $\mathrm{E}$ & $\mathrm{J}$ & UG/L & $06 / 19 / 2006$ & & DNT-330-06 \\
\hline GWM445012X & USGS-104 & 592 & Manganese & 0.56 & & B & & UG/L & 06/19/2006 & & DNT-330-06 \\
\hline GWM445012X & USGS-104 & 592 & Nickel & 1.2 & & B & & UG/L & 06/19/2006 & & DNT-330-06 \\
\hline GWM445012X & USGS-104 & 592 & Potassium & 2270 & & E & & UG/L & $06 / 19 / 2006$ & & DNT-330-06 \\
\hline GWM445012X & USGS-104 & 592 & Selenium & 1.3 & & $\bar{B}$ & $\mathrm{~J}$ & UG/L & $06 / 19 / 2006$ & & DNT-330-06 \\
\hline GWM445012X & USGS-104 & 592 & Silver & 0.2 & & U & & UG/L & $06 / 19 / 2006$ & & DNT-330-06 \\
\hline GWM445012X & USGS-104 & 592 & Sodium & 7750 & & & & UG/L & $06 / 19 / 2006$ & & DNT-330-06 \\
\hline GWM445012X & USGS-104 & 592 & Uranium & 1.8 & & & & UG/L & $06 / 19 / 2006$ & & DNT-330-06 \\
\hline GWM445012X & USGS-104 & 592 & Vanadium & 5.6 & & B & & UG/L & $06 / 19 / 2006$ & & DNT-330-06 \\
\hline GWM445012X & USGS-104 & 592 & Zinc & 11.1 & & & & UG/L & $06 / 19 / 2006$ & & DNT-330-06 \\
\hline GWM445012X & USGS-104 & 592 & Mercury & 0.093 & & U & & UG/L & 06/19/2006 & & DNT-330-06 \\
\hline GWM44501A1 & USGS-104 & 592 & Total Alkalinity & 122 & & & $\mathrm{~J}$ & MG/L & $06 / 19 / 2006$ & & DNT-281-06 \\
\hline GWM44501AN & USGS-104 & 592 & Chloride & 14.7 & & $\mathrm{~J}$ & & $\mathrm{MG} / \mathrm{L}$ & $06 / 19 / 2006$ & & DNT-281-06 \\
\hline GWM44501AN & USGS-104 & 592 & Fluoride & 0.19 & & & $\mathrm{~J}$ & MG/L & 06/19/2006 & & DNT-281-06 \\
\hline GWM44501AN & USGS-104 & 592 & Sulfate & 19.9 & & & & MG/L & 06/19/2006 & & DNT-281-06 \\
\hline GWM44501N2 & USGS-104 & 592 & Nitrate/Nitrite as $\mathrm{N}$ & 937 & & & & UG/L & $06 / 19 / 2006$ & & DNT-281-06 \\
\hline GWM44501VL & USGS-104 & 592 & Acetone & 2 & & U & $\mathrm{R}$ & UG/L & $06 / 19 / 2006$ & & JGJ-066-06 \\
\hline GWM44501VL & USGS-104 & 592 & Benzene & 1 & & $\mathrm{U}$ & & UG/L & 06/19/2006 & & JGJ-066-06 \\
\hline GWM44501VL & USGS-104 & 592 & Bromodichloromethane & 1 & & $\mathrm{u}$ & & UG/L & 06/19/2006 & & JGJ-066-06 \\
\hline GWM44501VL & USGS-104 & 592 & Bromoform & 1 & & U & & UG/L & 06/19/2006 & & JGJ-066-06 \\
\hline GWM44501VL & USGS-104 & 592 & Bromomethane & 2 & & $u$ & & UG/L & $06 / 19 / 2006$ & & JGJ-066-06 \\
\hline GWM44501VL & USGS-104 & 592 & 2-Butanone & 5 & & U & $\mathrm{R}$ & UG/L & $06 / 19 / 2006$ & & JGJ-066-06 \\
\hline GWM44501VL & USGS-104 & 592 & Carbon disulfide & 1 & & $\mathrm{U}$ & & UG/L & $06 / 19 / 2006$ & & JGJ-066-06 \\
\hline GWM44501VL & USGS-104 & 592 & Carbon tetrachloride & 1 & & $\mathrm{U}$ & & UG/L & 06/19/2006 & & JGJ-066-06 \\
\hline GWM44501VL & USGS-104 & 592 & Chlorobenzene & 1 & & $\mathrm{u}$ & & UG/L & $06 / 19 / 2006$ & & JGJ-066-06 \\
\hline GWM44501VL & USGS-104 & 592 & Dibromochloromethane & 1 & & $\mathrm{U}$ & & UG/L & $06 / 19 / 2006$ & & JGJ-066-06 \\
\hline GWM44501VL & USGS-104 & 592 & Chloromethane & 2 & & $\mathrm{U}$ & & UG/L & $06 / 19 / 2006$ & & JGJ-066-06 \\
\hline GWM44501VL & USGS-104 & 592 & Cyclohexane & 1 & & U & & UG/L & 06/19/2006 & & JGJ-066-06 \\
\hline GWM44501VL & USGS-104 & 592 & 1,2-Dibromoethane & 1 & & $\mathrm{u}$ & & UG/L & $06 / 19 / 2006$ & & JGJ-066-06 \\
\hline GWM44501VL & USGS-104 & 592 & 1,2-Dichlorobenzene & 1 & & $\mathrm{U}$ & & UG/L & 06/19/2006 & & JGJ-066-06 \\
\hline GWM44501VL & USGS-104 & 592 & 1,3-Dichlorobenzene & 1 & & $\mathrm{U}$ & & UG/L & 06/19/2006 & & JGJ-066-06 \\
\hline GWM44501VL & USGS-104 & 592 & 1,4-Dichlorobenzene & 1 & & $u$ & & UG/L & $06 / 19 / 2006$ & & JGJ-066-06 \\
\hline GWM44501VL & USGS-104 & 592 & Dichlorodifluoromethane & 2 & & $\mathrm{U}$ & & UG/L & $06 / 19 / 2006$ & & JGJ-066-06 \\
\hline GWM44501VL & USGS-104 & 592 & 1,1-Dichloroethane & 1 & & $\mathrm{U}$ & & UG/L & 06/19/2006 & & JGJ-066-06 \\
\hline GWM44501VL & USGS-104 & 592 & 1,2-Dichloroethane & 1 & & U & & UG/L & $06 / 19 / 2006$ & & JGJ-066-06 \\
\hline GWM44501VL & USGS-104 & 592 & 1,1-Dichloroethene & 1 & & $\mathrm{u}$ & & UG/L & $06 / 19 / 2006$ & & JGJ-066-06 \\
\hline GWM44501VL & USGS-104 & 592 & cis-1,2-Dichloroethene & 1 & & U & & UG/L & $06 / 19 / 2006$ & & JGJ-066-06 \\
\hline GWM44501VL & USGS-104 & 592 & trans-1,2-Dichloroethene & 1 & & U & & UG/L & $06 / 19 / 2006$ & & JGJ-066-06 \\
\hline GWM44501VL & USGS-104 & 592 & 1,2-Dichloropropane & 1 & & $\mathrm{u}$ & & UG/L & 06/19/2006 & & JGJ-066-06 \\
\hline GWM44501VL & USGS-104 & 592 & cis-1,3-Dichloropropene & 1 & & U & & UG/L & $06 / 19 / 2006$ & & JGJ-066-06 \\
\hline GWM44501VL & USGS-104 & 592 & trans-1,3-Dichloropropene & 1 & & U & & UG/L & $06 / 19 / 2006$ & & JGJ-066-06 \\
\hline GWM44501VL & USGS-104 & 592 & Ethylbenzene & 1 & & $\mathrm{u}$ & & UG/L & $06 / 19 / 2006$ & & JGJ-066-06 \\
\hline GWM44501VL & USGS-104 & 592 & Trichlorofluoromethane & 1 & & $\mathrm{U}$ & & UG/L & $06 / 19 / 2006$ & & JGJ-066-06 \\
\hline GWM44501VL & USGS-104 & 592 & 2-Hexanone & 5 & & $\mathrm{U}$ & $\mathrm{R}$ & UG/L & 06/19/2006 & & JGJ-066-06 \\
\hline GWM44501VL & USGS-104 & 592 & Isopropylbenzene & 1 & & $u$ & & UG/L & $06 / 19 / 2006$ & & JGJ-066-06 \\
\hline GWM44501VL & USGS-104 & 592 & Methyl acetate & 5 & & $\mathrm{U}$ & $\mathrm{R}$ & UG/L & $06 / 19 / 2006$ & & JGJ-066-06 \\
\hline GWM44501VL & USGS-104 & 592 & Methyl cyclohexane & 4 & & U & & UG/L & 06/19/2006 & & JGJ-066-06 \\
\hline GWM44501VL & USGS-104 & 592 & Methylene Chloride & 1 & & $u$ & & UG/L & $06 / 19 / 2006$ & & JGJ-066-06 \\
\hline GWM44501VL & USGS-104 & 592 & 4-Methyl-2-pentanone & 5 & & $\mathrm{U}$ & & UG/L & $06 / 19 / 2006$ & & JGJ-066-06 \\
\hline GWM44501VL & USGS-104 & 592 & Styrene & 1 & & U & & UG/L & 06/19/2006 & & JGJ-066-06 \\
\hline GWM44501VL & USGS-104 & 592 & 1,1,2,2-Tetrachloroethane & 1 & & U & & UG/L & $06 / 19 / 2006$ & & JGJ-066-06 \\
\hline GWM44501VL & USGS-104 & 592 & Tetrachloroethene & 1 & & $\mathrm{U}$ & & UG/L & $06 / 19 / 2006$ & & JGJ-066-06 \\
\hline GWM44501VL & USGS-104 & 592 & Toluene & 1 & & $\mathrm{U}$ & & UG/L & 06/19/2006 & & JGJ-066-06 \\
\hline GWM44501VL & USGS-104 & 592 & 1,2,4-Trichlorobenzene & 1 & & U & & UG/L & $06 / 19 / 2006$ & & JGJ-066-06 \\
\hline GWM44501VL & USGS-104 & 592 & 1,1,1-Trichloroethane & 1 & & $U$ & & UG/L & $06 / 19 / 2006$ & & JGJ-066-06 \\
\hline GWM44501VL & USGS-104 & 592 & 1,1,2-Trichloroethane & 1 & & $\mathrm{U}$ & & UG/L & 06/19/2006 & & JGJ-066-06 \\
\hline GWM44501VL & USGS-104 & 592 & Trichloroethene & 1 & & U & & UG/L & $06 / 19 / 2006$ & & JGJ-066-06 \\
\hline GWM44501VL & USGS-104 & 592 & 1,1,2-Trichloro-1,2,2-trifluoroethane & 1 & & $u$ & & UG/L & 06/19/2006 & & JGJ-066-06 \\
\hline GWM44501VL & USGS-104 & 592 & Vinyl Chloride & 2 & & U & & UG/L & 06/19/2006 & & JGJ-066-06 \\
\hline GWM44501VL & USGS-104 & 592 & Xylene (Total) & 3 & & $U$ & & UG/L & 06/19/2006 & & JGJ-066-06 \\
\hline GWM44501VL & USGS-104 & 592 & Methyl t-butyl ether & 2 & & $\mathrm{U}$ & & UG/L & $06 / 19 / 2006$ & & JGJ-066-06 \\
\hline
\end{tabular}


Table A-2. (continued).

\begin{tabular}{|c|c|c|c|c|c|c|c|c|c|c|c|}
\hline $\begin{array}{c}\text { Field Sample } \\
\text { Number }\end{array}$ & Location & Depth & Compound & $\begin{array}{c}\text { Sample } \\
\text { Result }\end{array}$ & $\begin{array}{c}\text { Sample } \\
\text { Error }\end{array}$ & $\begin{array}{c}\text { Result } \\
\text { Qualifier }\end{array}$ & $\begin{array}{c}\text { Validation } \\
\text { Flag }\end{array}$ & $\begin{array}{c}\text { Sample } \\
\text { Units }\end{array}$ & $\begin{array}{c}\text { Date Sample } \\
\text { Collected }\end{array}$ & MDA & $\begin{array}{l}\text { L\&V Report } \\
\text { Number }\end{array}$ \\
\hline GWM44501R8 & USGS-104 & 592 & Tritium & $1.08 \mathrm{E}+03$ & $1.62 \mathrm{E}+02$ & & & $\mathrm{PCl} / \mathrm{L}$ & $06 / 19 / 2006$ & $4.38 \mathrm{E}+02$ & SOS-TL199-06 \\
\hline GWM44501RH & USGS-104 & 592 & Gross Alpha & $1.11 \mathrm{E}+00$ & 3.12E-01 & & & $\mathrm{PCl} / \mathrm{L}$ & 06/19/2006 & $9.47 \mathrm{E}-01$ & SOS-TL198-06 \\
\hline GWM44501RH & USGS-104 & 592 & Gross Beta & $2.55 \mathrm{E}+00$ & 4.89E-01 & & & $\mathrm{PCl} / \mathrm{L}$ & 06/19/2006 & $1.67 \mathrm{E}+00$ & SOS-TL198-06 \\
\hline GWM44501RH & USGS-104 & 592 & Technetium-99 & $-5.96 E+00$ & $2.83 E+00$ & & U & $\mathrm{PCl} / \mathrm{L}$ & $06 / 19 / 2006$ & $9.64 \mathrm{E}+00$ & SOS-TL198-06 \\
\hline GWM44501UX & USGS-104 & 592 & lodine- 129 & $-9.36 \mathrm{E}-03$ & 2.94E-02 & & U & $\mathrm{PCI} / \mathrm{L}$ & $06 / 19 / 2006$ & $1.02 \mathrm{E}-01$ & SOS-TL197-06 \\
\hline GWM454012X & USGS-105 & 700 & Aluminum & 7.9 & & $U$ & & UG/L & 07/06/2006 & & DNT-335-06 \\
\hline GWM454012X & USGS-105 & 700 & Antimony & 0.5 & & $U$ & & UG/L & 07/06/2006 & & DNT-335-06 \\
\hline GWM454012X & USGS-105 & 700 & Barium & 35.5 & & & & UG/L & 07/06/2006 & & DNT-335-06 \\
\hline GWM454012X & USGS-105 & 700 & Beryllium & 0.088 & & $U$ & & UG/L & 07/06/2006 & & DNT-335-06 \\
\hline GWM454012X & USGS-105 & 700 & Cadmium & 0.057 & & $\mathrm{U}$ & & UG/L & 07/06/2006 & & DNT-335-06 \\
\hline GWM454012X & USGS-105 & 700 & Calcium & 37500 & & $\mathrm{E}$ & $\mathrm{J}$ & UG/L & 07/06/2006 & & DNT-335-06 \\
\hline GWM454012X & USGS-105 & 700 & Chromium & 8.2 & & & & UG/L & $07 / 06 / 2006$ & & DNT-335-06 \\
\hline GWM454012X & USGS-105 & 700 & Cobalt & 0.31 & & $\mathrm{U}$ & & UG/L & 07/06/2006 & & DNT-335-06 \\
\hline GWM454012X & USGS-105 & 700 & Copper & 0.51 & & B & $\mathrm{U}$ & UG/L & 07/06/2006 & & DNT-335-06 \\
\hline GWM454012X & USGS-105 & 700 & Iron & 9.4 & & $U$ & & UG/L & 07/06/2006 & & DNT-335-06 \\
\hline GWM454012X & USGS-105 & 700 & Lead & 0.49 & & U & & UG/L & 07/06/2006 & & DNT-335-06 \\
\hline GWM454012X & USGS-105 & 700 & Magnesium & 15000 & & & & UG/L & $07 / 06 / 2006$ & & DNT-335-06 \\
\hline GWM454012X & USGS-105 & 700 & Nickel & 1.5 & & B & & UG/L & 07/06/2006 & & DNT-335-06 \\
\hline GWM454012X & USGS-105 & 700 & Potassium & 2900 & & & & UG/L & 07/06/2006 & & DNT-335-06 \\
\hline GWM454012X & USGS-105 & 700 & Selenium & 2 & & B & & UG/L & 07/06/2006 & & DNT-335-06 \\
\hline GWM454012X & USGS-105 & 700 & Silver & 0.2 & & $\mathrm{U}$ & & UG/L & $07 / 06 / 2006$ & & DNT-335-06 \\
\hline GWM454012X & USGS-105 & 700 & Sodium & 12200 & & & & UG/L & 07/06/2006 & & DNT-335-06 \\
\hline GWM454012X & USGS-105 & 700 & Strontium & 233 & & & & UG/L & 07/06/2006 & & DNT-335-06 \\
\hline GWM454012X & USGS-105 & 700 & Thallium & 0.61 & & B & U & UG/L & $07 / 06 / 2006$ & & DNT-335-06 \\
\hline GWM454012X & USGS-105 & 700 & Uranium & 2 & & & & UG/L & 07/06/2006 & & DNT-335-06 \\
\hline GWM454012X & USGS-105 & 700 & Vanadium & 3.9 & & B & & UG/L & 07/06/2006 & & DNT-335-06 \\
\hline GWM454012X & USGS-105 & 700 & Zinc & 3.9 & & B & U & UG/L & 07/06/2006 & & DNT-335-06 \\
\hline GWM454012X & USGS-105 & 700 & Mercury & 0.093 & & $U^{*}$ & UJ & UG/L & 07/06/2006 & & DNT-335-06 \\
\hline GWM45401A1 & USGS-105 & 700 & Total Alkalinity & 136 & & & & $M G / L$ & 07/06/2006 & & DNT-329-06 \\
\hline GWM45401AN & USGS-105 & 700 & Chloride & 14.1 & & & & $M G / L$ & 07/06/2006 & & DNT-329-06 \\
\hline GWM45401AN & USGS-105 & 700 & Fluoride & 0.2 & & & & $\mathrm{MG} / \mathrm{L}$ & $07 / 06 / 2006$ & & DNT-329-06 \\
\hline GWM45401AN & USGS-105 & 700 & Sulfate & 24.1 & & & & $M G / L$ & 07/06/2006 & & DNT-329-06 \\
\hline GWM45401N2 & USGS-105 & 700 & Nitrate/Nitrite as $\mathrm{N}$ & 826 & & & & UG/L & 07/06/2006 & & DNT-329-06 \\
\hline GWM45401VL & USGS-105 & 700 & Acetone & 2 & & U & $\mathrm{R}$ & UG/L & 07/06/2006 & & JGJ-074-06 \\
\hline GWM45401VL & USGS-105 & 700 & Benzene & 1 & & $\mathrm{U}$ & & UG/L & 07/06/2006 & & JGJ-074-06 \\
\hline GWM45401VL & USGS-105 & 700 & Bromodichloromethane & 1 & & $\mathrm{U}$ & & UG/L & 07/06/2006 & & JGJ-074-06 \\
\hline GWM45401VL & USGS-105 & 700 & Bromoform & 1 & & $\mathrm{U}$ & & UG/L & $07 / 06 / 2006$ & & JGJ-074-06 \\
\hline GWM45401VL & USGS-105 & 700 & Carbon tetrachloride & 1 & & U & & UG/L & 07/06/2006 & & JGJ-074-06 \\
\hline GWM45401VL & USGS-105 & 700 & Chlorobenzene & 1 & & $\mathrm{U}$ & & UG/L & 07/06/2006 & & JGJ-074-06 \\
\hline GWM45401VL & USGS-105 & 700 & Dibromochloromethane & 1 & & $\mathrm{U}$ & & UG/L & 07/06/2006 & & JGJ-074-06 \\
\hline GWM45401VL & USGS-105 & 700 & 1,2-Dibromo-3-chloropropane & 1 & & $\mathrm{U}$ & $\mathrm{R}$ & UG/L & $07 / 06 / 2006$ & & JGJ-074-06 \\
\hline GWM45401VL & USGS-105 & 700 & Chloroethane & 2 & & $\mathrm{U}$ & & UG/L & 07/06/2006 & & JGJ-074-06 \\
\hline GWM45401VL & USGS-105 & 700 & Chloroform & 1 & & U & & UG/L & 07/06/2006 & & JGJ-074-06 \\
\hline GWM45401VL & USGS-105 & 700 & Chloromethane & 2 & & U & & UG/L & 07/06/2006 & & JGJ-074-06 \\
\hline GWM45401VL & USGS-105 & 700 & Cyclohexane & 1 & & $\mathrm{U}$ & & UG/L & $07 / 06 / 2006$ & & JGJ-074-06 \\
\hline GWM45401VL & USGS-105 & 700 & 1,2-Dibromoethane & 1 & & $\mathrm{U}$ & & UG/L & 07/06/2006 & & JGJ-074-06 \\
\hline GWM45401VL & USGS-105 & 700 & 1,2-Dichlorobenzene & 1 & & $U$ & & UG/L & $07 / 06 / 2006$ & & JGJ-074-06 \\
\hline GWM45401VL & USGS-105 & 700 & 1,3-Dichlorobenzene & 1 & & $\mathrm{U}$ & & UG/L & $07 / 06 / 2006$ & & JGJ-074-06 \\
\hline GWM45401VL & USGS-105 & 700 & 1,4-Dichlorobenzene & 1 & & $\mathrm{U}$ & & UG/L & 07/06/2006 & & JGJ-074-06 \\
\hline GWM45401VL & USGS-105 & 700 & Dichlorodifluoromethane & 2 & & U & & UG/L & $07 / 06 / 2006$ & & JGJ-074-06 \\
\hline GWM45401VL & USGS-105 & 700 & 1,1-Dichloroethane & 1 & & U & & UG/L & $07 / 06 / 2006$ & & JGJ-074-06 \\
\hline GWM45401VL & USGS-105 & 700 & 1,2-Dichloroethane & 1 & & U & & UG/L & 07/06/2006 & & JGJ-074-06 \\
\hline GWM45401VL & USGS-105 & 700 & 1,1-Dichloroethene & 1 & & U & & UG/L & 07/06/2006 & & JGJ-074-06 \\
\hline GWM45401VL & USGS-105 & 700 & cis-1,2-Dichloroethene & 1 & & $\mathrm{U}$ & & UG/L & $07 / 06 / 2006$ & & JGJ-074-06 \\
\hline GWM45401VL & USGS-105 & 700 & trans-1,2-Dichloroethene & 1 & & $\mathrm{U}$ & & UG/L & 07/06/2006 & & JGJ-074-06 \\
\hline GWM45401VL & USGS-105 & 700 & 1,2-Dichloropropane & 1 & & U & & UG/L & $07 / 06 / 2006$ & & JGJ-074-06 \\
\hline GWM45401VL & USGS-105 & 700 & cis-1,3-Dichloropropene & 1 & & U & & UG/L & 07/06/2006 & & JGJ-074-06 \\
\hline GWM45401VL & USGS-105 & 700 & trans-1,3-Dichloropropene & 1 & & $\mathrm{U}$ & & UG/L & $07 / 06 / 2006$ & & JGJ-074-06 \\
\hline GWM45401VL & USGS-105 & 700 & Ethylbenzene & 1 & & $\mathrm{U}$ & & UG/L & 07/06/2006 & & JGJ-074-06 \\
\hline GWM45401VL & USGS-105 & 700 & Trichlorofluoromethane & 1 & & U & & UG/L & $07 / 06 / 2006$ & & JGJ-074-06 \\
\hline GWM45401VL & USGS-105 & 700 & 2-Hexanone & 5 & & $\mathrm{U}$ & & UG/L & 07/06/2006 & & JGJ-074-06 \\
\hline GWM45401VL & USGS-105 & 700 & Isopropylbenzene & 1 & & U & & UG/L & 07/06/2006 & & JGJ-074-06 \\
\hline GWM45401VL & USGS-105 & 700 & Methyl acetate & 5 & & $U$ & $\mathrm{R}$ & UG/L & 07/06/2006 & & JGJ-074-06 \\
\hline GWM45401VL & USGS-105 & 700 & Methyl cyclohexane & 4 & & $U$ & & UG/L & 07/06/2006 & & JGJ-074-06 \\
\hline GWM45401VL & USGS-105 & 700 & Methylene Chloride & 1 & & $U$ & & UG/L & $07 / 06 / 2006$ & & JGJ-074-06 \\
\hline GWM45401VL & USGS-105 & 700 & 4-Methyl-2-pentanone & 5 & & U & & UG/L & 07/06/2006 & & JGJ-074-06 \\
\hline GWM45401VL & USGS-105 & 700 & Styrene & 1 & & $\mathrm{U}$ & & UG/L & $07 / 06 / 2006$ & & JGJ-074-06 \\
\hline GWM45401VL & USGS-105 & 700 & 1,1,2,2-Tetrachloroethane & 1 & & $\mathrm{U}$ & & UG/L & 07/06/2006 & & JGJ-074-06 \\
\hline GWM45401VL & USGS-105 & 700 & Tetrachloroethene & 1 & & U & & UG/L & 07/06/2006 & & JGJ-074-06 \\
\hline GWM45401VL & USGS-105 & 700 & Toluene & 4.8 & & & & UG/L & 07/06/2006 & & JGJ-074-06 \\
\hline GWM45401VL & USGS-105 & 700 & 1,2,4-Trichlorobenzene & 1 & & $U$ & & UG/L & 07/06/2006 & & JGJ-074-06 \\
\hline GWM45401VL & USGS-105 & 700 & 1,1,1-Trichloroethane & 1 & & U & & UG/L & 07/06/2006 & & JGJ-074-06 \\
\hline
\end{tabular}


Table A-2. (continued).

\begin{tabular}{|c|c|c|c|c|c|c|c|c|c|c|c|}
\hline $\begin{array}{l}\text { Field Sample } \\
\text { Number }\end{array}$ & Location & Depth & Compound & $\begin{array}{c}\text { Sample } \\
\text { Result }\end{array}$ & $\begin{array}{c}\text { Sample } \\
\text { Error }\end{array}$ & $\begin{array}{c}\text { Result } \\
\text { Qualifier }\end{array}$ & $\begin{array}{c}\text { Validation } \\
\text { Flag }\end{array}$ & $\begin{array}{c}\text { Sample } \\
\text { Units }\end{array}$ & $\begin{array}{c}\text { Date Sample } \\
\text { Collected }\end{array}$ & MDA & $\begin{array}{c}\text { L\&V Report } \\
\text { Number }\end{array}$ \\
\hline GWM45401VL & USGS-105 & 700 & 1,1,2-Trichloroethane & 1 & & U & & UG/L & $07 / 06 / 2006$ & & JGJ-074-06 \\
\hline GWM45401VL & USGS-105 & 700 & Trichloroethene & 1 & & $\mathrm{U}$ & & UG/L & $07 / 06 / 2006$ & & JGJ-074-06 \\
\hline GWM45401VL & USGS-105 & 700 & 1,1,2-Trichloro-1,2,2-trifluoroethane & 1 & & $\mathrm{U}$ & & UG/L & $07 / 06 / 2006$ & & JGJ-074-06 \\
\hline GWM45401VL & USGS-105 & 700 & Xylene (Total) & 3 & & $U$ & & UG/L & $07 / 06 / 2006$ & & JGJ-074-06 \\
\hline GWM45401VL & USGS-105 & 700 & Methyl t-butyl ether & 2 & & $\mathrm{U}$ & & UG/L & $07 / 06 / 2006$ & & JGJ-074-06 \\
\hline GWM45401R8 & USGS-105 & 700 & Tritium & $3.20 \mathrm{E}+02$ & $1.15 \mathrm{E}+02$ & & UJ & $\mathrm{PCI} / \mathrm{L}$ & $07 / 06 / 2006$ & $3.63 \mathrm{E}+02$ & SOS-TL228-06 \\
\hline GWM45401RH & USGS-105 & 700 & Gross Alpha & $2.23 \mathrm{E}+00$ & $6.78 \mathrm{E}-01$ & & & $\mathrm{PCl} / \mathrm{L}$ & $07 / 06 / 2006$ & $2.16 \mathrm{E}+00$ & SOS-TL227-06 \\
\hline GWM45401RH & USGS-105 & 700 & Strontium-90 & $2.88 \mathrm{E}-01$ & $1.15 \mathrm{E}-01$ & & UJ & $\mathrm{PCl} / \mathrm{L}$ & $07 / 06 / 2006$ & 4.45E-01 & SOS-TL227-06 \\
\hline GWM45401RH & USGS-105 & 700 & Technetium-99 & $-2.56 E+00$ & $1.92 \mathrm{E}+00$ & & $U$ & $\mathrm{PCl} / \mathrm{L}$ & $07 / 06 / 2006$ & $6.62 \mathrm{E}+00$ & SOS-TL227-06 \\
\hline GWM45401UX & USGS-105 & 700 & lodine-129 & $1.15 \mathrm{E}-02$ & $3.16 \mathrm{E}-02$ & & UJ & $\mathrm{PCl} / \mathrm{L}$ & $07 / 06 / 2006$ & 1.15E-01 & SOS-TL226-06 \\
\hline GWM446012X & USGS-106 & 609 & Aluminum & 7.9 & & $U$ & & UG/L & $06 / 21 / 2006$ & & DNT-330-06 \\
\hline GWM446012X & USGS-106 & 609 & Antimony & 0.5 & & $\mathrm{U}$ & & UG/L & $06 / 21 / 2006$ & & DNT-330-06 \\
\hline GWM446012X & USGS-106 & 609 & Arsenic & 2 & & U & & UG/L & $06 / 21 / 2006$ & & DNT-330-06 \\
\hline GWM446012X & USGS-106 & 609 & Barium & 55.1 & & & & UG/L & $06 / 21 / 2006$ & & DNT-330-06 \\
\hline GWM446012X & USGS-106 & 609 & Beryllium & 0.088 & & $U$ & & UG/L & $06 / 21 / 2006$ & & DNT-330-06 \\
\hline GWM446012X & USGS-106 & 609 & Cadmium & 0.071 & & B & & UG/L & $06 / 21 / 2006$ & & DNT-330-06 \\
\hline GWM446012X & USGS-106 & 609 & Calcium & 42800 & & & & UG/L & $06 / 21 / 2006$ & & DNT-330-06 \\
\hline GWM446012X & USGS-106 & 609 & Copper & 0.72 & & B & & UG/L & $06 / 21 / 2006$ & & DNT-330-06 \\
\hline GWM446012X & USGS-106 & 609 & Iron & 9.4 & & U & & UG/L & $06 / 21 / 2006$ & & DNT-330-06 \\
\hline GWM446012X & USGS-106 & 609 & Lead & 8.3 & & & & UG/L & $06 / 21 / 2006$ & & DNT-330-06 \\
\hline GWM446012X & USGS-106 & 609 & Magnesium & 15800 & & E & $\mathrm{J}$ & UG/L & $06 / 21 / 2006$ & & DNT-330-06 \\
\hline GWM446012X & USGS-106 & 609 & Manganese & 0.34 & & $\bar{U}$ & & UG/L & $06 / 21 / 2006$ & & DNT-330-06 \\
\hline GWM446012X & USGS-106 & 609 & Nickel & 1 & & B & & UG/L & $06 / 21 / 2006$ & & DNT-330-06 \\
\hline GWM446012X & USGS-106 & 609 & Potassium & 2170 & & $\mathrm{E}$ & $\mathrm{J}$ & UG/L & $06 / 21 / 2006$ & & DNT-330-06 \\
\hline GWM446012X & USGS-106 & 609 & Selenium & 1 & & $U$ & & UG/L & $06 / 21 / 2006$ & & DNT-330-06 \\
\hline GWM446012X & USGS-106 & 609 & Silver & 0.2 & & $U$ & & UG/L & $06 / 21 / 2006$ & & DNT-330-06 \\
\hline GWM446012X & USGS-106 & 609 & Sodium & 7380 & & & & UG/L & $06 / 21 / 2006$ & & DNT-330-06 \\
\hline GWM446012X & USGS-106 & 609 & Strontium & 237 & & $\mathrm{E}$ & $\mathrm{J}$ & UG/L & $06 / 21 / 2006$ & & DNT-330-06 \\
\hline GWM446012X & USGS-106 & 609 & Thallium & 0.32 & & $\bar{U}$ & & UG/L & $06 / 21 / 2006$ & & DNT-330-06 \\
\hline GWM446012X & USGS-106 & 609 & Uranium & 1.6 & & & & UG/L & $06 / 21 / 2006$ & & DNT-330-06 \\
\hline GWM446012X & USGS-106 & 609 & Vanadium & 3.1 & & B & & UG/L & $06 / 21 / 2006$ & & DNT-330-06 \\
\hline GWM446012X & USGS-106 & 609 & Zinc & 102 & & & & UG/L & $06 / 21 / 2006$ & & DNT-330-06 \\
\hline GWM446012X & USGS-106 & 609 & Mercury & 0.093 & & U & & UG/L & $06 / 21 / 2006$ & & DNT-330-06 \\
\hline GWM44601A1 & USGS-106 & 609 & Total Alkalinity & 158 & & & & $M G / L$ & $06 / 21 / 2006$ & & DNT-282-06 \\
\hline GWM44601AN & USGS-106 & 609 & Chloride & 15.8 & & $\mathrm{~J}$ & $\mathrm{~J}$ & $M G / L$ & $06 / 21 / 2006$ & & DNT-282-06 \\
\hline GWM44601AN & USGS-106 & 609 & Fluoride & 0.17 & & & $\mathrm{~J}$ & $M G / L$ & $06 / 21 / 2006$ & & DNT-282-06 \\
\hline GWM44601VL & USGS-106 & 609 & Benzene & 1 & & u & & UG/L & $06 / 21 / 2006$ & & JGJ-066-06 \\
\hline GWM44601VL & USGS-106 & 609 & Bromodichloromethane & 1 & & $\mathrm{U}$ & & UG/L & $06 / 21 / 2006$ & & JGJ-066-06 \\
\hline GWM44601VL & USGS-106 & 609 & Bromoform & 1 & & U & & UG/L & $06 / 21 / 2006$ & & JGJ-066-06 \\
\hline GWM44601VL & USGS-106 & 609 & Bromomethane & 2 & & $\mathrm{U}$ & & UG/L & $06 / 21 / 2006$ & & JGJ-066-06 \\
\hline GWM44601VL & USGS-106 & 609 & 2-Butanone & 5 & & $\mathrm{U}$ & $\mathrm{R}$ & UG/L & $06 / 21 / 2006$ & & JGJ-066-06 \\
\hline GWM44601VL & USGS-106 & 609 & Carbon disulfide & 1 & & U & & UG/L & $06 / 21 / 2006$ & & JGJ-066-06 \\
\hline GWM44601VL & USGS-106 & 609 & Carbon tetrachloride & 1 & & $\mathrm{U}$ & & UG/L & $06 / 21 / 2006$ & & JGJ-066-06 \\
\hline GWM44601VL & USGS-106 & 609 & Chlorobenzene & 1 & & $\mathrm{U}$ & & UG/L & $06 / 21 / 2006$ & & JGJ-066-06 \\
\hline GWM44601VL & USGS-106 & 609 & Dibromochloromethane & 1 & & $U$ & & UG/L & $06 / 21 / 2006$ & & JGJ-066-06 \\
\hline GWM44601VL & USGS-106 & 609 & 1,2-Dibromo-3-chloropropane & 1 & & U & $\mathrm{R}$ & UG/L & $06 / 21 / 2006$ & & JGJ-066-06 \\
\hline GWM44601VL & USGS-106 & 609 & Chloroethane & 2 & & $U$ & & UG/L & $06 / 21 / 2006$ & & JGJ-066-06 \\
\hline GWM44601VL & USGS-106 & 609 & Chloroform & 1 & & U & & UG/L & $06 / 21 / 2006$ & & JGJ-066-06 \\
\hline GWM44601VL & USGS-106 & 609 & Chloromethane & 2 & & $\mathrm{U}$ & & UG/L & $06 / 21 / 2006$ & & JGJ-066-06 \\
\hline GWM44601VL & USGS-106 & 609 & Cyclohexane & 1 & & $\mathrm{u}$ & & UG/L & $06 / 21 / 2006$ & & JGJ-066-06 \\
\hline GWM44601VL & USGS-106 & 609 & 1,2-Dibromoethane & 1 & & $\mathrm{U}$ & & UG/L & $06 / 21 / 2006$ & & JGJ-066-06 \\
\hline GWM44601VL & USGS-106 & 609 & 1,2-Dichlorobenzene & 1 & & U & & UG/L & $06 / 21 / 2006$ & & JGJ-066-06 \\
\hline GWM44601VL & USGS-106 & 609 & 1,3-Dichlorobenzene & 1 & & $\mathrm{U}$ & & UG/L & $06 / 21 / 2006$ & & JGJ-066-06 \\
\hline GWM44601VL & USGS-106 & 609 & 1,4-Dichlorobenzene & 1 & & $\mathrm{U}$ & & UG/L & $06 / 21 / 2006$ & & JGJ-066-06 \\
\hline GWM44601VL & USGS-106 & 609 & Dichlorodifluoromethane & 2 & & $U$ & & UG/L & $06 / 21 / 2006$ & & JGJ-066-06 \\
\hline GWM44601VL & USGS-106 & 609 & 1,1-Dichloroethane & 1 & & $\mathrm{U}$ & & UG/L & $06 / 21 / 2006$ & & JGJ-066-06 \\
\hline GWM44601VL & USGS-106 & 609 & 1,2-Dichloroethane & 1 & & $\mathrm{U}$ & & UG/L & $06 / 21 / 2006$ & & JGJ-066-06 \\
\hline GWM44601VL & USGS-106 & 609 & 1,1-Dichloroethene & 1 & & $\mathrm{U}$ & & UG/L & $06 / 21 / 2006$ & & JGJ-066-06 \\
\hline GWM44601VL & USGS-106 & 609 & cis-1,2-Dichloroethene & 1 & & $\mathrm{U}$ & & UG/L & $06 / 21 / 2006$ & & JGJ-066-06 \\
\hline GWM44601VL & USGS-106 & 609 & trans-1,2-Dichloroethene & 1 & & $\mathrm{U}$ & & UG/L & $06 / 21 / 2006$ & & JGJ-066-06 \\
\hline GWM44601VL & USGS-106 & 609 & 1,2-Dichloropropane & 1 & & U & & UG/L & $06 / 21 / 2006$ & & JGJ-066-06 \\
\hline GWM44601VL & USGS-106 & 609 & cis-1,3-Dichloropropene & 1 & & $U$ & & UG/L & $06 / 21 / 2006$ & & JGJ-066-06 \\
\hline GWM44601VL & USGS-106 & 609 & trans-1,3-Dichloropropene & 1 & & $\mathrm{U}$ & & UG/L & $06 / 21 / 2006$ & & JGJ-066-06 \\
\hline GWM44601VL & USGS-106 & 609 & Ethylbenzene & 1 & & U & & UG/L & $06 / 21 / 2006$ & & JGJ-066-06 \\
\hline GWM44601VL & USGS-106 & 609 & Trichlorofluoromethane & 1 & & $U$ & & UG/L & $06 / 21 / 2006$ & & JGJ-066-06 \\
\hline GWM44601VL & USGS-106 & 609 & 2-Hexanone & 5 & & $\mathrm{U}$ & $\mathrm{R}$ & UG/L & $06 / 21 / 2006$ & & JGJ-066-06 \\
\hline GWM44601VL & USGS-106 & 609 & Isopropylbenzene & 1 & & U & & UG/L & $06 / 21 / 2006$ & & JGJ-066-06 \\
\hline GWM44601VL & USGS-106 & 609 & Methyl acetate & 5 & & $U$ & $\mathrm{R}$ & UG/L & $06 / 21 / 2006$ & & JGJ-066-06 \\
\hline GWM44601VL & USGS-106 & 609 & Methyl cyclohexane & 4 & & $U$ & & UG/L & $06 / 21 / 2006$ & & JGJ-066-06 \\
\hline GWM44601VL & USGS-106 & 609 & Methylene Chloride & 1 & & $\mathrm{U}$ & & UG/L & $06 / 21 / 2006$ & & JGJ-066-06 \\
\hline GWM44601VL & USGS-106 & 609 & 4-Methyl-2-pentanone & 5 & & U & & UG/L & $06 / 21 / 2006$ & & JGJ-066-06 \\
\hline
\end{tabular}


Table A-2. (continued).

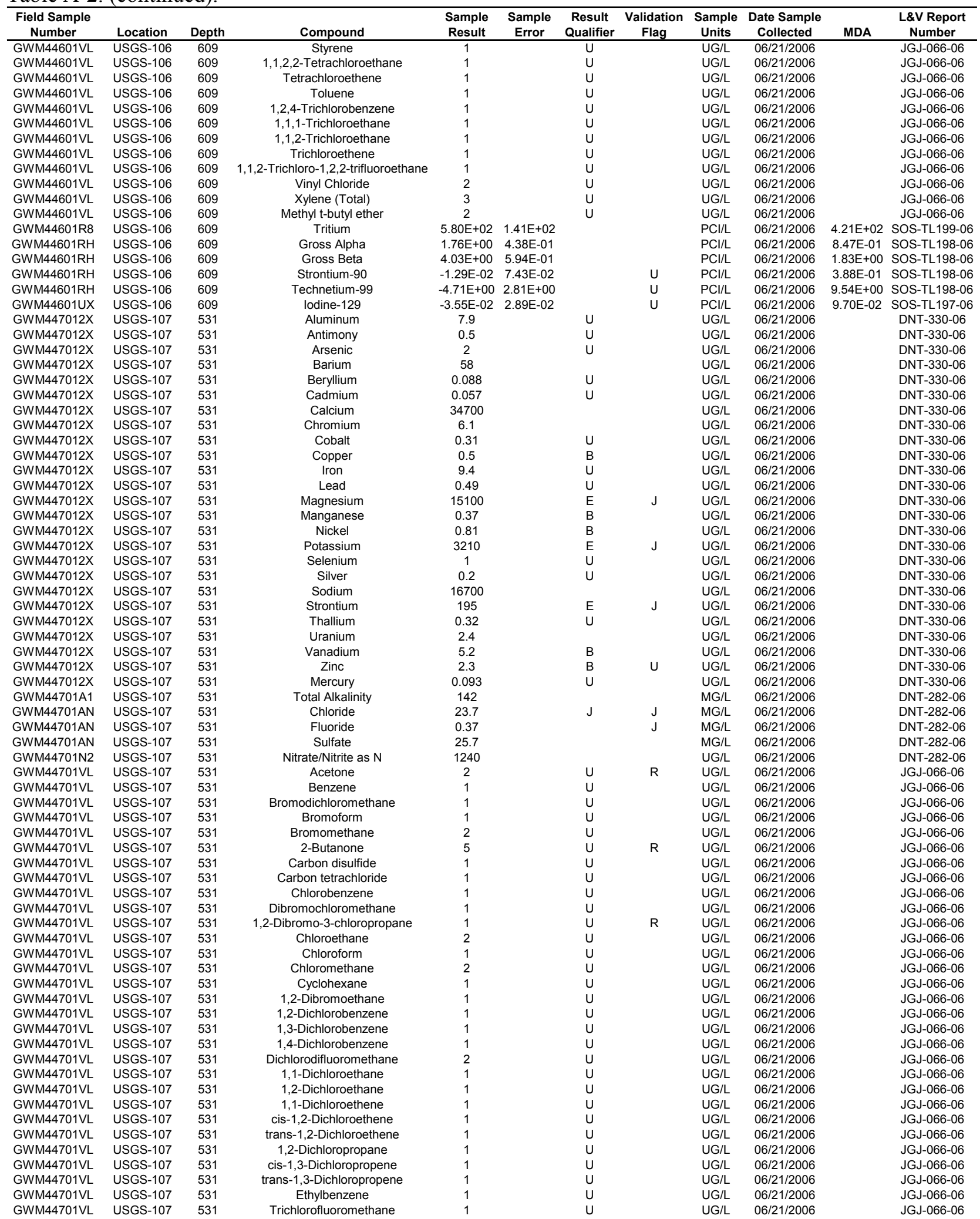


Table A-2. (continued).

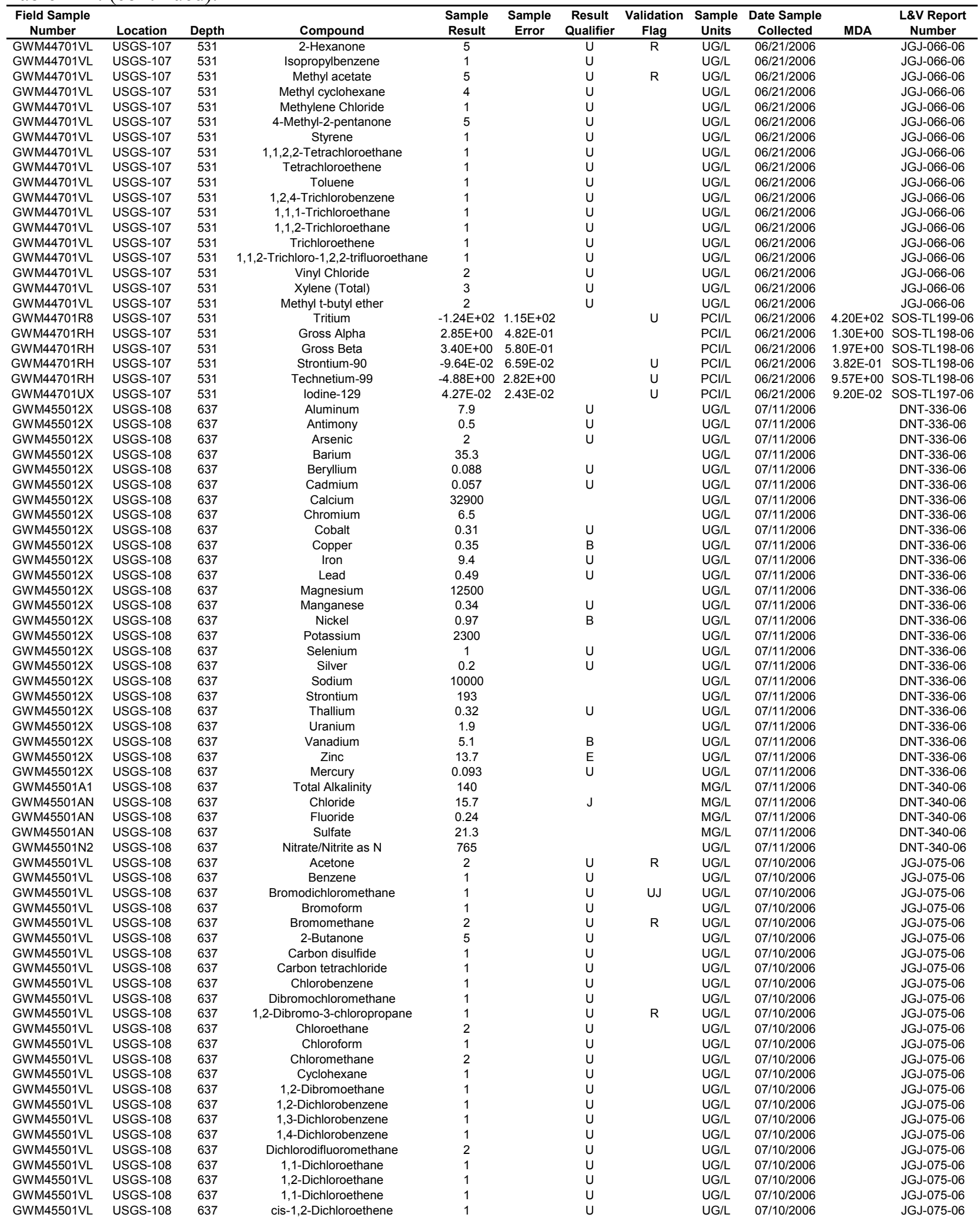


Table A-2. (continued).

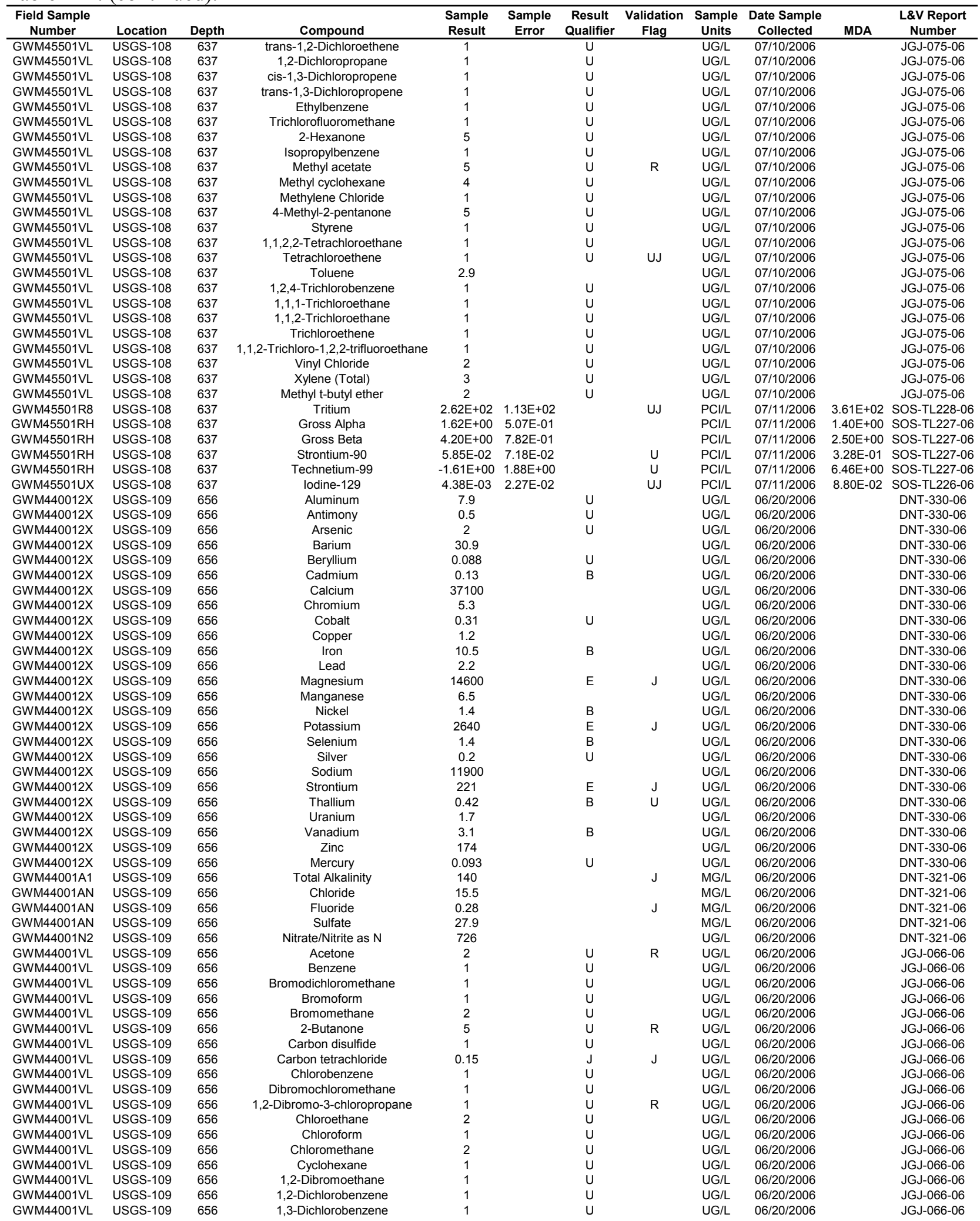


Table A-2. (continued).

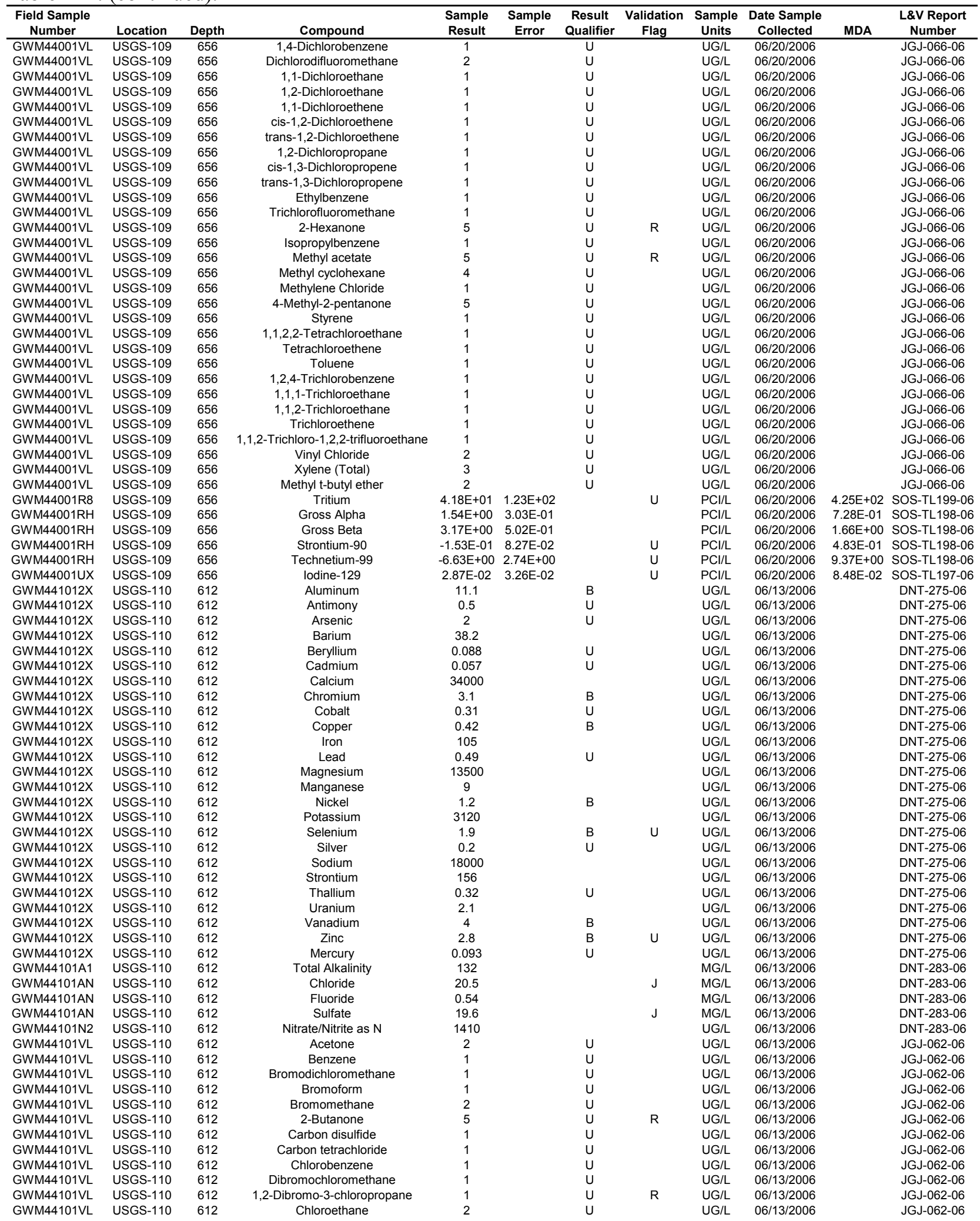


Table A-2. (continued).

\begin{tabular}{|c|c|c|c|c|c|c|c|c|c|c|c|}
\hline $\begin{array}{c}\text { Field Sample } \\
\text { Number }\end{array}$ & Location & Depth & Compound & $\begin{array}{c}\text { Sample } \\
\text { Result }\end{array}$ & $\begin{array}{l}\text { Sample } \\
\text { Error }\end{array}$ & $\begin{array}{c}\text { Result } \\
\text { Qualifier }\end{array}$ & $\begin{array}{c}\text { Validation } \\
\text { Flag }\end{array}$ & $\begin{array}{c}\text { Sample } \\
\text { Units }\end{array}$ & $\begin{array}{c}\text { Date Sample } \\
\text { Collected }\end{array}$ & MDA & $\begin{array}{c}\text { L\&V Report } \\
\text { Number }\end{array}$ \\
\hline GWM44101VL & USGS-110 & 612 & Chloroform & 1 & & $\mathrm{U}$ & & UG/L & $06 / 13 / 2006$ & & JGJ-062-06 \\
\hline GWM44101VL & USGS-110 & 612 & Chloromethane & 2 & & U & & UG/L & $06 / 13 / 2006$ & & JGJ-062-06 \\
\hline GWM44101VL & USGS-110 & 612 & Cyclohexane & 1 & & $\mathrm{U}$ & & UG/L & $06 / 13 / 2006$ & & JGJ-062-06 \\
\hline GWM44101VL & USGS-110 & 612 & 1,2-Dibromoethane & 1 & & U & & UG/L & $06 / 13 / 2006$ & & JGJ-062-06 \\
\hline GWM44101VL & USGS-110 & 612 & 1,2-Dichlorobenzene & 1 & & U & & UG/L & $06 / 13 / 2006$ & & JGJ-062-06 \\
\hline GWM44101VL & USGS-110 & 612 & 1,3-Dichlorobenzene & 1 & & $\mathrm{U}$ & & UG/L & $06 / 13 / 2006$ & & JGJ-062-06 \\
\hline GWM44101VL & USGS-110 & 612 & 1,4-Dichlorobenzene & 1 & & $U$ & & UG/L & $06 / 13 / 2006$ & & JGJ-062-06 \\
\hline GWM44101VL & USGS-110 & 612 & Dichlorodifluoromethane & 2 & & U & & UG/L & $06 / 13 / 2006$ & & JGJ-062-06 \\
\hline GWM44101VL & USGS-110 & 612 & 1,2-Dichloroethane & 1 & & $\mathrm{U}$ & & UG/L & $06 / 13 / 2006$ & & JGJ-062-06 \\
\hline GWM44101VL & USGS-110 & 612 & 1,1-Dichloroethene & 1 & & U & & UG/L & $06 / 13 / 2006$ & & JGJ-062-06 \\
\hline GWM44101VL & USGS-110 & 612 & cis-1,2-Dichloroethene & 1 & & $U$ & & UG/L & $06 / 13 / 2006$ & & JGJ-062-06 \\
\hline GWM44101VL & USGS-110 & 612 & trans-1,2-Dichloroethene & 1 & & $\mathrm{U}$ & & UG/L & $06 / 13 / 2006$ & & JGJ-062-06 \\
\hline GWM44101VL & USGS-110 & 612 & 1,2-Dichloropropane & 1 & & U & & UG/L & $06 / 13 / 2006$ & & JGJ-062-06 \\
\hline GWM44101VL & USGS-110 & 612 & cis-1,3-Dichloropropene & 1 & & U & & UG/L & $06 / 13 / 2006$ & & JGJ-062-06 \\
\hline GWM44101VL & USGS-110 & 612 & trans-1,3-Dichloropropene & 1 & & $\mathrm{U}$ & & UG/L & $06 / 13 / 2006$ & & JGJ-062-06 \\
\hline GWM44101VL & USGS-110 & 612 & Ethylbenzene & 1 & & $\mathrm{U}$ & & UG/L & $06 / 13 / 2006$ & & JGJ-062-06 \\
\hline GWM44101VL & USGS-110 & 612 & Trichlorofluoromethane & 1 & & U & & UG/L & $06 / 13 / 2006$ & & JGJ-062-06 \\
\hline GWM44101VL & USGS-110 & 612 & 2-Hexanone & 5 & & U & & UG/L & $06 / 13 / 2006$ & & JGJ-062-06 \\
\hline GWM44101VL & USGS-110 & 612 & Methyl acetate & 5 & & U & & UG/L & $06 / 13 / 2006$ & & JGJ-062-06 \\
\hline GWM44101VL & USGS-110 & 612 & Methyl cyclohexane & 4 & & U & & UG/L & $06 / 13 / 2006$ & & JGJ-062-06 \\
\hline GWM44101VL & USGS-110 & 612 & Methylene Chloride & 1 & & $\mathrm{U}$ & & UG/L & $06 / 13 / 2006$ & & JGJ-062-06 \\
\hline GWM44101VL & USGS-110 & 612 & 4-Methyl-2-pentanone & 5 & & $\mathrm{U}$ & & UG/L & $06 / 13 / 2006$ & & JGJ-062-06 \\
\hline GWM44101VL & USGS-110 & 612 & Styrene & 1 & & U & & UG/L & $06 / 13 / 2006$ & & JGJ-062-06 \\
\hline GWM44101VL & USGS-110 & 612 & 1,1,2,2-Tetrachloroethane & 1 & & $U$ & & UG/L & $06 / 13 / 2006$ & & JGJ-062-06 \\
\hline GWM44101VL & USGS-110 & 612 & Tetrachloroethene & 1 & & $\mathrm{U}$ & & UG/L & $06 / 13 / 2006$ & & JGJ-062-06 \\
\hline GWM44101VL & USGS-110 & 612 & Toluene & 4.3 & & B & & UG/L & $06 / 13 / 2006$ & & JGJ-062-06 \\
\hline GWM44101VL & USGS-110 & 612 & 1,2,4-Trichlorobenzene & 1 & & $U$ & & UG/L & $06 / 13 / 2006$ & & JGJ-062-06 \\
\hline GWM44101VL & USGS-110 & 612 & 1,1,1-Trichloroethane & 1 & & $\mathrm{U}$ & & UG/L & $06 / 13 / 2006$ & & JGJ-062-06 \\
\hline GWM44101VL & USGS-110 & 612 & 1,1,2-Trichloroethane & 1 & & $\mathrm{U}$ & & UG/L & $06 / 13 / 2006$ & & JGJ-062-06 \\
\hline GWM44101VL & USGS-110 & 612 & Trichloroethene & 1 & & U & & UG/L & $06 / 13 / 2006$ & & JGJ-062-06 \\
\hline GWM44101VL & USGS-110 & 612 & 1,1,2-Trichloro-1,2,2-trifluoroethane & 1 & & $\mathrm{U}$ & & UG/L & $06 / 13 / 2006$ & & JGJ-062-06 \\
\hline GWM44101VL & USGS-110 & 612 & Vinyl Chloride & 2 & & $\mathrm{U}$ & & UG/L & $06 / 13 / 2006$ & & JGJ-062-06 \\
\hline GWM44101VL & USGS-110 & 612 & Xylene (Total) & 3 & & $\mathrm{U}$ & & UG/L & $06 / 13 / 2006$ & & JGJ-062-06 \\
\hline GWM44101VL & USGS-110 & 612 & Methyl t-butyl ether & 2 & & $U$ & & UG/L & $06 / 13 / 2006$ & & JGJ-062-06 \\
\hline GWM44101R8 & USGS-110 & 612 & Tritium & $-4.34 \mathrm{E}+01$ & $1.25 \mathrm{E}+02$ & & $\mathrm{U}$ & $\mathrm{PCl} / \mathrm{L}$ & $06 / 13 / 2006$ & $4.41 \mathrm{E}+02$ & SOS-TL199-06 \\
\hline GWM44101RH & USGS-110 & 612 & Gross Alpha & $2.21 \mathrm{E}+00$ & $3.75 \mathrm{E}-01$ & & & $\mathrm{PCl} / \mathrm{L}$ & $06 / 13 / 2006$ & 7.76E-01 & SOS-TL198-06 \\
\hline GWM44101RH & USGS-110 & 612 & Gross Beta & $3.39 \mathrm{E}+00$ & $5.35 \mathrm{E}-01$ & & & $\mathrm{PCl} / \mathrm{L}$ & $06 / 13 / 2006$ & $1.65 \mathrm{E}+00$ & SOS-TL198-06 \\
\hline GWM44101RH & USGS-110 & 612 & Strontium-90 & $-7.43 E-02$ & 9.53E-02 & & $\mathrm{U}$ & $\mathrm{PCl} / \mathrm{L}$ & $06 / 13 / 2006$ & 4.93E-01 & SOS-TL198-06 \\
\hline GWM450012X & USGS-124 & 770 & Antimony & 0.5 & & $\mathrm{U}$ & & UG/L & $06 / 26 / 2006$ & & DNT-336-06 \\
\hline GWM450012X & USGS-124 & 770 & Arsenic & 2 & & U & & UG/L & $06 / 26 / 2006$ & & DNT-336-06 \\
\hline GWM450012X & USGS-124 & 770 & Barium & 26.6 & & & & UG/L & $06 / 26 / 2006$ & & DNT-336-06 \\
\hline GWM450012X & USGS-124 & 770 & Beryllium & 0.088 & & $U$ & & UG/L & $06 / 26 / 2006$ & & DNT-336-06 \\
\hline GWM450012X & USGS-124 & 770 & Cadmium & 0.057 & & U & & UG/L & $06 / 26 / 2006$ & & DNT-336-06 \\
\hline GWM450012X & USGS-124 & 770 & Calcium & 35400 & & & & UG/L & $06 / 26 / 2006$ & & DNT-336-06 \\
\hline GWM450012X & USGS-124 & 770 & Chromium & 5.9 & & & & UG/L & $06 / 26 / 2006$ & & DNT-336-06 \\
\hline GWM450012X & USGS-124 & 770 & Cobalt & 0.31 & & U & & UG/L & $06 / 26 / 2006$ & & DNT-336-06 \\
\hline GWM450012X & USGS-124 & 770 & Copper & 0.9 & & B & & UG/L & $06 / 26 / 2006$ & & DNT-336-06 \\
\hline GWM450012X & USGS-124 & 770 & Iron & 9.4 & & $U$ & & UG/L & 06/26/2006 & & DNT-336-06 \\
\hline GWM450012X & USGS-124 & 770 & Lead & 0.49 & & $U$ & & UG/L & $06 / 26 / 2006$ & & DNT-336-06 \\
\hline GWM450012X & USGS-124 & 770 & Magnesium & 13800 & & & & UG/L & $06 / 26 / 2006$ & & DNT-336-06 \\
\hline GWM450012X & USGS-124 & 770 & Manganese & 1.3 & & B & & UG/L & $06 / 26 / 2006$ & & DNT-336-06 \\
\hline GWM450012X & USGS-124 & 770 & Nickel & 1.2 & & B & & UG/L & $06 / 26 / 2006$ & & DNT-336-06 \\
\hline GWM450012X & USGS-124 & 770 & Potassium & 2110 & & & & UG/L & $06 / 26 / 2006$ & & DNT-336-06 \\
\hline GWM450012X & USGS-124 & 770 & Selenium & 1.1 & & B & & UG/L & $06 / 26 / 2006$ & & DNT-336-06 \\
\hline GWM450012X & USGS-124 & 770 & Silver & 0.2 & & $U$ & & UG/L & $06 / 26 / 2006$ & & DNT-336-06 \\
\hline GWM450012X & USGS-124 & 770 & Sodium & 8430 & & & & UG/L & $06 / 26 / 2006$ & & DNT-336-06 \\
\hline GWM450012X & USGS-124 & 770 & Strontium & 195 & & & & UG/L & $06 / 26 / 2006$ & & DNT-336-06 \\
\hline GWM450012X & USGS-124 & 770 & Thallium & 0.36 & & B & $U$ & UG/L & $06 / 26 / 2006$ & & DNT-336-06 \\
\hline GWM450012X & USGS-124 & 770 & Uranium & 1.7 & & & & UG/L & $06 / 26 / 2006$ & & DNT-336-06 \\
\hline GWM450012X & USGS-124 & 770 & Vanadium & 3.9 & & B & & UG/L & $06 / 26 / 2006$ & & DNT-336-06 \\
\hline GWM450012X & USGS-124 & 770 & Zinc & 2.4 & & $\mathrm{BE}$ & $U$ & UG/L & $06 / 26 / 2006$ & & DNT-336-06 \\
\hline GWM450012X & USGS-124 & 770 & Mercury & 0.093 & & $U$ & & UG/L & $06 / 26 / 2006$ & & DNT-336-06 \\
\hline GWM45001A1 & USGS-124 & 770 & Total Alkalinity & 144 & & & & $M G / L$ & $06 / 26 / 2006$ & & DNT-322-06 \\
\hline GWM45001AN & USGS-124 & 770 & Chloride & 15.4 & & $\mathrm{~J}$ & $\mathrm{~J}$ & $M G / L$ & $06 / 26 / 2006$ & & DNT-322-06 \\
\hline GWM45001AN & USGS-124 & 770 & Fluoride & 0.45 & & & & $M G / L$ & $06 / 26 / 2006$ & & DNT-322-06 \\
\hline GWM45001AN & USGS-124 & 770 & Sulfate & 21.4 & & & & $M G / L$ & $06 / 26 / 2006$ & & DNT-322-06 \\
\hline GWM45001N2 & USGS-124 & 770 & Nitrate/Nitrite as $\mathrm{N}$ & 783 & & & & UG/L & $06 / 26 / 2006$ & & DNT-322-06 \\
\hline GWM45001VL & USGS-124 & 770 & Acetone & 2 & & U & $\mathrm{R}$ & UG/L & $06 / 26 / 2006$ & & JGJ-067-06 \\
\hline GWM45001VL & USGS-124 & 770 & Benzene & 1 & & $U$ & & UG/L & $06 / 26 / 2006$ & & JGJ-067-06 \\
\hline GWM45001VL & USGS-124 & 770 & Bromodichloromethane & 1 & & $\mathrm{U}$ & & UG/L & $06 / 26 / 2006$ & & JGJ-067-06 \\
\hline GWM45001VL & USGS-124 & 770 & Bromoform & 1 & & $\mathrm{U}$ & & UG/L & $06 / 26 / 2006$ & & JGJ-067-06 \\
\hline GWM45001VL & USGS-124 & 770 & Bromomethane & 2 & & U & & UG/L & $06 / 26 / 2006$ & & JGJ-067-06 \\
\hline GWM45001VL & USGS-124 & 770 & 2-Butanone & 5 & & $\mathrm{U}$ & $\mathrm{R}$ & UG/L & $06 / 26 / 2006$ & & JGJ-067-06 \\
\hline
\end{tabular}


Table A-2. (continued).

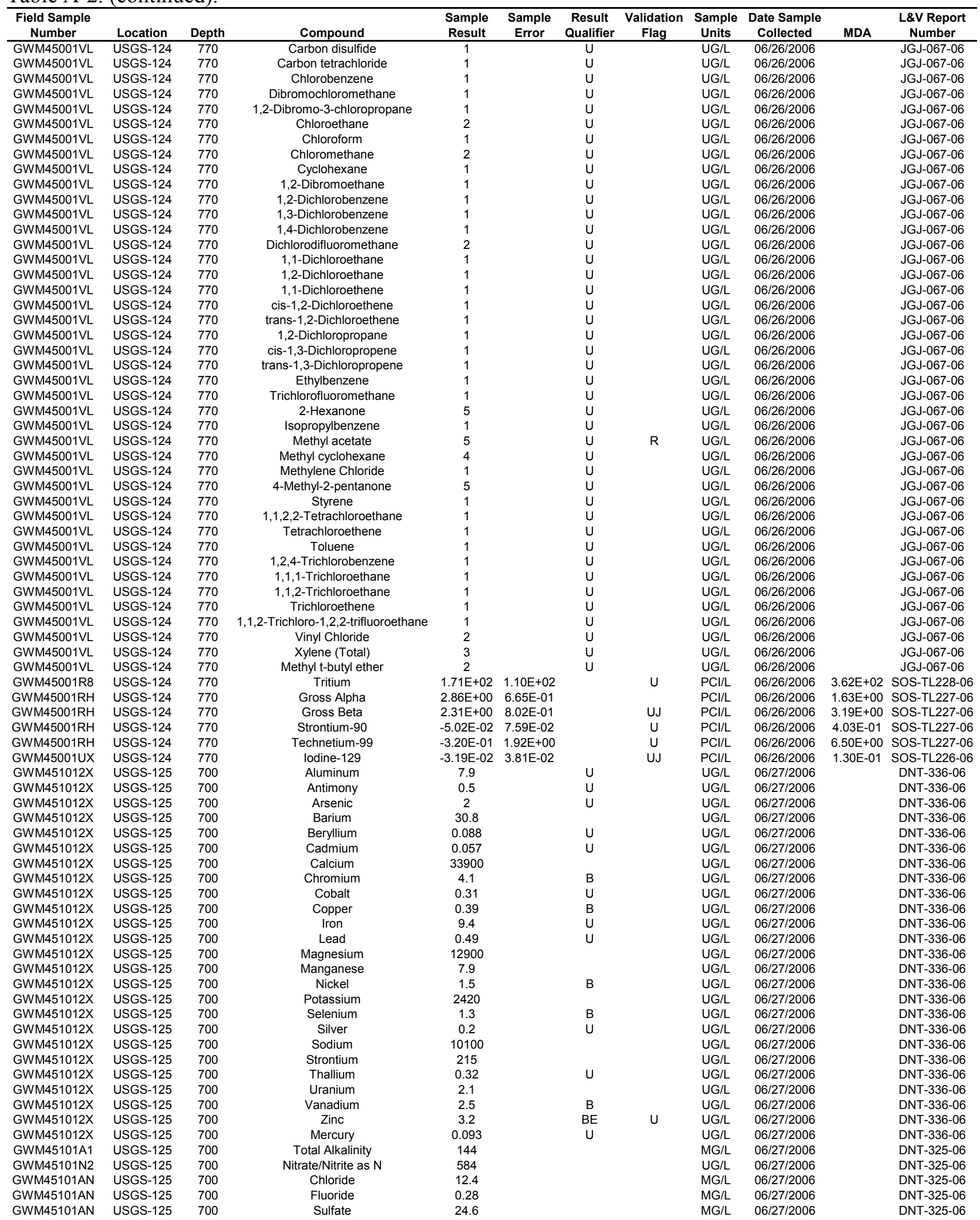


Table A-2. (continued).

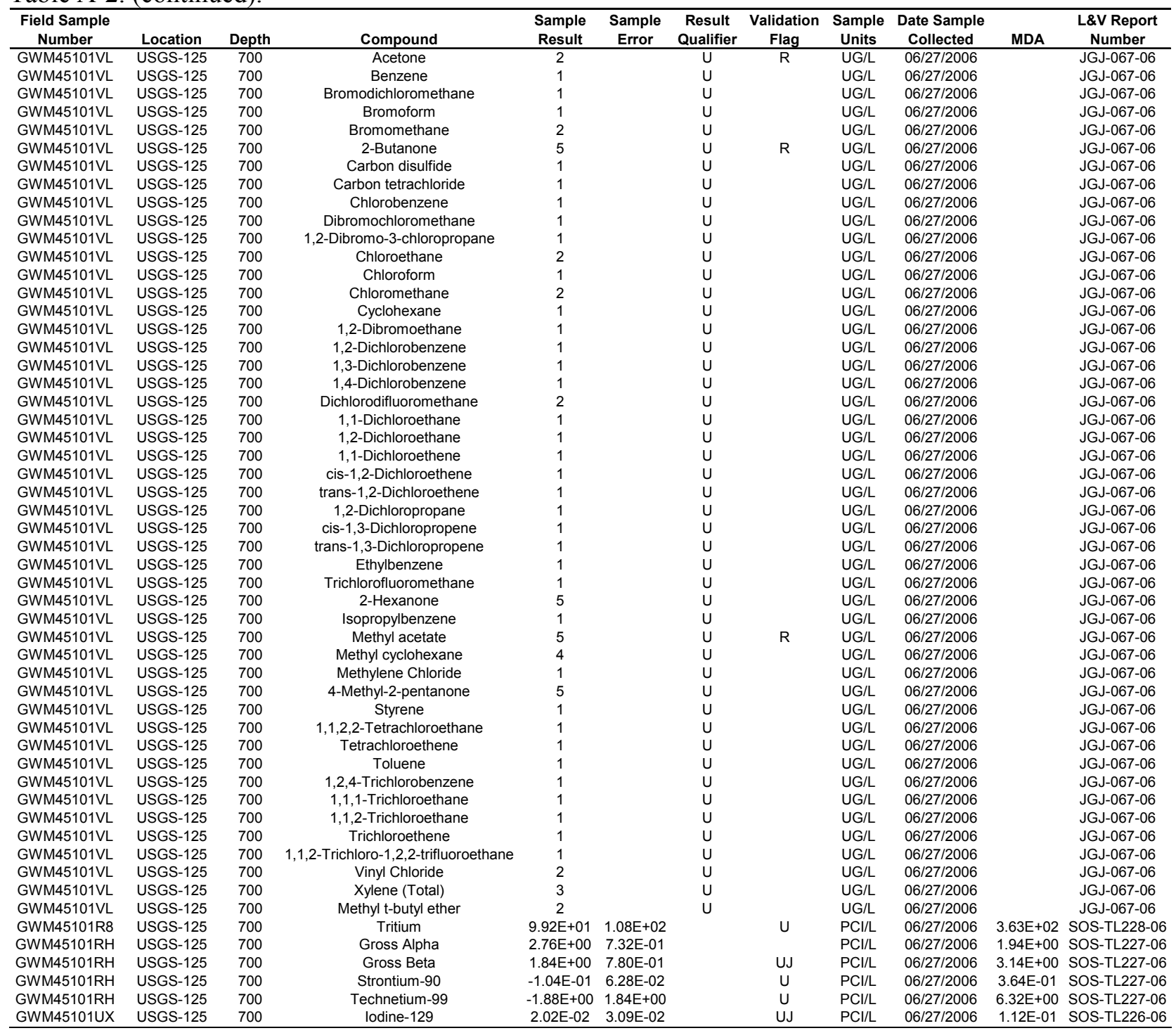


Table A-3. Waste Area Group 10 groundwater packer sampling data.

\begin{tabular}{|c|c|c|c|c|c|c|c|c|c|c|c|}
\hline $\begin{array}{l}\text { Field Sample } \\
\text { Number }\end{array}$ & Location & Depth & Compound & $\begin{array}{l}\text { Sample } \\
\text { Result }\end{array}$ & $\begin{array}{c}\text { Sample } \\
\text { Error }\end{array}$ & $\begin{array}{c}\text { Result } \\
\text { Qualifier }\end{array}$ & $\begin{array}{c}\text { Validation } \\
\text { Flag }\end{array}$ & $\begin{array}{c}\text { Sample } \\
\text { Units }\end{array}$ & $\begin{array}{c}\text { Date Sample } \\
\text { Collected }\end{array}$ & MDA & $\begin{array}{l}\text { L\&V Report } \\
\text { Number }\end{array}$ \\
\hline GWM459012X & FIELD BLANK & NA & Aluminum & 7.9 & & $\mathrm{U}$ & & UG/L & $07 / 11 / 2006$ & & DNT-337-06 \\
\hline GWM459012X & FIELD BLANK & NA & Antimony & 0.5 & & U & & UG/L & $07 / 11 / 2006$ & & DNT-337-06 \\
\hline GWM459012X & FIELD BLANK & NA & Arsenic & 2 & & u & & UG/L & $07 / 11 / 2006$ & & DNT-337-06 \\
\hline GWM459012X & FIELD BLANK & NA & Barium & 0.25 & & U & & UG/L & $07 / 11 / 2006$ & & DNT-337-06 \\
\hline GWM459012X & FIELD BLANK & NA & Beryllium & 0.088 & & U & & UG/L & $07 / 11 / 2006$ & & DNT-337-06 \\
\hline GWM459012X & FIELD BLANK & NA & Cadmium & 0.057 & & U & & UG/L & $07 / 11 / 2006$ & & DNT-337-06 \\
\hline GWM459012X & FIELD BLANK & NA & Calcium & 21 & & U & & UG/L & $07 / 11 / 2006$ & & DNT-337-06 \\
\hline GWM459012X & FIELD BLANK & NA & Chromium & 2.8 & & U & & UG/L & $07 / 11 / 2006$ & & DNT-337-06 \\
\hline GWM459012X & FIELD BLANK & NA & Cobalt & 0.31 & & U & & UG/L & $07 / 11 / 2006$ & & DNT-337-06 \\
\hline GWM459012X & FIELD BLANK & NA & Iron & 9.4 & & u & & UG/L & $07 / 11 / 2006$ & & DNT-337-06 \\
\hline GWM459012X & FIELD BLANK & NA & Lead & 0.49 & & U & & UG/L & $07 / 11 / 2006$ & & DNT-337-06 \\
\hline GWM459012X & FIELD BLANK & NA & Magnesium & 6.4 & & U & & UG/L & $07 / 11 / 2006$ & & DNT-337-06 \\
\hline GWM459012X & FIELD BLANK & NA & Manganese & 0.59 & & B & & UG/L & $07 / 11 / 2006$ & & DNT-337-06 \\
\hline GWM459012X & FIELD BLANK & NA & Nickel & 0.52 & & $u$ & & UG/L & $07 / 11 / 2006$ & & DNT-337-06 \\
\hline GWM459012X & FIELD BLANK & NA & Potassium & 10 & & $\mathrm{U}$ & & UG/L & $07 / 11 / 2006$ & & DNT-337-06 \\
\hline GWM459012X & FIELD BLANK & NA & Selenium & 1 & & U & & UG/L & $07 / 11 / 2006$ & & DNT-337-06 \\
\hline GWM459012X & FIELD BLANK & NA & Silver & 0.2 & & U & & UG/L & $07 / 11 / 2006$ & & DNT-337-06 \\
\hline GWM459012X & FIELD BLANK & NA & Sodium & 11 & & U & & UG/L & $07 / 11 / 2006$ & & DNT-337-06 \\
\hline GWM459012X & FIELD BLANK & NA & Strontium & 0.53 & & $u$ & & UG/L & $07 / 11 / 2006$ & & DNT-337-06 \\
\hline GWM459012X & FIELD BLANK & NA & Vanadium & 1.6 & & U & & UG/L & $07 / 11 / 2006$ & & DNT-337-06 \\
\hline GWM459012X & FIELD BLANK & NA & Zinc & 4.8 & & $\mathrm{BE}$ & U & UG/L & $07 / 11 / 2006$ & & DNT-337-06 \\
\hline GWM459012X & FIELD BLANK & NA & Mercury & 0.093 & & $\mathrm{U}$ & UJ & UG/L & $07 / 11 / 2006$ & & DNT-337-06 \\
\hline GWM45901A1 & FIELD BLANK & NA & Alkalinity & 5 & & U & UJ & MG/L & $07 / 11 / 2006$ & & DNT-341-06 \\
\hline GWM45901AN & FIELD BLANK & NA & Chloride & 0.075 & & $J B$ & u & $\mathrm{MG} / \mathrm{L}$ & $07 / 11 / 2006$ & & DNT-341-06 \\
\hline GWM45901AN & FIELD BLANK & NA & Fluoride & 0.1 & & U & & MG/L & $07 / 11 / 2006$ & & DNT-341-06 \\
\hline GWM45901AN & FIELD BLANK & NA & Sulfate & 0.5 & & $\mathrm{U}$ & & $\mathrm{MG} / \mathrm{L}$ & $07 / 11 / 2006$ & & DNT-341-06 \\
\hline GWM45901N2 & FIELD BLANK & NA & Nitrate/Nitrite as $\mathrm{N}$ & 50 & & u & & UG/L & $07 / 11 / 2006$ & & DNT-341-06 \\
\hline GWM45901VL & FIELD BLANK & NA & Acetone & 2 & & u & $\mathrm{R}$ & UG/L & $07 / 11 / 2006$ & & JGJ-076-06 \\
\hline GWM45901VL & FIELD BLANK & NA & Benzene & 1 & & u & & UG/L & $07 / 11 / 2006$ & & JGJ-076-06 \\
\hline GWM45901VL & FIELD BLANK & NA & Bromodichloromethane & 1 & & U & & UG/L & $07 / 11 / 2006$ & & JGJ-076-06 \\
\hline GWM45901VL & FIELD BLANK & NA & Bromoform & 1 & & u & UJ & UG/L & $07 / 11 / 2006$ & & JGJ-076-06 \\
\hline GWM45901VL & FIELD BLANK & NA & Bromomethane & 2 & & $\mathrm{u}$ & & UG/L & $07 / 11 / 2006$ & & JGJ-076-06 \\
\hline GWM45901VL & FIELD BLANK & NA & 2-Butanone & 5 & & $\mathrm{u}$ & $\mathrm{R}$ & UG/L & $07 / 11 / 2006$ & & JGJ-076-06 \\
\hline GWM45901VL & FIELD BLANK & NA & Carbon disulfide & 1 & & $\mathrm{u}$ & & UG/L & $07 / 11 / 2006$ & & JGJ-076-06 \\
\hline GWM45901VL & FIELD BLANK & NA & Carbon tetrachloride & 1 & & u & & UG/L & $07 / 11 / 2006$ & & JGJ-076-06 \\
\hline GWM45901VL & FIELD BLANK & NA & Chlorobenzene & 1 & & $\mathrm{u}$ & & UG/L & $07 / 11 / 2006$ & & JGJ-076-06 \\
\hline GWM45901VL & FIELD BLANK & NA & Dibromochloromethane & 1 & & $u$ & & UG/L & $07 / 11 / 2006$ & & JGJ-076-06 \\
\hline GWM45901VL & FIELD BLANK & NA & 1,2-Dibromo-3-chloropropane & 1 & & $\mathrm{u}$ & $\mathrm{R}$ & UG/L & $07 / 11 / 2006$ & & JGJ-076-06 \\
\hline GWM45901VL & FIELD BLANK & NA & Chloroethane & 2 & & $\mathrm{u}$ & & UG/L & $07 / 11 / 2006$ & & JGJ-076-06 \\
\hline GWM45901VL & FIELD BLANK & NA & 1,2-Dibromoethane & 1 & & $u$ & & UG/L & $07 / 11 / 2006$ & & JGJ-076-06 \\
\hline GWM45901VL & FIELD BLANK & NA & 1,2-Dichlorobenzene & 1 & & $\mathrm{u}$ & & UG/L & $07 / 11 / 2006$ & & JGJ-076-06 \\
\hline GWM45901VL & FIELD BLANK & NA & 1,3-Dichlorobenzene & 1 & & $\mathrm{u}$ & & UG/L & $07 / 11 / 2006$ & & JGJ-076-06 \\
\hline GWM45901VL & FIELD BLANK & NA & 1,4-Dichlorobenzene & 1 & & $\mathrm{u}$ & & UG/L & $07 / 11 / 2006$ & & JGJ-076-06 \\
\hline GWM45901VL & FIELD BLANK & NA & Dichlorodifluoromethane & 2 & & $\mathrm{U}$ & & UG/L & $07 / 11 / 2006$ & & JGJ-076-06 \\
\hline GWM45901VL & FIELD BLANK & NA & 1,1-Dichloroethane & 1 & & $\mathrm{u}$ & & UG/L & $07 / 11 / 2006$ & & JGJ-076-06 \\
\hline GWM45901VL & FIELD BLANK & NA & 1,2-Dichloroethane & 1 & & $\mathrm{u}$ & & UG/L & $07 / 11 / 2006$ & & JGJ-076-06 \\
\hline GWM45901VL & FIELD BLANK & NA & 1,1-Dichloroethene & 1 & & $\mathrm{u}$ & & UG/L & $07 / 11 / 2006$ & & JGJ-076-06 \\
\hline GWM45901VL & FIELD BLANK & NA & cis-1,2-Dichloroethene & 1 & & $\mathrm{u}$ & & UG/L & $07 / 11 / 2006$ & & JGJ-076-06 \\
\hline GWM45901VL & FIELD BLANK & NA & trans-1,2-Dichloroethene & 1 & & $\mathrm{u}$ & & UG/L & $07 / 11 / 2006$ & & JGJ-076-06 \\
\hline GWM45901VL & FIELD BLANK & NA & 1,2-Dichloropropane & 1 & & $\mathrm{u}$ & & UG/L & $07 / 11 / 2006$ & & JGJ-076-06 \\
\hline GWM45901VL & FIELD BLANK & NA & cis-1,3-Dichloropropene & 1 & & U & & UG/L & $07 / 11 / 2006$ & & JGJ-076-06 \\
\hline GWM45901VL & FIELD BLANK & NA & trans-1,3-Dichloropropene & 1 & & $\mathrm{U}$ & & UG/L & $07 / 11 / 2006$ & & JGJ-076-06 \\
\hline GWM45901VL & FIELD BLANK & NA & Ethylbenzene & 1 & & u & & UG/L & $07 / 11 / 2006$ & & JGJ-076-06 \\
\hline GWM45901VL & FIELD BLANK & NA & Trichlorofluoromethane & 1 & & u & & UG/L & $07 / 11 / 2006$ & & JGJ-076-06 \\
\hline GWM45901VL & FIELD BLANK & NA & 2-Hexanone & 5 & & u & & UG/L & $07 / 11 / 2006$ & & JGJ-076-06 \\
\hline GWM45901VL & FIELD BLANK & NA & Isopropylbenzene & 1 & & $\mathrm{u}$ & & UG/L & $07 / 11 / 2006$ & & JGJ-076-06 \\
\hline GWM45901VL & FIELD BLANK & NA & Methyl acetate & 5 & & u & $\mathrm{R}$ & UG/L & $07 / 11 / 2006$ & & JGJ-076-06 \\
\hline GWM45901VL & FIELD BLANK & NA & Methyl cyclohexane & 4 & & $\mathrm{U}$ & & UG/L & $07 / 11 / 2006$ & & JGJ-076-06 \\
\hline GWM45901VL & FIELD BLANK & NA & Methylene Chloride & 1 & & u & & UG/L & $07 / 11 / 2006$ & & JGJ-076-06 \\
\hline GWM45901VL & FIELD BLANK & NA & 4-Methyl-2-pentanone & 5 & & $\mathrm{u}$ & & UG/L & $07 / 11 / 2006$ & & JGJ-076-06 \\
\hline GWM45901VL & FIELD BLANK & NA & Styrene & 1 & & U & & UG/L & $07 / 11 / 2006$ & & JGJ-076-06 \\
\hline GWM45901VL & FIELD BLANK & NA & 1,1,2,2-Tetrachloroethane & 1 & & $\mathrm{u}$ & & UG/L & $07 / 11 / 2006$ & & JGJ-076-06 \\
\hline GWM45901VL & FIELD BLANK & NA & Tetrachloroethene & 1 & & U & UJ & UG/L & $07 / 11 / 2006$ & & JGJ-076-06 \\
\hline GWM45901VL & FIELD BLANK & NA & Toluene & 1 & & $\mathrm{u}$ & & UG/L & $07 / 11 / 2006$ & & JGJ-076-06 \\
\hline GWM45901VL & FIELD BLANK & NA & 1,2,4-Trichlorobenzene & 1 & & $\mathrm{u}$ & & UG/L & $07 / 11 / 2006$ & & JGJ-076-06 \\
\hline GWM45901VL & FIELD BLANK & NA & 1,1,1-Trichloroethane & 1 & & $\mathrm{u}$ & & UG/L & 07/11/2006 & & JGJ-076-06 \\
\hline GWM45901VL & FIELD BLANK & NA & 1,1,2-Trichloroethane & 1 & & $\mathrm{u}$ & & UG/L & 07/11/2006 & & JGJ-076-06 \\
\hline GWM45901VL & FIELD BLANK & NA & Trichloroethene & 1 & & $\mathrm{u}$ & & UG/L & $07 / 11 / 2006$ & & JGJ-076-06 \\
\hline GWM45901VL & FIELD BLANK & NA & 1,1,2-Trichloro-1,2,2-trifluoroethane & 1 & & u & & UG/L & $07 / 11 / 2006$ & & JGJ-076-06 \\
\hline GWM45901VL & FIELD BLANK & NA & Vinyl Chloride & 2 & & $\mathrm{u}$ & & UG/L & $07 / 11 / 2006$ & & JGJ-076-06 \\
\hline GWM45901VL & FIELD BLANK & NA & Xylene (Total) & 3 & & $\mathrm{u}$ & & UG/L & $07 / 11 / 2006$ & & JGJ-076-06 \\
\hline GWM45901VL & FIELD BLANK & NA & methyl tert-butyl ether & 2 & & $\mathrm{u}$ & & UG/L & $07 / 11 / 2006$ & & JGJ-076-06 \\
\hline GWM45901R8 & FIELD BLANK & NA & Tritium & $-3.45 E+01$ & $1.03 E+02$ & & $\mathrm{u}$ & $\mathrm{PCl} / \mathrm{L}$ & $07 / 11 / 2006$ & $3.61 \mathrm{E}+02$ & SOS-TL229-06 \\
\hline GWM45901UX & FIELD BLANK & NA & lodine-129 & $2.35 \mathrm{E}-02$ & $3.58 \mathrm{E}-02$ & & UJ & $\mathrm{PCl} / \mathrm{L}$ & $07 / 11 / 2006$ & 1.35E-01 & SOS-TL230-06 \\
\hline GWM45901RH & FIELD BLANK & NA & Gross Alpha & $1.08 \mathrm{E}-03$ & $9.26 \mathrm{E}-02$ & & u & $\mathrm{PCl} / \mathrm{L}$ & $07 / 11 / 2006$ & 3.47E-01 & SOS-TL231-06 \\
\hline GWM45901RH & FIELD BLANK & NA & Gross Beta & 1.31E-02 & $2.95 \mathrm{E}-01$ & & U & $\mathrm{PCl} / \mathrm{L}$ & $07 / 11 / 2006$ & $9.99 \mathrm{E}-01$ & SOS-TL231-06 \\
\hline
\end{tabular}


Table A-3. (continued).

\begin{tabular}{|c|c|c|c|c|c|c|c|c|c|c|c|}
\hline $\begin{array}{c}\text { Field Sample } \\
\text { Number }\end{array}$ & Location & Depth & Compound & $\begin{array}{c}\text { Sample } \\
\text { Result }\end{array}$ & $\begin{array}{c}\text { Sample } \\
\text { Error }\end{array}$ & $\begin{array}{c}\text { Result } \\
\text { Qualifier }\end{array}$ & $\begin{array}{c}\text { Validation } \\
\text { Flag }\end{array}$ & $\begin{array}{c}\text { Sample } \\
\text { Units }\end{array}$ & $\begin{array}{c}\text { Date Sample } \\
\text { Collected }\end{array}$ & MDA & $\begin{array}{c}\text { L\&V Report } \\
\text { Number }\end{array}$ \\
\hline GWM45901RH & FIELD BLANK & NA & Strontium-90 & $1.19 \mathrm{E}-01$ & $8.71 \mathrm{E}-02$ & & $U$ & $\mathrm{PCl} / \mathrm{L}$ & $07 / 11 / 2006$ & $3.73 \mathrm{E}-01$ & SOS-TL231-06 \\
\hline GWM45901RH & FIELD BLANK & NA & Technetium-99 & $-2.48 E+00$ & $1.85 \mathrm{E}+00$ & & $\mathrm{u}$ & $\mathrm{PCI} / \mathrm{L}$ & 07/11/2006 & $6.41 \mathrm{E}+00$ & SOS-TL231-06 \\
\hline GWM46002VL & TRIP BLANK & NA & Acetone & 2 & & $u$ & $\mathrm{R}$ & UG/L & 07/10/2006 & & JGJ-076-06 \\
\hline GWM46002VL & TRIP BLANK & NA & Benzene & 1 & & $u$ & & UG/L & $07 / 10 / 2006$ & & JGJ-076-06 \\
\hline GWM46002VL & TRIP BLANK & NA & Bromodichloromethane & 1 & & $\mathrm{U}$ & & UG/L & $07 / 10 / 2006$ & & JGJ-076-06 \\
\hline GWM46002VL & TRIP BLANK & NA & Bromoform & 1 & & $\mathrm{u}$ & UJ & UG/L & 07/10/2006 & & JGJ-076-06 \\
\hline GWM46002VL & TRIP BLANK & NA & Bromomethane & 2 & & $u$ & & UG/L & 07/10/2006 & & JGJ-076-06 \\
\hline GWM46002VL & TRIP BLANK & NA & 2-Butanone & 5 & & $\mathrm{u}$ & $\mathrm{R}$ & UG/L & 07/10/2006 & & JGJ-076-06 \\
\hline GWM46002VL & TRIP BLANK & NA & Carbon disulfide & 1 & & $u$ & & UG/L & 07/10/2006 & & JGJ-076-06 \\
\hline GWM46002VL & TRIP BLANK & NA & Chlorobenzene & 1 & & $\mathrm{u}$ & & UG/L & 07/10/2006 & & JGJ-076-06 \\
\hline GWM46002VL & TRIP BLANK & NA & Dibromochloromethane & 1 & & U & & UG/L & 07/10/2006 & & JGJ-076-06 \\
\hline GWM46002VL & TRIP BLANK & NA & 1,2-Dibromo-3-chloropropane & 1 & & $\mathrm{u}$ & $\mathrm{R}$ & UG/L & 07/10/2006 & & JGJ-076-06 \\
\hline GWM46002VL & TRIP BLANK & NA & Chloroethane & 2 & & $u$ & & UG/L & $07 / 10 / 2006$ & & JGJ-076-06 \\
\hline GWM46002VL & TRIP BLANK & NA & Chloroform & 1 & & $\mathrm{u}$ & & UG/L & $07 / 10 / 2006$ & & JGJ-076-06 \\
\hline GWM46002VL & TRIP BLANK & NA & Chloromethane & 2 & & $\mathrm{u}$ & & UG/L & 07/10/2006 & & JGJ-076-06 \\
\hline GWM46002VL & TRIP BLANK & NA & Cyclohexane & 1 & & $\mathrm{u}$ & & UG/L & 07/10/2006 & & JGJ-076-06 \\
\hline GWM46002VL & TRIP BLANK & NA & 1,2-Dibromoethane & 1 & & $u$ & & UG/L & 07/10/2006 & & JGJ-076-06 \\
\hline GWM46002VL & TRIP BLANK & NA & 1,2-Dichlorobenzene & 1 & & $\mathrm{u}$ & & UG/L & $07 / 10 / 2006$ & & JGJ-076-06 \\
\hline GWM46002VL & TRIP BLANK & NA & 1,3-Dichlorobenzene & 1 & & $\mathrm{u}$ & & UG/L & $07 / 10 / 2006$ & & JGJ-076-06 \\
\hline GWM46002VL & TRIP BLANK & NA & Dichlorodifluoromethane & 2 & & $\mathrm{u}$ & & UG/L & 07/10/2006 & & JGJ-076-06 \\
\hline GWM46002VL & TRIP BLANK & NA & 1,1-Dichloroethane & 1 & & $\mathrm{u}$ & & UG/L & 07/10/2006 & & JGJ-076-06 \\
\hline GWM46002VL & TRIP BLANK & NA & 1,2-Dichloroethane & 1 & & $\mathrm{u}$ & & UG/L & $07 / 10 / 2006$ & & JGJ-076-06 \\
\hline GWM46002VL & TRIP BLANK & NA & 1,1-Dichloroethene & 1 & & $\mathrm{u}$ & & UG/L & 07/10/2006 & & JGJ-076-06 \\
\hline GWM46002VL & TRIP BLANK & NA & cis-1,2-Dichloroethene & 1 & & $\mathrm{u}$ & & UG/L & 07/10/2006 & & JGJ-076-06 \\
\hline GWM46002VL & TRIP BLANK & NA & trans-1,2-Dichloroethene & 1 & & $u$ & & UG/L & 07/10/2006 & & JGJ-076-06 \\
\hline GWM46002VL & TRIP BLANK & NA & 1,2-Dichloropropane & 1 & & $u$ & & UG/L & 07/10/2006 & & JGJ-076-06 \\
\hline GWM46002VL & TRIP BLANK & NA & cis-1,3-Dichloropropene & 1 & & $u$ & & UG/L & 07/10/2006 & & JGJ-076-06 \\
\hline GWM46002VL & TRIP BLANK & NA & trans-1,3-Dichloropropene & 1 & & $\mathrm{u}$ & & UG/L & 07/10/2006 & & JGJ-076-06 \\
\hline GWM46002VL & TRIP BLANK & NA & Ethylbenzene & 1 & & $\mathrm{u}$ & & UG/L & $07 / 10 / 2006$ & & JGJ-076-06 \\
\hline GWM46002VL & TRIP BLANK & NA & Trichlorofluoromethane & 1 & & $u$ & & UG/L & $07 / 10 / 2006$ & & JGJ-076-06 \\
\hline GWM46002VL & TRIP BLANK & NA & 2-Hexanone & 5 & & $\mathrm{u}$ & & UG/L & 07/10/2006 & & JGJ-076-06 \\
\hline GWM46002VL & TRIP BLANK & NA & Isopropylbenzene & 1 & & $\mathrm{u}$ & & UG/L & $07 / 10 / 2006$ & & JGJ-076-06 \\
\hline GWM46002VL & TRIP BLANK & NA & Methyl acetate & 5 & & $\mathrm{u}$ & $\mathrm{R}$ & UG/L & $07 / 10 / 2006$ & & JGJ-076-06 \\
\hline GWM46002VL & TRIP BLANK & NA & Methyl cyclohexane & 4 & & $\mathrm{u}$ & & UG/L & 07/10/2006 & & JGJ-076-06 \\
\hline GWM46002VL & TRIP BLANK & NA & Methylene Chloride & 1 & & $\mathrm{u}$ & & UG/L & 07/10/2006 & & JGJ-076-06 \\
\hline GWM46002VL & TRIP BLANK & NA & 4-Methyl-2-pentanone & 5 & & $\mathrm{u}$ & & UG/L & $07 / 10 / 2006$ & & JGJ-076-06 \\
\hline GWM46002VL & TRIP BLANK & NA & Styrene & 1 & & $\mathrm{u}$ & & UG/L & $07 / 10 / 2006$ & & JGJ-076-06 \\
\hline GWM46002VL & TRIP BLANK & NA & 1,1,2,2-Tetrachloroethane & 1 & & $u$ & & UG/L & 07/10/2006 & & JGJ-076-06 \\
\hline GWM46002VL & TRIP BLANK & NA & Tetrachloroethene & 1 & & $\mathrm{u}$ & UJ & UG/L & 07/10/2006 & & JGJ-076-06 \\
\hline GWM46002VL & TRIP BLANK & NA & Toluene & 1 & & $\mathrm{u}$ & & UG/L & $07 / 10 / 2006$ & & JGJ-076-06 \\
\hline GWM46002VL & TRIP BLANK & NA & Trichloroethene & 1 & & $u$ & & UG/L & $07 / 10 / 2006$ & & JGJ-076-06 \\
\hline GWM46002VL & TRIP BLANK & NA & 1,1,2-Trichloro-1,2,2-trifluoroethane & 1 & & $\mathrm{u}$ & & UG/L & $07 / 10 / 2006$ & & JGJ-076-06 \\
\hline GWM46002VL & TRIP BLANK & NA & Vinyl Chloride & 2 & & $\mathrm{U}$ & & UG/L & $07 / 10 / 2006$ & & JGJ-076-06 \\
\hline GWM46002VL & TRIP BLANK & NA & Xylene (Total) & 3 & & $u$ & & UG/L & 07/10/2006 & & JGJ-076-06 \\
\hline GWM46002VL & TRIP BLANK & NA & methyl tert-butyl ether & 2 & & $u$ & & UG/L & 07/10/2006 & & JGJ-076-06 \\
\hline GWM46001VL & TRIP BLANK & NA & Acetone & 2 & & $\mathrm{U}$ & $\mathrm{R}$ & UG/L & 07/03/2006 & & JGJ-073-06 \\
\hline GWM46001VL & TRIP BLANK & NA & Benzene & 1 & & $u$ & & UG/L & 07/03/2006 & & JGJ-073-06 \\
\hline GWM46001VL & TRIP BLANK & NA & Bromodichloromethane & 1 & & $u$ & & UG/L & $07 / 03 / 2006$ & & JGJ-073-06 \\
\hline GWM46001VL & TRIP BLANK & NA & Bromoform & 1 & & $u$ & & UG/L & 07/03/2006 & & JGJ-073-06 \\
\hline GWM46001VL & TRIP BLANK & NA & Bromomethane & 2 & & U & & UG/L & 07/03/2006 & & JGJ-073-06 \\
\hline GWM46001VL & TRIP BLANK & NA & 2-Butanone & 5 & & $u$ & $\mathrm{R}$ & UG/L & 07/03/2006 & & JGJ-073-06 \\
\hline GWM46001VL & TRIP BLANK & NA & Carbon disulfide & 1 & & $u$ & & UG/L & $07 / 03 / 2006$ & & JGJ-073-06 \\
\hline GWM46001VL & TRIP BLANK & NA & Carbon tetrachloride & 1 & & $u$ & & UG/L & $07 / 03 / 2006$ & & JGJ-073-06 \\
\hline GWM46001VL & TRIP BLANK & NA & Chlorobenzene & 1 & & $u$ & & UG/L & 07/03/2006 & & JGJ-073-06 \\
\hline GWM46001VL & TRIP BLANK & NA & Dibromochloromethane & 1 & & U & & UG/L & $07 / 03 / 2006$ & & JGJ-073-06 \\
\hline GWM46001VL & TRIP BLANK & NA & 1,2-Dibromo-3-chloropropane & 1 & & $\mathrm{u}$ & $\mathrm{R}$ & UG/L & $07 / 03 / 2006$ & & JGJ-073-06 \\
\hline GWM46001VL & TRIP BLANK & NA & Chloroethane & 2 & & $u$ & & UG/L & 07/03/2006 & & JGJ-073-06 \\
\hline GWM46001VL & TRIP BLANK & NA & Chloroform & 1 & & $\mathrm{u}$ & & UG/L & $07 / 03 / 2006$ & & JGJ-073-06 \\
\hline GWM46001VL & TRIP BLANK & NA & Chloromethane & 2 & & $\mathrm{u}$ & & UG/L & $07 / 03 / 2006$ & & JGJ-073-06 \\
\hline GWM46001VL & TRIP BLANK & NA & Cyclohexane & 1 & & $\mathrm{u}$ & & UG/L & $07 / 03 / 2006$ & & JGJ-073-06 \\
\hline GWM46001VL & TRIP BLANK & NA & 1,2-Dibromoethane & 1 & & $\mathrm{u}$ & & UG/L & 07/03/2006 & & JGJ-073-06 \\
\hline GWM46001VL & TRIP BLANK & NA & 1,2-Dichlorobenzene & 1 & & $\mathrm{u}$ & & UG/L & $07 / 03 / 2006$ & & JGJ-073-06 \\
\hline GWM46001VL & TRIP BLANK & NA & 1,3-Dichlorobenzene & 1 & & $\mathrm{u}$ & & UG/L & 07/03/2006 & & JGJ-073-06 \\
\hline GWM46001VL & TRIP BLANK & NA & 1,4-Dichlorobenzene & 1 & & u & & UG/L & $07 / 03 / 2006$ & & JGJ-073-06 \\
\hline GWM46001VL & TRIP BLANK & NA & Dichlorodifluoromethane & 2 & & $\mathrm{u}$ & & UG/L & 07/03/2006 & & JGJ-073-06 \\
\hline GWM46001VL & TRIP BLANK & NA & 1,1-Dichloroethane & 1 & & $u$ & & UG/L & 07/03/2006 & & JGJ-073-06 \\
\hline GWM46001VL & TRIP BLANK & NA & 1,2-Dichloroethane & 1 & & $\mathrm{u}$ & & UG/L & 07/03/2006 & & JGJ-073-06 \\
\hline GWM46001VL & TRIP BLANK & NA & 1,1-Dichloroethene & 1 & & $u$ & & UG/L & $07 / 03 / 2006$ & & JGJ-073-06 \\
\hline GWM46001VL & TRIP BLANK & NA & cis-1,2-Dichloroethene & 1 & & $\mathrm{u}$ & & UG/L & $07 / 03 / 2006$ & & JGJ-073-06 \\
\hline GWM46001VL & TRIP BLANK & NA & trans-1,2-Dichloroethene & 1 & & $\mathrm{u}$ & & UG/L & $07 / 03 / 2006$ & & JGJ-073-06 \\
\hline GWM46001VL & TRIP BLANK & NA & 1,2-Dichloropropane & 1 & & $\mathrm{u}$ & & UG/L & 07/03/2006 & & JGJ-073-06 \\
\hline GWM46001VL & TRIP BLANK & NA & cis-1,3-Dichloropropene & 1 & & $\mathrm{u}$ & & UG/L & $07 / 03 / 2006$ & & JGJ-073-06 \\
\hline GWM46001VL & TRIP BLANK & NA & trans-1,3-Dichloropropene & 1 & & $\mathrm{u}$ & & UG/L & $07 / 03 / 2006$ & & JGJ-073-06 \\
\hline GWM46001VL & TRIP BLANK & NA & Ethylbenzene & 1 & & $\mathrm{u}$ & & UG/L & $07 / 03 / 2006$ & & JGJ-073-06 \\
\hline GWM46001VL & TRIP BLANK & NA & Trichlorofluoromethane & 1 & & $\mathrm{u}$ & & UG/L & 07/03/2006 & & JGJ-073-06 \\
\hline GWM46001VL & TRIP BLANK & NA & 2-Hexanone & 5 & & $u$ & & UG/L & $07 / 03 / 2006$ & & JGJ-073-06 \\
\hline GWM46001VL & TRIP BLANK & NA & Isopropylbenzene & 1 & & $u$ & & UG/L & 07/03/2006 & & JGJ-073-06 \\
\hline
\end{tabular}


Table A-3. (continued).

\begin{tabular}{|c|c|c|c|c|c|c|c|c|c|c|c|}
\hline $\begin{array}{c}\text { Field Sample } \\
\text { Number }\end{array}$ & Location & Depth & Compound & $\begin{array}{r}\text { Sample } \\
\text { Result }\end{array}$ & $\begin{array}{c}\text { Sample } \\
\text { Error }\end{array}$ & $\begin{array}{c}\text { Result } \\
\text { Qualifier }\end{array}$ & $\begin{array}{c}\text { Validation } \\
\text { Flag }\end{array}$ & $\begin{array}{c}\text { Sample } \\
\text { Units }\end{array}$ & $\begin{array}{c}\text { Date Sample } \\
\text { Collected }\end{array}$ & MDA & $\begin{array}{c}\text { L\&V Report } \\
\text { Number }\end{array}$ \\
\hline GWM46001VL & TRIP BLANK & NA & Methyl acetate & 5 & & $\mathrm{U}$ & $\mathrm{R}$ & UG/L & $07 / 03 / 2006$ & & JGJ-073-06 \\
\hline GWM46001VL & TRIP BLANK & NA & Methyl cyclohexane & 4 & & $\mathrm{u}$ & & UG/L & $07 / 03 / 2006$ & & JGJ-073-06 \\
\hline GWM46001VL & TRIP BLANK & NA & Methylene Chloride & 1 & & $u$ & & UG/L & 07/03/2006 & & JGJ-073-06 \\
\hline GWM46001VL & TRIP BLANK & NA & 4-Methyl-2-pentanone & 5 & & $u$ & & UG/L & 07/03/2006 & & JGJ-073-06 \\
\hline GWM46001VL & TRIP BLANK & NA & Styrene & 1 & & $\mathrm{u}$ & & UG/L & $07 / 03 / 2006$ & & JGJ-073-06 \\
\hline GWM46001VL & TRIP BLANK & NA & 1,1,2,2-Tetrachloroethane & 1 & & $\mathrm{u}$ & & UG/L & 07/03/2006 & & JGJ-073-06 \\
\hline GWM46001VL & TRIP BLANK & NA & Tetrachloroethene & 1 & & $u$ & & UG/L & 07/03/2006 & & JGJ-073-06 \\
\hline GWM46001VL & TRIP BLANK & NA & Toluene & 1 & & $u$ & & UG/L & $07 / 03 / 2006$ & & JGJ-073-06 \\
\hline GWM46001VL & TRIP BLANK & NA & 1,2,4-Trichlorobenzene & 1 & & $u$ & & UG/L & $07 / 03 / 2006$ & & JGJ-073-06 \\
\hline GWM46001VL & TRIP BLANK & NA & 1,1,2-Trichloroethane & 1 & & $\mathrm{u}$ & & UG/L & $07 / 03 / 2006$ & & JGJ-073-06 \\
\hline GWM46001VL & TRIP BLANK & NA & Trichloroethene & 1 & & $u$ & & UG/L & 07/03/2006 & & JGJ-073-06 \\
\hline GWM46001VL & TRIP BLANK & NA & 1,1,2-Trichloro-1,2,2-trifluoroethane & 1 & & $\mathrm{u}$ & & UG/L & $07 / 03 / 2006$ & & JGJ-073-06 \\
\hline GWM46001VL & TRIP BLANK & NA & Vinyl Chloride & 2 & & $\mathrm{u}$ & & UG/L & $07 / 03 / 2006$ & & JGJ-073-06 \\
\hline GWM46001VL & TRIP BLANK & NA & Xylene (Total) & 3 & & $u$ & & UG/L & $07 / 03 / 2006$ & & JGJ-073-06 \\
\hline GWM46001VL & TRIP BLANK & NA & methyl tert-butyl ether & 2 & & $\mathrm{u}$ & & UG/L & 07/03/2006 & & JGJ-073-06 \\
\hline GWM462012X & USGS-105 & 676-704 & Aluminum & 7.9 & & $\mathrm{u}$ & & UG/L & 07/05/2006 & & DNT-337-06 \\
\hline GWM462012X & USGS-105 & $676-704$ & Antimony & 0.5 & & $\mathrm{u}$ & & UG/L & 07/05/2006 & & DNT-337-06 \\
\hline GWM462012X & USGS-105 & 676-704 & Arsenic & 2 & & $\mathrm{u}$ & & UG/L & $07 / 05 / 2006$ & & DNT-337-06 \\
\hline GWM462012X & USGS-105 & 676-704 & Barium & 36.5 & & & & UG/L & $07 / 05 / 2006$ & & DNT-337-06 \\
\hline GWM462012X & USGS-105 & $676-704$ & Cadmium & 0.057 & & $\mathrm{u}$ & & UG/L & 07/05/2006 & & DNT-337-06 \\
\hline GWM462012X & USGS-105 & $676-704$ & Calcium & 36300 & & & & UG/L & $07 / 05 / 2006$ & & DNT-337-06 \\
\hline GWM462012X & USGS-105 & 676-704 & Chromium & 7.5 & & & & UG/L & $07 / 05 / 2006$ & & DNT-337-06 \\
\hline GWM462012X & USGS-105 & 676-704 & Cobalt & 0.31 & & $u$ & & UG/L & $07 / 05 / 2006$ & & DNT-337-06 \\
\hline GWM462012X & USGS-105 & 676-704 & Copper & 0.63 & & B & & UG/L & $07 / 05 / 2006$ & & DNT-337-06 \\
\hline GWM462012X & USGS-105 & 676-704 & Iron & 9.4 & & $u$ & & UG/L & $07 / 05 / 2006$ & & DNT-337-06 \\
\hline GWM462012X & USGS-105 & 676-704 & Lead & 0.49 & & $u$ & & UG/L & $07 / 05 / 2006$ & & DNT-337-06 \\
\hline GWM462012X & USGS-105 & 676-704 & Magnesium & 12700 & & & & UG/L & 07/05/2006 & & DNT-337-06 \\
\hline GWM462012X & USGS-105 & 676-704 & Manganese & 2.1 & & & & UG/L & 07/05/2006 & & DNT-337-06 \\
\hline GWM462012X & USGS-105 & 676-704 & Nickel & 1.1 & & B & & UG/L & $07 / 05 / 2006$ & & DNT-337-06 \\
\hline GWM462012X & USGS-105 & 676-704 & Potassium & 2530 & & & & UG/L & $07 / 05 / 2006$ & & DNT-337-06 \\
\hline GWM462012X & USGS-105 & $676-704$ & Selenium & 1.7 & & B & & UG/L & $07 / 05 / 2006$ & & DNT-337-06 \\
\hline GWM462012X & USGS-105 & 676-704 & Silver & 0.2 & & U & & UG/L & $07 / 05 / 2006$ & & DNT-337-06 \\
\hline GWM462012X & USGS-105 & $676-704$ & Sodium & 11200 & & & & UG/L & $07 / 05 / 2006$ & & DNT-337-06 \\
\hline GWM462012X & USGS-105 & 676-704 & Strontium & 232 & & & & UG/L & $07 / 05 / 2006$ & & DNT-337-06 \\
\hline GWM462012X & USGS-105 & $676-704$ & Thallium & 0.32 & & U & & UG/L & $07 / 05 / 2006$ & & DNT-337-06 \\
\hline GWM462012X & USGS-105 & 676-704 & Uranium & 2.2 & & & & UG/L & $07 / 05 / 2006$ & & DNT-337-06 \\
\hline GWM462012X & USGS-105 & $676-704$ & Vanadium & 4.3 & & B & & UG/L & $07 / 05 / 2006$ & & DNT-337-06 \\
\hline GWM462012X & USGS-105 & 676-704 & Zinc & 3.1 & & $\mathrm{BE}$ & $u$ & UG/L & 07/05/2006 & & DNT-337-06 \\
\hline GWM462012X & USGS-105 & $676-704$ & Mercury & 0.17 & & B & $\mathrm{J}$ & UG/L & 07/05/2006 & & DNT-337-06 \\
\hline GWM46201A1 & USGS-105 & $676-704$ & Alkalinity & 144 & & & & MG/L & $07 / 05 / 2006$ & & DNT-237-06 \\
\hline GWM46201N2 & USGS-105 & 676-704 & Nitrate/Nitrite as $\mathrm{N}$ & 1030 & & & & UG/L & $07 / 05 / 2006$ & & DNT-237-06 \\
\hline GWM46201VL & USGS-105 & $676-704$ & Acetone & 2 & & u & $\mathrm{R}$ & UG/L & 07/05/2006 & & JGJ-073-06 \\
\hline GWM46201VL & USGS-105 & $676-704$ & Benzene & 1 & & $\mathrm{u}$ & & UG/L & $07 / 05 / 2006$ & & JGJ-073-06 \\
\hline GWM46201VL & USGS-105 & $676-704$ & Bromodichloromethane & 1 & & $u$ & & UG/L & 07/05/2006 & & JGJ-073-06 \\
\hline GWM46201VL & USGS-105 & $676-704$ & Bromoform & 1 & & $u$ & & UG/L & 07/05/2006 & & JGJ-073-06 \\
\hline GWM46201VL & USGS-105 & $676-704$ & Bromomethane & 2 & & $\mathrm{U}$ & & UG/L & $07 / 05 / 2006$ & & JGJ-073-06 \\
\hline GWM46201VL & USGS-105 & $676-704$ & 2-Butanone & 5 & & $u$ & $\mathrm{R}$ & UG/L & $07 / 05 / 2006$ & & JGJ-073-06 \\
\hline GWM46201VL & USGS-105 & $676-704$ & Carbon disulfide & 1 & & $u$ & & UG/L & $07 / 05 / 2006$ & & JGJ-073-06 \\
\hline GWM46201VL & USGS-105 & $676-704$ & Carbon tetrachloride & 1 & & $u$ & & UG/L & $07 / 05 / 2006$ & & JGJ-073-06 \\
\hline GWM46201VL & USGS-105 & $676-704$ & Chlorobenzene & 1 & & U & & UG/L & $07 / 05 / 2006$ & & JGJ-073-06 \\
\hline GWM46201VL & USGS-105 & $676-704$ & Dibromochloromethane & 1 & & $u$ & & UG/L & 07/05/2006 & & JGJ-073-06 \\
\hline GWM46201VL & USGS-105 & $676-704$ & 1,2-Dibromo-3-chloropropane & 1 & & $\mathrm{u}$ & $\mathrm{R}$ & UG/L & $07 / 05 / 2006$ & & JGJ-073-06 \\
\hline GWM46201VL & USGS-105 & $676-704$ & Chloroethane & 2 & & $\mathrm{u}$ & & UG/L & $07 / 05 / 2006$ & & JGJ-073-06 \\
\hline GWM46201VL & USGS-105 & 676-704 & Chloroform & 1 & & $\mathrm{u}$ & & UG/L & $07 / 05 / 2006$ & & JGJ-073-06 \\
\hline GWM46201VL & USGS-105 & $676-704$ & Chloromethane & 2 & & $\mathrm{u}$ & & UG/L & $07 / 05 / 2006$ & & JGJ-073-06 \\
\hline GWM46201VL & USGS-105 & 676-704 & Cyclohexane & 1 & & $\mathrm{u}$ & & UG/L & $07 / 05 / 2006$ & & JGJ-073-06 \\
\hline GWM46201VL & USGS-105 & $676-704$ & 1,2-Dibromoethane & 1 & & $\mathrm{u}$ & & UG/L & $07 / 05 / 2006$ & & JGJ-073-06 \\
\hline GWM46201VL & USGS-105 & $676-704$ & 1,2-Dichlorobenzene & 1 & & $\mathrm{u}$ & & UG/L & 07/05/2006 & & JGJ-073-06 \\
\hline GWM46201VL & USGS-105 & $676-704$ & 1,3-Dichlorobenzene & 1 & & $\mathrm{u}$ & & UG/L & $07 / 05 / 2006$ & & JGJ-073-06 \\
\hline GWM46201VL & USGS-105 & $676-704$ & 1,4-Dichlorobenzene & 1 & & $\mathrm{u}$ & & UG/L & $07 / 05 / 2006$ & & JGJ-073-06 \\
\hline GWM46201VL & USGS-105 & $676-704$ & Dichlorodifluoromethane & 2 & & $\mathrm{u}$ & & UG/L & $07 / 05 / 2006$ & & JGJ-073-06 \\
\hline GWM46201VL & USGS-105 & $676-704$ & 1,1-Dichloroethane & 1 & & $\mathrm{u}$ & & UG/L & 07/05/2006 & & JGJ-073-06 \\
\hline GWM46201VL & USGS-105 & $676-704$ & 1,2-Dichloroethane & 1 & & $\mathrm{u}$ & & UG/L & $07 / 05 / 2006$ & & JGJ-073-06 \\
\hline GWM46201VL & USGS-105 & 676-704 & 1,1-Dichloroethene & 1 & & u & & UG/L & $07 / 05 / 2006$ & & JGJ-073-06 \\
\hline GWM46201VL & USGS-105 & $676-704$ & cis-1,2-Dichloroethene & 1 & & $\mathrm{u}$ & & UG/L & $07 / 05 / 2006$ & & JGJ-073-06 \\
\hline GWM46201VL & USGS-105 & $676-704$ & trans-1,2-Dichloroethene & 1 & & $u$ & & UG/L & 07/05/2006 & & JGJ-073-06 \\
\hline GWM46201VL & USGS-105 & $676-704$ & 1,2-Dichloropropane & 1 & & $\mathrm{u}$ & & UG/L & $07 / 05 / 2006$ & & JGJ-073-06 \\
\hline GWM46201VL & USGS-105 & 676-704 & cis-1,3-Dichloropropene & 1 & & $u$ & & UG/L & $07 / 05 / 2006$ & & JGJ-073-06 \\
\hline GWM46201VL & USGS-105 & $676-704$ & trans-1,3-Dichloropropene & 1 & & $\mathrm{u}$ & & UG/L & $07 / 05 / 2006$ & & JGJ-073-06 \\
\hline GWM46201VL & USGS-105 & $676-704$ & Ethylbenzene & 1 & & $\mathrm{u}$ & & UG/L & $07 / 05 / 2006$ & & JGJ-073-06 \\
\hline GWM46201VL & USGS-105 & $676-704$ & Trichlorofluoromethane & 1 & & $\mathrm{u}$ & & UG/L & $07 / 05 / 2006$ & & JGJ-073-06 \\
\hline GWM46201VL & USGS-105 & $676-704$ & 2-Hexanone & 5 & & $u$ & & UG/L & $07 / 05 / 2006$ & & JGJ-073-06 \\
\hline GWM46201VL & USGS-105 & $676-704$ & Isopropylbenzene & 1 & & $\mathrm{U}$ & & UG/L & $07 / 05 / 2006$ & & JGJ-073-06 \\
\hline GWM46201VL & USGS-105 & $676-704$ & Methyl acetate & 5 & & $\mathrm{u}$ & $\mathrm{R}$ & UG/L & $07 / 05 / 2006$ & & JGJ-073-06 \\
\hline GWM46201VL & USGS-105 & $676-704$ & Methyl cyclohexane & 4 & & $u$ & & UG/L & $07 / 05 / 2006$ & & JGJ-073-06 \\
\hline GWM46201VL & USGS-105 & $676-704$ & Methylene Chloride & 1 & & U & & UG/L & $07 / 05 / 2006$ & & JGJ-073-06 \\
\hline GWM46201VL & USGS-105 & $676-704$ & 4-Methyl-2-pentanone & 5 & & $u$ & & UG/L & $07 / 05 / 2006$ & & JGJ-073-06 \\
\hline
\end{tabular}


Table A-3. (continued).

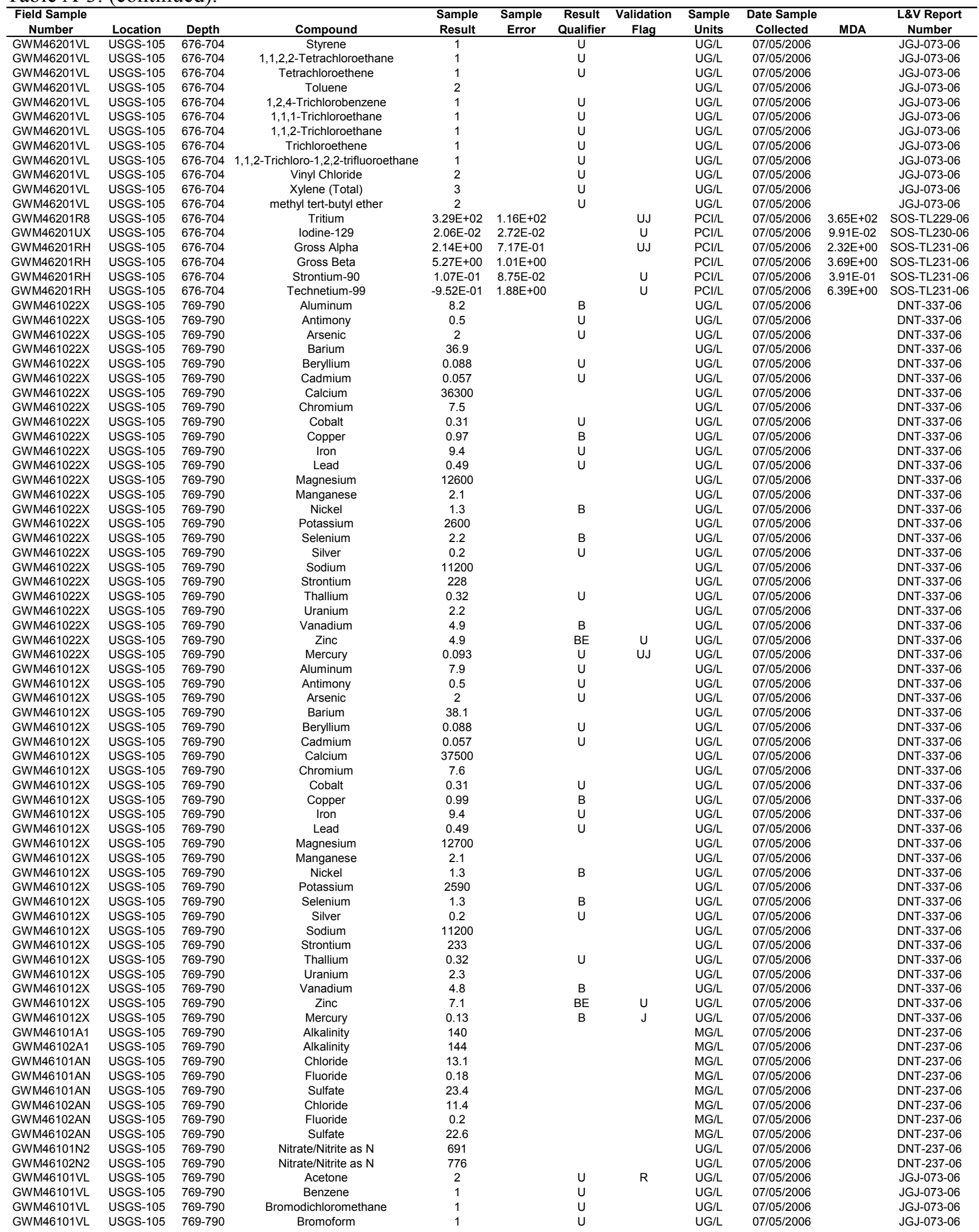


Table A-3. (continued).

\begin{tabular}{|c|c|c|c|c|c|c|c|c|c|c|c|}
\hline $\begin{array}{c}\text { Field Sample } \\
\text { Number }\end{array}$ & Location & Depth & Compound & $\begin{array}{c}\text { Sample } \\
\text { Result }\end{array}$ & $\begin{array}{c}\text { Sample } \\
\text { Error }\end{array}$ & $\begin{array}{c}\text { Result } \\
\text { Qualifier }\end{array}$ & $\begin{array}{c}\text { Validation } \\
\text { Flag }\end{array}$ & $\begin{array}{c}\text { Sample } \\
\text { Units }\end{array}$ & $\begin{array}{c}\text { Date Sample } \\
\text { Collected }\end{array}$ & MDA & $\begin{array}{c}\text { L\&V Report } \\
\text { Number }\end{array}$ \\
\hline GWM46101VL & USGS-105 & $769-790$ & Bromomethane & 2 & & $\bar{U}$ & & UG/L & $07 / 05 / 2006$ & & JGJ-073-06 \\
\hline GWM46101VL & USGS-105 & $769-790$ & 2-Butanone & 5 & & $\mathrm{u}$ & $\mathrm{R}$ & UG/L & $07 / 05 / 2006$ & & JGJ-073-06 \\
\hline GWM46101VL & USGS-105 & $769-790$ & Carbon disulfide & 1 & & $\mathrm{u}$ & & UG/L & $07 / 05 / 2006$ & & JGJ-073-06 \\
\hline GWM46101VL & USGS-105 & $769-790$ & Carbon tetrachloride & 1 & & $\mathrm{u}$ & & UG/L & $07 / 05 / 2006$ & & JGJ-073-06 \\
\hline GWM46101VL & USGS-105 & $769-790$ & Chlorobenzene & 1 & & $\mathrm{u}$ & & UG/L & $07 / 05 / 2006$ & & JGJ-073-06 \\
\hline GWM46101VL & USGS-105 & $769-790$ & Dibromochloromethane & 1 & & $\mathrm{u}$ & & UG/L & $07 / 05 / 2006$ & & JGJ-073-06 \\
\hline GWM46101VL & USGS-105 & $769-790$ & 1,2-Dibromo-3-chloropropane & 1 & & $\mathrm{u}$ & $\mathrm{R}$ & UG/L & 07/05/2006 & & JGJ-073-06 \\
\hline GWM46101VL & USGS-105 & $769-790$ & Chloroethane & 2 & & U & & UG/L & $07 / 05 / 2006$ & & JGJ-073-06 \\
\hline GWM46101VL & USGS-105 & $769-790$ & Chloroform & 1 & & $\mathrm{u}$ & & UG/L & $07 / 05 / 2006$ & & JGJ-073-06 \\
\hline GWM46101VL & USGS-105 & $769-790$ & Cyclohexane & 1 & & $\mathrm{u}$ & & UG/L & $07 / 05 / 2006$ & & JGJ-073-06 \\
\hline GWM46101VL & USGS-105 & $769-790$ & 1,2-Dibromoethane & 1 & & $u$ & & UG/L & $07 / 05 / 2006$ & & JGJ-073-06 \\
\hline GWM46101VL & USGS-105 & $769-790$ & 1,2-Dichlorobenzene & 1 & & $\mathrm{u}$ & & UG/L & 07/05/2006 & & JGJ-073-06 \\
\hline GWM46101VL & USGS-105 & $769-790$ & 1,3-Dichlorobenzene & 1 & & $u$ & & UG/L & $07 / 05 / 2006$ & & JGJ-073-06 \\
\hline GWM46101VL & USGS-105 & $769-790$ & 1,4-Dichlorobenzene & 1 & & $u$ & & UG/L & $07 / 05 / 2006$ & & JGJ-073-06 \\
\hline GWM46101VL & USGS-105 & $769-790$ & Dichlorodifluoromethane & 2 & & $\mathrm{u}$ & & UG/L & $07 / 05 / 2006$ & & JGJ-073-06 \\
\hline GWM46101VL & USGS-105 & $769-790$ & 1,1-Dichloroethane & 1 & & $u$ & & UG/L & 07/05/2006 & & JGJ-073-06 \\
\hline GWM46101VL & USGS-105 & $769-790$ & 1,2-Dichloroethane & 1 & & $u$ & & UG/L & 07/05/2006 & & JGJ-073-06 \\
\hline GWM46101VL & USGS-105 & $769-790$ & 1,1-Dichloroethene & 1 & & $\mathrm{u}$ & & UG/L & 07/05/2006 & & JGJ-073-06 \\
\hline GWM46101VL & USGS-105 & $769-790$ & cis-1,2-Dichloroethene & 1 & & $\mathrm{u}$ & & UG/L & $07 / 05 / 2006$ & & JGJ-073-06 \\
\hline GWM46101VL & USGS-105 & $769-790$ & cis-1,3-Dichloropropene & 1 & & $\mathrm{u}$ & & UG/L & $07 / 05 / 2006$ & & JGJ-073-06 \\
\hline GWM46101VL & USGS-105 & $769-790$ & trans-1,3-Dichloropropene & 1 & & $\mathrm{u}$ & & UG/L & $07 / 05 / 2006$ & & JGJ-073-06 \\
\hline GWM46101VL & USGS-105 & $769-790$ & Ethylbenzene & 1 & & $\mathrm{u}$ & & UG/L & $07 / 05 / 2006$ & & JGJ-073-06 \\
\hline GWM46101VL & USGS-105 & $769-790$ & Trichlorofluoromethane & 1 & & $\mathrm{u}$ & & UG/L & $07 / 05 / 2006$ & & JGJ-073-06 \\
\hline GWM46101VL & USGS-105 & $769-790$ & 2-Hexanone & 5 & & $u$ & & UG/L & 07/05/2006 & & JGJ-073-06 \\
\hline GWM46101VL & USGS-105 & $769-790$ & Isopropylbenzene & 1 & & $u$ & & UG/L & 07/05/2006 & & JGJ-073-06 \\
\hline GWM46101VL & USGS-105 & $769-790$ & Methyl acetate & 5 & & $u$ & $\mathrm{R}$ & UG/L & $07 / 05 / 2006$ & & JGJ-073-06 \\
\hline GWM46101VL & USGS-105 & $769-790$ & Methyl cyclohexane & 4 & & $\mathrm{u}$ & & UG/L & $07 / 05 / 2006$ & & JGJ-073-06 \\
\hline GWM46101VL & USGS-105 & $769-790$ & Methylene Chloride & 1 & & $u$ & & UG/L & $07 / 05 / 2006$ & & JGJ-073-06 \\
\hline GWM46101VL & USGS-105 & $769-790$ & 4-Methyl-2-pentanone & 5 & & $u$ & & UG/L & $07 / 05 / 2006$ & & JGJ-073-06 \\
\hline GWM46101VL & USGS-105 & $769-790$ & Styrene & 1 & & $u$ & & UG/L & $07 / 05 / 2006$ & & JGJ-073-06 \\
\hline GWM46101VL & USGS-105 & $769-790$ & 1,1,2,2-Tetrachloroethane & 1 & & $\mathrm{u}$ & & UG/L & $07 / 05 / 2006$ & & JGJ-073-06 \\
\hline GWM46101VL & USGS-105 & $769-790$ & Tetrachloroethene & 1 & & $u$ & & UG/L & $07 / 05 / 2006$ & & JGJ-073-06 \\
\hline GWM46101VL & USGS-105 & $769-790$ & Toluene & 1 & & $u$ & & UG/L & 07/05/2006 & & JGJ-073-06 \\
\hline GWM46101VL & USGS-105 & $769-790$ & 1,2,4-Trichlorobenzene & 1 & & $\mathrm{u}$ & & UG/L & $07 / 05 / 2006$ & & JGJ-073-06 \\
\hline GWM46101VL & USGS-105 & $769-790$ & 1,1,1-Trichloroethane & 1 & & $\mathrm{u}$ & & UG/L & 07/05/2006 & & JGJ-073-06 \\
\hline GWM46101VL & USGS-105 & $769-790$ & 1,1,2-Trichloroethane & 1 & & $\mathrm{u}$ & & UG/L & $07 / 05 / 2006$ & & JGJ-073-06 \\
\hline GWM46101VL & USGS-105 & $769-790$ & Trichloroethene & 1 & & $\mathrm{u}$ & & UG/L & 07/05/2006 & & JGJ-073-06 \\
\hline GWM46101VL & USGS-105 & $769-790$ & 1,1,2-Trichloro-1,2,2-trifluoroethane & 1 & & $\mathrm{u}$ & & UG/L & 07/05/2006 & & JGJ-073-06 \\
\hline GWM46101VL & USGS-105 & $769-790$ & Vinyl Chloride & 2 & & $\mathrm{u}$ & & UG/L & $07 / 05 / 2006$ & & JGJ-073-06 \\
\hline GWM46102VL & USGS-105 & $769-790$ & Benzene & 1 & & $u$ & & UG/L & $07 / 05 / 2006$ & & JGJ-073-06 \\
\hline GWM46102VL & USGS-105 & $769-790$ & Bromodichloromethane & 1 & & $\mathrm{u}$ & & UG/L & $07 / 05 / 2006$ & & JGJ-073-06 \\
\hline GWM46102VL & USGS-105 & $769-790$ & Bromoform & 1 & & $\mathrm{u}$ & & UG/L & $07 / 05 / 2006$ & & JGJ-073-06 \\
\hline GWM46102VL & USGS-105 & $769-790$ & Bromomethane & 2 & & $\mathrm{u}$ & & UG/L & $07 / 05 / 2006$ & & JGJ-073-06 \\
\hline GWM46102VL & USGS-105 & $769-790$ & 2-Butanone & 5 & & $u$ & & UG/L & 07/05/2006 & & JGJ-073-06 \\
\hline GWM46102VL & USGS-105 & $769-790$ & Carbon disulfide & 1 & & $\mathrm{u}$ & $\mathrm{R}$ & UG/L & $07 / 05 / 2006$ & & JGJ-073-06 \\
\hline GWM46102VL & USGS-105 & $769-790$ & Carbon tetrachloride & 1 & & $\mathrm{u}$ & & UG/L & $07 / 05 / 2006$ & & JGJ-073-06 \\
\hline GWM46102VL & USGS-105 & $769-790$ & Chlorobenzene & 1 & & $\mathrm{u}$ & & UG/L & $07 / 05 / 2006$ & & JGJ-073-06 \\
\hline GWM46102VL & USGS-105 & $769-790$ & Dibromochloromethane & 1 & & $\mathrm{u}$ & & UG/L & $07 / 05 / 2006$ & & JGJ-073-06 \\
\hline GWM46102VL & USGS-105 & $769-790$ & 1,2-Dibromo-3-chloropropane & 1 & & $\mathrm{u}$ & $\mathrm{R}$ & UG/L & $07 / 05 / 2006$ & & JGJ-073-06 \\
\hline GWM46102VL & USGS-105 & $769-790$ & Chloroethane & 2 & & $\mathrm{u}$ & & UG/L & 07/05/2006 & & JGJ-073-06 \\
\hline GWM46102VL & USGS-105 & $769-790$ & Chloroform & 1 & & $\mathrm{u}$ & & UG/L & $07 / 05 / 2006$ & & JGJ-073-06 \\
\hline GWM46102VL & USGS-105 & $769-790$ & Chloromethane & 2 & & $\mathrm{u}$ & & UG/L & $07 / 05 / 2006$ & & JGJ-073-06 \\
\hline GWM46102VL & USGS-105 & $769-790$ & Cyclohexane & 1 & & $\mathrm{u}$ & & UG/L & $07 / 05 / 2006$ & & JGJ-073-06 \\
\hline GWM46102VL & USGS-105 & $769-790$ & 1,2-Dibromoethane & 1 & & u & & UG/L & $07 / 05 / 2006$ & & JGJ-073-06 \\
\hline GWM46102VL & USGS-105 & $769-790$ & 1,2-Dichlorobenzene & 1 & & $u$ & & UG/L & $07 / 05 / 2006$ & & JGJ-073-06 \\
\hline GWM46102VL & USGS-105 & $769-790$ & 1,3-Dichlorobenzene & 1 & & $\mathrm{u}$ & & UG/L & 07/05/2006 & & JGJ-073-06 \\
\hline GWM46102VL & USGS-105 & $769-790$ & 1,4-Dichlorobenzene & 1 & & $u$ & & UG/L & 07/05/2006 & & JGJ-073-06 \\
\hline GWM46102VL & USGS-105 & $769-790$ & Dichlorodifluoromethane & 2 & & $u$ & & UG/L & $07 / 05 / 2006$ & & JGJ-073-06 \\
\hline GWM46102VL & USGS-105 & $769-790$ & 1,1-Dichloroethane & 1 & & $u$ & & UG/L & $07 / 05 / 2006$ & & JGJ-073-06 \\
\hline GWM46102VL & USGS-105 & $769-790$ & 1,2-Dichloroethane & 1 & & $\mathrm{u}$ & & UG/L & 07/05/2006 & & JGJ-073-06 \\
\hline GWM46102VL & USGS-105 & $769-790$ & 1,1-Dichloroethene & 1 & & $\mathrm{u}$ & & UG/L & 07/05/2006 & & JGJ-073-06 \\
\hline GWM46102VL & USGS-105 & $769-790$ & cis-1,2-Dichloroethene & 1 & & $\mathrm{u}$ & & UG/L & $07 / 05 / 2006$ & & JGJ-073-06 \\
\hline GWM46102VL & USGS-105 & $769-790$ & trans-1,2-Dichloroethene & 1 & & $u$ & & UG/L & $07 / 05 / 2006$ & & JGJ-073-06 \\
\hline GWM46102VL & USGS-105 & $769-790$ & 1,2-Dichloropropane & 1 & & $\mathrm{u}$ & & UG/L & $07 / 05 / 2006$ & & JGJ-073-06 \\
\hline GWM46102VL & USGS-105 & $769-790$ & cis-1,3-Dichloropropene & 1 & & $u$ & & UG/L & 07/05/2006 & & JGJ-073-06 \\
\hline GWM46102VL & USGS-105 & $769-790$ & trans-1,3-Dichloropropene & 1 & & $u$ & & UG/L & 07/05/2006 & & JGJ-073-06 \\
\hline GWM46102VL & USGS-105 & $769-790$ & Ethylbenzene & 1 & & $\mathrm{u}$ & & UG/L & $07 / 05 / 2006$ & & JGJ-073-06 \\
\hline GWM46102VL & USGS-105 & $769-790$ & Trichlorofluoromethane & 1 & & $\mathrm{u}$ & & UG/L & $07 / 05 / 2006$ & & JGJ-073-06 \\
\hline GWM46102VL & USGS-105 & $769-790$ & 2-Hexanone & 5 & & $u$ & & UG/L & $07 / 05 / 2006$ & & JGJ-073-06 \\
\hline GWM46102VL & USGS-105 & $769-790$ & Isopropylbenzene & 1 & & $\mathrm{u}$ & & UG/L & $07 / 05 / 2006$ & & JGJ-073-06 \\
\hline GWM46102VL & USGS-105 & $769-790$ & Methyl acetate & 5 & & $u$ & $\mathrm{R}$ & UG/L & $07 / 05 / 2006$ & & JGJ-073-06 \\
\hline GWM46102VL & USGS-105 & $769-790$ & Methyl cyclohexane & 4 & & $\mathrm{u}$ & & UG/L & $07 / 05 / 2006$ & & JGJ-073-06 \\
\hline GWM46102VL & USGS-105 & $769-790$ & Methylene Chloride & 1 & & $\mathrm{u}$ & & UG/L & $07 / 05 / 2006$ & & JGJ-073-06 \\
\hline GWM46102VL & USGS-105 & $769-790$ & 4-Methyl-2-pentanone & 5 & & $\mathrm{u}$ & & UG/L & $07 / 05 / 2006$ & & JGJ-073-06 \\
\hline GWM46102VL & USGS-105 & $769-790$ & Styrene & 1 & & $\mathrm{u}$ & & UG/L & $07 / 05 / 2006$ & & JGJ-073-06 \\
\hline GWM46102VL & USGS-105 & $769-790$ & 1,1,2,2-Tetrachloroethane & 1 & & $u$ & & UG/L & $07 / 05 / 2006$ & & JGJ-073-06 \\
\hline
\end{tabular}


Table A-3. (continued).

\begin{tabular}{|c|c|c|c|c|c|c|c|c|c|c|c|}
\hline $\begin{array}{c}\text { Field Sample } \\
\text { Number }\end{array}$ & Location & Depth & Compound & $\begin{array}{c}\text { Sample } \\
\text { Result }\end{array}$ & $\begin{array}{l}\text { Sample } \\
\text { Error }\end{array}$ & $\begin{array}{c}\text { Result } \\
\text { Qualifier }\end{array}$ & $\begin{array}{c}\text { Validation } \\
\text { Flag }\end{array}$ & $\begin{array}{c}\text { Sample } \\
\text { Units }\end{array}$ & $\begin{array}{c}\text { Date Sample } \\
\text { Collected }\end{array}$ & MDA & $\begin{array}{c}\text { L\&V Report } \\
\text { Number }\end{array}$ \\
\hline GWM46102VL & USGS-105 & $769-790$ & Tetrachloroethene & 1 & & $\mathrm{U}$ & & UG/L & $07 / 05 / 2006$ & & JGJ-073-06 \\
\hline GWM46102VL & USGS-105 & $769-790$ & Toluene & 1 & & $\mathrm{u}$ & & UG/L & $07 / 05 / 2006$ & & JGJ-073-06 \\
\hline GWM46102VL & USGS-105 & $769-790$ & 1,2,4-Trichlorobenzene & 1 & & $\mathrm{u}$ & & UG/L & $07 / 05 / 2006$ & & JGJ-073-06 \\
\hline GWM46102VL & USGS-105 & $769-790$ & 1,1,1-Trichloroethane & 1 & & $\mathrm{u}$ & & UG/L & $07 / 05 / 2006$ & & JGJ-073-06 \\
\hline GWM46102VL & USGS-105 & $769-790$ & 1,1,2-Trichloroethane & 1 & & $\mathrm{u}$ & & UG/L & $07 / 05 / 2006$ & & JGJ-073-06 \\
\hline GWM46102VL & USGS-105 & $769-790$ & Trichloroethene & 1 & & $\mathrm{u}$ & & UG/L & $07 / 05 / 2006$ & & JGJ-073-06 \\
\hline GWM46102VL & USGS-105 & $769-790$ & 1,1,2-Trichloro-1,2,2-trifluoroethane & 1 & & $\mathrm{u}$ & & UG/L & $07 / 05 / 2006$ & & JGJ-073-06 \\
\hline GWM46102VL & USGS-105 & $769-790$ & Vinyl Chloride & 2 & & $\mathrm{u}$ & & UG/L & $07 / 05 / 2006$ & & JGJ-073-06 \\
\hline GWM46102VL & USGS-105 & $769-790$ & Xylene (Total) & 3 & & $u$ & & UG/L & $07 / 05 / 2006$ & & JGJ-073-06 \\
\hline GWM46101R8 & USGS-105 & $769-790$ & Tritium & $2.06 \mathrm{E}+02$ & $1.11 \mathrm{E}+02$ & & u & $\mathrm{PCI} / \mathrm{L}$ & $07 / 05 / 2006$ & $3.63 E+02$ & SOS-TL229-06 \\
\hline GWM46102R8 & USGS-105 & $769-790$ & Tritium & $1.63 E+02$ & $1.09 \mathrm{E}+02$ & & U & $\mathrm{PCl} / \mathrm{L}$ & $07 / 05 / 2006$ & $3.61 \mathrm{E}+02$ & SOS-TL229-06 \\
\hline GWM46102UX & USGS-105 & $769-790$ & lodine-129 & $6.43 \mathrm{E}-02$ & $3.25 \mathrm{E}-02$ & & UJ & $\mathrm{PCI} / \mathrm{L}$ & $07 / 05 / 2006$ & 1.26E-01 & SOS-TL230-06 \\
\hline GWM46101UX & USGS-105 & $769-790$ & lodine-129 & $-2.78 \mathrm{E}-02$ & 3.99E-02 & & UJ & $\mathrm{PCI} / \mathrm{L}$ & $07 / 05 / 2006$ & 1.14E-01 & SOS-TL230-06 \\
\hline GWM46101RH & USGS-105 & $769-790$ & Gross Alpha & $1.50 \mathrm{E}+00$ & $5.68 \mathrm{E}-01$ & & UJ & $\mathrm{PCI} / \mathrm{L}$ & $07 / 05 / 2006$ & $1.76 \mathrm{E}+00$ & SOS-TL231-06 \\
\hline GWM46101RH & USGS-105 & $769-790$ & Gross Beta & $4.68 \mathrm{E}+00$ & $8.68 \mathrm{E}-01$ & & & $\mathrm{PCI} / \mathrm{L}$ & $07 / 05 / 2006$ & $2.95 \mathrm{E}+00$ & SOS-TL231-06 \\
\hline GWM46102RH & USGS-105 & $769-790$ & Gross Alpha & $2.57 \mathrm{E}+00$ & 7.13E-01 & & & $\mathrm{PCI} / \mathrm{L}$ & $07 / 05 / 2006$ & $1.93 \mathrm{E}+00$ & SOS-TL231-06 \\
\hline GWM46102RH & USGS-105 & $769-790$ & Gross Beta & $6.61 \mathrm{E}+00$ & $9.86 \mathrm{E}-01$ & & & $\mathrm{PCI} / \mathrm{L}$ & $07 / 05 / 2006$ & $3.14 \mathrm{E}+00$ & SOS-TL231-06 \\
\hline GWM46101RH & USGS-105 & $769-790$ & Strontium-90 & $1.41 \mathrm{E}-01$ & 7.69E-02 & & $u$ & $\mathrm{PCI} / \mathrm{L}$ & $07 / 05 / 2006$ & 3.22E-01 & SOS-TL231-06 \\
\hline GWM46102RH & USGS-105 & $769-790$ & Strontium-90 & $6.48 \mathrm{E}-01$ & $1.29 \mathrm{E}-01$ & & & $\mathrm{PCl} / \mathrm{L}$ & $07 / 05 / 2006$ & 4.09E-01 & SOS-TL231-06 \\
\hline GWM464012X & USGS-108 & $613-627$ & Aluminum & 9.8 & & B & & UG/L & $07 / 10 / 2006$ & & DNT-334-06 \\
\hline GWM464012X & USGS-108 & $613-627$ & Antimony & 0.5 & & U & & UG/L & $07 / 10 / 2006$ & & DNT-334-06 \\
\hline GWM464012X & USGS-108 & $613-627$ & Arsenic & 2.2 & & B & & UG/L & $07 / 10 / 2006$ & & DNT-334-06 \\
\hline GWM464012X & USGS-108 & $613-627$ & Barium & 39.7 & & & & UG/L & $07 / 10 / 2006$ & & DNT-334-06 \\
\hline GWM464012X & USGS-108 & 613-627 & Beryllium & 0.088 & & U & & UG/L & 07/10/2006 & & DNT-334-06 \\
\hline GWM464012X & USGS-108 & $613-627$ & Cadmium & 0.057 & & U & & UG/L & $07 / 10 / 2006$ & & DNT-334-06 \\
\hline GWM464012X & USGS-108 & $613-627$ & Calcium & 33700 & & $\mathrm{E}$ & $\mathrm{J}$ & UG/L & $07 / 10 / 2006$ & & DNT-334-06 \\
\hline GWM464012X & USGS-108 & $613-627$ & Chromium & 6.4 & & & & UG/L & $07 / 10 / 2006$ & & DNT-334-06 \\
\hline GWM464012X & USGS-108 & $613-627$ & Cobalt & 0.31 & & $u$ & & UG/L & $07 / 10 / 2006$ & & DNT-334-06 \\
\hline GWM464012X & USGS-108 & $613-627$ & Copper & 0.34 & & B & & UG/L & 07/10/2006 & & DNT-334-06 \\
\hline GWM464012X & USGS-108 & $613-627$ & Iron & 9.4 & & $u$ & & UG/L & $07 / 10 / 2006$ & & DNT-334-06 \\
\hline GWM464012X & USGS-108 & $613-627$ & Lead & 0.49 & & $\mathrm{u}$ & & UG/L & $07 / 10 / 2006$ & & DNT-334-06 \\
\hline GWM464012X & USGS-108 & $613-627$ & Magnesium & 13900 & & & & UG/L & $07 / 10 / 2006$ & & DNT-334-06 \\
\hline GWM464012X & USGS-108 & $613-627$ & Manganese & 0.45 & & B & & UG/L & $07 / 10 / 2006$ & & DNT-334-06 \\
\hline GWM464012X & USGS-108 & $613-627$ & Nickel & 1.1 & & B & & UG/L & $07 / 10 / 2006$ & & DNT-334-06 \\
\hline GWM464012X & USGS-108 & $613-627$ & Potassium & 2660 & & & & UG/L & 07/10/2006 & & DNT-334-06 \\
\hline GWM464012X & USGS-108 & $613-627$ & Selenium & 1.5 & & B & & UG/L & $07 / 10 / 2006$ & & DNT-334-06 \\
\hline GWM464012X & USGS-108 & 613-627 & Silver & 0.2 & & $u$ & & UG/L & 07/10/2006 & & DNT-334-06 \\
\hline GWM464012X & USGS-108 & $613-627$ & Sodium & 11300 & & & & UG/L & $07 / 10 / 2006$ & & DNT-334-06 \\
\hline GWM464012X & USGS-108 & $613-627$ & Strontium & 192 & & & & UG/L & 07/10/2006 & & DNT-334-06 \\
\hline GWM464012X & USGS-108 & 613-627 & Zinc & 6 & & $\mathrm{BN}$ & UJ & UG/L & 07/10/2006 & & DNT-334-06 \\
\hline GWM464012X & USGS-108 & $613-627$ & Mercury & 0.093 & & B & & UG/L & $07 / 10 / 2006$ & & DNT-334-06 \\
\hline GWM46401A1 & USGS-108 & $613-627$ & Alkalinity & 204 & & & & $\mathrm{MG} / \mathrm{L}$ & $07 / 10 / 2006$ & & DNT-328-06 \\
\hline GWM46401AN & USGS-108 & $613-627$ & Chloride & 16.3 & & $\mathrm{~J}$ & & MG/L & $07 / 10 / 2006$ & & DNT-328-06 \\
\hline GWM46401AN & USGS-108 & $613-627$ & Fluoride & 0.26 & & & & MG/L & $07 / 10 / 2006$ & & DNT-328-06 \\
\hline GWM46401AN & USGS-108 & $613-627$ & Sulfate & 22.2 & & & & MG/L & $07 / 10 / 2006$ & & DNT-328-06 \\
\hline GWM46401N2 & USGS-108 & $613-627$ & Nitrate/Nitrite as $\mathrm{N}$ & 752 & & & & UG/L & $07 / 10 / 2006$ & & DNT-328-06 \\
\hline GWM46401VL & USGS-108 & $613-627$ & Acetone & 2 & & $\mathrm{u}$ & $\mathrm{R}$ & & $07 / 10 / 2006$ & & JGJ-076-06 \\
\hline GWM46401VL & USGS-108 & $613-627$ & Benzene & 1 & & $\mathrm{u}$ & & & $07 / 10 / 2006$ & & JGJ-076-06 \\
\hline GWM46401VL & USGS-108 & $613-627$ & Bromodichloromethane & 1 & & $\mathrm{u}$ & & & $07 / 10 / 2006$ & & JGJ-076-06 \\
\hline GWM46401VL & USGS-108 & $613-627$ & Bromoform & 1 & & $\mathrm{u}$ & UJ & & 07/10/2006 & & JGJ-076-06 \\
\hline GWM46401VL & USGS-108 & $613-627$ & Bromomethane & 2 & & $u$ & & & 07/10/2006 & & JGJ-076-06 \\
\hline GWM46401VL & USGS-108 & $613-627$ & 2-Butanone & 5 & & $\mathrm{u}$ & $\mathrm{R}$ & & $07 / 10 / 2006$ & & JGJ-076-06 \\
\hline GWM46401VL & USGS-108 & $613-627$ & Carbon disulfide & 1 & & $\mathrm{u}$ & & & $07 / 10 / 2006$ & & JGJ-076-06 \\
\hline GWM46401VL & USGS-108 & $613-627$ & Carbon tetrachloride & 1 & & u & & & $07 / 10 / 2006$ & & JGJ-076-06 \\
\hline GWM46401VL & USGS-108 & $613-627$ & Chlorobenzene & 1 & & $u$ & & & 07/10/2006 & & JGJ-076-06 \\
\hline GWM46401VL & USGS-108 & $613-627$ & Dibromochloromethane & 1 & & $\mathrm{u}$ & & & $07 / 10 / 2006$ & & JGJ-076-06 \\
\hline GWM46401VL & USGS-108 & $613-627$ & 1,2-Dibromo-3-chloropropane & 1 & & $\mathrm{u}$ & $\mathrm{R}$ & & 07/10/2006 & & JGJ-076-06 \\
\hline GWM46401VL & USGS-108 & $613-627$ & Chloroethane & 2 & & $\mathrm{u}$ & & & 07/10/2006 & & JGJ-076-06 \\
\hline GWM46401VL & USGS-108 & $613-627$ & Chloroform & 1 & & $\mathrm{u}$ & & & 07/10/2006 & & JGJ-076-06 \\
\hline GWM46401VL & USGS-108 & $613-627$ & Chloromethane & 2 & & $\mathrm{u}$ & & & $07 / 10 / 2006$ & & JGJ-076-06 \\
\hline GWM46401VL & USGS-108 & $613-627$ & Cyclohexane & 1 & & $\mathrm{u}$ & & & 07/10/2006 & & JGJ-076-06 \\
\hline GWM46401VL & USGS-108 & $613-627$ & 1,2-Dibromoethane & 1 & & $\mathrm{u}$ & & & $07 / 10 / 2006$ & & JGJ-076-06 \\
\hline GWM46401VL & USGS-108 & $613-627$ & 1,2-Dichlorobenzene & 1 & & u & & & 07/10/2006 & & JGJ-076-06 \\
\hline GWM46401VL & USGS-108 & $613-627$ & 1,3-Dichlorobenzene & 1 & & $\mathrm{u}$ & & & $07 / 10 / 2006$ & & JGJ-076-06 \\
\hline GWM46401VL & USGS-108 & $613-627$ & 1,4-Dichlorobenzene & 1 & & $\mathrm{u}$ & & & 07/10/2006 & & JGJ-076-06 \\
\hline GWM46401VL & USGS-108 & $613-627$ & Dichlorodifluoromethane & 2 & & $\mathrm{u}$ & & & 07/10/2006 & & JGJ-076-06 \\
\hline GWM46401VL & USGS-108 & $613-627$ & 1,1-Dichloroethane & 1 & & $\mathrm{u}$ & & & $07 / 10 / 2006$ & & JGJ-076-06 \\
\hline GWM46401VL & USGS-108 & $613-627$ & 1,2-Dichloroethane & 1 & & $\mathrm{u}$ & & & $07 / 10 / 2006$ & & JGJ-076-06 \\
\hline GWM46401VL & USGS-108 & $613-627$ & 1,1-Dichloroethene & 1 & & $u$ & & & 07/10/2006 & & JGJ-076-06 \\
\hline GWM46401VL & USGS-108 & $613-627$ & cis-1,2-Dichloroethene & 1 & & $\mathrm{u}$ & & & $07 / 10 / 2006$ & & JGJ-076-06 \\
\hline GWM46401VL & USGS-108 & $613-627$ & trans-1,2-Dichloroethene & 1 & & $u$ & & & 07/10/2006 & & JGJ-076-06 \\
\hline GWM46401VL & USGS-108 & $613-627$ & 1,2-Dichloropropane & 1 & & $\mathrm{u}$ & & & $07 / 10 / 2006$ & & JGJ-076-06 \\
\hline GWM46401VL & USGS-108 & $613-627$ & cis-1,3-Dichloropropene & 1 & & $\mathrm{u}$ & & & $07 / 10 / 2006$ & & JGJ-076-06 \\
\hline GWM46401VL & USGS-108 & $613-627$ & trans-1,3-Dichloropropene & 1 & & $\mathrm{u}$ & & & $07 / 10 / 2006$ & & JGJ-076-06 \\
\hline GWM46401VL & USGS-108 & $613-627$ & Ethylbenzene & 1 & & $\mathrm{u}$ & & & 07/10/2006 & & JGJ-076-06 \\
\hline GWM46401VL & USGS-108 & $613-627$ & Trichlorofluoromethane & 1 & & $u$ & & & $07 / 10 / 2006$ & & JGJ-076-06 \\
\hline
\end{tabular}


Table A-3. (continued).

\begin{tabular}{|c|c|c|c|c|c|c|c|c|c|c|c|}
\hline $\begin{array}{c}\text { Field Sample } \\
\text { Number }\end{array}$ & Location & Depth & Compound & $\begin{array}{c}\text { Sample } \\
\text { Result }\end{array}$ & $\begin{array}{c}\text { Sample } \\
\text { Error }\end{array}$ & $\begin{array}{c}\text { Result } \\
\text { Qualifier }\end{array}$ & $\begin{array}{c}\text { Validation } \\
\text { Flag }\end{array}$ & $\begin{array}{c}\text { Sample } \\
\text { Units }\end{array}$ & $\begin{array}{c}\text { Date Sample } \\
\text { Collected }\end{array}$ & MDA & $\begin{array}{c}\text { L\&V Report } \\
\text { Number }\end{array}$ \\
\hline GWM46401VL & USGS-108 & $613-627$ & 2-Hexanone & 5 & & $\bar{U}$ & & & $07 / 10 / 2006$ & & JGJ-076-06 \\
\hline GWM46401VL & USGS-108 & $613-627$ & Isopropylbenzene & 1 & & $\mathrm{u}$ & & & $07 / 10 / 2006$ & & JGJ-076-06 \\
\hline GWM46401VL & USGS-108 & $613-627$ & Methyl acetate & 5 & & $u$ & $\mathrm{R}$ & & 07/10/2006 & & JGJ-076-06 \\
\hline GWM46401VL & USGS-108 & $613-627$ & Methyl cyclohexane & 4 & & $u$ & & & $07 / 10 / 2006$ & & JGJ-076-06 \\
\hline GWM46401VL & USGS-108 & $613-627$ & Methylene Chloride & 1 & & $\mathrm{u}$ & & & $07 / 10 / 2006$ & & JGJ-076-06 \\
\hline GWM46401VL & USGS-108 & $613-627$ & 4-Methyl-2-pentanone & 5 & & $\mathrm{u}$ & & & $07 / 10 / 2006$ & & JGJ-076-06 \\
\hline GWM46401VL & USGS-108 & $613-627$ & Styrene & 1 & & $u$ & & & $07 / 10 / 2006$ & & JGJ-076-06 \\
\hline GWM46401VL & USGS-108 & $613-627$ & 1,1,2,2-Tetrachloroethane & 1 & & $u$ & & & $07 / 10 / 2006$ & & JGJ-076-06 \\
\hline GWM46401VL & USGS-108 & $613-627$ & Tetrachloroethene & 1 & & $u$ & UJ & & $07 / 10 / 2006$ & & JGJ-076-06 \\
\hline GWM46401VL & USGS-108 & $613-627$ & 1,2,4-Trichlorobenzene & 1 & & $\mathrm{u}$ & & & $07 / 10 / 2006$ & & JGJ-076-06 \\
\hline GWM46401VL & USGS-108 & $613-627$ & 1,1,1-Trichloroethane & 1 & & $u$ & & & $07 / 10 / 2006$ & & JGJ-076-06 \\
\hline GWM46401VL & USGS-108 & $613-627$ & 1,1,2-Trichloroethane & 1 & & $\mathrm{u}$ & & & 07/10/2006 & & JGJ-076-06 \\
\hline GWM46401VL & USGS-108 & $613-627$ & Trichloroethene & 1 & & $u$ & & & 07/10/2006 & & JGJ-076-06 \\
\hline GWM46401VL & USGS-108 & $613-627$ & 1,1,2-Trichloro-1,2,2-trifluoroethane & 1 & & $u$ & & & $07 / 10 / 2006$ & & JGJ-076-06 \\
\hline GWM46401VL & USGS-108 & $613-627$ & Vinyl Chloride & 2 & & $\mathrm{u}$ & & & 07/10/2006 & & JGJ-076-06 \\
\hline GWM46401VL & USGS-108 & $613-627$ & Xylene (Total) & 3 & & $\mathrm{u}$ & & & 07/10/2006 & & JGJ-076-06 \\
\hline GWM46401VL & USGS-108 & $613-627$ & methyl tert-butyl ether & 2 & & $u$ & & & 07/10/2006 & & JGJ-076-06 \\
\hline GWM46401R8 & USGS-108 & $613-627$ & Tritium & $0.00 \mathrm{E}+00$ & $1.04 \mathrm{E}+02$ & & $\mathrm{U}$ & $\mathrm{PCl} / \mathrm{L}$ & $07 / 10 / 2006$ & $3.61 \mathrm{E}+02$ & SOS-TL229-06 \\
\hline GWM46401UX & USGS-108 & $613-627$ & lodine-129 & 1.57E-02 & $2.64 \mathrm{E}-02$ & & UJ & $\mathrm{PCI} / \mathrm{L}$ & 07/10/2006 & 1.02E-01 & SOS-TL230-06 \\
\hline GWM46401RH & USGS-108 & $613-627$ & Strontium-90 & $-8.78 \mathrm{E}-02$ & $8.24 \mathrm{E}-02$ & & $\mathrm{u}$ & $\mathrm{PCI} / \mathrm{L}$ & 07/10/2006 & 4.60E-01 & SOS-TL231-06 \\
\hline GWM46401RH & USGS-108 & $613-627$ & Technetium-99 & $-2.45 E+00$ & $1.83 E+00$ & & $\mathrm{u}$ & $\mathrm{PCI} / \mathrm{L}$ & 07/10/2006 & $6.32 \mathrm{E}+00$ & SOS-TL231-06 \\
\hline GWM463012X & USGS-108 & $657-760$ & Aluminum & 9.8 & & B & & UG/L & 07/10/2006 & & DNT-337-06 \\
\hline GWM463012X & USGS-108 & $657-760$ & Antimony & 0.5 & & U & & UG/L & 07/10/2006 & & DNT-337-06 \\
\hline GWM463012X & USGS-108 & $657-760$ & Arsenic & 2 & & $u$ & & UG/L & 07/10/2006 & & DNT-337-06 \\
\hline GWM463012X & USGS-108 & $657-760$ & Barium & 36.5 & & & & UG/L & 07/10/2006 & & DNT-337-06 \\
\hline GWM463012X & USGS-108 & $657-760$ & Beryllium & 0.088 & & $u$ & & UG/L & 07/10/2006 & & DNT-337-06 \\
\hline GWM463012X & USGS-108 & $657-760$ & Cadmium & 0.057 & & u & & UG/L & $07 / 10 / 2006$ & & DNT-337-06 \\
\hline GWM463012X & USGS-108 & $657-760$ & Calcium & 33200 & & & & UG/L & $07 / 10 / 2006$ & & DNT-337-06 \\
\hline GWM463012X & USGS-108 & $657-760$ & Chromium & 6.7 & & & & UG/L & 07/10/2006 & & DNT-337-06 \\
\hline GWM463012X & USGS-108 & $657-760$ & Cobalt & 0.31 & & $\mathrm{u}$ & & UG/L & $07 / 10 / 2006$ & & DNT-337-06 \\
\hline GWM463012X & USGS-108 & $657-760$ & Copper & 0.47 & & B & & UG/L & $07 / 10 / 2006$ & & DNT-337-06 \\
\hline GWM463012X & USGS-108 & $657-760$ & Iron & 9.4 & & $u$ & & UG/L & $07 / 10 / 2006$ & & DNT-337-06 \\
\hline GWM463012X & USGS-108 & $657-760$ & Lead & 0.49 & & $u$ & & UG/L & 07/10/2006 & & DNT-337-06 \\
\hline GWM463012X & USGS-108 & $657-760$ & Magnesium & 12700 & & & & UG/L & 07/10/2006 & & DNT-337-06 \\
\hline GWM463012X & USGS-108 & $657-760$ & Manganese & 0.84 & & B & & UG/L & 07/10/2006 & & DNT-337-06 \\
\hline GWM463012X & USGS-108 & $657-760$ & Nickel & 0.93 & & B & & UG/L & $07 / 10 / 2006$ & & DNT-337-06 \\
\hline GWM463012X & USGS-108 & $657-760$ & Potassium & 2310 & & & & UG/L & 07/10/2006 & & DNT-337-06 \\
\hline GWM463012X & USGS-108 & $657-760$ & Selenium & 2 & & B & & UG/L & 07/10/2006 & & DNT-337-06 \\
\hline GWM463012X & USGS-108 & $657-760$ & Silver & 0.2 & & $u$ & & UG/L & $07 / 10 / 2006$ & & DNT-337-06 \\
\hline GWM463012X & USGS-108 & $657-760$ & Uranium & 1.9 & & & & UG/L & 07/10/2006 & & DNT-337-06 \\
\hline GWM463012X & USGS-108 & $657-760$ & Vanadium & 4.7 & & B & & UG/L & 07/10/2006 & & DNT-337-06 \\
\hline GWM463012X & USGS-108 & $657-760$ & Zinc & 4.4 & & $\mathrm{BE}$ & $\mathrm{u}$ & UG/L & $07 / 10 / 2006$ & & DNT-337-06 \\
\hline GWM463012X & USGS-108 & $657-760$ & Mercury & 0.093 & & $\mathrm{U}$ & UJ & UG/L & 07/10/2006 & & DNT-337-06 \\
\hline GWM46301A1 & USGS-108 & $657-760$ & Alkalinity & 134 & & & $\mathrm{~J}$ & MG/L & 07/10/2006 & & DNT-341-06 \\
\hline GWM46301AN & USGS-108 & $657-760$ & Chloride & 15.4 & & $\mathrm{~J}$ & & $M G / L$ & $07 / 10 / 2006$ & & DNT-341-06 \\
\hline GWM46301AN & USGS-108 & $657-760$ & Fluoride & 0.26 & & & & $M G / L$ & 07/10/2006 & & DNT-341-06 \\
\hline GWM46301AN & USGS-108 & $657-760$ & Sulfate & 21.1 & & & & $M G / L$ & $07 / 10 / 2006$ & & DNT-341-06 \\
\hline GWM46301N2 & USGS-108 & $657-760$ & Nitrate/Nitrite as $\mathrm{N}$ & 776 & & & & UG/L & $07 / 10 / 2006$ & & DNT-341-06 \\
\hline GWM46301VL & USGS-108 & $657-760$ & Acetone & 2 & & U & $\mathrm{R}$ & & $07 / 10 / 2006$ & & JGJ-076-06 \\
\hline GWM46301VL & USGS-108 & $657-760$ & Benzene & 1 & & $u$ & & & 07/10/2006 & & JGJ-076-06 \\
\hline GWM46301VL & USGS-108 & $657-760$ & Bromodichloromethane & 1 & & $u$ & & & 07/10/2006 & & JGJ-076-06 \\
\hline GWM46301VL & USGS-108 & $657-760$ & Bromoform & 1 & & $u$ & UJ & & $07 / 10 / 2006$ & & JGJ-076-06 \\
\hline GWM46301VL & USGS-108 & $657-760$ & Bromomethane & 2 & & $u$ & & & 07/10/2006 & & JGJ-076-06 \\
\hline GWM46301VL & USGS-108 & $657-760$ & 2-Butanone & 5 & & U & $\mathrm{R}$ & & $07 / 10 / 2006$ & & JGJ-076-06 \\
\hline GWM46301VL & USGS-108 & $657-760$ & Carbon disulfide & 1 & & $u$ & & & 07/10/2006 & & JGJ-076-06 \\
\hline GWM46301VL & USGS-108 & $657-760$ & Carbon tetrachloride & 1 & & $\mathrm{u}$ & & & 07/10/2006 & & JGJ-076-06 \\
\hline GWM46301VL & USGS-108 & $657-760$ & Chlorobenzene & 1 & & $\mathrm{u}$ & & & $07 / 10 / 2006$ & & JGJ-076-06 \\
\hline GWM46301VL & USGS-108 & $657-760$ & Dibromochloromethane & 1 & & $\mathrm{u}$ & & & $07 / 10 / 2006$ & & JGJ-076-06 \\
\hline GWM46301VL & USGS-108 & $657-760$ & 1,2-Dibromo-3-chloropropane & 1 & & $\mathrm{u}$ & $\mathrm{R}$ & & $07 / 10 / 2006$ & & JGJ-076-06 \\
\hline GWM46301VL & USGS-108 & $657-760$ & Chloroethane & 2 & & $\mathrm{u}$ & & & 07/10/2006 & & JGJ-076-06 \\
\hline GWM46301VL & USGS-108 & $657-760$ & Chloroform & 1 & & $\mathrm{u}$ & & & $07 / 10 / 2006$ & & JGJ-076-06 \\
\hline GWM46301VL & USGS-108 & $657-760$ & Chloromethane & 2 & & $\mathrm{u}$ & & & 07/10/2006 & & JGJ-076-06 \\
\hline GWM46301VL & USGS-108 & $657-760$ & Cyclohexane & 1 & & u & & & $07 / 10 / 2006$ & & JGJ-076-06 \\
\hline GWM46301VL & USGS-108 & $657-760$ & 1,2-Dibromoethane & 1 & & $\mathrm{u}$ & & & 07/10/2006 & & JGJ-076-06 \\
\hline GWM46301VL & USGS-108 & $657-760$ & 1,2-Dichlorobenzene & 1 & & $\mathrm{u}$ & & & 07/10/2006 & & JGJ-076-06 \\
\hline GWM46301VL & USGS-108 & $657-760$ & 1,3-Dichlorobenzene & 1 & & $\mathrm{u}$ & & & 07/10/2006 & & JGJ-076-06 \\
\hline GWM46301VL & USGS-108 & $657-760$ & 1,4-Dichlorobenzene & 1 & & $u$ & & & $07 / 10 / 2006$ & & JGJ-076-06 \\
\hline GWM46301VL & USGS-108 & $657-760$ & Dichlorodifluoromethane & 2 & & $\mathrm{u}$ & & & $07 / 10 / 2006$ & & JGJ-076-06 \\
\hline GWM46301VL & USGS-108 & $657-760$ & 1,1-Dichloroethane & 1 & & $\mathrm{u}$ & & & 07/10/2006 & & JGJ-076-06 \\
\hline GWM46301VL & USGS-108 & $657-760$ & 1,2-Dichloroethane & 1 & & $\mathrm{u}$ & & & $07 / 10 / 2006$ & & JGJ-076-06 \\
\hline GWM46301VL & USGS-108 & $657-760$ & 1,1-Dichloroethene & 1 & & $u$ & & & $07 / 10 / 2006$ & & JGJ-076-06 \\
\hline GWM46301VL & USGS-108 & $657-760$ & cis-1,2-Dichloroethene & 1 & & $\mathrm{U}$ & & & $07 / 10 / 2006$ & & JGJ-076-06 \\
\hline GWM46301VL & USGS-108 & $657-760$ & trans-1,2-Dichloroethene & 1 & & $\mathrm{u}$ & & & 07/10/2006 & & JGJ-076-06 \\
\hline GWM46301VL & USGS-108 & $657-760$ & 1,2-Dichloropropane & 1 & & $\mathrm{u}$ & & & 07/10/2006 & & JGJ-076-06 \\
\hline GWM46301VL & USGS-108 & $657-760$ & cis-1,3-Dichloropropene & 1 & & $\mathrm{u}$ & & & 07/10/2006 & & JGJ-076-06 \\
\hline GWM46301VL & USGS-108 & $657-760$ & trans-1,3-Dichloropropene & 1 & & $u$ & & & $07 / 10 / 2006$ & & JGJ-076-06 \\
\hline
\end{tabular}


Table A-3. (continued).

\begin{tabular}{|c|c|c|c|c|c|c|c|c|c|c|c|}
\hline $\begin{array}{l}\text { Field Sample } \\
\text { Number }\end{array}$ & Location & Depth & Compound & $\begin{array}{l}\text { Sample } \\
\text { Result }\end{array}$ & $\begin{array}{c}\text { Sample } \\
\text { Error }\end{array}$ & $\begin{array}{c}\text { Result } \\
\text { Qualifier }\end{array}$ & $\begin{array}{l}\text { Validation } \\
\text { Flag }\end{array}$ & $\begin{array}{l}\text { Sample } \\
\text { Units }\end{array}$ & $\begin{array}{c}\text { Date Sample } \\
\text { Collected }\end{array}$ & MDA & $\begin{array}{l}\text { L\&V Report } \\
\text { Number }\end{array}$ \\
\hline GWM46301VL & USGS-108 & $657-760$ & Ethylbenzene & 1 & & $U$ & & & $07 / 10 / 2006$ & & JGJ-076-06 \\
\hline GWM46301VL & USGS-108 & $657-760$ & Trichlorofluoromethane & 1 & & U & & & $07 / 10 / 2006$ & & JGJ-076-06 \\
\hline GWM46301VL & USGS-108 & $657-760$ & 2-Hexanone & 5 & & $u$ & & & 07/10/2006 & & JGJ-076-06 \\
\hline GWM46301VL & USGS-108 & $657-760$ & Isopropylbenzene & 1 & & $\mathrm{U}$ & & & 07/10/2006 & & JGJ-076-06 \\
\hline GWM46301VL & USGS-108 & $657-760$ & Methyl acetate & 5 & & $\mathrm{u}$ & $\mathrm{R}$ & & 07/10/2006 & & JGJ-076-06 \\
\hline GWM46301VL & USGS-108 & $657-760$ & Methyl cyclohexane & 4 & & U & & & 07/10/2006 & & JGJ-076-06 \\
\hline GWM46301VL & USGS-108 & $657-760$ & Methylene Chloride & 1 & & $u$ & & & 07/10/2006 & & JGJ-076-06 \\
\hline GWM46301VL & USGS-108 & $657-760$ & 4-Methyl-2-pentanone & 5 & & u & & & 07/10/2006 & & JGJ-076-06 \\
\hline GWM46301VL & USGS-108 & $657-760$ & Styrene & 1 & & $u$ & & & 07/10/2006 & & JGJ-076-06 \\
\hline GWM46301VL & USGS-108 & $657-760$ & Tetrachloroethene & 1 & & $u$ & UJ & & 07/10/2006 & & JGJ-076-06 \\
\hline GWM46301VL & USGS-108 & $657-760$ & Toluene & 1 & & U & & & $07 / 10 / 2006$ & & JGJ-076-06 \\
\hline GWM46301VL & USGS-108 & $657-760$ & 1,2,4-Trichlorobenzene & 1 & & U & & & $07 / 10 / 2006$ & & JGJ-076-06 \\
\hline GWM46301VL & USGS-108 & $657-760$ & 1,1,1-Trichloroethane & 1 & & $u$ & & & $07 / 10 / 2006$ & & JGJ-076-06 \\
\hline GWM46301VL & USGS-108 & $657-760$ & 1,1,2-Trichloroethane & 1 & & U & & & $07 / 10 / 2006$ & & JGJ-076-06 \\
\hline GWM46301VL & USGS-108 & $657-760$ & Trichloroethene & 1 & & U & & & 07/10/2006 & & JGJ-076-06 \\
\hline GWM46301VL & USGS-108 & $657-760$ & 1,1,2-Trichloro-1,2,2-trifluoroethane & 1 & & $\mathrm{U}$ & & & $07 / 10 / 2006$ & & JGJ-076-06 \\
\hline GWM46301VL & USGS-108 & $657-760$ & Vinyl Chloride & 2 & & $\mathrm{u}$ & & & 07/10/2006 & & JGJ-076-06 \\
\hline GWM46301VL & USGS-108 & $657-760$ & Xylene (Total) & 3 & & $\mathrm{u}$ & & & $07 / 10 / 2006$ & & JGJ-076-06 \\
\hline GWM46301VL & USGS-108 & $657-760$ & methyl tert-butyl ether & 2 & & $\mathrm{u}$ & & & 07/10/2006 & & JGJ-076-06 \\
\hline GWM46301RH & USGS-108 & $657-760$ & Gross Alpha & $1.38 \mathrm{E}+00$ & $5.46 \mathrm{E}-01$ & & UJ & $\mathrm{PCI} / \mathrm{L}$ & $07 / 10 / 2006$ & $1.72 \mathrm{E}+00$ & SOS-TL231-06 \\
\hline GWM46301RH & USGS-108 & $657-760$ & Gross Beta & $3.45 \mathrm{E}+00$ & $8.52 \mathrm{E}-01$ & & & $\mathrm{PCI} / \mathrm{L}$ & 07/10/2006 & $3.16 \mathrm{E}+00$ & SOS-TL231-06 \\
\hline GWM46301RH & USGS-108 & $657-760$ & Strontium-90 & $-2.45 \mathrm{E}-01$ & 7.12E-02 & & U & $\mathrm{PCI} / \mathrm{L}$ & $07 / 10 / 2006$ & 4.64E-01 & SOS-TL231-06 \\
\hline GWM46301RH & USGS-108 & $657-760$ & Technetium-99 & $-2.62 \mathrm{E}+00$ & $1.86 \mathrm{E}+00$ & & U & $\mathrm{PCI} / \mathrm{L}$ & $07 / 10 / 2006$ & $6.45 \mathrm{E}+00$ & SOS-TL231-06 \\
\hline
\end{tabular}


Table A-4. Waste Area Group 10 Westbay monitoring data for 2005.

\begin{tabular}{|c|c|c|c|c|c|c|c|c|c|c|c|}
\hline $\begin{array}{c}\text { Field Sample } \\
\text { Number }\end{array}$ & Location & Depth & Compound & $\begin{array}{c}\text { Sample } \\
\text { Result }\end{array}$ & $\begin{array}{c}\text { Sample } \\
\text { Error }\end{array}$ & $\begin{array}{c}\text { Result } \\
\text { Qualifier }\end{array}$ & $\begin{array}{c}\text { Validation } \\
\text { Flag }\end{array}$ & $\begin{array}{c}\text { Sample } \\
\text { Units }\end{array}$ & $\begin{array}{c}\text { Date Sample } \\
\text { Collected }\end{array}$ & MDA & $\begin{array}{c}\text { L\&V Report } \\
\text { Number }\end{array}$ \\
\hline GWM429012X & Field Blank & NA & Antimony & 0.61 & & $\mathrm{U}$ & & UG/L & $09 / 30 / 2005$ & & DNT-001-06 \\
\hline GWM429012X & Field Blank & NA & Arsenic & 1.8 & & U & & UG/L & 09/30/2005 & & DNT-001-06 \\
\hline GWM429012X & Field Blank & NA & Beryllium & 0.13 & & $U$ & & UG/L & $09 / 30 / 2005$ & & DNT-001-06 \\
\hline GWM429012X & Field Blank & NA & Cadmium & 0.067 & & $\mathrm{U}$ & & UG/L & 09/30/2005 & & DNT-001-06 \\
\hline GWM429012X & Field Blank & NA & Chromium & 3.7 & & U & & UG/L & 09/30/2005 & & DNT-001-06 \\
\hline GWM429012X & Field Blank & NA & Manganese & 0.54 & & $u$ & & UG/L & 09/30/2005 & & DNT-001-06 \\
\hline GWM429012X & Field Blank & NA & Silver & 1.5 & & U & & UG/L & 09/30/2005 & & DNT-001-06 \\
\hline GWM429012X & Field Blank & NA & Thallium & 0.56 & & B & $u$ & UG/L & 09/30/2005 & & DNT-001-06 \\
\hline GWM429012X & Field Blank & NA & Uranium & 0.14 & & U & & UG/L & 09/30/2005 & & DNT-001-06 \\
\hline GWM429012X & Field Blank & NA & Mercury & 0.046 & & U & & UG/L & 09/30/2005 & & DNT-001-06 \\
\hline GWM429012X & Field Blank & NA & Aluminum & 22.1 & & U & & UG/L & 09/30/2005 & & DNT-001-06 \\
\hline GWM429012X & Field Blank & NA & Barium & 0.85 & & U & & UG/L & 09/30/2005 & & DNT-001-06 \\
\hline GWM429012X & Field Blank & NA & Calcium & 11.5 & & B & & UG/L & 09/30/2005 & & DNT-001-06 \\
\hline GWM429012X & Field Blank & NA & Cobalt & 2.8 & & $u$ & & UG/L & 09/30/2005 & & DNT-001-06 \\
\hline GWM429012X & Field Blank & NA & Copper & 1.1 & & $\mathrm{U}$ & & UG/L & 09/30/2005 & & DNT-001-06 \\
\hline GWM429012X & Field Blank & NA & Iron & 7.4 & & U & & UG/L & 09/30/2005 & & DNT-001-06 \\
\hline GWM429012X & Field Blank & NA & Lead & 1.1 & & U & & UG/L & 09/30/2005 & & DNT-001-06 \\
\hline GWM429012X & Field Blank & NA & Lithium & 3 & & U & & UG/L & 09/30/2005 & & DNT-001-06 \\
\hline GWM429012X & Field Blank & NA & Magnesium & 45.7 & & U & & UG/L & 09/30/2005 & & DNT-001-06 \\
\hline GWM429012X & Field Blank & NA & Molybdenum & 3.7 & & U & & UG/L & 09/30/2005 & & DNT-001-06 \\
\hline GWM429012X & Field Blank & NA & Nickel & 3.2 & & $\mathrm{U}$ & & UG/L & 09/30/2005 & & DNT-001-06 \\
\hline GWM429012X & Field Blank & NA & Potassium & 961 & & U & & UG/L & 09/30/2005 & & DNT-001-06 \\
\hline GWM429012X & Field Blank & NA & Selenium & 2 & & $\mathrm{U}$ & & UG/L & 09/30/2005 & & DNT-001-06 \\
\hline GWM429012X & Field Blank & NA & Sodium & 58.5 & & U & & UG/L & 09/30/2005 & & DNT-001-06 \\
\hline GWM429012X & Field Blank & NA & Strontium & 0.63 & & U & & UG/L & 09/30/2005 & & DNT-001-06 \\
\hline GWM429012X & Field Blank & NA & Tin & 3.9 & & U & & UG/L & 09/30/2005 & & DNT-001-06 \\
\hline GWM429012X & Field Blank & NA & Vanadium & 2.5 & & $\mathrm{U}$ & & UG/L & 09/30/2005 & & DNT-001-06 \\
\hline GWM429012X & Field Blank & NA & Zinc & 1.5 & & B & u & UG/L & 09/30/2005 & & DNT-001-06 \\
\hline GWM42901A1 & Field Blank & NA & Total Alkalinity & 5 & & U & & MG/L & 09/30/2005 & & DNT-499-05 \\
\hline GWM42901AN & Field Blank & NA & Bromide & 0.25 & & U & & $M G / L$ & 09/30/2005 & & DNT-499-05 \\
\hline GWM42901AN & Field Blank & NA & Chloride & 0.051 & & $\mathrm{JB}$ & $\mathrm{R}$ & $M G / L$ & 09/30/2005 & & DNT-499-05 \\
\hline GWM42901AN & Field Blank & NA & Fluoride & 0.1 & & U & & $M G / L$ & $09 / 30 / 2005$ & & DNT-499-05 \\
\hline GWM42901AN & Field Blank & NA & Sulfate & 0.5 & & $\mathrm{U}$ & & $M G / L$ & 09/30/2005 & & DNT-499-05 \\
\hline GWM42901N2 & Field Blank & NA & Nitrate/Nitrite as $\mathrm{N}$ & 0.05 & & u & & $M G / L$ & 09/30/2005 & & DNT-499-05 \\
\hline GWM42901AV & Field Blank & NA & Acetone & 10 & & U & $\mathrm{R}$ & UG/L & 09/30/2005 & & HCJ-099-05 \\
\hline GWM42901AV & Field Blank & NA & Acetonitrile & 20 & & U & & UG/L & 09/30/2005 & & HCJ-099-05 \\
\hline GWM42901AV & Field Blank & NA & Acrolein & 5 & & $\mathrm{U}$ & $\mathrm{R}$ & UG/L & 09/30/2005 & & HCJ-099-05 \\
\hline GWM42901AV & Field Blank & NA & Acrylonitrile & 1 & & U & $\mathrm{R}$ & UG/L & 09/30/2005 & & HCJ-099-05 \\
\hline GWM42901AV & Field Blank & NA & Benzene & 1 & & $\mathrm{U}$ & & UG/L & 09/30/2005 & & HCJ-099-05 \\
\hline GWM42901AV & Field Blank & NA & Bromodichloromethane & 1 & & u & & UG/L & 09/30/2005 & & HCJ-099-05 \\
\hline GWM42901AV & Field Blank & NA & Bromoform & 2 & & $\mathrm{U}$ & & UG/L & 09/30/2005 & & HCJ-099-05 \\
\hline GWM42901AV & Field Blank & NA & Bromomethane & 1 & & U & & UG/L & 09/30/2005 & & HCJ-099-05 \\
\hline GWM42901AV & Field Blank & NA & 2-Butanone & 10 & & U & $\mathrm{R}$ & UG/L & 09/30/2005 & & HCJ-099-05 \\
\hline GWM42901AV & Field Blank & NA & Carbon disulfide & 5 & & U & & UG/L & 09/30/2005 & & HCJ-099-05 \\
\hline GWM42901AV & Field Blank & NA & Carbon tetrachloride & 1 & & $\mathrm{U}$ & & UG/L & 09/30/2005 & & HCJ-099-05 \\
\hline GWM42901AV & Field Blank & NA & Chlorobenzene & 1 & & U & & UG/L & 09/30/2005 & & HCJ-099-05 \\
\hline GWM42901AV & Field Blank & NA & Chloroprene & 5 & & $\mathrm{U}$ & & UG/L & 09/30/2005 & & HCJ-099-05 \\
\hline GWM42901AV & Field Blank & NA & Dibromochloromethane & 1 & & u & & UG/L & 09/30/2005 & & HCJ-099-05 \\
\hline GWM42901AV & Field Blank & NA & Chloroethane & 1 & & $\mathrm{u}$ & & UG/L & 09/30/2005 & & HCJ-099-05 \\
\hline GWM42901AV & Field Blank & NA & Chloroform & 1 & & u & & UG/L & 09/30/2005 & & HCJ-099-05 \\
\hline GWM42901AV & Field Blank & NA & Chloromethane & 5 & & $\mathrm{u}$ & & UG/L & 09/30/2005 & & HCJ-099-05 \\
\hline GWM42901AV & Field Blank & NA & Allyl chloride & 5 & & U & & UG/L & 09/30/2005 & & HCJ-099-05 \\
\hline GWM42901AV & Field Blank & NA & 1,2-Dibromo-3-chloropropane & 5 & & $\mathrm{u}$ & & UG/L & 09/30/2005 & & HCJ-099-05 \\
\hline GWM42901AV & Field Blank & NA & 1,2-Dibromoethane & 1 & & u & & UG/L & 09/30/2005 & & HCJ-099-05 \\
\hline GWM42901AV & Field Blank & NA & Dibromomethane & 1 & & $\mathrm{U}$ & & UG/L & 09/30/2005 & & HCJ-099-05 \\
\hline GWM42901AV & Field Blank & NA & trans-1,4-Dichloro-2-butene & 5 & & $\mathrm{u}$ & & UG/L & 09/30/2005 & & HCJ-099-05 \\
\hline GWM42901AV & Field Blank & NA & Dichlorodifluoromethane & 5 & & $\mathrm{u}$ & & UG/L & 09/30/2005 & & HCJ-099-05 \\
\hline GWM42901AV & Field Blank & NA & 1,1-Dichloroethane & 1 & & u & & UG/L & 09/30/2005 & & HCJ-099-05 \\
\hline GWM42901AV & Field Blank & NA & 1,2-Dichloroethane & 1 & & $\mathrm{u}$ & & UG/L & 09/30/2005 & & HCJ-099-05 \\
\hline GWM42901AV & Field Blank & NA & cis-1,2-Dichloroethene & 1 & & U & & UG/L & 09/30/2005 & & HCJ-099-05 \\
\hline GWM42901AV & Field Blank & NA & trans-1,2-Dichloroethene & 1 & & $\mathrm{U}$ & & UG/L & 09/30/2005 & & HCJ-099-05 \\
\hline GWM42901AV & Field Blank & NA & 1,1-Dichloroethene & 1 & & U & & UG/L & 09/30/2005 & & HCJ-099-05 \\
\hline GWM42901AV & Field Blank & NA & 1,2-Dichloropropane & 1 & & $\mathrm{u}$ & & UG/L & 09/30/2005 & & HCJ-099-05 \\
\hline GWM42901AV & Field Blank & NA & cis-1,3-Dichloropropene & 1 & & U & & UG/L & 09/30/2005 & & HCJ-099-05 \\
\hline GWM42901AV & Field Blank & NA & trans-1,3-Dichloropropene & 1 & & $\mathrm{U}$ & & UG/L & 09/30/2005 & & HCJ-099-05 \\
\hline GWM42901AV & Field Blank & NA & 1,4-Dioxane & 80 & & u & $\mathrm{R}$ & UG/L & 09/30/2005 & & HCJ-099-05 \\
\hline GWM42901AV & Field Blank & NA & Ethylbenzene & 1 & & $\mathrm{U}$ & & UG/L & 09/30/2005 & & HCJ-099-05 \\
\hline GWM42901AV & Field Blank & NA & 2-Hexanone & 5 & & u & & UG/L & 09/30/2005 & & HCJ-099-05 \\
\hline GWM42901AV & Field Blank & NA & Methyl iodide & 2 & & $\mathrm{U}$ & & UG/L & 09/30/2005 & & HCJ-099-05 \\
\hline GWM42901AV & Field Blank & NA & Isobutyl alcohol & 80 & & U & $\mathrm{R}$ & UG/L & 09/30/2005 & & HCJ-099-05 \\
\hline GWM42901AV & Field Blank & NA & Methacrylonitrile & 5 & & $\mathrm{U}$ & & UG/L & 09/30/2005 & & HCJ-099-05 \\
\hline GWM42901AV & Field Blank & NA & Methylene Chloride & 1 & & U & & UG/L & 09/30/2005 & & HCJ-099-05 \\
\hline GWM42901AV & Field Blank & NA & Methylmethacrylate & 1 & & $\mathrm{U}$ & & UG/L & 09/30/2005 & & HCJ-099-05 \\
\hline GWM42901AV & Field Blank & NA & Methyl isobutyl ketone & 5 & & u & & UG/L & 09/30/2005 & & HCJ-099-05 \\
\hline GWM42901AV & Field Blank & NA & Propionitrile & 5 & & $\mathrm{U}$ & $\mathrm{R}$ & UG/L & 09/30/2005 & & HCJ-099-05 \\
\hline GWM42901AV & Field Blank & NA & Styrene & 1 & & U & & UG/L & 09/30/2005 & & HCJ-099-05 \\
\hline GWM42901AV & Field Blank & NA & 1,1,1,2-Tetrachloroethane & 1 & & $U$ & & UG/L & $09 / 30 / 2005$ & & HCJ-099-05 \\
\hline GWM42901AV & Field Blank & NA & 1,1,2,2-Tetrachloroethane & 1 & & U & & UG/L & 09/30/2005 & & HCJ-099-05 \\
\hline GWM42901AV & Field Blank & NA & Tetrachloroethene & 1 & & $\mathrm{U}$ & & UG/L & 09/30/2005 & & HCJ-099-05 \\
\hline GWM42901AV & Field Blank & NA & Toluene & 1 & & u & & UG/L & 09/30/2005 & & HCJ-099-05 \\
\hline GWM42901AV & Field Blank & NA & 1,1,1-Trichloroethane & 1 & & u & & UG/L & 09/30/2005 & & HCJ-099-05 \\
\hline GWM42901AV & Field Blank & NA & 1,1,2-Trichloroethane & 1 & & U & & UG/L & 09/30/2005 & & HCJ-099-05 \\
\hline GWM42901AV & Field Blank & NA & Trichloroethene & 0.48 & & $\mathrm{~J}$ & $\mathrm{~J}$ & UG/L & 09/30/2005 & & HCJ-099-05 \\
\hline GWM42901AV & Field Blank & NA & Trichlorofluoromethane & 1 & & U & & UG/L & 09/30/2005 & & HCJ-099-05 \\
\hline GWM42901AV & Field Blank & NA & 1,2,3-Trichloropropane & 1 & & $\mathrm{U}$ & & UG/L & 09/30/2005 & & HCJ-099-05 \\
\hline GWM42901AV & Field Blank & NA & Vinyl Acetate & 2 & & U & & UG/L & 09/30/2005 & & HCJ-099-05 \\
\hline
\end{tabular}


Table A-4. (continued).

\begin{tabular}{|c|c|c|c|c|c|c|c|c|c|c|c|}
\hline $\begin{array}{c}\text { Field Sample } \\
\text { Number }\end{array}$ & Location & Depth & Compound & $\begin{array}{l}\text { Sample } \\
\text { Result }\end{array}$ & $\begin{array}{l}\text { Sample } \\
\text { Error }\end{array}$ & $\begin{array}{c}\text { Result } \\
\text { Qualifier }\end{array}$ & $\begin{array}{c}\text { Validation } \\
\text { Flag }\end{array}$ & $\begin{array}{c}\text { Sample } \\
\text { Units }\end{array}$ & $\begin{array}{c}\text { Date Sample } \\
\text { Collected }\end{array}$ & MDA & $\begin{array}{l}\text { L\&V Report } \\
\text { Number }\end{array}$ \\
\hline GWM42901AV & Field Blank & NA & Vinyl Chloride & 1 & & $\bar{U}$ & & $\overline{U G / L}$ & $09 / 30 / 2005$ & & HCJ-099-05 \\
\hline GWM42901AV & Field Blank & NA & Xylenes & 3 & & U & & UG/L & 09/30/2005 & & HCJ-099-05 \\
\hline GWM42901R8 & Field Blank & NA & Tritium & $-9.60 \mathrm{E}+01$ & $1.09 \mathrm{E}+02$ & & U & $\mathrm{PCl} / \mathrm{L}$ & 09/30/2005 & $3.96 \mathrm{E}+02$ & SOS-TL342-05 \\
\hline GWM42901RH & Field Blank & NA & Uranium-233/234 & $9.88 \mathrm{E}-02$ & $3.95 \mathrm{E}-02$ & & $\mathrm{~J}$ & $\mathrm{PCl} / \mathrm{L}$ & 09/30/2005 & 7.72E-02 & SOS-TL351-05 \\
\hline GWM42901RH & Field Blank & NA & Uranium-238 & $7.60 \mathrm{E}-03$ & $1.54 \mathrm{E}-02$ & & U & $\mathrm{PCl} / \mathrm{L}$ & 09/30/2005 & $9.10 \mathrm{E}-02$ & SOS-TL351-05 \\
\hline GWM42901RH & Field Blank & NA & Uranium-235 & $0.00 \mathrm{E}+00$ & $1.00 \mathrm{E}+00$ & & U & $\mathrm{PCI} / \mathrm{L}$ & 09/30/2005 & $5.42 \mathrm{E}-02$ & SOS-TL351-05 \\
\hline GWM42901RH & Field Blank & NA & Gross Alpha & 3.37E-01 & $5.83 \mathrm{E}-01$ & & U & $\mathrm{PCI} / \mathrm{L}$ & 09/30/2005 & $2.52 \mathrm{E}+00$ & SOS-TL351-05 \\
\hline GWM42901RH & Field Blank & NA & Gross Beta & $1.56 \mathrm{E}+00$ & $1.02 \mathrm{E}+00$ & & U & $\mathrm{PCI} / \mathrm{L}$ & 09/30/2005 & $4.20 \mathrm{E}+00$ & SOS-TL351-05 \\
\hline GWM42901RH & Field Blank & NA & Strontium-90 & $6.23 \mathrm{E}-03$ & $1.42 \mathrm{E}-01$ & & U & $\mathrm{PCl} / \mathrm{L}$ & 09/30/2005 & $6.89 \mathrm{E}-01$ & SOS-TL351-05 \\
\hline GWM42901RH & Field Blank & NA & Silver-108m & $-7.46 \mathrm{E}-01$ & $1.30 \mathrm{E}+00$ & & U & $\mathrm{PCI} / \mathrm{L}$ & 09/30/2005 & $4.68 \mathrm{E}+00$ & SOS-TL351-05 \\
\hline GWM42901RH & Field Blank & NA & Silver-110m & $2.18 \mathrm{E}+00$ & 1.13E+00 & & U & $\mathrm{PCl} / \mathrm{L}$ & 09/30/2005 & $4.92 \mathrm{E}+00$ & SOS-TL351-05 \\
\hline GWM42901RH & Field Blank & NA & Cerium-144 & $1.47 \mathrm{E}+00$ & $9.24 \mathrm{E}+00$ & & U & $\mathrm{PCI} / \mathrm{L}$ & 09/30/2005 & $3.32 \mathrm{E}+01$ & SOS-TL351-05 \\
\hline GWM42901RH & Field Blank & NA & Cobalt-60 & $5.80 \mathrm{E}-01$ & $1.41 \mathrm{E}+00$ & & U & $\mathrm{PCI} / \mathrm{L}$ & 09/30/2005 & $5.73 \mathrm{E}+00$ & SOS-TL351-05 \\
\hline GWM42901RH & Field Blank & NA & Cesium-134 & $-1.64 E+00$ & $1.64 \mathrm{E}+00$ & & U & $\mathrm{PCI} / \mathrm{L}$ & 09/30/2005 & $5.49 \mathrm{E}+00$ & SOS-TL351-05 \\
\hline GWM42901RH & Field Blank & NA & Cesium-137 & $-1.01 \mathrm{E}+00$ & $1.05 E+00$ & & U & $\mathrm{PCl} / \mathrm{L}$ & 09/30/2005 & $3.65 \mathrm{E}+00$ & SOS-TL351-05 \\
\hline GWM42901RH & Field Blank & NA & Europium-152 & $1.00 \mathrm{E}+00$ & $4.10 \mathrm{E}+00$ & & U & $\mathrm{PCl} / \mathrm{L}$ & 09/30/2005 & $1.44 \mathrm{E}+01$ & SOS-TL351-05 \\
\hline GWM42901RH & Field Blank & NA & Europium-154 & $2.12 \mathrm{E}-01$ & $3.82 \mathrm{E}+00$ & & U & $\mathrm{PCI} / \mathrm{L}$ & 09/30/2005 & $1.34 \mathrm{E}+01$ & SOS-TL351-05 \\
\hline GWM42901RH & Field Blank & NA & Europium-155 & $5.68 \mathrm{E}+00$ & $4.70 \mathrm{E}+00$ & & U & $\mathrm{PCI} / \mathrm{L}$ & 09/30/2005 & $1.77 \mathrm{E}+01$ & SOS-TL351-05 \\
\hline GWM42901RH & Field Blank & NA & Manganese-54 & $1.02 \mathrm{E}+00$ & $1.27 \mathrm{E}+00$ & & U & $\mathrm{PCI} / \mathrm{L}$ & 09/30/2005 & $5.06 \mathrm{E}+00$ & SOS-TL351-05 \\
\hline GWM42901RH & Field Blank & NA & Ruthenium-106 & $1.03 E+01$ & $1.30 \mathrm{E}+01$ & & U & $\mathrm{PCI} / \mathrm{L}$ & 09/30/2005 & $5.02 \mathrm{E}+01$ & SOS-TL351-05 \\
\hline GWM42901RH & Field Blank & NA & Antimony-125 & $1.12 \mathrm{E}+00$ & $3.42 \mathrm{E}+00$ & & U & $\mathrm{PCl} / \mathrm{L}$ & 09/30/2005 & $1.31 \mathrm{E}+01$ & SOS-TL351-05 \\
\hline GWM42901RH & Field Blank & NA & Zinc-65 & $-2.61 \mathrm{E}+00$ & $3.35 E+00$ & & U & $\mathrm{PCl} / \mathrm{L}$ & 09/30/2005 & $1.01 \mathrm{E}+01$ & SOS-TL351-05 \\
\hline GWM42901RH & Field Blank & NA & Technetium-99 & $3.26 \mathrm{E}-01$ & $1.60 \mathrm{E}+00$ & & U & $\mathrm{PCl} / \mathrm{L}$ & 09/30/2005 & $5.43 \mathrm{E}+00$ & SOS-TL351-05 \\
\hline GWM418012X & MIDDLE-2050A & 474 & Antimony & 0.61 & & $\mathrm{U}$ & & UG/L & 09/30/2005 & & DNT-001-06 \\
\hline GWM418012X & MIDDLE-2050A & 474 & Arsenic & 1.8 & & U & & UG/L & 09/30/2005 & & DNT-001-06 \\
\hline GWM418012X & MIDDLE-2050A & 474 & Beryllium & 0.13 & & U & & UG/L & 09/30/2005 & & DNT-001-06 \\
\hline GWM418012X & MIDDLE-2050A & 474 & Cadmium & 1.4 & & & & UG/L & 09/30/2005 & & DNT-001-06 \\
\hline GWM418012X & MIDDLE-2050A & 474 & Chromium & 3.7 & & U & & UG/L & 09/30/2005 & & DNT-001-06 \\
\hline GWM418012X & MIDDLE-2050A & 474 & Manganese & 17.4 & & & & UG/L & 09/30/2005 & & DNT-001-06 \\
\hline GWM418012X & MIDDLE-2050A & 474 & Silver & 1.5 & & $\mathrm{U}$ & & UG/L & 09/30/2005 & & DNT-001-06 \\
\hline GWM418012X & MIDDLE-2050A & 474 & Thallium & 1.4 & & & U & UG/L & 09/30/2005 & & DNT-001-06 \\
\hline GWM418012X & MIDDLE-2050A & 474 & Uranium & 1.8 & & B & & UG/L & $09 / 30 / 2005$ & & DNT-001-06 \\
\hline GWM418012X & MIDDLE-2050A & 474 & Mercury & 0.046 & & U & & UG/L & 09/30/2005 & & DNT-001-06 \\
\hline GWM418012X & MIDDLE-2050A & 474 & Aluminum & 22.1 & & U & & UG/L & 09/30/2005 & & DNT-001-06 \\
\hline GWM418012X & MIDDLE-2050A & 474 & Barium & 86.6 & & B & & UG/L & 09/30/2005 & & DNT-001-06 \\
\hline GWM418012X & MIDDLE-2050A & 474 & Calcium & 56100 & & & & UG/L & 09/30/2005 & & DNT-001-06 \\
\hline GWM418012X & MIDDLE-2050A & 474 & Cobalt & 2.8 & & U & & UG/L & 09/30/2005 & & DNT-001-06 \\
\hline GWM418012X & MIDDLE-2050A & 474 & Copper & 3.8 & & B & & UG/L & 09/30/2005 & & DNT-001-06 \\
\hline GWM418012X & MIDDLE-2050A & 474 & Iron & 10.4 & & B & & UG/L & 09/30/2005 & & DNT-001-06 \\
\hline GWM418012X & MIDDLE-2050A & 474 & Lead & 1.1 & & $u$ & & UG/L & 09/30/2005 & & DNT-001-06 \\
\hline GWM418012X & MIDDLE-2050A & 474 & Lithium & 6.1 & & B & & UG/L & 09/30/2005 & & DNT-001-06 \\
\hline GWM418012X & MIDDLE-2050A & 474 & Magnesium & 17900 & & & & UG/L & 09/30/2005 & & DNT-001-06 \\
\hline GWM418012X & MIDDLE-2050A & 474 & Molybdenum & 3.7 & & U & & UG/L & 09/30/2005 & & DNT-001-06 \\
\hline GWM418012X & MIDDLE-2050A & 474 & Nickel & 3.2 & & U & & UG/L & 09/30/2005 & & DNT-001-06 \\
\hline GWM418012X & MIDDLE-2050A & 474 & Potassium & 2600 & & B & & UG/L & 09/30/2005 & & DNT-001-06 \\
\hline GWM418012X & MIDDLE-2050A & 474 & Selenium & 2.6 & & B & & UG/L & 09/30/2005 & & DNT-001-06 \\
\hline GWM418012X & MIDDLE-2050A & 474 & Sodium & 10100 & & & & UG/L & 09/30/2005 & & DNT-001-06 \\
\hline GWM418012X & MIDDLE-2050A & 474 & Strontium & 326 & & & & UG/L & 09/30/2005 & & DNT-001-06 \\
\hline GWM418012X & MIDDLE-2050A & 474 & Tin & 3.9 & & U & & UG/L & 09/30/2005 & & DNT-001-06 \\
\hline GWM418012X & MIDDLE-2050A & 474 & Vanadium & 2.6 & & B & & UG/L & 09/30/2005 & & DNT-001-06 \\
\hline GWM418012X & MIDDLE-2050A & 474 & Zinc & 1790 & & & & UG/L & 09/30/2005 & & DNT-001-06 \\
\hline GWM41801AN & MIDDLE-2050A & 474 & Bromide & 0.25 & & $\mathrm{U}$ & & MG/L & 09/30/2005 & & DNT-499-05 \\
\hline GWM41801AN & MIDDLE-2050A & 474 & Chloride & 15.3 & & $\mathrm{~J}$ & & MG/L & 09/30/2005 & & DNT-499-05 \\
\hline GWM41801AN & MIDDLE-2050A & 474 & Fluoride & 0.15 & & & & MG/L & 09/30/2005 & & DNT-499-05 \\
\hline GWM41801AN & MIDDLE-2050A & 474 & Sulfate & 23.6 & & & & MG/L & 09/30/2005 & & DNT-499-05 \\
\hline GWM41801A1 & MIDDLE-2050A & 474 & Total Alkalinity & 168 & & & & $\mathrm{MG} / \mathrm{L}$ & 09/30/2005 & & DNT-499-05 \\
\hline GWM41801N2 & MIDDLE-2050A & 474 & Nitrate/Nitrite as $\mathrm{N}$ & 1.1 & & & & MG/L & 09/30/2005 & & DNT-499-05 \\
\hline GWM41801AV & MIDDLE-2050A & 474 & Acetone & 10 & & $\mathrm{U}$ & $\mathrm{R}$ & UG/L & $09 / 30 / 2005$ & & HCJ-099-05 \\
\hline GWM41801AV & MIDDLE-2050A & 474 & Acetonitrile & 20 & & U & & UG/L & 09/30/2005 & & HCJ-099-05 \\
\hline GWM41801AV & MIDDLE-2050A & 474 & Acrolein & 5 & & U & $\mathrm{R}$ & UG/L & 09/30/2005 & & HCJ-099-05 \\
\hline GWM41801AV & MIDDLE-2050A & 474 & Acrylonitrile & 1 & & U & $\mathrm{R}$ & UG/L & 09/30/2005 & & HCJ-099-05 \\
\hline GWM41801AV & MIDDLE-2050A & 474 & Benzene & 1 & & U & & UG/L & 09/30/2005 & & HCJ-099-05 \\
\hline GWM41801AV & MIDDLE-2050A & 474 & Bromodichloromethane & 1 & & $\mathrm{U}$ & & UG/L & 09/30/2005 & & HCJ-099-05 \\
\hline GWM41801AV & MIDDLE-2050A & 474 & Bromoform & 2 & & $U$ & & UG/L & 09/30/2005 & & HCJ-099-05 \\
\hline GWM41801AV & MIDDLE-2050A & 474 & Bromomethane & 1 & & $\mathrm{U}$ & & UG/L & 09/30/2005 & & HCJ-099-05 \\
\hline GWM41801AV & MIDDLE-2050A & 474 & 2-Butanone & 10 & & u & $\mathrm{R}$ & UG/L & 09/30/2005 & & HCJ-099-05 \\
\hline GWM41801AV & MIDDLE-2050A & 474 & Carbon disulfide & 5 & & U & & UG/L & 09/30/2005 & & HCJ-099-05 \\
\hline GWM41801AV & MIDDLE-2050A & 474 & Carbon tetrachloride & 1 & & $\mathrm{U}$ & & UG/L & 09/30/2005 & & HCJ-099-05 \\
\hline GWM41801AV & MIDDLE-2050A & 474 & Chlorobenzene & 1 & & $\mathrm{U}$ & & UG/L & 09/30/2005 & & HCJ-099-05 \\
\hline GWM41801AV & MIDDLE-2050A & 474 & Chloroprene & 5 & & U & & UG/L & 09/30/2005 & & HCJ-099-05 \\
\hline GWM41801AV & MIDDLE-2050A & 474 & Dibromochloromethane & 1 & & $\mathrm{U}$ & & UG/L & 09/30/2005 & & HCJ-099-05 \\
\hline GWM41801AV & MIDDLE-2050A & 474 & Chloroethane & 1 & & $U$ & & UG/L & 09/30/2005 & & HCJ-099-05 \\
\hline GWM41801AV & MIDDLE-2050A & 474 & Chloroform & 1 & & $\mathrm{U}$ & & UG/L & 09/30/2005 & & HCJ-099-05 \\
\hline GWM41801AV & MIDDLE-2050A & 474 & Chloromethane & 5 & & u & & UG/L & 09/30/2005 & & HCJ-099-05 \\
\hline GWM41801AV & MIDDLE-2050A & 474 & Allyl chloride & 5 & & U & & UG/L & 09/30/2005 & & HCJ-099-05 \\
\hline GWM41801AV & MIDDLE-2050A & 474 & 1,2-Dibromo-3-chloropropane & 5 & & $U$ & $\mathrm{R}$ & UG/L & 09/30/2005 & & HCJ-099-05 \\
\hline GWM41801AV & MIDDLE-2050A & 474 & 1,2-Dibromoethane & 1 & & U & & UG/L & 09/30/2005 & & HCJ-099-05 \\
\hline GWM41801AV & MIDDLE-2050A & 474 & Dibromomethane & 1 & & $\mathrm{U}$ & & UG/L & 09/30/2005 & & HCJ-099-05 \\
\hline GWM41801AV & MIDDLE-2050A & 474 & trans-1,4-Dichloro-2-butene & 5 & & $\mathrm{U}$ & & UG/L & 09/30/2005 & & HCJ-099-05 \\
\hline GWM41801AV & MIDDLE-2050A & 474 & Dichlorodifluoromethane & 5 & & $u$ & & UG/L & 09/30/2005 & & HCJ-099-05 \\
\hline GWM41801AV & MIDDLE-2050A & 474 & 1,1-Dichloroethane & 1 & & $\mathrm{U}$ & & UG/L & 09/30/2005 & & HCJ-099-05 \\
\hline GWM41801AV & MIDDLE-2050A & 474 & 1,2-Dichloroethane & 1 & & $\mathrm{U}$ & & UG/L & 09/30/2005 & & HCJ-099-05 \\
\hline GWM41801AV & MIDDLE-2050A & 474 & cis-1,2-Dichloroethene & 1 & & $\mathrm{U}$ & & UG/L & 09/30/2005 & & HCJ-099-05 \\
\hline GWM41801AV & MIDDLE-2050A & 474 & trans-1,2-Dichloroethene & 1 & & $\mathrm{U}$ & & UG/L & 09/30/2005 & & HCJ-099-05 \\
\hline GWM41801AV & MIDDLE-2050A & 474 & 1,1-Dichloroethene & 1 & & $\mathrm{U}$ & & UG/L & 09/30/2005 & & HCJ-099-05 \\
\hline GWM41801AV & MIDDLE-2050A & 474 & 1,2-Dichloropropane & 1 & & U & & UG/L & 09/30/2005 & & HCJ-099-05 \\
\hline
\end{tabular}


Table A-4. (continued).

\begin{tabular}{|c|c|c|c|c|c|c|c|c|c|c|c|}
\hline $\begin{array}{c}\text { Field Sample } \\
\text { Number }\end{array}$ & Location & Depth & Compound & $\begin{array}{c}\text { Sample } \\
\text { Result }\end{array}$ & $\begin{array}{l}\text { Sample } \\
\text { Error }\end{array}$ & $\begin{array}{c}\text { Result } \\
\text { Qualifier }\end{array}$ & $\begin{array}{c}\text { Validation } \\
\text { Flag }\end{array}$ & $\begin{array}{c}\text { Sample } \\
\text { Units }\end{array}$ & $\begin{array}{c}\text { Date Sample } \\
\text { Collected }\end{array}$ & MDA & $\begin{array}{l}\text { L\&V Report } \\
\text { Number }\end{array}$ \\
\hline GWM41801AV & MIDDLE-2050A & 474 & cis-1,3-Dichloropropene & 1 & & $\bar{U}$ & & UG/L & $09 / 30 / 2005$ & & HCJ-099-05 \\
\hline GWM41801AV & MIDDLE-2050A & 474 & trans-1,3-Dichloropropene & 1 & & u & & UG/L & 09/30/2005 & & HCJ-099-05 \\
\hline GWM41801AV & MIDDLE-2050A & 474 & 1,4-Dioxane & 80 & & $\mathrm{u}$ & $\mathrm{R}$ & UG/L & 09/30/2005 & & HCJ-099-05 \\
\hline GWM41801AV & MIDDLE-2050A & 474 & Ethylbenzene & 1 & & $\mathrm{u}$ & & UG/L & 09/30/2005 & & HCJ-099-05 \\
\hline GWM41801AV & MIDDLE-2050A & 474 & 2-Hexanone & 5 & & $\mathrm{u}$ & & UG/L & 09/30/2005 & & HCJ-099-05 \\
\hline GWM41801AV & MIDDLE-2050A & 474 & Methyl iodide & 2 & & $\mathrm{u}$ & & UG/L & 09/30/2005 & & HCJ-099-05 \\
\hline GWM41801AV & MIDDLE-2050A & 474 & Isobutyl alcohol & 80 & & $\mathrm{u}$ & $\mathrm{R}$ & UG/L & 09/30/2005 & & HCJ-099-05 \\
\hline GWM41801AV & MIDDLE-2050A & 474 & Methacrylonitrile & 5 & & $\mathrm{u}$ & & UG/L & 09/30/2005 & & HCJ-099-05 \\
\hline GWM41801AV & MIDDLE-2050A & 474 & Methylene Chloride & 1 & & $\mathrm{u}$ & & UG/L & 09/30/2005 & & HCJ-099-05 \\
\hline GWM41801AV & MIDDLE-2050A & 474 & Methylmethacrylate & 1 & & $\mathrm{u}$ & & UG/L & 09/30/2005 & & HCJ-099-05 \\
\hline GWM41801AV & MIDDLE-2050A & 474 & Methyl isobutyl ketone & 5 & & $\mathrm{u}$ & & UG/L & 09/30/2005 & & HCJ-099-05 \\
\hline GWM41801AV & MIDDLE-2050A & 474 & Propionitrile & 5 & & $\mathrm{u}$ & $\mathrm{R}$ & UG/L & 09/30/2005 & & HCJ-099-05 \\
\hline GWM41801AV & MIDDLE-2050A & 474 & Styrene & 1 & & u & & UG/L & 09/30/2005 & & HCJ-099-05 \\
\hline GWM41801AV & MIDDLE-2050A & 474 & 1,1,1,2-Tetrachloroethane & 1 & & $\mathrm{u}$ & & UG/L & 09/30/2005 & & HCJ-099-05 \\
\hline GWM41801AV & MIDDLE-2050A & 474 & 1,1,2,2-Tetrachloroethane & 1 & & $\mathrm{u}$ & & UG/L & 09/30/2005 & & HCJ-099-05 \\
\hline GWM41801AV & MIDDLE-2050A & 474 & Tetrachloroethene & 1 & & $\mathrm{u}$ & & UG/L & 09/30/2005 & & HCJ-099-05 \\
\hline GWM41801AV & MIDDLE-2050A & 474 & Toluene & 1 & & $\mathrm{u}$ & & UG/L & 09/30/2005 & & HCJ-099-05 \\
\hline GWM41801AV & MIDDLE-2050A & 474 & 1,1,1-Trichloroethane & 1 & & $\mathrm{u}$ & & UG/L & 09/30/2005 & & HCJ-099-05 \\
\hline GWM41801AV & MIDDLE-2050A & 474 & 1,1,2-Trichloroethane & 1 & & $\mathrm{u}$ & & UG/L & 09/30/2005 & & HCJ-099-05 \\
\hline GWM41801AV & MIDDLE-2050A & 474 & Trichloroethene & 1 & & $\mathrm{u}$ & & UG/L & $09 / 30 / 2005$ & & HCJ-099-05 \\
\hline GWM41801AV & MIDDLE-2050A & 474 & Trichlorofluoromethane & 1 & & u & & UG/L & 09/30/2005 & & HCJ-099-05 \\
\hline GWM41801AV & MIDDLE-2050A & 474 & 1,2,3-Trichloropropane & 1 & & $\mathrm{u}$ & & UG/L & 09/30/2005 & & HCJ-099-05 \\
\hline GWM41801AV & MIDDLE-2050A & 474 & Vinyl Acetate & 2 & & $\mathrm{u}$ & & UG/L & 09/30/2005 & & HCJ-099-05 \\
\hline GWM41801AV & MIDDLE-2050A & 474 & Vinyl Chloride & 1 & & $\mathrm{u}$ & & UG/L & 09/30/2005 & & HCJ-099-05 \\
\hline GWM41801AV & MIDDLE-2050A & 474 & Xylenes & 3 & & u & & UG/L & 09/30/2005 & & HCJ-099-05 \\
\hline GWM41801R8 & MIDDLE-2050A & 474 & Tritium & $3.10 \mathrm{E}+02$ & $1.04 \mathrm{E}+02$ & & UJ & $\mathrm{PCl} / \mathrm{L}$ & 09/30/2005 & $3.29 \mathrm{E}+02$ & SOS-TL342-05 \\
\hline GWM41801RH & MIDDLE-2050A & 474 & Uranium-233/234 & $1.25 \mathrm{E}+00$ & $1.41 \mathrm{E}-01$ & & & $\mathrm{PCl} / \mathrm{L}$ & 09/30/2005 & $6.62 \mathrm{E}-02$ & SOS-TL351-05 \\
\hline GWM41801RH & MIDDLE-2050A & 474 & Uranium-238 & $6.44 \mathrm{E}-01$ & $9.31 \mathrm{E}-02$ & & & $\mathrm{PCl} / \mathrm{L}$ & 09/30/2005 & $6.62 \mathrm{E}-02$ & SOS-TL351-05 \\
\hline GWM41801RH & MIDDLE-2050A & 474 & Uranium-235 & $1.31 \mathrm{E}-01$ & $4.24 \mathrm{E}-02$ & & & $\mathrm{PCl} / \mathrm{L}$ & 09/30/2005 & $3.94 \mathrm{E}-02$ & SOS-TL351-05 \\
\hline GWM41801RH & MIDDLE-2050A & 474 & Gross Alpha & 4.02E-01 & $6.00 \mathrm{E}-01$ & & u & $\mathrm{PCl} / \mathrm{L}$ & 09/30/2005 & $2.35 \mathrm{E}+00$ & SOS-TL351-05 \\
\hline GWM41801RH & MIDDLE-2050A & 474 & Gross Beta & $3.40 \mathrm{E}+00$ & $9.53 \mathrm{E}-01$ & & & $\mathrm{PCl} / \mathrm{L}$ & 09/30/2005 & $3.57 E+00$ & SOS-TL351-05 \\
\hline GWM41801RH & MIDDLE-2050A & 474 & Strontium-90 & $-3.00 \mathrm{E}-02$ & 1.33E-01 & & $\mathrm{U}$ & $\mathrm{PCl} / \mathrm{L}$ & 09/30/2005 & $6.67 \mathrm{E}-01$ & SOS-TL351-05 \\
\hline GWM41801RH & MIDDLE-2050A & 474 & Silver-108m & $-6.38 \mathrm{E}-01$ & $1.34 \mathrm{E}+00$ & & u & $\mathrm{PCl} / \mathrm{L}$ & 09/30/2005 & $4.70 \mathrm{E}+00$ & SOS-TL351-05 \\
\hline GWM41801RH & MIDDLE-2050A & 474 & Silver-110m & $1.19 \mathrm{E}+00$ & $1.29 \mathrm{E}+00$ & & u & $\mathrm{PCl} / \mathrm{L}$ & 09/30/2005 & $5.23 \mathrm{E}+00$ & SOS-TL351-05 \\
\hline GWM41801RH & MIDDLE-2050A & 474 & Cerium-144 & $-1.09 \mathrm{E}+01$ & $9.41 \mathrm{E}+00$ & & $u$ & $\mathrm{PCl} / \mathrm{L}$ & 09/30/2005 & $3.14 \mathrm{E}+01$ & SOS-TL351-05 \\
\hline GWM41801RH & MIDDLE-2050A & 474 & Cobalt-60 & $1.04 \mathrm{E}+00$ & $1.49 \mathrm{E}+00$ & & u & $\mathrm{PCl} / \mathrm{L}$ & 09/30/2005 & $6.21 \mathrm{E}+00$ & SOS-TL351-05 \\
\hline GWM41801RH & MIDDLE-2050A & 474 & Cesium-134 & $3.02 E+00$ & $1.15 \mathrm{E}+00$ & & $u$ & $\mathrm{PCl} / \mathrm{L}$ & 09/30/2005 & $5.60 \mathrm{E}+00$ & SOS-TL351-05 \\
\hline GWM41801RH & MIDDLE-2050A & 474 & Cesium-137 & 1.23E-01 & $1.55 \mathrm{E}+00$ & & $\mathrm{U}$ & $\mathrm{PCl} / \mathrm{L}$ & 09/30/2005 & $5.82 \mathrm{E}+00$ & SOS-TL351-05 \\
\hline GWM41801RH & MIDDLE-2050A & 474 & Europium-152 & $1.85 \mathrm{E}+00$ & $4.32 \mathrm{E}+00$ & & $u$ & $\mathrm{PCl} / \mathrm{L}$ & 09/30/2005 & $1.59 \mathrm{E}+01$ & SOS-TL351-05 \\
\hline GWM41801RH & MIDDLE-2050A & 474 & Europium-154 & $-1.68 \mathrm{E}+00$ & $3.88 \mathrm{E}+00$ & & u & $\mathrm{PCl} / \mathrm{L}$ & 09/30/2005 & $1.47 \mathrm{E}+01$ & SOS-TL351-05 \\
\hline GWM41801RH & MIDDLE-2050A & 474 & Europium-155 & $9.32 \mathrm{E}-01$ & $4.26 \mathrm{E}+00$ & & u & $\mathrm{PCl} / \mathrm{L}$ & 09/30/2005 & $1.53 E+01$ & SOS-TL351-05 \\
\hline GWM41801RH & MIDDLE-2050A & 474 & Manganese-54 & $1.52 \mathrm{E}+00$ & $1.39 \mathrm{E}+00$ & & u & $\mathrm{PCl} / \mathrm{L}$ & 09/30/2005 & $5.68 \mathrm{E}+00$ & SOS-TL351-05 \\
\hline GWM41801RH & MIDDLE-2050A & 474 & Ruthenium-106 & $-5.20 E+00$ & $1.36 \mathrm{E}+01$ & & $u$ & $\mathrm{PCl} / \mathrm{L}$ & 09/30/2005 & $4.98 \mathrm{E}+01$ & SOS-TL351-05 \\
\hline GWM41801RH & MIDDLE-2050A & 474 & Antimony-125 & $2.07 \mathrm{E}+00$ & $3.88 \mathrm{E}+00$ & & $\mathrm{u}$ & $\mathrm{PCl} / \mathrm{L}$ & 09/30/2005 & $1.44 \mathrm{E}+01$ & SOS-TL351-05 \\
\hline GWM41801RH & MIDDLE-2050A & 474 & Zinc-65 & $-4.15 E+00$ & $2.90 \mathrm{E}+00$ & & $u$ & $\mathrm{PCl} / \mathrm{L}$ & 09/30/2005 & $9.88 \mathrm{E}+00$ & SOS-TL351-05 \\
\hline GWM41801RH & MIDDLE-2050A & 474 & Technetium-99 & $-1.37 E+00$ & $1.55 \mathrm{E}+00$ & & $\mathrm{U}$ & $\mathrm{PCl} / \mathrm{L}$ & 09/30/2005 & $5.38 \mathrm{E}+00$ & SOS-TL351-05 \\
\hline GWM419022X & MIDDLE-2050A & 647 & Antimony & 0.61 & & u & & UG/L & 09/30/2005 & & DNT-001-06 \\
\hline GWM419022X & MIDDLE-2050A & 647 & Arsenic & 1.8 & & u & & UG/L & 09/30/2005 & & DNT-001-06 \\
\hline GWM419022X & MIDDLE-2050A & 647 & Beryllium & 0.13 & & u & & UG/L & 09/30/2005 & & DNT-001-06 \\
\hline GWM419022X & MIDDLE-2050A & 647 & Cadmium & 0.067 & & u & & UG/L & 09/30/2005 & & DNT-001-06 \\
\hline GWM419022X & MIDDLE-2050A & 647 & Chromium & 5.6 & & & & UG/L & 09/30/2005 & & DNT-001-06 \\
\hline GWM419022X & MIDDLE-2050A & 647 & Manganese & 4.3 & & B & & UG/L & 09/30/2005 & & DNT-001-06 \\
\hline GWM419022X & MIDDLE-2050A & 647 & Silver & 1.5 & & u & & UG/L & 09/30/2005 & & DNT-001-06 \\
\hline GWM419022X & MIDDLE-2050A & 647 & Thallium & 0.28 & & B & u & UG/L & 09/30/2005 & & DNT-001-06 \\
\hline GWM419022X & MIDDLE-2050A & 647 & Uranium & 2.2 & & B & & UG/L & 09/30/2005 & & DNT-001-06 \\
\hline GWM419022X & MIDDLE-2050A & 647 & Mercury & 0.046 & & $u$ & & UG/L & 09/30/2005 & & DNT-001-06 \\
\hline GWM419022X & MIDDLE-2050A & 647 & Aluminum & 22.1 & & u & & UG/L & 09/30/2005 & & DNT-001-06 \\
\hline GWM419022X & MIDDLE-2050A & 647 & Barium & 86.4 & & B & & UG/L & 09/30/2005 & & DNT-001-06 \\
\hline GWM419022X & MIDDLE-2050A & 647 & Calcium & 56700 & & & & UG/L & 09/30/2005 & & DNT-001-06 \\
\hline GWM419022X & MIDDLE-2050A & 647 & Cobalt & 2.8 & & $\mathrm{u}$ & & UG/L & 09/30/2005 & & DNT-001-06 \\
\hline GWM419022X & MIDDLE-2050A & 647 & Copper & 1.1 & & $u$ & & UG/L & 09/30/2005 & & DNT-001-06 \\
\hline GWM419022X & MIDDLE-2050A & 647 & Iron & 11.9 & & B & & UG/L & 09/30/2005 & & DNT-001-06 \\
\hline GWM419022X & MIDDLE-2050A & 647 & Lead & 1.1 & & u & & UG/L & 09/30/2005 & & DNT-001-06 \\
\hline GWM419022X & MIDDLE-2050A & 647 & Lithium & 3.8 & & B & & UG/L & 09/30/2005 & & DNT-001-06 \\
\hline GWM419022X & MIDDLE-2050A & 647 & Magnesium & 17100 & & & & UG/L & 09/30/2005 & & DNT-001-06 \\
\hline GWM419022X & MIDDLE-2050A & 647 & Molybdenum & 3.7 & & $\mathrm{u}$ & & UG/L & 09/30/2005 & & DNT-001-06 \\
\hline GWM419022X & MIDDLE-2050A & 647 & Nickel & 3.2 & & u & & UG/L & 09/30/2005 & & DNT-001-06 \\
\hline GWM419022X & MIDDLE-2050A & 647 & Potassium & 2990 & & B & & UG/L & 09/30/2005 & & DNT-001-06 \\
\hline GWM419022X & MIDDLE-2050A & 647 & Selenium & 2 & & B & & UG/L & 09/30/2005 & & DNT-001-06 \\
\hline GWM419022X & MIDDLE-2050A & 647 & Sodium & 8590 & & & & UG/L & $09 / 30 / 2005$ & & DNT-001-06 \\
\hline GWM419022X & MIDDLE-2050A & 647 & Strontium & 317 & & & & UG/L & 09/30/2005 & & DNT-001-06 \\
\hline GWM419022X & MIDDLE-2050A & 647 & Tin & 3.9 & & $\mathrm{U}$ & & UG/L & 09/30/2005 & & DNT-001-06 \\
\hline GWM419022X & MIDDLE-2050A & 647 & Vanadium & 4.1 & & B & & UG/L & 09/30/2005 & & DNT-001-06 \\
\hline GWM419022X & MIDDLE-2050A & 647 & Zinc & 10.3 & & B & $\mathrm{u}$ & UG/L & 09/30/2005 & & DNT-001-06 \\
\hline GWM41902A1 & MIDDLE-2050A & 647 & Total Alkalinity & 159 & & & & MG/L & 09/30/2005 & & DNT-499-05 \\
\hline GWM41902N2 & MIDDLE-2050A & 647 & Nitrate/Nitrite as N & 0.79 & & & & $\mathrm{MG} / \mathrm{L}$ & 09/30/2005 & & DNT-499-05 \\
\hline GWM419012X & MIDDLE-2050A & 647 & Antimony & 0.61 & & u & & UG/L & 09/30/2005 & & DNT-001-06 \\
\hline GWM419012X & MIDDLE-2050A & 647 & Arsenic & 1.8 & & $\mathrm{u}$ & & UG/L & 09/30/2005 & & DNT-001-06 \\
\hline GWM419012X & MIDDLE-2050A & 647 & Beryllium & 0.13 & & u & & UG/L & 09/30/2005 & & DNT-001-06 \\
\hline GWM419012X & MIDDLE-2050A & 647 & Cadmium & 0.067 & & $\mathrm{u}$ & & UG/L & 09/30/2005 & & DNT-001-06 \\
\hline GWM419012X & MIDDLE-2050A & 647 & Chromium & 5.4 & & & & UG/L & 09/30/2005 & & DNT-001-06 \\
\hline GWM419012X & MIDDLE-2050A & 647 & Manganese & 4.3 & & B & & UG/L & 09/30/2005 & & DNT-001-06 \\
\hline GWM419012X & MIDDLE-2050A & 647 & Silver & 1.5 & & u & & UG/L & 09/30/2005 & & DNT-001-06 \\
\hline GWM419012X & MIDDLE-2050A & 647 & Thallium & 0.66 & & B & $\mathrm{u}$ & UG/L & 09/30/2005 & & DNT-001-06 \\
\hline GWM419012X & MIDDLE-2050A & 647 & Uranium & 2.3 & & B & & UG/L & 09/30/2005 & & DNT-001-06 \\
\hline GWM419012X & MIDDLE-2050A & 647 & Mercury & 0.046 & & $u$ & & UG/L & 09/30/2005 & & DNT-001-06 \\
\hline
\end{tabular}


Table A-4. (continued).

\begin{tabular}{|c|c|c|c|c|c|c|c|c|c|c|c|}
\hline $\begin{array}{c}\text { Field Sample } \\
\text { Number }\end{array}$ & Location & Depth & Compound & $\begin{array}{l}\text { Sample } \\
\text { Result }\end{array}$ & $\begin{array}{l}\text { Sample } \\
\text { Error }\end{array}$ & $\begin{array}{c}\text { Result } \\
\text { Qualifier }\end{array}$ & $\begin{array}{c}\text { Validation } \\
\text { Flag }\end{array}$ & $\begin{array}{c}\text { Sample } \\
\text { Units }\end{array}$ & $\begin{array}{c}\text { Date Sample } \\
\text { Collected }\end{array}$ & MDA & $\begin{array}{l}\text { L\&V Report } \\
\text { Number }\end{array}$ \\
\hline GWM419012X & MIDDLE-2050A & 647 & Aluminum & 22.1 & & $\bar{U}$ & & UG/L & $09 / 30 / 2005$ & & DNT-001-06 \\
\hline GWM419012X & MIDDLE-2050A & 647 & Barium & 87 & & B & & UG/L & 09/30/2005 & & DNT-001-06 \\
\hline GWM419012X & MIDDLE-2050A & 647 & Calcium & 57300 & & & & UG/L & 09/30/2005 & & DNT-001-06 \\
\hline GWM419012X & MIDDLE-2050A & 647 & Cobalt & 2.8 & & $u$ & & UG/L & 09/30/2005 & & DNT-001-06 \\
\hline GWM419012X & MIDDLE-2050A & 647 & Copper & 1.1 & & u & & UG/L & 09/30/2005 & & DNT-001-06 \\
\hline GWM419012X & MIDDLE-2050A & 647 & Iron & 7.4 & & u & & UG/L & 09/30/2005 & & DNT-001-06 \\
\hline GWM419012X & MIDDLE-2050A & 647 & Lead & 1.1 & & u & & UG/L & 09/30/2005 & & DNT-001-06 \\
\hline GWM419012X & MIDDLE-2050A & 647 & Lithium & 3 & & u & & UG/L & 09/30/2005 & & DNT-001-06 \\
\hline GWM419012X & MIDDLE-2050A & 647 & Magnesium & 17300 & & & & UG/L & 09/30/2005 & & DNT-001-06 \\
\hline GWM419012X & MIDDLE-2050A & 647 & Molybdenum & 3.7 & & $u$ & & UG/L & 09/30/2005 & & DNT-001-06 \\
\hline GWM419012X & MIDDLE-2050A & 647 & Nickel & 3.2 & & u & & UG/L & 09/30/2005 & & DNT-001-06 \\
\hline GWM419012X & MIDDLE-2050A & 647 & Potassium & 2470 & & B & & UG/L & 09/30/2005 & & DNT-001-06 \\
\hline GWM419012X & MIDDLE-2050A & 647 & Selenium & 2 & & u & & UG/L & 09/30/2005 & & DNT-001-06 \\
\hline GWM419012X & MIDDLE-2050A & 647 & Sodium & 8560 & & & & UG/L & 09/30/2005 & & DNT-001-06 \\
\hline GWM419012X & MIDDLE-2050A & 647 & Strontium & 320 & & & & UG/L & 09/30/2005 & & DNT-001-06 \\
\hline GWM419012X & MIDDLE-2050A & 647 & Tin & 3.9 & & $u$ & & UG/L & 09/30/2005 & & DNT-001-06 \\
\hline GWM419012X & MIDDLE-2050A & 647 & Vanadium & 4.2 & & B & & UG/L & 09/30/2005 & & DNT-001-06 \\
\hline GWM419012X & MIDDLE-2050A & 647 & Zinc & 7.5 & & B & $u$ & UG/L & 09/30/2005 & & DNT-001-06 \\
\hline GWM41901A1 & MIDDLE-2050A & 647 & Total Alkalinity & 166 & & & & MG/L & 09/30/2005 & & DNT-499-05 \\
\hline GWM41901N2 & MIDDLE-2050A & 647 & Nitrate/Nitrite as $\mathrm{N}$ & 0.81 & & & & MG/L & 09/30/2005 & & DNT-499-05 \\
\hline GWM41901AN & MIDDLE-2050A & 647 & Bromide & 0.25 & & u & & MG/L & $10 / 14 / 2005$ & & DNT-501-05 \\
\hline GWM41901AN & MIDDLE-2050A & 647 & Chloride & 15.5 & & $\mathrm{~J}$ & & $M G / L$ & $10 / 14 / 2005$ & & DNT-501-05 \\
\hline GWM41901AN & MIDDLE-2050A & 647 & Fluoride & 0.15 & & & & MG/L & $10 / 14 / 2005$ & & DNT-501-05 \\
\hline GWM41901AN & MIDDLE-2050A & 647 & Sulfate & 23.4 & & & & $\mathrm{MG} / \mathrm{L}$ & $10 / 14 / 2005$ & & DNT-501-05 \\
\hline GWM41901AV & MIDDLE-2050A & 647 & Acetone & 10 & & $\mathrm{u}$ & $\mathrm{R}$ & UG/L & $10 / 14 / 2005$ & & HCJ-101-05 \\
\hline GWM41901AV & MIDDLE-2050A & 647 & Acetonitrile & 20 & & $\mathrm{u}$ & & UG/L & $10 / 14 / 2005$ & & HCJ-101-05 \\
\hline GWM41901AV & MIDDLE-2050A & 647 & Acrolein & 5 & & $\mathrm{u}$ & $\mathrm{R}$ & UG/L & $10 / 14 / 2005$ & & HCJ-101-05 \\
\hline GWM41901AV & MIDDLE-2050A & 647 & Acrylonitrile & 1 & & $\mathrm{u}$ & $\mathrm{R}$ & UG/L & $10 / 14 / 2005$ & & HCJ-101-05 \\
\hline GWM41901AV & MIDDLE-2050A & 647 & Benzene & 1 & & $\mathrm{u}$ & & UG/L & $10 / 14 / 2005$ & & HCJ-101-05 \\
\hline GWM41901AV & MIDDLE-2050A & 647 & Bromodichloromethane & 1 & & $\mathrm{u}$ & & UG/L & $10 / 14 / 2005$ & & HCJ-101-05 \\
\hline GWM41901AV & MIDDLE-2050A & 647 & Bromoform & 2 & & u & & UG/L & $10 / 14 / 2005$ & & HCJ-101-05 \\
\hline GWM41901AV & MIDDLE-2050A & 647 & Bromomethane & 1 & & $\mathrm{u}$ & & UG/L & $10 / 14 / 2005$ & & HCJ-101-05 \\
\hline GWM41901AV & MIDDLE-2050A & 647 & 2-Butanone & 10 & & $\mathrm{u}$ & $\mathrm{R}$ & UG/L & $10 / 14 / 2005$ & & HCJ-101-05 \\
\hline GWM41901AV & MIDDLE-2050A & 647 & Carbon disulfide & 5 & & $\mathrm{u}$ & & UG/L & $10 / 14 / 2005$ & & HCJ-101-05 \\
\hline GWM41901AV & MIDDLE-2050A & 647 & Carbon tetrachloride & 1 & & $\mathrm{u}$ & & UG/L & $10 / 14 / 2005$ & & HCJ-101-05 \\
\hline GWM41901AV & MIDDLE-2050A & 647 & Chlorobenzene & 1 & & $\mathrm{u}$ & & UG/L & $10 / 14 / 2005$ & & HCJ-101-05 \\
\hline GWM41901AV & MIDDLE-2050A & 647 & Chloroprene & 5 & & $\mathrm{u}$ & & UG/L & $10 / 14 / 2005$ & & HCJ-101-05 \\
\hline GWM41901AV & MIDDLE-2050A & 647 & Dibromochloromethane & 1 & & $\mathrm{u}$ & & UG/L & $10 / 14 / 2005$ & & HCJ-101-05 \\
\hline GWM41901AV & MIDDLE-2050A & 647 & Chloroethane & 1 & & u & & UG/L & $10 / 14 / 2005$ & & HCJ-101-05 \\
\hline GWM41901AV & MIDDLE-2050A & 647 & Chloroform & 1 & & u & & UG/L & $10 / 14 / 2005$ & & HCJ-101-05 \\
\hline GWM41901AV & MIDDLE-2050A & 647 & Chloromethane & 5 & & $\mathrm{u}$ & & UG/L & $10 / 14 / 2005$ & & HCJ-101-05 \\
\hline GWM41901AV & MIDDLE-2050A & 647 & Allyl chloride & 5 & & $\mathrm{u}$ & & UG/L & $10 / 14 / 2005$ & & HCJ-101-05 \\
\hline GWM41901AV & MIDDLE-2050A & 647 & 1,2-Dibromo-3-chloropropane & 5 & & $\mathrm{u}$ & $\mathrm{R}$ & UG/L & $10 / 14 / 2005$ & & HCJ-101-05 \\
\hline GWM41901AV & MIDDLE-2050A & 647 & 1,2-Dibromoethane & 1 & & $\mathrm{u}$ & & UG/L & $10 / 14 / 2005$ & & HCJ-101-05 \\
\hline GWM41901AV & MIDDLE-2050A & 647 & Dibromomethane & 1 & & $\mathrm{u}$ & & UG/L & $10 / 14 / 2005$ & & HCJ-101-05 \\
\hline GWM41901AV & MIDDLE-2050A & 647 & trans-1,4-Dichloro-2-butene & 5 & & $\mathrm{u}$ & & UG/L & $10 / 14 / 2005$ & & HCJ-101-05 \\
\hline GWM41901AV & MIDDLE-2050A & 647 & Dichlorodifluoromethane & 5 & & $\mathrm{u}$ & & UG/L & $10 / 14 / 2005$ & & HCJ-101-05 \\
\hline GWM41901AV & MIDDLE-2050A & 647 & 1,1-Dichloroethane & 1 & & $\mathrm{u}$ & & UG/L & $10 / 14 / 2005$ & & HCJ-101-05 \\
\hline GWM41901AV & MIDDLE-2050A & 647 & 1,2-Dichloroethane & 1 & & $\mathrm{u}$ & & UG/L & $10 / 14 / 2005$ & & HCJ-101-05 \\
\hline GWM41901AV & MIDDLE-2050A & 647 & cis-1,2-Dichloroethene & 1 & & $\mathrm{u}$ & & UG/L & $10 / 14 / 2005$ & & HCJ-101-05 \\
\hline GWM41901AV & MIDDLE-2050A & 647 & trans-1,2-Dichloroethene & 1 & & $\mathrm{u}$ & & UG/L & $10 / 14 / 2005$ & & HCJ-101-05 \\
\hline GWM41901AV & MIDDLE-2050A & 647 & 1,1-Dichloroethene & 1 & & $\mathrm{u}$ & & UG/L & $10 / 14 / 2005$ & & HCJ-101-05 \\
\hline GWM41901AV & MIDDLE-2050A & 647 & 1,2-Dichloropropane & 1 & & $\mathrm{u}$ & & UG/L & $10 / 14 / 2005$ & & HCJ-101-05 \\
\hline GWM41901AV & MIDDLE-2050A & 647 & cis-1,3-Dichloropropene & 1 & & $\mathrm{u}$ & & UG/L & $10 / 14 / 2005$ & & HCJ-101-05 \\
\hline GWM41901AV & MIDDLE-2050A & 647 & trans-1,3-Dichloropropene & 1 & & $\mathrm{u}$ & & UG/L & $10 / 14 / 2005$ & & HCJ-101-05 \\
\hline GWM41901AV & MIDDLE-2050A & 647 & 1,4-Dioxane & 80 & & $\mathrm{u}$ & $\mathrm{R}$ & UG/L & $10 / 14 / 2005$ & & HCJ-101-05 \\
\hline GWM41901AV & MIDDLE-2050A & 647 & Ethylbenzene & 1 & & $\mathrm{u}$ & & UG/L & $10 / 14 / 2005$ & & HCJ-101-05 \\
\hline GWM41901AV & MIDDLE-2050A & 647 & 2-Hexanone & 5 & & $\mathrm{u}$ & & UG/L & $10 / 14 / 2005$ & & HCJ-101-05 \\
\hline GWM41901AV & MIDDLE-2050A & 647 & Methyl iodide & 2 & & u & & UG/L & $10 / 14 / 2005$ & & HCJ-101-05 \\
\hline GWM41901AV & MIDDLE-2050A & 647 & Isobutyl alcohol & 80 & & $\mathrm{u}$ & $\mathrm{R}$ & UG/L & $10 / 14 / 2005$ & & HCJ-101-05 \\
\hline GWM41901AV & MIDDLE-2050A & 647 & Methacrylonitrile & 5 & & $\mathrm{u}$ & $\mathrm{R}$ & UG/L & $10 / 14 / 2005$ & & HCJ-101-05 \\
\hline GWM41901AV & MIDDLE-2050A & 647 & Methylene Chloride & 1 & & $\mathrm{u}$ & & UG/L & $10 / 14 / 2005$ & & HCJ-101-05 \\
\hline GWM41901AV & MIDDLE-2050A & 647 & Methylmethacrylate & 1 & & $\mathrm{u}$ & $\mathrm{R}$ & UG/L & $10 / 14 / 2005$ & & HCJ-101-05 \\
\hline GWM41901AV & MIDDLE-2050A & 647 & Methyl isobutyl ketone & 5 & & u & $\mathrm{R}$ & UG/L & $10 / 14 / 2005$ & & HCJ-101-05 \\
\hline GWM41901AV & MIDDLE-2050A & 647 & Propionitrile & 5 & & $\mathrm{u}$ & $\mathrm{R}$ & UG/L & $10 / 14 / 2005$ & & HCJ-101-05 \\
\hline GWM41901AV & MIDDLE-2050A & 647 & Styrene & 1 & & $\mathrm{u}$ & & UG/L & $10 / 14 / 2005$ & & HCJ-101-05 \\
\hline GWM41901AV & MIDDLE-2050A & 647 & 1,1,1,2-Tetrachloroethane & 1 & & u & & UG/L & $10 / 14 / 2005$ & & HCJ-101-05 \\
\hline GWM41901AV & MIDDLE-2050A & 647 & 1,1,2,2-Tetrachloroethane & 1 & & u & & UG/L & $10 / 14 / 2005$ & & HCJ-101-05 \\
\hline GWM41901AV & MIDDLE-2050A & 647 & Tetrachloroethene & 1 & & u & & UG/L & $10 / 14 / 2005$ & & HCJ-101-05 \\
\hline GWM41901AV & MIDDLE-2050A & 647 & Toluene & 1 & & $\mathrm{u}$ & & UG/L & $10 / 14 / 2005$ & & HCJ-101-05 \\
\hline GWM41901AV & MIDDLE-2050A & 647 & 1,1,1-Trichloroethane & 1 & & $\mathrm{u}$ & & UG/L & $10 / 14 / 2005$ & & HCJ-101-05 \\
\hline GWM41901AV & MIDDLE-2050A & 647 & $1,1,2$-Trichloroethane & 1 & & $u$ & & UG/L & $10 / 14 / 2005$ & & HCJ-101-05 \\
\hline GWM41901AV & MIDDLE-2050A & 647 & Trichloroethene & 1 & & $\mathrm{u}$ & & UG/L & $10 / 14 / 2005$ & & HCJ-101-05 \\
\hline GWM41901AV & MIDDLE-2050A & 647 & Trichlorofluoromethane & 1 & & $\mathrm{u}$ & & UG/L & $10 / 14 / 2005$ & & HCJ-101-05 \\
\hline GWM41901AV & MIDDLE-2050A & 647 & 1,2,3-Trichloropropane & 1 & & $\mathrm{u}$ & & UG/L & $10 / 14 / 2005$ & & HCJ-101-05 \\
\hline GWM41901AV & MIDDLE-2050A & 647 & Vinyl Acetate & 2 & & $\mathrm{u}$ & & UG/L & $10 / 14 / 2005$ & & HCJ-101-05 \\
\hline GWM41901AV & MIDDLE-2050A & 647 & Vinyl Chloride & 1 & & u & & UG/L & $10 / 14 / 2005$ & & HCJ-101-05 \\
\hline GWM41901AV & MIDDLE-2050A & 647 & Xylenes & 3 & & $\mathrm{u}$ & & UG/L & $10 / 14 / 2005$ & & HCJ-101-05 \\
\hline GWM41901RH & MIDDLE-2050A & 647 & Uranium-233/234 & $2.04 \mathrm{E}+00$ & $2.52 \mathrm{E}-01$ & & & $\mathrm{PCl} / \mathrm{L}$ & $10 / 14 / 2005$ & $5.51 \mathrm{E}-02$ & SOS-TL352-05 \\
\hline GWM41901RH & MIDDLE-2050A & 647 & Uranium-238 & $9.46 \mathrm{E}-01$ & $1.52 \mathrm{E}-01$ & & & $\mathrm{PCl} / \mathrm{L}$ & $10 / 14 / 2005$ & 1.14E-01 & SOS-TL352-05 \\
\hline GWM41901RH & MIDDLE-2050A & 647 & Uranium-235 & 1.36E-01 & $5.66 \mathrm{E}-02$ & & $\mathrm{~J}$ & $\mathrm{PCl} / \mathrm{L}$ & $10 / 14 / 2005$ & $6.81 \mathrm{E}-02$ & SOS-TL352-05 \\
\hline GWM41901RH & MIDDLE-2050A & 647 & Gross Alpha & $3.17 \mathrm{E}+00$ & $1.06 \mathrm{E}+00$ & & UJ & $\mathrm{PCl} / \mathrm{L}$ & $10 / 14 / 2005$ & $3.69 \mathrm{E}+00$ & SOS-TL352-05 \\
\hline GWM41901RH & MIDDLE-2050A & 647 & Gross Beta & $2.13 \mathrm{E}+00$ & $1.06 \mathrm{E}+00$ & & UJ & $\mathrm{PCl} / \mathrm{L}$ & $10 / 14 / 2005$ & $4.26 \mathrm{E}+00$ & SOS-TL352-05 \\
\hline GWM41901RH & MIDDLE-2050A & 647 & Strontium-90 & $2.49 \mathrm{E}-01$ & $1.52 \mathrm{E}-01$ & & $u$ & $\mathrm{PCl} / \mathrm{L}$ & $10 / 14 / 2005$ & $6.32 \mathrm{E}-01$ & SOS-TL352-05 \\
\hline GWM41901RH & MIDDLE-2050A & 647 & Silver-108m & $-5.25 \mathrm{E}-01$ & $1.31 \mathrm{E}+00$ & & $u$ & $\mathrm{PCl} / \mathrm{L}$ & $10 / 14 / 2005$ & $4.65 \mathrm{E}+00$ & SOS-TL352-05 \\
\hline GWM41901RH & MIDDLE-2050A & 647 & Silver-110m & $-1.37 \mathrm{E}-03$ & $1.39 \mathrm{E}+00$ & & u & $\mathrm{PCl} / \mathrm{L}$ & $10 / 14 / 2005$ & $4.44 \mathrm{E}+00$ & SOS-TL352-05 \\
\hline
\end{tabular}


Table A-4. (continued).

\begin{tabular}{|c|c|c|c|c|c|c|c|c|c|c|c|}
\hline $\begin{array}{c}\text { Field Sample } \\
\text { Number }\end{array}$ & Location & Depth & Compound & $\begin{array}{l}\text { Sample } \\
\text { Result }\end{array}$ & $\begin{array}{l}\text { Sample } \\
\text { Error }\end{array}$ & $\begin{array}{c}\text { Result } \\
\text { Qualifier }\end{array}$ & $\begin{array}{l}\text { Validation } \\
\text { Flag }\end{array}$ & $\begin{array}{l}\text { Sample } \\
\text { Units }\end{array}$ & $\begin{array}{c}\text { Date Sample } \\
\text { Collected }\end{array}$ & MDA & $\begin{array}{l}\text { L\&V Report } \\
\text { Number }\end{array}$ \\
\hline GWM41901RH & MIDDLE-2050A & 647 & Cerium-144 & $-5.48 \mathrm{E}+00$ & $9.32 \mathrm{E}+00$ & & $U$ & $\mathrm{PCl} / \mathrm{L}$ & $10 / 14 / 2005$ & $3.13 \mathrm{E}+01$ & SOS-TL352-05 \\
\hline GWM41901RH & MIDDLE-2050A & 647 & Cobalt- 60 & $-7.77 \mathrm{E}-01$ & $1.16 \mathrm{E}+00$ & & u & $\mathrm{PCl} / \mathrm{L}$ & $10 / 14 / 2005$ & $4.27 \mathrm{E}+00$ & SOS-TL352-05 \\
\hline GWM41901RH & MIDDLE-2050A & 647 & Cesium-134 & $5.11 \mathrm{E}-01$ & $1.40 \mathrm{E}+00$ & & $u$ & $\mathrm{PCl} / \mathrm{L}$ & 10/14/2005 & $4.73 E+00$ & SOS-TL352-05 \\
\hline GWM41901RH & MIDDLE-2050A & 647 & Cesium-137 & $8.82 \mathrm{E}-01$ & $1.52 \mathrm{E}+00$ & & u & $\mathrm{PCl} / \mathrm{L}$ & $10 / 14 / 2005$ & $5.10 \mathrm{E}+00$ & SOS-TL352-05 \\
\hline GWM41901RH & MIDDLE-2050A & 647 & Europium-152 & $-1.59 E+00$ & $3.87 \mathrm{E}+00$ & & u & $\mathrm{PCl} / \mathrm{L}$ & $10 / 14 / 2005$ & $1.38 \mathrm{E}+01$ & SOS-TL352-05 \\
\hline GWM41901RH & MIDDLE-2050A & 647 & Europium-154 & $-2.70 \mathrm{E}+00$ & $3.05 E+00$ & & u & $\mathrm{PCl} / \mathrm{L}$ & 10/14/2005 & $1.10 \mathrm{E}+01$ & SOS-TL352-05 \\
\hline GWM41901RH & MIDDLE-2050A & 647 & Europium-155 & $-3.45 \mathrm{E}+00$ & $4.87 \mathrm{E}+00$ & & u & $\mathrm{PCl} / \mathrm{L}$ & 10/14/2005 & $1.64 \mathrm{E}+01$ & SOS-TL352-05 \\
\hline GWM41901RH & MIDDLE-2050A & 647 & Manganese-54 & $-2.31 \mathrm{E}+00$ & $1.32 \mathrm{E}+00$ & & u & $\mathrm{PCl} / \mathrm{L}$ & 10/14/2005 & $4.04 \mathrm{E}+00$ & SOS-TL352-05 \\
\hline GWM41901RH & MIDDLE-2050A & 647 & Ruthenium-106 & $1.88 \mathrm{E}+00$ & $1.10 \mathrm{E}+01$ & & $u$ & $\mathrm{PCl} / \mathrm{L}$ & $10 / 14 / 2005$ & $4.09 \mathrm{E}+01$ & SOS-TL352-05 \\
\hline GWM41901RH & MIDDLE-2050A & 647 & Antimony-125 & $-4.45 \mathrm{E}+00$ & $3.37 E+00$ & & $u$ & $\mathrm{PCl} / \mathrm{L}$ & $10 / 14 / 2005$ & $1.14 \mathrm{E}+01$ & SOS-TL352-05 \\
\hline GWM41901RH & MIDDLE-2050A & 647 & Zinc-65 & $-3.13 \mathrm{E}-02$ & $2.43 E+00$ & & $u$ & $\mathrm{PCl} / \mathrm{L}$ & $10 / 14 / 2005$ & $8.41 \mathrm{E}+00$ & SOS-TL352-05 \\
\hline GWM41901RH & MIDDLE-2050A & 647 & Technetium-99 & $-7.07 \mathrm{E}-02$ & $1.63 E+00$ & & u & $\mathrm{PCl} / \mathrm{L}$ & $10 / 14 / 2005$ & $5.56 \mathrm{E}+00$ & SOS-TL352-05 \\
\hline GWM41901R8 & MIDDLE-2050A & 647 & Tritium & $2.30 \mathrm{E}+02$ & $8.63 E+01$ & & UJ & $\mathrm{PCl} / \mathrm{L}$ & 10/14/2005 & $2.79 \mathrm{E}+02$ & SOS-TL352-05 \\
\hline GWM42001AN & MIDDLE-2050A & 790 & Bromide & 0.25 & & u & & MG/L & 10/14/2005 & & DNT-501-05 \\
\hline GWM42001AN & MIDDLE-2050A & 790 & Chloride & 14.1 & & $\mathrm{~J}$ & & MG/L & 10/14/2005 & & DNT-501-05 \\
\hline GWM42001AN & MIDDLE-2050A & 790 & Fluoride & 0.12 & & & & MG/L & 10/14/2005 & & DNT-501-05 \\
\hline GWM42001AN & MIDDLE-2050A & 790 & Sulfate & 22.9 & & & & MG/L & $10 / 14 / 2005$ & & DNT-501-05 \\
\hline GWM42001A1 & MIDDLE-2050A & 790 & Total Alkalinity & 158 & & & & MG/L & $10 / 14 / 2005$ & & DNT-501-05 \\
\hline GWM42001N2 & MIDDLE-2050A & 790 & Nitrate/Nitrite as $\mathrm{N}$ & 0.94 & & & & MG/L & $10 / 14 / 2005$ & & DNT-501-05 \\
\hline GWM420012X & MIDDLE-2050A & 790 & Antimony & 0.61 & & $\mathrm{u}$ & & UG/L & $10 / 14 / 2005$ & & DNT-007-06 \\
\hline GWM420012X & MIDDLE-2050A & 790 & Arsenic & 1.8 & & u & & UG/L & $10 / 14 / 2005$ & & DNT-007-06 \\
\hline GWM420012X & MIDDLE-2050A & 790 & Beryllium & 0.13 & & $\mathrm{u}$ & & UG/L & $10 / 14 / 2005$ & & DNT-007-06 \\
\hline GWM420012X & MIDDLE-2050A & 790 & Cadmium & 0.067 & & $\mathrm{u}$ & & UG/L & $10 / 14 / 2005$ & & DNT-007-06 \\
\hline GWM420012X & MIDDLE-2050A & 790 & Chromium & 3.7 & & $u$ & & UG/L & $10 / 14 / 2005$ & & DNT-007-06 \\
\hline GWM420012X & MIDDLE-2050A & 790 & Manganese & 174 & & & & UG/L & $10 / 14 / 2005$ & & DNT-007-06 \\
\hline GWM420012X & MIDDLE-2050A & 790 & Silver & 1.5 & & $\mathrm{u}$ & & UG/L & $10 / 14 / 2005$ & & DNT-007-06 \\
\hline GWM420012X & MIDDLE-2050A & 790 & Thallium & 0.61 & & B & u & UG/L & 10/14/2005 & & DNT-007-06 \\
\hline GWM420012X & MIDDLE-2050A & 790 & Uranium & 1.4 & & B & & UG/L & $10 / 14 / 2005$ & & DNT-007-06 \\
\hline GWM420012X & MIDDLE-2050A & 790 & Mercury & 0.046 & & $u$ & & UG/L & $10 / 14 / 2005$ & & DNT-007-06 \\
\hline GWM420012X & MIDDLE-2050A & 790 & Aluminum & 22.1 & & $\mathrm{u}$ & & UG/L & $10 / 14 / 2005$ & & DNT-007-06 \\
\hline GWM420012X & MIDDLE-2050A & 790 & Barium & 116 & & & & UG/L & $10 / 14 / 2005$ & & DNT-007-06 \\
\hline GWM420012X & MIDDLE-2050A & 790 & Calcium & 52000 & & & & UG/L & $10 / 14 / 2005$ & & DNT-007-06 \\
\hline GWM420012X & MIDDLE-2050A & 790 & Cobalt & 4.3 & & B & & UG/L & 10/14/2005 & & DNT-007-06 \\
\hline GWM420012X & MIDDLE-2050A & 790 & Copper & 1.1 & & $u$ & & UG/L & $10 / 14 / 2005$ & & DNT-007-06 \\
\hline GWM420012X & MIDDLE-2050A & 790 & Iron & 18.7 & & B & $u$ & UG/L & 10/14/2005 & & DNT-007-06 \\
\hline GWM420012X & MIDDLE-2050A & 790 & Lead & 1.1 & & $u$ & & UG/L & $10 / 14 / 2005$ & & DNT-007-06 \\
\hline GWM420012X & MIDDLE-2050A & 790 & Lithium & 3 & & u & & UG/L & 10/14/2005 & & DNT-007-06 \\
\hline GWM420012X & MIDDLE-2050A & 790 & Magnesium & 16500 & & & & UG/L & $10 / 14 / 2005$ & & DNT-007-06 \\
\hline GWM420012X & MIDDLE-2050A & 790 & Molybdenum & 11.9 & & B & & UG/L & $10 / 14 / 2005$ & & DNT-007-06 \\
\hline GWM420012X & MIDDLE-2050A & 790 & Nickel & 3.2 & & B & & UG/L & $10 / 14 / 2005$ & & DNT-007-06 \\
\hline GWM420012X & MIDDLE-2050A & 790 & Potassium & 2870 & & B & u & UG/L & 10/14/2005 & & DNT-007-06 \\
\hline GWM420012X & MIDDLE-2050A & 790 & Selenium & 2 & & $u$ & & UG/L & $10 / 14 / 2005$ & & DNT-007-06 \\
\hline GWM420012X & MIDDLE-2050A & 790 & Sodium & 11900 & & & & UG/L & $10 / 14 / 2005$ & & DNT-007-06 \\
\hline GWM420012X & MIDDLE-2050A & 790 & Strontium & 388 & & & & UG/L & $10 / 14 / 2005$ & & DNT-007-06 \\
\hline GWM420012X & MIDDLE-2050A & 790 & Tin & 3.9 & & u & & UG/L & 10/14/2005 & & DNT-007-06 \\
\hline GWM420012X & MIDDLE-2050A & 790 & Vanadium & 2.5 & & u & $\mathrm{U}$ & UG/L & $10 / 14 / 2005$ & & DNT-007-06 \\
\hline GWM420012X & MIDDLE-2050A & 790 & Zinc & 9 & & B & & UG/L & $10 / 14 / 2005$ & & DNT-007-06 \\
\hline GWM42001AV & MIDDLE-2050A & 790 & Acetone & 10 & & $u$ & $\mathrm{R}$ & UG/L & $10 / 14 / 2005$ & & HCJ-101-05 \\
\hline GWM42001AV & MIDDLE-2050A & 790 & Acetonitrile & 20 & & u & & UG/L & $10 / 14 / 2005$ & & HCJ-101-05 \\
\hline GWM42001AV & MIDDLE-2050A & 790 & Acrolein & 5 & & u & $\mathrm{R}$ & UG/L & $10 / 14 / 2005$ & & HCJ-101-05 \\
\hline GWM42001AV & MIDDLE-2050A & 790 & Acrylonitrile & 1 & & u & $\mathrm{R}$ & UG/L & $10 / 14 / 2005$ & & HCJ-101-05 \\
\hline GWM42001AV & MIDDLE-2050A & 790 & Benzene & 1 & & $\mathrm{u}$ & & UG/L & $10 / 14 / 2005$ & & HCJ-101-05 \\
\hline GWM42001AV & MIDDLE-2050A & 790 & Bromodichloromethane & 1 & & $\mathrm{u}$ & & UG/L & $10 / 14 / 2005$ & & HCJ-101-05 \\
\hline GWM42001AV & MIDDLE-2050A & 790 & Bromoform & 2 & & $\mathrm{u}$ & & UG/L & $10 / 14 / 2005$ & & HCJ-101-05 \\
\hline GWM42001AV & MIDDLE-2050A & 790 & Bromomethane & 1 & & $\mathrm{u}$ & & UG/L & $10 / 14 / 2005$ & & HCJ-101-05 \\
\hline GWM42001AV & MIDDLE-2050A & 790 & 2-Butanone & 10 & & $\mathrm{u}$ & $\mathrm{R}$ & UG/L & $10 / 14 / 2005$ & & HCJ-101-05 \\
\hline GWM42001AV & MIDDLE-2050A & 790 & Carbon disulfide & 5 & & $\mathrm{u}$ & & UG/L & $10 / 14 / 2005$ & & HCJ-101-05 \\
\hline GWM42001AV & MIDDLE-2050A & 790 & Carbon tetrachloride & 1 & & $\mathrm{u}$ & & UG/L & $10 / 14 / 2005$ & & HCJ-101-05 \\
\hline GWM42001AV & MIDDLE-2050A & 790 & Chlorobenzene & 1 & & u & & UG/L & $10 / 14 / 2005$ & & HCJ-101-05 \\
\hline GWM42001AV & MIDDLE-2050A & 790 & Chloroprene & 5 & & $\mathrm{u}$ & & UG/L & $10 / 14 / 2005$ & & HCJ-101-05 \\
\hline GWM42001AV & MIDDLE-2050A & 790 & Dibromochloromethane & 1 & & $\mathrm{u}$ & & UG/L & $10 / 14 / 2005$ & & HCJ-101-05 \\
\hline GWM42001AV & MIDDLE-2050A & 790 & Chloroethane & 1 & & $\mathrm{u}$ & & UG/L & $10 / 14 / 2005$ & & HCJ-101-05 \\
\hline GWM42001AV & MIDDLE-2050A & 790 & Chloroform & 1 & & $\mathrm{u}$ & & UG/L & $10 / 14 / 2005$ & & HCJ-101-05 \\
\hline GWM42001AV & MIDDLE-2050A & 790 & Chloromethane & 5 & & $\mathrm{u}$ & & UG/L & $10 / 14 / 2005$ & & HCJ-101-05 \\
\hline GWM42001AV & MIDDLE-2050A & 790 & Allyl chloride & 5 & & $\mathrm{u}$ & & UG/L & $10 / 14 / 2005$ & & HCJ-101-05 \\
\hline GWM42001AV & MIDDLE-2050A & 790 & 1,2-Dibromo-3-chloropropane & 5 & & $\mathrm{u}$ & $\mathrm{R}$ & UG/L & $10 / 14 / 2005$ & & HCJ-101-05 \\
\hline GWM42001AV & MIDDLE-2050A & 790 & 1,2-Dibromoethane & 1 & & $\mathrm{u}$ & & UG/L & $10 / 14 / 2005$ & & HCJ-101-05 \\
\hline GWM42001AV & MIDDLE-2050A & 790 & Dibromomethane & 1 & & $\mathrm{u}$ & & UG/L & $10 / 14 / 2005$ & & HCJ-101-05 \\
\hline GWM42001AV & MIDDLE-2050A & 790 & trans-1,4-Dichloro-2-butene & 5 & & $\mathrm{u}$ & & UG/L & $10 / 14 / 2005$ & & HCJ-101-05 \\
\hline GWM42001AV & MIDDLE-2050A & 790 & Dichlorodifluoromethane & 5 & & $\mathrm{u}$ & & UG/L & $10 / 14 / 2005$ & & HCJ-101-05 \\
\hline GWM42001AV & MIDDLE-2050A & 790 & 1,1-Dichloroethane & 1 & & $\mathrm{u}$ & & UG/L & 10/14/2005 & & HCJ-101-05 \\
\hline GWM42001AV & MIDDLE-2050A & 790 & 1,2-Dichloroethane & 1 & & $\mathrm{u}$ & & UG/L & $10 / 14 / 2005$ & & HCJ-101-05 \\
\hline GWM42001AV & MIDDLE-2050A & 790 & cis-1,2-Dichloroethene & 1 & & $\mathrm{u}$ & & UG/L & $10 / 14 / 2005$ & & HCJ-101-05 \\
\hline GWM42001AV & MIDDLE-2050A & 790 & trans-1,2-Dichloroethene & 1 & & $\mathrm{u}$ & & UG/L & $10 / 14 / 2005$ & & HCJ-101-05 \\
\hline GWM42001AV & MIDDLE-2050A & 790 & 1,1-Dichloroethene & 1 & & $\mathrm{u}$ & & UG/L & $10 / 14 / 2005$ & & HCJ-101-05 \\
\hline GWM42001AV & MIDDLE-2050A & 790 & 1,2-Dichloropropane & 1 & & $\mathrm{u}$ & & UG/L & $10 / 14 / 2005$ & & HCJ-101-05 \\
\hline GWM42001AV & MIDDLE-2050A & 790 & cis-1,3-Dichloropropene & 1 & & u & & UG/L & $10 / 14 / 2005$ & & HCJ-101-05 \\
\hline GWM42001AV & MIDDLE-2050A & 790 & trans-1,3-Dichloropropene & 1 & & $\mathrm{u}$ & & UG/L & $10 / 14 / 2005$ & & HCJ-101-05 \\
\hline GWM42001AV & MIDDLE-2050A & 790 & 1,4-Dioxane & 80 & & $\mathrm{u}$ & $\mathrm{R}$ & UG/L & $10 / 14 / 2005$ & & HCJ-101-05 \\
\hline GWM42001AV & MIDDLE-2050A & 790 & Ethylbenzene & 1 & & $\mathrm{u}$ & & UG/L & $10 / 14 / 2005$ & & HCJ-101-05 \\
\hline GWM42001AV & MIDDLE-2050A & 790 & 2-Hexanone & 5 & & $u$ & & UG/L & $10 / 14 / 2005$ & & HCJ-101-05 \\
\hline GWM42001AV & MIDDLE-2050A & 790 & Methyl iodide & 2 & & $\mathrm{u}$ & & UG/L & $10 / 14 / 2005$ & & HCJ-101-05 \\
\hline GWM42001AV & MIDDLE-2050A & 790 & Isobutyl alcohol & 80 & & $\mathrm{u}$ & $\mathrm{R}$ & UG/L & $10 / 14 / 2005$ & & HCJ-101-05 \\
\hline GWM42001AV & MIDDLE-2050A & 790 & Methacrylonitrile & 5 & & $\mathrm{u}$ & $\mathrm{R}$ & UG/L & $10 / 14 / 2005$ & & HCJ-101-05 \\
\hline GWM42001AV & MIDDLE-2050A & 790 & Methylene Chloride & 1 & & u & & UG/L & $10 / 14 / 2005$ & & HCJ-101-05 \\
\hline GWM42001AV & MIDDLE-2050A & 790 & Methylmethacrylate & 1 & & $\mathrm{u}$ & $\mathrm{R}$ & UG/L & $10 / 14 / 2005$ & & HCJ-101-05 \\
\hline
\end{tabular}


Table A-4. (continued).

\begin{tabular}{|c|c|c|c|c|c|c|c|c|c|c|c|}
\hline $\begin{array}{c}\text { Field Sample } \\
\text { Number }\end{array}$ & Location & Depth & Compound & $\begin{array}{c}\text { Sample } \\
\text { Result }\end{array}$ & $\begin{array}{l}\text { Sample } \\
\text { Error }\end{array}$ & $\begin{array}{c}\text { Result } \\
\text { Qualifier }\end{array}$ & $\begin{array}{c}\text { Validation } \\
\text { Flag }\end{array}$ & $\begin{array}{c}\text { Sample } \\
\text { Units }\end{array}$ & $\begin{array}{c}\text { Date Sample } \\
\text { Collected }\end{array}$ & MDA & $\begin{array}{l}\text { L\&V Report } \\
\text { Number }\end{array}$ \\
\hline GWM42001AV & MIDDLE-2050A & 790 & Methyl isobutyl ketone & 5 & & $\bar{U}$ & $\mathrm{R}$ & UG/L & $10 / 14 / 2005$ & & HCJ-101-05 \\
\hline GWM42001AV & MIDDLE-2050A & 790 & Propionitrile & 5 & & u & $\mathrm{R}$ & UG/L & $10 / 14 / 2005$ & & HCJ-101-05 \\
\hline GWM42001AV & MIDDLE-2050A & 790 & Styrene & 1 & & $\mathrm{u}$ & & UG/L & $10 / 14 / 2005$ & & HCJ-101-05 \\
\hline GWM42001AV & MIDDLE-2050A & 790 & 1,1,1,2-Tetrachloroethane & 1 & & $\mathrm{u}$ & & UG/L & $10 / 14 / 2005$ & & HCJ-101-05 \\
\hline GWM42001AV & MIDDLE-2050A & 790 & 1,1,2,2-Tetrachloroethane & 1 & & $\mathrm{u}$ & & UG/L & $10 / 14 / 2005$ & & HCJ-101-05 \\
\hline GWM42001AV & MIDDLE-2050A & 790 & Tetrachloroethene & 1 & & $\mathrm{u}$ & & UG/L & $10 / 14 / 2005$ & & HCJ-101-05 \\
\hline GWM42001AV & MIDDLE-2050A & 790 & Toluene & 1 & & $\mathrm{u}$ & & UG/L & $10 / 14 / 2005$ & & HCJ-101-05 \\
\hline GWM42001AV & MIDDLE-2050A & 790 & 1,1,1-Trichloroethane & 1 & & $\mathrm{u}$ & & UG/L & $10 / 14 / 2005$ & & HCJ-101-05 \\
\hline GWM42001AV & MIDDLE-2050A & 790 & 1,1,2-Trichloroethane & 1 & & $\mathrm{u}$ & & UG/L & $10 / 14 / 2005$ & & HCJ-101-05 \\
\hline GWM42001AV & MIDDLE-2050A & 790 & Trichloroethene & 1 & & $\mathrm{u}$ & & UG/L & $10 / 14 / 2005$ & & HCJ-101-05 \\
\hline GWM42001AV & MIDDLE-2050A & 790 & Trichlorofluoromethane & 1 & & $\mathrm{u}$ & & UG/L & $10 / 14 / 2005$ & & HCJ-101-05 \\
\hline GWM42001AV & MIDDLE-2050A & 790 & 1,2,3-Trichloropropane & 1 & & $\mathrm{u}$ & & UG/L & $10 / 14 / 2005$ & & HCJ-101-05 \\
\hline GWM42001AV & MIDDLE-2050A & 790 & Vinyl Acetate & 2 & & u & & UG/L & $10 / 14 / 2005$ & & HCJ-101-05 \\
\hline GWM42001AV & MIDDLE-2050A & 790 & Vinyl Chloride & 1 & & $\mathrm{u}$ & & UG/L & $10 / 14 / 2005$ & & HCJ-101-05 \\
\hline GWM42001AV & MIDDLE-2050A & 790 & Xylenes & 3 & & u & & UG/L & $10 / 14 / 2005$ & & HCJ-101-05 \\
\hline GWM42001RH & MIDDLE-2050A & 790 & Uranium-233/234 & $1.41 \mathrm{E}+00$ & $1.63 \mathrm{E}-01$ & & & $\mathrm{PCl} / \mathrm{L}$ & $10 / 14 / 2005$ & $9.23 \mathrm{E}-02$ & SOS-TL352-05 \\
\hline GWM42001RH & MIDDLE-2050A & 790 & Uranium-235 & 1.06E-01 & 4.39E-02 & & UJ & $\mathrm{PCl} / \mathrm{L}$ & $10 / 14 / 2005$ & $1.14 \mathrm{E}-01$ & SOS-TL352-05 \\
\hline GWM42001RH & MIDDLE-2050A & 790 & Uranium-238 & $6.82 \mathrm{E}-01$ & $1.03 \mathrm{E}-01$ & & & $\mathrm{PCl} / \mathrm{L}$ & $10 / 14 / 2005$ & $9.89 \mathrm{E}-02$ & SOS-TL352-05 \\
\hline GWM42001RH & MIDDLE-2050A & 790 & Gross Alpha & $1.91 \mathrm{E}+00$ & $5.33 \mathrm{E}-01$ & & & $\mathrm{PCl} / \mathrm{L}$ & $10 / 14 / 2005$ & $1.55 E+00$ & SOS-TL352-05 \\
\hline GWM42001RH & MIDDLE-2050A & 790 & Gross Beta & $3.00 \mathrm{E}+00$ & $7.97 \mathrm{E}-01$ & & & $\mathrm{PCl} / \mathrm{L}$ & $10 / 14 / 2005$ & $2.90 \mathrm{E}+00$ & SOS-TL352-05 \\
\hline GWM42001RH & MIDDLE-2050A & 790 & Strontium-90 & $-6.45 \mathrm{E}-02$ & $1.12 \mathrm{E}-01$ & & u & $\mathrm{PCl} / \mathrm{L}$ & $10 / 14 / 2005$ & $5.97 \mathrm{E}-01$ & SOS-TL352-05 \\
\hline GWM42001RH & MIDDLE-2050A & 790 & Silver-108m & 2.64E-01 & $1.18 \mathrm{E}+00$ & & u & $\mathrm{PCl} / \mathrm{L}$ & $10 / 14 / 2005$ & $3.61 \mathrm{E}+00$ & SOS-TL352-05 \\
\hline GWM42001RH & MIDDLE-2050A & 790 & Silver-110m & $9.09 \mathrm{E}-01$ & 1.10E+00 & & $\mathrm{u}$ & $\mathrm{PCl} / \mathrm{L}$ & $10 / 14 / 2005$ & $3.75 E+00$ & SOS-TL352-05 \\
\hline GWM42001RH & MIDDLE-2050A & 790 & Cerium-144 & $2.13 E+00$ & $8.18 \mathrm{E}+00$ & & $\mathrm{u}$ & $\mathrm{PCl} / \mathrm{L}$ & $10 / 14 / 2005$ & $2.85 E+01$ & SOS-TL352-05 \\
\hline GWM42001RH & MIDDLE-2050A & 790 & Cobalt- 60 & $1.21 \mathrm{E}+00$ & $1.10 \mathrm{E}+00$ & & u & $\mathrm{PCl} / \mathrm{L}$ & 10/14/2005 & $4.43 E+00$ & SOS-TL352-05 \\
\hline GWM42001RH & MIDDLE-2050A & 790 & Cesium-134 & $-9.40 \mathrm{E}-01$ & $9.37 \mathrm{E}-01$ & & $\mathrm{u}$ & $\mathrm{PCl} / \mathrm{L}$ & $10 / 14 / 2005$ & $3.26 \mathrm{E}+00$ & SOS-TL352-05 \\
\hline GWM42001RH & MIDDLE-2050A & 790 & Cesium-137 & $1.53 \mathrm{E}-01$ & $1.04 \mathrm{E}+00$ & & $u$ & $\mathrm{PCl} / \mathrm{L}$ & 10/14/2005 & $3.84 \mathrm{E}+00$ & SOS-TL352-05 \\
\hline GWM42001RH & MIDDLE-2050A & 790 & Europium-152 & $-1.36 \mathrm{E}+00$ & $3.51 \mathrm{E}+00$ & & u & $\mathrm{PCl} / \mathrm{L}$ & $10 / 14 / 2005$ & $1.19 \mathrm{E}+01$ & SOS-TL352-05 \\
\hline GWM42001RH & MIDDLE-2050A & 790 & Europium-154 & $-2.13 E+00$ & $2.88 \mathrm{E}+00$ & & $u$ & $\mathrm{PCl} / \mathrm{L}$ & $10 / 14 / 2005$ & $1.01 \mathrm{E}+01$ & SOS-TL352-05 \\
\hline GWM42001RH & MIDDLE-2050A & 790 & Europium-155 & $-5.20 \mathrm{E}+00$ & 4.47E+00 & & u & $\mathrm{PCl} / \mathrm{L}$ & $10 / 14 / 2005$ & $1.51 \mathrm{E}+01$ & SOS-TL352-05 \\
\hline GWM42001RH & MIDDLE-2050A & 790 & Manganese-54 & 4.91E-02 & $1.36 \mathrm{E}+00$ & & u & $\mathrm{PCl} / \mathrm{L}$ & $10 / 14 / 2005$ & $4.27 \mathrm{E}+00$ & SOS-TL352-05 \\
\hline GWM42001RH & MIDDLE-2050A & 790 & Ruthenium-106 & $-1.36 \mathrm{E}+01$ & $9.37 \mathrm{E}+00$ & & $\mathrm{U}$ & $\mathrm{PCl} / \mathrm{L}$ & $10 / 14 / 2005$ & $3.17 E+01$ & SOS-TL352-05 \\
\hline GWM42001RH & MIDDLE-2050A & 790 & Antimony-125 & $-8.20 \mathrm{E}-01$ & $3.37 E+00$ & & u & $\mathrm{PCl} / \mathrm{L}$ & 10/14/2005 & $1.00 \mathrm{E}+01$ & SOS-TL352-05 \\
\hline GWM42001RH & MIDDLE-2050A & 790 & Zinc-65 & $9.85 \mathrm{E}-01$ & $2.40 \mathrm{E}+00$ & & UJ & $\mathrm{PCl} / \mathrm{L}$ & $10 / 14 / 2005$ & $9.01 \mathrm{E}+00$ & SOS-TL352-05 \\
\hline GWM42001RH & MIDDLE-2050A & 790 & Technetium-99 & $-1.44 \mathrm{E}+00$ & $1.56 \mathrm{E}+00$ & & U & $\mathrm{PCl} / \mathrm{L}$ & 10/14/2005 & $5.39 E+00$ & SOS-TL352-05 \\
\hline GWM42001R8 & MIDDLE-2050A & 790 & Tritium & $2.23 \mathrm{E}+02$ & $8.48 \mathrm{E}+01$ & & UJ & $\mathrm{PCl} / \mathrm{L}$ & $10 / 14 / 2005$ & $2.74 \mathrm{E}+02$ & SOS-TL352-05 \\
\hline GWM421022X & MIDDLE-2050A & 1005 & Antimony & 0.61 & & U & & UG/L & $10 / 07 / 2005$ & & DNT-508-05 \\
\hline GWM421022X & MIDDLE-2050A & 1005 & Arsenic & 0.9 & & u & & UG/L & $10 / 07 / 2005$ & & DNT-508-05 \\
\hline GWM421022X & MIDDLE-2050A & 1005 & Beryllium & 0.13 & & u & & UG/L & $10 / 07 / 2005$ & & DNT-508-05 \\
\hline GWM421022X & MIDDLE-2050A & 1005 & Cadmium & 0.067 & & u & & UG/L & $10 / 07 / 2005$ & & DNT-508-05 \\
\hline GWM421022X & MIDDLE-2050A & 1005 & Chromium & 5.7 & & & & UG/L & $10 / 07 / 2005$ & & DNT-508-05 \\
\hline GWM421022X & MIDDLE-2050A & 1005 & Manganese & 7.3 & & & & UG/L & $10 / 07 / 2005$ & & DNT-508-05 \\
\hline GWM421022X & MIDDLE-2050A & 1005 & Silver & 1.5 & & u & & UG/L & $10 / 07 / 2005$ & & DNT-508-05 \\
\hline GWM421022X & MIDDLE-2050A & 1005 & Thallium & 0.56 & & B & $\mathrm{u}$ & UG/L & $10 / 07 / 2005$ & & DNT-508-05 \\
\hline GWM421022X & MIDDLE-2050A & 1005 & Uranium & 2 & & B & $\mathrm{J}$ & UG/L & 10/07/2005 & & DNT-508-05 \\
\hline GWM421022X & MIDDLE-2050A & 1005 & Mercury & 0.046 & & u & UJ & UG/L & $10 / 07 / 2005$ & & DNT-508-05 \\
\hline GWM421022X & MIDDLE-2050A & 1005 & Aluminum & 22.1 & & u & UJ & UG/L & $10 / 07 / 2005$ & & DNT-508-05 \\
\hline GWM421022X & MIDDLE-2050A & 1005 & Barium & 66.3 & & B & & UG/L & $10 / 07 / 2005$ & & DNT-508-05 \\
\hline GWM421022X & MIDDLE-2050A & 1005 & Calcium & 49100 & & & & UG/L & $10 / 07 / 2005$ & & DNT-508-05 \\
\hline GWM421022X & MIDDLE-2050A & 1005 & Cobalt & 2.8 & & $\mathrm{u}$ & & UG/L & $10 / 07 / 2005$ & & DNT-508-05 \\
\hline GWM421022X & MIDDLE-2050A & 1005 & Copper & 1.1 & & u & & UG/L & $10 / 07 / 2005$ & & DNT-508-05 \\
\hline GWM421022X & MIDDLE-2050A & 1005 & Iron & 9.6 & & B & & UG/L & $10 / 07 / 2005$ & & DNT-508-05 \\
\hline GWM421022X & MIDDLE-2050A & 1005 & Lead & 1.1 & & u & & UG/L & $10 / 07 / 2005$ & & DNT-508-05 \\
\hline GWM421022X & MIDDLE-2050A & 1005 & Lithium & 3 & & u & & UG/L & $10 / 07 / 2005$ & & DNT-508-05 \\
\hline GWM421022X & MIDDLE-2050A & 1005 & Magnesium & 18200 & & & & UG/L & 10/07/2005 & & DNT-508-05 \\
\hline GWM421022X & MIDDLE-2050A & 1005 & Molybdenum & 3.7 & & $\mathrm{U}$ & & UG/L & $10 / 07 / 2005$ & & DNT-508-05 \\
\hline GWM421022X & MIDDLE-2050A & 1005 & Nickel & 3.2 & & u & & UG/L & $10 / 07 / 2005$ & & DNT-508-05 \\
\hline GWM421022X & MIDDLE-2050A & 1005 & Potassium & 2290 & & B & & UG/L & $10 / 07 / 2005$ & & DNT-508-05 \\
\hline GWM421022X & MIDDLE-2050A & 1005 & Selenium & 3.5 & & B & u & UG/L & 10/07/2005 & & DNT-508-05 \\
\hline GWM421022X & MIDDLE-2050A & 1005 & Sodium & 8440 & & & & UG/L & $10 / 07 / 2005$ & & DNT-508-05 \\
\hline GWM421022X & MIDDLE-2050A & 1005 & Strontium & 257 & & & & UG/L & 10/07/2005 & & DNT-508-05 \\
\hline GWM421022X & MIDDLE-2050A & 1005 & Tin & 3.9 & & $\mathrm{U}$ & & UG/L & $10 / 07 / 2005$ & & DNT-508-05 \\
\hline GWM421022X & MIDDLE-2050A & 1005 & Vanadium & 3.8 & & B & & UG/L & 10/07/2005 & & DNT-508-05 \\
\hline GWM421022X & MIDDLE-2050A & 1005 & Zinc & 13.6 & & B & $\mathrm{u}$ & UG/L & $10 / 07 / 2005$ & & DNT-508-05 \\
\hline GWM421012X & MIDDLE-2050A & 1005 & Antimony & 0.61 & & u & & UG/L & $10 / 07 / 2005$ & & DNT-508-05 \\
\hline GWM421012X & MIDDLE-2050A & 1005 & Arsenic & 0.99 & & B & $\mathrm{u}$ & UG/L & $10 / 07 / 2005$ & & DNT-508-05 \\
\hline GWM421012X & MIDDLE-2050A & 1005 & Beryllium & 0.13 & & u & & UG/L & $10 / 07 / 2005$ & & DNT-508-05 \\
\hline GWM421012X & MIDDLE-2050A & 1005 & Cadmium & 0.067 & & u & & UG/L & $10 / 07 / 2005$ & & DNT-508-05 \\
\hline GWM421012X & MIDDLE-2050A & 1005 & Chromium & 5.8 & & & & UG/L & $10 / 07 / 2005$ & & DNT-508-05 \\
\hline GWM421012X & MIDDLE-2050A & 1005 & Manganese & 7 & & & & UG/L & $10 / 07 / 2005$ & & DNT-508-05 \\
\hline GWM421012X & MIDDLE-2050A & 1005 & Silver & 1.5 & & u & & UG/L & $10 / 07 / 2005$ & & DNT-508-05 \\
\hline GWM421012X & MIDDLE-2050A & 1005 & Thallium & 1.1 & & & $\mathrm{u}$ & UG/L & $10 / 07 / 2005$ & & DNT-508-05 \\
\hline GWM421012X & MIDDLE-2050A & 1005 & Uranium & 1.8 & & B & $\mathrm{J}$ & UG/L & 10/07/2005 & & DNT-508-05 \\
\hline GWM421012X & MIDDLE-2050A & 1005 & Mercury & 0.046 & & $u$ & UJ & UG/L & $10 / 07 / 2005$ & & DNT-508-05 \\
\hline GWM421012X & MIDDLE-2050A & 1005 & Aluminum & 22.1 & & u & UJ & UG/L & $10 / 07 / 2005$ & & DNT-508-05 \\
\hline GWM421012X & MIDDLE-2050A & 1005 & Barium & 65.6 & & B & & UG/L & $10 / 07 / 2005$ & & DNT-508-05 \\
\hline GWM421012X & MIDDLE-2050A & 1005 & Calcium & 49000 & & & & UG/L & $10 / 07 / 2005$ & & DNT-508-05 \\
\hline GWM421012X & MIDDLE-2050A & 1005 & Cobalt & 2.8 & & $\mathrm{u}$ & & UG/L & $10 / 07 / 2005$ & & DNT-508-05 \\
\hline GWM421012X & MIDDLE-2050A & 1005 & Copper & 1.1 & & u & & UG/L & $10 / 07 / 2005$ & & DNT-508-05 \\
\hline GWM421012X & MIDDLE-2050A & 1005 & Iron & 7.4 & & u & & UG/L & $10 / 07 / 2005$ & & DNT-508-05 \\
\hline GWM421012X & MIDDLE-2050A & 1005 & Lead & 1.1 & & $u$ & & UG/L & 10/07/2005 & & DNT-508-05 \\
\hline GWM421012X & MIDDLE-2050A & 1005 & Lithium & 3 & & $\mathrm{U}$ & & UG/L & $10 / 07 / 2005$ & & DNT-508-05 \\
\hline GWM421012X & MIDDLE-2050A & 1005 & Magnesium & 18100 & & & & UG/L & $10 / 07 / 2005$ & & DNT-508-05 \\
\hline GWM421012X & MIDDLE-2050A & 1005 & Molybdenum & 3.7 & & $\mathrm{U}$ & & UG/L & $10 / 07 / 2005$ & & DNT-508-05 \\
\hline GWM421012X & MIDDLE-2050A & 1005 & Nickel & 3.2 & & u & & UG/L & $10 / 07 / 2005$ & & DNT-508-05 \\
\hline GWM421012X & MIDDLE-2050A & 1005 & Potassium & 3030 & & B & & UG/L & $10 / 07 / 2005$ & & DNT-508-05 \\
\hline
\end{tabular}


Table A-4. (continued).

\begin{tabular}{|c|c|c|c|c|c|c|c|c|c|c|c|}
\hline $\begin{array}{c}\text { Field Sample } \\
\text { Number }\end{array}$ & Location & Depth & Compound & $\begin{array}{c}\text { Sample } \\
\text { Result }\end{array}$ & $\begin{array}{l}\text { Sample } \\
\text { Error }\end{array}$ & $\begin{array}{c}\text { Result } \\
\text { Qualifier }\end{array}$ & $\begin{array}{c}\text { Validation } \\
\text { Flag }\end{array}$ & $\begin{array}{c}\text { Sample } \\
\text { Units }\end{array}$ & $\begin{array}{c}\text { Date Sample } \\
\text { Collected }\end{array}$ & MDA & $\begin{array}{c}\text { L\&V Report } \\
\text { Number }\end{array}$ \\
\hline GWM421012X & MIDDLE-2050A & 1005 & Selenium & 5.5 & & & $\mathrm{U}$ & UG/L & $10 / 07 / 2005$ & & DNT-508-05 \\
\hline GWM421012X & MIDDLE-2050A & 1005 & Sodium & 8400 & & & & UG/L & 10/07/2005 & & DNT-508-05 \\
\hline GWM421012X & MIDDLE-2050A & 1005 & Strontium & 256 & & & & UG/L & $10 / 07 / 2005$ & & DNT-508-05 \\
\hline GWM421012X & MIDDLE-2050A & 1005 & Tin & 3.9 & & $u$ & & UG/L & 10/07/2005 & & DNT-508-05 \\
\hline GWM421012X & MIDDLE-2050A & 1005 & Vanadium & 3.1 & & B & & UG/L & $10 / 07 / 2005$ & & DNT-508-05 \\
\hline GWM421012X & MIDDLE-2050A & 1005 & Zinc & 10.6 & & B & $u$ & UG/L & 10/07/2005 & & DNT-508-05 \\
\hline GWM42101AN & MIDDLE-2050A & 1005 & Bromide & 0.25 & & u & & MG/L & 10/07/2005 & & DNT-006-06 \\
\hline GWM42101AN & MIDDLE-2050A & 1005 & Chloride & 10.2 & & $\mathrm{~J}$ & & MG/L & 10/07/2005 & & DNT-006-06 \\
\hline GWM42101AN & MIDDLE-2050A & 1005 & Fluoride & 0.12 & & & $\mathrm{~J}$ & MG/L & $10 / 07 / 2005$ & & DNT-006-06 \\
\hline GWM42101AN & MIDDLE-2050A & 1005 & Sulfate & 19.8 & & & & MG/L & 10/07/2005 & & DNT-006-06 \\
\hline GWM42101A1 & MIDDLE-2050A & 1005 & Total Alkalinity & 162 & & & & $\mathrm{MG} / \mathrm{L}$ & $10 / 07 / 2005$ & & DNT-006-06 \\
\hline GWM42101N2 & MIDDLE-2050A & 1005 & Nitrate/Nitrite as $\mathrm{N}$ & 0.66 & & & $\mathrm{~J}$ & MG/L & 10/07/2005 & & DNT-006-06 \\
\hline GWM42102N2 & MIDDLE-2050A & 1005 & Nitrate/Nitrite as $\mathrm{N}$ & 0.71 & & & $\mathrm{~J}$ & MG/L & $10 / 07 / 2005$ & & DNT-006-06 \\
\hline GWM42102AN & MIDDLE-2050A & 1005 & Bromide & 0.25 & & $u$ & & MG/L & 10/07/2005 & & DNT-006-06 \\
\hline GWM42102AN & MIDDLE-2050A & 1005 & Chloride & 9.8 & & $\mathrm{~J}$ & $\mathrm{R}$ & MG/L & 10/07/2005 & & DNT-006-06 \\
\hline GWM42102AN & MIDDLE-2050A & 1005 & Fluoride & 0.12 & & & & MG/L & 10/07/2005 & & DNT-006-06 \\
\hline GWM42102AN & MIDDLE-2050A & 1005 & Sulfate & 19.3 & & & & $\mathrm{MG} / \mathrm{L}$ & $10 / 07 / 2005$ & & DNT-006-06 \\
\hline GWM42102A1 & MIDDLE-2050A & 1005 & Total Alkalinity & 168 & & & & MG/L & 10/07/2005 & & DNT-006-06 \\
\hline GWM42101AV & MIDDLE-2050A & 1005 & Acetone & 0.8 & & $\mathrm{~J}$ & $\mathrm{~J}$ & UG/L & $10 / 07 / 2005$ & & HCJ-100-05 \\
\hline GWM42101AV & MIDDLE-2050A & 1005 & Acetonitrile & 20 & & $u$ & $\mathrm{R}$ & UG/L & 10/07/2005 & & HCJ-100-05 \\
\hline GWM42101AV & MIDDLE-2050A & 1005 & Acrolein & 5 & & u & $\mathrm{R}$ & UG/L & $10 / 07 / 2005$ & & HCJ-100-05 \\
\hline GWM42101AV & MIDDLE-2050A & 1005 & Acrylonitrile & 1 & & $u$ & & UG/L & 10/07/2005 & & HCJ-100-05 \\
\hline GWM42101AV & MIDDLE-2050A & 1005 & Benzene & 1 & & $\mathrm{u}$ & & UG/L & $10 / 07 / 2005$ & & HCJ-100-05 \\
\hline GWM42101AV & MIDDLE-2050A & 1005 & Bromodichloromethane & 1 & & u & & UG/L & 10/07/2005 & & HCJ-100-05 \\
\hline GWM42101AV & MIDDLE-2050A & 1005 & Bromoform & 2 & & u & & UG/L & $10 / 07 / 2005$ & & HCJ-100-05 \\
\hline GWM42101AV & MIDDLE-2050A & 1005 & Bromomethane & 0.43 & & $\mathrm{~J}$ & $\mathrm{~J}$ & UG/L & 10/07/2005 & & HCJ-100-05 \\
\hline GWM42101AV & MIDDLE-2050A & 1005 & 2-Butanone & 10 & & u & $\mathrm{R}$ & UG/L & 10/07/2005 & & HCJ-100-05 \\
\hline GWM42101AV & MIDDLE-2050A & 1005 & Carbon disulfide & 5 & & $u$ & & UG/L & 10/07/2005 & & HCJ-100-05 \\
\hline GWM42101AV & MIDDLE-2050A & 1005 & Carbon tetrachloride & 0.19 & & $\mathrm{~J}$ & $\mathrm{~J}$ & UG/L & $10 / 07 / 2005$ & & HCJ-100-05 \\
\hline GWM42101AV & MIDDLE-2050A & 1005 & Chlorobenzene & 1 & & $\mathrm{u}$ & & UG/L & 10/07/2005 & & HCJ-100-05 \\
\hline GWM42101AV & MIDDLE-2050A & 1005 & Chloroprene & 5 & & $\mathrm{u}$ & & UG/L & $10 / 07 / 2005$ & & HCJ-100-05 \\
\hline GWM42101AV & MIDDLE-2050A & 1005 & Dibromochloromethane & 1 & & $u$ & & UG/L & 10/07/2005 & & HCJ-100-05 \\
\hline GWM42101AV & MIDDLE-2050A & 1005 & Chloroethane & 1 & & $\mathrm{u}$ & & UG/L & $10 / 07 / 2005$ & & HCJ-100-05 \\
\hline GWM42101AV & MIDDLE-2050A & 1005 & Chloroform & 1 & & u & & UG/L & 10/07/2005 & & HCJ-100-05 \\
\hline GWM42101AV & MIDDLE-2050A & 1005 & Chloromethane & 0.32 & & $\mathrm{~J}$ & $\mathrm{~J}$ & UG/L & $10 / 07 / 2005$ & & HCJ-100-05 \\
\hline GWM42101AV & MIDDLE-2050A & 1005 & Allyl chloride & 5 & & u & & UG/L & 10/07/2005 & & HCJ-100-05 \\
\hline GWM42101AV & MIDDLE-2050A & 1005 & 1,2-Dibromo-3-chloropropane & 5 & & $\mathrm{u}$ & $\mathrm{R}$ & UG/L & 10/07/2005 & & HCJ-100-05 \\
\hline GWM42101AV & MIDDLE-2050A & 1005 & 1,2-Dibromoethane & 1 & & u & & UG/L & $10 / 07 / 2005$ & & HCJ-100-05 \\
\hline GWM42101AV & MIDDLE-2050A & 1005 & Dibromomethane & 1 & & $\mathrm{u}$ & & UG/L & $10 / 07 / 2005$ & & HCJ-100-05 \\
\hline GWM42101AV & MIDDLE-2050A & 1005 & trans-1,4-Dichloro-2-butene & 5 & & $\mathrm{u}$ & $\mathrm{R}$ & UG/L & 10/07/2005 & & HCJ-100-05 \\
\hline GWM42101AV & MIDDLE-2050A & 1005 & Dichlorodifluoromethane & 5 & & $\mathrm{u}$ & & UG/L & $10 / 07 / 2005$ & & HCJ-100-05 \\
\hline GWM42101AV & MIDDLE-2050A & 1005 & 1,1-Dichloroethane & 1 & & $u$ & & UG/L & 10/07/2005 & & HCJ-100-05 \\
\hline GWM42101AV & MIDDLE-2050A & 1005 & 1,2-Dichloroethane & 1 & & $\mathrm{u}$ & & UG/L & $10 / 07 / 2005$ & & HCJ-100-05 \\
\hline GWM42101AV & MIDDLE-2050A & 1005 & cis-1,2-Dichloroethene & 1 & & $\mathrm{u}$ & & UG/L & $10 / 07 / 2005$ & & HCJ-100-05 \\
\hline GWM42101AV & MIDDLE-2050A & 1005 & trans-1,2-Dichloroethene & 1 & & $\mathrm{u}$ & & UG/L & $10 / 07 / 2005$ & & HCJ-100-05 \\
\hline GWM42101AV & MIDDLE-2050A & 1005 & 1,1-Dichloroethene & 1 & & U & & UG/L & $10 / 07 / 2005$ & & HCJ-100-05 \\
\hline GWM42101AV & MIDDLE-2050A & 1005 & 1,2-Dichloropropane & 1 & & $\mathrm{u}$ & & UG/L & $10 / 07 / 2005$ & & HCJ-100-05 \\
\hline GWM42101AV & MIDDLE-2050A & 1005 & cis-1,3-Dichloropropene & 1 & & $u$ & & UG/L & 10/07/2005 & & HCJ-100-05 \\
\hline GWM42101AV & MIDDLE-2050A & 1005 & trans-1,3-Dichloropropene & 1 & & $\mathrm{u}$ & UJ & UG/L & $10 / 07 / 2005$ & & HCJ-100-05 \\
\hline GWM42101AV & MIDDLE-2050A & 1005 & 1,4-Dioxane & 80 & & U & $\mathrm{R}$ & UG/L & 10/07/2005 & & HCJ-100-05 \\
\hline GWM42101AV & MIDDLE-2050A & 1005 & Ethylbenzene & 1 & & $\mathrm{U}$ & & UG/L & $10 / 07 / 2005$ & & HCJ-100-05 \\
\hline GWM42101AV & MIDDLE-2050A & 1005 & 2-Hexanone & 5 & & u & & UG/L & $10 / 07 / 2005$ & & HCJ-100-05 \\
\hline GWM42101AV & MIDDLE-2050A & 1005 & Methyl iodide & 2 & & $\mathrm{u}$ & & UG/L & $10 / 07 / 2005$ & & HCJ-100-05 \\
\hline GWM42101AV & MIDDLE-2050A & 1005 & Isobutyl alcohol & 80 & & $\mathrm{u}$ & $\mathrm{R}$ & UG/L & $10 / 07 / 2005$ & & HCJ-100-05 \\
\hline GWM42101AV & MIDDLE-2050A & 1005 & Methacrylonitrile & 5 & & $\mathrm{u}$ & $\mathrm{R}$ & UG/L & $10 / 07 / 2005$ & & HCJ-100-05 \\
\hline GWM42101AV & MIDDLE-2050A & 1005 & Methylene Chloride & 1 & & $\mathrm{u}$ & & UG/L & $10 / 07 / 2005$ & & HCJ-100-05 \\
\hline GWM42101AV & MIDDLE-2050A & 1005 & Methylmethacrylate & 1 & & $\mathrm{u}$ & UJ & UG/L & $10 / 07 / 2005$ & & HCJ-100-05 \\
\hline GWM42101AV & MIDDLE-2050A & 1005 & Methyl isobutyl ketone & 5 & & $\mathrm{u}$ & & UG/L & $10 / 07 / 2005$ & & HCJ-100-05 \\
\hline GWM42101AV & MIDDLE-2050A & 1005 & Propionitrile & 5 & & u & $\mathrm{R}$ & UG/L & $10 / 07 / 2005$ & & HCJ-100-05 \\
\hline GWM42101AV & MIDDLE-2050A & 1005 & Styrene & 1 & & $u$ & & UG/L & 10/07/2005 & & HCJ-100-05 \\
\hline GWM42101AV & MIDDLE-2050A & 1005 & 1,1,1,2-Tetrachloroethane & 1 & & $\mathrm{u}$ & & UG/L & $10 / 07 / 2005$ & & HCJ-100-05 \\
\hline GWM42101AV & MIDDLE-2050A & 1005 & 1,1,2,2-Tetrachloroethane & 1 & & $\mathrm{u}$ & & UG/L & $10 / 07 / 2005$ & & HCJ-100-05 \\
\hline GWM42101AV & MIDDLE-2050A & 1005 & Tetrachloroethene & 1 & & $\mathrm{u}$ & & UG/L & $10 / 07 / 2005$ & & HCJ-100-05 \\
\hline GWM42101AV & MIDDLE-2050A & 1005 & Toluene & 0.16 & & $\mathrm{~J}$ & $\mathrm{~J}$ & UG/L & 10/07/2005 & & HCJ-100-05 \\
\hline GWM42101AV & MIDDLE-2050A & 1005 & 1,1,1-Trichloroethane & 1 & & $u$ & & UG/L & $10 / 07 / 2005$ & & HCJ-100-05 \\
\hline GWM42101AV & MIDDLE-2050A & 1005 & 1,1,2-Trichloroethane & 1 & & u & & UG/L & $10 / 07 / 2005$ & & HCJ-100-05 \\
\hline GWM42101AV & MIDDLE-2050A & 1005 & Trichloroethene & 1 & & u & & UG/L & $10 / 07 / 2005$ & & HCJ-100-05 \\
\hline GWM42101AV & MIDDLE-2050A & 1005 & Trichlorofluoromethane & 1 & & u & & UG/L & 10/07/2005 & & HCJ-100-05 \\
\hline GWM42101AV & MIDDLE-2050A & 1005 & 1,2,3-Trichloropropane & 1 & & u & & UG/L & $10 / 07 / 2005$ & & HCJ-100-05 \\
\hline GWM42101AV & MIDDLE-2050A & 1005 & Vinyl Acetate & 2 & & $\mathrm{u}$ & & UG/L & $10 / 07 / 2005$ & & HCJ-100-05 \\
\hline GWM42101AV & MIDDLE-2050A & 1005 & Vinyl Chloride & 1 & & u & & UG/L & $10 / 07 / 2005$ & & HCJ-100-05 \\
\hline GWM42101AV & MIDDLE-2050A & 1005 & Xylenes & 3 & & $u$ & & UG/L & 10/07/2005 & & HCJ-100-05 \\
\hline GWM42102AV & MIDDLE-2050A & 1005 & Acetone & 0.75 & & $\mathrm{~J}$ & $\mathrm{~J}$ & UG/L & $10 / 07 / 2005$ & & HCJ-100-05 \\
\hline GWM42102AV & MIDDLE-2050A & 1005 & Acetonitrile & 20 & & u & $\mathrm{R}$ & UG/L & $10 / 07 / 2005$ & & HCJ-100-05 \\
\hline GWM42102AV & MIDDLE-2050A & 1005 & Acrolein & 5 & & u & $\mathrm{R}$ & UG/L & 10/07/2005 & & HCJ-100-05 \\
\hline GWM42102AV & MIDDLE-2050A & 1005 & Acrylonitrile & 1 & & $u$ & & UG/L & 10/07/2005 & & HCJ-100-05 \\
\hline GWM42102AV & MIDDLE-2050A & 1005 & Benzene & 1 & & u & & UG/L & $10 / 07 / 2005$ & & HCJ-100-05 \\
\hline GWM42102AV & MIDDLE-2050A & 1005 & Bromodichloromethane & 1 & & $\mathrm{u}$ & & UG/L & $10 / 07 / 2005$ & & HCJ-100-05 \\
\hline GWM42102AV & MIDDLE-2050A & 1005 & Bromoform & 2 & & u & & UG/L & $10 / 07 / 2005$ & & HCJ-100-05 \\
\hline GWM42102AV & MIDDLE-2050A & 1005 & Bromomethane & 0.48 & & $\mathrm{~J}$ & $\mathrm{~J}$ & UG/L & $10 / 07 / 2005$ & & HCJ-100-05 \\
\hline GWM42102AV & MIDDLE-2050A & 1005 & 2-Butanone & 10 & & $u$ & $\mathrm{R}$ & UG/L & $10 / 07 / 2005$ & & HCJ-100-05 \\
\hline GWM42102AV & MIDDLE-2050A & 1005 & Carbon disulfide & 5 & & $\mathrm{u}$ & & UG/L & $10 / 07 / 2005$ & & HCJ-100-05 \\
\hline GWM42102AV & MIDDLE-2050A & 1005 & Carbon tetrachloride & 1 & & $\mathrm{u}$ & & UG/L & 10/07/2005 & & HCJ-100-05 \\
\hline GWM42102AV & MIDDLE-2050A & 1005 & Chlorobenzene & 1 & & $\mathrm{U}$ & & UG/L & $10 / 07 / 2005$ & & HCJ-100-05 \\
\hline GWM42102AV & MIDDLE-2050A & 1005 & Chloroprene & 5 & & $\mathrm{u}$ & & UG/L & 10/07/2005 & & HCJ-100-05 \\
\hline GWM42102AV & MIDDLE-2050A & 1005 & Dibromochloromethane & 1 & & $\mathrm{u}$ & & UG/L & $10 / 07 / 2005$ & & HCJ-100-05 \\
\hline
\end{tabular}


Table A-4. (continued).

\begin{tabular}{|c|c|c|c|c|c|c|c|c|c|c|c|}
\hline $\begin{array}{c}\text { Field Sample } \\
\text { Number }\end{array}$ & Location & Depth & Compound & $\begin{array}{c}\text { Sample } \\
\text { Result }\end{array}$ & $\begin{array}{c}\text { Sample } \\
\text { Error }\end{array}$ & $\begin{array}{c}\text { Result } \\
\text { Qualifier }\end{array}$ & $\begin{array}{c}\text { Validation } \\
\text { Flag }\end{array}$ & $\begin{array}{c}\text { Sample } \\
\text { Units }\end{array}$ & $\begin{array}{c}\text { Date Sample } \\
\text { Collected }\end{array}$ & MDA & $\begin{array}{l}\text { L\&V Report } \\
\text { Number }\end{array}$ \\
\hline GWM42102AV & MIDDLE-2050A & 1005 & Chloroethane & 1 & & $\bar{U}$ & & UG/L & $10 / 07 / 2005$ & & HCJ-100-05 \\
\hline GWM42102AV & MIDDLE-2050A & 1005 & Chloroform & 1 & & $\mathrm{u}$ & & UG/L & 10/07/2005 & & HCJ-100-05 \\
\hline GWM42102AV & MIDDLE-2050A & 1005 & Chloromethane & 5 & & $\mathrm{u}$ & & UG/L & $10 / 07 / 2005$ & & HCJ-100-05 \\
\hline GWM42102AV & MIDDLE-2050A & 1005 & Allyl chloride & 5 & & $\mathrm{u}$ & & UG/L & $10 / 07 / 2005$ & & HCJ-100-05 \\
\hline GWM42102AV & MIDDLE-2050A & 1005 & 1,2-Dibromo-3-chloropropane & 5 & & $\mathrm{u}$ & $\mathrm{R}$ & UG/L & $10 / 07 / 2005$ & & HCJ-100-05 \\
\hline GWM42102AV & MIDDLE-2050A & 1005 & 1,2-Dibromoethane & 1 & & $\mathrm{u}$ & & UG/L & $10 / 07 / 2005$ & & HCJ-100-05 \\
\hline GWM42102AV & MIDDLE-2050A & 1005 & Dibromomethane & 1 & & $\mathrm{u}$ & & UG/L & $10 / 07 / 2005$ & & HCJ-100-05 \\
\hline GWM42102AV & MIDDLE-2050A & 1005 & trans-1,4-Dichloro-2-butene & 5 & & $u$ & $\mathrm{R}$ & UG/L & $10 / 07 / 2005$ & & HCJ-100-05 \\
\hline GWM42102AV & MIDDLE-2050A & 1005 & Dichlorodifluoromethane & 5 & & $\mathrm{u}$ & & UG/L & $10 / 07 / 2005$ & & HCJ-100-05 \\
\hline GWM42102AV & MIDDLE-2050A & 1005 & 1,1-Dichloroethane & 1 & & $\mathrm{u}$ & & UG/L & $10 / 07 / 2005$ & & HCJ-100-05 \\
\hline GWM42102AV & MIDDLE-2050A & 1005 & 1,2-Dichloroethane & 1 & & $\mathrm{u}$ & & UG/L & $10 / 07 / 2005$ & & HCJ-100-05 \\
\hline GWM42102AV & MIDDLE-2050A & 1005 & cis-1,2-Dichloroethene & 1 & & $\mathrm{u}$ & & UG/L & $10 / 07 / 2005$ & & HCJ-100-05 \\
\hline GWM42102AV & MIDDLE-2050A & 1005 & trans-1,2-Dichloroethene & 1 & & u & & UG/L & 10/07/2005 & & HCJ-100-05 \\
\hline GWM42102AV & MIDDLE-2050A & 1005 & 1,1-Dichloroethene & 1 & & $\mathrm{u}$ & & UG/L & $10 / 07 / 2005$ & & HCJ-100-05 \\
\hline GWM42102AV & MIDDLE-2050A & 1005 & 1,2-Dichloropropane & 1 & & $\mathrm{u}$ & & UG/L & 10/07/2005 & & HCJ-100-05 \\
\hline GWM42102AV & MIDDLE-2050A & 1005 & cis-1,3-Dichloropropene & 1 & & $\mathrm{u}$ & & UG/L & $10 / 07 / 2005$ & & HCJ-100-05 \\
\hline GWM42102AV & MIDDLE-2050A & 1005 & trans-1,3-Dichloropropene & 1 & & $\mathrm{u}$ & UJ & UG/L & $10 / 07 / 2005$ & & HCJ-100-05 \\
\hline GWM42102AV & MIDDLE-2050A & 1005 & 1,4-Dioxane & 80 & & $\mathrm{u}$ & $\mathrm{R}$ & UG/L & $10 / 07 / 2005$ & & HCJ-100-05 \\
\hline GWM42102AV & MIDDLE-2050A & 1005 & Ethylbenzene & 1 & & $\mathrm{u}$ & & UG/L & $10 / 07 / 2005$ & & HCJ-100-05 \\
\hline GWM42102AV & MIDDLE-2050A & 1005 & 2-Hexanone & 5 & & $\mathrm{u}$ & & UG/L & $10 / 07 / 2005$ & & HCJ-100-05 \\
\hline GWM42102AV & MIDDLE-2050A & 1005 & Methyl iodide & 2 & & u & & UG/L & $10 / 07 / 2005$ & & HCJ-100-05 \\
\hline GWM42102AV & MIDDLE-2050A & 1005 & Isobutyl alcohol & 80 & & $\mathrm{~J}$ & $\mathrm{R}$ & UG/L & $10 / 07 / 2005$ & & HCJ-100-05 \\
\hline GWM42102AV & MIDDLE-2050A & 1005 & Methacrylonitrile & 5 & & $\mathrm{u}$ & $\mathrm{R}$ & UG/L & $10 / 07 / 2005$ & & HCJ-100-05 \\
\hline GWM42102AV & MIDDLE-2050A & 1005 & Methylene Chloride & 1 & & $\mathrm{u}$ & & UG/L & $10 / 07 / 2005$ & & HCJ-100-05 \\
\hline GWM42102AV & MIDDLE-2050A & 1005 & Methylmethacrylate & 1 & & $\mathrm{u}$ & UJ & UG/L & 10/07/2005 & & HCJ-100-05 \\
\hline GWM42102AV & MIDDLE-2050A & 1005 & Methyl isobutyl ketone & 5 & & $\mathrm{u}$ & & UG/L & $10 / 07 / 2005$ & & HCJ-100-05 \\
\hline GWM42102AV & MIDDLE-2050A & 1005 & Propionitrile & 5 & & $\mathrm{u}$ & $\mathrm{R}$ & UG/L & 10/07/2005 & & HCJ-100-05 \\
\hline GWM42102AV & MIDDLE-2050A & 1005 & Styrene & 1 & & $\mathrm{u}$ & & UG/L & $10 / 07 / 2005$ & & HCJ-100-05 \\
\hline GWM42102AV & MIDDLE-2050A & 1005 & 1,1,1,2-Tetrachloroethane & 1 & & $u$ & & UG/L & $10 / 07 / 2005$ & & HCJ-100-05 \\
\hline GWM42102AV & MIDDLE-2050A & 1005 & 1,1,2,2-Tetrachloroethane & 1 & & $\mathrm{u}$ & & UG/L & $10 / 07 / 2005$ & & HCJ-100-05 \\
\hline GWM42102AV & MIDDLE-2050A & 1005 & Tetrachloroethene & 1 & & $\mathrm{u}$ & & UG/L & $10 / 07 / 2005$ & & HCJ-100-05 \\
\hline GWM42102AV & MIDDLE-2050A & 1005 & Toluene & 1 & & $\mathrm{u}$ & & UG/L & $10 / 07 / 2005$ & & HCJ-100-05 \\
\hline GWM42102AV & MIDDLE-2050A & 1005 & 1,1,1-Trichloroethane & 1 & & u & & UG/L & 10/07/2005 & & HCJ-100-05 \\
\hline GWM42102AV & MIDDLE-2050A & 1005 & $1,1,2$-Trichloroethane & 1 & & $\mathrm{u}$ & & UG/L & $10 / 07 / 2005$ & & HCJ-100-05 \\
\hline GWM42102AV & MIDDLE-2050A & 1005 & Trichloroethene & 1 & & $\mathrm{u}$ & & UG/L & $10 / 07 / 2005$ & & HCJ-100-05 \\
\hline GWM42102AV & MIDDLE-2050A & 1005 & Trichlorofluoromethane & 1 & & $\mathrm{u}$ & & UG/L & $10 / 07 / 2005$ & & HCJ-100-05 \\
\hline GWM42102AV & MIDDLE-2050A & 1005 & 1,2,3-Trichloropropane & 1 & & u & & UG/L & 10/07/2005 & & HCJ-100-05 \\
\hline GWM42102AV & MIDDLE-2050A & 1005 & Vinyl Acetate & 2 & & $\mathrm{u}$ & & UG/L & $10 / 07 / 2005$ & & HCJ-100-05 \\
\hline GWM42102AV & MIDDLE-2050A & 1005 & Vinyl Chloride & 1 & & u & & UG/L & $10 / 07 / 2005$ & & HCJ-100-05 \\
\hline GWM42102AV & MIDDLE-2050A & 1005 & Xylenes & 3 & & $\mathrm{u}$ & & UG/L & $10 / 07 / 2005$ & & HCJ-100-05 \\
\hline GWM42101RH & MIDDLE-2050A & 1005 & Uranium-233/234 & $1.17 \mathrm{E}+00$ & $1.11 \mathrm{E}-01$ & & & $\mathrm{PCl} / \mathrm{L}$ & $10 / 07 / 2005$ & $7.24 \mathrm{E}-02$ & SOS-TL342-05 \\
\hline GWM42101RH & MIDDLE-2050A & 1005 & Uranium-238 & 4.47E-01 & $6.05 \mathrm{E}-02$ & & & $\mathrm{PCl} / \mathrm{L}$ & $10 / 07 / 2005$ & $5.69 \mathrm{E}-02$ & SOS-TL342-05 \\
\hline GWM42101RH & MIDDLE-2050A & 1005 & Uranium-235 & 4.02E-02 & $2.07 \mathrm{E}-02$ & & $\mathrm{u}$ & $\mathrm{PCl} / \mathrm{L}$ & $10 / 07 / 2005$ & $6.19 \mathrm{E}-02$ & SOS-TL342-05 \\
\hline GWM42102RH & MIDDLE-2050A & 1005 & Uranium-233/234 & $1.36 \mathrm{E}+00$ & $1.15 \mathrm{E}-01$ & & & $\mathrm{PCl} / \mathrm{L}$ & $10 / 07 / 2005$ & 4.32E-02 & SOS-TL342-05 \\
\hline GWM42102RH & MIDDLE-2050A & 1005 & Uranium-238 & $5.15 \mathrm{E}-01$ & $6.13 \mathrm{E}-02$ & & & $\mathrm{PCl} / \mathrm{L}$ & $10 / 07 / 2005$ & 4.91E-02 & SOS-TL342-05 \\
\hline GWM42102RH & MIDDLE-2050A & 1005 & Uranium-235 & $9.04 \mathrm{E}-02$ & $2.56 \mathrm{E}-02$ & & & $\mathrm{PCl} / \mathrm{L}$ & $10 / 07 / 2005$ & $2.09 \mathrm{E}-02$ & SOS-TL342-05 \\
\hline GWM42101RH & MIDDLE-2050A & 1005 & Gross Alpha & $7.72 \mathrm{E}-01$ & 7.81E-01 & & u & $\mathrm{PCl} / \mathrm{L}$ & $10 / 07 / 2005$ & $3.13 E+00$ & SOS-TL342-05 \\
\hline GWM42101RH & MIDDLE-2050A & 1005 & Gross Beta & $-7.32 \mathrm{E}-01$ & $9.30 \mathrm{E}-01$ & & u & $\mathrm{PCl} / \mathrm{L}$ & $10 / 07 / 2005$ & $3.93 E+00$ & SOS-TL342-05 \\
\hline GWM42102RH & MIDDLE-2050A & 1005 & Gross Alpha & $1.89 \mathrm{E}+00$ & $5.86 \mathrm{E}-01$ & & & $\mathrm{PCl} / \mathrm{L}$ & $10 / 07 / 2005$ & $1.82 \mathrm{E}+00$ & SOS-TL342-05 \\
\hline GWM42102RH & MIDDLE-2050A & 1005 & Gross Beta & $1.70 \mathrm{E}+00$ & $8.46 \mathrm{E}-01$ & & UJ & $\mathrm{PCl} / \mathrm{L}$ & $10 / 07 / 2005$ & $3.29 \mathrm{E}+00$ & SOS-TL342-05 \\
\hline GWM42101RH & MIDDLE-2050A & 1005 & Strontium-90 & $5.15 \mathrm{E}-01$ & $1.87 \mathrm{E}-01$ & & UJ & $\mathrm{PCl} / \mathrm{L}$ & $10 / 07 / 2005$ & 7.10E-01 & SOS-TL342-05 \\
\hline GWM42102RH & MIDDLE-2050A & 1005 & Strontium-90 & $2.79 \mathrm{E}-01$ & $1.75 \mathrm{E}-01$ & & u & $\mathrm{PCl} / \mathrm{L}$ & $10 / 07 / 2005$ & $7.40 \mathrm{E}-01$ & SOS-TL342-05 \\
\hline GWM42101RH & MIDDLE-2050A & 1005 & Silver-108m & $-2.90 \mathrm{E}-01$ & $1.37 \mathrm{E}+00$ & & u & $\mathrm{PCl} / \mathrm{L}$ & $10 / 07 / 2005$ & $4.93 E+00$ & SOS-TL342-05 \\
\hline GWM42101RH & MIDDLE-2050A & 1005 & Silver-110m & $1.94 \mathrm{E}+00$ & $1.26 \mathrm{E}+00$ & & u & $\mathrm{PCl} / \mathrm{L}$ & $10 / 07 / 2005$ & $5.36 \mathrm{E}+00$ & SOS-TL342-05 \\
\hline GWM42101RH & MIDDLE-2050A & 1005 & Cerium-144 & $-9.39 E+00$ & $1.07 \mathrm{E}+01$ & & $u$ & $\mathrm{PCl} / \mathrm{L}$ & $10 / 07 / 2005$ & $3.50 \mathrm{E}+01$ & SOS-TL342-05 \\
\hline GWM42101RH & MIDDLE-2050A & 1005 & Cobalt- 60 & $2.39 \mathrm{E}+00$ & $1.43 \mathrm{E}+00$ & & $\mathrm{U}$ & $\mathrm{PCl} / \mathrm{L}$ & $10 / 07 / 2005$ & $6.55 \mathrm{E}+00$ & SOS-TL342-05 \\
\hline GWM42101RH & MIDDLE-2050A & 1005 & Cesium-134 & $1.26 \mathrm{E}+00$ & $1.18 \mathrm{E}+00$ & & UJ & $\mathrm{PCl} / \mathrm{L}$ & $10 / 07 / 2005$ & $6.01 \mathrm{E}+00$ & SOS-TL342-05 \\
\hline GWM42101RH & MIDDLE-2050A & 1005 & Cesium-137 & $1.86 \mathrm{E}+00$ & $1.51 \mathrm{E}+00$ & & $u$ & $\mathrm{PCl} / \mathrm{L}$ & $10 / 07 / 2005$ & $5.97 \mathrm{E}+00$ & SOS-TL342-05 \\
\hline GWM42101RH & MIDDLE-2050A & 1005 & Europium-152 & $-4.64 \mathrm{E}+00$ & $3.75 E+00$ & & u & $\mathrm{PCl} / \mathrm{L}$ & $10 / 07 / 2005$ & $1.28 \mathrm{E}+01$ & SOS-TL342-05 \\
\hline GWM42101RH & MIDDLE-2050A & 1005 & Europium-154 & $2.82 \mathrm{E}-01$ & $4.01 \mathrm{E}+00$ & & u & $\mathrm{PCl} / \mathrm{L}$ & $10 / 07 / 2005$ & $1.59 \mathrm{E}+01$ & SOS-TL342-05 \\
\hline GWM42101RH & MIDDLE-2050A & 1005 & Europium-155 & $-5.18 \mathrm{E}+00$ & $5.15 E+00$ & & $u$ & $\mathrm{PCl} / \mathrm{L}$ & $10 / 07 / 2005$ & $1.70 \mathrm{E}+01$ & SOS-TL342-05 \\
\hline GWM42101RH & MIDDLE-2050A & 1005 & Manganese-54 & $-1.47 \mathrm{E}+00$ & $1.36 \mathrm{E}+00$ & & u & $\mathrm{PCl} / \mathrm{L}$ & $10 / 07 / 2005$ & $4.52 \mathrm{E}+00$ & SOS-TL342-05 \\
\hline GWM42101RH & MIDDLE-2050A & 1005 & Ruthenium-106 & $-1.88 \mathrm{E}+01$ & $1.29 \mathrm{E}+01$ & & $u$ & $\mathrm{PCl} / \mathrm{L}$ & $10 / 07 / 2005$ & $4.22 \mathrm{E}+01$ & SOS-TL342-05 \\
\hline GWM42101RH & MIDDLE-2050A & 1005 & Antimony-125 & $-2.84 \mathrm{E}+00$ & $3.57 \mathrm{E}+00$ & & $\mathrm{u}$ & $\mathrm{PCl} / \mathrm{L}$ & $10 / 07 / 2005$ & $1.25 \mathrm{E}+01$ & SOS-TL342-05 \\
\hline GWM42101RH & MIDDLE-2050A & 1005 & Zinc-65 & $-3.93 E+00$ & $3.22 \mathrm{E}+00$ & & $u$ & $\mathrm{PCl} / \mathrm{L}$ & $10 / 07 / 2005$ & $1.12 \mathrm{E}+01$ & SOS-TL342-05 \\
\hline GWM42102RH & MIDDLE-2050A & 1005 & Silver-108m & $-2.73 E-01$ & $1.57 \mathrm{E}+00$ & & u & $\mathrm{PCl} / \mathrm{L}$ & $10 / 07 / 2005$ & $4.89 E+00$ & SOS-TL342-05 \\
\hline GWM42102RH & MIDDLE-2050A & 1005 & Silver-110m & $-6.21 \mathrm{E}-01$ & $1.47 \mathrm{E}+00$ & & u & $\mathrm{PCl} / \mathrm{L}$ & $10 / 07 / 2005$ & $5.36 \mathrm{E}+00$ & SOS-TL342-05 \\
\hline GWM42102RH & MIDDLE-2050A & 1005 & Cerium-144 & $-2.92 \mathrm{E}+00$ & $9.23 \mathrm{E}+00$ & & u & $\mathrm{PCl} / \mathrm{L}$ & $10 / 07 / 2005$ & $3.19 \mathrm{E}+01$ & SOS-TL342-05 \\
\hline GWM42102RH & MIDDLE-2050A & 1005 & Cobalt- 60 & $5.37 \mathrm{E}-02$ & 1.47E+00 & & u & $\mathrm{PCl} / \mathrm{L}$ & $10 / 07 / 2005$ & $5.12 \mathrm{E}+00$ & SOS-TL342-05 \\
\hline GWM42102RH & MIDDLE-2050A & 1005 & Cesium-134 & $5.35 \mathrm{E}-01$ & $1.52 \mathrm{E}+00$ & & u & $\mathrm{PCl} / \mathrm{L}$ & $10 / 07 / 2005$ & $5.88 \mathrm{E}+00$ & SOS-TL342-05 \\
\hline GWM42102RH & MIDDLE-2050A & 1005 & Cesium-137 & $-3.67 \mathrm{E}-01$ & $1.63 \mathrm{E}+00$ & & u & $\mathrm{PCI} / \mathrm{L}$ & $10 / 07 / 2005$ & $5.99 \mathrm{E}+00$ & SOS-TL342-05 \\
\hline GWM42102RH & MIDDLE-2050A & 1005 & Europium-152 & $6.85 E+00$ & $5.25 \mathrm{E}+00$ & & $\mathrm{u}$ & $\mathrm{PCl} / \mathrm{L}$ & $10 / 07 / 2005$ & $1.58 \mathrm{E}+01$ & SOS-TL342-05 \\
\hline GWM42102RH & MIDDLE-2050A & 1005 & Europium-154 & $-2.20 \mathrm{E}+00$ & $4.14 \mathrm{E}+00$ & & $u$ & $\mathrm{PCl} / \mathrm{L}$ & $10 / 07 / 2005$ & $1.31 \mathrm{E}+01$ & SOS-TL342-05 \\
\hline GWM42102RH & MIDDLE-2050A & 1005 & Europium-155 & $2.69 \mathrm{E}+00$ & 4.83E+00 & & u & $\mathrm{PCl} / \mathrm{L}$ & $10 / 07 / 2005$ & $1.75 \mathrm{E}+01$ & SOS-TL342-05 \\
\hline GWM42102RH & MIDDLE-2050A & 1005 & Manganese-54 & $7.59 \mathrm{E}-01$ & $1.30 \mathrm{E}+00$ & & $u$ & $\mathrm{PCl} / \mathrm{L}$ & $10 / 07 / 2005$ & $5.16 \mathrm{E}+00$ & SOS-TL342-05 \\
\hline GWM42102RH & MIDDLE-2050A & 1005 & Ruthenium-106 & $4.89 \mathrm{E}+00$ & $1.10 \mathrm{E}+01$ & & u & $\mathrm{PCl} / \mathrm{L}$ & $10 / 07 / 2005$ & $4.37 \mathrm{E}+01$ & SOS-TL342-05 \\
\hline GWM42102RH & MIDDLE-2050A & 1005 & Antimony-125 & $5.01 \mathrm{E}+00$ & $3.79 \mathrm{E}+00$ & & $u$ & $\mathrm{PCl} / \mathrm{L}$ & $10 / 07 / 2005$ & $1.47 \mathrm{E}+01$ & SOS-TL342-05 \\
\hline GWM42102RH & MIDDLE-2050A & 1005 & Zinc-65 & $4.09 \mathrm{E}+00$ & $2.86 \mathrm{E}+00$ & & $\mathrm{u}$ & $\mathrm{PCl} / \mathrm{L}$ & $10 / 07 / 2005$ & $1.26 \mathrm{E}+01$ & SOS-TL342-05 \\
\hline GWM42101R8 & MIDDLE-2050A & 1005 & Tritium & $-8.57 E+00$ & $1.14 \mathrm{E}+02$ & & u & $\mathrm{PCI} / \mathrm{L}$ & $10 / 07 / 2005$ & $4.01 \mathrm{E}+02$ & SOS-TL342-05 \\
\hline GWM42102R8 & MIDDLE-2050A & 1005 & Tritium & $-8.66 \mathrm{E}+01$ & $1.11 \mathrm{E}+02$ & & u & $\mathrm{PCl} / \mathrm{L}$ & $10 / 07 / 2005$ & $4.05 \mathrm{E}+02$ & SOS-TL342-05 \\
\hline GWM42101RH & MIDDLE-2050A & 1005 & Technetium-99 & $1.29 \mathrm{E}-01$ & $1.59 \mathrm{E}+00$ & & U & $\mathrm{PCl} / \mathrm{L}$ & $10 / 07 / 2005$ & $5.39 E+00$ & SOS-TL342-05 \\
\hline GWM42102RH & MIDDLE-2050A & 1005 & Technetium-99 & $-6.08 \mathrm{E}-01$ & $1.60 \mathrm{E}+00$ & & $\mathrm{u}$ & $\mathrm{PCl} / \mathrm{L}$ & $10 / 07 / 2005$ & $5.50 \mathrm{E}+00$ & SOS-TL342-05 \\
\hline GWM422012X & MIDDLE-2050A & 1212 & Antimony & 0.61 & & u & & UG/L & 10/21/2005 & & DNT-007-06 \\
\hline GWM422012X & MIDDLE-2050A & 1212 & Arsenic & 1.8 & & u & & UG/L & $10 / 21 / 2005$ & & DNT-007-06 \\
\hline GWM422012X & MIDDLE-2050A & 1212 & Beryllium & 0.13 & & u & & UG/L & $10 / 21 / 2005$ & & DNT-007-06 \\
\hline GWM422012X & MIDDLE-2050A & 1212 & Cadmium & 0.067 & & u & & UG/L & $10 / 21 / 2005$ & & DNT-007-06 \\
\hline
\end{tabular}


Table A-4. (continued).

\begin{tabular}{|c|c|c|c|c|c|c|c|c|c|c|c|}
\hline $\begin{array}{c}\text { Field Sample } \\
\text { Number }\end{array}$ & Location & Depth & Compound & $\begin{array}{l}\text { Sample } \\
\text { Result }\end{array}$ & $\begin{array}{l}\text { Sample } \\
\text { Error }\end{array}$ & $\begin{array}{c}\text { Result } \\
\text { Qualifier }\end{array}$ & $\begin{array}{c}\text { Validation } \\
\text { Flag }\end{array}$ & $\begin{array}{c}\text { Sample } \\
\text { Units }\end{array}$ & $\begin{array}{c}\text { Date Sample } \\
\text { Collected }\end{array}$ & MDA & $\begin{array}{l}\text { L\&V Report } \\
\text { Number }\end{array}$ \\
\hline GWM422012X & MIDDLE-2050A & 1212 & Chromium & 7.1 & & & & UG/L & $10 / 21 / 2005$ & & DNT-007-06 \\
\hline GWM422012X & MIDDLE-2050A & 1212 & Manganese & 3.5 & & B & & UG/L & $10 / 21 / 2005$ & & DNT-007-06 \\
\hline GWM422012X & MIDDLE-2050A & 1212 & Silver & 1.5 & & $u$ & & UG/L & $10 / 21 / 2005$ & & DNT-007-06 \\
\hline GWM422012X & MIDDLE-2050A & 1212 & Thallium & 0.22 & & $u$ & & UG/L & 10/21/2005 & & DNT-007-06 \\
\hline GWM422012X & MIDDLE-2050A & 1212 & Uranium & 2.1 & & B & & UG/L & $10 / 21 / 2005$ & & DNT-007-06 \\
\hline GWM422012X & MIDDLE-2050A & 1212 & Mercury & 0.046 & & u & & UG/L & $10 / 21 / 2005$ & & DNT-007-06 \\
\hline GWM422012X & MIDDLE-2050A & 1212 & Aluminum & 26.3 & & B & $u$ & UG/L & 10/21/2005 & & DNT-007-06 \\
\hline GWM422012X & MIDDLE-2050A & 1212 & Barium & 71.7 & & B & & UG/L & 10/21/2005 & & DNT-007-06 \\
\hline GWM422012X & MIDDLE-2050A & 1212 & Calcium & 52100 & & & & UG/L & $10 / 21 / 2005$ & & DNT-007-06 \\
\hline GWM422012X & MIDDLE-2050A & 1212 & Cobalt & 2.8 & & $u$ & & UG/L & $10 / 21 / 2005$ & & DNT-007-06 \\
\hline GWM422012X & MIDDLE-2050A & 1212 & Copper & 1.1 & & u & & UG/L & $10 / 21 / 2005$ & & DNT-007-06 \\
\hline GWM422012X & MIDDLE-2050A & 1212 & Iron & 12.6 & & B & $u$ & UG/L & $10 / 21 / 2005$ & & DNT-007-06 \\
\hline GWM422012X & MIDDLE-2050A & 1212 & Lead & 1.1 & & u & & UG/L & $10 / 21 / 2005$ & & DNT-007-06 \\
\hline GWM422012X & MIDDLE-2050A & 1212 & Lithium & 3 & & u & & UG/L & $10 / 21 / 2005$ & & DNT-007-06 \\
\hline GWM422012X & MIDDLE-2050A & 1212 & Magnesium & 16600 & & & & UG/L & $10 / 21 / 2005$ & & DNT-007-06 \\
\hline GWM422012X & MIDDLE-2050A & 1212 & Molybdenum & 3.7 & & $u$ & & UG/L & $10 / 21 / 2005$ & & DNT-007-06 \\
\hline GWM422012X & MIDDLE-2050A & 1212 & Nickel & 3.2 & & u & & UG/L & $10 / 21 / 2005$ & & DNT-007-06 \\
\hline GWM422012X & MIDDLE-2050A & 1212 & Potassium & 2900 & & B & $\mathrm{u}$ & UG/L & $10 / 21 / 2005$ & & DNT-007-06 \\
\hline GWM422012X & MIDDLE-2050A & 1212 & Selenium & 2 & & u & & UG/L & $10 / 21 / 2005$ & & DNT-007-06 \\
\hline GWM422012X & MIDDLE-2050A & 1212 & Sodium & 7760 & & & & UG/L & $10 / 21 / 2005$ & & DNT-007-06 \\
\hline GWM422012X & MIDDLE-2050A & 1212 & Strontium & 279 & & & & UG/L & $10 / 21 / 2005$ & & DNT-007-06 \\
\hline GWM422012X & MIDDLE-2050A & 1212 & Tin & 3.9 & & $\mathrm{u}$ & & UG/L & $10 / 21 / 2005$ & & DNT-007-06 \\
\hline GWM422012X & MIDDLE-2050A & 1212 & Vanadium & 4.1 & & B & & UG/L & $10 / 21 / 2005$ & & DNT-007-06 \\
\hline GWM422012X & MIDDLE-2050A & 1212 & Zinc & 13.9 & & B & $\mathrm{u}$ & UG/L & $10 / 21 / 2005$ & & DNT-007-06 \\
\hline GWM42201AN & MIDDLE-2050A & 1212 & Bromide & 0.25 & & u & & MG/L & 10/21/2005 & & DNT-005-06 \\
\hline GWM42201AN & MIDDLE-2050A & 1212 & Chloride & 17 & & & & $\mathrm{MG} / \mathrm{L}$ & $10 / 21 / 2005$ & & DNT-005-06 \\
\hline GWM42201AN & MIDDLE-2050A & 1212 & Fluoride & 0.13 & & & & MG/L & 10/21/2005 & & DNT-005-06 \\
\hline GWM42201AN & MIDDLE-2050A & 1212 & Sulfate & 23.1 & & & & MG/L & $10 / 21 / 2005$ & & DNT-005-06 \\
\hline GWM42201A1 & MIDDLE-2050A & 1212 & Total Alkalinity & 154 & & & & MG/L & $10 / 21 / 2005$ & & DNT-005-06 \\
\hline GWM42201N2 & MIDDLE-2050A & 1212 & Nitrate/Nitrite as N & 0.05 & & $\mathrm{u}$ & $\mathrm{u}$ & $M G / L$ & $10 / 21 / 2005$ & & DNT-005-06 \\
\hline GWM42201AV & MIDDLE-2050A & 1212 & Acetone & 1.5 & & $\mathrm{~J}$ & $\mathrm{~J}$ & UG/L & $10 / 21 / 2005$ & & DMG-217-05 \\
\hline GWM42201AV & MIDDLE-2050A & 1212 & Acetonitrile & 20 & & $\mathrm{u}$ & $\mathrm{R}$ & UG/L & $10 / 21 / 2005$ & & DMG-217-05 \\
\hline GWM42201AV & MIDDLE-2050A & 1212 & Acrolein & 5 & & $\mathrm{u}$ & $\mathrm{R}$ & UG/L & $10 / 21 / 2005$ & & DMG-217-05 \\
\hline GWM42201AV & MIDDLE-2050A & 1212 & Acrylonitrile & 1 & & $\mathrm{u}$ & & UG/L & $10 / 21 / 2005$ & & DMG-217-05 \\
\hline GWM42201AV & MIDDLE-2050A & 1212 & Benzene & 1 & & $\mathrm{u}$ & & UG/L & $10 / 21 / 2005$ & & DMG-217-05 \\
\hline GWM42201AV & MIDDLE-2050A & 1212 & Bromodichloromethane & 1 & & $\mathrm{u}$ & & UG/L & $10 / 21 / 2005$ & & DMG-217-05 \\
\hline GWM42201AV & MIDDLE-2050A & 1212 & Bromoform & 2 & & $\mathrm{u}$ & & UG/L & 10/21/2005 & & DMG-217-05 \\
\hline GWM42201AV & MIDDLE-2050A & 1212 & Bromomethane & 1 & & $\mathrm{JB}$ & $\mathrm{u}$ & UG/L & $10 / 21 / 2005$ & & DMG-217-05 \\
\hline GWM42201AV & MIDDLE-2050A & 1212 & 2-Butanone & 10 & & $u$ & $\mathrm{R}$ & UG/L & $10 / 21 / 2005$ & & DMG-217-05 \\
\hline GWM42201AV & MIDDLE-2050A & 1212 & Carbon disulfide & 5 & & u & & UG/L & $10 / 21 / 2005$ & & DMG-217-05 \\
\hline GWM42201AV & MIDDLE-2050A & 1212 & Carbon tetrachloride & 1 & & $\mathrm{u}$ & & UG/L & $10 / 21 / 2005$ & & DMG-217-05 \\
\hline GWM42201AV & MIDDLE-2050A & 1212 & Chlorobenzene & 1 & & $\mathrm{u}$ & & UG/L & $10 / 21 / 2005$ & & DMG-217-05 \\
\hline GWM42201AV & MIDDLE-2050A & 1212 & Chloroprene & 5 & & $\mathrm{u}$ & & UG/L & $10 / 21 / 2005$ & & DMG-217-05 \\
\hline GWM42201AV & MIDDLE-2050A & 1212 & Dibromochloromethane & 1 & & $\mathrm{u}$ & & UG/L & $10 / 21 / 2005$ & & DMG-217-05 \\
\hline GWM42201AV & MIDDLE-2050A & 1212 & Chloroethane & 1 & & $\mathrm{u}$ & & UG/L & 10/21/2005 & & DMG-217-05 \\
\hline GWM42201AV & MIDDLE-2050A & 1212 & Chloroform & 1 & & $\mathrm{u}$ & & UG/L & $10 / 21 / 2005$ & & DMG-217-05 \\
\hline GWM42201AV & MIDDLE-2050A & 1212 & Chloromethane & 5 & & $\mathrm{u}$ & & UG/L & $10 / 21 / 2005$ & & DMG-217-05 \\
\hline GWM42201AV & MIDDLE-2050A & 1212 & Allyl chloride & 5 & & $u$ & & UG/L & $10 / 21 / 2005$ & & DMG-217-05 \\
\hline GWM42201AV & MIDDLE-2050A & 1212 & 1,2-Dibromo-3-chloropropane & 5 & & $\mathrm{u}$ & $\mathrm{R}$ & UG/L & $10 / 21 / 2005$ & & DMG-217-05 \\
\hline GWM42201AV & MIDDLE-2050A & 1212 & 1,2-Dibromoethane & 1 & & $\mathrm{u}$ & & UG/L & $10 / 21 / 2005$ & & DMG-217-05 \\
\hline GWM42201AV & MIDDLE-2050A & 1212 & Dibromomethane & 1 & & $\mathrm{u}$ & & UG/L & $10 / 21 / 2005$ & & DMG-217-05 \\
\hline GWM42201AV & MIDDLE-2050A & 1212 & trans-1,4-Dichloro-2-butene & 5 & & $\mathrm{u}$ & $\mathrm{R}$ & UG/L & $10 / 21 / 2005$ & & DMG-217-05 \\
\hline GWM42201AV & MIDDLE-2050A & 1212 & Dichlorodifluoromethane & 5 & & $\mathrm{u}$ & & UG/L & $10 / 21 / 2005$ & & DMG-217-05 \\
\hline GWM42201AV & MIDDLE-2050A & 1212 & 1,1-Dichloroethane & 1 & & $\mathrm{u}$ & & UG/L & $10 / 21 / 2005$ & & DMG-217-05 \\
\hline GWM42201AV & MIDDLE-2050A & 1212 & 1,2-Dichloroethane & 1 & & $\mathrm{u}$ & & UG/L & $10 / 21 / 2005$ & & DMG-217-05 \\
\hline GWM42201AV & MIDDLE-2050A & 1212 & cis-1,2-Dichloroethene & 1 & & $\mathrm{u}$ & & UG/L & $10 / 21 / 2005$ & & DMG-217-05 \\
\hline GWM42201AV & MIDDLE-2050A & 1212 & trans-1,2-Dichloroethene & 1 & & $\mathrm{u}$ & & UG/L & $10 / 21 / 2005$ & & DMG-217-05 \\
\hline GWM42201AV & MIDDLE-2050A & 1212 & 1,1-Dichloroethene & 1 & & $\mathrm{u}$ & & UG/L & $10 / 21 / 2005$ & & DMG-217-05 \\
\hline GWM42201AV & MIDDLE-2050A & 1212 & 1,2-Dichloropropane & 1 & & u & & UG/L & $10 / 21 / 2005$ & & DMG-217-05 \\
\hline GWM42201AV & MIDDLE-2050A & 1212 & cis-1,3-Dichloropropene & 1 & & $\mathrm{u}$ & & UG/L & $10 / 21 / 2005$ & & DMG-217-05 \\
\hline GWM42201AV & MIDDLE-2050A & 1212 & trans-1,3-Dichloropropene & 1 & & $\mathrm{u}$ & & UG/L & $10 / 21 / 2005$ & & DMG-217-05 \\
\hline GWM42201AV & MIDDLE-2050A & 1212 & 1,4-Dioxane & 80 & & $\mathrm{u}$ & $\mathrm{R}$ & UG/L & $10 / 21 / 2005$ & & DMG-217-05 \\
\hline GWM42201AV & MIDDLE-2050A & 1212 & Ethylbenzene & 1 & & u & & UG/L & $10 / 21 / 2005$ & & DMG-217-05 \\
\hline GWM42201AV & MIDDLE-2050A & 1212 & 2-Hexanone & 5 & & $\mathrm{u}$ & & UG/L & $10 / 21 / 2005$ & & DMG-217-05 \\
\hline GWM42201AV & MIDDLE-2050A & 1212 & Methyl iodide & 2 & & $\mathrm{u}$ & & UG/L & $10 / 21 / 2005$ & & DMG-217-05 \\
\hline GWM42201AV & MIDDLE-2050A & 1212 & Isobutyl alcohol & 80 & & $\mathrm{u}$ & $\mathrm{R}$ & UG/L & $10 / 21 / 2005$ & & DMG-217-05 \\
\hline GWM42201AV & MIDDLE-2050A & 1212 & Methacrylonitrile & 5 & & u & $\mathrm{R}$ & UG/L & $10 / 21 / 2005$ & & DMG-217-05 \\
\hline GWM42201AV & MIDDLE-2050A & 1212 & Methylene Chloride & 1 & & u & & UG/L & 10/21/2005 & & DMG-217-05 \\
\hline GWM42201AV & MIDDLE-2050A & 1212 & Methylmethacrylate & 1 & & u & & UG/L & $10 / 21 / 2005$ & & DMG-217-05 \\
\hline GWM42201AV & MIDDLE-2050A & 1212 & Methyl isobutyl ketone & 5 & & $\mathrm{u}$ & & UG/L & $10 / 21 / 2005$ & & DMG-217-05 \\
\hline GWM42201AV & MIDDLE-2050A & 1212 & Propionitrile & 5 & & $\mathrm{u}$ & $\mathrm{R}$ & UG/L & $10 / 21 / 2005$ & & DMG-217-05 \\
\hline GWM42201AV & MIDDLE-2050A & 1212 & Styrene & 1 & & $u$ & & UG/L & 10/21/2005 & & DMG-217-05 \\
\hline GWM42201AV & MIDDLE-2050A & 1212 & 1,1,1,2-Tetrachloroethane & 1 & & $\mathrm{u}$ & & UG/L & $10 / 21 / 2005$ & & DMG-217-05 \\
\hline GWM42201AV & MIDDLE-2050A & 1212 & 1,1,2,2-Tetrachloroethane & 1 & & $\mathrm{u}$ & & UG/L & $10 / 21 / 2005$ & & DMG-217-05 \\
\hline GWM42201AV & MIDDLE-2050A & 1212 & Tetrachloroethene & 1 & & $\mathrm{u}$ & & UG/L & $10 / 21 / 2005$ & & DMG-217-05 \\
\hline GWM42201AV & MIDDLE-2050A & 1212 & Toluene & 0.17 & & $\mathrm{~J}$ & $\mathrm{~J}$ & UG/L & $10 / 21 / 2005$ & & DMG-217-05 \\
\hline GWM42201AV & MIDDLE-2050A & 1212 & 1,1,1-Trichloroethane & 1 & & $u$ & & UG/L & $10 / 21 / 2005$ & & DMG-217-05 \\
\hline GWM42201AV & MIDDLE-2050A & 1212 & 1,1,2-Trichloroethane & 1 & & $\mathrm{u}$ & & UG/L & $10 / 21 / 2005$ & & DMG-217-05 \\
\hline GWM42201AV & MIDDLE-2050A & 1212 & Trichloroethene & 1 & & u & & UG/L & $10 / 21 / 2005$ & & DMG-217-05 \\
\hline GWM42201AV & MIDDLE-2050A & 1212 & Trichlorofluoromethane & 1 & & $u$ & & UG/L & 10/21/2005 & & DMG-217-05 \\
\hline GWM42201AV & MIDDLE-2050A & 1212 & 1,2,3-Trichloropropane & 1 & & $u$ & & UG/L & $10 / 21 / 2005$ & & DMG-217-05 \\
\hline GWM42201AV & MIDDLE-2050A & 1212 & Vinyl Acetate & 2 & & $\mathrm{U}$ & & UG/L & $10 / 21 / 2005$ & & DMG-217-05 \\
\hline GWM42201AV & MIDDLE-2050A & 1212 & Vinyl Chloride & 1 & & u & & UG/L & 10/21/2005 & & DMG-217-05 \\
\hline GWM42201AV & MIDDLE-2050A & 1212 & Xylenes & 3 & & U & & UG/L & $10 / 21 / 2005$ & & DMG-217-05 \\
\hline GWM42201RH & MIDDLE-2050A & 1212 & Uranium-233/234 & $9.60 \mathrm{E}-01$ & $1.30 \mathrm{E}-01$ & & & $\mathrm{PCl} / \mathrm{L}$ & $10 / 21 / 2005$ & $6.87 \mathrm{E}-02$ & SOS-TL352-05 \\
\hline GWM42201RH & MIDDLE-2050A & 1212 & Uranium-238 & 4.30E-01 & $8.18 \mathrm{E}-02$ & & & $\mathrm{PCl} / \mathrm{L}$ & $10 / 21 / 2005$ & $9.84 \mathrm{E}-02$ & SOS-TL352-05 \\
\hline
\end{tabular}


Table A-4. (continued).

\begin{tabular}{|c|c|c|c|c|c|c|c|c|c|c|c|}
\hline $\begin{array}{c}\text { Field Sample } \\
\text { Number }\end{array}$ & Location & Depth & Compound & $\begin{array}{c}\text { Sample } \\
\text { Result }\end{array}$ & $\begin{array}{c}\text { Sample } \\
\text { Error }\end{array}$ & $\begin{array}{c}\text { Result } \\
\text { Qualifier }\end{array}$ & $\begin{array}{l}\text { Validation } \\
\text { Flag }\end{array}$ & $\begin{array}{l}\text { Sample } \\
\text { Units }\end{array}$ & $\begin{array}{c}\text { Date Sample } \\
\text { Collected }\end{array}$ & MDA & $\begin{array}{l}\text { L\&V Report } \\
\text { Number }\end{array}$ \\
\hline GWM42201RH & MIDDLE-2050A & 1212 & Uranium-235 & $1.13 \mathrm{E}-01$ & $4.33 \mathrm{E}-02$ & & $\mathrm{~J}$ & $\mathrm{PCl} / \mathrm{L}$ & $10 / 21 / 2005$ & $4.83 \mathrm{E}-02$ & SOS-TL352-05 \\
\hline GWM42201RH & MIDDLE-2050A & 1212 & Gross Alpha & $2.10 \mathrm{E}+00$ & 7.26E-01 & & UJ & $\mathrm{PCl} / \mathrm{L}$ & $10 / 21 / 2005$ & $2.31 \mathrm{E}+00$ & SOS-TL352-05 \\
\hline GWM42201RH & MIDDLE-2050A & 1212 & Gross Beta & $5.12 \mathrm{E}+00$ & $1.04 \mathrm{E}+00$ & & & $\mathrm{PCl} / \mathrm{L}$ & $10 / 21 / 2005$ & $3.66 \mathrm{E}+00$ & SOS-TL352-05 \\
\hline GWM42201RH & MIDDLE-2050A & 1212 & Strontium-90 & $-1.77 \mathrm{E}-01$ & $9.77 \mathrm{E}-02$ & & $\mathrm{U}$ & $\mathrm{PCl} / \mathrm{L}$ & $10 / 21 / 2005$ & 5.99E-01 & SOS-TL352-05 \\
\hline GWM42201RH & MIDDLE-2050A & 1212 & Silver-108m & $-1.43 E+00$ & $1.40 \mathrm{E}+00$ & & $u$ & $\mathrm{PCl} / \mathrm{L}$ & $10 / 21 / 2005$ & $4.70 \mathrm{E}+00$ & SOS-TL352-05 \\
\hline GWM42201RH & MIDDLE-2050A & 1212 & Silver-110m & $-1.63 E+00$ & $1.56 \mathrm{E}+00$ & & $u$ & $\mathrm{PCl} / \mathrm{L}$ & 10/21/2005 & $5.53 \mathrm{E}+00$ & SOS-TL352-05 \\
\hline GWM42201RH & MIDDLE-2050A & 1212 & Cerium-144 & $-4.38 \mathrm{E}+00$ & $8.50 \mathrm{E}+00$ & & $u$ & $\mathrm{PCl} / \mathrm{L}$ & $10 / 21 / 2005$ & $2.78 \mathrm{E}+01$ & SOS-TL352-05 \\
\hline GWM42201RH & MIDDLE-2050A & 1212 & Cobalt-60 & $-3.38 \mathrm{E}-02$ & $1.40 \mathrm{E}+00$ & & $u$ & $\mathrm{PCl} / \mathrm{L}$ & $10 / 21 / 2005$ & $5.81 \mathrm{E}+00$ & SOS-TL352-05 \\
\hline GWM42201RH & MIDDLE-2050A & 1212 & Cesium-134 & $8.72 \mathrm{E}-01$ & $1.87 \mathrm{E}+00$ & & $u$ & $\mathrm{PCl} / \mathrm{L}$ & $10 / 21 / 2005$ & $7.30 \mathrm{E}+00$ & SOS-TL352-05 \\
\hline GWM42201RH & MIDDLE-2050A & 1212 & Cesium-137 & $1.62 \mathrm{E}+00$ & $1.63 \mathrm{E}+00$ & & $u$ & $\mathrm{PCl} / \mathrm{L}$ & $10 / 21 / 2005$ & $6.57 \mathrm{E}+00$ & SOS-TL352-05 \\
\hline GWM42201RH & MIDDLE-2050A & 1212 & Europium-152 & $-5.22 \mathrm{E}+00$ & $3.89 \mathrm{E}+00$ & & $u$ & $\mathrm{PCl} / \mathrm{L}$ & $10 / 21 / 2005$ & $1.30 \mathrm{E}+01$ & SOS-TL352-05 \\
\hline GWM42201RH & MIDDLE-2050A & 1212 & Europium-154 & $-1.25 \mathrm{E}+00$ & $5.47 \mathrm{E}+00$ & & u & $\mathrm{PCl} / \mathrm{L}$ & $10 / 21 / 2005$ & $2.00 \mathrm{E}+01$ & SOS-TL352-05 \\
\hline GWM42201RH & MIDDLE-2050A & 1212 & Europium-155 & $1.38 \mathrm{E}+00$ & $4.24 \mathrm{E}+00$ & & u & $\mathrm{PCI} / \mathrm{L}$ & $10 / 21 / 2005$ & $1.46 \mathrm{E}+01$ & SOS-TL352-05 \\
\hline GWM42201RH & MIDDLE-2050A & 1212 & Manganese-54 & $-1.81 \mathrm{E}+00$ & $1.49 \mathrm{E}+00$ & & u & $\mathrm{PCl} / \mathrm{L}$ & $10 / 21 / 2005$ & $5.13 \mathrm{E}+00$ & SOS-TL352-05 \\
\hline GWM42201RH & MIDDLE-2050A & 1212 & Ruthenium-106 & $2.07 \mathrm{E}+00$ & $1.44 \mathrm{E}+01$ & & $u$ & $\mathrm{PCl} / \mathrm{L}$ & 10/21/2005 & $5.52 \mathrm{E}+01$ & SOS-TL352-05 \\
\hline GWM42201RH & MIDDLE-2050A & 1212 & Antimony-125 & $-2.73 E+00$ & $4.24 \mathrm{E}+00$ & & u & $\mathrm{PCl} / \mathrm{L}$ & $10 / 21 / 2005$ & $1.46 \mathrm{E}+01$ & SOS-TL352-05 \\
\hline GWM42201RH & MIDDLE-2050A & 1212 & Zinc-65 & $9.47 \mathrm{E}+00$ & $4.00 \mathrm{E}+00$ & & $u$ & $\mathrm{PCl} / \mathrm{L}$ & $10 / 21 / 2005$ & $1.75 \mathrm{E}+01$ & SOS-TL352-05 \\
\hline GWM42201RH & MIDDLE-2050A & 1212 & Technetium-99 & $-1.66 \mathrm{E}+00$ & $1.65 \mathrm{E}+00$ & & $u$ & $\mathrm{PCl} / \mathrm{L}$ & $10 / 21 / 2005$ & $5.73 E+00$ & SOS-TL352-05 \\
\hline GWM42201R8 & MIDDLE-2050A & 1212 & Tritium & $-2.78 \mathrm{E}+01$ & $7.85 \mathrm{E}+01$ & & $u$ & $\mathrm{PCl} / \mathrm{L}$ & $10 / 21 / 2005$ & $2.67 \mathrm{E}+02$ & SOS-TL352-05 \\
\hline GWM424012X & MIDDLE-2051 & 608 & Antimony & 0.61 & & $\mathrm{U}$ & & UG/L & $09 / 27 / 2005$ & & DNT-001-06 \\
\hline GWM424012X & MIDDLE-2051 & 608 & Arsenic & 1.8 & & u & & UG/L & 09/27/2005 & & DNT-001-06 \\
\hline GWM424012X & MIDDLE-2051 & 608 & Beryllium & 0.13 & & u & & UG/L & 09/27/2005 & & DNT-001-06 \\
\hline GWM424012X & MIDDLE-2051 & 608 & Cadmium & 0.067 & & u & & UG/L & 09/27/2005 & & DNT-001-06 \\
\hline GWM424012X & MIDDLE-2051 & 608 & Chromium & 3.7 & & u & & UG/L & $09 / 27 / 2005$ & & DNT-001-06 \\
\hline GWM424012X & MIDDLE-2051 & 608 & Manganese & 5.5 & & & & UG/L & 09/27/2005 & & DNT-001-06 \\
\hline GWM424012X & MIDDLE-2051 & 608 & Silver & 1.5 & & $\mathrm{U}$ & & UG/L & $09 / 27 / 2005$ & & DNT-001-06 \\
\hline GWM424012X & MIDDLE-2051 & 608 & Thallium & 0.29 & & B & $u$ & UG/L & 09/27/2005 & & DNT-001-06 \\
\hline GWM424012X & MIDDLE-2051 & 608 & Uranium & 2.3 & & B & & UG/L & 09/27/2005 & & DNT-001-06 \\
\hline GWM424012X & MIDDLE-2051 & 608 & Mercury & 0.046 & & $u$ & & UG/L & 09/27/2005 & & DNT-001-06 \\
\hline GWM424012X & MIDDLE-2051 & 608 & Aluminum & 22.1 & & u & & UG/L & 09/27/2005 & & DNT-001-06 \\
\hline GWM424012X & MIDDLE-2051 & 608 & Barium & 60.5 & & B & & UG/L & 09/27/2005 & & DNT-001-06 \\
\hline GWM424012X & MIDDLE-2051 & 608 & Calcium & 53300 & & & & UG/L & $09 / 27 / 2005$ & & DNT-001-06 \\
\hline GWM424012X & MIDDLE-2051 & 608 & Cobalt & 2.8 & & U & & UG/L & 09/27/2005 & & DNT-001-06 \\
\hline GWM424012X & MIDDLE-2051 & 608 & Copper & 1.1 & & u & & UG/L & 09/27/2005 & & DNT-001-06 \\
\hline GWM424012X & MIDDLE-2051 & 608 & Iron & 8.1 & & B & & UG/L & 09/27/2005 & & DNT-001-06 \\
\hline GWM424012X & MIDDLE-2051 & 608 & Lead & 1.1 & & $u$ & & UG/L & $09 / 27 / 2005$ & & DNT-001-06 \\
\hline GWM424012X & MIDDLE-2051 & 608 & Lithium & 3 & & u & & UG/L & 09/27/2005 & & DNT-001-06 \\
\hline GWM424012X & MIDDLE-2051 & 608 & Magnesium & 12700 & & & & UG/L & $09 / 27 / 2005$ & & DNT-001-06 \\
\hline GWM424012X & MIDDLE-2051 & 608 & Molybdenum & 3.7 & & u & & UG/L & 09/27/2005 & & DNT-001-06 \\
\hline GWM424012X & MIDDLE-2051 & 608 & Nickel & 3.2 & & u & & UG/L & 09/27/2005 & & DNT-001-06 \\
\hline GWM424012X & MIDDLE-2051 & 608 & Potassium & 2760 & & B & & UG/L & 09/27/2005 & & DNT-001-06 \\
\hline GWM424012X & MIDDLE-2051 & 608 & Selenium & 2 & & $u$ & & UG/L & $09 / 27 / 2005$ & & DNT-001-06 \\
\hline GWM424012X & MIDDLE-2051 & 608 & Sodium & 6140 & & & & UG/L & 09/27/2005 & & DNT-001-06 \\
\hline GWM424012X & MIDDLE-2051 & 608 & Strontium & 329 & & & & UG/L & 09/27/2005 & & DNT-001-06 \\
\hline GWM424012X & MIDDLE-2051 & 608 & Tin & 3.9 & & u & & UG/L & 09/27/2005 & & DNT-001-06 \\
\hline GWM424012X & MIDDLE-2051 & 608 & Vanadium & 4.2 & & B & & UG/L & $09 / 27 / 2005$ & & DNT-001-06 \\
\hline GWM424012X & MIDDLE-2051 & 608 & Zinc & 6 & & B & $u$ & UG/L & 09/27/2005 & & DNT-001-06 \\
\hline GWM42401AN & MIDDLE-2051 & 608 & Bromide & 0.25 & & $u$ & & $\mathrm{MG} / \mathrm{L}$ & $09 / 27 / 2005$ & & DNT-500-05 \\
\hline GWM42401AN & MIDDLE-2051 & 608 & Chloride & 6.7 & & $\mathrm{~J}$ & & MG/L & 09/27/2005 & & DNT-500-05 \\
\hline GWM42401AN & MIDDLE-2051 & 608 & Fluoride & 0.23 & & & & MG/L & $09 / 27 / 2005$ & & DNT-500-05 \\
\hline GWM42401AN & MIDDLE-2051 & 608 & Sulfate & 22.6 & & & & MG/L & 09/27/2005 & & DNT-500-05 \\
\hline GWM42401A1 & MIDDLE-2051 & 608 & Total Alkalinity & 150 & & & & $\mathrm{MG} / \mathrm{L}$ & $09 / 27 / 2005$ & & DNT-500-05 \\
\hline GWM42401N2 & MIDDLE-2051 & 608 & Nitrate/Nitrite as $\mathrm{N}$ & 0.29 & & & $\mathrm{~J}$ & MG/L & 09/27/2005 & & DNT-500-05 \\
\hline GWM42401AV & MIDDLE-2051 & 608 & Acetone & 10 & & $\mathrm{u}$ & $\mathrm{R}$ & UG/L & $09 / 27 / 2005$ & & JGJ-071-05 \\
\hline GWM42401AV & MIDDLE-2051 & 608 & Acetonitrile & 20 & & $u$ & & UG/L & 09/27/2005 & & JGJ-071-05 \\
\hline GWM42401AV & MIDDLE-2051 & 608 & Acrolein & 5 & & $\mathrm{u}$ & $\mathrm{R}$ & UG/L & 09/27/2005 & & JGJ-071-05 \\
\hline GWM42401AV & MIDDLE-2051 & 608 & Acrylonitrile & 1 & & $u$ & $\mathrm{R}$ & UG/L & 09/27/2005 & & JGJ-071-05 \\
\hline GWM42401AV & MIDDLE-2051 & 608 & Benzene & 1 & & $\mathrm{u}$ & & UG/L & $09 / 27 / 2005$ & & JGJ-071-05 \\
\hline GWM42401AV & MIDDLE-2051 & 608 & Bromodichloromethane & 1 & & u & & UG/L & 09/27/2005 & & JGJ-071-05 \\
\hline GWM42401AV & MIDDLE-2051 & 608 & Bromoform & 2 & & $\mathrm{u}$ & & UG/L & $09 / 27 / 2005$ & & JGJ-071-05 \\
\hline GWM42401AV & MIDDLE-2051 & 608 & Bromomethane & 1 & & $\mathrm{u}$ & & UG/L & 09/27/2005 & & JGJ-071-05 \\
\hline GWM42401AV & MIDDLE-2051 & 608 & 2-Butanone & 10 & & $\mathrm{u}$ & $\mathrm{R}$ & UG/L & 09/27/2005 & & JGJ-071-05 \\
\hline GWM42401AV & MIDDLE-2051 & 608 & Carbon disulfide & 5 & & $\mathrm{u}$ & & UG/L & 09/27/2005 & & JGJ-071-05 \\
\hline GWM42401AV & MIDDLE-2051 & 608 & Carbon tetrachloride & 1 & & $\mathrm{u}$ & & UG/L & $09 / 27 / 2005$ & & JGJ-071-05 \\
\hline GWM42401AV & MIDDLE-2051 & 608 & Chlorobenzene & 1 & & $\mathrm{u}$ & & UG/L & 09/27/2005 & & JGJ-071-05 \\
\hline GWM42401AV & MIDDLE-2051 & 608 & Chloroprene & 5 & & $\mathrm{u}$ & & UG/L & 09/27/2005 & & JGJ-071-05 \\
\hline GWM42401AV & MIDDLE-2051 & 608 & Dibromochloromethane & 1 & & $\mathrm{u}$ & & UG/L & 09/27/2005 & & JGJ-071-05 \\
\hline GWM42401AV & MIDDLE-2051 & 608 & Chloroethane & 1 & & $\mathrm{u}$ & & UG/L & $09 / 27 / 2005$ & & JGJ-071-05 \\
\hline GWM42401AV & MIDDLE-2051 & 608 & Chloroform & 1 & & $\mathrm{u}$ & & UG/L & 09/27/2005 & & JGJ-071-05 \\
\hline GWM42401AV & MIDDLE-2051 & 608 & Chloromethane & 5 & & $\mathrm{u}$ & & UG/L & $09 / 27 / 2005$ & & JGJ-071-05 \\
\hline GWM42401AV & MIDDLE-2051 & 608 & Allyl chloride & 5 & & $\mathrm{u}$ & & UG/L & 09/27/2005 & & JGJ-071-05 \\
\hline GWM42401AV & MIDDLE-2051 & 608 & 1,2-Dibromo-3-chloropropane & 5 & & $\mathrm{u}$ & $\mathrm{R}$ & UG/L & $09 / 27 / 2005$ & & JGJ-071-05 \\
\hline GWM42401AV & MIDDLE-2051 & 608 & 1,2-Dibromoethane & 1 & & $\mathrm{u}$ & & UG/L & 09/27/2005 & & JGJ-071-05 \\
\hline GWM42401AV & MIDDLE-2051 & 608 & Dibromomethane & 1 & & $\mathrm{u}$ & & UG/L & 09/27/2005 & & JGJ-071-05 \\
\hline GWM42401AV & MIDDLE-2051 & 608 & trans-1,4-Dichloro-2-butene & 5 & & $\mathrm{u}$ & & UG/L & 09/27/2005 & & JGJ-071-05 \\
\hline GWM42401AV & MIDDLE-2051 & 608 & Dichlorodifluoromethane & 5 & & $\mathrm{u}$ & & UG/L & $09 / 27 / 2005$ & & JGJ-071-05 \\
\hline GWM42401AV & MIDDLE-2051 & 608 & 1,1-Dichloroethane & 1 & & u & & UG/L & 09/27/2005 & & JGJ-071-05 \\
\hline GWM42401AV & MIDDLE-2051 & 608 & 1,2-Dichloroethane & 1 & & $\mathrm{u}$ & & UG/L & $09 / 27 / 2005$ & & JGJ-071-05 \\
\hline GWM42401AV & MIDDLE-2051 & 608 & cis-1,2-Dichloroethene & 1 & & $\mathrm{u}$ & & UG/L & 09/27/2005 & & JGJ-071-05 \\
\hline GWM42401AV & MIDDLE-2051 & 608 & trans-1,2-Dichloroethene & 1 & & $\mathrm{u}$ & & UG/L & $09 / 27 / 2005$ & & JGJ-071-05 \\
\hline GWM42401AV & MIDDLE-2051 & 608 & 1,1-Dichloroethene & 1 & & $\mathrm{u}$ & & UG/L & $09 / 27 / 2005$ & & JGJ-071-05 \\
\hline GWM42401AV & MIDDLE-2051 & 608 & 1,2-Dichloropropane & 1 & & $\mathrm{u}$ & & UG/L & 09/27/2005 & & JGJ-071-05 \\
\hline GWM42401AV & MIDDLE-2051 & 608 & cis-1,3-Dichloropropene & 1 & & $\mathrm{u}$ & & UG/L & 09/27/2005 & & JGJ-071-05 \\
\hline GWM42401AV & MIDDLE-2051 & 608 & trans-1,3-Dichloropropene & 1 & & $\mathrm{u}$ & & UG/L & $09 / 27 / 2005$ & & JGJ-071-05 \\
\hline GWM42401AV & MIDDLE-2051 & 608 & 1,4-Dioxane & 80 & & $\mathrm{u}$ & $\mathrm{R}$ & UG/L & 09/27/2005 & & JGJ-071-05 \\
\hline GWM42401AV & MIDDLE-2051 & 608 & Ethylbenzene & 1 & & u & & UG/L & 09/27/2005 & & JGJ-071-05 \\
\hline
\end{tabular}


Table A-4. (continued).

\begin{tabular}{|c|c|c|c|c|c|c|c|c|c|c|c|}
\hline $\begin{array}{c}\text { Field Sample } \\
\text { Number }\end{array}$ & Location & Depth & Compound & $\begin{array}{c}\text { Sample } \\
\text { Result }\end{array}$ & $\begin{array}{l}\text { Sample } \\
\text { Error }\end{array}$ & $\begin{array}{c}\text { Result } \\
\text { Qualifier }\end{array}$ & $\begin{array}{c}\text { Validation } \\
\text { Flag }\end{array}$ & $\begin{array}{c}\text { Sample } \\
\text { Units }\end{array}$ & $\begin{array}{c}\text { Date Sample } \\
\text { Collected }\end{array}$ & MDA & $\begin{array}{l}\text { L\&V Report } \\
\text { Number }\end{array}$ \\
\hline GWM42401AV & MIDDLE-2051 & 608 & 2-Hexanone & 5 & & $\bar{U}$ & & UG/L & $09 / 27 / 2005$ & & JGJ-071-05 \\
\hline GWM42401AV & MIDDLE-2051 & 608 & Methyl iodide & 2 & & u & & UG/L & $09 / 27 / 2005$ & & JGJ-071-05 \\
\hline GWM42401AV & MIDDLE-2051 & 608 & Isobutyl alcohol & 80 & & $u$ & $\mathrm{R}$ & UG/L & 09/27/2005 & & JGJ-071-05 \\
\hline GWM42401AV & MIDDLE-2051 & 608 & Methacrylonitrile & 5 & & $\mathrm{u}$ & $\mathrm{R}$ & UG/L & 09/27/2005 & & JGJ-071-05 \\
\hline GWM42401AV & MIDDLE-2051 & 608 & Methylene Chloride & 1 & & $\mathrm{u}$ & & UG/L & 09/27/2005 & & JGJ-071-05 \\
\hline GWM42401AV & MIDDLE-2051 & 608 & Methylmethacrylate & 1 & & $\mathrm{u}$ & & UG/L & 09/27/2005 & & JGJ-071-05 \\
\hline GWM42401AV & MIDDLE-2051 & 608 & Methyl isobutyl ketone & 5 & & $\mathrm{u}$ & & UG/L & 09/27/2005 & & JGJ-071-05 \\
\hline GWM42401AV & MIDDLE-2051 & 608 & Propionitrile & 5 & & $\mathrm{u}$ & $\mathrm{R}$ & UG/L & $09 / 27 / 2005$ & & JGJ-071-05 \\
\hline GWM42401AV & MIDDLE-2051 & 608 & Styrene & 1 & & $\mathrm{u}$ & & UG/L & 09/27/2005 & & JGJ-071-05 \\
\hline GWM42401AV & MIDDLE-2051 & 608 & 1,1,1,2-Tetrachloroethane & 1 & & $\mathrm{u}$ & & UG/L & 09/27/2005 & & JGJ-071-05 \\
\hline GWM42401AV & MIDDLE-2051 & 608 & 1,1,2,2-Tetrachloroethane & 1 & & $\mathrm{u}$ & & UG/L & 09/27/2005 & & JGJ-071-05 \\
\hline GWM42401AV & MIDDLE-2051 & 608 & Tetrachloroethene & 1 & & $\mathrm{u}$ & & UG/L & 09/27/2005 & & JGJ-071-05 \\
\hline GWM42401AV & MIDDLE-2051 & 608 & Toluene & 1 & & u & & UG/L & 09/27/2005 & & JGJ-071-05 \\
\hline GWM42401AV & MIDDLE-2051 & 608 & 1,1,1-Trichloroethane & 1 & & $\mathrm{u}$ & & UG/L & 09/27/2005 & & JGJ-071-05 \\
\hline GWM42401AV & MIDDLE-2051 & 608 & 1,1,2-Trichloroethane & 1 & & u & & UG/L & 09/27/2005 & & JGJ-071-05 \\
\hline GWM42401AV & MIDDLE-2051 & 608 & Trichloroethene & 1 & & $\mathrm{u}$ & & UG/L & 09/27/2005 & & JGJ-071-05 \\
\hline GWM42401AV & MIDDLE-2051 & 608 & Trichlorofluoromethane & 1 & & $\mathrm{u}$ & & UG/L & 09/27/2005 & & JGJ-071-05 \\
\hline GWM42401AV & MIDDLE-2051 & 608 & 1,2,3-Trichloropropane & 1 & & $\mathrm{u}$ & & UG/L & $09 / 27 / 2005$ & & JGJ-071-05 \\
\hline GWM42401AV & MIDDLE-2051 & 608 & Vinyl Acetate & 2 & & $u$ & & UG/L & $09 / 27 / 2005$ & & JGJ-071-05 \\
\hline GWM42401AV & MIDDLE-2051 & 608 & Vinyl Chloride & 1 & & $\mathrm{u}$ & & UG/L & $09 / 27 / 2005$ & & JGJ-071-05 \\
\hline GWM42401AV & MIDDLE-2051 & 608 & Xylenes & 3 & & u & & UG/L & 09/27/2005 & & JGJ-071-05 \\
\hline GWM42401RH & MIDDLE-2051 & 608 & Uranium-233/234 & $9.64 \mathrm{E}-01$ & $8.16 \mathrm{E}-02$ & & & $\mathrm{PCl} / \mathrm{L}$ & 09/27/2005 & 4.41E-02 & SOS-TL342-05 \\
\hline GWM42401RH & MIDDLE-2051 & 608 & Uranium-238 & $5.72 \mathrm{E}-01$ & $5.83 \mathrm{E}-02$ & & & $\mathrm{PCl} / \mathrm{L}$ & 09/27/2005 & 2.89E-02 & SOS-TL342-05 \\
\hline GWM42401RH & MIDDLE-2051 & 608 & Uranium-235 & 3.97E-02 & $1.67 \mathrm{E}-02$ & & UJ & $\mathrm{PCl} / \mathrm{L}$ & 09/27/2005 & 4.35E-02 & SOS-TL342-05 \\
\hline GWM42401RH & MIDDLE-2051 & 608 & Gross Alpha & $1.75 \mathrm{E}+00$ & $6.51 \mathrm{E}-01$ & & $\mathrm{~J}$ & $\mathrm{PCl} / \mathrm{L}$ & 09/27/2005 & $1.71 \mathrm{E}+00$ & SOS-TL342-05 \\
\hline GWM42401RH & MIDDLE-2051 & 608 & Gross Beta & $3.37 \mathrm{E}+00$ & $1.06 \mathrm{E}+00$ & & $\mathrm{~J}$ & $\mathrm{PCl} / \mathrm{L}$ & $09 / 27 / 2005$ & $4.03 E+00$ & SOS-TL342-05 \\
\hline GWM42401RH & MIDDLE-2051 & 608 & Strontium-90 & $3.96 \mathrm{E}-01$ & $1.89 \mathrm{E}-01$ & & UJ & $\mathrm{PCl} / \mathrm{L}$ & 09/27/2005 & 7.68E-01 & SOS-TL342-05 \\
\hline GWM42401RH & MIDDLE-2051 & 608 & Silver-108m & $-1.03 E+00$ & $1.58 \mathrm{E}+00$ & & u & $\mathrm{PCl} / \mathrm{L}$ & 09/27/2005 & $5.33 \mathrm{E}+00$ & SOS-TL342-05 \\
\hline GWM42401RH & MIDDLE-2051 & 608 & Silver-110m & $2.65 \mathrm{E}-01$ & $1.46 \mathrm{E}+00$ & & $u$ & $\mathrm{PCl} / \mathrm{L}$ & 09/27/2005 & $5.48 \mathrm{E}+00$ & SOS-TL342-05 \\
\hline GWM42401RH & MIDDLE-2051 & 608 & Cerium-144 & $-6.91 E+00$ & $1.21 \mathrm{E}+01$ & & u & $\mathrm{PCl} / \mathrm{L}$ & 09/27/2005 & $3.73 \mathrm{E}+01$ & SOS-TL342-05 \\
\hline GWM42401RH & MIDDLE-2051 & 608 & Cobalt-60 & 7.12E-01 & $1.34 \mathrm{E}+00$ & & u & $\mathrm{PCl} / \mathrm{L}$ & 09/27/2005 & $5.56 \mathrm{E}+00$ & SOS-TL342-05 \\
\hline GWM42401RH & MIDDLE-2051 & 608 & Cesium-134 & $2.80 \mathrm{E}+00$ & $1.15 E+00$ & & $\mathrm{U}$ & $\mathrm{PCl} / \mathrm{L}$ & 09/27/2005 & $5.21 \mathrm{E}+00$ & SOS-TL342-05 \\
\hline GWM42401RH & MIDDLE-2051 & 608 & Cesium-137 & $6.87 \mathrm{E}-03$ & $1.38 \mathrm{E}+00$ & & U & $\mathrm{PCl} / \mathrm{L}$ & 09/27/2005 & $5.18 \mathrm{E}+00$ & SOS-TL342-05 \\
\hline GWM42401RH & MIDDLE-2051 & 608 & Europium-152 & $5.61 \mathrm{E}-01$ & $4.61 \mathrm{E}+00$ & & u & $\mathrm{PCl} / \mathrm{L}$ & 09/27/2005 & $1.64 \mathrm{E}+01$ & SOS-TL342-05 \\
\hline GWM42401RH & MIDDLE-2051 & 608 & Europium-154 & $6.54 \mathrm{E}+00$ & $4.11 \mathrm{E}+00$ & & $u$ & $\mathrm{PCl} / \mathrm{L}$ & 09/27/2005 & $1.81 \mathrm{E}+01$ & SOS-TL342-05 \\
\hline GWM42401RH & MIDDLE-2051 & 608 & Europium-155 & $-1.37 E+00$ & $6.26 \mathrm{E}+00$ & & u & $\mathrm{PCl} / \mathrm{L}$ & 09/27/2005 & $2.08 \mathrm{E}+01$ & SOS-TL342-05 \\
\hline GWM42401RH & MIDDLE-2051 & 608 & Manganese-54 & $-2.24 \mathrm{E}+00$ & $1.36 \mathrm{E}+00$ & & $u$ & $\mathrm{PCl} / \mathrm{L}$ & 09/27/2005 & $4.36 \mathrm{E}+00$ & SOS-TL342-05 \\
\hline GWM42401RH & MIDDLE-2051 & 608 & Ruthenium-106 & $5.54 \mathrm{E}+00$ & $1.40 \mathrm{E}+01$ & & $\mathrm{u}$ & $\mathrm{PCl} / \mathrm{L}$ & 09/27/2005 & $5.29 \mathrm{E}+01$ & SOS-TL342-05 \\
\hline GWM42401RH & MIDDLE-2051 & 608 & Antimony-125 & $4.33 E+00$ & $4.54 \mathrm{E}+00$ & & $u$ & $\mathrm{PCl} / \mathrm{L}$ & 09/27/2005 & $1.67 \mathrm{E}+01$ & SOS-TL342-05 \\
\hline GWM42401RH & MIDDLE-2051 & 608 & Zinc-65 & $-6.23 E+00$ & $3.14 \mathrm{E}+00$ & & u & $\mathrm{PCl} / \mathrm{L}$ & 09/27/2005 & $1.01 \mathrm{E}+01$ & SOS-TL342-05 \\
\hline GWM42401R8 & MIDDLE-2051 & 608 & Tritium & $6.80 \mathrm{E}+01$ & $1.16 \mathrm{E}+02$ & & u & $\mathrm{PCl} / \mathrm{L}$ & 09/27/2005 & $3.98 \mathrm{E}+02$ & SOS-TL342-05 \\
\hline GWM42401RH & MIDDLE-2051 & 608 & Technetium-99 & $-2.40 \mathrm{E}+00$ & $1.58 \mathrm{E}+00$ & & u & $\mathrm{PCl} / \mathrm{L}$ & 09/27/2005 & $5.55 \mathrm{E}+00$ & SOS-TL342-05 \\
\hline GWM425012X & MIDDLE-2051 & 748 & Antimony & 0.61 & & u & & UG/L & 09/28/2005 & & DNT-001-06 \\
\hline GWM425012X & MIDDLE-2051 & 748 & Arsenic & 1.8 & & $\mathrm{U}$ & & UG/L & 09/28/2005 & & DNT-001-06 \\
\hline GWM425012X & MIDDLE-2051 & 748 & Beryllium & 0.13 & & $u$ & & UG/L & 09/28/2005 & & DNT-001-06 \\
\hline GWM425012X & MIDDLE-2051 & 748 & Cadmium & 0.067 & & U & & UG/L & 09/28/2005 & & DNT-001-06 \\
\hline GWM425012X & MIDDLE-2051 & 748 & Chromium & 9.6 & & & & UG/L & 09/28/2005 & & DNT-001-06 \\
\hline GWM425012X & MIDDLE-2051 & 748 & Manganese & 1.3 & & B & & UG/L & 09/28/2005 & & DNT-001-06 \\
\hline GWM425012X & MIDDLE-2051 & 748 & Silver & 1.5 & & u & & UG/L & 09/28/2005 & & DNT-001-06 \\
\hline GWM425012X & MIDDLE-2051 & 748 & Thallium & 0.22 & & u & & UG/L & 09/28/2005 & & DNT-001-06 \\
\hline GWM425012X & MIDDLE-2051 & 748 & Uranium & 2 & & B & & UG/L & 09/28/2005 & & DNT-001-06 \\
\hline GWM425012X & MIDDLE-2051 & 748 & Mercury & 0.046 & & u & & UG/L & 09/28/2005 & & DNT-001-06 \\
\hline GWM425012X & MIDDLE-2051 & 748 & Aluminum & 22.1 & & u & & UG/L & 09/28/2005 & & DNT-001-06 \\
\hline GWM425012X & MIDDLE-2051 & 748 & Barium & 57.1 & & B & & UG/L & 09/28/2005 & & DNT-001-06 \\
\hline GWM425012X & MIDDLE-2051 & 748 & Calcium & 48700 & & & & UG/L & 09/28/2005 & & DNT-001-06 \\
\hline GWM425012X & MIDDLE-2051 & 748 & Cobalt & 2.8 & & U & & UG/L & 09/28/2005 & & DNT-001-06 \\
\hline GWM425012X & MIDDLE-2051 & 748 & Copper & 1.1 & & $u$ & & UG/L & 09/28/2005 & & DNT-001-06 \\
\hline GWM425012X & MIDDLE-2051 & 748 & Iron & 7.4 & & u & & UG/L & 09/28/2005 & & DNT-001-06 \\
\hline GWM425012X & MIDDLE-2051 & 748 & Lead & 1.1 & & U & & UG/L & $09 / 28 / 2005$ & & DNT-001-06 \\
\hline GWM425012X & MIDDLE-2051 & 748 & Lithium & 3 & & u & & UG/L & 09/28/2005 & & DNT-001-06 \\
\hline GWM425012X & MIDDLE-2051 & 748 & Magnesium & 16900 & & & & UG/L & 09/28/2005 & & DNT-001-06 \\
\hline GWM425012X & MIDDLE-2051 & 748 & Molybdenum & 3.7 & & $\mathrm{U}$ & & UG/L & 09/28/2005 & & DNT-001-06 \\
\hline GWM425012X & MIDDLE-2051 & 748 & Nickel & 3.2 & & u & & UG/L & 09/28/2005 & & DNT-001-06 \\
\hline GWM425012X & MIDDLE-2051 & 748 & Potassium & 3050 & & B & & UG/L & 09/28/2005 & & DNT-001-06 \\
\hline GWM425012X & MIDDLE-2051 & 748 & Selenium & 2 & & U & & UG/L & 09/28/2005 & & DNT-001-06 \\
\hline GWM425012X & MIDDLE-2051 & 748 & Sodium & 8330 & & & & UG/L & 09/28/2005 & & DNT-001-06 \\
\hline GWM425012X & MIDDLE-2051 & 748 & Strontium & 283 & & & & UG/L & 09/28/2005 & & DNT-001-06 \\
\hline GWM425012X & MIDDLE-2051 & 748 & Tin & 3.9 & & $\mathrm{u}$ & & UG/L & $09 / 28 / 2005$ & & DNT-001-06 \\
\hline GWM425012X & MIDDLE-2051 & 748 & Vanadium & 4.6 & & B & & UG/L & 09/28/2005 & & DNT-001-06 \\
\hline GWM425012X & MIDDLE-2051 & 748 & Zinc & 7.8 & & B & $\mathrm{U}$ & UG/L & $09 / 28 / 2005$ & & DNT-001-06 \\
\hline GWM42501AN & MIDDLE-2051 & 748 & Bromide & 0.25 & & U & & MG/L & 09/28/2005 & & DNT-500-05 \\
\hline GWM42501AN & MIDDLE-2051 & 748 & Chloride & 11.1 & & $\mathrm{~J}$ & & $\mathrm{MG} / \mathrm{L}$ & 09/28/2005 & & DNT-500-05 \\
\hline GWM42501AN & MIDDLE-2051 & 748 & Fluoride & 0.14 & & & & MG/L & 09/28/2005 & & DNT-500-05 \\
\hline GWM42501AN & MIDDLE-2051 & 748 & Sulfate & 24.7 & & & & $\mathrm{MG} / \mathrm{L}$ & $09 / 28 / 2005$ & & DNT-500-05 \\
\hline GWM42501A1 & MIDDLE-2051 & 748 & Total Alkalinity & 152 & & & & MG/L & 09/28/2005 & & DNT-500-05 \\
\hline GWM42501N2 & MIDDLE-2051 & 748 & Nitrate/Nitrite as N & 0.59 & & & $\mathrm{~J}$ & $\mathrm{MG} / \mathrm{L}$ & $09 / 27 / 2005$ & & DNT-500-05 \\
\hline GWM42501AV & MIDDLE-2051 & 748 & Acetone & 10 & & u & $\mathrm{R}$ & UG/L & 09/28/2005 & & JGJ-071-05 \\
\hline GWM42501AV & MIDDLE-2051 & 748 & Acetonitrile & 20 & & u & & UG/L & $09 / 28 / 2005$ & & JGJ-071-05 \\
\hline GWM42501AV & MIDDLE-2051 & 748 & Acrolein & 5 & & u & $\mathrm{R}$ & UG/L & 09/28/2005 & & JGJ-071-05 \\
\hline GWM42501AV & MIDDLE-2051 & 748 & Acrylonitrile & 1 & & u & $\mathrm{R}$ & UG/L & $09 / 28 / 2005$ & & JGJ-071-05 \\
\hline GWM42501AV & MIDDLE-2051 & 748 & Benzene & 1 & & $u$ & & UG/L & 09/28/2005 & & JGJ-071-05 \\
\hline GWM42501AV & MIDDLE-2051 & 748 & Bromodichloromethane & 1 & & $\mathrm{U}$ & & UG/L & 09/28/2005 & & JGJ-071-05 \\
\hline GWM42501AV & MIDDLE-2051 & 748 & Bromoform & 2 & & u & & UG/L & 09/28/2005 & & JGJ-071-05 \\
\hline GWM42501AV & MIDDLE-2051 & 748 & Bromomethane & 1 & & u & & UG/L & $09 / 28 / 2005$ & & JGJ-071-05 \\
\hline GWM42501AV & MIDDLE-2051 & 748 & 2-Butanone & 10 & & u & $\mathrm{R}$ & UG/L & 09/28/2005 & & JGJ-071-05 \\
\hline GWM42501AV & MIDDLE-2051 & 748 & Carbon disulfide & 5 & & $\mathrm{u}$ & & UG/L & $09 / 28 / 2005$ & & JGJ-071-05 \\
\hline
\end{tabular}


Table A-4. (continued).

\begin{tabular}{|c|c|c|c|c|c|c|c|c|c|c|c|}
\hline $\begin{array}{c}\text { Field Sample } \\
\text { Number }\end{array}$ & Location & Depth & Compound & $\begin{array}{l}\text { Sample } \\
\text { Result }\end{array}$ & $\begin{array}{l}\text { Sample } \\
\text { Error }\end{array}$ & $\begin{array}{c}\text { Result } \\
\text { Qualifier }\end{array}$ & $\begin{array}{c}\text { Validation } \\
\text { Flag }\end{array}$ & $\begin{array}{c}\text { Sample } \\
\text { Units }\end{array}$ & $\begin{array}{c}\text { Date Sample } \\
\text { Collected }\end{array}$ & MDA & $\begin{array}{l}\text { L\&V Report } \\
\text { Number }\end{array}$ \\
\hline GWM42501AV & MIDDLE-2051 & 748 & Carbon tetrachloride & 1 & & $\mathrm{U}$ & & $\overline{U G / L}$ & $09 / 28 / 2005$ & & JGJ-071-05 \\
\hline GWM42501AV & MIDDLE-2051 & 748 & Chlorobenzene & 1 & & $\mathrm{U}$ & & UG/L & 09/28/2005 & & JGJ-071-05 \\
\hline GWM42501AV & MIDDLE-2051 & 748 & Chloroprene & 5 & & $\mathrm{U}$ & & UG/L & 09/28/2005 & & JGJ-071-05 \\
\hline GWM42501AV & MIDDLE-2051 & 748 & Dibromochloromethane & 1 & & $\mathrm{u}$ & & UG/L & 09/28/2005 & & JGJ-071-05 \\
\hline GWM42501AV & MIDDLE-2051 & 748 & Chloroethane & 1 & & $\mathrm{U}$ & & UG/L & 09/28/2005 & & JGJ-071-05 \\
\hline GWM42501AV & MIDDLE-2051 & 748 & Chloroform & 1 & & $\mathrm{U}$ & & UG/L & 09/28/2005 & & JGJ-071-05 \\
\hline GWM42501AV & MIDDLE-2051 & 748 & Chloromethane & 5 & & $\mathrm{U}$ & & UG/L & 09/28/2005 & & JGJ-071-05 \\
\hline GWM42501AV & MIDDLE-2051 & 748 & Allyl chloride & 5 & & $\mathrm{U}$ & & UG/L & 09/28/2005 & & JGJ-071-05 \\
\hline GWM42501AV & MIDDLE-2051 & 748 & 1,2-Dibromo-3-chloropropane & 5 & & $\mathrm{u}$ & $\mathrm{R}$ & UG/L & 09/28/2005 & & JGJ-071-05 \\
\hline GWM42501AV & MIDDLE-2051 & 748 & 1,2-Dibromoethane & 1 & & U & & UG/L & 09/28/2005 & & JGJ-071-05 \\
\hline GWM42501AV & MIDDLE-2051 & 748 & Dibromomethane & 1 & & U & & UG/L & 09/28/2005 & & JGJ-071-05 \\
\hline GWM42501AV & MIDDLE-2051 & 748 & trans-1,4-Dichloro-2-butene & 5 & & $\mathrm{U}$ & & UG/L & 09/28/2005 & & JGJ-071-05 \\
\hline GWM42501AV & MIDDLE-2051 & 748 & Dichlorodifluoromethane & 5 & & U & & UG/L & 09/28/2005 & & JGJ-071-05 \\
\hline GWM42501AV & MIDDLE-2051 & 748 & 1,1-Dichloroethane & 1 & & $\mathrm{u}$ & & UG/L & 09/28/2005 & & JGJ-071-05 \\
\hline GWM42501AV & MIDDLE-2051 & 748 & 1,2-Dichloroethane & 1 & & $\mathrm{U}$ & & UG/L & 09/28/2005 & & JGJ-071-05 \\
\hline GWM42501AV & MIDDLE-2051 & 748 & cis-1,2-Dichloroethene & 1 & & $\mathrm{U}$ & & UG/L & 09/28/2005 & & JGJ-071-05 \\
\hline GWM42501AV & MIDDLE-2051 & 748 & trans-1,2-Dichloroethene & 1 & & $\mathrm{U}$ & & UG/L & 09/28/2005 & & JGJ-071-05 \\
\hline GWM42501AV & MIDDLE-2051 & 748 & 1,1-Dichloroethene & 1 & & U & & UG/L & 09/28/2005 & & JGJ-071-05 \\
\hline GWM42501AV & MIDDLE-2051 & 748 & 1,2-Dichloropropane & 1 & & U & & UG/L & 09/28/2005 & & JGJ-071-05 \\
\hline GWM42501AV & MIDDLE-2051 & 748 & cis-1,3-Dichloropropene & 1 & & U & & UG/L & 09/28/2005 & & JGJ-071-05 \\
\hline GWM42501AV & MIDDLE-2051 & 748 & trans-1,3-Dichloropropene & 1 & & $\mathrm{U}$ & & UG/L & 09/28/2005 & & JGJ-071-05 \\
\hline GWM42501AV & MIDDLE-2051 & 748 & 1,4-Dioxane & 80 & & U & $\mathrm{R}$ & UG/L & 09/28/2005 & & JGJ-071-05 \\
\hline GWM42501AV & MIDDLE-2051 & 748 & Ethylbenzene & 1 & & $\mathrm{U}$ & & UG/L & 09/28/2005 & & JGJ-071-05 \\
\hline GWM42501AV & MIDDLE-2051 & 748 & 2-Hexanone & 5 & & u & & UG/L & 09/28/2005 & & JGJ-071-05 \\
\hline GWM42501AV & MIDDLE-2051 & 748 & Methyl iodide & 2 & & $\mathrm{U}$ & & UG/L & 09/28/2005 & & JGJ-071-05 \\
\hline GWM42501AV & MIDDLE-2051 & 748 & Isobutyl alcohol & 80 & & U & $\mathrm{R}$ & UG/L & 09/28/2005 & & JGJ-071-05 \\
\hline GWM42501AV & MIDDLE-2051 & 748 & Methacrylonitrile & 5 & & U & $\mathrm{R}$ & UG/L & 09/28/2005 & & JGJ-071-05 \\
\hline GWM42501AV & MIDDLE-2051 & 748 & Methylene Chloride & 1 & & $\mathrm{U}$ & & UG/L & 09/28/2005 & & JGJ-071-05 \\
\hline GWM42501AV & MIDDLE-2051 & 748 & Methylmethacrylate & 1 & & U & & UG/L & 09/28/2005 & & JGJ-071-05 \\
\hline GWM42501AV & MIDDLE-2051 & 748 & Methyl isobutyl ketone & 5 & & U & & UG/L & 09/28/2005 & & JGJ-071-05 \\
\hline GWM42501AV & MIDDLE-2051 & 748 & Propionitrile & 5 & & $\mathrm{U}$ & $\mathrm{R}$ & UG/L & 09/28/2005 & & JGJ-071-05 \\
\hline GWM42501AV & MIDDLE-2051 & 748 & Styrene & 1 & & U & & UG/L & 09/28/2005 & & JGJ-071-05 \\
\hline GWM42501AV & MIDDLE-2051 & 748 & 1,1,1,2-Tetrachloroethane & 1 & & U & & UG/L & 09/28/2005 & & JGJ-071-05 \\
\hline GWM42501AV & MIDDLE-2051 & 748 & $1,1,2,2$-Tetrachloroethane & 1 & & U & & UG/L & 09/28/2005 & & JGJ-071-05 \\
\hline GWM42501AV & MIDDLE-2051 & 748 & Tetrachloroethene & 1 & & $\mathrm{U}$ & & UG/L & 09/28/2005 & & JGJ-071-05 \\
\hline GWM42501AV & MIDDLE-2051 & 748 & Toluene & 1 & & U & & UG/L & 09/28/2005 & & JGJ-071-05 \\
\hline GWM42501AV & MIDDLE-2051 & 748 & 1,1,1-Trichloroethane & 1 & & U & & UG/L & 09/28/2005 & & JGJ-071-05 \\
\hline GWM42501AV & MIDDLE-2051 & 748 & 1,1,2-Trichloroethane & 1 & & $\mathrm{u}$ & & UG/L & 09/28/2005 & & JGJ-071-05 \\
\hline GWM42501AV & MIDDLE-2051 & 748 & Trichloroethene & 1 & & U & & UG/L & 09/28/2005 & & JGJ-071-05 \\
\hline GWM42501AV & MIDDLE-2051 & 748 & Trichlorofluoromethane & 1 & & $\mathrm{u}$ & & UG/L & 09/28/2005 & & JGJ-071-05 \\
\hline GWM42501AV & MIDDLE-2051 & 748 & 1,2,3-Trichloropropane & 1 & & $\mathrm{U}$ & & UG/L & 09/28/2005 & & JGJ-071-05 \\
\hline GWM42501AV & MIDDLE-2051 & 748 & Vinyl Acetate & 2 & & U & & UG/L & 09/28/2005 & & JGJ-071-05 \\
\hline GWM42501AV & MIDDLE-2051 & 748 & Vinyl Chloride & 1 & & U & & UG/L & 09/28/2005 & & JGJ-071-05 \\
\hline GWM42501AV & MIDDLE-2051 & 748 & Xylenes & 3 & & u & & UG/L & 09/28/2005 & & JGJ-071-05 \\
\hline GWM42501R8 & MIDDLE-2051 & 748 & Tritium & $6.32 \mathrm{E}+02$ & $1.36 \mathrm{E}+02$ & & & $\mathrm{PCI} / \mathrm{L}$ & 09/28/2005 & $3.92 \mathrm{E}+02$ & SOS-TL342-05 \\
\hline GWM42501RH & MIDDLE-2051 & 748 & Uranium-233/234 & $1.41 \mathrm{E}+00$ & $1.61 \mathrm{E}-01$ & & & $\mathrm{PCl} / \mathrm{L}$ & 09/27/2005 & 8.93E-02 & SOS-TL351-05 \\
\hline GWM42501RH & MIDDLE-2051 & 748 & Uranium-238 & $6.38 \mathrm{E}-01$ & $9.69 \mathrm{E}-02$ & & & $\mathrm{PCI} / \mathrm{L}$ & 09/27/2005 & $3.54 \mathrm{E}-02$ & SOS-TL351-05 \\
\hline GWM42501RH & MIDDLE-2051 & 748 & Uranium-235 & $9.52 \mathrm{E}-02$ & 3.95E-02 & & $\mathrm{J}$ & $\mathrm{PCl} / \mathrm{L}$ & 09/27/2005 & $9.08 \mathrm{E}-02$ & SOS-TL351-05 \\
\hline GWM42501RH & MIDDLE-2051 & 748 & Gross Alpha & $1.41 \mathrm{E}+00$ & $6.03 \mathrm{E}-01$ & & UJ & $\mathrm{PCl} / \mathrm{L}$ & 09/27/2005 & $2.05 \mathrm{E}+00$ & SOS-TL351-05 \\
\hline GWM42501RH & MIDDLE-2051 & 748 & Gross Beta & $5.62 \mathrm{E}-01$ & $9.47 \mathrm{E}-01$ & & U & $\mathrm{PCl} / \mathrm{L}$ & 09/27/2005 & $4.06 \mathrm{E}+00$ & SOS-TL351-05 \\
\hline GWM42501RH & MIDDLE-2051 & 748 & Strontium-90 & $-9.97 \mathrm{E}-02$ & $1.07 \mathrm{E}-01$ & & U & $\mathrm{PCI} / \mathrm{L}$ & 09/27/2005 & $5.99 \mathrm{E}-01$ & SOS-TL351-05 \\
\hline GWM42501RH & MIDDLE-2051 & 748 & Silver-108m & 4.37E-01 & $1.38 \mathrm{E}+00$ & & U & $\mathrm{PCl} / \mathrm{L}$ & 09/27/2005 & $5.22 \mathrm{E}+00$ & SOS-TL351-05 \\
\hline GWM42501RH & MIDDLE-2051 & 748 & Silver-110m & $1.60 \mathrm{E}+00$ & $8.84 \mathrm{E}-01$ & & U & $\mathrm{PCl} / \mathrm{L}$ & 09/27/2005 & $5.19 \mathrm{E}+00$ & SOS-TL351-05 \\
\hline GWM42501RH & MIDDLE-2051 & 748 & Cerium-144 & $1.66 \mathrm{E}+00$ & $9.86 \mathrm{E}+00$ & & U & $\mathrm{PCl} / \mathrm{L}$ & 09/27/2005 & $3.58 \mathrm{E}+01$ & SOS-TL351-05 \\
\hline GWM42501RH & MIDDLE-2051 & 748 & Cobalt-60 & $-9.34 \mathrm{E}-01$ & $1.30 \mathrm{E}+00$ & & U & $\mathrm{PCl} / \mathrm{L}$ & 09/27/2005 & $4.76 \mathrm{E}+00$ & SOS-TL351-05 \\
\hline GWM42501RH & MIDDLE-2051 & 748 & Cesium-134 & $1.35 \mathrm{E}+00$ & $1.30 \mathrm{E}+00$ & & U & $\mathrm{PCl} / \mathrm{L}$ & 09/27/2005 & $5.40 \mathrm{E}+00$ & SOS-TL351-05 \\
\hline GWM42501RH & MIDDLE-2051 & 748 & Cesium-137 & $1.63 \mathrm{E}+00$ & 1.16E+00 & & U & $\mathrm{PCI} / \mathrm{L}$ & 09/27/2005 & $4.91 \mathrm{E}+00$ & SOS-TL351-05 \\
\hline GWM42501RH & MIDDLE-2051 & 748 & Europium-152 & $-6.41 E+00$ & 4. $35 \mathrm{E}+00$ & & U & $\mathrm{PCl} / \mathrm{L}$ & 09/27/2005 & $1.38 \mathrm{E}+01$ & SOS-TL351-05 \\
\hline GWM42501RH & MIDDLE-2051 & 748 & Europium-154 & $-3.66 \mathrm{E}+00$ & $4.04 \mathrm{E}+00$ & & U & $\mathrm{PCI} / \mathrm{L}$ & 09/27/2005 & $1.44 \mathrm{E}+01$ & SOS-TL351-05 \\
\hline GWM42501RH & MIDDLE-2051 & 748 & Europium-155 & $2.44 \mathrm{E}+00$ & $4.71 \mathrm{E}+00$ & & U & $\mathrm{PCl} / \mathrm{L}$ & 09/27/2005 & $1.76 \mathrm{E}+01$ & SOS-TL351-05 \\
\hline GWM42501RH & MIDDLE-2051 & 748 & Manganese-54 & $-8.65 \mathrm{E}-02$ & $1.32 \mathrm{E}+00$ & & UJ & $\mathrm{PCl} / \mathrm{L}$ & 09/27/2005 & $4.91 \mathrm{E}+00$ & SOS-TL351-05 \\
\hline GWM42501RH & MIDDLE-2051 & 748 & Ruthenium-106 & $-1.14 E+01$ & $1.28 \mathrm{E}+01$ & & U & $\mathrm{PCl} / \mathrm{L}$ & 09/27/2005 & $3.81 \mathrm{E}+01$ & SOS-TL351-05 \\
\hline GWM42501RH & MIDDLE-2051 & 748 & Antimony-125 & $9.34 \mathrm{E}+00$ & $3.97 \mathrm{E}+00$ & & U & $\mathrm{PCl} / \mathrm{L}$ & 09/27/2005 & $1.29 \mathrm{E}+01$ & SOS-TL351-05 \\
\hline GWM42501RH & MIDDLE-2051 & 748 & Zinc-65 & $-8.39 \mathrm{E}-01$ & $2.88 \mathrm{E}+00$ & & & $\mathrm{PCI} / \mathrm{L}$ & 09/27/2005 & $1.11 \mathrm{E}+01$ & SOS-TL351-05 \\
\hline GWM42501RH & MIDDLE-2051 & 748 & Technetium-99 & $5.21 \mathrm{E}-01$ & $1.60 \mathrm{E}+00$ & & U & $\mathrm{PCI} / \mathrm{L}$ & 09/27/2005 & $5.42 \mathrm{E}+00$ & SOS-TL351-05 \\
\hline GWM426012X & MIDDLE-2051 & 834 & Antimony & 0.61 & & $\mathrm{U}$ & & UG/L & 09/28/2005 & & DNT-001-06 \\
\hline GWM426012X & MIDDLE-2051 & 834 & Arsenic & 1.8 & & U & & UG/L & 09/28/2005 & & DNT-001-06 \\
\hline GWM426012X & MIDDLE-2051 & 834 & Beryllium & 0.13 & & $U$ & & UG/L & $09 / 28 / 2005$ & & DNT-001-06 \\
\hline GWM426012X & MIDDLE-2051 & 834 & Cadmium & 0.067 & & U & & UG/L & 09/28/2005 & & DNT-001-06 \\
\hline GWM426012X & MIDDLE-2051 & 834 & Chromium & 4.8 & & B & & UG/L & 09/28/2005 & & DNT-001-06 \\
\hline GWM426012X & MIDDLE-2051 & 834 & Manganese & 29.5 & & & & UG/L & 09/28/2005 & & DNT-001-06 \\
\hline GWM426012X & MIDDLE-2051 & 834 & Silver & 1.5 & & $U$ & & UG/L & 09/28/2005 & & DNT-001-06 \\
\hline GWM426012X & MIDDLE-2051 & 834 & Thallium & 0.22 & & U & & UG/L & 09/28/2005 & & DNT-001-06 \\
\hline GWM426012X & MIDDLE-2051 & 834 & Uranium & 1.8 & & B & $\mathrm{J}$ & UG/L & 09/28/2005 & & DNT-001-06 \\
\hline GWM426012X & MIDDLE-2051 & 834 & Mercury & 0.046 & & U & & UG/L & 09/28/2005 & & DNT-001-06 \\
\hline GWM426012X & MIDDLE-2051 & 834 & Aluminum & 22.1 & & $U$ & & UG/L & $09 / 28 / 2005$ & & DNT-001-06 \\
\hline GWM426012X & MIDDLE-2051 & 834 & Barium & 58.2 & & B & & UG/L & 09/28/2005 & & DNT-001-06 \\
\hline GWM426012X & MIDDLE-2051 & 834 & Calcium & 48300 & & & & UG/L & 09/28/2005 & & DNT-001-06 \\
\hline GWM426012X & MIDDLE-2051 & 834 & Cobalt & 2.8 & & $U$ & & UG/L & 09/28/2005 & & DNT-001-06 \\
\hline GWM426012X & MIDDLE-2051 & 834 & Copper & 1.4 & & B & & UG/L & $09 / 28 / 2005$ & & DNT-001-06 \\
\hline GWM426012X & MIDDLE-2051 & 834 & Iron & 10.9 & & B & & UG/L & 09/28/2005 & & DNT-001-06 \\
\hline GWM426012X & MIDDLE-2051 & 834 & Lead & 1.1 & & $u$ & & UG/L & 09/28/2005 & & DNT-001-06 \\
\hline GWM426012X & MIDDLE-2051 & 834 & Lithium & 5.9 & & B & & UG/L & 09/28/2005 & & DNT-001-06 \\
\hline GWM426012X & MIDDLE-2051 & 834 & Magnesium & 18200 & & & & UG/L & 09/28/2005 & & DNT-001-06 \\
\hline GWM426012X & MIDDLE-2051 & 834 & Molybdenum & 3.7 & & U & & UG/L & 09/28/2005 & & DNT-001-06 \\
\hline GWM426012X & MIDDLE-2051 & 834 & Nickel & 3.5 & & B & & UG/L & $09 / 28 / 2005$ & & DNT-001-06 \\
\hline
\end{tabular}


Table A-4. (continued).

\begin{tabular}{|c|c|c|c|c|c|c|c|c|c|c|c|}
\hline $\begin{array}{c}\text { Field Sample } \\
\text { Number }\end{array}$ & Location & Depth & Compound & $\begin{array}{c}\text { Sample } \\
\text { Result }\end{array}$ & $\begin{array}{c}\text { Sample } \\
\text { Error }\end{array}$ & $\begin{array}{c}\text { Result } \\
\text { Qualifier }\end{array}$ & $\begin{array}{c}\text { Validation } \\
\text { Flag }\end{array}$ & $\begin{array}{c}\text { Sample } \\
\text { Units }\end{array}$ & $\begin{array}{c}\text { Date Sample } \\
\text { Collected }\end{array}$ & MDA & $\begin{array}{l}\text { L\&V Report } \\
\text { Number }\end{array}$ \\
\hline GWM426012X & MIDDLE-2051 & 834 & Potassium & 2830 & & $\mathrm{~B}$ & & UG/L & $09 / 28 / 2005$ & & DNT-001-06 \\
\hline GWM426012X & MIDDLE-2051 & 834 & Selenium & 2 & & u & & UG/L & 09/28/2005 & & DNT-001-06 \\
\hline GWM426012X & MIDDLE-2051 & 834 & Sodium & 8540 & & & & UG/L & 09/28/2005 & & DNT-001-06 \\
\hline GWM426012X & MIDDLE-2051 & 834 & Strontium & 295 & & & & UG/L & 09/28/2005 & & DNT-001-06 \\
\hline GWM426012X & MIDDLE-2051 & 834 & Tin & 3.9 & & $\mathrm{u}$ & & UG/L & 09/28/2005 & & DNT-001-06 \\
\hline GWM426012X & MIDDLE-2051 & 834 & Vanadium & 2.5 & & u & & UG/L & 09/28/2005 & & DNT-001-06 \\
\hline GWM426012X & MIDDLE-2051 & 834 & Zinc & 29.2 & & & & UG/L & 09/28/2005 & & DNT-001-06 \\
\hline GWM42601AN & MIDDLE-2051 & 834 & Bromide & 0.059 & & B & & MG/L & 09/28/2005 & & DNT-500-05 \\
\hline GWM42601AN & MIDDLE-2051 & 834 & Chloride & 11.6 & & $\mathrm{~J}$ & & $\mathrm{MG} / \mathrm{L}$ & 09/28/2005 & & DNT-500-05 \\
\hline GWM42601AN & MIDDLE-2051 & 834 & Fluoride & 0.11 & & & & MG/L & $09 / 28 / 2005$ & & DNT-500-05 \\
\hline GWM42601AN & MIDDLE-2051 & 834 & Sulfate & 26.3 & & & & MG/L & $09 / 28 / 2005$ & & DNT-500-05 \\
\hline GWM42601A1 & MIDDLE-2051 & 834 & Total Alkalinity & 158 & & & & MG/L & $09 / 28 / 2005$ & & DNT-500-05 \\
\hline GWM42601N2 & MIDDLE-2051 & 834 & Nitrate/Nitrite as $\mathrm{N}$ & 0.4 & & & $\mathrm{~J}$ & MG/L & 09/28/2005 & & DNT-500-05 \\
\hline GWM42601AV & MIDDLE-2051 & 834 & Acetone & 10 & & $\mathrm{u}$ & $\mathrm{R}$ & UG/L & 09/28/2005 & & JGJ-071-05 \\
\hline GWM42601AV & MIDDLE-2051 & 834 & Acetonitrile & 20 & & u & & UG/L & 09/28/2005 & & JGJ-071-05 \\
\hline GWM42601AV & MIDDLE-2051 & 834 & Acrolein & 5 & & $\mathrm{u}$ & $\mathrm{R}$ & UG/L & 09/28/2005 & & JGJ-071-05 \\
\hline GWM42601AV & MIDDLE-2051 & 834 & Acrylonitrile & 1 & & $\mathrm{u}$ & $\mathrm{R}$ & UG/L & 09/28/2005 & & JGJ-071-05 \\
\hline GWM42601AV & MIDDLE-2051 & 834 & Benzene & 1 & & $\mathrm{u}$ & & UG/L & 09/28/2005 & & JGJ-071-05 \\
\hline GWM42601AV & MIDDLE-2051 & 834 & Bromodichloromethane & 1 & & $u$ & & UG/L & 09/28/2005 & & JGJ-071-05 \\
\hline GWM42601AV & MIDDLE-2051 & 834 & Bromoform & 2 & & $\mathrm{u}$ & & UG/L & $09 / 28 / 2005$ & & JGJ-071-05 \\
\hline GWM42601AV & MIDDLE-2051 & 834 & Bromomethane & 1 & & u & & UG/L & 09/28/2005 & & JGJ-071-05 \\
\hline GWM42601AV & MIDDLE-2051 & 834 & 2-Butanone & 0.51 & & $\mathrm{~J}$ & $\mathrm{~J}$ & UG/L & 09/28/2005 & & JGJ-071-05 \\
\hline GWM42601AV & MIDDLE-2051 & 834 & Carbon disulfide & 5 & & $\mathrm{u}$ & & UG/L & 09/28/2005 & & JGJ-071-05 \\
\hline GWM42601AV & MIDDLE-2051 & 834 & Carbon tetrachloride & 1 & & $\mathrm{u}$ & & UG/L & 09/28/2005 & & JGJ-071-05 \\
\hline GWM42601AV & MIDDLE-2051 & 834 & Chlorobenzene & 1 & & $\mathrm{u}$ & & UG/L & 09/28/2005 & & JGJ-071-05 \\
\hline GWM42601AV & MIDDLE-2051 & 834 & Chloroprene & 5 & & $\mathrm{u}$ & & UG/L & 09/28/2005 & & JGJ-071-05 \\
\hline GWM42601AV & MIDDLE-2051 & 834 & Dibromochloromethane & 1 & & $\mathrm{u}$ & & UG/L & 09/28/2005 & & JGJ-071-05 \\
\hline GWM42601AV & MIDDLE-2051 & 834 & Chloroethane & 1 & & $\mathrm{u}$ & & UG/L & 09/28/2005 & & JGJ-071-05 \\
\hline GWM42601AV & MIDDLE-2051 & 834 & Chloroform & 1 & & $\mathrm{u}$ & & UG/L & 09/28/2005 & & JGJ-071-05 \\
\hline GWM42601AV & MIDDLE-2051 & 834 & Chloromethane & 5 & & $\mathrm{u}$ & & UG/L & $09 / 28 / 2005$ & & JGJ-071-05 \\
\hline GWM42601AV & MIDDLE-2051 & 834 & Allyl chloride & 5 & & $\mathrm{u}$ & & UG/L & $09 / 28 / 2005$ & & JGJ-071-05 \\
\hline GWM42601AV & MIDDLE-2051 & 834 & 1,2-Dibromo-3-chloropropane & 5 & & $\mathrm{u}$ & $\mathrm{R}$ & UG/L & 09/28/2005 & & JGJ-071-05 \\
\hline GWM42601AV & MIDDLE-2051 & 834 & 1,2-Dibromoethane & 1 & & $\mathrm{u}$ & & UG/L & $09 / 28 / 2005$ & & JGJ-071-05 \\
\hline GWM42601AV & MIDDLE-2051 & 834 & Dibromomethane & 1 & & $\mathrm{u}$ & & UG/L & $09 / 28 / 2005$ & & JGJ-071-05 \\
\hline GWM42601AV & MIDDLE-2051 & 834 & trans-1,4-Dichloro-2-butene & 5 & & $\mathrm{u}$ & & UG/L & 09/28/2005 & & JGJ-071-05 \\
\hline GWM42601AV & MIDDLE-2051 & 834 & Dichlorodifluoromethane & 5 & & $\mathrm{u}$ & & UG/L & 09/28/2005 & & JGJ-071-05 \\
\hline GWM42601AV & MIDDLE-2051 & 834 & 1,1-Dichloroethane & 1 & & $\mathrm{u}$ & & UG/L & 09/28/2005 & & JGJ-071-05 \\
\hline GWM42601AV & MIDDLE-2051 & 834 & 1,2-Dichloroethane & 1 & & $\mathrm{u}$ & & UG/L & 09/28/2005 & & JGJ-071-05 \\
\hline GWM42601AV & MIDDLE-2051 & 834 & cis-1,2-Dichloroethene & 1 & & u & & UG/L & 09/28/2005 & & JGJ-071-05 \\
\hline GWM42601AV & MIDDLE-2051 & 834 & trans-1,2-Dichloroethene & 1 & & $\mathrm{u}$ & & UG/L & 09/28/2005 & & JGJ-071-05 \\
\hline GWM42601AV & MIDDLE-2051 & 834 & 1,1-Dichloroethene & 1 & & u & & UG/L & 09/28/2005 & & JGJ-071-05 \\
\hline GWM42601AV & MIDDLE-2051 & 834 & 1,2-Dichloropropane & 1 & & $\mathrm{u}$ & & UG/L & $09 / 28 / 2005$ & & JGJ-071-05 \\
\hline GWM42601AV & MIDDLE-2051 & 834 & cis-1,3-Dichloropropene & 1 & & $\mathrm{u}$ & & UG/L & 09/28/2005 & & JGJ-071-05 \\
\hline GWM42601AV & MIDDLE-2051 & 834 & trans-1,3-Dichloropropene & 1 & & $\mathrm{u}$ & & UG/L & 09/28/2005 & & JGJ-071-05 \\
\hline GWM42601AV & MIDDLE-2051 & 834 & 1,4-Dioxane & 80 & & $\mathrm{u}$ & $\mathrm{R}$ & UG/L & 09/28/2005 & & JGJ-071-05 \\
\hline GWM42601AV & MIDDLE-2051 & 834 & Ethylbenzene & 1 & & $\mathrm{u}$ & & UG/L & 09/28/2005 & & JGJ-071-05 \\
\hline GWM42601AV & MIDDLE-2051 & 834 & 2-Hexanone & 5 & & $\mathrm{u}$ & & UG/L & $09 / 28 / 2005$ & & JGJ-071-05 \\
\hline GWM42601AV & MIDDLE-2051 & 834 & Methyl iodide & 2 & & $u$ & & UG/L & 09/28/2005 & & JGJ-071-05 \\
\hline GWM42601AV & MIDDLE-2051 & 834 & Isobutyl alcohol & 80 & & $\mathrm{u}$ & $\mathrm{R}$ & UG/L & $09 / 28 / 2005$ & & JGJ-071-05 \\
\hline GWM42601AV & MIDDLE-2051 & 834 & Methacrylonitrile & 5 & & $\mathrm{u}$ & $\mathrm{R}$ & UG/L & $09 / 28 / 2005$ & & JGJ-071-05 \\
\hline GWM42601AV & MIDDLE-2051 & 834 & Methylene Chloride & 1 & & u & & UG/L & 09/28/2005 & & JGJ-071-05 \\
\hline GWM42601AV & MIDDLE-2051 & 834 & Methylmethacrylate & 1 & & $\mathrm{u}$ & & UG/L & $09 / 28 / 2005$ & & JGJ-071-05 \\
\hline GWM42601AV & MIDDLE-2051 & 834 & Methyl isobutyl ketone & 5 & & u & & UG/L & 09/28/2005 & & JGJ-071-05 \\
\hline GWM42601AV & MIDDLE-2051 & 834 & Propionitrile & 5 & & $\mathrm{u}$ & $\mathrm{R}$ & UG/L & $09 / 28 / 2005$ & & JGJ-071-05 \\
\hline GWM42601AV & MIDDLE-2051 & 834 & Styrene & 1 & & $u$ & & UG/L & 09/28/2005 & & JGJ-071-05 \\
\hline GWM42601AV & MIDDLE-2051 & 834 & 1,1,1,2-Tetrachloroethane & 1 & & $\mathrm{u}$ & & UG/L & 09/28/2005 & & JGJ-071-05 \\
\hline GWM42601AV & MIDDLE-2051 & 834 & 1,1,2,2-Tetrachloroethane & 1 & & $u$ & & UG/L & 09/28/2005 & & JGJ-071-05 \\
\hline GWM42601AV & MIDDLE-2051 & 834 & Tetrachloroethene & 1 & & $\mathrm{u}$ & & UG/L & $09 / 28 / 2005$ & & JGJ-071-05 \\
\hline GWM42601AV & MIDDLE-2051 & 834 & Toluene & 1 & & u & & UG/L & 09/28/2005 & & JGJ-071-05 \\
\hline GWM42601AV & MIDDLE-2051 & 834 & 1,1,1-Trichloroethane & 1 & & $u$ & & UG/L & $09 / 28 / 2005$ & & JGJ-071-05 \\
\hline GWM42601AV & MIDDLE-2051 & 834 & 1,1,2-Trichloroethane & 1 & & $\mathrm{u}$ & & UG/L & 09/28/2005 & & JGJ-071-05 \\
\hline GWM42601AV & MIDDLE-2051 & 834 & Trichloroethene & 1 & & $\mathrm{u}$ & & UG/L & 09/28/2005 & & JGJ-071-05 \\
\hline GWM42601AV & MIDDLE-2051 & 834 & Trichlorofluoromethane & 1 & & u & & UG/L & 09/28/2005 & & JGJ-071-05 \\
\hline GWM42601AV & MIDDLE-2051 & 834 & 1,2,3-Trichloropropane & 1 & & $\mathrm{u}$ & & UG/L & $09 / 28 / 2005$ & & JGJ-071-05 \\
\hline GWM42601AV & MIDDLE-2051 & 834 & Vinyl Acetate & 2 & & $u$ & & UG/L & 09/28/2005 & & JGJ-071-05 \\
\hline GWM42601AV & MIDDLE-2051 & 834 & Vinyl Chloride & 1 & & u & & UG/L & 09/28/2005 & & JGJ-071-05 \\
\hline GWM42601AV & MIDDLE-2051 & 834 & Xylenes & 1.2 & & $\mathrm{~J}$ & $\mathrm{~J}$ & UG/L & 09/28/2005 & & JGJ-071-05 \\
\hline GWM42601R8 & MIDDLE-2051 & 834 & Tritium & $7.45 \mathrm{E}+02$ & $1.41 \mathrm{E}+02$ & & & $\mathrm{PCl} / \mathrm{L}$ & $09 / 28 / 2005$ & $3.97 \mathrm{E}+02$ & SOS-TL342-05 \\
\hline GWM42601RH & MIDDLE-2051 & 834 & Uranium-233/234 & $1.49 \mathrm{E}+00$ & $1.50 \mathrm{E}-01$ & & & $\mathrm{PCl} / \mathrm{L}$ & 09/28/2005 & $6.92 \mathrm{E}-02$ & SOS-TL351-05 \\
\hline GWM42601RH & MIDDLE-2051 & 834 & Uranium-238 & $5.66 \mathrm{E}-01$ & 8.03E-02 & & & $\mathrm{PCl} / \mathrm{L}$ & $09 / 28 / 2005$ & 4.84E-02 & SOS-TL351-05 \\
\hline GWM42601RH & MIDDLE-2051 & 834 & Uranium-235 & 4.26E-02 & $2.30 \mathrm{E}-02$ & & U & $\mathrm{PCl} / \mathrm{L}$ & $09 / 28 / 2005$ & $5.98 \mathrm{E}-02$ & SOS-TL351-05 \\
\hline GWM42601RH & MIDDLE-2051 & 834 & Gross Alpha & $3.01 \mathrm{E}+00$ & $8.10 \mathrm{E}-01$ & & & $\mathrm{PCl} / \mathrm{L}$ & $09 / 28 / 2005$ & $2.44 \mathrm{E}+00$ & SOS-TL351-05 \\
\hline GWM42601RH & MIDDLE-2051 & 834 & Gross Beta & $2.41 \mathrm{E}+00$ & $9.86 \mathrm{E}-01$ & & UJ & $\mathrm{PCl} / \mathrm{L}$ & 09/28/2005 & $3.91 \mathrm{E}+00$ & SOS-TL351-05 \\
\hline GWM42601RH & MIDDLE-2051 & 834 & Strontium-90 & $-2.61 \mathrm{E}-01$ & $1.35 \mathrm{E}-01$ & & u & $\mathrm{PCl} / \mathrm{L}$ & $09 / 28 / 2005$ & 7.68E-01 & SOS-TL351-05 \\
\hline GWM42601RH & MIDDLE-2051 & 834 & Silver-108m & $1.19 \mathrm{E}+00$ & $1.53 \mathrm{E}+00$ & & $u$ & $\mathrm{PCl} / \mathrm{L}$ & 09/28/2005 & $5.59 \mathrm{E}+00$ & SOS-TL351-05 \\
\hline GWM42601RH & MIDDLE-2051 & 834 & Silver-110m & $-3.55 \mathrm{E}-01$ & $1.54 \mathrm{E}+00$ & & $u$ & $\mathrm{PCl} / \mathrm{L}$ & $09 / 28 / 2005$ & $5.65 \mathrm{E}+00$ & SOS-TL351-05 \\
\hline GWM42601RH & MIDDLE-2051 & 834 & Cerium-144 & $-1.78 \mathrm{E}-02$ & $1.12 \mathrm{E}+01$ & & $u$ & $\mathrm{PCl} / \mathrm{L}$ & $09 / 28 / 2005$ & $4.02 \mathrm{E}+01$ & SOS-TL351-05 \\
\hline GWM42601RH & MIDDLE-2051 & 834 & Cobalt-60 & $1.89 \mathrm{E}+00$ & $1.38 \mathrm{E}+00$ & & $\mathrm{u}$ & $\mathrm{PCl} / \mathrm{L}$ & $09 / 28 / 2005$ & $6.29 \mathrm{E}+00$ & SOS-TL351-05 \\
\hline GWM42601RH & MIDDLE-2051 & 834 & Cesium-134 & $-1.50 \mathrm{E}+00$ & $1.52 \mathrm{E}+00$ & & u & $\mathrm{PCl} / \mathrm{L}$ & $09 / 28 / 2005$ & $5.24 \mathrm{E}+00$ & SOS-TL351-05 \\
\hline GWM42601RH & MIDDLE-2051 & 834 & Cesium-137 & $9.15 \mathrm{E}-01$ & $1.37 \mathrm{E}+00$ & & U & $\mathrm{PCl} / \mathrm{L}$ & $09 / 28 / 2005$ & $5.42 \mathrm{E}+00$ & SOS-TL351-05 \\
\hline GWM42601RH & MIDDLE-2051 & 834 & Europium-152 & $5.18 \mathrm{E}+00$ & $4.76 \mathrm{E}+00$ & & U & $\mathrm{PCl} / \mathrm{L}$ & 09/28/2005 & $1.61 \mathrm{E}+01$ & SOS-TL351-05 \\
\hline GWM42601RH & MIDDLE-2051 & 834 & Europium-154 & $-2.70 \mathrm{E}+00$ & 4.67E+00 & & $\mathrm{u}$ & $\mathrm{PCl} / \mathrm{L}$ & $09 / 28 / 2005$ & $1.73 \mathrm{E}+01$ & SOS-TL351-05 \\
\hline GWM42601RH & MIDDLE-2051 & 834 & Europium-155 & $2.24 \mathrm{E}+00$ & $5.90 \mathrm{E}+00$ & & $u$ & $\mathrm{PCl} / \mathrm{L}$ & 09/28/2005 & $2.16 \mathrm{E}+01$ & SOS-TL351-05 \\
\hline GWM42601RH & MIDDLE-2051 & 834 & Manganese- 54 & $1.61 \mathrm{E}+00$ & $1.41 \mathrm{E}+00$ & & u & $\mathrm{PCl} / \mathrm{L}$ & $09 / 28 / 2005$ & $5.78 \mathrm{E}+00$ & SOS-TL351-05 \\
\hline GWM42601RH & MIDDLE-2051 & 834 & Ruthenium-106 & $-7.74 \mathrm{E}+00$ & $1.38 \mathrm{E}+01$ & & $u$ & $\mathrm{PCl} / \mathrm{L}$ & 09/28/2005 & $4.97 \mathrm{E}+01$ & SOS-TL351-05 \\
\hline GWM42601RH & MIDDLE-2051 & 834 & Antimony-125 & $-1.20 \mathrm{E}+00$ & 4.12E+00 & & UJ & $\mathrm{PCl} / \mathrm{L}$ & $09 / 28 / 2005$ & $1.42 \mathrm{E}+01$ & SOS-TL351-05 \\
\hline
\end{tabular}


Table A-4. (continued).

\begin{tabular}{|c|c|c|c|c|c|c|c|c|c|c|c|}
\hline $\begin{array}{c}\text { Field Sample } \\
\text { Number }\end{array}$ & Location & Depth & Compound & $\begin{array}{c}\text { Sample } \\
\text { Result }\end{array}$ & $\begin{array}{c}\text { Sample } \\
\text { Error }\end{array}$ & $\begin{array}{c}\text { Result } \\
\text { Qualifier }\end{array}$ & $\begin{array}{c}\text { Validation } \\
\text { Flag }\end{array}$ & $\begin{array}{c}\text { Sample } \\
\text { Units }\end{array}$ & $\begin{array}{c}\text { Date Sample } \\
\text { Collected }\end{array}$ & MDA & $\begin{array}{l}\text { L\&V Report } \\
\text { Number }\end{array}$ \\
\hline GWM42601RH & MIDDLE-2051 & 834 & Zinc-65 & $-8.32 \mathrm{E}-01$ & $3.88 \mathrm{E}+00$ & & $\bar{U}$ & $\mathrm{PCl} / \mathrm{L}$ & $09 / 28 / 2005$ & $1.46 \mathrm{E}+01$ & SOS-TL351-05 \\
\hline GWM42601RH & MIDDLE-2051 & 834 & Technetium-99 & $6.51 \mathrm{E}-01$ & $1.61 \mathrm{E}+00$ & & u & $\mathrm{PCl} / \mathrm{L}$ & 09/28/2005 & $5.43 E+00$ & SOS-TL351-05 \\
\hline GWM427012X & MIDDLE-2051 & 1098 & Antimony & 0.61 & & $u$ & & UG/L & 09/28/2005 & & DNT-001-06 \\
\hline GWM427012X & MIDDLE-2051 & 1098 & Arsenic & 1.8 & & $u$ & & UG/L & 09/28/2005 & & DNT-001-06 \\
\hline GWM427012X & MIDDLE-2051 & 1098 & Beryllium & 0.13 & & u & & UG/L & 09/28/2005 & & DNT-001-06 \\
\hline GWM427012X & MIDDLE-2051 & 1098 & Cadmium & 0.067 & & u & & UG/L & 09/28/2005 & & DNT-001-06 \\
\hline GWM427012X & MIDDLE-2051 & 1098 & Chromium & 9.2 & & & & UG/L & 09/28/2005 & & DNT-001-06 \\
\hline GWM427012X & MIDDLE-2051 & 1098 & Manganese & 0.93 & & B & & UG/L & 09/28/2005 & & DNT-001-06 \\
\hline GWM427012X & MIDDLE-2051 & 1098 & Silver & 1.5 & & $u$ & & UG/L & 09/28/2005 & & DNT-001-06 \\
\hline GWM427012X & MIDDLE-2051 & 1098 & Thallium & 0.22 & & $u$ & & UG/L & $09 / 28 / 2005$ & & DNT-001-06 \\
\hline GWM427012X & MIDDLE-2051 & 1098 & Uranium & 2.1 & & B & $\mathrm{J}$ & UG/L & $09 / 28 / 2005$ & & DNT-001-06 \\
\hline GWM427012X & MIDDLE-2051 & 1098 & Mercury & 0.046 & & u & & UG/L & $09 / 28 / 2005$ & & DNT-001-06 \\
\hline GWM427012X & MIDDLE-2051 & 1098 & Aluminum & 22.1 & & U & & UG/L & 09/28/2005 & & DNT-001-06 \\
\hline GWM427012X & MIDDLE-2051 & 1098 & Barium & 44.7 & & B & & UG/L & 09/28/2005 & & DNT-001-06 \\
\hline GWM427012X & MIDDLE-2051 & 1098 & Calcium & 42100 & & & & UG/L & 09/28/2005 & & DNT-001-06 \\
\hline GWM427012X & MIDDLE-2051 & 1098 & Cobalt & 2.8 & & $u$ & & UG/L & 09/28/2005 & & DNT-001-06 \\
\hline GWM427012X & MIDDLE-2051 & 1098 & Copper & 1.1 & & u & & UG/L & $09 / 28 / 2005$ & & DNT-001-06 \\
\hline GWM427012X & MIDDLE-2051 & 1098 & Iron & 7.4 & & u & & UG/L & 09/28/2005 & & DNT-001-06 \\
\hline GWM427012X & MIDDLE-2051 & 1098 & Lead & 1.1 & & $u$ & & UG/L & 09/28/2005 & & DNT-001-06 \\
\hline GWM427012X & MIDDLE-2051 & 1098 & Lithium & 3 & & $u$ & & UG/L & $09 / 28 / 2005$ & & DNT-001-06 \\
\hline GWM427012X & MIDDLE-2051 & 1098 & Magnesium & 19600 & & & & UG/L & $09 / 28 / 2005$ & & DNT-001-06 \\
\hline GWM427012X & MIDDLE-2051 & 1098 & Molybdenum & 3.7 & & u & & UG/L & 09/28/2005 & & DNT-001-06 \\
\hline GWM427012X & MIDDLE-2051 & 1098 & Nickel & 3.2 & & $u$ & & UG/L & 09/28/2005 & & DNT-001-06 \\
\hline GWM427012X & MIDDLE-2051 & 1098 & Potassium & 2090 & & B & & UG/L & 09/28/2005 & & DNT-001-06 \\
\hline GWM427012X & MIDDLE-2051 & 1098 & Selenium & 2 & & u & & UG/L & 09/28/2005 & & DNT-001-06 \\
\hline GWM427012X & MIDDLE-2051 & 1098 & Sodium & 7970 & & & & UG/L & 09/28/2005 & & DNT-001-06 \\
\hline GWM427012X & MIDDLE-2051 & 1098 & Strontium & 277 & & & & UG/L & 09/28/2005 & & DNT-001-06 \\
\hline GWM427012X & MIDDLE-2051 & 1098 & Tin & 3.9 & & U & & UG/L & $09 / 28 / 2005$ & & DNT-001-06 \\
\hline GWM427012X & MIDDLE-2051 & 1098 & Vanadium & 6.1 & & B & & UG/L & 09/28/2005 & & DNT-001-06 \\
\hline GWM427012X & MIDDLE-2051 & 1098 & Zinc & 13.4 & & B & & UG/L & 09/28/2005 & & DNT-001-06 \\
\hline GWM42701AN & MIDDLE-2051 & 1098 & Bromide & 0.25 & & U & & MG/L & 09/29/2005 & & DNT-500-05 \\
\hline GWM42701AN & MIDDLE-2051 & 1098 & Chloride & 15.6 & & $\mathrm{~J}$ & & $\mathrm{MG} / \mathrm{L}$ & $09 / 29 / 2005$ & & DNT-500-05 \\
\hline GWM42701AN & MIDDLE-2051 & 1098 & Fluoride & 0.18 & & & & MG/L & 09/29/2005 & & DNT-500-05 \\
\hline GWM42701AN & MIDDLE-2051 & 1098 & Sulfate & 22.7 & & & & $\mathrm{MG} / \mathrm{L}$ & 09/29/2005 & & DNT-500-05 \\
\hline GWM42701A1 & MIDDLE-2051 & 1098 & Total Alkalinity & 166 & & & & MG/L & 09/28/2005 & & DNT-500-05 \\
\hline GWM42701N2 & MIDDLE-2051 & 1098 & Nitrate/Nitrite as $\mathrm{N}$ & 0.56 & & & $\mathrm{~J}$ & MG/L & 09/29/2005 & & DNT-500-05 \\
\hline GWM42701AV & MIDDLE-2051 & 1098 & Acetone & 10 & & $\mathrm{u}$ & $\mathrm{R}$ & UG/L & 09/29/2005 & & JGJ-071-05 \\
\hline GWM42701AV & MIDDLE-2051 & 1098 & Acetonitrile & 20 & & $\mathrm{u}$ & & UG/L & 09/29/2005 & & JGJ-071-05 \\
\hline GWM42701AV & MIDDLE-2051 & 1098 & Acrolein & 5 & & u & $\mathrm{R}$ & UG/L & 09/29/2005 & & JGJ-071-05 \\
\hline GWM42701AV & MIDDLE-2051 & 1098 & Acrylonitrile & 1 & & u & $\mathrm{R}$ & UG/L & 09/29/2005 & & JGJ-071-05 \\
\hline GWM42701AV & MIDDLE-2051 & 1098 & Benzene & 1 & & u & & UG/L & 09/29/2005 & & JGJ-071-05 \\
\hline GWM42701AV & MIDDLE-2051 & 1098 & Bromodichloromethane & 1 & & $\mathrm{u}$ & & UG/L & 09/29/2005 & & JGJ-071-05 \\
\hline GWM42701AV & MIDDLE-2051 & 1098 & Bromoform & 2 & & $\mathrm{u}$ & & UG/L & 09/29/2005 & & JGJ-071-05 \\
\hline GWM42701AV & MIDDLE-2051 & 1098 & Bromomethane & 1 & & $\mathrm{u}$ & & UG/L & 09/29/2005 & & JGJ-071-05 \\
\hline GWM42701AV & MIDDLE-2051 & 1098 & 2-Butanone & 10 & & $\mathrm{u}$ & $\mathrm{R}$ & UG/L & 09/29/2005 & & JGJ-071-05 \\
\hline GWM42701AV & MIDDLE-2051 & 1098 & Carbon disulfide & 5 & & $\mathrm{u}$ & & UG/L & 09/29/2005 & & JGJ-071-05 \\
\hline GWM42701AV & MIDDLE-2051 & 1098 & Carbon tetrachloride & 1 & & $u$ & & UG/L & 09/29/2005 & & JGJ-071-05 \\
\hline GWM42701AV & MIDDLE-2051 & 1098 & Chlorobenzene & 1 & & $\mathrm{u}$ & & UG/L & 09/29/2005 & & JGJ-071-05 \\
\hline GWM42701AV & MIDDLE-2051 & 1098 & Chloroprene & 5 & & $\mathrm{u}$ & & UG/L & 09/29/2005 & & JGJ-071-05 \\
\hline GWM42701AV & MIDDLE-2051 & 1098 & Dibromochloromethane & 1 & & $\mathrm{u}$ & & UG/L & 09/29/2005 & & JGJ-071-05 \\
\hline GWM42701AV & MIDDLE-2051 & 1098 & Chloroethane & 1 & & $\mathrm{u}$ & & UG/L & 09/29/2005 & & JGJ-071-05 \\
\hline GWM42701AV & MIDDLE-2051 & 1098 & Chloroform & 1 & & $\mathrm{u}$ & & UG/L & 09/29/2005 & & JGJ-071-05 \\
\hline GWM42701AV & MIDDLE-2051 & 1098 & Chloromethane & 0.27 & & $\mathrm{~J}$ & $\mathrm{~J}$ & UG/L & 09/29/2005 & & JGJ-071-05 \\
\hline GWM42701AV & MIDDLE-2051 & 1098 & Allyl chloride & 5 & & u & & UG/L & 09/29/2005 & & JGJ-071-05 \\
\hline GWM42701AV & MIDDLE-2051 & 1098 & 1,2-Dibromo-3-chloropropane & 5 & & $\mathrm{u}$ & $\mathrm{R}$ & UG/L & 09/29/2005 & & JGJ-071-05 \\
\hline GWM42701AV & MIDDLE-2051 & 1098 & 1,2-Dibromoethane & 1 & & $\mathrm{u}$ & & UG/L & 09/29/2005 & & JGJ-071-05 \\
\hline GWM42701AV & MIDDLE-2051 & 1098 & Dibromomethane & 1 & & $\mathrm{u}$ & & UG/L & 09/29/2005 & & JGJ-071-05 \\
\hline GWM42701AV & MIDDLE-2051 & 1098 & trans-1,4-Dichloro-2-butene & 5 & & $\mathrm{u}$ & & UG/L & 09/29/2005 & & JGJ-071-05 \\
\hline GWM42701AV & MIDDLE-2051 & 1098 & Dichlorodifluoromethane & 5 & & u & & UG/L & 09/29/2005 & & JGJ-071-05 \\
\hline GWM42701AV & MIDDLE-2051 & 1098 & 1,1-Dichloroethane & 1 & & $\mathrm{u}$ & & UG/L & 09/29/2005 & & JGJ-071-05 \\
\hline GWM42701AV & MIDDLE-2051 & 1098 & 1,2-Dichloroethane & 1 & & $\mathrm{u}$ & & UG/L & 09/29/2005 & & JGJ-071-05 \\
\hline GWM42701AV & MIDDLE-2051 & 1098 & cis-1,2-Dichloroethene & 1 & & $\mathrm{u}$ & & UG/L & 09/29/2005 & & JGJ-071-05 \\
\hline GWM42701AV & MIDDLE-2051 & 1098 & trans-1,2-Dichloroethene & 1 & & $\mathrm{u}$ & & UG/L & 09/29/2005 & & JGJ-071-05 \\
\hline GWM42701AV & MIDDLE-2051 & 1098 & 1,1-Dichloroethene & 1 & & $\mathrm{u}$ & & UG/L & 09/29/2005 & & JGJ-071-05 \\
\hline GWM42701AV & MIDDLE-2051 & 1098 & 1,2-Dichloropropane & 1 & & $\mathrm{u}$ & & UG/L & 09/29/2005 & & JGJ-071-05 \\
\hline GWM42701AV & MIDDLE-2051 & 1098 & cis-1,3-Dichloropropene & 1 & & $\mathrm{u}$ & & UG/L & 09/29/2005 & & JGJ-071-05 \\
\hline GWM42701AV & MIDDLE-2051 & 1098 & trans-1,3-Dichloropropene & 1 & & $u$ & & UG/L & 09/29/2005 & & JGJ-071-05 \\
\hline GWM42701AV & MIDDLE-2051 & 1098 & 1,4-Dioxane & 80 & & u & $\mathrm{R}$ & UG/L & 09/29/2005 & & JGJ-071-05 \\
\hline GWM42701AV & MIDDLE-2051 & 1098 & Ethylbenzene & 1 & & $u$ & & UG/L & 09/29/2005 & & JGJ-071-05 \\
\hline GWM42701AV & MIDDLE-2051 & 1098 & 2-Hexanone & 5 & & $\mathrm{u}$ & & UG/L & 09/29/2005 & & JGJ-071-05 \\
\hline GWM42701AV & MIDDLE-2051 & 1098 & Methyl iodide & 2 & & $\mathrm{u}$ & & UG/L & 09/29/2005 & & JGJ-071-05 \\
\hline GWM42701AV & MIDDLE-2051 & 1098 & Isobutyl alcohol & 80 & & $u$ & $\mathrm{R}$ & UG/L & 09/29/2005 & & JGJ-071-05 \\
\hline GWM42701AV & MIDDLE-2051 & 1098 & Methacrylonitrile & 5 & & $\mathrm{u}$ & $\mathrm{R}$ & UG/L & 09/29/2005 & & JGJ-071-05 \\
\hline GWM42701AV & MIDDLE-2051 & 1098 & Methylene Chloride & 1 & & $\mathrm{u}$ & & UG/L & 09/29/2005 & & JGJ-071-05 \\
\hline GWM42701AV & MIDDLE-2051 & 1098 & Methylmethacrylate & 1 & & $\mathrm{u}$ & & UG/L & 09/29/2005 & & JGJ-071-05 \\
\hline GWM42701AV & MIDDLE-2051 & 1098 & Methyl isobutyl ketone & 5 & & $\mathrm{u}$ & & UG/L & 09/29/2005 & & JGJ-071-05 \\
\hline GWM42701AV & MIDDLE-2051 & 1098 & Propionitrile & 5 & & $u$ & $\mathrm{R}$ & UG/L & 09/29/2005 & & JGJ-071-05 \\
\hline GWM42701AV & MIDDLE-2051 & 1098 & Styrene & 1 & & $\mathrm{u}$ & & UG/L & 09/29/2005 & & JGJ-071-05 \\
\hline GWM42701AV & MIDDLE-2051 & 1098 & 1,1,1,2-Tetrachloroethane & 1 & & u & & UG/L & 09/29/2005 & & JGJ-071-05 \\
\hline GWM42701AV & MIDDLE-2051 & 1098 & $1,1,2,2$-Tetrachloroethane & 1 & & u & & UG/L & 09/29/2005 & & JGJ-071-05 \\
\hline GWM42701AV & MIDDLE-2051 & 1098 & Tetrachloroethene & 1 & & $u$ & & UG/L & 09/29/2005 & & JGJ-071-05 \\
\hline GWM42701AV & MIDDLE-2051 & 1098 & Toluene & 1 & & $\mathrm{U}$ & & UG/L & 09/29/2005 & & JGJ-071-05 \\
\hline GWM42701AV & MIDDLE-2051 & 1098 & 1,1,1-Trichloroethane & 1 & & u & & UG/L & 09/29/2005 & & JGJ-071-05 \\
\hline GWM42701AV & MIDDLE-2051 & 1098 & 1,1,2-Trichloroethane & 1 & & $u$ & & UG/L & $09 / 29 / 2005$ & & JGJ-071-05 \\
\hline GWM42701AV & MIDDLE-2051 & 1098 & Trichloroethene & 1 & & $u$ & & UG/L & 09/29/2005 & & JGJ-071-05 \\
\hline GWM42701AV & MIDDLE-2051 & 1098 & Trichlorofluoromethane & 1 & & u & & UG/L & $09 / 29 / 2005$ & & JGJ-071-05 \\
\hline
\end{tabular}


Table A-4. (continued).

\begin{tabular}{|c|c|c|c|c|c|c|c|c|c|c|c|}
\hline $\begin{array}{c}\text { Field Sample } \\
\text { Number }\end{array}$ & Location & Depth & Compound & $\begin{array}{l}\text { Sample } \\
\text { Result }\end{array}$ & $\begin{array}{l}\text { Sample } \\
\text { Error }\end{array}$ & $\begin{array}{c}\text { Result } \\
\text { Qualifier }\end{array}$ & $\begin{array}{c}\text { Validation } \\
\text { Flag }\end{array}$ & $\begin{array}{c}\text { Sample } \\
\text { Units }\end{array}$ & $\begin{array}{c}\text { Date Sample } \\
\text { Collected }\end{array}$ & MDA & $\begin{array}{l}\text { L\&V Report } \\
\text { Number }\end{array}$ \\
\hline GWM42701AV & MIDDLE-2051 & 1098 & 1,2,3-Trichloropropane & 1 & & $\bar{U}$ & & $\overline{U G / L}$ & $09 / 29 / 2005$ & & JGJ-071-05 \\
\hline GWM42701AV & MIDDLE-2051 & 1098 & Vinyl Acetate & 2 & & U & & UG/L & 09/29/2005 & & JGJ-071-05 \\
\hline GWM42701AV & MIDDLE-2051 & 1098 & Vinyl Chloride & 1 & & $\mathrm{U}$ & & UG/L & 09/29/2005 & & JGJ-071-05 \\
\hline GWM42701AV & MIDDLE-2051 & 1098 & Xylenes & 3 & & U & & UG/L & 09/29/2005 & & JGJ-071-05 \\
\hline GWM42701R8 & MIDDLE-2051 & 1098 & Tritium & $1.23 \mathrm{E}+02$ & 1.16E+02 & & U & $\mathrm{PCl} / \mathrm{L}$ & 09/29/2005 & $3.92 \mathrm{E}+02$ & SOS-TL342-05 \\
\hline GWM42701RH & MIDDLE-2051 & 1098 & Uranium-233/234 & $1.27 \mathrm{E}+00$ & $1.59 \mathrm{E}-01$ & & & $\mathrm{PCI} / \mathrm{L}$ & 09/28/2005 & $9.35 \mathrm{E}-02$ & SOS-TL351-05 \\
\hline GWM42701RH & MIDDLE-2051 & 1098 & Uranium-238 & $5.08 \mathrm{E}-01$ & $9.04 \mathrm{E}-02$ & & & $\mathrm{PCI} / \mathrm{L}$ & 09/28/2005 & 7.10E-02 & SOS-TL351-05 \\
\hline GWM42701RH & MIDDLE-2051 & 1098 & Uranium-235 & $4.66 \mathrm{E}-03$ & $1.80 \mathrm{E}-02$ & & U & $\mathrm{PCI} / \mathrm{L}$ & 09/28/2005 & 1.16E-01 & SOS-TL351-05 \\
\hline GWM42701RH & MIDDLE-2051 & 1098 & Gross Alpha & $5.03 \mathrm{E}-01$ & $5.93 \mathrm{E}-01$ & & u & $\mathrm{PCl} / \mathrm{L}$ & 09/28/2005 & $2.16 \mathrm{E}+00$ & SOS-TL351-05 \\
\hline GWM42701RH & MIDDLE-2051 & 1098 & Gross Beta & $2.41 \mathrm{E}+00$ & $9.38 \mathrm{E}-01$ & & UJ & $\mathrm{PCI} / \mathrm{L}$ & 09/28/2005 & $3.66 \mathrm{E}+00$ & SOS-TL351-05 \\
\hline GWM42701RH & MIDDLE-2051 & 1098 & Strontium-90 & $-5.90 \mathrm{E}-02$ & $1.11 \mathrm{E}-01$ & & U & $\mathrm{PCI} / \mathrm{L}$ & 09/28/2005 & $5.85 \mathrm{E}-01$ & SOS-TL351-05 \\
\hline GWM42701RH & MIDDLE-2051 & 1098 & Silver-108m & $4.68 \mathrm{E}-01$ & $1.24 \mathrm{E}+00$ & & $\mathrm{U}$ & $\mathrm{PCI} / \mathrm{L}$ & 09/28/2005 & $4.67 \mathrm{E}+00$ & SOS-TL351-05 \\
\hline GWM42701RH & MIDDLE-2051 & 1098 & Silver-110m & $1.59 \mathrm{E}-01$ & $1.59 \mathrm{E}+00$ & & U & $\mathrm{PCI} / \mathrm{L}$ & 09/28/2005 & $5.81 \mathrm{E}+00$ & SOS-TL351-05 \\
\hline GWM42701RH & MIDDLE-2051 & 1098 & Cerium-144 & $4.38 \mathrm{E}+00$ & $9.97 \mathrm{E}+00$ & & U & $\mathrm{PCI} / \mathrm{L}$ & 09/28/2005 & $3.46 \mathrm{E}+01$ & SOS-TL351-05 \\
\hline GWM42701RH & MIDDLE-2051 & 1098 & Cobalt-60 & $-4.29 \mathrm{E}-01$ & $1.43 \mathrm{E}+00$ & & U & $\mathrm{PCl} / \mathrm{L}$ & 09/28/2005 & $5.50 \mathrm{E}+00$ & SOS-TL351-05 \\
\hline GWM42701RH & MIDDLE-2051 & 1098 & Cesium-134 & $1.10 \mathrm{E}+00$ & $1.58 \mathrm{E}+00$ & & U & $\mathrm{PCl} / \mathrm{L}$ & 09/28/2005 & $5.63 \mathrm{E}+00$ & SOS-TL351-05 \\
\hline GWM42701RH & MIDDLE-2051 & 1098 & Cesium-137 & $9.98 \mathrm{E}-01$ & $1.48 \mathrm{E}+00$ & & U & $\mathrm{PCI} / \mathrm{L}$ & 09/28/2005 & $5.67 \mathrm{E}+00$ & SOS-TL351-05 \\
\hline GWM42701RH & MIDDLE-2051 & 1098 & Europium-152 & $1.52 \mathrm{E}+00$ & $3.72 \mathrm{E}+00$ & & U & $\mathrm{PCI} / \mathrm{L}$ & 09/28/2005 & $1.39 \mathrm{E}+01$ & SOS-TL351-05 \\
\hline GWM42701RH & MIDDLE-2051 & 1098 & Europium-154 & $-4.62 E+00$ & $2.81 E+00$ & & U & $\mathrm{PCI} / \mathrm{L}$ & 09/28/2005 & $8.85 \mathrm{E}+00$ & SOS-TL351-05 \\
\hline GWM42701RH & MIDDLE-2051 & 1098 & Europium-155 & $-4.63 E+00$ & $5.43 E+00$ & & U & $\mathrm{PCI} / \mathrm{L}$ & 09/28/2005 & $1.80 \mathrm{E}+01$ & SOS-TL351-05 \\
\hline GWM42701RH & MIDDLE-2051 & 1098 & Manganese-54 & $-1.80 E+00$ & $1.42 \mathrm{E}+00$ & & U & $\mathrm{PCI} / \mathrm{L}$ & 09/28/2005 & $4.62 \mathrm{E}+00$ & SOS-TL351-05 \\
\hline GWM42701RH & MIDDLE-2051 & 1098 & Ruthenium-106 & $-7.38 \mathrm{E}+00$ & $1.17 \mathrm{E}+01$ & & U & $\mathrm{PCl} / \mathrm{L}$ & 09/28/2005 & $4.11 \mathrm{E}+01$ & SOS-TL351-05 \\
\hline GWM42701RH & MIDDLE-2051 & 1098 & Antimony-125 & $-8.97 \mathrm{E}-01$ & $3.33 E+00$ & & U & $\mathrm{PCl} / \mathrm{L}$ & 09/28/2005 & $1.21 \mathrm{E}+01$ & SOS-TL351-05 \\
\hline GWM42701RH & MIDDLE-2051 & 1098 & Zinc-65 & $6.70 \mathrm{E}-01$ & $3.30 \mathrm{E}+00$ & & U & $\mathrm{PCl} / \mathrm{L}$ & 09/28/2005 & $1.30 \mathrm{E}+01$ & SOS-TL351-05 \\
\hline GWM42701RH & MIDDLE-2051 & 1098 & Technetium-99 & $-7.39 \mathrm{E}-01$ & $1.60 \mathrm{E}+00$ & & U & $\mathrm{PCl} / \mathrm{L}$ & 09/28/2005 & $5.50 \mathrm{E}+00$ & SOS-TL351-05 \\
\hline GWM428012X & MIDDLE-2051 & 1148 & Antimony & 0.61 & & $\mathrm{U}$ & & UG/L & 09/29/2005 & & DNT-001-06 \\
\hline GWM428012X & MIDDLE-2051 & 1148 & Arsenic & 1.8 & & U & & UG/L & 09/29/2005 & & DNT-001-06 \\
\hline GWM428012X & MIDDLE-2051 & 1148 & Beryllium & 0.13 & & U & & UG/L & 09/29/2005 & & DNT-001-06 \\
\hline GWM428012X & MIDDLE-2051 & 1148 & Cadmium & 0.14 & & B & & UG/L & 09/29/2005 & & DNT-001-06 \\
\hline GWM428012X & MIDDLE-2051 & 1148 & Chromium & 3.7 & & U & & UG/L & 09/29/2005 & & DNT-001-06 \\
\hline GWM428012X & MIDDLE-2051 & 1148 & Manganese & 64.7 & & & & UG/L & 09/29/2005 & & DNT-001-06 \\
\hline GWM428012X & MIDDLE-2051 & 1148 & Silver & 1.5 & & U & & UG/L & 09/29/2005 & & DNT-001-06 \\
\hline GWM428012X & MIDDLE-2051 & 1148 & Thallium & 0.22 & & U & & UG/L & 09/29/2005 & & DNT-001-06 \\
\hline GWM428012X & MIDDLE-2051 & 1148 & Uranium & 2.1 & & B & $\mathrm{J}$ & UG/L & 09/29/2005 & & DNT-001-06 \\
\hline GWM428012X & MIDDLE-2051 & 1148 & Mercury & 0.046 & & U & & UG/L & 09/29/2005 & & DNT-001-06 \\
\hline GWM428012X & MIDDLE-2051 & 1148 & Aluminum & 27.6 & & B & & UG/L & 09/29/2005 & & DNT-001-06 \\
\hline GWM428012X & MIDDLE-2051 & 1148 & Barium & 80.5 & & B & & UG/L & 09/29/2005 & & DNT-001-06 \\
\hline GWM428012X & MIDDLE-2051 & 1148 & Calcium & 54700 & & & & UG/L & 09/29/2005 & & DNT-001-06 \\
\hline GWM428012X & MIDDLE-2051 & 1148 & Cobalt & 2.8 & & $U$ & & UG/L & 09/29/2005 & & DNT-001-06 \\
\hline GWM428012X & MIDDLE-2051 & 1148 & Copper & 3.8 & & B & & UG/L & 09/29/2005 & & DNT-001-06 \\
\hline GWM428012X & MIDDLE-2051 & 1148 & Iron & 88.6 & & B & & UG/L & 09/29/2005 & & DNT-001-06 \\
\hline GWM428012X & MIDDLE-2051 & 1148 & Lead & 1.1 & & U & & UG/L & 09/29/2005 & & DNT-001-06 \\
\hline GWM428012X & MIDDLE-2051 & 1148 & Lithium & 3 & & U & & UG/L & 09/29/2005 & & DNT-001-06 \\
\hline GWM428012X & MIDDLE-2051 & 1148 & Magnesium & 17600 & & & & UG/L & 09/29/2005 & & DNT-001-06 \\
\hline GWM428012X & MIDDLE-2051 & 1148 & Molybdenum & 3.7 & & U & & UG/L & 09/29/2005 & & DNT-001-06 \\
\hline GWM428012X & MIDDLE-2051 & 1148 & Nickel & 8.5 & & B & & UG/L & 09/29/2005 & & DNT-001-06 \\
\hline GWM428012X & MIDDLE-2051 & 1148 & Potassium & 1760 & & B & & UG/L & 09/29/2005 & & DNT-001-06 \\
\hline GWM428012X & MIDDLE-2051 & 1148 & Selenium & 2 & & $u$ & & UG/L & $09 / 29 / 2005$ & & DNT-001-06 \\
\hline GWM428012X & MIDDLE-2051 & 1148 & Sodium & 9720 & & & & UG/L & 09/29/2005 & & DNT-001-06 \\
\hline GWM428012X & MIDDLE-2051 & 1148 & Strontium & 325 & & & & UG/L & 09/29/2005 & & DNT-001-06 \\
\hline GWM428012X & MIDDLE-2051 & 1148 & Tin & 3.9 & & U & & UG/L & 09/29/2005 & & DNT-001-06 \\
\hline GWM428012X & MIDDLE-2051 & 1148 & Vanadium & 2.5 & & U & & UG/L & 09/29/2005 & & DNT-001-06 \\
\hline GWM428012X & MIDDLE-2051 & 1148 & Zinc & 401 & & & & UG/L & 09/29/2005 & & DNT-001-06 \\
\hline GWM42801AN & MIDDLE-2051 & 1148 & Bromide & 0.13 & & B & & MG/L & 09/29/2005 & & DNT-500-05 \\
\hline GWM42801AN & MIDDLE-2051 & 1148 & Chloride & 17.1 & & $\mathrm{~J}$ & & MG/L & 09/29/2005 & & DNT-500-05 \\
\hline GWM42801AN & MIDDLE-2051 & 1148 & Fluoride & 0.12 & & & & $\mathrm{MG} / \mathrm{L}$ & 09/29/2005 & & DNT-500-05 \\
\hline GWM42801AN & MIDDLE-2051 & 1148 & Sulfate & 22.6 & & & & MG/L & 09/29/2005 & & DNT-500-05 \\
\hline GWM42801A1 & MIDDLE-2051 & 1148 & Total Alkalinity & 167 & & & & MG/L & 09/29/2005 & & DNT-500-05 \\
\hline GWM42801N2 & MIDDLE-2051 & 1148 & Nitrate/Nitrite as N & 0.17 & & & $\mathrm{~J}$ & MG/L & 09/29/2005 & & DNT-500-05 \\
\hline GWM42801AV & MIDDLE-2051 & 1148 & Acetone & 10 & & $\mathrm{U}$ & $\mathrm{R}$ & UG/L & 09/29/2005 & & JGJ-071-05 \\
\hline GWM42801AV & MIDDLE-2051 & 1148 & Acetonitrile & 20 & & U & & UG/L & 09/29/2005 & & JGJ-071-05 \\
\hline GWM42801AV & MIDDLE-2051 & 1148 & Acrolein & 5 & & U & $\mathrm{R}$ & UG/L & 09/29/2005 & & JGJ-071-05 \\
\hline GWM42801AV & MIDDLE-2051 & 1148 & Acrylonitrile & 1 & & $\mathrm{U}$ & $\mathrm{R}$ & UG/L & 09/29/2005 & & JGJ-071-05 \\
\hline GWM42801AV & MIDDLE-2051 & 1148 & Benzene & 1 & & U & & UG/L & 09/29/2005 & & JGJ-071-05 \\
\hline GWM42801AV & MIDDLE-2051 & 1148 & Bromodichloromethane & 1 & & $\mathrm{U}$ & & UG/L & 09/29/2005 & & JGJ-071-05 \\
\hline GWM42801AV & MIDDLE-2051 & 1148 & Bromoform & 2 & & u & & UG/L & 09/29/2005 & & JGJ-071-05 \\
\hline GWM42801AV & MIDDLE-2051 & 1148 & Bromomethane & 1 & & U & & UG/L & 09/29/2005 & & JGJ-071-05 \\
\hline GWM42801AV & MIDDLE-2051 & 1148 & 2-Butanone & 10 & & $U$ & $\mathrm{R}$ & UG/L & $09 / 29 / 2005$ & & JGJ-071-05 \\
\hline GWM42801AV & MIDDLE-2051 & 1148 & Carbon disulfide & 1.2 & & $\mathrm{~J}$ & $\mathrm{~J}$ & UG/L & 09/29/2005 & & JGJ-071-05 \\
\hline GWM42801AV & MIDDLE-2051 & 1148 & Carbon tetrachloride & 1 & & U & & UG/L & 09/29/2005 & & JGJ-071-05 \\
\hline GWM42801AV & MIDDLE-2051 & 1148 & Chlorobenzene & 1 & & U & & UG/L & 09/29/2005 & & JGJ-071-05 \\
\hline GWM42801AV & MIDDLE-2051 & 1148 & Chloroprene & 5 & & $U$ & & UG/L & 09/29/2005 & & JGJ-071-05 \\
\hline GWM42801AV & MIDDLE-2051 & 1148 & Dibromochloromethane & 1 & & $\mathrm{U}$ & & UG/L & 09/29/2005 & & JGJ-071-05 \\
\hline GWM42801AV & MIDDLE-2051 & 1148 & Chloroethane & 1 & & u & & UG/L & 09/29/2005 & & JGJ-071-05 \\
\hline GWM42801AV & MIDDLE-2051 & 1148 & Chloroform & 1 & & U & & UG/L & 09/29/2005 & & JGJ-071-05 \\
\hline GWM42801AV & MIDDLE-2051 & 1148 & Chloromethane & 0.32 & & $\mathrm{~J}$ & $\mathrm{~J}$ & UG/L & 09/29/2005 & & JGJ-071-05 \\
\hline GWM42801AV & MIDDLE-2051 & 1148 & Allyl chloride & 5 & & $\mathrm{U}$ & & UG/L & 09/29/2005 & & JGJ-071-05 \\
\hline GWM42801AV & MIDDLE-2051 & 1148 & 1,2-Dibromo-3-chloropropane & 5 & & $\mathrm{u}$ & $\mathrm{R}$ & UG/L & 09/29/2005 & & JGJ-071-05 \\
\hline GWM42801AV & MIDDLE-2051 & 1148 & 1,2-Dibromoethane & 1 & & U & & UG/L & 09/29/2005 & & JGJ-071-05 \\
\hline GWM42801AV & MIDDLE-2051 & 1148 & Dibromomethane & 1 & & $U$ & & UG/L & $09 / 29 / 2005$ & & JGJ-071-05 \\
\hline GWM42801AV & MIDDLE-2051 & 1148 & trans-1,4-Dichloro-2-butene & 5 & & $\mathrm{U}$ & & UG/L & 09/29/2005 & & JGJ-071-05 \\
\hline GWM42801AV & MIDDLE-2051 & 1148 & Dichlorodifluoromethane & 5 & & $\mathrm{U}$ & & UG/L & 09/29/2005 & & JGJ-071-05 \\
\hline GWM42801AV & MIDDLE-2051 & 1148 & 1,1-Dichloroethane & 1 & & $\mathrm{U}$ & & UG/L & 09/29/2005 & & JGJ-071-05 \\
\hline GWM42801AV & MIDDLE-2051 & 1148 & 1,2-Dichloroethane & 1 & & $\mathrm{U}$ & & UG/L & 09/29/2005 & & JGJ-071-05 \\
\hline GWM42801AV & MIDDLE-2051 & 1148 & cis-1,2-Dichloroethene & 1 & & $\mathrm{U}$ & & UG/L & 09/29/2005 & & JGJ-071-05 \\
\hline GWM42801AV & MIDDLE-2051 & 1148 & trans-1,2-Dichloroethene & 1 & & $\mathrm{u}$ & & UG/L & 09/29/2005 & & JGJ-071-05 \\
\hline
\end{tabular}


Table A-4. (continued).

\begin{tabular}{|c|c|c|c|c|c|c|c|c|c|c|c|}
\hline $\begin{array}{c}\text { Field Sample } \\
\text { Number }\end{array}$ & Location & Depth & Compound & $\begin{array}{c}\text { Sample } \\
\text { Result }\end{array}$ & $\begin{array}{l}\text { Sample } \\
\text { Error }\end{array}$ & $\begin{array}{c}\text { Result } \\
\text { Qualifier }\end{array}$ & $\begin{array}{c}\text { Validation } \\
\text { Flag }\end{array}$ & $\begin{array}{c}\text { Sample } \\
\text { Units }\end{array}$ & $\begin{array}{c}\text { Date Sample } \\
\text { Collected }\end{array}$ & MDA & $\begin{array}{l}\text { L\&V Report } \\
\text { Number }\end{array}$ \\
\hline GWM42801AV & MIDDLE-2051 & 1148 & 1,1-Dichloroethene & 1 & & $\mathrm{U}$ & & UG/L & 09/29/2005 & & JGJ-071-05 \\
\hline GWM42801AV & MIDDLE-2051 & 1148 & 1,2-Dichloropropane & 1 & & $u$ & & UG/L & 09/29/2005 & & JGJ-071-05 \\
\hline GWM42801AV & MIDDLE-2051 & 1148 & cis-1,3-Dichloropropene & 1 & & $\mathrm{u}$ & & UG/L & 09/29/2005 & & JGJ-071-05 \\
\hline GWM42801AV & MIDDLE-2051 & 1148 & trans-1,3-Dichloropropene & 1 & & $u$ & & UG/L & 09/29/2005 & & JGJ-071-05 \\
\hline GWM42801AV & MIDDLE-2051 & 1148 & 1,4-Dioxane & 80 & & $\mathrm{u}$ & $\mathrm{R}$ & UG/L & 09/29/2005 & & JGJ-071-05 \\
\hline GWM42801AV & MIDDLE-2051 & 1148 & Ethylbenzene & 1 & & $u$ & & UG/L & 09/29/2005 & & JGJ-071-05 \\
\hline GWM42801AV & MIDDLE-2051 & 1148 & 2-Hexanone & 5 & & $\mathrm{u}$ & & UG/L & 09/29/2005 & & JGJ-071-05 \\
\hline GWM42801AV & MIDDLE-2051 & 1148 & Methyl iodide & 2 & & $u$ & & UG/L & 09/29/2005 & & JGJ-071-05 \\
\hline GWM42801AV & MIDDLE-2051 & 1148 & Isobutyl alcohol & 80 & & $\mathrm{u}$ & $\mathrm{R}$ & UG/L & 09/29/2005 & & JGJ-071-05 \\
\hline GWM42801AV & MIDDLE-2051 & 1148 & Methacrylonitrile & 5 & & $u$ & $\mathrm{R}$ & UG/L & 09/29/2005 & & JGJ-071-05 \\
\hline GWM42801AV & MIDDLE-2051 & 1148 & Methylene Chloride & 1 & & $\mathrm{u}$ & & UG/L & 09/29/2005 & & JGJ-071-05 \\
\hline GWM42801AV & MIDDLE-2051 & 1148 & Methylmethacrylate & 1 & & u & & UG/L & 09/29/2005 & & JGJ-071-05 \\
\hline GWM42801AV & MIDDLE-2051 & 1148 & Methyl isobutyl ketone & 5 & & u & & UG/L & 09/29/2005 & & JGJ-071-05 \\
\hline GWM42801AV & MIDDLE-2051 & 1148 & Propionitrile & 5 & & $u$ & $\mathrm{R}$ & UG/L & 09/29/2005 & & JGJ-071-05 \\
\hline GWM42801AV & MIDDLE-2051 & 1148 & Styrene & 1 & & u & & UG/L & 09/29/2005 & & JGJ-071-05 \\
\hline GWM42801AV & MIDDLE-2051 & 1148 & 1,1,1,2-Tetrachloroethane & 1 & & $u$ & & UG/L & 09/29/2005 & & JGJ-071-05 \\
\hline GWM42801AV & MIDDLE-2051 & 1148 & 1,1,2,2-Tetrachloroethane & 1 & & $\mathrm{u}$ & & UG/L & 09/29/2005 & & JGJ-071-05 \\
\hline GWM42801AV & MIDDLE-2051 & 1148 & Tetrachloroethene & 1 & & u & & UG/L & 09/29/2005 & & JGJ-071-05 \\
\hline GWM42801AV & MIDDLE-2051 & 1148 & Toluene & 1 & & $u$ & & UG/L & 09/29/2005 & & JGJ-071-05 \\
\hline GWM42801AV & MIDDLE-2051 & 1148 & 1,1,1-Trichloroethane & 1 & & $u$ & & UG/L & 09/29/2005 & & JGJ-071-05 \\
\hline GWM42801AV & MIDDLE-2051 & 1148 & 1,1,2-Trichloroethane & 1 & & u & & UG/L & 09/29/2005 & & JGJ-071-05 \\
\hline GWM42801AV & MIDDLE-2051 & 1148 & Trichloroethene & 1 & & $u$ & & UG/L & 09/29/2005 & & JGJ-071-05 \\
\hline GWM42801AV & MIDDLE-2051 & 1148 & Trichlorofluoromethane & 1 & & $\mathrm{u}$ & & UG/L & 09/29/2005 & & JGJ-071-05 \\
\hline GWM42801AV & MIDDLE-2051 & 1148 & 1,2,3-Trichloropropane & 1 & & u & & UG/L & 09/29/2005 & & JGJ-071-05 \\
\hline GWM42801AV & MIDDLE-2051 & 1148 & Vinyl Acetate & 2 & & u & & UG/L & 09/29/2005 & & JGJ-071-05 \\
\hline GWM42801AV & MIDDLE-2051 & 1148 & Vinyl Chloride & 1 & & $u$ & & UG/L & 09/29/2005 & & JGJ-071-05 \\
\hline GWM42801AV & MIDDLE-2051 & 1148 & Xylenes & 3 & & $u$ & & UG/L & 09/29/2005 & & JGJ-071-05 \\
\hline GWM42801R8 & MIDDLE-2051 & 1148 & Tritium & $1.72 \mathrm{E}+02$ & $1.20 \mathrm{E}+02$ & & $u$ & $\mathrm{PCI} / \mathrm{L}$ & 09/29/2005 & $3.96 \mathrm{E}+02$ & SOS-TL342-05 \\
\hline GWM42801RH & MIDDLE-2051 & 1148 & Uranium-233/234 & $1.66 \mathrm{E}+00$ & $1.72 \mathrm{E}-01$ & & & $\mathrm{PCl} / \mathrm{L}$ & 09/29/2005 & $6.61 \mathrm{E}-02$ & SOS-TL351-05 \\
\hline GWM42801RH & MIDDLE-2051 & 1148 & Uranium-238 & 4.73E-01 & 7.77E-02 & & $u$ & $\mathrm{PCl} / \mathrm{L}$ & 09/29/2005 & $6.61 \mathrm{E}-02$ & SOS-TL351-05 \\
\hline GWM42801RH & MIDDLE-2051 & 1148 & Uranium-235 & $5.25 \mathrm{E}-02$ & $2.65 \mathrm{E}-02$ & & & $\mathrm{PCl} / \mathrm{L}$ & 09/29/2005 & $3.94 \mathrm{E}-02$ & SOS-TL351-05 \\
\hline GWM42801RH & MIDDLE-2051 & 1148 & Gross Alpha & $1.21 \mathrm{E}+00$ & $6.12 \mathrm{E}-01$ & & $u$ & $\mathrm{PCI} / \mathrm{L}$ & 09/29/2005 & $1.92 \mathrm{E}+00$ & SOS-TL351-05 \\
\hline GWM42801RH & MIDDLE-2051 & 1148 & Gross Beta & $3.90 \mathrm{E}+00$ & $9.95 \mathrm{E}-01$ & & & $\mathrm{PCl} / \mathrm{L}$ & 09/29/2005 & $3.67 \mathrm{E}+00$ & SOS-TL351-05 \\
\hline GWM42801RH & MIDDLE-2051 & 1148 & Strontium-90 & $5.42 \mathrm{E}-01$ & 1.67E-01 & & $\mathrm{J}$ & $\mathrm{PCl} / \mathrm{L}$ & 09/29/2005 & $5.88 \mathrm{E}-01$ & SOS-TL351-05 \\
\hline GWM42801RH & MIDDLE-2051 & 1148 & Silver-108m & $2.15 \mathrm{E}+00$ & $1.43 \mathrm{E}+00$ & & $u$ & $\mathrm{PCl} / \mathrm{L}$ & 09/29/2005 & $5.51 \mathrm{E}+00$ & SOS-TL351-05 \\
\hline GWM42801RH & MIDDLE-2051 & 1148 & Silver-110m & $-1.22 \mathrm{E}+00$ & $1.70 \mathrm{E}+00$ & & $u$ & $\mathrm{PCl} / \mathrm{L}$ & 09/29/2005 & $5.98 \mathrm{E}+00$ & SOS-TL351-05 \\
\hline GWM42801RH & MIDDLE-2051 & 1148 & Cerium-144 & $-2.13 E+00$ & $1.15 \mathrm{E}+01$ & & $u$ & $\mathrm{PCl} / \mathrm{L}$ & 09/29/2005 & $3.90 E+01$ & SOS-TL351-05 \\
\hline GWM42801RH & MIDDLE-2051 & 1148 & Cobalt-60 & 1.75E-01 & $1.13 E+00$ & & $u$ & $\mathrm{PCl} / \mathrm{L}$ & 09/29/2005 & $4.70 \mathrm{E}+00$ & SOS-TL351-05 \\
\hline GWM42801RH & MIDDLE-2051 & 1148 & Cesium-134 & $2.23 E+00$ & $1.47 \mathrm{E}+00$ & & UJ & $\mathrm{PCl} / \mathrm{L}$ & 09/29/2005 & $6.19 E+00$ & SOS-TL351-05 \\
\hline GWM42801RH & MIDDLE-2051 & 1148 & Cesium-137 & 3.07E+00 & $1.59 \mathrm{E}+00$ & & $u$ & $\mathrm{PCl} / \mathrm{L}$ & 09/29/2005 & $6.56 \mathrm{E}+00$ & SOS-TL351-05 \\
\hline GWM42801RH & MIDDLE-2051 & 1148 & Europium-152 & $5.22 \mathrm{E}+00$ & $4.60 \mathrm{E}+00$ & & u & $\mathrm{PCl} / \mathrm{L}$ & 09/29/2005 & $1.72 \mathrm{E}+01$ & SOS-TL351-05 \\
\hline GWM42801RH & MIDDLE-2051 & 1148 & Europium-154 & $-4.35 \mathrm{E}+00$ & $4.09 \mathrm{E}+00$ & & $u$ & $\mathrm{PCl} / \mathrm{L}$ & 09/29/2005 & $1.42 \mathrm{E}+01$ & SOS-TL351-05 \\
\hline GWM42801RH & MIDDLE-2051 & 1148 & Europium-155 & $-5.50 E+00$ & $5.54 \mathrm{E}+00$ & & $u$ & $\mathrm{PCl} / \mathrm{L}$ & 09/29/2005 & $1.85 \mathrm{E}+01$ & SOS-TL351-05 \\
\hline GWM42801RH & MIDDLE-2051 & 1148 & Manganese-54 & $3.75 \mathrm{E}+00$ & $1.29 \mathrm{E}+00$ & & $u$ & $\mathrm{PCl} / \mathrm{L}$ & 09/29/2005 & $4.51 \mathrm{E}+00$ & SOS-TL351-05 \\
\hline GWM42801RH & MIDDLE-2051 & 1148 & Ruthenium-106 & $9.41 \mathrm{E}-01$ & $1.34 \mathrm{E}+01$ & & $u$ & $\mathrm{PCl} / \mathrm{L}$ & 09/29/2005 & $5.03 E+01$ & SOS-TL351-05 \\
\hline GWM42801RH & MIDDLE-2051 & 1148 & Antimony-125 & $4.29 \mathrm{E}+00$ & $3.61 \mathrm{E}+00$ & & $u$ & $\mathrm{PCl} / \mathrm{L}$ & 09/29/2005 & $1.40 \mathrm{E}+01$ & SOS-TL351-05 \\
\hline GWM42801RH & MIDDLE-2051 & 1148 & Zinc-65 & $9.28 \mathrm{E}+00$ & $2.06 \mathrm{E}+00$ & & $u$ & $\mathrm{PCl} / \mathrm{L}$ & 09/29/2005 & $1.03 E+01$ & SOS-TL351-05 \\
\hline GWM42801RH & MIDDLE-2051 & 1148 & Technetium-99 & $1.28 \mathrm{E}+00$ & $1.67 \mathrm{E}+00$ & & $u$ & $\mathrm{PCl} / \mathrm{L}$ & 09/29/2005 & $5.58 \mathrm{E}+00$ & SOS-TL351-05 \\
\hline GWM43101AV & Trip Blank & NA & Acetone & 10 & & u & $\mathrm{R}$ & UG/L & 09/30/2005 & & HCJ-099-05 \\
\hline GWM43101AV & Trip Blank & NA & Acetonitrile & 20 & & U & & UG/L & 09/30/2005 & & HCJ-099-05 \\
\hline GWM43101AV & Trip Blank & NA & Acrolein & 5 & & u & $\mathrm{R}$ & UG/L & 09/30/2005 & & HCJ-099-05 \\
\hline GWM43101AV & Trip Blank & NA & Acrylonitrile & 1 & & $u$ & $\mathrm{R}$ & UG/L & 09/30/2005 & & HCJ-099-05 \\
\hline GWM43101AV & Trip Blank & NA & Benzene & 1 & & $\mathrm{u}$ & & UG/L & 09/30/2005 & & HCJ-099-05 \\
\hline GWM43101AV & Trip Blank & NA & Bromodichloromethane & 1 & & $\mathrm{u}$ & & UG/L & 09/30/2005 & & HCJ-099-05 \\
\hline GWM43101AV & Trip Blank & NA & Bromoform & 2 & & $\mathrm{u}$ & & UG/L & 09/30/2005 & & HCJ-099-05 \\
\hline GWM43101AV & Trip Blank & NA & Bromomethane & 1 & & $u$ & & UG/L & 09/30/2005 & & HCJ-099-05 \\
\hline GWM43101AV & Trip Blank & NA & 2-Butanone & 10 & & $\mathrm{u}$ & $\mathrm{R}$ & UG/L & 09/30/2005 & & HCJ-099-05 \\
\hline GWM43101AV & Trip Blank & NA & Carbon disulfide & 5 & & $\mathrm{u}$ & & UG/L & 09/30/2005 & & HCJ-099-05 \\
\hline GWM43101AV & Trip Blank & NA & Carbon tetrachloride & 1 & & u & & UG/L & 09/30/2005 & & HCJ-099-05 \\
\hline GWM43101AV & Trip Blank & NA & Chlorobenzene & 1 & & $u$ & & UG/L & 09/30/2005 & & HCJ-099-05 \\
\hline GWM43101AV & Trip Blank & NA & Chloroprene & 5 & & $\mathrm{u}$ & & UG/L & 09/30/2005 & & HCJ-099-05 \\
\hline GWM43101AV & Trip Blank & NA & Dibromochloromethane & 1 & & $\mathrm{u}$ & & UG/L & 09/30/2005 & & HCJ-099-05 \\
\hline GWM43101AV & Trip Blank & NA & Chloroethane & 1 & & $\mathrm{u}$ & & UG/L & 09/30/2005 & & HCJ-099-05 \\
\hline GWM43101AV & Trip Blank & NA & Chloroform & 1 & & $u$ & & UG/L & 09/30/2005 & & HCJ-099-05 \\
\hline GWM43101AV & Trip Blank & NA & Chloromethane & 5 & & $\mathrm{u}$ & & UG/L & 09/30/2005 & & HCJ-099-05 \\
\hline GWM43101AV & Trip Blank & NA & Allyl chloride & 5 & & $\mathrm{u}$ & & UG/L & 09/30/2005 & & HCJ-099-05 \\
\hline GWM43101AV & Trip Blank & NA & 1,2-Dibromo-3-chloropropane & 5 & & $\mathrm{u}$ & & UG/L & 09/30/2005 & & HCJ-099-05 \\
\hline GWM43101AV & Trip Blank & NA & 1,2-Dibromoethane & 1 & & U & & UG/L & 09/30/2005 & & HCJ-099-05 \\
\hline GWM43101AV & Trip Blank & NA & Dibromomethane & 1 & & $\mathrm{u}$ & & UG/L & 09/30/2005 & & HCJ-099-05 \\
\hline GWM43101AV & Trip Blank & NA & trans-1,4-Dichloro-2-butene & 5 & & $\mathrm{u}$ & & UG/L & 09/30/2005 & & HCJ-099-05 \\
\hline GWM43101AV & Trip Blank & NA & Dichlorodifluoromethane & 5 & & $\mathrm{u}$ & & UG/L & 09/30/2005 & & HCJ-099-05 \\
\hline GWM43101AV & Trip Blank & NA & 1,1-Dichloroethane & 1 & & u & & UG/L & 09/30/2005 & & HCJ-099-05 \\
\hline GWM43101AV & Trip Blank & NA & 1,2-Dichloroethane & 1 & & $\mathrm{u}$ & & UG/L & 09/30/2005 & & HCJ-099-05 \\
\hline GWM43101AV & Trip Blank & NA & cis-1,2-Dichloroethene & 1 & & $\mathrm{u}$ & & UG/L & 09/30/2005 & & HCJ-099-05 \\
\hline GWM43101AV & Trip Blank & NA & trans-1,2-Dichloroethene & 1 & & $\mathrm{u}$ & & UG/L & 09/30/2005 & & HCJ-099-05 \\
\hline GWM43101AV & Trip Blank & NA & 1,1-Dichloroethene & 1 & & $u$ & & UG/L & 09/30/2005 & & HCJ-099-05 \\
\hline GWM43101AV & Trip Blank & NA & 1,2-Dichloropropane & 1 & & u & & UG/L & 09/30/2005 & & HCJ-099-05 \\
\hline GWM43101AV & Trip Blank & NA & cis-1,3-Dichloropropene & 1 & & $\mathrm{u}$ & & UG/L & 09/30/2005 & & HCJ-099-05 \\
\hline GWM43101AV & Trip Blank & NA & trans-1,3-Dichloropropene & 1 & & $\mathrm{u}$ & & UG/L & 09/30/2005 & & HCJ-099-05 \\
\hline GWM43101AV & Trip Blank & NA & 1,4-Dioxane & 80 & & $u$ & $\mathrm{R}$ & UG/L & 09/30/2005 & & HCJ-099-05 \\
\hline GWM43101AV & Trip Blank & NA & Ethylbenzene & 1 & & $u$ & & UG/L & 09/30/2005 & & HCJ-099-05 \\
\hline GWM43101AV & Trip Blank & NA & 2-Hexanone & 5 & & $\mathrm{u}$ & & UG/L & 09/30/2005 & & HCJ-099-05 \\
\hline GWM43101AV & Trip Blank & NA & Methyl iodide & 2 & & $\mathrm{u}$ & & UG/L & 09/30/2005 & & HCJ-099-05 \\
\hline GWM43101AV & Trip Blank & NA & Isobutyl alcohol & 80 & & u & $R$ & UG/L & 09/30/2005 & & HCJ-099-05 \\
\hline GWM43101AV & Trip Blank & NA & Methacrylonitrile & 5 & & $\mathrm{u}$ & & UG/L & 09/30/2005 & & HCJ-099-05 \\
\hline GWM43101AV & Trip Blank & NA & Methylene Chloride & 1 & & $\mathrm{u}$ & & UG/L & 09/30/2005 & & HCJ-099-05 \\
\hline
\end{tabular}


Table A-4. (continued).

\begin{tabular}{|c|c|c|c|c|c|c|c|c|c|c|c|}
\hline $\begin{array}{c}\text { Field Sample } \\
\text { Number }\end{array}$ & Location & Depth & Compound & $\begin{array}{c}\text { Sample } \\
\text { Result }\end{array}$ & $\begin{array}{l}\text { Sample } \\
\text { Error }\end{array}$ & $\begin{array}{c}\text { Result } \\
\text { Qualifier }\end{array}$ & $\begin{array}{c}\text { Validation } \\
\text { Flag }\end{array}$ & $\begin{array}{c}\text { Sample } \\
\text { Units }\end{array}$ & $\begin{array}{c}\text { Date Sample } \\
\text { Collected }\end{array}$ & MDA & $\begin{array}{c}\text { L\&V Report } \\
\text { Number }\end{array}$ \\
\hline GWM43101AV & Trip Blank & NA & Methylmethacrylate & 1 & & $\mathrm{U}$ & & UG/L & $09 / 30 / 2005$ & & HCJ-099-05 \\
\hline GWM43101AV & Trip Blank & NA & Methyl isobutyl ketone & 5 & & u & & UG/L & 09/30/2005 & & HCJ-099-05 \\
\hline GWM43101AV & Trip Blank & NA & Propionitrile & 5 & & $\mathrm{u}$ & & UG/L & 09/30/2005 & & HCJ-099-05 \\
\hline GWM43101AV & Trip Blank & NA & Styrene & 1 & & $\mathrm{u}$ & & UG/L & 09/30/2005 & & HCJ-099-05 \\
\hline GWM43101AV & Trip Blank & NA & 1,1,1,2-Tetrachloroethane & 1 & & $\mathrm{u}$ & & UG/L & 09/30/2005 & & HCJ-099-05 \\
\hline GWM43101AV & Trip Blank & NA & 1,1,2,2-Tetrachloroethane & 1 & & $\mathrm{u}$ & & UG/L & 09/30/2005 & & HCJ-099-05 \\
\hline GWM43101AV & Trip Blank & NA & Tetrachloroethene & 1 & & $\mathrm{u}$ & & UG/L & 09/30/2005 & & HCJ-099-05 \\
\hline GWM43101AV & Trip Blank & NA & Toluene & 1 & & $\mathrm{u}$ & & UG/L & 09/30/2005 & & HCJ-099-05 \\
\hline GWM43101AV & Trip Blank & NA & 1,1,1-Trichloroethane & 1 & & $\mathrm{u}$ & & UG/L & 09/30/2005 & & HCJ-099-05 \\
\hline GWM43101AV & Trip Blank & NA & 1,1,2-Trichloroethane & 1 & & $\mathrm{u}$ & & UG/L & 09/30/2005 & & HCJ-099-05 \\
\hline GWM43101AV & Trip Blank & NA & Trichloroethene & 0.29 & & $\mathrm{~J}$ & $\mathrm{~J}$ & UG/L & 09/30/2005 & & HCJ-099-05 \\
\hline GWM43101AV & Trip Blank & NA & Trichlorofluoromethane & 1 & & $\mathrm{u}$ & & UG/L & 09/30/2005 & & HCJ-099-05 \\
\hline GWM43101AV & Trip Blank & NA & 1,2,3-Trichloropropane & 1 & & u & & UG/L & 09/30/2005 & & HCJ-099-05 \\
\hline GWM43101AV & Trip Blank & NA & Vinyl Acetate & 2 & & $\mathrm{u}$ & & UG/L & 09/30/2005 & & HCJ-099-05 \\
\hline GWM43101AV & Trip Blank & NA & Vinyl Chloride & 1 & & $\mathrm{u}$ & & UG/L & 09/30/2005 & & HCJ-099-05 \\
\hline GWM43101AV & Trip Blank & NA & Xylenes & 3 & & $\mathrm{u}$ & & UG/L & 09/30/2005 & & HCJ-099-05 \\
\hline GWM43301AV & Trip Blank & NA & Acetone & 10 & & $\mathrm{u}$ & $\mathrm{R}$ & UG/L & $10 / 14 / 2005$ & & HCJ-101-05 \\
\hline GWM43301AV & Trip Blank & NA & Acetonitrile & 20 & & $\mathrm{u}$ & & UG/L & $10 / 14 / 2005$ & & HCJ-101-05 \\
\hline GWM43301AV & Trip Blank & NA & Acrolein & 5 & & $\mathrm{u}$ & $\mathrm{R}$ & UG/L & $10 / 14 / 2005$ & & HCJ-101-05 \\
\hline GWM43301AV & Trip Blank & NA & Acrylonitrile & 1 & & $\mathrm{u}$ & $\mathrm{R}$ & UG/L & $10 / 14 / 2005$ & & HCJ-101-05 \\
\hline GWM43301AV & Trip Blank & NA & Benzene & 1 & & u & & UG/L & $10 / 14 / 2005$ & & HCJ-101-05 \\
\hline GWM43301AV & Trip Blank & NA & Bromodichloromethane & 1 & & $\mathrm{u}$ & & UG/L & $10 / 14 / 2005$ & & HCJ-101-05 \\
\hline GWM43301AV & Trip Blank & NA & Bromoform & 2 & & $\mathrm{u}$ & & UG/L & $10 / 14 / 2005$ & & HCJ-101-05 \\
\hline GWM43301AV & Trip Blank & NA & Bromomethane & 1 & & $\mathrm{u}$ & & UG/L & $10 / 14 / 2005$ & & HCJ-101-05 \\
\hline GWM43301AV & Trip Blank & NA & 2-Butanone & 10 & & $\mathrm{u}$ & $\mathrm{R}$ & UG/L & $10 / 14 / 2005$ & & HCJ-101-05 \\
\hline GWM43301AV & Trip Blank & NA & Carbon disulfide & 5 & & $\mathrm{u}$ & & UG/L & $10 / 14 / 2005$ & & HCJ-101-05 \\
\hline GWM43301AV & Trip Blank & NA & Carbon tetrachloride & 1 & & $\mathrm{u}$ & & UG/L & $10 / 14 / 2005$ & & HCJ-101-05 \\
\hline GWM43301AV & Trip Blank & NA & Chlorobenzene & 1 & & $\mathrm{u}$ & & UG/L & $10 / 14 / 2005$ & & HCJ-101-05 \\
\hline GWM43301AV & Trip Blank & NA & Chloroprene & 5 & & $\mathrm{u}$ & & UG/L & $10 / 14 / 2005$ & & HCJ-101-05 \\
\hline GWM43301AV & Trip Blank & NA & Dibromochloromethane & 1 & & $\mathrm{u}$ & & UG/L & $10 / 14 / 2005$ & & HCJ-101-05 \\
\hline GWM43301AV & Trip Blank & NA & Chloroethane & 1 & & u & & UG/L & $10 / 14 / 2005$ & & HCJ-101-05 \\
\hline GWM43301AV & Trip Blank & NA & Chloroform & 1 & & $\mathrm{u}$ & & UG/L & $10 / 14 / 2005$ & & HCJ-101-05 \\
\hline GWM43301AV & Trip Blank & NA & Chloromethane & 5 & & u & & UG/L & $10 / 14 / 2005$ & & HCJ-101-05 \\
\hline GWM43301AV & Trip Blank & NA & Allyl chloride & 5 & & $\mathrm{u}$ & & UG/L & $10 / 14 / 2005$ & & HCJ-101-05 \\
\hline GWM43301AV & Trip Blank & NA & 1,2-Dibromo-3-chloropropane & 5 & & $\mathrm{u}$ & $\mathrm{R}$ & UG/L & $10 / 14 / 2005$ & & HCJ-101-05 \\
\hline GWM43301AV & Trip Blank & NA & 1,2-Dibromoethane & 1 & & $\mathrm{u}$ & & UG/L & $10 / 14 / 2005$ & & HCJ-101-05 \\
\hline GWM43301AV & Trip Blank & NA & Dibromomethane & 1 & & $\mathrm{u}$ & & UG/L & $10 / 14 / 2005$ & & HCJ-101-05 \\
\hline GWM43301AV & Trip Blank & NA & trans-1,4-Dichloro-2-butene & 5 & & $\mathrm{u}$ & & UG/L & $10 / 14 / 2005$ & & HCJ-101-05 \\
\hline GWM43301AV & Trip Blank & NA & Dichlorodifluoromethane & 5 & & $\mathrm{u}$ & & UG/L & $10 / 14 / 2005$ & & HCJ-101-05 \\
\hline GWM43301AV & Trip Blank & NA & 1,1-Dichloroethane & 1 & & $\mathrm{u}$ & & UG/L & $10 / 14 / 2005$ & & HCJ-101-05 \\
\hline GWM43301AV & Trip Blank & NA & 1,2-Dichloroethane & 1 & & $\mathrm{u}$ & & UG/L & $10 / 14 / 2005$ & & HCJ-101-05 \\
\hline GWM43301AV & Trip Blank & NA & cis-1,2-Dichloroethene & 1 & & $\mathrm{u}$ & & UG/L & $10 / 14 / 2005$ & & HCJ-101-05 \\
\hline GWM43301AV & Trip Blank & NA & trans-1,2-Dichloroethene & 1 & & $\mathrm{u}$ & & UG/L & $10 / 14 / 2005$ & & HCJ-101-05 \\
\hline GWM43301AV & Trip Blank & NA & 1,1-Dichloroethene & 1 & & $\mathrm{u}$ & & UG/L & $10 / 14 / 2005$ & & HCJ-101-05 \\
\hline GWM43301AV & Trip Blank & NA & 1,2-Dichloropropane & 1 & & $\mathrm{u}$ & & UG/L & $10 / 14 / 2005$ & & HCJ-101-05 \\
\hline GWM43301AV & Trip Blank & NA & cis-1,3-Dichloropropene & 1 & & $\mathrm{u}$ & & UG/L & $10 / 14 / 2005$ & & HCJ-101-05 \\
\hline GWM43301AV & Trip Blank & NA & trans-1,3-Dichloropropene & 1 & & $\mathrm{u}$ & & UG/L & $10 / 14 / 2005$ & & HCJ-101-05 \\
\hline GWM43301AV & Trip Blank & NA & 1,4-Dioxane & 80 & & $\mathrm{u}$ & $\mathrm{R}$ & UG/L & $10 / 14 / 2005$ & & HCJ-101-05 \\
\hline GWM43301AV & Trip Blank & NA & Ethylbenzene & 1 & & $\mathrm{u}$ & & UG/L & $10 / 14 / 2005$ & & HCJ-101-05 \\
\hline GWM43301AV & Trip Blank & NA & 2-Hexanone & 5 & & $\mathrm{u}$ & & UG/L & $10 / 14 / 2005$ & & HCJ-101-05 \\
\hline GWM43301AV & Trip Blank & NA & Methyl iodide & 2 & & u & & UG/L & $10 / 14 / 2005$ & & HCJ-101-05 \\
\hline GWM43301AV & Trip Blank & NA & Isobutyl alcohol & 80 & & $\mathrm{u}$ & $\mathrm{R}$ & UG/L & $10 / 14 / 2005$ & & HCJ-101-05 \\
\hline GWM43301AV & Trip Blank & NA & Methacrylonitrile & 5 & & $\mathrm{u}$ & $\mathrm{R}$ & UG/L & $10 / 14 / 2005$ & & HCJ-101-05 \\
\hline GWM43301AV & Trip Blank & NA & Methylene Chloride & 1 & & $\mathrm{u}$ & & UG/L & $10 / 14 / 2005$ & & HCJ-101-05 \\
\hline GWM43301AV & Trip Blank & NA & Methylmethacrylate & 1 & & $\mathrm{u}$ & $\mathrm{R}$ & UG/L & $10 / 14 / 2005$ & & HCJ-101-05 \\
\hline GWM43301AV & Trip Blank & NA & Methyl isobutyl ketone & 5 & & $\mathrm{u}$ & $\mathrm{R}$ & UG/L & $10 / 14 / 2005$ & & HCJ-101-05 \\
\hline GWM43301AV & Trip Blank & NA & Propionitrile & 5 & & $\mathrm{u}$ & $\mathrm{R}$ & UG/L & $10 / 14 / 2005$ & & HCJ-101-05 \\
\hline GWM43301AV & Trip Blank & NA & Styrene & 1 & & $\mathrm{u}$ & & UG/L & $10 / 14 / 2005$ & & HCJ-101-05 \\
\hline GWM43301AV & Trip Blank & NA & 1,1,1,2-Tetrachloroethane & 1 & & u & & UG/L & $10 / 14 / 2005$ & & HCJ-101-05 \\
\hline GWM43301AV & Trip Blank & NA & 1,1,2,2-Tetrachloroethane & 1 & & $\mathrm{u}$ & & UG/L & $10 / 14 / 2005$ & & HCJ-101-05 \\
\hline GWM43301AV & Trip Blank & NA & Tetrachloroethene & 1 & & $\mathrm{u}$ & & UG/L & $10 / 14 / 2005$ & & HCJ-101-05 \\
\hline GWM43301AV & Trip Blank & NA & Toluene & 1 & & $\mathrm{u}$ & & UG/L & $10 / 14 / 2005$ & & HCJ-101-05 \\
\hline GWM43301AV & Trip Blank & NA & 1,1,1-Trichloroethane & 1 & & $\mathrm{u}$ & & UG/L & $10 / 14 / 2005$ & & HCJ-101-05 \\
\hline GWM43301AV & Trip Blank & NA & 1,1,2-Trichloroethane & 1 & & $\mathrm{u}$ & & UG/L & $10 / 14 / 2005$ & & HCJ-101-05 \\
\hline GWM43301AV & Trip Blank & NA & Trichloroethene & 1 & & $\mathrm{u}$ & & UG/L & $10 / 14 / 2005$ & & HCJ-101-05 \\
\hline GWM43301AV & Trip Blank & NA & Trichlorofluoromethane & 1 & & $\mathrm{u}$ & & UG/L & $10 / 14 / 2005$ & & HCJ-101-05 \\
\hline GWM43301AV & Trip Blank & NA & 1,2,3-Trichloropropane & 1 & & u & & UG/L & $10 / 14 / 2005$ & & HCJ-101-05 \\
\hline GWM43301AV & Trip Blank & NA & Vinyl Acetate & 2 & & $\mathrm{u}$ & & UG/L & $10 / 14 / 2005$ & & HCJ-101-05 \\
\hline GWM43301AV & Trip Blank & NA & Vinyl Chloride & 1 & & u & & UG/L & $10 / 14 / 2005$ & & HCJ-101-05 \\
\hline GWM43301AV & Trip Blank & NA & Xylenes & 3 & & $\mathrm{u}$ & & UG/L & $10 / 14 / 2005$ & & HCJ-101-05 \\
\hline GWM43201AV & Trip Blank & NA & Acetone & 10 & & $\mathrm{u}$ & $\mathrm{R}$ & UG/L & $10 / 07 / 2005$ & & HCJ-100-05 \\
\hline GWM43201AV & Trip Blank & NA & Acetonitrile & 20 & & $\mathrm{u}$ & $\mathrm{R}$ & UG/L & $10 / 07 / 2005$ & & HCJ-100-05 \\
\hline GWM43201AV & Trip Blank & NA & Acrolein & 5 & & $\mathrm{u}$ & $\mathrm{R}$ & UG/L & $10 / 07 / 2005$ & & HCJ-100-05 \\
\hline GWM43201AV & Trip Blank & NA & Acrylonitrile & 1 & & $\mathrm{u}$ & & UG/L & $10 / 07 / 2005$ & & HCJ-100-05 \\
\hline GWM43201AV & Trip Blank & NA & Benzene & 1 & & $\mathrm{u}$ & & UG/L & $10 / 07 / 2005$ & & HCJ-100-05 \\
\hline GWM43201AV & Trip Blank & NA & Bromodichloromethane & 1 & & $\mathrm{u}$ & & UG/L & $10 / 07 / 2005$ & & HCJ-100-05 \\
\hline GWM43201AV & Trip Blank & NA & Bromoform & 2 & & u & & UG/L & $10 / 07 / 2005$ & & HCJ-100-05 \\
\hline GWM43201AV & Trip Blank & NA & Bromomethane & 1 & & $\mathrm{u}$ & & UG/L & $10 / 07 / 2005$ & & HCJ-100-05 \\
\hline GWM43201AV & Trip Blank & NA & 2-Butanone & 10 & & $\mathrm{u}$ & $\mathrm{R}$ & UG/L & $10 / 07 / 2005$ & & HCJ-100-05 \\
\hline GWM43201AV & Trip Blank & NA & Carbon disulfide & 5 & & $\mathrm{u}$ & & UG/L & $10 / 07 / 2005$ & & HCJ-100-05 \\
\hline GWM43201AV & Trip Blank & NA & Carbon tetrachloride & 1 & & u & & UG/L & $10 / 07 / 2005$ & & HCJ-100-05 \\
\hline GWM43201AV & Trip Blank & NA & Chlorobenzene & 1 & & $\mathrm{u}$ & & UG/L & $10 / 07 / 2005$ & & HCJ-100-05 \\
\hline GWM43201AV & Trip Blank & NA & Chloroprene & 5 & & $\mathrm{u}$ & & UG/L & 10/07/2005 & & HCJ-100-05 \\
\hline GWM43201AV & Trip Blank & NA & Dibromochloromethane & 1 & & $\mathrm{u}$ & & UG/L & $10 / 07 / 2005$ & & HCJ-100-05 \\
\hline GWM43201AV & Trip Blank & NA & Chloroethane & 1 & & $\mathrm{u}$ & & UG/L & $10 / 07 / 2005$ & & HCJ-100-05 \\
\hline GWM43201AV & Trip Blank & NA & Chloroform & 1 & & $\mathrm{u}$ & & UG/L & $10 / 07 / 2005$ & & HCJ-100-05 \\
\hline
\end{tabular}


Table A-4. (continued).

\begin{tabular}{|c|c|c|c|c|c|c|c|c|c|c|c|}
\hline $\begin{array}{c}\text { Field Sample } \\
\text { Number }\end{array}$ & Location & Depth & Compound & $\begin{array}{c}\text { Sample } \\
\text { Result }\end{array}$ & $\begin{array}{l}\text { Sample } \\
\text { Error }\end{array}$ & $\begin{array}{c}\text { Result } \\
\text { Qualifier }\end{array}$ & $\begin{array}{c}\text { Validation } \\
\text { Flag }\end{array}$ & $\begin{array}{c}\text { Sample } \\
\text { Units }\end{array}$ & $\begin{array}{c}\text { Date Sample } \\
\text { Collected }\end{array}$ & MDA & $\begin{array}{c}\text { L\&V Report } \\
\text { Number }\end{array}$ \\
\hline GWM43201AV & Trip Blank & NA & Chloromethane & 5 & & $\mathrm{U}$ & & UG/L & $10 / 07 / 2005$ & & HCJ-100-05 \\
\hline GWM43201AV & Trip Blank & NA & Allyl chloride & 5 & & $\mathrm{u}$ & & UG/L & 10/07/2005 & & HCJ-100-05 \\
\hline GWM43201AV & Trip Blank & NA & 1,2-Dibromo-3-chloropropane & 5 & & $\mathrm{u}$ & $\mathrm{R}$ & UG/L & $10 / 07 / 2005$ & & HCJ-100-05 \\
\hline GWM43201AV & Trip Blank & NA & 1,2-Dibromoethane & 1 & & $\mathrm{u}$ & & UG/L & $10 / 07 / 2005$ & & HCJ-100-05 \\
\hline GWM43201AV & Trip Blank & NA & Dibromomethane & 1 & & $\mathrm{u}$ & & UG/L & $10 / 07 / 2005$ & & HCJ-100-05 \\
\hline GWM43201AV & Trip Blank & NA & trans-1,4-Dichloro-2-butene & 5 & & $\mathrm{u}$ & $\mathrm{R}$ & UG/L & $10 / 07 / 2005$ & & HCJ-100-05 \\
\hline GWM43201AV & Trip Blank & NA & Dichlorodifluoromethane & 5 & & $\mathrm{u}$ & & UG/L & $10 / 07 / 2005$ & & HCJ-100-05 \\
\hline GWM43201AV & Trip Blank & NA & 1,1-Dichloroethane & 1 & & $\mathrm{u}$ & & UG/L & $10 / 07 / 2005$ & & HCJ-100-05 \\
\hline GWM43201AV & Trip Blank & NA & 1,2-Dichloroethane & 1 & & $\mathrm{u}$ & & UG/L & $10 / 07 / 2005$ & & HCJ-100-05 \\
\hline GWM43201AV & Trip Blank & NA & cis-1,2-Dichloroethene & 1 & & $\mathrm{u}$ & & UG/L & $10 / 07 / 2005$ & & HCJ-100-05 \\
\hline GWM43201AV & Trip Blank & NA & trans-1,2-Dichloroethene & 1 & & $\mathrm{u}$ & & UG/L & $10 / 07 / 2005$ & & HCJ-100-05 \\
\hline GWM43201AV & Trip Blank & NA & 1,1-Dichloroethene & 1 & & $\mathrm{u}$ & & UG/L & $10 / 07 / 2005$ & & HCJ-100-05 \\
\hline GWM43201AV & Trip Blank & NA & 1,2-Dichloropropane & 1 & & u & & UG/L & 10/07/2005 & & HCJ-100-05 \\
\hline GWM43201AV & Trip Blank & NA & cis-1,3-Dichloropropene & 1 & & $\mathrm{u}$ & & UG/L & $10 / 07 / 2005$ & & HCJ-100-05 \\
\hline GWM43201AV & Trip Blank & NA & trans-1,3-Dichloropropene & 1 & & $\mathrm{u}$ & UJ & UG/L & 10/07/2005 & & HCJ-100-05 \\
\hline GWM43201AV & Trip Blank & NA & 1,4-Dioxane & 80 & & $\mathrm{u}$ & $\mathrm{R}$ & UG/L & $10 / 07 / 2005$ & & HCJ-100-05 \\
\hline GWM43201AV & Trip Blank & NA & Ethylbenzene & 1 & & $\mathrm{u}$ & & UG/L & $10 / 07 / 2005$ & & HCJ-100-05 \\
\hline GWM43201AV & Trip Blank & NA & 2-Hexanone & 5 & & $\mathrm{u}$ & & UG/L & $10 / 07 / 2005$ & & HCJ-100-05 \\
\hline GWM43201AV & Trip Blank & NA & Methyl iodide & 2 & & $\mathrm{u}$ & & UG/L & 10/07/2005 & & HCJ-100-05 \\
\hline GWM43201AV & Trip Blank & NA & Isobutyl alcohol & 17 & & $\mathrm{~J}$ & $\mathrm{~J}$ & UG/L & $10 / 07 / 2005$ & & HCJ-100-05 \\
\hline GWM43201AV & Trip Blank & NA & Methacrylonitrile & 5 & & u & $\mathrm{R}$ & UG/L & 10/07/2005 & & HCJ-100-05 \\
\hline GWM43201AV & Trip Blank & NA & Methylene Chloride & 1 & & $\mathrm{u}$ & & UG/L & $10 / 07 / 2005$ & & HCJ-100-05 \\
\hline GWM43201AV & Trip Blank & NA & Methylmethacrylate & 1 & & $\mathrm{u}$ & UJ & UG/L & $10 / 07 / 2005$ & & HCJ-100-05 \\
\hline GWM43201AV & Trip Blank & NA & Methyl isobutyl ketone & 5 & & $\mathrm{u}$ & & UG/L & $10 / 07 / 2005$ & & HCJ-100-05 \\
\hline GWM43201AV & Trip Blank & NA & Propionitrile & 5 & & $\mathrm{u}$ & $\mathrm{R}$ & UG/L & 10/07/2005 & & HCJ-100-05 \\
\hline GWM43201AV & Trip Blank & NA & Styrene & 1 & & $\mathrm{u}$ & & UG/L & $10 / 07 / 2005$ & & HCJ-100-05 \\
\hline GWM43201AV & Trip Blank & NA & 1,1,1,2-Tetrachloroethane & 1 & & $\mathrm{u}$ & & UG/L & 10/07/2005 & & HCJ-100-05 \\
\hline GWM43201AV & Trip Blank & NA & $1,1,2,2$-Tetrachloroethane & 1 & & $\mathrm{u}$ & & UG/L & $10 / 07 / 2005$ & & HCJ-100-05 \\
\hline GWM43201AV & Trip Blank & NA & Tetrachloroethene & 1 & & $\mathrm{u}$ & & UG/L & $10 / 07 / 2005$ & & HCJ-100-05 \\
\hline GWM43201AV & Trip Blank & NA & Toluene & 1 & & $\mathrm{u}$ & & UG/L & $10 / 07 / 2005$ & & HCJ-100-05 \\
\hline GWM43201AV & Trip Blank & NA & 1,1,1-Trichloroethane & 1 & & u & & UG/L & $10 / 07 / 2005$ & & HCJ-100-05 \\
\hline GWM43201AV & Trip Blank & NA & 1,1,2-Trichloroethane & 1 & & $\mathrm{u}$ & & UG/L & $10 / 07 / 2005$ & & HCJ-100-05 \\
\hline GWM43201AV & Trip Blank & NA & Trichloroethene & 1 & & $\mathrm{u}$ & & UG/L & 10/07/2005 & & HCJ-100-05 \\
\hline GWM43201AV & Trip Blank & NA & Trichlorofluoromethane & 1 & & $\mathrm{u}$ & & UG/L & $10 / 07 / 2005$ & & HCJ-100-05 \\
\hline GWM43201AV & Trip Blank & NA & 1,2,3-Trichloropropane & 1 & & $\mathrm{u}$ & & UG/L & $10 / 07 / 2005$ & & HCJ-100-05 \\
\hline GWM43201AV & Trip Blank & NA & Vinyl Acetate & 2 & & $\mathrm{u}$ & & UG/L & $10 / 07 / 2005$ & & HCJ-100-05 \\
\hline GWM43201AV & Trip Blank & NA & Vinyl Chloride & 1 & & $\mathrm{u}$ & & UG/L & 10/07/2005 & & HCJ-100-05 \\
\hline GWM43201AV & Trip Blank & NA & Xylenes & 3 & & $\mathrm{u}$ & & UG/L & $10 / 07 / 2005$ & & HCJ-100-05 \\
\hline GWM43101AV(1) & Trip Blank & NA & Acetone & 10 & & u & $\mathrm{R}$ & UG/L & $10 / 21 / 2005$ & & DMG-217-05 \\
\hline GWM43101AV(1) & Trip Blank & NA & Acetonitrile & 20 & & $\mathrm{u}$ & $\mathrm{R}$ & UG/L & $10 / 21 / 2005$ & & DMG-217-05 \\
\hline GWM43101AV(1) & Trip Blank & NA & Acrolein & 5 & & $\mathrm{u}$ & $\mathrm{R}$ & UG/L & $10 / 21 / 2005$ & & DMG-217-05 \\
\hline GWM43101AV(1) & Trip Blank & NA & Acrylonitrile & 1 & & $\mathrm{u}$ & & UG/L & $10 / 21 / 2005$ & & DMG-217-05 \\
\hline GWM43101AV(1) & Trip Blank & NA & Benzene & 1 & & $\mathrm{u}$ & & UG/L & $10 / 21 / 2005$ & & DMG-217-05 \\
\hline GWM43101AV(1) & Trip Blank & NA & Bromodichloromethane & 1 & & $\mathrm{u}$ & & UG/L & $10 / 21 / 2005$ & & DMG-217-05 \\
\hline GWM43101AV(1) & Trip Blank & NA & Bromoform & 2 & & $\mathrm{u}$ & & UG/L & 10/21/2005 & & DMG-217-05 \\
\hline GWM43101AV(1) & Trip Blank & NA & Bromomethane & 1 & & $\mathrm{u}$ & & UG/L & $10 / 21 / 2005$ & & DMG-217-05 \\
\hline GWM43101AV(1) & Trip Blank & NA & 2-Butanone & 10 & & $\mathrm{u}$ & $\mathrm{R}$ & UG/L & $10 / 21 / 2005$ & & DMG-217-05 \\
\hline GWM43101AV(1) & Trip Blank & NA & Carbon disulfide & 5 & & $\mathrm{u}$ & & UG/L & $10 / 21 / 2005$ & & DMG-217-05 \\
\hline GWM43101AV(1) & Trip Blank & NA & Carbon tetrachloride & 1 & & $\mathrm{u}$ & & UG/L & $10 / 21 / 2005$ & & DMG-217-05 \\
\hline GWM43101AV(1) & Trip Blank & NA & Chlorobenzene & 1 & & $\mathrm{u}$ & & UG/L & $10 / 21 / 2005$ & & DMG-217-05 \\
\hline GWM43101AV(1) & Trip Blank & NA & Chloroprene & 5 & & u & & UG/L & $10 / 21 / 2005$ & & DMG-217-05 \\
\hline GWM43101AV(1) & Trip Blank & NA & Dibromochloromethane & 1 & & $\mathrm{u}$ & & UG/L & $10 / 21 / 2005$ & & DMG-217-05 \\
\hline GWM43101AV(1) & Trip Blank & NA & Chloroethane & 1 & & $\mathrm{u}$ & & UG/L & $10 / 21 / 2005$ & & DMG-217-05 \\
\hline GWM43101AV(1) & Trip Blank & NA & Chloroform & 0.16 & & $\mathrm{~J}$ & $\mathrm{~J}$ & UG/L & $10 / 21 / 2005$ & & DMG-217-05 \\
\hline GWM43101AV(1) & Trip Blank & NA & Chloromethane & 5 & & $\mathrm{u}$ & & UG/L & $10 / 21 / 2005$ & & DMG-217-05 \\
\hline GWM43101AV(1) & Trip Blank & NA & Allyl chloride & 5 & & $\mathrm{u}$ & & UG/L & $10 / 21 / 2005$ & & DMG-217-05 \\
\hline GWM43101AV(1) & Trip Blank & NA & 1,2-Dibromo-3-chloropropane & 5 & & $\mathrm{u}$ & $\mathrm{R}$ & UG/L & $10 / 21 / 2005$ & & DMG-217-05 \\
\hline GWM43101AV(1) & Trip Blank & NA & 1,2-Dibromoethane & 1 & & $\mathrm{u}$ & & UG/L & $10 / 21 / 2005$ & & DMG-217-05 \\
\hline GWM43101AV(1) & Trip Blank & NA & Dibromomethane & 1 & & $\mathrm{u}$ & & UG/L & $10 / 21 / 2005$ & & DMG-217-05 \\
\hline GWM43101AV(1) & Trip Blank & NA & trans-1,4-Dichloro-2-butene & 5 & & $\mathrm{u}$ & $\mathrm{R}$ & UG/L & $10 / 21 / 2005$ & & DMG-217-05 \\
\hline GWM43101AV(1) & Trip Blank & NA & Dichlorodifluoromethane & 5 & & $\mathrm{u}$ & & UG/L & $10 / 21 / 2005$ & & DMG-217-05 \\
\hline GWM43101AV(1) & Trip Blank & NA & 1,1-Dichloroethane & 1 & & $\mathrm{u}$ & & UG/L & $10 / 21 / 2005$ & & DMG-217-05 \\
\hline GWM43101AV(1) & Trip Blank & NA & 1,2-Dichloroethane & 1 & & $\mathrm{u}$ & & UG/L & $10 / 21 / 2005$ & & DMG-217-05 \\
\hline GWM43101AV(1) & Trip Blank & NA & cis-1,2-Dichloroethene & 1 & & $\mathrm{u}$ & & UG/L & $10 / 21 / 2005$ & & DMG-217-05 \\
\hline GWM43101AV(1) & Trip Blank & NA & trans-1,2-Dichloroethene & 1 & & $\mathrm{u}$ & & UG/L & $10 / 21 / 2005$ & & DMG-217-05 \\
\hline GWM43101AV(1) & Trip Blank & NA & 1,1-Dichloroethene & 1 & & $\mathrm{u}$ & & UG/L & $10 / 21 / 2005$ & & DMG-217-05 \\
\hline GWM43101AV(1) & Trip Blank & NA & 1,2-Dichloropropane & 1 & & u & & UG/L & $10 / 21 / 2005$ & & DMG-217-05 \\
\hline GWM43101AV(1) & Trip Blank & NA & cis-1,3-Dichloropropene & 1 & & $\mathrm{u}$ & & UG/L & $10 / 21 / 2005$ & & DMG-217-05 \\
\hline GWM43101AV(1) & Trip Blank & NA & trans-1,3-Dichloropropene & 1 & & u & & UG/L & $10 / 21 / 2005$ & & DMG-217-05 \\
\hline GWM43101AV(1) & Trip Blank & NA & 1,4-Dioxane & 80 & & $\mathrm{u}$ & $\mathrm{R}$ & UG/L & $10 / 21 / 2005$ & & DMG-217-05 \\
\hline GWM43101AV(1) & Trip Blank & NA & Ethylbenzene & 1 & & $\mathrm{u}$ & & UG/L & $10 / 21 / 2005$ & & DMG-217-05 \\
\hline GWM43101AV(1) & Trip Blank & NA & 2-Hexanone & 5 & & $\mathrm{u}$ & & UG/L & $10 / 21 / 2005$ & & DMG-217-05 \\
\hline GWM43101AV(1) & Trip Blank & NA & Methyl iodide & 2 & & $\mathrm{u}$ & & UG/L & $10 / 21 / 2005$ & & DMG-217-05 \\
\hline GWM43101AV(1) & Trip Blank & NA & Isobutyl alcohol & 80 & & $\mathrm{u}$ & $\mathrm{R}$ & UG/L & $10 / 21 / 2005$ & & DMG-217-05 \\
\hline GWM43101AV(1) & Trip Blank & NA & Methacrylonitrile & 5 & & $\mathrm{u}$ & $\mathrm{R}$ & UG/L & 10/21/2005 & & DMG-217-05 \\
\hline GWM43101AV(1) & Trip Blank & NA & Methylene Chloride & 1 & & $\mathrm{u}$ & & UG/L & $10 / 21 / 2005$ & & DMG-217-05 \\
\hline GWM43101AV(1) & Trip Blank & NA & Methylmethacrylate & 1 & & u & & UG/L & $10 / 21 / 2005$ & & DMG-217-05 \\
\hline GWM43101AV(1) & Trip Blank & NA & Methyl isobutyl ketone & 5 & & $\mathrm{u}$ & & UG/L & $10 / 21 / 2005$ & & DMG-217-05 \\
\hline GWM43101AV(1) & Trip Blank & NA & Propionitrile & 5 & & $\mathrm{u}$ & $\mathrm{R}$ & UG/L & $10 / 21 / 2005$ & & DMG-217-05 \\
\hline GWM43101AV(1) & Trip Blank & NA & Styrene & 1 & & $\mathrm{u}$ & & UG/L & $10 / 21 / 2005$ & & DMG-217-05 \\
\hline GWM43101AV(1) & Trip Blank & NA & 1,1,1,2-Tetrachloroethane & 1 & & $u$ & & UG/L & $10 / 21 / 2005$ & & DMG-217-05 \\
\hline GWM43101AV(1) & Trip Blank & NA & 1,1,2,2-Tetrachloroethane & 1 & & $\mathrm{u}$ & & UG/L & $10 / 21 / 2005$ & & DMG-217-05 \\
\hline GWM43101AV(1) & Trip Blank & NA & Tetrachloroethene & 1 & & $\mathrm{u}$ & & UG/L & $10 / 21 / 2005$ & & DMG-217-05 \\
\hline GWM43101AV(1) & Trip Blank & NA & Toluene & 1 & & $\mathrm{u}$ & & UG/L & $10 / 21 / 2005$ & & DMG-217-05 \\
\hline GWM43101AV(1) & Trip Blank & NA & 1,1,1-Trichloroethane & 1 & & $u$ & & UG/L & $10 / 21 / 2005$ & & DMG-217-05 \\
\hline GWM43101AV(1) & Trip Blank & NA & $1,1,2$-Trichloroethane & 1 & & $\mathrm{u}$ & & UG/L & $10 / 21 / 2005$ & & DMG-217-05 \\
\hline
\end{tabular}


Table A-4. (continued).

\begin{tabular}{|c|c|c|c|c|c|c|c|c|c|c|c|}
\hline $\begin{array}{l}\text { Field Sample } \\
\text { Number }\end{array}$ & Location & Depth & Compound & $\begin{array}{c}\text { Sample } \\
\text { Result }\end{array}$ & $\begin{array}{c}\text { Sample } \\
\text { Error }\end{array}$ & $\begin{array}{c}\text { Result } \\
\text { Qualifier }\end{array}$ & $\begin{array}{c}\text { Validation } \\
\text { Flag }\end{array}$ & $\begin{array}{c}\text { Sample } \\
\text { Units }\end{array}$ & $\begin{array}{c}\text { Date Sample } \\
\text { Collected }\end{array}$ & MDA & $\begin{array}{c}\text { L\&V Report } \\
\text { Number }\end{array}$ \\
\hline GWM43101AV(1) & Trip Blank & NA & Trichloroethene & 1 & & $\mathrm{U}$ & & UG/L & $10 / 21 / 2005$ & & DMG-217-05 \\
\hline GWM43101AV(1) & Trip Blank & NA & Trichlorofluoromethane & 1 & & $\mathrm{U}$ & & UG/L & $10 / 21 / 2005$ & & DMG-217-05 \\
\hline GWM43101AV(1) & Trip Blank & NA & 1,2,3-Trichloropropane & 1 & & $\mathrm{u}$ & & UG/L & $10 / 21 / 2005$ & & DMG-217-05 \\
\hline GWM43101AV(1) & Trip Blank & NA & Vinyl Acetate & 2 & & $\mathrm{u}$ & & UG/L & $10 / 21 / 2005$ & & DMG-217-05 \\
\hline GWM43101AV(1) & Trip Blank & NA & Vinyl Chloride & 1 & & $\mathrm{u}$ & & UG/L & $10 / 21 / 2005$ & & DMG-217-05 \\
\hline GWM43101AV(1) & Trip Blank & NA & Xylenes & 3 & & 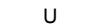 & & UG/L & $10 / 21 / 2005$ & & DMG-217-05 \\
\hline GWM43001AV & Trip Blank & NA & Acetone & 10 & & u & $\mathrm{R}$ & UG/L & 09/27/2005 & & JGJ-071-05 \\
\hline GWM43001AV & Trip Blank & NA & Acetonitrile & 20 & & $\mathrm{U}$ & & UG/L & 09/27/2005 & & JGJ-071-05 \\
\hline GWM43001AV & Trip Blank & NA & Acrolein & 5 & & u & $\mathrm{R}$ & UG/L & $09 / 27 / 2005$ & & JGJ-071-05 \\
\hline GWM43001AV & Trip Blank & NA & Acrylonitrile & 1 & & $\mathrm{U}$ & $\mathrm{R}$ & UG/L & 09/27/2005 & & JGJ-071-05 \\
\hline GWM43001AV & Trip Blank & NA & Benzene & 1 & & $\mathrm{U}$ & & UG/L & $09 / 27 / 2005$ & & JGJ-071-05 \\
\hline GWM43001AV & Trip Blank & NA & Bromodichloromethane & 1 & & U & & UG/L & $09 / 27 / 2005$ & & JGJ-071-05 \\
\hline GWM43001AV & Trip Blank & NA & Bromoform & 2 & & u & & UG/L & 09/27/2005 & & JGJ-071-05 \\
\hline GWM43001AV & Trip Blank & NA & Bromomethane & 1 & & $\mathrm{u}$ & & UG/L & 09/27/2005 & & JGJ-071-05 \\
\hline GWM43001AV & Trip Blank & NA & 2-Butanone & 10 & & u & $\mathrm{R}$ & UG/L & 09/27/2005 & & JGJ-071-05 \\
\hline GWM43001AV & Trip Blank & NA & Carbon disulfide & 5 & & $\mathrm{u}$ & & UG/L & 09/27/2005 & & JGJ-071-05 \\
\hline GWM43001AV & Trip Blank & NA & Carbon tetrachloride & 1 & & u & & UG/L & $09 / 27 / 2005$ & & JGJ-071-05 \\
\hline GWM43001AV & Trip Blank & NA & Chlorobenzene & 1 & & $\mathrm{u}$ & & UG/L & $09 / 27 / 2005$ & & JGJ-071-05 \\
\hline GWM43001AV & Trip Blank & NA & Chloroprene & 5 & & u & & UG/L & 09/27/2005 & & JGJ-071-05 \\
\hline GWM43001AV & Trip Blank & NA & Dibromochloromethane & 1 & & $\mathrm{u}$ & & UG/L & $09 / 27 / 2005$ & & JGJ-071-05 \\
\hline GWM43001AV & Trip Blank & NA & Chloroethane & 1 & & u & & UG/L & 09/27/2005 & & JGJ-071-05 \\
\hline GWM43001AV & Trip Blank & NA & Chloroform & 1 & & $\mathrm{u}$ & & UG/L & $09 / 27 / 2005$ & & JGJ-071-05 \\
\hline GWM43001AV & Trip Blank & NA & Chloromethane & 5 & & u & & UG/L & $09 / 27 / 2005$ & & JGJ-071-05 \\
\hline GWM43001AV & Trip Blank & NA & Allyl chloride & 5 & & $\mathrm{u}$ & & UG/L & $09 / 27 / 2005$ & & JGJ-071-05 \\
\hline GWM43001AV & Trip Blank & NA & 1,2-Dibromo-3-chloropropane & 5 & & u & $\mathrm{R}$ & UG/L & 09/27/2005 & & JGJ-071-05 \\
\hline GWM43001AV & Trip Blank & NA & 1,2-Dibromoethane & 1 & & $\mathrm{u}$ & & UG/L & 09/27/2005 & & JGJ-071-05 \\
\hline GWM43001AV & Trip Blank & NA & Dibromomethane & 1 & & u & & UG/L & 09/27/2005 & & JGJ-071-05 \\
\hline GWM43001AV & Trip Blank & NA & trans-1,4-Dichloro-2-butene & 5 & & $\mathrm{u}$ & & UG/L & 09/27/2005 & & JGJ-071-05 \\
\hline GWM43001AV & Trip Blank & NA & Dichlorodifluoromethane & 5 & & u & & UG/L & $09 / 27 / 2005$ & & JGJ-071-05 \\
\hline GWM43001AV & Trip Blank & NA & 1,1-Dichloroethane & 1 & & $\mathrm{U}$ & & UG/L & 09/27/2005 & & JGJ-071-05 \\
\hline GWM43001AV & Trip Blank & NA & 1,2-Dichloroethane & 1 & & u & & UG/L & 09/27/2005 & & JGJ-071-05 \\
\hline GWM43001AV & Trip Blank & NA & cis-1,2-Dichloroethene & 1 & & $\mathrm{u}$ & & UG/L & $09 / 27 / 2005$ & & JGJ-071-05 \\
\hline GWM43001AV & Trip Blank & NA & trans-1,2-Dichloroethene & 1 & & u & & UG/L & 09/27/2005 & & JGJ-071-05 \\
\hline GWM43001AV & Trip Blank & NA & 1,1-Dichloroethene & 1 & & $\mathrm{u}$ & & UG/L & 09/27/2005 & & JGJ-071-05 \\
\hline GWM43001AV & Trip Blank & NA & 1,2-Dichloropropane & 1 & & u & & UG/L & $09 / 27 / 2005$ & & JGJ-071-05 \\
\hline GWM43001AV & Trip Blank & NA & cis-1,3-Dichloropropene & 1 & & $\mathrm{u}$ & & UG/L & 09/27/2005 & & JGJ-071-05 \\
\hline GWM43001AV & Trip Blank & NA & trans-1,3-Dichloropropene & 1 & & u & & UG/L & 09/27/2005 & & JGJ-071-05 \\
\hline GWM43001AV & Trip Blank & NA & 1,4-Dioxane & 80 & & $\mathrm{u}$ & $\mathrm{R}$ & UG/L & 09/27/2005 & & JGJ-071-05 \\
\hline GWM43001AV & Trip Blank & NA & Ethylbenzene & 1 & & u & & UG/L & 09/27/2005 & & JGJ-071-05 \\
\hline GWM43001AV & Trip Blank & NA & 2-Hexanone & 5 & & $\mathrm{u}$ & & UG/L & $09 / 27 / 2005$ & & JGJ-071-05 \\
\hline GWM43001AV & Trip Blank & NA & Methyl iodide & 2 & & u & & UG/L & 09/27/2005 & & JGJ-071-05 \\
\hline GWM43001AV & Trip Blank & NA & Isobutyl alcohol & 80 & & $\mathrm{u}$ & $\mathrm{R}$ & UG/L & 09/27/2005 & & JGJ-071-05 \\
\hline GWM43001AV & Trip Blank & NA & Methacrylonitrile & 5 & & u & $\mathrm{R}$ & UG/L & 09/27/2005 & & JGJ-071-05 \\
\hline GWM43001AV & Trip Blank & NA & Methylene Chloride & 1 & & $\mathrm{u}$ & & UG/L & 09/27/2005 & & JGJ-071-05 \\
\hline GWM43001AV & Trip Blank & NA & Methylmethacrylate & 1 & & u & & UG/L & 09/27/2005 & & JGJ-071-05 \\
\hline GWM43001AV & Trip Blank & NA & Methyl isobutyl ketone & 5 & & u & & UG/L & 09/27/2005 & & JGJ-071-05 \\
\hline GWM43001AV & Trip Blank & NA & Propionitrile & 5 & & u & $\mathrm{R}$ & UG/L & 09/27/2005 & & JGJ-071-05 \\
\hline GWM43001AV & Trip Blank & NA & Styrene & 1 & & $\mathrm{u}$ & & UG/L & $09 / 27 / 2005$ & & JGJ-071-05 \\
\hline GWM43001AV & Trip Blank & NA & 1,1,1,2-Tetrachloroethane & 1 & & u & & UG/L & 09/27/2005 & & JGJ-071-05 \\
\hline GWM43001AV & Trip Blank & NA & 1,1,2,2-Tetrachloroethane & 1 & & u & & UG/L & 09/27/2005 & & JGJ-071-05 \\
\hline GWM43001AV & Trip Blank & NA & Tetrachloroethene & 1 & & u & & UG/L & 09/27/2005 & & JGJ-071-05 \\
\hline GWM43001AV & Trip Blank & NA & Toluene & 1 & & $\mathrm{u}$ & & UG/L & 09/27/2005 & & JGJ-071-05 \\
\hline GWM43001AV & Trip Blank & NA & 1,1,1-Trichloroethane & 1 & & u & & UG/L & 09/27/2005 & & JGJ-071-05 \\
\hline GWM43001AV & Trip Blank & NA & $1,1,2$-Trichloroethane & 1 & & u & & UG/L & 09/27/2005 & & JGJ-071-05 \\
\hline GWM43001AV & Trip Blank & NA & Trichloroethene & 1 & & u & & UG/L & $09 / 27 / 2005$ & & JGJ-071-05 \\
\hline GWM43001AV & Trip Blank & NA & Trichlorofluoromethane & 1 & & u & & UG/L & 09/27/2005 & & JGJ-071-05 \\
\hline GWM43001AV & Trip Blank & NA & 1,2,3-Trichloropropane & 1 & & u & & UG/L & 09/27/2005 & & JGJ-071-05 \\
\hline GWM43001AV & Trip Blank & NA & Vinyl Acetate & 2 & & u & & UG/L & 09/27/2005 & & JGJ-071-05 \\
\hline GWM43001AV & Trip Blank & NA & Vinyl Chloride & 1 & & U & & UG/L & 09/27/2005 & & JGJ-071-05 \\
\hline GWM43001AV & Trip Blank & NA & Xylenes & 3 & & $u$ & & UG/L & 09/27/2005 & & JGJ-071-05 \\
\hline
\end{tabular}


Table A-5. Waste Area Group 10 Westbay monitoring data for 2006.

\begin{tabular}{|c|c|c|c|c|c|c|c|c|c|c|c|}
\hline $\begin{array}{l}\text { Field Sample } \\
\text { Number }\end{array}$ & Location & Depth & Compound & $\begin{array}{c}\text { Sample } \\
\text { Result }\end{array}$ & $\begin{array}{l}\text { Sample } \\
\text { Error }\end{array}$ & $\begin{array}{c}\text { Result } \\
\text { Qualifier }\end{array}$ & $\begin{array}{l}\text { Validation } \\
\text { Flag }\end{array}$ & $\begin{array}{c}\text { Sample } \\
\text { Units }\end{array}$ & $\begin{array}{c}\text { Date Sample } \\
\text { Collected }\end{array}$ & MDA & $\begin{array}{l}\text { L\&V Report } \\
\text { Number }\end{array}$ \\
\hline GWM468012X & FIELD BLANK & NA & Aluminum & 7.9 & & $U$ & & UG/L & $05 / 31 / 2006$ & & DNT-333-06 \\
\hline GWM468012X & FIELD BLANK & NA & Antimony & 0.5 & & u & & UG/L & $05 / 31 / 2006$ & & DNT-333-06 \\
\hline GWM468012X & FIELD BLANK & NA & Arsenic & 2 & & $u$ & & UG/L & 05/31/2006 & & DNT-333-06 \\
\hline GWM468012X & FIELD BLANK & NA & Barium & 0.25 & & u & & UG/L & $05 / 31 / 2006$ & & DNT-333-06 \\
\hline GWM468012X & FIELD BLANK & NA & Beryllium & 0.088 & & u & & UG/L & $05 / 31 / 2006$ & & DNT-333-06 \\
\hline GWM468012X & FIELD BLANK & NA & Cadmium & 0.057 & & u & & UG/L & $05 / 31 / 2006$ & & DNT-333-06 \\
\hline GWM468012X & FIELD BLANK & NA & Calcium & 21 & & $u$ & & UG/L & $05 / 31 / 2006$ & & DNT-333-06 \\
\hline GWM468012X & FIELD BLANK & NA & Chromium & 2.8 & & u & & UG/L & $05 / 31 / 2006$ & & DNT-333-06 \\
\hline GWM468012X & FIELD BLANK & NA & Cobalt & 0.31 & & $\mathrm{U}$ & & UG/L & $05 / 31 / 2006$ & & DNT-333-06 \\
\hline GWM468012X & FIELD BLANK & NA & Copper & 9 & & & & UG/L & $05 / 31 / 2006$ & & DNT-333-06 \\
\hline GWM468012X & FIELD BLANK & NA & Iron & 9.4 & & U & & UG/L & $05 / 31 / 2006$ & & DNT-333-06 \\
\hline GWM468012X & FIELD BLANK & NA & Lead & 0.84 & & B & & UG/L & $05 / 31 / 2006$ & & DNT-333-06 \\
\hline GWM468012X & FIELD BLANK & NA & Magnesium & 6.4 & & $u$ & & UG/L & $05 / 31 / 2006$ & & DNT-333-06 \\
\hline GWM468012X & FIELD BLANK & NA & Manganese & 0.34 & & u & & UG/L & $05 / 31 / 2006$ & & DNT-333-06 \\
\hline GWM468012X & FIELD BLANK & NA & Nickel & 0.52 & & $u$ & & UG/L & $05 / 31 / 2006$ & & DNT-333-06 \\
\hline GWM468012X & FIELD BLANK & NA & Potassium & 10 & & $\mathrm{U}$ & & UG/L & $05 / 31 / 2006$ & & DNT-333-06 \\
\hline GWM468012X & FIELD BLANK & NA & Selenium & 1 & & U & & UG/L & $05 / 31 / 2006$ & & DNT-333-06 \\
\hline GWM468012X & FIELD BLANK & NA & Silver & 0.2 & & u & & UG/L & $05 / 31 / 2006$ & & DNT-333-06 \\
\hline GWM468012X & FIELD BLANK & NA & Sodium & 11 & & $\mathrm{u}$ & & UG/L & $05 / 31 / 2006$ & & DNT-333-06 \\
\hline GWM468012X & FIELD BLANK & NA & Strontium & 0.53 & & $u$ & & UG/L & $05 / 31 / 2006$ & & DNT-333-06 \\
\hline GWM468012X & FIELD BLANK & NA & Thallium & 0.95 & & B & $\mathrm{U}$ & UG/L & $05 / 31 / 2006$ & & DNT-333-06 \\
\hline GWM468012X & FIELD BLANK & NA & Uranium & 0.21 & & $u$ & & UG/L & $05 / 31 / 2006$ & & DNT-333-06 \\
\hline GWM468012X & FIELD BLANK & NA & Vanadium & 1.6 & & u & & UG/L & $05 / 31 / 2006$ & & DNT-333-06 \\
\hline GWM468012X & FIELD BLANK & NA & Zinc & 5.3 & & B & $u$ & UG/L & $05 / 31 / 2006$ & & DNT-333-06 \\
\hline GWM468012X & FIELD BLANK & NA & Mercury & 0.093 & & $u$ & & UG/L & $05 / 31 / 2006$ & & DNT-333-06 \\
\hline GWM46801A1 & FIELD BLANK & NA & Total Alkalinity & 5 & & $u$ & & MG/L & $05 / 31 / 2006$ & & DNT-278-06 \\
\hline GWM46801AN & FIELD BLANK & NA & Chloride & 0.2 & & JU & & MG/L & $05 / 31 / 2006$ & & DNT-278-06 \\
\hline GWM46801AN & FIELD BLANK & NA & Fluoride & 0.1 & & $u$ & & MG/L & $05 / 31 / 2006$ & & DNT-278-06 \\
\hline GWM46801AN & FIELD BLANK & NA & Sulfate & 0.5 & & u & & $\mathrm{MG} / \mathrm{L}$ & $05 / 31 / 2006$ & & DNT-278-06 \\
\hline GWM46801N2 & FIELD BLANK & NA & Nitrate/Nitrite as $\mathrm{N}$ & 50 & & $u$ & & UG/L & $05 / 31 / 2006$ & & DNT-278-06 \\
\hline GWM46801VL & FIELD BLANK & NA & Acetone & 2 & & u & $\mathrm{R}$ & UG/L & $05 / 31 / 2006$ & & JGJ-084-06 \\
\hline GWM46801VL & FIELD BLANK & NA & Benzene & 1 & & $\mathrm{u}$ & & UG/L & $05 / 31 / 2006$ & & JGJ-084-06 \\
\hline GWM46801VL & FIELD BLANK & NA & Bromodichloromethane & 1 & & $\mathrm{u}$ & & UG/L & $05 / 31 / 2006$ & & JGJ-084-06 \\
\hline GWM46801VL & FIELD BLANK & NA & Bromoform & 1 & & $\mathrm{u}$ & & UG/L & $05 / 31 / 2006$ & & JGJ-084-06 \\
\hline GWM46801VL & FIELD BLANK & NA & Bromomethane & 2 & & $\mathrm{u}$ & & UG/L & $05 / 31 / 2006$ & & JGJ-084-06 \\
\hline GWM46801VL & FIELD BLANK & NA & 2-Butanone & 5 & & $\mathrm{u}$ & $\mathrm{R}$ & UG/L & $05 / 31 / 2006$ & & JGJ-084-06 \\
\hline GWM46801VL & FIELD BLANK & NA & Carbon disulfide & 1 & & $\mathrm{u}$ & & UG/L & $05 / 31 / 2006$ & & JGJ-084-06 \\
\hline GWM46801VL & FIELD BLANK & NA & Carbon tetrachloride & 1 & & u & & UG/L & $05 / 31 / 2006$ & & JGJ-084-06 \\
\hline GWM46801VL & FIELD BLANK & NA & Chlorobenzene & 1 & & $u$ & & UG/L & $05 / 31 / 2006$ & & JGJ-084-06 \\
\hline GWM46801VL & FIELD BLANK & NA & Dibromochloromethane & 1 & & u & & UG/L & $05 / 31 / 2006$ & & JGJ-084-06 \\
\hline GWM46801VL & FIELD BLANK & NA & 1,2-Dibromo-3-chloropropane & 1 & & $\mathrm{u}$ & $\mathrm{R}$ & UG/L & $05 / 31 / 2006$ & & JGJ-084-06 \\
\hline GWM46801VL & FIELD BLANK & NA & Chloroethane & 2 & & u & & UG/L & $05 / 31 / 2006$ & & JGJ-084-06 \\
\hline GWM46801VL & FIELD BLANK & NA & Chloroform & 1 & & $\mathrm{u}$ & & UG/L & $05 / 31 / 2006$ & & JGJ-084-06 \\
\hline GWM46801VL & FIELD BLANK & NA & Chloromethane & 2 & & u & & UG/L & $05 / 31 / 2006$ & & JGJ-084-06 \\
\hline GWM46801VL & FIELD BLANK & NA & Cyclohexane & 1 & & $\mathrm{U}$ & & UG/L & $05 / 31 / 2006$ & & JGJ-084-06 \\
\hline GWM46801VL & FIELD BLANK & NA & 1.2-Dibromoethane & 1 & & u & & UG/L & $05 / 31 / 2006$ & & JGJ-084-06 \\
\hline GWM46801VL & FIELD BLANK & NA & 1,2-Dichlorobenzene & 1 & & $\mathrm{u}$ & & UG/L & $05 / 31 / 2006$ & & JGJ-084-06 \\
\hline GWM46801VL & FIELD BLANK & NA & 1,3-Dichlorobenzene & 1 & & u & & UG/L & $05 / 31 / 2006$ & & JGJ-084-06 \\
\hline GWM46801VL & FIELD BLANK & NA & 1,4-Dichlorobenzene & 1 & & $\mathrm{u}$ & & UG/L & $05 / 31 / 2006$ & & JGJ-084-06 \\
\hline GWM46801VL & FIELD BLANK & NA & Dichlorodifluoromethane & 2 & & $\mathrm{u}$ & & UG/L & $05 / 31 / 2006$ & & JGJ-084-06 \\
\hline GWM46801VL & FIELD BLANK & NA & 1,1-Dichloroethane & 1 & & $\mathrm{u}$ & & UG/L & $05 / 31 / 2006$ & & JGJ-084-06 \\
\hline GWM46801VL & FIELD BLANK & NA & 1,2-Dichloroethane & 1 & & $\mathrm{u}$ & & UG/L & $05 / 31 / 2006$ & & JGJ-084-06 \\
\hline GWM46801VL & FIELD BLANK & NA & 1,1-Dichloroethene & 1 & & u & & UG/L & $05 / 31 / 2006$ & & JGJ-084-06 \\
\hline GWM46801VL & FIELD BLANK & NA & cis-1,2-Dichloroethene & 1 & & $u$ & & UG/L & $05 / 31 / 2006$ & & JGJ-084-06 \\
\hline GWM46801VL & FIELD BLANK & NA & trans-1,2-Dichloroethene & 1 & & u & & UG/L & $05 / 31 / 2006$ & & JGJ-084-06 \\
\hline GWM46801VL & FIELD BLANK & NA & 1,2-Dichloropropane & 1 & & $u$ & & UG/L & $05 / 31 / 2006$ & & JGJ-084-06 \\
\hline GWM46801VL & FIELD BLANK & NA & cis-1,3-Dichloropropene & 1 & & u & & UG/L & $05 / 31 / 2006$ & & JGJ-084-06 \\
\hline GWM46801VL & FIELD BLANK & NA & trans-1,3-Dichloropropene & 1 & & $u$ & & UG/L & $05 / 31 / 2006$ & & JGJ-084-06 \\
\hline GWM46801VL & FIELD BLANK & NA & Ethylbenzene & 1 & & u & & UG/L & $05 / 31 / 2006$ & & JGJ-084-06 \\
\hline GWM46801VL & FIELD BLANK & NA & Trichlorofluoromethane & 1 & & $\mathrm{u}$ & & UG/L & $05 / 31 / 2006$ & & JGJ-084-06 \\
\hline GWM46801VL & FIELD BLANK & NA & 2-Hexanone & 5 & & u & $\mathrm{R}$ & UG/L & $05 / 31 / 2006$ & & JGJ-084-06 \\
\hline GWM46801VL & FIELD BLANK & NA & Isopropylbenzene & 1 & & u & & UG/L & $05 / 31 / 2006$ & & JGJ-084-06 \\
\hline GWM46801VL & FIELD BLANK & NA & Methyl acetate & 5 & & $u$ & $\mathrm{R}$ & UG/L & $05 / 31 / 2006$ & & JGJ-084-06 \\
\hline GWM46801VL & FIELD BLANK & NA & Methyl cyclohexane & 4 & & u & & UG/L & $05 / 31 / 2006$ & & JGJ-084-06 \\
\hline GWM46801VL & FIELD BLANK & NA & Methylene Chloride & 1 & & $u$ & & UG/L & $05 / 31 / 2006$ & & JGJ-084-06 \\
\hline GWM46801VL & FIELD BLANK & NA & 4-Methyl-2-pentanone & 5 & & u & & UG/L & $05 / 31 / 2006$ & & JGJ-084-06 \\
\hline GWM46801VL & FIELD BLANK & NA & Styrene & 1 & & $u$ & & UG/L & $05 / 31 / 2006$ & & JGJ-084-06 \\
\hline GWM46801VL & FIELD BLANK & NA & 1,1,2,2-Tetrachloroethane & 1 & & u & & UG/L & $05 / 31 / 2006$ & & JGJ-084-06 \\
\hline GWM46801VL & FIELD BLANK & NA & Tetrachloroethene & 1 & & $\mathrm{u}$ & & UG/L & $05 / 31 / 2006$ & & JGJ-084-06 \\
\hline GWM46801VL & FIELD BLANK & NA & Toluene & 1 & & u & & UG/L & $05 / 31 / 2006$ & & JGJ-084-06 \\
\hline GWM46801VL & FIELD BLANK & NA & 1,2,4-Trichlorobenzene & 1 & & $\mathrm{u}$ & & UG/L & $05 / 31 / 2006$ & & JGJ-084-06 \\
\hline GWM46801VL & FIELD BLANK & NA & 1,1,1-Trichloroethane & 1 & & $\mathrm{u}$ & & UG/L & $05 / 31 / 2006$ & & JGJ-084-06 \\
\hline GWM46801VL & FIELD BLANK & NA & $1,1,2$-Trichloroethane & 1 & & $\mathrm{u}$ & & UG/L & $05 / 31 / 2006$ & & JGJ-084-06 \\
\hline GWM46801VL & FIELD BLANK & NA & Trichloroethene & 1 & & $\mathrm{u}$ & & UG/L & $05 / 31 / 2006$ & & JGJ-084-06 \\
\hline GWM46801VL & FIELD BLANK & NA & 1,1,2-Trichloro-1,2,2-trifluoroethane & 1 & & u & & UG/L & $05 / 31 / 2006$ & & JGJ-084-06 \\
\hline GWM46801VL & FIELD BLANK & NA & Vinyl Chloride & 2 & & $\mathrm{u}$ & & UG/L & $05 / 31 / 2006$ & & JGJ-084-06 \\
\hline GWM46801VL & FIELD BLANK & NA & Xylenes & 3 & & $u$ & & UG/L & $05 / 31 / 2006$ & & JGJ-084-06 \\
\hline GWM46801VL & FIELD BLANK & NA & Methyl t-butyl ether & 2 & & $\mathrm{u}$ & & UG/L & $05 / 31 / 2006$ & & JGJ-084-06 \\
\hline GWM46801CW & FIELD BLANK & NA & Carbon-14 & $-2.35 \mathrm{E}+00$ & $1.52 \mathrm{E}+00$ & & $\mathrm{u}$ & $\mathrm{PCl} / \mathrm{L}$ & $05 / 31 / 2006$ & $5.11 \mathrm{E}+00$ & SOS-TL175-06 \\
\hline GWM46801R8 & FIELD BLANK & NA & Tritium & $-2.54 \mathrm{E}+01$ & $1.13 E+02$ & & U & $\mathrm{PCl} / \mathrm{L}$ & $05 / 31 / 2006$ & $3.86 \mathrm{E}+02$ & SOS-TL176-06 \\
\hline GWM46801RH & FIELD BLANK & NA & Gross Alpha & 5.31E-01 & $3.52 \mathrm{E}-01$ & & UJ & $\mathrm{PCl} / \mathrm{L}$ & $05 / 31 / 2006$ & $1.36 \mathrm{E}+00$ & SOS-TL177-06 \\
\hline GWM46801RH & FIELD BLANK & NA & Gross Beta & $4.58 \mathrm{E}-01$ & 6.49E-01 & & U & $\mathrm{PCl} / \mathrm{L}$ & $05 / 31 / 2006$ & $2.86 \mathrm{E}+00$ & SOS-TL177-06 \\
\hline GWM46801RH & FIELD BLANK & NA & Strontium-90 & 4.83E-02 & 7.15E-02 & & u & $\mathrm{PCl} / \mathrm{L}$ & $05 / 31 / 2006$ & $3.21 \mathrm{E}-01$ & SOS-TL177-06 \\
\hline GWM46801RH & FIELD BLANK & NA & Technetium-99 & $3.40 \mathrm{E}+00$ & $1.72 \mathrm{E}+00$ & & U & $\mathrm{PCl} / \mathrm{L}$ & $05 / 31 / 2006$ & $5.61 \mathrm{E}+00$ & SOS-TL177-06 \\
\hline GWM46801UX & FIELD BLANK & NA & lodine-129 & $1.74 \mathrm{E}-02$ & $2.00 \mathrm{E}-02$ & & U & $\mathrm{PCl} / \mathrm{L}$ & $05 / 31 / 2006$ & $8.46 \mathrm{E}-02$ & SOS-TL174-06 \\
\hline
\end{tabular}


Table A-5. (continued).

\begin{tabular}{|c|c|c|c|c|c|c|c|c|c|c|c|}
\hline $\begin{array}{l}\text { Field Sample } \\
\text { Number }\end{array}$ & Location & Depth & Compound & $\begin{array}{c}\text { Sample } \\
\text { Result }\end{array}$ & $\begin{array}{l}\text { Sample } \\
\text { Error }\end{array}$ & $\begin{array}{c}\text { Result } \\
\text { Qualifier }\end{array}$ & $\begin{array}{l}\text { Validation } \\
\text { Flag }\end{array}$ & $\begin{array}{l}\text { Sample } \\
\text { Units }\end{array}$ & $\begin{array}{c}\text { Date Sample } \\
\text { Collected }\end{array}$ & MDA & $\begin{array}{l}\text { L\&V Report } \\
\text { Number }\end{array}$ \\
\hline GWM470012X & MIDDLE-2050A & 474 & Aluminum & 7.9 & & $U$ & & UG/L & $05 / 25 / 2006$ & & DNT-284-06 \\
\hline GWM470012X & MIDDLE-2050A & 474 & Antimony & 0.5 & & $u$ & & UG/L & $05 / 25 / 2006$ & & DNT-284-06 \\
\hline GWM470012X & MIDDLE-2050A & 474 & Arsenic & 2 & & U & & UG/L & $05 / 25 / 2006$ & & DNT-284-06 \\
\hline GWM470012X & MIDDLE-2050A & 474 & Barium & 83.3 & & & & UG/L & $05 / 25 / 2006$ & & DNT-284-06 \\
\hline GWM470012X & MIDDLE-2050A & 474 & Beryllium & 0.088 & & $u$ & & UG/L & $05 / 25 / 2006$ & & DNT-284-06 \\
\hline GWM470012X & MIDDLE-2050A & 474 & Cadmium & 0.057 & & u & & UG/L & 05/25/2006 & & DNT-284-06 \\
\hline GWM470012X & MIDDLE-2050A & 474 & Calcium & 48400 & & $\mathrm{E}$ & $\mathrm{J}$ & UG/L & $05 / 25 / 2006$ & & DNT-284-06 \\
\hline GWM470012X & MIDDLE-2050A & 474 & Chromium & 9 & & & & UG/L & $05 / 25 / 2006$ & & DNT-284-06 \\
\hline GWM470012X & MIDDLE-2050A & 474 & Cobalt & 0.31 & & $u$ & & UG/L & $05 / 25 / 2006$ & & DNT-284-06 \\
\hline GWM470012X & MIDDLE-2050A & 474 & Copper & 0.48 & & B & & UG/L & 05/25/2006 & & DNT-284-06 \\
\hline GWM470012X & MIDDLE-2050A & 474 & Iron & 9.4 & & U & & UG/L & $05 / 25 / 2006$ & & DNT-284-06 \\
\hline GWM470012X & MIDDLE-2050A & 474 & Lead & 0.49 & & U & & UG/L & $05 / 25 / 2006$ & & DNT-284-06 \\
\hline GWM470012X & MIDDLE-2050A & 474 & Magnesium & 15200 & & $\mathrm{E}$ & $\mathrm{J}$ & UG/L & $05 / 25 / 2006$ & & DNT-284-06 \\
\hline GWM470012X & MIDDLE-2050A & 474 & Manganese & 0.42 & & B & $\mathrm{U}$ & UG/L & $05 / 25 / 2006$ & & DNT-284-06 \\
\hline GWM470012X & MIDDLE-2050A & 474 & Nickel & 1.4 & & B & & UG/L & $05 / 25 / 2006$ & & DNT-284-06 \\
\hline GWM470012X & MIDDLE-2050A & 474 & Potassium & 2150 & & & & UG/L & $05 / 25 / 2006$ & & DNT-284-06 \\
\hline GWM470012X & MIDDLE-2050A & 474 & Selenium & 1 & & $u$ & & UG/L & $05 / 25 / 2006$ & & DNT-284-06 \\
\hline GWM470012X & MIDDLE-2050A & 474 & Silver & 0.2 & & u & & UG/L & $05 / 25 / 2006$ & & DNT-284-06 \\
\hline GWM470012X & MIDDLE-2050A & 474 & Sodium & 8060 & & E & $\mathrm{J}$ & UG/L & $05 / 25 / 2006$ & & DNT-284-06 \\
\hline GWM470012X & MIDDLE-2050A & 474 & Strontium & 268 & & $\mathrm{E}$ & $\mathrm{J}$ & UG/L & $05 / 25 / 2006$ & & DNT-284-06 \\
\hline GWM470012X & MIDDLE-2050A & 474 & Thallium & 1.1 & & B & $\mathrm{U}$ & UG/L & $05 / 25 / 2006$ & & DNT-284-06 \\
\hline GWM470012X & MIDDLE-2050A & 474 & Uranium & 2.1 & & & & UG/L & $05 / 25 / 2006$ & & DNT-284-06 \\
\hline GWM470012X & MIDDLE-2050A & 474 & Vanadium & 4.5 & & B & & UG/L & $05 / 25 / 2006$ & & DNT-284-06 \\
\hline GWM470012X & MIDDLE-2050A & 474 & Zinc & 12.3 & & & & UG/L & $05 / 25 / 2006$ & & DNT-284-06 \\
\hline GWM470012X & MIDDLE-2050A & 474 & Mercury & 0.093 & & u & & UG/L & $05 / 25 / 2006$ & & DNT-284-06 \\
\hline GWM47001A1 & MIDDLE-2050A & 474 & Total Alkalinity & 170 & & & & $\mathrm{MG} / \mathrm{L}$ & $05 / 25 / 2006$ & & DNT-271-06 \\
\hline GWM47001AN & MIDDLE-2050A & 474 & Chloride & 16.5 & & & & MG/L & $05 / 25 / 2006$ & & DNT-271-06 \\
\hline GWM47001AN & MIDDLE-2050A & 474 & Fluoride & 0.16 & & & $\mathrm{~J}$ & $\mathrm{MG} / \mathrm{L}$ & $05 / 25 / 2006$ & & DNT-271-06 \\
\hline GWM47001AN & MIDDLE-2050A & 474 & Sulfate & 27.7 & & & & MG/L & $05 / 25 / 2006$ & & DNT-271-06 \\
\hline GWM47001N2 & MIDDLE-2050A & 474 & Nitrate/Nitrite as $\mathrm{N}$ & 931 & & & & UG/L & $05 / 25 / 2006$ & & DNT-271-06 \\
\hline GWM47001VL & MIDDLE-2050A & 474 & Acetone & 2 & & $u$ & $\mathrm{R}$ & UG/L & $05 / 26 / 2006$ & & JGJ-083-06 \\
\hline GWM47001VL & MIDDLE-2050A & 474 & Benzene & 1 & & $u$ & & UG/L & $05 / 26 / 2006$ & & JGJ-083-06 \\
\hline GWM47001VL & MIDDLE-2050A & 474 & Bromodichloromethane & 1 & & $\mathrm{u}$ & & UG/L & $05 / 26 / 2006$ & & JGJ-083-06 \\
\hline GWM47001VL & MIDDLE-2050A & 474 & Bromoform & 1 & & $u$ & & UG/L & $05 / 26 / 2006$ & & JGJ-083-06 \\
\hline GWM47001VL & MIDDLE-2050A & 474 & Bromomethane & 2 & & u & & UG/L & $05 / 26 / 2006$ & & JGJ-083-06 \\
\hline GWM47001VL & MIDDLE-2050A & 474 & 2-Butanone & 5 & & $\mathrm{u}$ & $\mathrm{R}$ & UG/L & $05 / 26 / 2006$ & & JGJ-083-06 \\
\hline GWM47001VL & MIDDLE-2050A & 474 & Carbon disulfide & 1 & & $\mathrm{u}$ & & UG/L & $05 / 26 / 2006$ & & JGJ-083-06 \\
\hline GWM47001VL & MIDDLE-2050A & 474 & Carbon tetrachloride & 1 & & $\mathrm{u}$ & & UG/L & $05 / 26 / 2006$ & & JGJ-083-06 \\
\hline GWM47001VL & MIDDLE-2050A & 474 & Chlorobenzene & 1 & & u & & UG/L & $05 / 26 / 2006$ & & JGJ-083-06 \\
\hline GWM47001VL & MIDDLE-2050A & 474 & Dibromochloromethane & 1 & & $\mathrm{u}$ & & UG/L & $05 / 26 / 2006$ & & JGJ-083-06 \\
\hline GWM47001VL & MIDDLE-2050A & 474 & 1,2-Dibromo-3-chloropropane & 1 & & u & $\mathrm{R}$ & UG/L & $05 / 26 / 2006$ & & JGJ-083-06 \\
\hline GWM47001VL & MIDDLE-2050A & 474 & Chloroethane & 2 & & $\mathrm{u}$ & & UG/L & $05 / 26 / 2006$ & & JGJ-083-06 \\
\hline GWM47001VL & MIDDLE-2050A & 474 & Chloroform & 1 & & u & & UG/L & $05 / 26 / 2006$ & & JGJ-083-06 \\
\hline GWM47001VL & MIDDLE-2050A & 474 & Chloromethane & 2 & & $\mathrm{u}$ & & UG/L & $05 / 26 / 2006$ & & JGJ-083-06 \\
\hline GWM47001VL & MIDDLE-2050A & 474 & Cyclohexane & 1 & & $\mathrm{u}$ & & UG/L & $05 / 26 / 2006$ & & JGJ-083-06 \\
\hline GWM47001VL & MIDDLE-2050A & 474 & 1,2-Dibromoethane & 1 & & U & & UG/L & $05 / 26 / 2006$ & & JGJ-083-06 \\
\hline GWM47001VL & MIDDLE-2050A & 474 & 1,2-Dichlorobenzene & 1 & & $\mathrm{U}$ & & UG/L & $05 / 26 / 2006$ & & JGJ-083-06 \\
\hline GWM47001VL & MIDDLE-2050A & 474 & 1,3-Dichlorobenzene & 1 & & $\mathrm{u}$ & & UG/L & $05 / 26 / 2006$ & & JGJ-083-06 \\
\hline GWM47001VL & MIDDLE-2050A & 474 & 1,4-Dichlorobenzene & 1 & & U & & UG/L & $05 / 26 / 2006$ & & JGJ-083-06 \\
\hline GWM47001VL & MIDDLE-2050A & 474 & Dichlorodifluoromethane & 2 & & u & & UG/L & $05 / 26 / 2006$ & & JGJ-083-06 \\
\hline GWM47001VL & MIDDLE-2050A & 474 & 1,1-Dichloroethane & 1 & & $u$ & & UG/L & $05 / 26 / 2006$ & & JGJ-083-06 \\
\hline GWM47001VL & MIDDLE-2050A & 474 & 1,2-Dichloroethane & 1 & & $\mathrm{u}$ & & UG/L & $05 / 26 / 2006$ & & JGJ-083-06 \\
\hline GWM47001VL & MIDDLE-2050A & 474 & 1,1-Dichloroethene & 1 & & $\mathrm{U}$ & & UG/L & $05 / 26 / 2006$ & & JGJ-083-06 \\
\hline GWM47001VL & MIDDLE-2050A & 474 & cis-1,2-Dichloroethene & 1 & & u & & UG/L & $05 / 26 / 2006$ & & JGJ-083-06 \\
\hline GWM47001VL & MIDDLE-2050A & 474 & trans-1,2-Dichloroethene & 1 & & $u$ & & UG/L & $05 / 26 / 2006$ & & JGJ-083-06 \\
\hline GWM47001VL & MIDDLE-2050A & 474 & 1,2-Dichloropropane & 1 & & $u$ & & UG/L & $05 / 26 / 2006$ & & JGJ-083-06 \\
\hline GWM47001VL & MIDDLE-2050A & 474 & cis-1,3-Dichloropropene & 1 & & $\mathrm{U}$ & & UG/L & $05 / 26 / 2006$ & & JGJ-083-06 \\
\hline GWM47001VL & MIDDLE-2050A & 474 & trans-1,3-Dichloropropene & 1 & & $\mathrm{u}$ & & UG/L & $05 / 26 / 2006$ & & JGJ-083-06 \\
\hline GWM47001VL & MIDDLE-2050A & 474 & Ethylbenzene & 1 & & $\mathrm{u}$ & & UG/L & $05 / 26 / 2006$ & & JGJ-083-06 \\
\hline GWM47001VL & MIDDLE-2050A & 474 & Trichlorofluoromethane & 1 & & u & & UG/L & $05 / 26 / 2006$ & & JGJ-083-06 \\
\hline GWM47001VL & MIDDLE-2050A & 474 & 2-Hexanone & 5 & & $\mathrm{u}$ & & UG/L & $05 / 26 / 2006$ & & JGJ-083-06 \\
\hline GWM47001VL & MIDDLE-2050A & 474 & Isopropylbenzene & 1 & & $\mathrm{u}$ & & UG/L & $05 / 26 / 2006$ & & JGJ-083-06 \\
\hline GWM47001VL & MIDDLE-2050A & 474 & Methyl acetate & 5 & & u & $\mathrm{R}$ & UG/L & $05 / 26 / 2006$ & & JGJ-083-06 \\
\hline GWM47001VL & MIDDLE-2050A & 474 & Methyl cyclohexane & 4 & & U & & UG/L & $05 / 26 / 2006$ & & JGJ-083-06 \\
\hline GWM47001VL & MIDDLE-2050A & 474 & Methylene Chloride & 1 & & $\mathrm{u}$ & & UG/L & $05 / 26 / 2006$ & & JGJ-083-06 \\
\hline GWM47001VL & MIDDLE-2050A & 474 & 4-Methyl-2-pentanone & 5 & & $u$ & & UG/L & $05 / 26 / 2006$ & & JGJ-083-06 \\
\hline GWM47001VL & MIDDLE-2050A & 474 & Styrene & 1 & & u & & UG/L & $05 / 26 / 2006$ & & JGJ-083-06 \\
\hline GWM47001VL & MIDDLE-2050A & 474 & 1,1,2,2-Tetrachloroethane & 1 & & $u$ & & UG/L & $05 / 26 / 2006$ & & JGJ-083-06 \\
\hline GWM47001VL & MIDDLE-2050A & 474 & Tetrachloroethene & 1 & & u & & UG/L & $05 / 26 / 2006$ & & JGJ-083-06 \\
\hline GWM47001VL & MIDDLE-2050A & 474 & Toluene & 1 & & $\mathrm{u}$ & & UG/L & $05 / 26 / 2006$ & & JGJ-083-06 \\
\hline GWM47001VL & MIDDLE-2050A & 474 & 1,2,4-Trichlorobenzene & 1 & & $U$ & & UG/L & $05 / 26 / 2006$ & & JGJ-083-06 \\
\hline GWM47001VL & MIDDLE-2050A & 474 & 1,1,1-Trichloroethane & 1 & & $\mathrm{U}$ & & UG/L & $05 / 26 / 2006$ & & JGJ-083-06 \\
\hline GWM47001VL & MIDDLE-2050A & 474 & $1,1,2$-Trichloroethane & 1 & & u & & UG/L & $05 / 26 / 2006$ & & JGJ-083-06 \\
\hline GWM47001VL & MIDDLE-2050A & 474 & Trichloroethene & 1 & & $\mathrm{u}$ & & UG/L & $05 / 26 / 2006$ & & JGJ-083-06 \\
\hline GWM47001VL & MIDDLE-2050A & 474 & 1,1,2-Trichloro-1,2,2-trifluoroethane & 1 & & $U$ & & UG/L & $05 / 26 / 2006$ & & JGJ-083-06 \\
\hline GWM47001VL & MIDDLE-2050A & 474 & Vinyl Chloride & 2 & & $\mathrm{u}$ & & UG/L & $05 / 26 / 2006$ & & JGJ-083-06 \\
\hline GWM47001VL & MIDDLE-2050A & 474 & Xylenes & 3 & & u & & UG/L & $05 / 26 / 2006$ & & JGJ-083-06 \\
\hline GWM47001VL & MIDDLE-2050A & 474 & Methyl t-butyl ether & 2 & & u & & UG/L & $05 / 26 / 2006$ & & JGJ-083-06 \\
\hline GWM47001R8 & MIDDLE-2050A & 474 & Tritium & $-1.40 \mathrm{E}+02$ & $1.12 \mathrm{E}+02$ & & $u$ & $\mathrm{PCl} / \mathrm{L}$ & $05 / 25 / 2006$ & $3.88 \mathrm{E}+02$ & SOS-TL176-06 \\
\hline GWM47001RH & MIDDLE-2050A & 474 & Gross Alpha & $1.83 E+00$ & 3.33E-01 & & & $\mathrm{PCl} / \mathrm{L}$ & $05 / 25 / 2006$ & 7.88E-01 & SOS-TL177-06 \\
\hline GWM47001RH & MIDDLE-2050A & 474 & Gross Beta & $3.42 \mathrm{E}+00$ & 3.97E-01 & & & $\mathrm{PCl} / \mathrm{L}$ & $05 / 25 / 2006$ & 1.15E+00 & SOS-TL177-06 \\
\hline GWM47001RH & MIDDLE-2050A & 474 & Strontium-90 & $-1.57 \mathrm{E}-01$ & $8.36 \mathrm{E}-02$ & & U & $\mathrm{PCl} / \mathrm{L}$ & $05 / 25 / 2006$ & 4.33E-01 & SOS-TL177-06 \\
\hline GWM47001RH & MIDDLE-2050A & 474 & Technetium-99 & $2.44 \mathrm{E}+00$ & $1.68 \mathrm{E}+00$ & & $U$ & $\mathrm{PCl} / \mathrm{L}$ & $05 / 25 / 2006$ & $5.54 \mathrm{E}+00$ & SOS-TL177-06 \\
\hline GWM47001UX & MIDDLE-2050A & 474 & lodine-129 & $-1.97 \mathrm{E}-02$ & 2.33E-02 & & $u$ & $\mathrm{PCl} / \mathrm{L}$ & $05 / 26 / 2006$ & 8.19E-02 & SOS-TL174-06 \\
\hline GWM471012X & MIDDLE-2050A & 647 & Aluminum & 11.9 & & B & & UG/L & $05 / 25 / 2006$ & & DNT-284-06 \\
\hline GWM471012X & MIDDLE-2050A & 647 & Antimony & 0.5 & & u & & UG/L & $05 / 25 / 2006$ & & DNT-284-06 \\
\hline
\end{tabular}


Table A-5. (continued).

\begin{tabular}{|c|c|c|c|c|c|c|c|c|c|c|c|}
\hline $\begin{array}{c}\text { Field Sample } \\
\text { Number }\end{array}$ & Location & Depth & Compound & $\begin{array}{l}\text { Sample } \\
\text { Result }\end{array}$ & $\begin{array}{l}\text { Sample } \\
\text { Error }\end{array}$ & $\begin{array}{c}\text { Result } \\
\text { Qualifier }\end{array}$ & $\begin{array}{c}\text { Validation } \\
\text { Flag }\end{array}$ & $\begin{array}{c}\text { Sample } \\
\text { Units }\end{array}$ & $\begin{array}{c}\text { Date Sample } \\
\text { Collected }\end{array}$ & MDA & $\begin{array}{l}\text { L\&V Report } \\
\text { Number }\end{array}$ \\
\hline GWM471012X & MIDDLE-2050A & 647 & Arsenic & 2 & & $\mathrm{U}$ & & UG/L & $05 / 25 / 2006$ & & DNT-284-06 \\
\hline GWM471012X & MIDDLE-2050A & 647 & Barium & 47.5 & & & & UG/L & $05 / 25 / 2006$ & & DNT-284-06 \\
\hline GWM471012X & MIDDLE-2050A & 647 & Beryllium & 0.088 & & $u$ & & UG/L & 05/25/2006 & & DNT-284-06 \\
\hline GWM471012X & MIDDLE-2050A & 647 & Cadmium & 0.057 & & $\mathrm{U}$ & & UG/L & $05 / 25 / 2006$ & & DNT-284-06 \\
\hline GWM471012X & MIDDLE-2050A & 647 & Calcium & 38200 & & E & $\mathrm{J}$ & UG/L & $05 / 25 / 2006$ & & DNT-284-06 \\
\hline GWM471012X & MIDDLE-2050A & 647 & Chromium & 2.8 & & U & & UG/L & $05 / 25 / 2006$ & & DNT-284-06 \\
\hline GWM471012X & MIDDLE-2050A & 647 & Cobalt & 0.31 & & 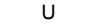 & & UG/L & 05/25/2006 & & DNT-284-06 \\
\hline GWM471012X & MIDDLE-2050A & 647 & Copper & 0.51 & & B & & UG/L & $05 / 25 / 2006$ & & DNT-284-06 \\
\hline GWM471012X & MIDDLE-2050A & 647 & Iron & 9.4 & & $u$ & & UG/L & 05/25/2006 & & DNT-284-06 \\
\hline GWM471012X & MIDDLE-2050A & 647 & Lead & 0.49 & & U & & UG/L & $05 / 25 / 2006$ & & DNT-284-06 \\
\hline GWM471012X & MIDDLE-2050A & 647 & Magnesium & 13600 & & E & $\mathrm{J}$ & UG/L & 05/25/2006 & & DNT-284-06 \\
\hline GWM471012X & MIDDLE-2050A & 647 & Manganese & 2 & & & U & UG/L & $05 / 25 / 2006$ & & DNT-284-06 \\
\hline GWM471012X & MIDDLE-2050A & 647 & Nickel & 1.4 & & B & & UG/L & 05/25/2006 & & DNT-284-06 \\
\hline GWM471012X & MIDDLE-2050A & 647 & Potassium & 2040 & & & & UG/L & $05 / 25 / 2006$ & & DNT-284-06 \\
\hline GWM471012X & MIDDLE-2050A & 647 & Selenium & 1 & & U & & UG/L & $05 / 25 / 2006$ & & DNT-284-06 \\
\hline GWM471012X & MIDDLE-2050A & 647 & Silver & 0.2 & & U & & UG/L & $05 / 25 / 2006$ & & DNT-284-06 \\
\hline GWM471012X & MIDDLE-2050A & 647 & Sodium & 7110 & & E & $\mathrm{J}$ & UG/L & $05 / 25 / 2006$ & & DNT-284-06 \\
\hline GWM471012X & MIDDLE-2050A & 647 & Strontium & 219 & & E & $\mathrm{J}$ & UG/L & $05 / 25 / 2006$ & & DNT-284-06 \\
\hline GWM471012X & MIDDLE-2050A & 647 & Thallium & 0.56 & & B & & UG/L & $05 / 25 / 2006$ & & DNT-284-06 \\
\hline GWM471012X & MIDDLE-2050A & 647 & Uranium & 1.5 & & & & UG/L & 05/25/2006 & & DNT-284-06 \\
\hline GWM471012X & MIDDLE-2050A & 647 & Vanadium & 1.6 & & $\mathrm{U}$ & & UG/L & $05 / 25 / 2006$ & & DNT-284-06 \\
\hline GWM471012X & MIDDLE-2050A & 647 & Zinc & 13.8 & & & & UG/L & $05 / 25 / 2006$ & & DNT-284-06 \\
\hline GWM471012X & MIDDLE-2050A & 647 & Mercury & 0.14 & & B & & UG/L & $05 / 25 / 2006$ & & DNT-284-06 \\
\hline GWM47101A1 & MIDDLE-2050A & 647 & Total Alkalinity & 140 & & & & MG/L & 05/25/2006 & & DNT-271-06 \\
\hline GWM47101AN & MIDDLE-2050A & 647 & Chloride & 12.3 & & & & MG/L & $05 / 25 / 2006$ & & DNT-271-06 \\
\hline GWM47101AN & MIDDLE-2050A & 647 & Fluoride & 0.18 & & & $\mathrm{~J}$ & MG/L & 05/25/2006 & & DNT-271-06 \\
\hline GWM47101AN & MIDDLE-2050A & 647 & Sulfate & 19.2 & & & & MG/L & $05 / 25 / 2006$ & & DNT-271-06 \\
\hline GWM47101N2 & MIDDLE-2050A & 647 & Nitrate/Nitrite as $\mathrm{N}$ & 162 & & & & UG/L & 05/25/2006 & & DNT-271-06 \\
\hline GWM47101VL & MIDDLE-2050A & 647 & Acetone & 2 & & $\mathrm{U}$ & $\mathrm{R}$ & UG/L & $05 / 25 / 2006$ & & JGJ-083-06 \\
\hline GWM47101VL & MIDDLE-2050A & 647 & Benzene & 1 & & $\mathrm{U}$ & & UG/L & 05/25/2006 & & JGJ-083-06 \\
\hline GWM47101VL & MIDDLE-2050A & 647 & Bromodichloromethane & 1 & & $\mathrm{U}$ & & UG/L & $05 / 25 / 2006$ & & JGJ-083-06 \\
\hline GWM47101VL & MIDDLE-2050A & 647 & Bromoform & 1 & & $\mathrm{U}$ & & UG/L & 05/25/2006 & & JGJ-083-06 \\
\hline GWM47101VL & MIDDLE-2050A & 647 & Bromomethane & 2 & & $\mathrm{U}$ & & UG/L & $05 / 25 / 2006$ & & JGJ-083-06 \\
\hline GWM47101VL & MIDDLE-2050A & 647 & 2-Butanone & 5 & & $\mathrm{U}$ & $\mathrm{R}$ & UG/L & 05/25/2006 & & JGJ-083-06 \\
\hline GWM47101VL & MIDDLE-2050A & 647 & Carbon disulfide & 1 & & $\mathrm{U}$ & & UG/L & $05 / 25 / 2006$ & & JGJ-083-06 \\
\hline GWM47101VL & MIDDLE-2050A & 647 & Carbon tetrachloride & 1 & & $\mathrm{U}$ & & UG/L & $05 / 25 / 2006$ & & JGJ-083-06 \\
\hline GWM47101VL & MIDDLE-2050A & 647 & Chlorobenzene & 1 & & $\mathrm{U}$ & & UG/L & 05/25/2006 & & JGJ-083-06 \\
\hline GWM47101VL & MIDDLE-2050A & 647 & Dibromochloromethane & 1 & & $\mathrm{U}$ & & UG/L & $05 / 25 / 2006$ & & JGJ-083-06 \\
\hline GWM47101VL & MIDDLE-2050A & 647 & 1,2-Dibromo-3-chloropropane & 1 & & $\mathrm{U}$ & $\mathrm{R}$ & UG/L & $05 / 25 / 2006$ & & JGJ-083-06 \\
\hline GWM47101VL & MIDDLE-2050A & 647 & Chloroethane & 2 & & $\mathrm{U}$ & & UG/L & $05 / 25 / 2006$ & & JGJ-083-06 \\
\hline GWM47101VL & MIDDLE-2050A & 647 & Chloroform & 1 & & $\mathrm{U}$ & & UG/L & 05/25/2006 & & JGJ-083-06 \\
\hline GWM47101VL & MIDDLE-2050A & 647 & Chloromethane & 2 & & $\mathrm{U}$ & & UG/L & $05 / 25 / 2006$ & & JGJ-083-06 \\
\hline GWM47101VL & MIDDLE-2050A & 647 & Cyclohexane & 1 & & $\mathrm{U}$ & & UG/L & 05/25/2006 & & JGJ-083-06 \\
\hline GWM47101VL & MIDDLE-2050A & 647 & 1,2-Dibromoethane & 1 & & $\mathrm{U}$ & & UG/L & $05 / 25 / 2006$ & & JGJ-083-06 \\
\hline GWM47101VL & MIDDLE-2050A & 647 & 1,2-Dichlorobenzene & 1 & & $\mathrm{u}$ & & UG/L & 05/25/2006 & & JGJ-083-06 \\
\hline GWM47101VL & MIDDLE-2050A & 647 & 1,3-Dichlorobenzene & 1 & & $\mathrm{U}$ & & UG/L & $05 / 25 / 2006$ & & JGJ-083-06 \\
\hline GWM47101VL & MIDDLE-2050A & 647 & 1,4-Dichlorobenzene & 1 & & $\mathrm{U}$ & & UG/L & 05/25/2006 & & JGJ-083-06 \\
\hline GWM47101VL & MIDDLE-2050A & 647 & Dichlorodifluoromethane & 2 & & $\mathrm{U}$ & & UG/L & $05 / 25 / 2006$ & & JGJ-083-06 \\
\hline GWM47101VL & MIDDLE-2050A & 647 & 1,1-Dichloroethane & 1 & & $\mathrm{U}$ & & UG/L & 05/25/2006 & & JGJ-083-06 \\
\hline GWM47101VL & MIDDLE-2050A & 647 & 1,2-Dichloroethane & 1 & & $\mathrm{U}$ & & UG/L & $05 / 25 / 2006$ & & JGJ-083-06 \\
\hline GWM47101VL & MIDDLE-2050A & 647 & 1,1-Dichloroethene & 1 & & $\mathrm{U}$ & & UG/L & $05 / 25 / 2006$ & & JGJ-083-06 \\
\hline GWM47101VL & MIDDLE-2050A & 647 & cis-1,2-Dichloroethene & 1 & & $\mathrm{U}$ & & UG/L & $05 / 25 / 2006$ & & JGJ-083-06 \\
\hline GWM47101VL & MIDDLE-2050A & 647 & trans-1,2-Dichloroethene & 1 & & $\mathrm{U}$ & & UG/L & 05/25/2006 & & JGJ-083-06 \\
\hline GWM47101VL & MIDDLE-2050A & 647 & 1,2-Dichloropropane & 1 & & $\mathrm{U}$ & & UG/L & $05 / 25 / 2006$ & & JGJ-083-06 \\
\hline GWM47101VL & MIDDLE-2050A & 647 & cis-1,3-Dichloropropene & 1 & & $\mathrm{U}$ & & UG/L & $05 / 25 / 2006$ & & JGJ-083-06 \\
\hline GWM47101VL & MIDDLE-2050A & 647 & trans-1,3-Dichloropropene & 1 & & $\mathrm{U}$ & & UG/L & 05/25/2006 & & JGJ-083-06 \\
\hline GWM47101VL & MIDDLE-2050A & 647 & Ethylbenzene & 1 & & $\mathrm{U}$ & & UG/L & $05 / 25 / 2006$ & & JGJ-083-06 \\
\hline GWM47101VL & MIDDLE-2050A & 647 & Trichlorofluoromethane & 1 & & $\mathrm{U}$ & & UG/L & 05/25/2006 & & JGJ-083-06 \\
\hline GWM47101VL & MIDDLE-2050A & 647 & 2-Hexanone & 5 & & $\mathrm{U}$ & & UG/L & $05 / 25 / 2006$ & & JGJ-083-06 \\
\hline GWM47101VL & MIDDLE-2050A & 647 & Isopropylbenzene & 1 & & $\mathrm{U}$ & & UG/L & 05/25/2006 & & JGJ-083-06 \\
\hline GWM47101VL & MIDDLE-2050A & 647 & Methyl acetate & 5 & & $\mathrm{u}$ & $\mathrm{R}$ & UG/L & $05 / 25 / 2006$ & & JGJ-083-06 \\
\hline GWM47101VL & MIDDLE-2050A & 647 & Methyl cyclohexane & 4 & & $\mathrm{U}$ & & UG/L & 05/25/2006 & & JGJ-083-06 \\
\hline GWM47101VL & MIDDLE-2050A & 647 & Methylene Chloride & 1 & & $\mathrm{U}$ & & UG/L & $05 / 25 / 2006$ & & JGJ-083-06 \\
\hline GWM47101VL & MIDDLE-2050A & 647 & 4-Methyl-2-pentanone & 5 & & $\mathrm{U}$ & & UG/L & 05/25/2006 & & JGJ-083-06 \\
\hline GWM47101VL & MIDDLE-2050A & 647 & Styrene & 1 & & $\mathrm{U}$ & & UG/L & $05 / 25 / 2006$ & & JGJ-083-06 \\
\hline GWM47101VL & MIDDLE-2050A & 647 & 1,1,2,2-Tetrachloroethane & 1 & & $\mathrm{U}$ & & UG/L & $05 / 25 / 2006$ & & JGJ-083-06 \\
\hline GWM47101VL & MIDDLE-2050A & 647 & Tetrachloroethene & 1 & & $\mathrm{U}$ & & UG/L & $05 / 25 / 2006$ & & JGJ-083-06 \\
\hline GWM47101VL & MIDDLE-2050A & 647 & Toluene & 1 & & $\mathrm{U}$ & & UG/L & 05/25/2006 & & JGJ-083-06 \\
\hline GWM47101VL & MIDDLE-2050A & 647 & 1,2,4-Trichlorobenzene & 1 & & $\mathrm{u}$ & & UG/L & $05 / 25 / 2006$ & & JGJ-083-06 \\
\hline GWM47101VL & MIDDLE-2050A & 647 & 1,1,1-Trichloroethane & 1 & & $\mathrm{u}$ & & UG/L & $05 / 25 / 2006$ & & JGJ-083-06 \\
\hline GWM47101VL & MIDDLE-2050A & 647 & 1,1,2-Trichloroethane & 1 & & $\mathrm{U}$ & & UG/L & $05 / 25 / 2006$ & & JGJ-083-06 \\
\hline GWM47101VL & MIDDLE-2050A & 647 & Trichloroethene & 1 & & $\mathrm{U}$ & & UG/L & 05/25/2006 & & JGJ-083-06 \\
\hline GWM47101VL & MIDDLE-2050A & 647 & 1,1,2-Trichloro-1,2,2-trifluoroethane & 1 & & $\mathrm{u}$ & & UG/L & 05/25/2006 & & JGJ-083-06 \\
\hline GWM47101VL & MIDDLE-2050A & 647 & Vinyl Chloride & 2 & & $\mathrm{U}$ & & UG/L & $05 / 25 / 2006$ & & JGJ-083-06 \\
\hline GWM47101VL & MIDDLE-2050A & 647 & Xylenes & 3 & & $\mathrm{U}$ & & UG/L & $05 / 25 / 2006$ & & JGJ-083-06 \\
\hline GWM47101VL & MIDDLE-2050A & 647 & Methyl t-butyl ether & 2 & & $\mathrm{U}$ & & UG/L & 05/25/2006 & & JGJ-083-06 \\
\hline GWM47101R8 & MIDDLE-2050A & 647 & Tritium & $1.80 \mathrm{E}+01$ & $1.17 \mathrm{E}+02$ & & U & $\mathrm{PCI} / \mathrm{L}$ & 05/25/2006 & $3.95 \mathrm{E}+02$ & SOS-TL176-06 \\
\hline GWM47101RH & MIDDLE-2050A & 647 & Gross Alpha & $1.22 \mathrm{E}+00$ & $5.41 \mathrm{E}-01$ & & UJ & $\mathrm{PCI} / \mathrm{L}$ & $05 / 25 / 2006$ & $1.84 \mathrm{E}+00$ & SOS-TL177-06 \\
\hline GWM47101RH & MIDDLE-2050A & 647 & Gross Beta & $3.44 \mathrm{E}+00$ & 8.36E-01 & & & $\mathrm{PCI} / \mathrm{L}$ & 05/25/2006 & $3.08 \mathrm{E}+00$ & SOS-TL177-06 \\
\hline GWM47101RH & MIDDLE-2050A & 647 & Strontium-90 & $5.70 \mathrm{E}-02$ & $6.96 \mathrm{E}-02$ & & $U$ & $\mathrm{PCI} / \mathrm{L}$ & 05/25/2006 & 3.09E-01 & SOS-TL177-06 \\
\hline GWM47101RH & MIDDLE-2050A & 647 & Technetium-99 & $3.38 \mathrm{E}+00$ & $1.74 \mathrm{E}+00$ & & U & $\mathrm{PCl} / \mathrm{L}$ & 05/25/2006 & $5.70 \mathrm{E}+00$ & SOS-TL177-06 \\
\hline GWM47101UX & MIDDLE-2050A & 647 & lodine-129 & $-3.22 \mathrm{E}-02$ & $2.54 \mathrm{E}-02$ & & U & $\mathrm{PCI} / \mathrm{L}$ & $05 / 25 / 2006$ & 8.34E-02 & SOS-TL174-06 \\
\hline GWM472012X & MIDDLE-2050A & 790 & Aluminum & 7.9 & & U & & UG/L & 05/24/2006 & & DNT-284-06 \\
\hline GWM472012X & MIDDLE-2050A & 790 & Antimony & 0.5 & & U & & UG/L & $05 / 24 / 2006$ & & DNT-284-06 \\
\hline GWM472012X & MIDDLE-2050A & 790 & Arsenic & 2 & & U & & UG/L & 05/24/2006 & & DNT-284-06 \\
\hline GWM472012X & MIDDLE-2050A & 790 & Barium & 58.4 & & & & UG/L & $05 / 24 / 2006$ & & DNT-284-06 \\
\hline
\end{tabular}


Table A-5. (continued).

\begin{tabular}{|c|c|c|c|c|c|c|c|c|c|c|c|}
\hline $\begin{array}{l}\text { Field Sample } \\
\text { Number }\end{array}$ & Location & Depth & Compound & $\begin{array}{l}\text { Sample } \\
\text { Result }\end{array}$ & $\begin{array}{l}\text { Sample } \\
\text { Error }\end{array}$ & $\begin{array}{c}\text { Result } \\
\text { Qualifier }\end{array}$ & $\begin{array}{l}\text { Validation } \\
\text { Flag }\end{array}$ & $\begin{array}{l}\text { Sample } \\
\text { Units }\end{array}$ & $\begin{array}{c}\text { Date Sample } \\
\text { Collected }\end{array}$ & MDA & $\begin{array}{l}\text { L\&V Report } \\
\text { Number }\end{array}$ \\
\hline GWM472012X & MIDDLE-2050A & 790 & Beryllium & 0.088 & & $U$ & & UG/L & $05 / 24 / 2006$ & & DNT-284-06 \\
\hline GWM472012X & MIDDLE-2050A & 790 & Cadmium & 0.057 & & U & & UG/L & $05 / 24 / 2006$ & & DNT-284-06 \\
\hline GWM472012X & MIDDLE-2050A & 790 & Calcium & 43000 & & $\mathrm{E}$ & $\mathrm{J}$ & UG/L & $05 / 24 / 2006$ & & DNT-284-06 \\
\hline GWM472012X & MIDDLE-2050A & 790 & Chromium & 4.2 & & B & & UG/L & 05/24/2006 & & DNT-284-06 \\
\hline GWM472012X & MIDDLE-2050A & 790 & Cobalt & 0.31 & & u & & UG/L & $05 / 24 / 2006$ & & DNT-284-06 \\
\hline GWM472012X & MIDDLE-2050A & 790 & Copper & 0.38 & & B & & UG/L & $05 / 24 / 2006$ & & DNT-284-06 \\
\hline GWM472012X & MIDDLE-2050A & 790 & Iron & 9.4 & & u & & UG/L & $05 / 24 / 2006$ & & DNT-284-06 \\
\hline GWM472012X & MIDDLE-2050A & 790 & Lead & 0.49 & & $u$ & & UG/L & 05/24/2006 & & DNT-284-06 \\
\hline GWM472012X & MIDDLE-2050A & 790 & Magnesium & 16600 & & $\mathrm{E}$ & $\mathrm{J}$ & UG/L & $05 / 24 / 2006$ & & DNT-284-06 \\
\hline GWM472012X & MIDDLE-2050A & 790 & Manganese & 0.47 & & B & $u$ & UG/L & $05 / 24 / 2006$ & & DNT-284-06 \\
\hline GWM472012X & MIDDLE-2050A & 790 & Nickel & 1.4 & & B & & UG/L & $05 / 24 / 2006$ & & DNT-284-06 \\
\hline GWM472012X & MIDDLE-2050A & 790 & Potassium & 1800 & & & & UG/L & 05/24/2006 & & DNT-284-06 \\
\hline GWM472012X & MIDDLE-2050A & 790 & Selenium & 1.1 & & B & & UG/L & $05 / 24 / 2006$ & & DNT-284-06 \\
\hline GWM472012X & MIDDLE-2050A & 790 & Silver & 0.2 & & $u$ & & UG/L & $05 / 24 / 2006$ & & DNT-284-06 \\
\hline GWM472012X & MIDDLE-2050A & 790 & Sodium & 7820 & & $\mathrm{E}$ & $\mathrm{J}$ & UG/L & $05 / 24 / 2006$ & & DNT-284-06 \\
\hline GWM472012X & MIDDLE-2050A & 790 & Strontium & 213 & & E & $\mathrm{J}$ & UG/L & $05 / 24 / 2006$ & & DNT-284-06 \\
\hline GWM472012X & MIDDLE-2050A & 790 & Thallium & 0.32 & & $u$ & & UG/L & 05/24/2006 & & DNT-284-06 \\
\hline GWM472012X & MIDDLE-2050A & 790 & Uranium & 1.6 & & & & UG/L & $05 / 24 / 2006$ & & DNT-284-06 \\
\hline GWM472012X & MIDDLE-2050A & 790 & Vanadium & 4.1 & & B & & UG/L & $05 / 24 / 2006$ & & DNT-284-06 \\
\hline GWM472012X & MIDDLE-2050A & 790 & Zinc & 13.4 & & & & UG/L & $05 / 24 / 2006$ & & DNT-284-06 \\
\hline GWM472012X & MIDDLE-2050A & 790 & Mercury & 0.093 & & $\mathrm{u}$ & & UG/L & $05 / 24 / 2006$ & & DNT-284-06 \\
\hline GWM47201A1 & MIDDLE-2050A & 790 & Total Alkalinity & 182 & & & & $\mathrm{MG} / \mathrm{L}$ & $05 / 24 / 2006$ & & DNT-326-06 \\
\hline GWM47201AN & MIDDLE-2050A & 790 & Chloride & 11.6 & & & & MG/L & $05 / 24 / 2006$ & & DNT-326-06 \\
\hline GWM47201AN & MIDDLE-2050A & 790 & Fluoride & 0.13 & & & & MG/L & $05 / 24 / 2006$ & & DNT-326-06 \\
\hline GWM47201AN & MIDDLE-2050A & 790 & Sulfate & 19.7 & & & & MG/L & $05 / 24 / 2006$ & & DNT-326-06 \\
\hline GWM47201N2 & MIDDLE-2050A & 790 & Nitrate/Nitrite as $\mathrm{N}$ & 917 & & & & UG/L & $05 / 24 / 2006$ & & DNT-326-06 \\
\hline GWM47201VL & MIDDLE-2050A & 790 & Acetone & 2 & & $u$ & $R$ & UG/L & $05 / 24 / 2006$ & & JGJ-083-06 \\
\hline GWM47201VL & MIDDLE-2050A & 790 & Benzene & 1 & & U & & UG/L & $05 / 24 / 2006$ & & JGJ-083-06 \\
\hline GWM47201VL & MIDDLE-2050A & 790 & Bromodichloromethane & 1 & & $\mathrm{U}$ & & UG/L & 05/24/2006 & & JGJ-083-06 \\
\hline GWM47201VL & MIDDLE-2050A & 790 & Bromoform & 1 & & $\mathrm{U}$ & & UG/L & $05 / 24 / 2006$ & & JGJ-083-06 \\
\hline GWM47201VL & MIDDLE-2050A & 790 & Bromomethane & 2 & & u & & UG/L & $05 / 24 / 2006$ & & JGJ-083-06 \\
\hline GWM47201VL & MIDDLE-2050A & 790 & 2-Butanone & 5 & & $\mathrm{U}$ & $\mathrm{R}$ & UG/L & $05 / 24 / 2006$ & & JGJ-083-06 \\
\hline GWM47201VL & MIDDLE-2050A & 790 & Carbon disulfide & 1 & & U & & UG/L & $05 / 24 / 2006$ & & JGJ-083-06 \\
\hline GWM47201VL & MIDDLE-2050A & 790 & Carbon tetrachloride & 1 & & $\mathrm{u}$ & & UG/L & $05 / 24 / 2006$ & & JGJ-083-06 \\
\hline GWM47201VL & MIDDLE-2050A & 790 & Chlorobenzene & 1 & & u & & UG/L & $05 / 24 / 2006$ & & JGJ-083-06 \\
\hline GWM47201VL & MIDDLE-2050A & 790 & Dibromochloromethane & 1 & & $\mathrm{u}$ & & UG/L & $05 / 24 / 2006$ & & JGJ-083-06 \\
\hline GWM47201VL & MIDDLE-2050A & 790 & 1,2-Dibromo-3-chloropropane & 1 & & u & $\mathrm{R}$ & UG/L & $05 / 24 / 2006$ & & JGJ-083-06 \\
\hline GWM47201VL & MIDDLE-2050A & 790 & Chloroethane & 2 & & $\mathrm{u}$ & & UG/L & $05 / 24 / 2006$ & & JGJ-083-06 \\
\hline GWM47201VL & MIDDLE-2050A & 790 & Chloroform & 1 & & $\mathrm{u}$ & & UG/L & $05 / 24 / 2006$ & & JGJ-083-06 \\
\hline GWM47201VL & MIDDLE-2050A & 790 & Chloromethane & 2 & & $\mathrm{u}$ & & UG/L & $05 / 24 / 2006$ & & JGJ-083-06 \\
\hline GWM47201VL & MIDDLE-2050A & 790 & Cyclohexane & 1 & & $\mathrm{u}$ & & UG/L & $05 / 24 / 2006$ & & JGJ-083-06 \\
\hline GWM47201VL & MIDDLE-2050A & 790 & 1,2-Dibromoethane & 1 & & $\mathrm{U}$ & & UG/L & $05 / 24 / 2006$ & & JGJ-083-06 \\
\hline GWM47201VL & MIDDLE-2050A & 790 & 1,2-Dichlorobenzene & 1 & & $\mathrm{u}$ & & UG/L & $05 / 24 / 2006$ & & JGJ-083-06 \\
\hline GWM47201VL & MIDDLE-2050A & 790 & 1,3-Dichlorobenzene & 1 & & $\mathrm{u}$ & & UG/L & $05 / 24 / 2006$ & & JGJ-083-06 \\
\hline GWM47201VL & MIDDLE-2050A & 790 & 1,4-Dichlorobenzene & 1 & & $\mathrm{u}$ & & UG/L & $05 / 24 / 2006$ & & JGJ-083-06 \\
\hline GWM47201VL & MIDDLE-2050A & 790 & Dichlorodifluoromethane & 2 & & u & & UG/L & $05 / 24 / 2006$ & & JGJ-083-06 \\
\hline GWM47201VL & MIDDLE-2050A & 790 & 1,1-Dichloroethane & 1 & & $\mathrm{U}$ & & UG/L & $05 / 24 / 2006$ & & JGJ-083-06 \\
\hline GWM47201VL & MIDDLE-2050A & 790 & 1,2-Dichloroethane & 1 & & $\mathrm{u}$ & & UG/L & $05 / 24 / 2006$ & & JGJ-083-06 \\
\hline GWM47201VL & MIDDLE-2050A & 790 & 1,1-Dichloroethene & 1 & & $\mathrm{u}$ & & UG/L & $05 / 24 / 2006$ & & JGJ-083-06 \\
\hline GWM47201VL & MIDDLE-2050A & 790 & cis-1,2-Dichloroethene & 1 & & u & & UG/L & $05 / 24 / 2006$ & & JGJ-083-06 \\
\hline GWM47201VL & MIDDLE-2050A & 790 & trans-1,2-Dichloroethene & 1 & & $u$ & & UG/L & $05 / 24 / 2006$ & & JGJ-083-06 \\
\hline GWM47201VL & MIDDLE-2050A & 790 & 1,2-Dichloropropane & 1 & & u & & UG/L & $05 / 24 / 2006$ & & JGJ-083-06 \\
\hline GWM47201VL & MIDDLE-2050A & 790 & cis-1,3-Dichloropropene & 1 & & $\mathrm{u}$ & & UG/L & 05/24/2006 & & JGJ-083-06 \\
\hline GWM47201VL & MIDDLE-2050A & 790 & trans-1,3-Dichloropropene & 1 & & u & & UG/L & $05 / 24 / 2006$ & & JGJ-083-06 \\
\hline GWM47201VL & MIDDLE-2050A & 790 & Ethylbenzene & 1 & & $\mathrm{U}$ & & UG/L & $05 / 24 / 2006$ & & JGJ-083-06 \\
\hline GWM47201VL & MIDDLE-2050A & 790 & Trichlorofluoromethane & 1 & & u & & UG/L & $05 / 24 / 2006$ & & JGJ-083-06 \\
\hline GWM47201VL & MIDDLE-2050A & 790 & 2-Hexanone & 5 & & $\mathrm{u}$ & & UG/L & $05 / 24 / 2006$ & & JGJ-083-06 \\
\hline GWM47201VL & MIDDLE-2050A & 790 & Isopropylbenzene & 1 & & $\mathrm{U}$ & & UG/L & $05 / 24 / 2006$ & & JGJ-083-06 \\
\hline GWM47201VL & MIDDLE-2050A & 790 & Methyl acetate & 5 & & $\mathrm{U}$ & $\mathrm{R}$ & UG/L & $05 / 24 / 2006$ & & JGJ-083-06 \\
\hline GWM47201VL & MIDDLE-2050A & 790 & Methyl cyclohexane & 4 & & $\mathrm{u}$ & & UG/L & $05 / 24 / 2006$ & & JGJ-083-06 \\
\hline GWM47201VL & MIDDLE-2050A & 790 & Methylene Chloride & 1 & & u & & UG/L & $05 / 24 / 2006$ & & JGJ-083-06 \\
\hline GWM47201VL & MIDDLE-2050A & 790 & 4-Methyl-2-pentanone & 5 & & $\mathrm{U}$ & & UG/L & $05 / 24 / 2006$ & & JGJ-083-06 \\
\hline GWM47201VL & MIDDLE-2050A & 790 & Styrene & 1 & & $\mathrm{U}$ & & UG/L & $05 / 24 / 2006$ & & JGJ-083-06 \\
\hline GWM47201VL & MIDDLE-2050A & 790 & 1,1,2,2-Tetrachloroethane & 1 & & $\mathrm{U}$ & & UG/L & $05 / 24 / 2006$ & & JGJ-083-06 \\
\hline GWM47201VL & MIDDLE-2050A & 790 & Tetrachloroethene & 1 & & u & & UG/L & $05 / 24 / 2006$ & & JGJ-083-06 \\
\hline GWM47201VL & MIDDLE-2050A & 790 & Toluene & 1 & & $\mathrm{u}$ & & UG/L & $05 / 24 / 2006$ & & JGJ-083-06 \\
\hline GWM47201VL & MIDDLE-2050A & 790 & 1,2,4-Trichlorobenzene & 1 & & $\mathrm{U}$ & & UG/L & $05 / 24 / 2006$ & & JGJ-083-06 \\
\hline GWM47201VL & MIDDLE-2050A & 790 & 1,1,1-Trichloroethane & 1 & & U & & UG/L & $05 / 24 / 2006$ & & JGJ-083-06 \\
\hline GWM47201VL & MIDDLE-2050A & 790 & $1,1,2$-Trichloroethane & 1 & & u & & UG/L & $05 / 24 / 2006$ & & JGJ-083-06 \\
\hline GWM47201VL & MIDDLE-2050A & 790 & Trichloroethene & 1 & & $u$ & & UG/L & $05 / 24 / 2006$ & & JGJ-083-06 \\
\hline GWM47201VL & MIDDLE-2050A & 790 & 1,1,2-Trichloro-1,2,2-trifluoroethane & 1 & & u & & UG/L & $05 / 24 / 2006$ & & JGJ-083-06 \\
\hline GWM47201VL & MIDDLE-2050A & 790 & Vinyl Chloride & 2 & & $\mathrm{U}$ & & UG/L & $05 / 24 / 2006$ & & JGJ-083-06 \\
\hline GWM47201VL & MIDDLE-2050A & 790 & Xylenes & 3 & & u & & UG/L & $05 / 24 / 2006$ & & JGJ-083-06 \\
\hline GWM47201VL & MIDDLE-2050A & 790 & Methyl t-butyl ether & 2 & & $\mathrm{U}$ & & UG/L & $05 / 24 / 2006$ & & JGJ-083-06 \\
\hline GWM47201R8 & MIDDLE-2050A & 790 & Tritium & $-7.41 \mathrm{E}+01$ & $1.12 \mathrm{E}+02$ & & $\mathrm{u}$ & $\mathrm{PCI} / \mathrm{L}$ & $05 / 24 / 2006$ & $3.85 \mathrm{E}+02$ & SOS-TL176-06 \\
\hline GWM47201RH & MIDDLE-2050A & 790 & Gross Alpha & $1.77 \mathrm{E}+00$ & $3.02 \mathrm{E}-01$ & & & $\mathrm{PCI} / \mathrm{L}$ & $05 / 24 / 2006$ & $6.49 \mathrm{E}-01$ & SOS-TL177-06 \\
\hline GWM47201RH & MIDDLE-2050A & 790 & Gross Beta & $5.50 \mathrm{E}+00$ & 4.23E-01 & & & $\mathrm{PCl} / \mathrm{L}$ & $05 / 24 / 2006$ & $1.09 \mathrm{E}+00$ & SOS-TL177-06 \\
\hline GWM47201RH & MIDDLE-2050A & 790 & Strontium-90 & $1.34 \mathrm{E}-01$ & $9.59 \mathrm{E}-02$ & & $\mathrm{U}$ & $\mathrm{PCI} / \mathrm{L}$ & $05 / 24 / 2006$ & 4.11E-01 & SOS-TL177-06 \\
\hline GWM47201RH & MIDDLE-2050A & 790 & Technetium-99 & $-1.97 \mathrm{E}-01$ & $1.62 \mathrm{E}+00$ & & U & $\mathrm{PCI} / \mathrm{L}$ & $05 / 24 / 2006$ & $5.51 \mathrm{E}+00$ & SOS-TL177-06 \\
\hline GWM47201UX & MIDDLE-2050A & 790 & lodine-129 & 2.16E-03 & 2.60E-02 & & u & $\mathrm{PCI} / \mathrm{L}$ & $05 / 24 / 2006$ & $9.31 \mathrm{E}-02$ & SOS-TL174-06 \\
\hline GWM473012X & MIDDLE-2050A & 1005 & Aluminum & 7.9 & & $\mathrm{U}$ & & UG/L & $05 / 24 / 2006$ & & DNT-284-06 \\
\hline GWM473012X & MIDDLE-2050A & 1005 & Antimony & 0.5 & & u & & UG/L & $05 / 24 / 2006$ & & DNT-284-06 \\
\hline GWM473012X & MIDDLE-2050A & 1005 & Arsenic & 2 & & $u$ & & UG/L & $05 / 24 / 2006$ & & DNT-284-06 \\
\hline GWM473012X & MIDDLE-2050A & 1005 & Barium & 49.5 & & & & UG/L & $05 / 24 / 2006$ & & DNT-284-06 \\
\hline GWM473012X & MIDDLE-2050A & 1005 & Beryllium & 0.088 & & $\mathrm{U}$ & & UG/L & $05 / 24 / 2006$ & & DNT-284-06 \\
\hline GWM473012X & MIDDLE-2050A & 1005 & Cadmium & 0.057 & & U & $\mathrm{J}$ & UG/L & $05 / 24 / 2006$ & & DNT-284-06 \\
\hline
\end{tabular}


Table A-5. (continued).

\begin{tabular}{|c|c|c|c|c|c|c|c|c|c|c|c|}
\hline $\begin{array}{l}\text { Field Sample } \\
\text { Number }\end{array}$ & Location & Depth & Compound & $\begin{array}{l}\text { Sample } \\
\text { Result }\end{array}$ & $\begin{array}{l}\text { Sample } \\
\text { Error }\end{array}$ & $\begin{array}{c}\text { Result } \\
\text { Qualifier }\end{array}$ & $\begin{array}{l}\text { Validation } \\
\text { Flag }\end{array}$ & $\begin{array}{l}\text { Sample } \\
\text { Units }\end{array}$ & $\begin{array}{c}\text { Date Sample } \\
\text { Collected }\end{array}$ & MDA & $\begin{array}{l}\text { L\&V Report } \\
\text { Number }\end{array}$ \\
\hline GWM473012X & MIDDLE-2050A & 1005 & Calcium & 40100 & & $E$ & & UG/L & $05 / 24 / 2006$ & & DNT-284-06 \\
\hline GWM473012X & MIDDLE-2050A & 1005 & Chromium & 4 & & B & & UG/L & $05 / 24 / 2006$ & & DNT-284-06 \\
\hline GWM473012X & MIDDLE-2050A & 1005 & Cobalt & 0.31 & & U & & UG/L & $05 / 24 / 2006$ & & DNT-284-06 \\
\hline GWM473012X & MIDDLE-2050A & 1005 & Copper & 0.61 & & B & & UG/L & 05/24/2006 & & DNT-284-06 \\
\hline GWM473012X & MIDDLE-2050A & 1005 & Iron & 9.4 & & u & & UG/L & $05 / 24 / 2006$ & & DNT-284-06 \\
\hline GWM473012X & MIDDLE-2050A & 1005 & Lead & 0.49 & & U & & UG/L & 05/24/2006 & & DNT-284-06 \\
\hline GWM473012X & MIDDLE-2050A & 1005 & Magnesium & 16700 & & $\mathrm{E}$ & $\mathrm{J}$ & UG/L & $05 / 24 / 2006$ & & DNT-284-06 \\
\hline GWM473012X & MIDDLE-2050A & 1005 & Manganese & 0.52 & & B & $u$ & UG/L & 05/24/2006 & & DNT-284-06 \\
\hline GWM473012X & MIDDLE-2050A & 1005 & Nickel & 1.3 & & B & & UG/L & $05 / 24 / 2006$ & & DNT-284-06 \\
\hline GWM473012X & MIDDLE-2050A & 1005 & Potassium & 1630 & & & & UG/L & $05 / 24 / 2006$ & & DNT-284-06 \\
\hline GWM473012X & MIDDLE-2050A & 1005 & Selenium & 1 & & U & & UG/L & $05 / 24 / 2006$ & & DNT-284-06 \\
\hline GWM473012X & MIDDLE-2050A & 1005 & Silver & 0.2 & & U & & UG/L & $05 / 24 / 2006$ & & DNT-284-06 \\
\hline GWM473012X & MIDDLE-2050A & 1005 & Sodium & 7770 & & E & $\mathrm{J}$ & UG/L & $05 / 24 / 2006$ & & DNT-284-06 \\
\hline GWM473012X & MIDDLE-2050A & 1005 & Strontium & 205 & & $\mathrm{E}$ & $\mathrm{J}$ & UG/L & $05 / 24 / 2006$ & & DNT-284-06 \\
\hline GWM473012X & MIDDLE-2050A & 1005 & Thallium & 0.32 & & $\bar{u}$ & & UG/L & $05 / 24 / 2006$ & & DNT-284-06 \\
\hline GWM473012X & MIDDLE-2050A & 1005 & Uranium & 1.5 & & & & UG/L & $05 / 24 / 2006$ & & DNT-284-06 \\
\hline GWM473012X & MIDDLE-2050A & 1005 & Vanadium & 2.7 & & B & & UG/L & 05/24/2006 & & DNT-284-06 \\
\hline GWM473012X & MIDDLE-2050A & 1005 & Zinc & 13.7 & & & & UG/L & $05 / 24 / 2006$ & & DNT-284-06 \\
\hline GWM473012X & MIDDLE-2050A & 1005 & Mercury & 0.093 & & $\mathrm{U}$ & & UG/L & $05 / 24 / 2006$ & & DNT-284-06 \\
\hline GWM47301A1 & MIDDLE-2050A & 1005 & Total Alkalinity & 164 & & & & $\mathrm{MG} / \mathrm{L}$ & $05 / 24 / 2006$ & & DNT-326-06 \\
\hline GWM47301AN & MIDDLE-2050A & 1005 & Chloride & 9.7 & & & & MG/L & $05 / 24 / 2006$ & & DNT-326-06 \\
\hline GWM47301AN & MIDDLE-2050A & 1005 & Fluoride & 0.15 & & & & $\mathrm{MG} / \mathrm{L}$ & $05 / 24 / 2006$ & & DNT-326-06 \\
\hline GWM47301AN & MIDDLE-2050A & 1005 & Sulfate & 19.7 & & & & MG/L & $05 / 24 / 2006$ & & DNT-326-06 \\
\hline GWM47301N2 & MIDDLE-2050A & 1005 & Nitrate/Nitrite as N & 907 & & & & UG/L & $05 / 24 / 2006$ & & DNT-326-06 \\
\hline GWM47301VL & MIDDLE-2050A & 1005 & Acetone & 2 & & $\mathrm{U}$ & $\mathrm{R}$ & UG/L & $05 / 24 / 2006$ & & JGJ-083-06 \\
\hline GWM47301VL & MIDDLE-2050A & 1005 & Benzene & 1 & & u & & UG/L & $05 / 24 / 2006$ & & JGJ-083-06 \\
\hline GWM47301VL & MIDDLE-2050A & 1005 & Bromodichloromethane & 1 & & $\mathrm{U}$ & & UG/L & 05/24/2006 & & JGJ-083-06 \\
\hline GWM47301VL & MIDDLE-2050A & 1005 & Bromoform & 1 & & U & & UG/L & $05 / 24 / 2006$ & & JGJ-083-06 \\
\hline GWM47301VL & MIDDLE-2050A & 1005 & Bromomethane & 2 & & $\mathrm{U}$ & & UG/L & $05 / 24 / 2006$ & & JGJ-083-06 \\
\hline GWM47301VL & MIDDLE-2050A & 1005 & 2-Butanone & 5 & & U & $\mathrm{R}$ & UG/L & $05 / 24 / 2006$ & & JGJ-083-06 \\
\hline GWM47301VL & MIDDLE-2050A & 1005 & Carbon disulfide & 1 & & u & & UG/L & $05 / 24 / 2006$ & & JGJ-083-06 \\
\hline GWM47301VL & MIDDLE-2050A & 1005 & Carbon tetrachloride & 1 & & $\mathrm{U}$ & & UG/L & $05 / 24 / 2006$ & & JGJ-083-06 \\
\hline GWM47301VL & MIDDLE-2050A & 1005 & Chlorobenzene & 1 & & $\mathrm{u}$ & & UG/L & $05 / 24 / 2006$ & & JGJ-083-06 \\
\hline GWM47301VL & MIDDLE-2050A & 1005 & Dibromochloromethane & 1 & & $\mathrm{u}$ & & UG/L & $05 / 24 / 2006$ & & JGJ-083-06 \\
\hline GWM47301VL & MIDDLE-2050A & 1005 & 1,2-Dibromo-3-chloropropane & 1 & & u & $\mathrm{R}$ & UG/L & $05 / 24 / 2006$ & & JGJ-083-06 \\
\hline GWM47301VL & MIDDLE-2050A & 1005 & Chloroethane & 2 & & $\mathrm{u}$ & & UG/L & $05 / 24 / 2006$ & & JGJ-083-06 \\
\hline GWM47301VL & MIDDLE-2050A & 1005 & Chloroform & 1 & & u & & UG/L & $05 / 24 / 2006$ & & JGJ-083-06 \\
\hline GWM47301VL & MIDDLE-2050A & 1005 & Chloromethane & 2 & & $\mathrm{u}$ & & UG/L & $05 / 24 / 2006$ & & JGJ-083-06 \\
\hline GWM47301VL & MIDDLE-2050A & 1005 & Cyclohexane & 1 & & u & & UG/L & $05 / 24 / 2006$ & & JGJ-083-06 \\
\hline GWM47301VL & MIDDLE-2050A & 1005 & 1,2-Dibromoethane & 1 & & $\mathrm{u}$ & & UG/L & $05 / 24 / 2006$ & & JGJ-083-06 \\
\hline GWM47301VL & MIDDLE-2050A & 1005 & 1,2-Dichlorobenzene & 1 & & $\mathrm{u}$ & & UG/L & $05 / 24 / 2006$ & & JGJ-083-06 \\
\hline GWM47301VL & MIDDLE-2050A & 1005 & 1,3-Dichlorobenzene & 1 & & $\mathrm{U}$ & & UG/L & $05 / 24 / 2006$ & & JGJ-083-06 \\
\hline GWM47301VL & MIDDLE-2050A & 1005 & 1,4-Dichlorobenzene & 1 & & $\mathrm{u}$ & & UG/L & $05 / 24 / 2006$ & & JGJ-083-06 \\
\hline GWM47301VL & MIDDLE-2050A & 1005 & Dichlorodifluoromethane & 2 & & $\mathrm{u}$ & & UG/L & $05 / 24 / 2006$ & & JGJ-083-06 \\
\hline GWM47301VL & MIDDLE-2050A & 1005 & 1,1-Dichloroethane & 1 & & $\mathrm{u}$ & & UG/L & $05 / 24 / 2006$ & & JGJ-083-06 \\
\hline GWM47301VL & MIDDLE-2050A & 1005 & 1,2-Dichloroethane & 1 & & u & & UG/L & $05 / 24 / 2006$ & & JGJ-083-06 \\
\hline GWM47301VL & MIDDLE-2050A & 1005 & 1,1-Dichloroethene & 1 & & $\mathrm{U}$ & & UG/L & $05 / 24 / 2006$ & & JGJ-083-06 \\
\hline GWM47301VL & MIDDLE-2050A & 1005 & cis-1,2-Dichloroethene & 1 & & $\mathrm{u}$ & & UG/L & $05 / 24 / 2006$ & & JGJ-083-06 \\
\hline GWM47301VL & MIDDLE-2050A & 1005 & trans-1,2-Dichloroethene & 1 & & $\mathrm{U}$ & & UG/L & $05 / 24 / 2006$ & & JGJ-083-06 \\
\hline GWM47301VL & MIDDLE-2050A & 1005 & 1,2-Dichloropropane & 1 & & u & & UG/L & $05 / 24 / 2006$ & & JGJ-083-06 \\
\hline GWM47301VL & MIDDLE-2050A & 1005 & cis-1,3-Dichloropropene & 1 & & $\mathrm{U}$ & & UG/L & $05 / 24 / 2006$ & & JGJ-083-06 \\
\hline GWM47301VL & MIDDLE-2050A & 1005 & trans-1,3-Dichloropropene & 1 & & $\mathrm{u}$ & & UG/L & $05 / 24 / 2006$ & & JGJ-083-06 \\
\hline GWM47301VL & MIDDLE-2050A & 1005 & Ethylbenzene & 1 & & $\mathrm{u}$ & & UG/L & $05 / 24 / 2006$ & & JGJ-083-06 \\
\hline GWM47301VL & MIDDLE-2050A & 1005 & Trichlorofluoromethane & 1 & & u & & UG/L & $05 / 24 / 2006$ & & JGJ-083-06 \\
\hline GWM47301VL & MIDDLE-2050A & 1005 & 2-Hexanone & 5 & & $\mathrm{U}$ & & UG/L & $05 / 24 / 2006$ & & JGJ-083-06 \\
\hline GWM47301VL & MIDDLE-2050A & 1005 & Isopropylbenzene & 1 & & u & & UG/L & $05 / 24 / 2006$ & & JGJ-083-06 \\
\hline GWM47301VL & MIDDLE-2050A & 1005 & Methyl acetate & 5 & & $\mathrm{u}$ & $\mathrm{R}$ & UG/L & $05 / 24 / 2006$ & & JGJ-083-06 \\
\hline GWM47301VL & MIDDLE-2050A & 1005 & Methyl cyclohexane & 4 & & $\mathrm{U}$ & & UG/L & $05 / 24 / 2006$ & & JGJ-083-06 \\
\hline GWM47301VL & MIDDLE-2050A & 1005 & Methylene Chloride & 1 & & $\mathrm{U}$ & & UG/L & $05 / 24 / 2006$ & & JGJ-083-06 \\
\hline GWM47301VL & MIDDLE-2050A & 1005 & 4-Methyl-2-pentanone & 5 & & u & & UG/L & $05 / 24 / 2006$ & & JGJ-083-06 \\
\hline GWM47301VL & MIDDLE-2050A & 1005 & Styrene & 1 & & u & & UG/L & $05 / 24 / 2006$ & & JGJ-083-06 \\
\hline GWM47301VL & MIDDLE-2050A & 1005 & 1,1,2,2-Tetrachloroethane & 1 & & $\mathrm{U}$ & & UG/L & $05 / 24 / 2006$ & & JGJ-083-06 \\
\hline GWM47301VL & MIDDLE-2050A & 1005 & Tetrachloroethene & 1 & & $\mathrm{U}$ & & UG/L & $05 / 24 / 2006$ & & JGJ-083-06 \\
\hline GWM47301VL & MIDDLE-2050A & 1005 & Toluene & 1 & & $\mathrm{U}$ & & UG/L & $05 / 24 / 2006$ & & JGJ-083-06 \\
\hline GWM47301VL & MIDDLE-2050A & 1005 & 1,2,4-Trichlorobenzene & 1 & & u & & UG/L & $05 / 24 / 2006$ & & JGJ-083-06 \\
\hline GWM47301VL & MIDDLE-2050A & 1005 & 1,1,1-Trichloroethane & 1 & & u & & UG/L & $05 / 24 / 2006$ & & JGJ-083-06 \\
\hline GWM47301VL & MIDDLE-2050A & 1005 & 1,1,2-Trichloroethane & 1 & & U & & UG/L & $05 / 24 / 2006$ & & JGJ-083-06 \\
\hline GWM47301VL & MIDDLE-2050A & 1005 & Trichloroethene & 1 & & $\mathrm{U}$ & & UG/L & 05/24/2006 & & JGJ-083-06 \\
\hline GWM47301VL & MIDDLE-2050A & 1005 & 1,1,2-Trichloro-1,2,2-trifluoroethane & 1 & & u & & UG/L & $05 / 24 / 2006$ & & JGJ-083-06 \\
\hline GWM47301VL & MIDDLE-2050A & 1005 & Vinyl Chloride & 2 & & $\mathrm{u}$ & & UG/L & $05 / 24 / 2006$ & & JGJ-083-06 \\
\hline GWM47301VL & MIDDLE-2050A & 1005 & Xylenes & 3 & & u & & UG/L & $05 / 24 / 2006$ & & JGJ-083-06 \\
\hline GWM47301VL & MIDDLE-2050A & 1005 & Methyl t-butyl ether & 2 & & $\mathrm{u}$ & & UG/L & 05/24/2006 & & JGJ-083-06 \\
\hline GWM47301R8 & MIDDLE-2050A & 1005 & Tritium & $-3.53 E+01$ & $1.13 \mathrm{E}+02$ & & $\mathrm{u}$ & $\mathrm{PCI} / \mathrm{L}$ & 05/24/2006 & $3.87 \mathrm{E}+02$ & SOS-TL176-06 \\
\hline GWM47301RH & MIDDLE-2050A & 1005 & Gross Alpha & $1.84 \mathrm{E}+00$ & 3.18E-01 & & & $\mathrm{PCI} / \mathrm{L}$ & 05/24/2006 & $6.42 \mathrm{E}-01$ & SOS-TL177-06 \\
\hline GWM47301RH & MIDDLE-2050A & 1005 & Gross Beta & $3.91 \mathrm{E}+00$ & 3.97E-01 & & & $\mathrm{PCl} / \mathrm{L}$ & 05/24/2006 & $1.08 \mathrm{E}+00$ & SOS-TL177-06 \\
\hline GWM47301RH & MIDDLE-2050A & 1005 & Strontium-90 & 4.23E-01 & $1.23 \mathrm{E}-01$ & & $\mathrm{~J}$ & $\mathrm{PCI} / \mathrm{L}$ & $05 / 24 / 2006$ & 4.66E-01 & SOS-TL177-06 \\
\hline GWM47301RH & MIDDLE-2050A & 1005 & Technetium-99 & $2.63 \mathrm{E}+00$ & $1.68 \mathrm{E}+00$ & & u & $\mathrm{PCI} / \mathrm{L}$ & $05 / 24 / 2006$ & $5.52 \mathrm{E}+00$ & SOS-TL177-06 \\
\hline GWM47301UX & MIDDLE-2050A & 1005 & lodine-129 & 1.35E-02 & $1.84 \mathrm{E}-02$ & & $u$ & $\mathrm{PCI} / \mathrm{L}$ & 05/24/2006 & 7.87E-02 & SOS-TL174-06 \\
\hline GWM474012X & MIDDLE-2050A & 1212 & Aluminum & 7.9 & & U & & UG/L & 05/23/2006 & & DNT-272-06 \\
\hline GWM474012X & MIDDLE-2050A & 1212 & Antimony & 0.5 & & U & & UG/L & $05 / 23 / 2006$ & & DNT-272-06 \\
\hline GWM474012X & MIDDLE-2050A & 1212 & Arsenic & 2 & & $u$ & & UG/L & $05 / 23 / 2006$ & & DNT-272-06 \\
\hline GWM474012X & MIDDLE-2050A & 1212 & Barium & 87.7 & & & & UG/L & 05/23/2006 & & DNT-272-06 \\
\hline GWM474012X & MIDDLE-2050A & 1212 & Beryllium & 0.088 & & $\mathrm{U}$ & & UG/L & $05 / 23 / 2006$ & & DNT-272-06 \\
\hline GWM474012X & MIDDLE-2050A & 1212 & Cadmium & 0.06 & & B & & UG/L & $05 / 23 / 2006$ & & DNT-272-06 \\
\hline GWM474012X & MIDDLE-2050A & 1212 & Calcium & 41200 & & & & UG/L & $05 / 23 / 2006$ & & DNT-272-06 \\
\hline GWM474012X & MIDDLE-2050A & 1212 & Chromium & 2.8 & & $\mathrm{U}$ & & UG/L & $05 / 23 / 2006$ & & DNT-272-06 \\
\hline
\end{tabular}


Table A-5. (continued).

\begin{tabular}{|c|c|c|c|c|c|c|c|c|c|c|c|}
\hline $\begin{array}{l}\text { Field Sample } \\
\text { Number }\end{array}$ & Location & Depth & Compound & $\begin{array}{l}\text { Sample } \\
\text { Result }\end{array}$ & $\begin{array}{c}\text { Sample } \\
\text { Error }\end{array}$ & $\begin{array}{c}\text { Result } \\
\text { Qualifier }\end{array}$ & $\begin{array}{l}\text { Validation } \\
\text { Flag }\end{array}$ & $\begin{array}{l}\text { Sample } \\
\text { Units }\end{array}$ & $\begin{array}{c}\text { Date Sample } \\
\text { Collected }\end{array}$ & MDA & $\begin{array}{l}\text { L\&V Report } \\
\text { Number }\end{array}$ \\
\hline GWM474012X & MIDDLE-2050A & 1212 & Cobalt & 2.8 & & & & UG/L & $05 / 23 / 2006$ & & DNT-272-06 \\
\hline GWM474012X & MIDDLE-2050A & 1212 & Copper & 0.41 & & B & & UG/L & $05 / 23 / 2006$ & & DNT-272-06 \\
\hline GWM474012X & MIDDLE-2050A & 1212 & Iron & 9.4 & & U & & UG/L & $05 / 23 / 2006$ & & DNT-272-06 \\
\hline GWM474012X & MIDDLE-2050A & 1212 & Lead & 0.49 & & $\mathrm{U}$ & & UG/L & 05/23/2006 & & DNT-272-06 \\
\hline GWM474012X & MIDDLE-2050A & 1212 & Magnesium & 16000 & & & & UG/L & $05 / 23 / 2006$ & & DNT-272-06 \\
\hline GWM474012X & MIDDLE-2050A & 1212 & Manganese & 424 & & & & UG/L & 05/23/2006 & & DNT-272-06 \\
\hline GWM474012X & MIDDLE-2050A & 1212 & Nickel & 2.4 & & B & & UG/L & $05 / 23 / 2006$ & & DNT-272-06 \\
\hline GWM474012X & MIDDLE-2050A & 1212 & Potassium & 2450 & & & & UG/L & 05/23/2006 & & DNT-272-06 \\
\hline GWM474012X & MIDDLE-2050A & 1212 & Selenium & 1 & & $\mathrm{U}$ & & UG/L & $05 / 23 / 2006$ & & DNT-272-06 \\
\hline GWM474012X & MIDDLE-2050A & 1212 & Silver & 0.2 & & U & & UG/L & $05 / 23 / 2006$ & & DNT-272-06 \\
\hline GWM474012X & MIDDLE-2050A & 1212 & Sodium & 13100 & & & & UG/L & $05 / 23 / 2006$ & & DNT-272-06 \\
\hline GWM474012X & MIDDLE-2050A & 1212 & Strontium & 291 & & & & UG/L & $05 / 23 / 2006$ & & DNT-272-06 \\
\hline GWM474012X & MIDDLE-2050A & 1212 & Thallium & 1.1 & & B & $u$ & UG/L & $05 / 23 / 2006$ & & DNT-272-06 \\
\hline GWM474012X & MIDDLE-2050A & 1212 & Uranium & 0.55 & & B & & UG/L & $05 / 23 / 2006$ & & DNT-272-06 \\
\hline GWM474012X & MIDDLE-2050A & 1212 & Vanadium & 1.6 & & u & & UG/L & $05 / 23 / 2006$ & & DNT-272-06 \\
\hline GWM474012X & MIDDLE-2050A & 1212 & Zinc & 10.9 & & & & UG/L & $05 / 23 / 2006$ & & DNT-272-06 \\
\hline GWM474012X & MIDDLE-2050A & 1212 & Mercury & 0.093 & & $u$ & & UG/L & 05/23/2006 & & DNT-272-06 \\
\hline GWM47401A1 & MIDDLE-2050A & 1212 & Total Alkalinity & 172 & & & & MG/L & 05/23/2006 & & DNT-270-06 \\
\hline GWM47401AN & MIDDLE-2050A & 1212 & Chloride & 16 & & $\mathrm{~J}$ & & $M G / L$ & $05 / 23 / 2006$ & & DNT-270-06 \\
\hline GWM47401AN & MIDDLE-2050A & 1212 & Fluoride & 0.18 & & & & $\mathrm{MG} / \mathrm{L}$ & $05 / 23 / 2006$ & & DNT-270-06 \\
\hline GWM47401AN & MIDDLE-2050A & 1212 & Sulfate & 17.4 & & $\mathrm{~J}$ & $\mathrm{~J}$ & MG/L & $05 / 23 / 2006$ & & DNT-270-06 \\
\hline GWM47401N2 & MIDDLE-2050A & 1212 & Nitrate/Nitrite as $\mathrm{N}$ & 50 & & U & & UG/L & $05 / 23 / 2006$ & & DNT-270-06 \\
\hline GWM47401VL & MIDDLE-2050A & 1212 & Acetone & 2 & & $u$ & $\mathrm{R}$ & UG/L & $05 / 23 / 2006$ & & JGJ-083-06 \\
\hline GWM47401VL & MIDDLE-2050A & 1212 & Benzene & 1 & & U & & UG/L & $05 / 23 / 2006$ & & JGJ-083-06 \\
\hline GWM47401VL & MIDDLE-2050A & 1212 & Bromodichloromethane & 1 & & $\mathrm{U}$ & & UG/L & 05/23/2006 & & JGJ-083-06 \\
\hline GWM47401VL & MIDDLE-2050A & 1212 & Bromoform & 1 & & u & & UG/L & $05 / 23 / 2006$ & & JGJ-083-06 \\
\hline GWM47401VL & MIDDLE-2050A & 1212 & Bromomethane & 2 & & u & & UG/L & $05 / 23 / 2006$ & & JGJ-083-06 \\
\hline GWM47401VL & MIDDLE-2050A & 1212 & 2-Butanone & 5 & & U & $\mathrm{R}$ & UG/L & $05 / 23 / 2006$ & & JGJ-083-06 \\
\hline GWM47401VL & MIDDLE-2050A & 1212 & Carbon disulfide & 1 & & $\mathrm{U}$ & & UG/L & 05/23/2006 & & JGJ-083-06 \\
\hline GWM47401VL & MIDDLE-2050A & 1212 & Carbon tetrachloride & 1 & & U & & UG/L & $05 / 23 / 2006$ & & JGJ-083-06 \\
\hline GWM47401VL & MIDDLE-2050A & 1212 & Chlorobenzene & 1 & & u & & UG/L & $05 / 23 / 2006$ & & JGJ-083-06 \\
\hline GWM47401VL & MIDDLE-2050A & 1212 & Dibromochloromethane & 1 & & $\mathrm{u}$ & & UG/L & $05 / 23 / 2006$ & & JGJ-083-06 \\
\hline GWM47401VL & MIDDLE-2050A & 1212 & 1,2-Dibromo-3-chloropropane & 1 & & $\mathrm{u}$ & $\mathrm{R}$ & UG/L & $05 / 23 / 2006$ & & JGJ-083-06 \\
\hline GWM47401VL & MIDDLE-2050A & 1212 & Chloroethane & 2 & & $\mathrm{u}$ & & UG/L & $05 / 23 / 2006$ & & JGJ-083-06 \\
\hline GWM47401VL & MIDDLE-2050A & 1212 & Chloroform & 1 & & u & & UG/L & $05 / 23 / 2006$ & & JGJ-083-06 \\
\hline GWM47401VL & MIDDLE-2050A & 1212 & Chloromethane & 0.35 & & $\mathrm{~J}$ & $\mathrm{~J}$ & UG/L & $05 / 23 / 2006$ & & JGJ-083-06 \\
\hline GWM47401VL & MIDDLE-2050A & 1212 & Cyclohexane & 1 & & u & & UG/L & $05 / 23 / 2006$ & & JGJ-083-06 \\
\hline GWM47401VL & MIDDLE-2050A & 1212 & 1,2-Dibromoethane & 1 & & $\mathrm{u}$ & & UG/L & $05 / 23 / 2006$ & & JGJ-083-06 \\
\hline GWM47401VL & MIDDLE-2050A & 1212 & 1,2-Dichlorobenzene & 1 & & $\mathrm{u}$ & & UG/L & $05 / 23 / 2006$ & & JGJ-083-06 \\
\hline GWM47401VL & MIDDLE-2050A & 1212 & 1,3-Dichlorobenzene & 1 & & $\mathrm{u}$ & & UG/L & 05/23/2006 & & JGJ-083-06 \\
\hline GWM47401VL & MIDDLE-2050A & 1212 & 1,4-Dichlorobenzene & 1 & & $\mathrm{u}$ & & UG/L & 05/23/2006 & & JGJ-083-06 \\
\hline GWM47401VL & MIDDLE-2050A & 1212 & Dichlorodifluoromethane & 2 & & $\mathrm{U}$ & & UG/L & $05 / 23 / 2006$ & & JGJ-083-06 \\
\hline GWM47401VL & MIDDLE-2050A & 1212 & 1,1-Dichloroethane & 1 & & $\mathrm{u}$ & & UG/L & $05 / 23 / 2006$ & & JGJ-083-06 \\
\hline GWM47401VL & MIDDLE-2050A & 1212 & 1,2-Dichloroethane & 1 & & $\mathrm{u}$ & & UG/L & $05 / 23 / 2006$ & & JGJ-083-06 \\
\hline GWM47401VL & MIDDLE-2050A & 1212 & 1,1-Dichloroethene & 1 & & $\mathrm{u}$ & & UG/L & $05 / 23 / 2006$ & & JGJ-083-06 \\
\hline GWM47401VL & MIDDLE-2050A & 1212 & cis-1,2-Dichloroethene & 1 & & u & & UG/L & $05 / 23 / 2006$ & & JGJ-083-06 \\
\hline GWM47401VL & MIDDLE-2050A & 1212 & trans-1,2-Dichloroethene & 1 & & $\mathrm{u}$ & & UG/L & $05 / 23 / 2006$ & & JGJ-083-06 \\
\hline GWM47401VL & MIDDLE-2050A & 1212 & 1,2-Dichloropropane & 1 & & u & & UG/L & $05 / 23 / 2006$ & & JGJ-083-06 \\
\hline GWM47401VL & MIDDLE-2050A & 1212 & cis-1,3-Dichloropropene & 1 & & $\mathrm{u}$ & & UG/L & $05 / 23 / 2006$ & & JGJ-083-06 \\
\hline GWM47401VL & MIDDLE-2050A & 1212 & trans-1,3-Dichloropropene & 1 & & u & & UG/L & $05 / 23 / 2006$ & & JGJ-083-06 \\
\hline GWM47401VL & MIDDLE-2050A & 1212 & Ethylbenzene & 1 & & $u$ & & UG/L & $05 / 23 / 2006$ & & JGJ-083-06 \\
\hline GWM47401VL & MIDDLE-2050A & 1212 & Trichlorofluoromethane & 1 & & u & & UG/L & $05 / 23 / 2006$ & & JGJ-083-06 \\
\hline GWM47401VL & MIDDLE-2050A & 1212 & 2-Hexanone & 5 & & $\mathrm{u}$ & & UG/L & $05 / 23 / 2006$ & & JGJ-083-06 \\
\hline GWM47401VL & MIDDLE-2050A & 1212 & Isopropylbenzene & 1 & & u & & UG/L & $05 / 23 / 2006$ & & JGJ-083-06 \\
\hline GWM47401VL & MIDDLE-2050A & 1212 & Methyl acetate & 5 & & $u$ & $\mathrm{R}$ & UG/L & $05 / 23 / 2006$ & & JGJ-083-06 \\
\hline GWM47401VL & MIDDLE-2050A & 1212 & Methyl cyclohexane & 4 & & u & & UG/L & $05 / 23 / 2006$ & & JGJ-083-06 \\
\hline GWM47401VL & MIDDLE-2050A & 1212 & Methylene Chloride & 1 & & $\mathrm{u}$ & & UG/L & $05 / 23 / 2006$ & & JGJ-083-06 \\
\hline GWM47401VL & MIDDLE-2050A & 1212 & 4-Methyl-2-pentanone & 5 & & $\mathrm{U}$ & & UG/L & $05 / 23 / 2006$ & & JGJ-083-06 \\
\hline GWM47401VL & MIDDLE-2050A & 1212 & Styrene & 1 & & $\mathrm{U}$ & & UG/L & $05 / 23 / 2006$ & & JGJ-083-06 \\
\hline GWM47401VL & MIDDLE-2050A & 1212 & 1,1,2,2-Tetrachloroethane & 1 & & u & & UG/L & $05 / 23 / 2006$ & & JGJ-083-06 \\
\hline GWM47401VL & MIDDLE-2050A & 1212 & Tetrachloroethene & 1 & & $\mathrm{u}$ & & UG/L & $05 / 23 / 2006$ & & JGJ-083-06 \\
\hline GWM47401VL & MIDDLE-2050A & 1212 & Toluene & 1 & & $\mathrm{U}$ & & UG/L & 05/23/2006 & & JGJ-083-06 \\
\hline GWM47401VL & MIDDLE-2050A & 1212 & 1,2,4-Trichlorobenzene & 1 & & $\mathrm{U}$ & & UG/L & 05/23/2006 & & JGJ-083-06 \\
\hline GWM47401VL & MIDDLE-2050A & 1212 & 1,1,1-Trichloroethane & 1 & & $\mathrm{U}$ & & UG/L & 05/23/2006 & & JGJ-083-06 \\
\hline GWM47401VL & MIDDLE-2050A & 1212 & 1,1,2-Trichloroethane & 1 & & u & & UG/L & $05 / 23 / 2006$ & & JGJ-083-06 \\
\hline GWM47401VL & MIDDLE-2050A & 1212 & Trichloroethene & 1 & & u & & UG/L & $05 / 23 / 2006$ & & JGJ-083-06 \\
\hline GWM47401VL & MIDDLE-2050A & 1212 & 1,1,2-Trichloro-1,2,2-trifluoroethane & 1 & & u & & UG/L & 05/23/2006 & & JGJ-083-06 \\
\hline GWM47401VL & MIDDLE-2050A & 1212 & Vinyl Chloride & 2 & & U & & UG/L & 05/23/2006 & & JGJ-083-06 \\
\hline GWM47401VL & MIDDLE-2050A & 1212 & Xylenes & 3 & & u & & UG/L & $05 / 23 / 2006$ & & JGJ-083-06 \\
\hline GWM47401VL & MIDDLE-2050A & 1212 & Methyl t-butyl ether & 2 & & $u$ & & UG/L & 05/23/2006 & & JGJ-083-06 \\
\hline GWM47401R8 & MIDDLE-2050A & 1212 & Tritium & $-1.62 E+02$ & $1.12 \mathrm{E}+02$ & & $u$ & $\mathrm{PCI} / \mathrm{L}$ & $05 / 23 / 2006$ & $3.90 \mathrm{E}+02$ & SOS-TL176-06 \\
\hline GWM47401RH & MIDDLE-2050A & 1212 & Gross Alpha & 8.37E-01 & $2.47 \mathrm{E}-01$ & & $\mathrm{~J}$ & $\mathrm{PCI} / \mathrm{L}$ & 05/23/2006 & $6.07 \mathrm{E}-01$ & SOS-TL177-06 \\
\hline GWM47401RH & MIDDLE-2050A & 1212 & Gross Beta & $5.72 \mathrm{E}+00$ & 4.29E-01 & & $\mathrm{J}$ & $\mathrm{PCl} / \mathrm{L}$ & 05/23/2006 & $1.12 \mathrm{E}+00$ & SOS-TL177-06 \\
\hline GWM47401RH & MIDDLE-2050A & 1212 & Strontium-90 & 3.71E-01 & 1.19E-01 & & $\mathrm{J}$ & $\mathrm{PCI} / \mathrm{L}$ & 05/23/2006 & 4.56E-01 & SOS-TL177-06 \\
\hline GWM47401RH & MIDDLE-2050A & 1212 & Technetium-99 & 1.97E-01 & $1.63 \mathrm{E}+00$ & & UJ & $\mathrm{PCI} / \mathrm{L}$ & 05/23/2006 & $5.53 \mathrm{E}+00$ & SOS-TL177-06 \\
\hline GWM47401UX & MIDDLE-2050A & 1212 & lodine-129 & 5.36E-02 & 3.03E-02 & & u & $\mathrm{PCI} / \mathrm{L}$ & $05 / 23 / 2006$ & $1.09 \mathrm{E}-01$ & SOS-TL174-06 \\
\hline GWM475012X & MIDDLE-2051 & 608 & Aluminum & 7.9 & & $\mathrm{u}$ & & UG/L & $06 / 02 / 2006$ & & DNT-274-06 \\
\hline GWM475012X & MIDDLE-2051 & 608 & Antimony & 0.59 & & B & & UG/L & $06 / 02 / 2006$ & & DNT-274-06 \\
\hline GWM475012X & MIDDLE-2051 & 608 & Arsenic & 2 & & $u$ & & UG/L & 06/02/2006 & & DNT-274-06 \\
\hline GWM475012X & MIDDLE-2051 & 608 & Barium & 56.8 & & & & UG/L & $06 / 02 / 2006$ & & DNT-274-06 \\
\hline GWM475012X & MIDDLE-2051 & 608 & Beryllium & 0.088 & & $\mathrm{U}$ & & UG/L & $06 / 02 / 2006$ & & DNT-274-06 \\
\hline GWM475012X & MIDDLE-2051 & 608 & Cadmium & 0.057 & & $\mathrm{U}$ & & UG/L & 06/02/2006 & & DNT-274-06 \\
\hline GWM475012X & MIDDLE-2051 & 608 & Calcium & 45600 & & $\mathrm{E}$ & $\mathrm{J}$ & UG/L & $06 / 02 / 2006$ & & DNT-274-06 \\
\hline GWM475012X & MIDDLE-2051 & 608 & Chromium & 40 & & $\mathrm{~N}$ & $\mathrm{~J}$ & UG/L & $06 / 02 / 2006$ & & DNT-274-06 \\
\hline GWM475012X & MIDDLE-2051 & 608 & Cobalt & 0.31 & & U & & UG/L & $06 / 02 / 2006$ & & DNT-274-06 \\
\hline GWM475012X & MIDDLE-2051 & 608 & Copper & 2.3 & & & & UG/L & $06 / 02 / 2006$ & & DNT-274-06 \\
\hline
\end{tabular}


Table A-5. (continued).

\begin{tabular}{|c|c|c|c|c|c|c|c|c|c|c|c|}
\hline $\begin{array}{c}\text { Field Sample } \\
\text { Number }\end{array}$ & Location & Depth & Compound & $\begin{array}{l}\text { Sample } \\
\text { Result }\end{array}$ & $\begin{array}{l}\text { Sample } \\
\text { Error }\end{array}$ & $\begin{array}{c}\text { Result } \\
\text { Qualifier }\end{array}$ & $\begin{array}{c}\text { Validation } \\
\text { Flag }\end{array}$ & $\begin{array}{l}\text { Sample } \\
\text { Units }\end{array}$ & $\begin{array}{c}\text { Date Sample } \\
\text { Collected }\end{array}$ & MDA & $\begin{array}{l}\text { L\&V Report } \\
\text { Number }\end{array}$ \\
\hline GWM475012X & MIDDLE-2051 & 608 & Iron & 209 & & $\mathrm{~N}$ & $\mathrm{~J}$ & UG/L & 06/02/2006 & & DNT-274-06 \\
\hline GWM475012X & MIDDLE-2051 & 608 & Lead & 0.49 & & $\mathrm{U}$ & & UG/L & $06 / 02 / 2006$ & & DNT-274-06 \\
\hline GWM475012X & MIDDLE-2051 & 608 & Magnesium & 11200 & & E & & UG/L & $06 / 02 / 2006$ & & DNT-274-06 \\
\hline GWM475012X & MIDDLE-2051 & 608 & Manganese & 4.3 & & & & UG/L & $06 / 02 / 2006$ & & DNT-274-06 \\
\hline GWM475012X & MIDDLE-2051 & 608 & Nickel & 1.8 & & B & & UG/L & $06 / 02 / 2006$ & & DNT-274-06 \\
\hline GWM475012X & MIDDLE-2051 & 608 & Potassium & 2200 & & & & UG/L & $06 / 02 / 2006$ & & DNT-274-06 \\
\hline GWM475012X & MIDDLE-2051 & 608 & Selenium & 2.1 & & B & $u$ & UG/L & $06 / 02 / 2006$ & & DNT-274-06 \\
\hline GWM475012X & MIDDLE-2051 & 608 & Silver & 0.2 & & $u$ & & UG/L & 06/02/2006 & & DNT-274-06 \\
\hline GWM475012X & MIDDLE-2051 & 608 & Sodium & 5820 & & & & UG/L & $06 / 02 / 2006$ & & DNT-274-06 \\
\hline GWM475012X & MIDDLE-2051 & 608 & Strontium & 267 & & E & $\mathrm{J}$ & UG/L & $06 / 02 / 2006$ & & DNT-274-06 \\
\hline GWM475012X & MIDDLE-2051 & 608 & Thallium & 0.92 & & B & $u$ & UG/L & $06 / 02 / 2006$ & & DNT-274-06 \\
\hline GWM475012X & MIDDLE-2051 & 608 & Uranium & 2.2 & & & & UG/L & $06 / 02 / 2006$ & & DNT-274-06 \\
\hline GWM475012X & MIDDLE-2051 & 608 & Vanadium & 4.3 & & B & & UG/L & $06 / 02 / 2006$ & & DNT-274-06 \\
\hline GWM475012X & MIDDLE-2051 & 608 & Zinc & 9.8 & & B & & UG/L & $06 / 02 / 2006$ & & DNT-274-06 \\
\hline GWM475012X & MIDDLE-2051 & 608 & Mercury & 0.093 & & U & & UG/L & $06 / 02 / 2006$ & & DNT-274-06 \\
\hline GWM47501A1 & MIDDLE-2051 & 608 & Total Alkalinity & 150 & & & $\mathrm{~J}$ & MG/L & $06 / 02 / 2006$ & & DNT-279-06 \\
\hline GWM47501AN & MIDDLE-2051 & 608 & Chloride & 6.9 & & $\mathrm{~J}$ & & MG/L & 06/02/2006 & & DNT-279-06 \\
\hline GWM47501AN & MIDDLE-2051 & 608 & Fluoride & 0.2 & & & & MG/L & $06 / 02 / 2006$ & & DNT-279-06 \\
\hline GWM47501AN & MIDDLE-2051 & 608 & Sulfate & 20.2 & & & $\mathrm{~J}$ & MG/L & $06 / 02 / 2006$ & & DNT-279-06 \\
\hline GWM47501N2 & MIDDLE-2051 & 608 & Nitrate/Nitrite as $\mathrm{N}$ & 322 & & & $\mathrm{~J}$ & UG/L & $06 / 02 / 2006$ & & DNT-279-06 \\
\hline GWM47501VL & MIDDLE-2051 & 608 & Acetone & 2 & & $\mathrm{U}$ & $\mathrm{R}$ & UG/L & $06 / 02 / 2006$ & & JGJ-084-06 \\
\hline GWM47501VL & MIDDLE-2051 & 608 & Benzene & 1 & & $\mathrm{U}$ & & UG/L & $06 / 02 / 2006$ & & JGJ-084-06 \\
\hline GWM47501VL & MIDDLE-2051 & 608 & Bromodichloromethane & 1 & & $\mathrm{U}$ & & UG/L & 06/02/2006 & & JGJ-084-06 \\
\hline GWM47501VL & MIDDLE-2051 & 608 & Bromoform & 1 & & $\mathrm{U}$ & & UG/L & $06 / 02 / 2006$ & & JGJ-084-06 \\
\hline GWM47501VL & MIDDLE-2051 & 608 & Bromomethane & 2 & & $\mathrm{U}$ & & UG/L & $06 / 02 / 2006$ & & JGJ-084-06 \\
\hline GWM47501VL & MIDDLE-2051 & 608 & 2-Butanone & 5 & & $\mathrm{u}$ & $\mathrm{R}$ & UG/L & $06 / 02 / 2006$ & & JGJ-084-06 \\
\hline GWM47501VL & MIDDLE-2051 & 608 & Carbon disulfide & 1 & & $\mathrm{U}$ & & UG/L & $06 / 02 / 2006$ & & JGJ-084-06 \\
\hline GWM47501VL & MIDDLE-2051 & 608 & Carbon tetrachloride & 1 & & $\mathrm{U}$ & & UG/L & $06 / 02 / 2006$ & & JGJ-084-06 \\
\hline GWM47501VL & MIDDLE-2051 & 608 & Chlorobenzene & 1 & & $\mathrm{U}$ & & UG/L & $06 / 02 / 2006$ & & JGJ-084-06 \\
\hline GWM47501VL & MIDDLE-2051 & 608 & Dibromochloromethane & 1 & & $\mathrm{u}$ & & UG/L & $06 / 02 / 2006$ & & JGJ-084-06 \\
\hline GWM47501VL & MIDDLE-2051 & 608 & 1,2-Dibromo-3-chloropropane & 1 & & $\mathrm{U}$ & $\mathrm{R}$ & UG/L & $06 / 02 / 2006$ & & JGJ-084-06 \\
\hline GWM47501VL & MIDDLE-2051 & 608 & Chloroethane & 2 & & $\mathrm{U}$ & & UG/L & $06 / 02 / 2006$ & & JGJ-084-06 \\
\hline GWM47501VL & MIDDLE-2051 & 608 & Chloroform & 1 & & $\mathrm{U}$ & & UG/L & $06 / 02 / 2006$ & & JGJ-084-06 \\
\hline GWM47501VL & MIDDLE-2051 & 608 & Chloromethane & 2 & & $\mathrm{U}$ & & UG/L & $06 / 02 / 2006$ & & JGJ-084-06 \\
\hline GWM47501VL & MIDDLE-2051 & 608 & Cyclohexane & 1 & & $\mathrm{U}$ & & UG/L & $06 / 02 / 2006$ & & JGJ-084-06 \\
\hline GWM47501VL & MIDDLE-2051 & 608 & 1,2-Dibromoethane & 1 & & $\mathrm{u}$ & & UG/L & $06 / 02 / 2006$ & & JGJ-084-06 \\
\hline GWM47501VL & MIDDLE-2051 & 608 & 1,2-Dichlorobenzene & 1 & & $\mathrm{U}$ & & UG/L & $06 / 02 / 2006$ & & JGJ-084-06 \\
\hline GWM47501VL & MIDDLE-2051 & 608 & 1,3-Dichlorobenzene & 1 & & $\mathrm{U}$ & & UG/L & $06 / 02 / 2006$ & & JGJ-084-06 \\
\hline GWM47501VL & MIDDLE-2051 & 608 & 1,4-Dichlorobenzene & 1 & & $\mathrm{U}$ & & UG/L & $06 / 02 / 2006$ & & JGJ-084-06 \\
\hline GWM47501VL & MIDDLE-2051 & 608 & Dichlorodifluoromethane & 2 & & $\mathrm{U}$ & & UG/L & 06/02/2006 & & JGJ-084-06 \\
\hline GWM47501VL & MIDDLE-2051 & 608 & 1,1-Dichloroethane & 1 & & $\mathrm{U}$ & & UG/L & $06 / 02 / 2006$ & & JGJ-084-06 \\
\hline GWM47501VL & MIDDLE-2051 & 608 & 1,2-Dichloroethane & 1 & & $\mathrm{U}$ & & UG/L & $06 / 02 / 2006$ & & JGJ-084-06 \\
\hline GWM47501VL & MIDDLE-2051 & 608 & 1,1-Dichloroethene & 1 & & $\mathrm{U}$ & & UG/L & $06 / 02 / 2006$ & & JGJ-084-06 \\
\hline GWM47501VL & MIDDLE-2051 & 608 & cis-1,2-Dichloroethene & 1 & & $\mathrm{U}$ & & UG/L & $06 / 02 / 2006$ & & JGJ-084-06 \\
\hline GWM47501VL & MIDDLE-2051 & 608 & trans-1,2-Dichloroethene & 1 & & $\mathrm{u}$ & & UG/L & $06 / 02 / 2006$ & & JGJ-084-06 \\
\hline GWM47501VL & MIDDLE-2051 & 608 & 1,2-Dichloropropane & 1 & & $\mathrm{U}$ & & UG/L & $06 / 02 / 2006$ & & JGJ-084-06 \\
\hline GWM47501VL & MIDDLE-2051 & 608 & cis-1,3-Dichloropropene & 1 & & $\mathrm{U}$ & & UG/L & $06 / 02 / 2006$ & & JGJ-084-06 \\
\hline GWM47501VL & MIDDLE-2051 & 608 & trans-1,3-Dichloropropene & 1 & & $\mathrm{U}$ & & UG/L & $06 / 02 / 2006$ & & JGJ-084-06 \\
\hline GWM47501VL & MIDDLE-2051 & 608 & Ethylbenzene & 1 & & $\mathrm{U}$ & & UG/L & $06 / 02 / 2006$ & & JGJ-084-06 \\
\hline GWM47501VL & MIDDLE-2051 & 608 & Trichlorofluoromethane & 1 & & $\mathrm{U}$ & & UG/L & $06 / 02 / 2006$ & & JGJ-084-06 \\
\hline GWM47501VL & MIDDLE-2051 & 608 & 2-Hexanone & 5 & & $\mathrm{U}$ & $\mathrm{R}$ & UG/L & 06/02/2006 & & JGJ-084-06 \\
\hline GWM47501VL & MIDDLE-2051 & 608 & Isopropylbenzene & 1 & & $\mathrm{U}$ & & UG/L & $06 / 02 / 2006$ & & JGJ-084-06 \\
\hline GWM47501VL & MIDDLE-2051 & 608 & Methyl acetate & 5 & & $u$ & $\mathrm{R}$ & UG/L & $06 / 02 / 2006$ & & JGJ-084-06 \\
\hline GWM47501VL & MIDDLE-2051 & 608 & Methyl cyclohexane & 4 & & $\mathrm{U}$ & & UG/L & $06 / 02 / 2006$ & & JGJ-084-06 \\
\hline GWM47501VL & MIDDLE-2051 & 608 & Methylene Chloride & 1 & & $\mathrm{U}$ & & UG/L & 06/02/2006 & & JGJ-084-06 \\
\hline GWM47501VL & MIDDLE-2051 & 608 & 4-Methyl-2-pentanone & 5 & & $\mathrm{U}$ & & UG/L & $06 / 02 / 2006$ & & JGJ-084-06 \\
\hline GWM47501VL & MIDDLE-2051 & 608 & Styrene & 1 & & $\mathrm{U}$ & & UG/L & $06 / 02 / 2006$ & & JGJ-084-06 \\
\hline GWM47501VL & MIDDLE-2051 & 608 & 1,1,2,2-Tetrachloroethane & 1 & & $\mathrm{U}$ & & UG/L & $06 / 02 / 2006$ & & JGJ-084-06 \\
\hline GWM47501VL & MIDDLE-2051 & 608 & Tetrachloroethene & 1 & & $\mathrm{U}$ & & UG/L & $06 / 02 / 2006$ & & JGJ-084-06 \\
\hline GWM47501VL & MIDDLE-2051 & 608 & Toluene & 1 & & $\mathrm{U}$ & & UG/L & $06 / 02 / 2006$ & & JGJ-084-06 \\
\hline GWM47501VL & MIDDLE-2051 & 608 & 1,2,4-Trichlorobenzene & 1 & & $\mathrm{U}$ & & UG/L & 06/02/2006 & & JGJ-084-06 \\
\hline GWM47501VL & MIDDLE-2051 & 608 & 1,1,1-Trichloroethane & 1 & & $\mathrm{U}$ & & UG/L & $06 / 02 / 2006$ & & JGJ-084-06 \\
\hline GWM47501VL & MIDDLE-2051 & 608 & 1,1,2-Trichloroethane & 1 & & $\mathrm{U}$ & & UG/L & $06 / 02 / 2006$ & & JGJ-084-06 \\
\hline GWM47501VL & MIDDLE-2051 & 608 & Trichloroethene & 1 & & $\mathrm{U}$ & & UG/L & $06 / 02 / 2006$ & & JGJ-084-06 \\
\hline GWM47501VL & MIDDLE-2051 & 608 & 1,1,2-Trichloro-1,2,2-trifluoroethane & 1 & & $\mathrm{U}$ & & UG/L & $06 / 02 / 2006$ & & JGJ-084-06 \\
\hline GWM47501VL & MIDDLE-2051 & 608 & Vinyl Chloride & 2 & & $\mathrm{U}$ & & UG/L & 06/02/2006 & & JGJ-084-06 \\
\hline GWM47501VL & MIDDLE-2051 & 608 & Xylenes & 3 & & $\mathrm{U}$ & & UG/L & 06/02/2006 & & JGJ-084-06 \\
\hline GWM47501VL & MIDDLE-2051 & 608 & Methyl t-butyl ether & 2 & & $\mathrm{U}$ & & UG/L & 06/02/2006 & & JGJ-084-06 \\
\hline GWM47501CW & MIDDLE-2051 & 608 & Carbon-14 & $-2.55 E+00$ & $1.51 \mathrm{E}+00$ & & U & $\mathrm{PCl} / \mathrm{L}$ & 06/02/2006 & $5.08 \mathrm{E}+00$ & SOS-TL175-06 \\
\hline GWM47501R8 & MIDDLE-2051 & 608 & Tritium & $7.72 E+00$ & $1.12 \mathrm{E}+02$ & & U & $\mathrm{PCI} / \mathrm{L}$ & 06/02/2006 & $3.81 \mathrm{E}+02$ & SOS-TL176-06 \\
\hline GWM47501RH & MIDDLE-2051 & 608 & Gross Alpha & $1.84 \mathrm{E}+00$ & $6.23 \mathrm{E}-01$ & & UJ & $\mathrm{PCI} / \mathrm{L}$ & 06/02/2006 & $1.89 \mathrm{E}+00$ & SOS-TL177-06 \\
\hline GWM47501RH & MIDDLE-2051 & 608 & Gross Beta & $4.67 \mathrm{E}+00$ & 8.75E-01 & & & $\mathrm{PCI} / \mathrm{L}$ & 06/02/2006 & $2.99 \mathrm{E}+00$ & SOS-TL177-06 \\
\hline GWM47501RH & MIDDLE-2051 & 608 & Strontium-90 & $4.38 \mathrm{E}-03$ & 7.33E-02 & & $\mathrm{U}$ & $\mathrm{PCI} / \mathrm{L}$ & 06/02/2006 & $3.40 \mathrm{E}-01$ & SOS-TL177-06 \\
\hline GWM47501RH & MIDDLE-2051 & 608 & Technetium-99 & $2.40 \mathrm{E}+00$ & $1.70 \mathrm{E}+00$ & & U & $\mathrm{PCI} / \mathrm{L}$ & 06/02/2006 & $5.62 \mathrm{E}+00$ & SOS-TL177-06 \\
\hline GWM47501UX & MIDDLE-2051 & 608 & lodine-129 & $8.50 \mathrm{E}-02$ & $3.59 \mathrm{E}-02$ & & UJ & $\mathrm{PCl} / \mathrm{L}$ & 06/02/2006 & $8.46 \mathrm{E}-02$ & SOS-TL174-06 \\
\hline GWM476012X & MIDDLE-2051 & 748 & Aluminum & 7.9 & & B & & UG/L & $05 / 31 / 2006$ & & DNT-333-06 \\
\hline GWM476012X & MIDDLE-2051 & 748 & Antimony & 0.5 & & $\mathrm{U}$ & & UG/L & 05/31/2006 & & DNT-333-06 \\
\hline GWM476012X & MIDDLE-2051 & 748 & Arsenic & 2 & & U & & UG/L & 05/31/2006 & & DNT-333-06 \\
\hline GWM476012X & MIDDLE-2051 & 748 & Barium & 63.2 & & & & UG/L & 05/31/2006 & & DNT-333-06 \\
\hline GWM476012X & MIDDLE-2051 & 748 & Beryllium & 0.088 & & $u$ & & UG/L & $05 / 31 / 2006$ & & DNT-333-06 \\
\hline GWM476012X & MIDDLE-2051 & 748 & Cadmium & 0.057 & & $u$ & & UG/L & 05/31/2006 & & DNT-333-06 \\
\hline GWM476012X & MIDDLE-2051 & 748 & Calcium & 44300 & & & & UG/L & 05/31/2006 & & DNT-333-06 \\
\hline GWM476012X & MIDDLE-2051 & 748 & Chromium & 7.1 & & & & UG/L & 05/31/2006 & & DNT-333-06 \\
\hline GWM476012X & MIDDLE-2051 & 748 & Cobalt & 0.31 & & U & & UG/L & $05 / 31 / 2006$ & & DNT-333-06 \\
\hline GWM476012X & MIDDLE-2051 & 748 & Copper & 0.45 & & B & & UG/L & 05/31/2006 & & DNT-333-06 \\
\hline GWM476012X & MIDDLE-2051 & 748 & Iron & 9.4 & & $\mathrm{U}$ & & UG/L & $05 / 31 / 2006$ & & DNT-333-06 \\
\hline
\end{tabular}


Table A-5. (continued).

\begin{tabular}{|c|c|c|c|c|c|c|c|c|c|c|c|}
\hline $\begin{array}{l}\text { Field Sample } \\
\text { Number }\end{array}$ & Location & Depth & Compound & $\begin{array}{l}\text { Sample } \\
\text { Result }\end{array}$ & $\begin{array}{l}\text { Sample } \\
\text { Error }\end{array}$ & $\begin{array}{c}\text { Result } \\
\text { Qualifier }\end{array}$ & $\begin{array}{l}\text { Validation } \\
\text { Flag }\end{array}$ & $\begin{array}{l}\text { Sample } \\
\text { Units }\end{array}$ & $\begin{array}{c}\text { Date Sample } \\
\text { Collected }\end{array}$ & MDA & $\begin{array}{l}\text { L\&V Report } \\
\text { Number }\end{array}$ \\
\hline GWM476012X & MIDDLE-2051 & 748 & Lead & 0.49 & & $U$ & & UG/L & $05 / 31 / 2006$ & & DNT-333-06 \\
\hline GWM476012X & MIDDLE-2051 & 748 & Magnesium & 15800 & & & & UG/L & $05 / 31 / 2006$ & & DNT-333-06 \\
\hline GWM476012X & MIDDLE-2051 & 748 & Manganese & 0.37 & & B & & UG/L & $05 / 31 / 2006$ & & DNT-333-06 \\
\hline GWM476012X & MIDDLE-2051 & 748 & Nickel & 1.1 & & B & & UG/L & 05/31/2006 & & DNT-333-06 \\
\hline GWM476012X & MIDDLE-2051 & 748 & Potassium & 2240 & & & & UG/L & $05 / 31 / 2006$ & & DNT-333-06 \\
\hline GWM476012X & MIDDLE-2051 & 748 & Selenium & 1 & & U & & UG/L & 05/31/2006 & & DNT-333-06 \\
\hline GWM476012X & MIDDLE-2051 & 748 & Silver & 0.2 & & U & & UG/L & $05 / 31 / 2006$ & & DNT-333-06 \\
\hline GWM476012X & MIDDLE-2051 & 748 & Sodium & 8490 & & & & UG/L & 05/31/2006 & & DNT-333-06 \\
\hline GWM476012X & MIDDLE-2051 & 748 & Strontium & 243 & & & & UG/L & $05 / 31 / 2006$ & & DNT-333-06 \\
\hline GWM476012X & MIDDLE-2051 & 748 & Thallium & 0.48 & & B & $u$ & UG/L & 05/31/2006 & & DNT-333-06 \\
\hline GWM476012X & MIDDLE-2051 & 748 & Uranium & 1.9 & & & & UG/L & $05 / 31 / 2006$ & & DNT-333-06 \\
\hline GWM476012X & MIDDLE-2051 & 748 & Vanadium & 4.1 & & B & & UG/L & 05/31/2006 & & DNT-333-06 \\
\hline GWM476012X & MIDDLE-2051 & 748 & Zinc & 17.8 & & & & UG/L & $05 / 31 / 2006$ & & DNT-333-06 \\
\hline GWM476012X & MIDDLE-2051 & 748 & Mercury & 0.093 & & U & & UG/L & $05 / 31 / 2006$ & & DNT-333-06 \\
\hline GWM47601A1 & MIDDLE-2051 & 748 & Total Alkalinity & 160 & & & $\mathrm{~J}$ & $\mathrm{MG} / \mathrm{L}$ & $06 / 01 / 2006$ & & DNT-277-06 \\
\hline GWM47601AN & MIDDLE-2051 & 748 & Chloride & 11.3 & & $\mathrm{~J}$ & & MG/L & $06 / 01 / 2006$ & & DNT-277-06 \\
\hline GWM47601AN & MIDDLE-2051 & 748 & Fluoride & 0.17 & & & & MG/L & 06/01/2006 & & DNT-277-06 \\
\hline GWM47601AN & MIDDLE-2051 & 748 & Sulfate & 25.4 & & & & MG/L & $06 / 01 / 2006$ & & DNT-277-06 \\
\hline GWM47601N2 & MIDDLE-2051 & 748 & Nitrate/Nitrite as $\mathrm{N}$ & 759 & & & & UG/L & $06 / 01 / 2006$ & & DNT-277-06 \\
\hline GWM47601VL & MIDDLE-2051 & 748 & Acetone & 2 & & $\mathrm{u}$ & $\mathrm{R}$ & UG/L & $06 / 01 / 2006$ & & JGJ-084-06 \\
\hline GWM47601VL & MIDDLE-2051 & 748 & Benzene & 1 & & $\mathrm{u}$ & & UG/L & 06/01/2006 & & JGJ-084-06 \\
\hline GWM47601VL & MIDDLE-2051 & 748 & Bromodichloromethane & 1 & & $\mathrm{u}$ & & UG/L & $06 / 01 / 2006$ & & JGJ-084-06 \\
\hline GWM47601VL & MIDDLE-2051 & 748 & Bromoform & 1 & & $\mathrm{u}$ & & UG/L & 06/01/2006 & & JGJ-084-06 \\
\hline GWM47601VL & MIDDLE-2051 & 748 & Bromomethane & 2 & & u & & UG/L & $06 / 01 / 2006$ & & JGJ-084-06 \\
\hline GWM47601VL & MIDDLE-2051 & 748 & 2-Butanone & 5 & & $\mathrm{u}$ & $\mathrm{R}$ & UG/L & 06/01/2006 & & JGJ-084-06 \\
\hline GWM47601VL & MIDDLE-2051 & 748 & Carbon disulfide & 1 & & $\mathrm{u}$ & & UG/L & $06 / 01 / 2006$ & & JGJ-084-06 \\
\hline GWM47601VL & MIDDLE-2051 & 748 & Carbon tetrachloride & 1 & & u & & UG/L & $06 / 01 / 2006$ & & JGJ-084-06 \\
\hline GWM47601VL & MIDDLE-2051 & 748 & Chlorobenzene & 1 & & $\mathrm{U}$ & & UG/L & $06 / 01 / 2006$ & & JGJ-084-06 \\
\hline GWM47601VL & MIDDLE-2051 & 748 & Dibromochloromethane & 1 & & $\mathrm{U}$ & & UG/L & 06/01/2006 & & JGJ-084-06 \\
\hline GWM47601VL & MIDDLE-2051 & 748 & 1,2-Dibromo-3-chloropropane & 1 & & $\mathrm{u}$ & $\mathrm{R}$ & UG/L & 06/01/2006 & & JGJ-084-06 \\
\hline GWM47601VL & MIDDLE-2051 & 748 & Chloroethane & 2 & & u & & UG/L & $06 / 01 / 2006$ & & JGJ-084-06 \\
\hline GWM47601VL & MIDDLE-2051 & 748 & Chloroform & 1 & & $\mathrm{u}$ & & UG/L & 06/01/2006 & & JGJ-084-06 \\
\hline GWM47601VL & MIDDLE-2051 & 748 & Chloromethane & 2 & & $\mathrm{u}$ & & UG/L & $06 / 01 / 2006$ & & JGJ-084-06 \\
\hline GWM47601VL & MIDDLE-2051 & 748 & Cyclohexane & 1 & & $\mathrm{u}$ & & UG/L & $06 / 01 / 2006$ & & JGJ-084-06 \\
\hline GWM47601VL & MIDDLE-2051 & 748 & 1,2-Dibromoethane & 1 & & u & & UG/L & $06 / 01 / 2006$ & & JGJ-084-06 \\
\hline GWM47601VL & MIDDLE-2051 & 748 & 1,2-Dichlorobenzene & 1 & & $\mathrm{u}$ & & UG/L & 06/01/2006 & & JGJ-084-06 \\
\hline GWM47601VL & MIDDLE-2051 & 748 & 1,3-Dichlorobenzene & 1 & & $\mathrm{u}$ & & UG/L & $06 / 01 / 2006$ & & JGJ-084-06 \\
\hline GWM47601VL & MIDDLE-2051 & 748 & 1,4-Dichlorobenzene & 1 & & $\mathrm{u}$ & & UG/L & 06/01/2006 & & JGJ-084-06 \\
\hline GWM47601VL & MIDDLE-2051 & 748 & Dichlorodifluoromethane & 2 & & $\mathrm{u}$ & & UG/L & $06 / 01 / 2006$ & & JGJ-084-06 \\
\hline GWM47601VL & MIDDLE-2051 & 748 & 1,1-Dichloroethane & 1 & & $\mathrm{u}$ & & UG/L & 06/01/2006 & & JGJ-084-06 \\
\hline GWM47601VL & MIDDLE-2051 & 748 & 1,2-Dichloroethane & 1 & & $\mathrm{u}$ & & UG/L & 06/01/2006 & & JGJ-084-06 \\
\hline GWM47601VL & MIDDLE-2051 & 748 & 1,1-Dichloroethene & 1 & & $\mathrm{U}$ & & UG/L & 06/01/2006 & & JGJ-084-06 \\
\hline GWM47601VL & MIDDLE-2051 & 748 & cis-1,2-Dichloroethene & 1 & & $\mathrm{u}$ & & UG/L & $06 / 01 / 2006$ & & JGJ-084-06 \\
\hline GWM47601VL & MIDDLE-2051 & 748 & trans-1,2-Dichloroethene & 1 & & $\mathrm{u}$ & & UG/L & 06/01/2006 & & JGJ-084-06 \\
\hline GWM47601VL & MIDDLE-2051 & 748 & 1,2-Dichloropropane & 1 & & $\mathrm{u}$ & & UG/L & 06/01/2006 & & JGJ-084-06 \\
\hline GWM47601VL & MIDDLE-2051 & 748 & cis-1,3-Dichloropropene & 1 & & $\mathrm{U}$ & & UG/L & 06/01/2006 & & JGJ-084-06 \\
\hline GWM47601VL & MIDDLE-2051 & 748 & trans-1,3-Dichloropropene & 1 & & $\mathrm{U}$ & & UG/L & $06 / 01 / 2006$ & & JGJ-084-06 \\
\hline GWM47601VL & MIDDLE-2051 & 748 & Ethylbenzene & 1 & & u & & UG/L & $06 / 01 / 2006$ & & JGJ-084-06 \\
\hline GWM47601VL & MIDDLE-2051 & 748 & Trichlorofluoromethane & 1 & & $\mathrm{U}$ & & UG/L & $06 / 01 / 2006$ & & JGJ-084-06 \\
\hline GWM47601VL & MIDDLE-2051 & 748 & 2-Hexanone & 5 & & u & $\mathrm{R}$ & UG/L & $06 / 01 / 2006$ & & JGJ-084-06 \\
\hline GWM47601VL & MIDDLE-2051 & 748 & Isopropylbenzene & 1 & & $\mathrm{U}$ & & UG/L & $06 / 01 / 2006$ & & JGJ-084-06 \\
\hline GWM47601VL & MIDDLE-2051 & 748 & Methyl acetate & 5 & & u & $\mathrm{R}$ & UG/L & $06 / 01 / 2006$ & & JGJ-084-06 \\
\hline GWM47601VL & MIDDLE-2051 & 748 & Methyl cyclohexane & 4 & & $\mathrm{u}$ & & UG/L & 06/01/2006 & & JGJ-084-06 \\
\hline GWM47601VL & MIDDLE-2051 & 748 & Methylene Chloride & 1 & & u & & UG/L & $06 / 01 / 2006$ & & JGJ-084-06 \\
\hline GWM47601VL & MIDDLE-2051 & 748 & 4-Methyl-2-pentanone & 5 & & $\mathrm{U}$ & & UG/L & 06/01/2006 & & JGJ-084-06 \\
\hline GWM47601VL & MIDDLE-2051 & 748 & Styrene & 1 & & u & & UG/L & $06 / 01 / 2006$ & & JGJ-084-06 \\
\hline GWM47601VL & MIDDLE-2051 & 748 & 1,1,2,2-Tetrachloroethane & 1 & & $\mathrm{u}$ & & UG/L & 06/01/2006 & & JGJ-084-06 \\
\hline GWM47601VL & MIDDLE-2051 & 748 & Tetrachloroethene & 1 & & $\mathrm{u}$ & & UG/L & 06/01/2006 & & JGJ-084-06 \\
\hline GWM47601VL & MIDDLE-2051 & 748 & Toluene & 1 & & $\mathrm{U}$ & & UG/L & 06/01/2006 & & JGJ-084-06 \\
\hline GWM47601VL & MIDDLE-2051 & 748 & 1,2,4-Trichlorobenzene & 1 & & $\mathrm{u}$ & & UG/L & $06 / 01 / 2006$ & & JGJ-084-06 \\
\hline GWM47601VL & MIDDLE-2051 & 748 & 1,1,1-Trichloroethane & 1 & & $\mathrm{u}$ & & UG/L & 06/01/2006 & & JGJ-084-06 \\
\hline GWM47601VL & MIDDLE-2051 & 748 & 1,1,2-Trichloroethane & 1 & & $\mathrm{U}$ & & UG/L & $06 / 01 / 2006$ & & JGJ-084-06 \\
\hline GWM47601VL & MIDDLE-2051 & 748 & Trichloroethene & 1 & & $\mathrm{U}$ & & UG/L & 06/01/2006 & & JGJ-084-06 \\
\hline GWM47601VL & MIDDLE-2051 & 748 & 1,1,2-Trichloro-1,2,2-trifluoroethane & 1 & & $\mathrm{u}$ & & UG/L & 06/01/2006 & & JGJ-084-06 \\
\hline GWM47601VL & MIDDLE-2051 & 748 & Vinyl Chloride & 2 & & $\mathrm{u}$ & & UG/L & 06/01/2006 & & JGJ-084-06 \\
\hline GWM47601VL & MIDDLE-2051 & 748 & Xylenes & 3 & & $\mathrm{u}$ & & UG/L & 06/01/2006 & & JGJ-084-06 \\
\hline GWM47601VL & MIDDLE-2051 & 748 & Methyl t-butyl ether & 2 & & $\mathrm{U}$ & & UG/L & $06 / 01 / 2006$ & & JGJ-084-06 \\
\hline GWM47601CW & MIDDLE-2051 & 748 & Carbon-14 & $-3.81 \mathrm{E}-01$ & $1.52 \mathrm{E}+00$ & & $\mathrm{u}$ & $\mathrm{PCI} / \mathrm{L}$ & 06/01/2006 & $5.09 \mathrm{E}+00$ & SOS-TL175-06 \\
\hline GWM47601R8 & MIDDLE-2051 & 748 & Tritium & $4.49 \mathrm{E}+02$ & $1.23 \mathrm{E}+02$ & & & $\mathrm{PCI} / \mathrm{L}$ & $06 / 01 / 2006$ & $3.90 \mathrm{E}+02$ & SOS-TL176-06 \\
\hline GWM47601RH & MIDDLE-2051 & 748 & Gross Alpha & $1.14 \mathrm{E}+00$ & $5.54 \mathrm{E}-01$ & & UJ & $\mathrm{PCI} / \mathrm{L}$ & 06/01/2006 & $1.84 \mathrm{E}+00$ & SOS-TL177-06 \\
\hline GWM47601RH & MIDDLE-2051 & 748 & Gross Beta & $3.62 \mathrm{E}+00$ & $1.04 \mathrm{E}+00$ & & & $\mathrm{PCI} / \mathrm{L}$ & $06 / 01 / 2006$ & $3.99 \mathrm{E}+00$ & SOS-TL177-06 \\
\hline GWM47601RH & MIDDLE-2051 & 748 & Strontium-90 & $-6.92 \mathrm{E}-02$ & 8.47E-02 & & U & $\mathrm{PCI} / \mathrm{L}$ & 06/01/2006 & 4.07E-01 & SOS-TL177-06 \\
\hline GWM47601RH & MIDDLE-2051 & 748 & Technetium-99 & $1.12 \mathrm{E}+00$ & $1.66 \mathrm{E}+00$ & & U & $\mathrm{PCl} / \mathrm{L}$ & 06/01/2006 & $5.58 \mathrm{E}+00$ & SOS-TL177-06 \\
\hline GWM47601UX & MIDDLE-2051 & 748 & lodine-129 & 4.64E-03 & 1.82E-02 & & $u$ & $\mathrm{PCI} / \mathrm{L}$ & 06/01/2006 & $6.70 \mathrm{E}-02$ & SOS-TL174-06 \\
\hline GWM477012X & MIDDLE-2051 & 834 & Aluminum & 9.1 & & B & & UG/L & $05 / 31 / 2006$ & & DNT-333-06 \\
\hline GWM477012X & MIDDLE-2051 & 834 & Antimony & 0.5 & & $u$ & & UG/L & $05 / 31 / 2006$ & & DNT-333-06 \\
\hline GWM477012X & MIDDLE-2051 & 834 & Arsenic & 2 & & $\mathrm{u}$ & & UG/L & $05 / 31 / 2006$ & & DNT-333-06 \\
\hline GWM477012X & MIDDLE-2051 & 834 & Barium & 69.6 & & & & UG/L & 05/31/2006 & & DNT-333-06 \\
\hline GWM477012X & MIDDLE-2051 & 834 & Beryllium & 0.088 & & U & & UG/L & $05 / 31 / 2006$ & & DNT-333-06 \\
\hline GWM477012X & MIDDLE-2051 & 834 & Cadmium & 0.057 & & U & & UG/L & $05 / 31 / 2006$ & & DNT-333-06 \\
\hline GWM477012X & MIDDLE-2051 & 834 & Calcium & 44000 & & & & UG/L & $05 / 31 / 2006$ & & DNT-333-06 \\
\hline GWM477012X & MIDDLE-2051 & 834 & Chromium & 7.9 & & & & UG/L & 05/31/2006 & & DNT-333-06 \\
\hline GWM477012X & MIDDLE-2051 & 834 & Cobalt & 0.31 & & U & & UG/L & $05 / 31 / 2006$ & & DNT-333-06 \\
\hline GWM477012X & MIDDLE-2051 & 834 & Copper & 0.45 & & B & & UG/L & $05 / 31 / 2006$ & & DNT-333-06 \\
\hline GWM477012X & MIDDLE-2051 & 834 & Iron & 9.4 & & U & & UG/L & $05 / 31 / 2006$ & & DNT-333-06 \\
\hline GWM477012X & MIDDLE-2051 & 834 & Lead & 0.49 & & U & & UG/L & $05 / 31 / 2006$ & & DNT-333-06 \\
\hline
\end{tabular}


Table A-5. (continued).

\begin{tabular}{|c|c|c|c|c|c|c|c|c|c|c|c|}
\hline $\begin{array}{l}\text { Field Sample } \\
\text { Number }\end{array}$ & Location & Depth & Compound & $\begin{array}{c}\text { Sample } \\
\text { Result }\end{array}$ & $\begin{array}{l}\text { Sample } \\
\text { Error }\end{array}$ & $\begin{array}{c}\text { Result } \\
\text { Qualifier }\end{array}$ & $\begin{array}{c}\text { Validation } \\
\text { Flag }\end{array}$ & $\begin{array}{l}\text { Sample } \\
\text { Units }\end{array}$ & $\begin{array}{c}\text { Date Sample } \\
\text { Collected }\end{array}$ & MDA & $\begin{array}{c}\text { L\&V Report } \\
\text { Number }\end{array}$ \\
\hline GWM477012X & MIDDLE-2051 & 834 & Magnesium & 15400 & & & & UG/L & $05 / 31 / 2006$ & & DNT-333-06 \\
\hline GWM477012X & MIDDLE-2051 & 834 & Manganese & 0.34 & & U & & UG/L & $05 / 31 / 2006$ & & DNT-333-06 \\
\hline GWM477012X & MIDDLE-2051 & 834 & Nickel & 1.1 & & B & & UG/L & $05 / 31 / 2006$ & & DNT-333-06 \\
\hline GWM477012X & MIDDLE-2051 & 834 & Potassium & 2090 & & & & UG/L & 05/31/2006 & & DNT-333-06 \\
\hline GWM477012X & MIDDLE-2051 & 834 & Selenium & 1.7 & & B & $\mathrm{U}$ & UG/L & $05 / 31 / 2006$ & & DNT-333-06 \\
\hline GWM477012X & MIDDLE-2051 & 834 & Silver & 0.2 & & U & & UG/L & 05/31/2006 & & DNT-333-06 \\
\hline GWM477012X & MIDDLE-2051 & 834 & Sodium & 8420 & & & & UG/L & $05 / 31 / 2006$ & & DNT-333-06 \\
\hline GWM477012X & MIDDLE-2051 & 834 & Strontium & 235 & & & & UG/L & 05/31/2006 & & DNT-333-06 \\
\hline GWM477012X & MIDDLE-2051 & 834 & Thallium & 0.32 & & $\mathrm{U}$ & & UG/L & $05 / 31 / 2006$ & & DNT-333-06 \\
\hline GWM477012X & MIDDLE-2051 & 834 & Uranium & 1.8 & & & & UG/L & 05/31/2006 & & DNT-333-06 \\
\hline GWM477012X & MIDDLE-2051 & 834 & Vanadium & 4 & & B & & UG/L & $05 / 31 / 2006$ & & DNT-333-06 \\
\hline GWM477012X & MIDDLE-2051 & 834 & Zinc & 21 & & & & UG/L & 05/31/2006 & & DNT-333-06 \\
\hline GWM477012X & MIDDLE-2051 & 834 & Mercury & 0.093 & & U & & UG/L & $05 / 31 / 2006$ & & DNT-333-06 \\
\hline GWM477022X & MIDDLE-2051 & 834 & Aluminum & 7.9 & & $u$ & & UG/L & $05 / 31 / 2006$ & & DNT-333-06 \\
\hline GWM477022X & MIDDLE-2051 & 834 & Antimony & 0.5 & & U & & UG/L & $05 / 31 / 2006$ & & DNT-333-06 \\
\hline GWM477022X & MIDDLE-2051 & 834 & Arsenic & 2 & & U & & UG/L & $05 / 31 / 2006$ & & DNT-333-06 \\
\hline GWM477022X & MIDDLE-2051 & 834 & Barium & 70.4 & & & & UG/L & 05/31/2006 & & DNT-333-06 \\
\hline GWM477022X & MIDDLE-2051 & 834 & Beryllium & 0.088 & & $\mathrm{U}$ & & UG/L & $05 / 31 / 2006$ & & DNT-333-06 \\
\hline GWM477022X & MIDDLE-2051 & 834 & Cadmium & 0.057 & & $u$ & & UG/L & $05 / 31 / 2006$ & & DNT-333-06 \\
\hline GWM477022X & MIDDLE-2051 & 834 & Calcium & 43800 & & & & UG/L & $05 / 31 / 2006$ & & DNT-333-06 \\
\hline GWM477022X & MIDDLE-2051 & 834 & Chromium & 8 & & & & UG/L & 05/31/2006 & & DNT-333-06 \\
\hline GWM477022X & MIDDLE-2051 & 834 & Cobalt & 0.31 & & $\mathrm{U}$ & & UG/L & $05 / 31 / 2006$ & & DNT-333-06 \\
\hline GWM477022X & MIDDLE-2051 & 834 & Copper & 0.43 & & B & & UG/L & 05/31/2006 & & DNT-333-06 \\
\hline GWM477022X & MIDDLE-2051 & 834 & Iron & 9.4 & & U & & UG/L & $05 / 31 / 2006$ & & DNT-333-06 \\
\hline GWM477022X & MIDDLE-2051 & 834 & Lead & 0.49 & & 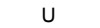 & & UG/L & 05/31/2006 & & DNT-333-06 \\
\hline GWM477022X & MIDDLE-2051 & 834 & Magnesium & 15200 & & & & UG/L & $05 / 31 / 2006$ & & DNT-333-06 \\
\hline GWM477022X & MIDDLE-2051 & 834 & Manganese & 0.48 & & B & & UG/L & 05/31/2006 & & DNT-333-06 \\
\hline GWM477022X & MIDDLE-2051 & 834 & Nickel & 1.2 & & B & & UG/L & $05 / 31 / 2006$ & & DNT-333-06 \\
\hline GWM477022X & MIDDLE-2051 & 834 & Potassium & 2090 & & & & UG/L & 05/31/2006 & & DNT-333-06 \\
\hline GWM477022X & MIDDLE-2051 & 834 & Selenium & 2 & & B & U & UG/L & $05 / 31 / 2006$ & & DNT-333-06 \\
\hline GWM477022X & MIDDLE-2051 & 834 & Silver & 0.2 & & U & & UG/L & $05 / 31 / 2006$ & & DNT-333-06 \\
\hline GWM477022X & MIDDLE-2051 & 834 & Sodium & 8280 & & & & UG/L & $05 / 31 / 2006$ & & DNT-333-06 \\
\hline GWM477022X & MIDDLE-2051 & 834 & Strontium & 234 & & & & UG/L & $05 / 31 / 2006$ & & DNT-333-06 \\
\hline GWM477022X & MIDDLE-2051 & 834 & Thallium & 0.32 & & U & & UG/L & $05 / 31 / 2006$ & & DNT-333-06 \\
\hline GWM477022X & MIDDLE-2051 & 834 & Uranium & 1.9 & & & & UG/L & $05 / 31 / 2006$ & & DNT-333-06 \\
\hline GWM477022X & MIDDLE-2051 & 834 & Vanadium & 4 & & B & & UG/L & 05/31/2006 & & DNT-333-06 \\
\hline GWM477022X & MIDDLE-2051 & 834 & Zinc & 18.3 & & & & UG/L & $05 / 31 / 2006$ & & DNT-333-06 \\
\hline GWM477022X & MIDDLE-2051 & 834 & Mercury & 0.093 & & $\mathrm{U}$ & & UG/L & 05/31/2006 & & DNT-333-06 \\
\hline GWM47701A1 & MIDDLE-2051 & 834 & Total Alkalinity & 150 & & & & $\mathrm{MG} / \mathrm{L}$ & $05 / 31 / 2006$ & & DNT-278-06 \\
\hline GWM47702A1 & MIDDLE-2051 & 834 & Total Alkalinity & 152 & & & & MG/L & 05/31/2006 & & DNT-278-06 \\
\hline GWM47701AN & MIDDLE-2051 & 834 & Chloride & 11.8 & & $\mathrm{~J}$ & & $\mathrm{MG} / \mathrm{L}$ & $05 / 31 / 2006$ & & DNT-278-06 \\
\hline GWM47701AN & MIDDLE-2051 & 834 & Fluoride & 0.17 & & & & MG/L & 05/31/2006 & & DNT-278-06 \\
\hline GWM47701AN & MIDDLE-2051 & 834 & Sulfate & 26.3 & & & & $\mathrm{MG} / \mathrm{L}$ & $05 / 31 / 2006$ & & DNT-278-06 \\
\hline GWM47702AN & MIDDLE-2051 & 834 & Chloride & 13.1 & & $\mathrm{~J}$ & & MG/L & $05 / 31 / 2006$ & & DNT-278-06 \\
\hline GWM47702AN & MIDDLE-2051 & 834 & Fluoride & 0.18 & & & & $\mathrm{MG} / \mathrm{L}$ & $05 / 31 / 2006$ & & DNT-278-06 \\
\hline GWM47702AN & MIDDLE-2051 & 834 & Sulfate & 26.9 & & & & MG/L & $05 / 31 / 2006$ & & DNT-278-06 \\
\hline GWM47701N2 & MIDDLE-2051 & 834 & Nitrate/Nitrite as $\mathrm{N}$ & 834 & & & & UG/L & $05 / 31 / 2006$ & & DNT-278-06 \\
\hline GWM47702N2 & MIDDLE-2051 & 834 & Nitrate/Nitrite as $\mathrm{N}$ & 840 & & & & UG/L & $05 / 31 / 2006$ & & DNT-278-06 \\
\hline GWM47701VL & MIDDLE-2051 & 834 & Acetone & 2 & & $\mathrm{U}$ & $\mathrm{R}$ & UG/L & $05 / 31 / 2006$ & & JGJ-084-06 \\
\hline GWM47701VL & MIDDLE-2051 & 834 & Benzene & 1 & & u & & UG/L & $05 / 31 / 2006$ & & JGJ-084-06 \\
\hline GWM47701VL & MIDDLE-2051 & 834 & Bromodichloromethane & 1 & & $u$ & & UG/L & $05 / 31 / 2006$ & & JGJ-084-06 \\
\hline GWM47701VL & MIDDLE-2051 & 834 & Bromoform & 1 & & U & & UG/L & $05 / 31 / 2006$ & & JGJ-084-06 \\
\hline GWM47701VL & MIDDLE-2051 & 834 & Bromomethane & 2 & & $\mathrm{u}$ & & UG/L & $05 / 31 / 2006$ & & JGJ-084-06 \\
\hline GWM47701VL & MIDDLE-2051 & 834 & 2-Butanone & 5 & & u & $\mathrm{R}$ & UG/L & $05 / 31 / 2006$ & & JGJ-084-06 \\
\hline GWM47701VL & MIDDLE-2051 & 834 & Carbon disulfide & 1 & & $u$ & & UG/L & $05 / 31 / 2006$ & & JGJ-084-06 \\
\hline GWM47701VL & MIDDLE-2051 & 834 & Carbon tetrachloride & 1 & & u & & UG/L & $05 / 31 / 2006$ & & JGJ-084-06 \\
\hline GWM47701VL & MIDDLE-2051 & 834 & Chlorobenzene & 1 & & $\mathrm{u}$ & & UG/L & $05 / 31 / 2006$ & & JGJ-084-06 \\
\hline GWM47701VL & MIDDLE-2051 & 834 & Dibromochloromethane & 1 & & $\mathrm{U}$ & & UG/L & $05 / 31 / 2006$ & & JGJ-084-06 \\
\hline GWM47701VL & MIDDLE-2051 & 834 & 1,2-Dibromo-3-chloropropane & 1 & & $u$ & $\mathrm{R}$ & UG/L & $05 / 31 / 2006$ & & JGJ-084-06 \\
\hline GWM47701VL & MIDDLE-2051 & 834 & Chloroethane & 2 & & $\mathrm{u}$ & & UG/L & $05 / 31 / 2006$ & & JGJ-084-06 \\
\hline GWM47701VL & MIDDLE-2051 & 834 & Chloroform & 1 & & u & & UG/L & $05 / 31 / 2006$ & & JGJ-084-06 \\
\hline GWM47701VL & MIDDLE-2051 & 834 & Chloromethane & 2 & & $\mathrm{u}$ & & UG/L & $05 / 31 / 2006$ & & JGJ-084-06 \\
\hline GWM47701VL & MIDDLE-2051 & 834 & Cyclohexane & 1 & & $\mathrm{U}$ & & UG/L & 05/31/2006 & & JGJ-084-06 \\
\hline GWM47701VL & MIDDLE-2051 & 834 & 1,2-Dibromoethane & 1 & & $\mathrm{U}$ & & UG/L & $05 / 31 / 2006$ & & JGJ-084-06 \\
\hline GWM47701VL & MIDDLE-2051 & 834 & 1.2-Dichlorobenzene & 1 & & u & & UG/L & $05 / 31 / 2006$ & & JGJ-084-06 \\
\hline GWM47701VL & MIDDLE-2051 & 834 & 1,3-Dichlorobenzene & 1 & & $\mathrm{u}$ & & UG/L & $05 / 31 / 2006$ & & JGJ-084-06 \\
\hline GWM47701VL & MIDDLE-2051 & 834 & 1,4-Dichlorobenzene & 1 & & u & & UG/L & $05 / 31 / 2006$ & & JGJ-084-06 \\
\hline GWM47701VL & MIDDLE-2051 & 834 & Dichlorodifluoromethane & 2 & & $\mathrm{u}$ & & UG/L & $05 / 31 / 2006$ & & JGJ-084-06 \\
\hline GWM47701VL & MIDDLE-2051 & 834 & 1,1-Dichloroethane & 1 & & u & & UG/L & $05 / 31 / 2006$ & & JGJ-084-06 \\
\hline GWM47701VL & MIDDLE-2051 & 834 & 1,2-Dichloroethane & 1 & & $\mathrm{u}$ & & UG/L & $05 / 31 / 2006$ & & JGJ-084-06 \\
\hline GWM47701VL & MIDDLE-2051 & 834 & 1,1-Dichloroethene & 1 & & u & & UG/L & $05 / 31 / 2006$ & & JGJ-084-06 \\
\hline GWM47701VL & MIDDLE-2051 & 834 & cis-1,2-Dichloroethene & 1 & & u & & UG/L & $05 / 31 / 2006$ & & JGJ-084-06 \\
\hline GWM47701VL & MIDDLE-2051 & 834 & trans-1,2-Dichloroethene & 1 & & $\mathrm{u}$ & & UG/L & $05 / 31 / 2006$ & & JGJ-084-06 \\
\hline GWM47701VL & MIDDLE-2051 & 834 & 1,2-Dichloropropane & 1 & & $\mathrm{u}$ & & UG/L & $05 / 31 / 2006$ & & JGJ-084-06 \\
\hline GWM47701VL & MIDDLE-2051 & 834 & cis-1,3-Dichloropropene & 1 & & $\mathrm{u}$ & & UG/L & $05 / 31 / 2006$ & & JGJ-084-06 \\
\hline GWM47701VL & MIDDLE-2051 & 834 & trans-1,3-Dichloropropene & 1 & & u & & UG/L & $05 / 31 / 2006$ & & JGJ-084-06 \\
\hline GWM47701VL & MIDDLE-2051 & 834 & Ethylbenzene & 1 & & $\mathrm{u}$ & & UG/L & $05 / 31 / 2006$ & & JGJ-084-06 \\
\hline GWM47701VL & MIDDLE-2051 & 834 & Trichlorofluoromethane & 1 & & $\mathrm{U}$ & & UG/L & $05 / 31 / 2006$ & & JGJ-084-06 \\
\hline GWM47701VL & MIDDLE-2051 & 834 & 2-Hexanone & 5 & & $\mathrm{U}$ & $\mathrm{R}$ & UG/L & $05 / 31 / 2006$ & & JGJ-084-06 \\
\hline GWM47701VL & MIDDLE-2051 & 834 & Isopropylbenzene & 1 & & u & & UG/L & $05 / 31 / 2006$ & & JGJ-084-06 \\
\hline GWM47701VL & MIDDLE-2051 & 834 & Methyl acetate & 5 & & $\mathrm{u}$ & $\mathrm{R}$ & UG/L & $05 / 31 / 2006$ & & JGJ-084-06 \\
\hline GWM47701VL & MIDDLE-2051 & 834 & Methyl cyclohexane & 4 & & $\mathrm{U}$ & & UG/L & 05/31/2006 & & JGJ-084-06 \\
\hline GWM47701VL & MIDDLE-2051 & 834 & Methylene Chloride & 1 & & $\mathrm{U}$ & & UG/L & $05 / 31 / 2006$ & & JGJ-084-06 \\
\hline GWM47701VL & MIDDLE-2051 & 834 & 4-Methyl-2-pentanone & 5 & & u & & UG/L & $05 / 31 / 2006$ & & JGJ-084-06 \\
\hline GWM47701VL & MIDDLE-2051 & 834 & Styrene & 1 & & $\mathrm{U}$ & & UG/L & $05 / 31 / 2006$ & & JGJ-084-06 \\
\hline GWM47701VL & MIDDLE-2051 & 834 & 1,1,2,2-Tetrachloroethane & 1 & & u & & UG/L & $05 / 31 / 2006$ & & JGJ-084-06 \\
\hline
\end{tabular}


Table A-5. (continued).

\begin{tabular}{|c|c|c|c|c|c|c|c|c|c|c|c|}
\hline $\begin{array}{l}\text { Field Sample } \\
\text { Number }\end{array}$ & Location & Depth & Compound & $\begin{array}{l}\text { Sample } \\
\text { Result }\end{array}$ & $\begin{array}{c}\text { Sample } \\
\text { Error }\end{array}$ & $\begin{array}{c}\text { Result } \\
\text { Qualifier }\end{array}$ & $\begin{array}{l}\text { Validation } \\
\text { Flag }\end{array}$ & $\begin{array}{l}\text { Sample } \\
\text { Units }\end{array}$ & $\begin{array}{c}\text { Date Sample } \\
\text { Collected }\end{array}$ & MDA & $\begin{array}{l}\text { L\&V Report } \\
\text { Number }\end{array}$ \\
\hline GWM47701VL & MIDDLE-2051 & 834 & Tetrachloroethene & 1 & & $U$ & & UG/L & $05 / 31 / 2006$ & & JGJ-084-06 \\
\hline GWM47701VL & MIDDLE-2051 & 834 & Toluene & 1 & & $\mathrm{u}$ & & UG/L & $05 / 31 / 2006$ & & JGJ-084-06 \\
\hline GWM47701VL & MIDDLE-2051 & 834 & 1,2,4-Trichlorobenzene & 1 & & $\mathrm{u}$ & & UG/L & $05 / 31 / 2006$ & & JGJ-084-06 \\
\hline GWM47701VL & MIDDLE-2051 & 834 & 1,1,1-Trichloroethane & 1 & & $\mathrm{u}$ & & UG/L & $05 / 31 / 2006$ & & JGJ-084-06 \\
\hline GWM47701VL & MIDDLE-2051 & 834 & $1,1,2$-Trichloroethane & 1 & & u & & UG/L & $05 / 31 / 2006$ & & JGJ-084-06 \\
\hline GWM47701VL & MIDDLE-2051 & 834 & Trichloroethene & 1 & & $\mathrm{U}$ & & UG/L & $05 / 31 / 2006$ & & JGJ-084-06 \\
\hline GWM47701VL & MIDDLE-2051 & 834 & 1,1,2-Trichloro-1,2,2-trifluoroethane & 1 & & u & & UG/L & $05 / 31 / 2006$ & & JGJ-084-06 \\
\hline GWM47701VL & MIDDLE-2051 & 834 & Vinyl Chloride & 2 & & $\mathrm{u}$ & & UG/L & 05/31/2006 & & JGJ-084-06 \\
\hline GWM47701VL & MIDDLE-2051 & 834 & Xylenes & 3 & & $\mathrm{u}$ & & UG/L & $05 / 31 / 2006$ & & JGJ-084-06 \\
\hline GWM47701VL & MIDDLE-2051 & 834 & Methyl t-butyl ether & 2 & & $\mathrm{U}$ & & UG/L & 05/31/2006 & & JGJ-084-06 \\
\hline GWM47702VL & MIDDLE-2051 & 834 & Acetone & 2 & & U & $\mathrm{R}$ & UG/L & $05 / 31 / 2006$ & & JGJ-084-06 \\
\hline GWM47702VL & MIDDLE-2051 & 834 & Benzene & 1 & & $\mathrm{U}$ & & UG/L & 05/31/2006 & & JGJ-084-06 \\
\hline GWM47702VL & MIDDLE-2051 & 834 & Bromodichloromethane & 1 & & $\mathrm{u}$ & & UG/L & $05 / 31 / 2006$ & & JGJ-084-06 \\
\hline GWM47702VL & MIDDLE-2051 & 834 & Bromoform & 1 & & $\mathrm{U}$ & & UG/L & $05 / 31 / 2006$ & & JGJ-084-06 \\
\hline GWM47702VL & MIDDLE-2051 & 834 & Bromomethane & 2 & & u & & UG/L & $05 / 31 / 2006$ & & JGJ-084-06 \\
\hline GWM47702VL & MIDDLE-2051 & 834 & 2-Butanone & 5 & & u & $\mathrm{R}$ & UG/L & $05 / 31 / 2006$ & & JGJ-084-06 \\
\hline GWM47702VL & MIDDLE-2051 & 834 & Carbon disulfide & 1 & & $\mathrm{U}$ & & UG/L & 05/31/2006 & & JGJ-084-06 \\
\hline GWM47702VL & MIDDLE-2051 & 834 & Carbon tetrachloride & 1 & & u & & UG/L & $05 / 31 / 2006$ & & JGJ-084-06 \\
\hline GWM47702VL & MIDDLE-2051 & 834 & Chlorobenzene & 1 & & U & & UG/L & $05 / 31 / 2006$ & & JGJ-084-06 \\
\hline GWM47702VL & MIDDLE-2051 & 834 & Dibromochloromethane & 1 & & u & & UG/L & $05 / 31 / 2006$ & & JGJ-084-06 \\
\hline GWM47702VL & MIDDLE-2051 & 834 & 1,2-Dibromo-3-chloropropane & 1 & & $\mathrm{u}$ & $\mathrm{R}$ & UG/L & $05 / 31 / 2006$ & & JGJ-084-06 \\
\hline GWM47702VL & MIDDLE-2051 & 834 & Chloroethane & 2 & & u & & UG/L & $05 / 31 / 2006$ & & JGJ-084-06 \\
\hline GWM47702VL & MIDDLE-2051 & 834 & Chloroform & 1 & & $\mathrm{u}$ & & UG/L & $05 / 31 / 2006$ & & JGJ-084-06 \\
\hline GWM47702VL & MIDDLE-2051 & 834 & Chloromethane & 2 & & $\mathrm{u}$ & & UG/L & $05 / 31 / 2006$ & & JGJ-084-06 \\
\hline GWM47702VL & MIDDLE-2051 & 834 & Cyclohexane & 1 & & $\mathrm{U}$ & & UG/L & $05 / 31 / 2006$ & & JGJ-084-06 \\
\hline GWM47702VL & MIDDLE-2051 & 834 & 1,2-Dibromoethane & 1 & & u & & UG/L & $05 / 31 / 2006$ & & JGJ-084-06 \\
\hline GWM47702VL & MIDDLE-2051 & 834 & 1,2-Dichlorobenzene & 1 & & $\mathrm{u}$ & & UG/L & $05 / 31 / 2006$ & & JGJ-084-06 \\
\hline GWM47702VL & MIDDLE-2051 & 834 & 1,3-Dichlorobenzene & 1 & & $\mathrm{u}$ & & UG/L & $05 / 31 / 2006$ & & JGJ-084-06 \\
\hline GWM47702VL & MIDDLE-2051 & 834 & 1,4-Dichlorobenzene & 1 & & $\mathrm{U}$ & & UG/L & $05 / 31 / 2006$ & & JGJ-084-06 \\
\hline GWM47702VL & MIDDLE-2051 & 834 & Dichlorodifluoromethane & 2 & & $\mathrm{u}$ & & UG/L & $05 / 31 / 2006$ & & JGJ-084-06 \\
\hline GWM47702VL & MIDDLE-2051 & 834 & 1,1-Dichloroethane & 1 & & $\mathrm{u}$ & & UG/L & $05 / 31 / 2006$ & & JGJ-084-06 \\
\hline GWM47702VL & MIDDLE-2051 & 834 & 1,2-Dichloroethane & 1 & & $\mathrm{u}$ & & UG/L & $05 / 31 / 2006$ & & JGJ-084-06 \\
\hline GWM47702VL & MIDDLE-2051 & 834 & 1,1-Dichloroethene & 1 & & $\mathrm{U}$ & & UG/L & $05 / 31 / 2006$ & & JGJ-084-06 \\
\hline GWM47702VL & MIDDLE-2051 & 834 & cis-1,2-Dichloroethene & 1 & & $u$ & & UG/L & $05 / 31 / 2006$ & & JGJ-084-06 \\
\hline GWM47702VL & MIDDLE-2051 & 834 & trans-1,2-Dichloroethene & 1 & & u & & UG/L & $05 / 31 / 2006$ & & JGJ-084-06 \\
\hline GWM47702VL & MIDDLE-2051 & 834 & 1,2-Dichloropropane & 1 & & $\mathrm{u}$ & & UG/L & 05/31/2006 & & JGJ-084-06 \\
\hline GWM47702VL & MIDDLE-2051 & 834 & cis-1,3-Dichloropropene & 1 & & u & & UG/L & $05 / 31 / 2006$ & & JGJ-084-06 \\
\hline GWM47702VL & MIDDLE-2051 & 834 & trans-1,3-Dichloropropene & 1 & & $\mathrm{u}$ & & UG/L & 05/31/2006 & & JGJ-084-06 \\
\hline GWM47702VL & MIDDLE-2051 & 834 & Ethylbenzene & 1 & & u & & UG/L & $05 / 31 / 2006$ & & JGJ-084-06 \\
\hline GWM47702VL & MIDDLE-2051 & 834 & Trichlorofluoromethane & 1 & & $\mathrm{u}$ & & UG/L & 05/31/2006 & & JGJ-084-06 \\
\hline GWM47702VL & MIDDLE-2051 & 834 & 2-Hexanone & 5 & & $\mathrm{u}$ & $\mathrm{R}$ & UG/L & $05 / 31 / 2006$ & & JGJ-084-06 \\
\hline GWM47702VL & MIDDLE-2051 & 834 & Isopropylbenzene & 1 & & $\mathrm{U}$ & & UG/L & $05 / 31 / 2006$ & & JGJ-084-06 \\
\hline GWM47702VL & MIDDLE-2051 & 834 & Methyl acetate & 5 & & u & $\mathrm{R}$ & UG/L & $05 / 31 / 2006$ & & JGJ-084-06 \\
\hline GWM47702VL & MIDDLE-2051 & 834 & Methyl cyclohexane & 4 & & $\mathrm{u}$ & & UG/L & 05/31/2006 & & JGJ-084-06 \\
\hline GWM47702VL & MIDDLE-2051 & 834 & Methylene Chloride & 1 & & U & & UG/L & $05 / 31 / 2006$ & & JGJ-084-06 \\
\hline GWM47702VL & MIDDLE-2051 & 834 & 4-Methyl-2-pentanone & 5 & & $\mathrm{U}$ & & UG/L & 05/31/2006 & & JGJ-084-06 \\
\hline GWM47702VL & MIDDLE-2051 & 834 & Styrene & 1 & & $\mathrm{u}$ & & UG/L & $05 / 31 / 2006$ & & JGJ-084-06 \\
\hline GWM47702VL & MIDDLE-2051 & 834 & 1,1,2,2-Tetrachloroethane & 1 & & $\mathrm{u}$ & & UG/L & $05 / 31 / 2006$ & & JGJ-084-06 \\
\hline GWM47702VL & MIDDLE-2051 & 834 & Tetrachloroethene & 1 & & $\mathrm{u}$ & & UG/L & $05 / 31 / 2006$ & & JGJ-084-06 \\
\hline GWM47702VL & MIDDLE-2051 & 834 & Toluene & 1 & & u & & UG/L & $05 / 31 / 2006$ & & JGJ-084-06 \\
\hline GWM47702VL & MIDDLE-2051 & 834 & 1,2,4-Trichlorobenzene & 1 & & $\mathrm{U}$ & & UG/L & $05 / 31 / 2006$ & & JGJ-084-06 \\
\hline GWM47702VL & MIDDLE-2051 & 834 & 1,1,1-Trichloroethane & 1 & & u & & UG/L & $05 / 31 / 2006$ & & JGJ-084-06 \\
\hline GWM47702VL & MIDDLE-2051 & 834 & 1,1,2-Trichloroethane & 1 & & $\mathrm{u}$ & & UG/L & $05 / 31 / 2006$ & & JGJ-084-06 \\
\hline GWM47702VL & MIDDLE-2051 & 834 & Trichloroethene & 1 & & u & & UG/L & $05 / 31 / 2006$ & & JGJ-084-06 \\
\hline GWM47702VL & MIDDLE-2051 & 834 & 1,1,2-Trichloro-1,2,2-trifluoroethane & 1 & & $\mathrm{u}$ & & UG/L & $05 / 31 / 2006$ & & JGJ-084-06 \\
\hline GWM47702VL & MIDDLE-2051 & 834 & Vinyl Chloride & 2 & & u & & UG/L & $05 / 31 / 2006$ & & JGJ-084-06 \\
\hline GWM47702VL & MIDDLE-2051 & 834 & Xylenes & 3 & & $\mathrm{u}$ & & UG/L & $05 / 31 / 2006$ & & JGJ-084-06 \\
\hline GWM47702VL & MIDDLE-2051 & 834 & Methyl t-butyl ether & 2 & & $\mathrm{u}$ & & UG/L & $05 / 31 / 2006$ & & JGJ-084-06 \\
\hline GWM47701CW & MIDDLE-2051 & 834 & Carbon-14 & $-1.04 E+00$ & $1.52 \mathrm{E}+00$ & & $\mathrm{u}$ & $\mathrm{PCI} / \mathrm{L}$ & $05 / 31 / 2006$ & $5.10 E+00$ & SOS-TL175-06 \\
\hline GWM47702CW & MIDDLE-2051 & 834 & Carbon-14 & $-4.10 E+00$ & $5.39 \mathrm{E}+00$ & & $\mathrm{u}$ & $\mathrm{PCl} / \mathrm{L}$ & $05 / 31 / 2006$ & $1.81 \mathrm{E}+01$ & SOS-TL175-06 \\
\hline GWM47701R8 & MIDDLE-2051 & 834 & Tritium & $5.65 \mathrm{E}+02$ & $1.28 \mathrm{E}+02$ & & & $\mathrm{PCl} / \mathrm{L}$ & $05 / 31 / 2006$ & $3.98 \mathrm{E}+02$ & SOS-TL176-06 \\
\hline GWM47702R8 & MIDDLE-2051 & 834 & Tritium & $5.76 \mathrm{E}+02$ & $1.26 \mathrm{E}+02$ & & & $\mathrm{PCI} / \mathrm{L}$ & $05 / 31 / 2006$ & $3.93 \mathrm{E}+02$ & SOS-TL176-06 \\
\hline GWM47701RH & MIDDLE-2051 & 834 & Gross Alpha & $2.08 \mathrm{E}+00$ & $6.78 \mathrm{E}-01$ & & & $\mathrm{PCI} / \mathrm{L}$ & 05/31/2006 & $1.57 \mathrm{E}+00$ & SOS-TL177-06 \\
\hline GWM47701RH & MIDDLE-2051 & 834 & Gross Beta & $2.22 \mathrm{E}+00$ & 8.33E-01 & & UJ & $\mathrm{PCl} / \mathrm{L}$ & $05 / 31 / 2006$ & $3.30 E+00$ & SOS-TL177-06 \\
\hline GWM47702RH & MIDDLE-2051 & 834 & Gross Alpha & $1.92 \mathrm{E}+00$ & $6.88 \mathrm{E}-01$ & & UJ & $\mathrm{PCI} / \mathrm{L}$ & $05 / 31 / 2006$ & $1.99 \mathrm{E}+00$ & SOS-TL177-06 \\
\hline GWM47702RH & MIDDLE-2051 & 834 & Gross Beta & $3.03 \mathrm{E}+00$ & 9.04E-01 & & & $\mathrm{PCl} / \mathrm{L}$ & $05 / 31 / 2006$ & $3.42 \mathrm{E}+00$ & SOS-TL177-06 \\
\hline GWM47701RH & MIDDLE-2051 & 834 & Strontium-90 & $-1.26 \mathrm{E}-01$ & 7.94E-02 & & $\mathrm{U}$ & $\mathrm{PCI} / \mathrm{L}$ & $05 / 31 / 2006$ & 4.08E-01 & SOS-TL177-06 \\
\hline GWM47702RH & MIDDLE-2051 & 834 & Strontium-90 & 3.09E-02 & 7.54E-02 & & U & $\mathrm{PCl} / \mathrm{L}$ & $05 / 31 / 2006$ & 3.44E-01 & SOS-TL177-06 \\
\hline GWM47701RH & MIDDLE-2051 & 834 & Technetium-99 & $1.12 \mathrm{E}+00$ & $1.65 \mathrm{E}+00$ & & u & $\mathrm{PCl} / \mathrm{L}$ & $05 / 31 / 2006$ & $5.54 \mathrm{E}+00$ & SOS-TL177-06 \\
\hline GWM47702RH & MIDDLE-2051 & 834 & Technetium-99 & $2.19 \mathrm{E}+00$ & $1.68 \mathrm{E}+00$ & & $u$ & $\mathrm{PCI} / \mathrm{L}$ & $05 / 31 / 2006$ & $5.57 \mathrm{E}+00$ & SOS-TL177-06 \\
\hline GWM47701UX & MIDDLE-2051 & 834 & lodine-129 & $-1.26 \mathrm{E}-02$ & 2.33E-02 & & U & $\mathrm{PCI} / \mathrm{L}$ & $05 / 31 / 2006$ & 7.95E-02 & SOS-TL174-06 \\
\hline GWM47702UX & MIDDLE-2051 & 834 & lodine-129 & $-1.04 \mathrm{E}-02$ & 1.12E-02 & & U & $\mathrm{PCI} / \mathrm{L}$ & $05 / 31 / 2006$ & $3.78 \mathrm{E}-02$ & SOS-TL174-06 \\
\hline GWM478012X & MIDDLE-2051 & 1098 & Aluminum & 11 & & B & & UG/L & 05/30/2006 & & DNT-237-06 \\
\hline GWM478012X & MIDDLE-2051 & 1098 & Antimony & 0.5 & & U & & UG/L & 05/30/2006 & & DNT-237-06 \\
\hline GWM478012X & MIDDLE-2051 & 1098 & Arsenic & 2 & & u & & UG/L & 05/30/2006 & & DNT-237-06 \\
\hline GWM478012X & MIDDLE-2051 & 1098 & Barium & 38.4 & & & & UG/L & 05/30/2006 & & DNT-237-06 \\
\hline GWM478012X & MIDDLE-2051 & 1098 & Beryllium & 0.088 & & $\mathrm{U}$ & & UG/L & 05/30/2006 & & DNT-237-06 \\
\hline GWM478012X & MIDDLE-2051 & 1098 & Cadmium & 0.057 & & U & & UG/L & 05/30/2006 & & DNT-237-06 \\
\hline GWM478012X & MIDDLE-2051 & 1098 & Calcium & 33700 & & & & UG/L & 05/30/2006 & & DNT-237-06 \\
\hline GWM478012X & MIDDLE-2051 & 1098 & Chromium & 7.5 & & & & UG/L & $05 / 30 / 2006$ & & DNT-237-06 \\
\hline GWM478012X & MIDDLE-2051 & 1098 & Cobalt & 0.31 & & $\mathrm{U}$ & & UG/L & 05/30/2006 & & DNT-237-06 \\
\hline GWM478012X & MIDDLE-2051 & 1098 & Copper & 0.69 & & B & & UG/L & 05/30/2006 & & DNT-237-06 \\
\hline GWM478012X & MIDDLE-2051 & 1098 & Iron & 9.4 & & $u$ & & UG/L & 05/30/2006 & & DNT-237-06 \\
\hline GWM478012X & MIDDLE-2051 & 1098 & Lead & 0.49 & & u & & UG/L & $05 / 30 / 2006$ & & DNT-237-06 \\
\hline GWM478012X & MIDDLE-2051 & 1098 & Magnesium & 17100 & & & & UG/L & 05/30/2006 & & DNT-237-06 \\
\hline GWM478012X & MIDDLE-2051 & 1098 & Manganese & 0.58 & & B & & UG/L & 05/30/2006 & & DNT-237-06 \\
\hline
\end{tabular}


Table A-5. (continued).

\begin{tabular}{|c|c|c|c|c|c|c|c|c|c|c|c|}
\hline $\begin{array}{l}\text { Field Sample } \\
\text { Number }\end{array}$ & Location & Depth & Compound & $\begin{array}{c}\text { Sample } \\
\text { Result }\end{array}$ & $\begin{array}{l}\text { Sample } \\
\text { Error }\end{array}$ & $\begin{array}{c}\text { Result } \\
\text { Qualifier }\end{array}$ & $\begin{array}{l}\text { Validation } \\
\text { Flag }\end{array}$ & $\begin{array}{c}\text { Sample } \\
\text { Units }\end{array}$ & $\begin{array}{c}\text { Date Sample } \\
\text { Collected }\end{array}$ & MDA & $\begin{array}{l}\text { L\&V Report } \\
\text { Number }\end{array}$ \\
\hline GWM478012X & MIDDLE-2051 & 1098 & Nickel & 1.5 & & $\mathrm{~B}$ & & UG/L & $05 / 30 / 2006$ & & DNT-237-06 \\
\hline GWM478012X & MIDDLE-2051 & 1098 & Potassium & 2270 & & & & UG/L & 05/30/2006 & & DNT-237-06 \\
\hline GWM478012X & MIDDLE-2051 & 1098 & Selenium & 1.1 & & B & & UG/L & $05 / 30 / 2006$ & & DNT-237-06 \\
\hline GWM478012X & MIDDLE-2051 & 1098 & Silver & 0.2 & & $u$ & & UG/L & 05/30/2006 & & DNT-237-06 \\
\hline GWM478012X & MIDDLE-2051 & 1098 & Sodium & 7320 & & & & UG/L & 05/30/2006 & & DNT-237-06 \\
\hline GWM478012X & MIDDLE-2051 & 1098 & Strontium & 215 & & E & $\mathrm{J}$ & UG/L & 05/30/2006 & & DNT-237-06 \\
\hline GWM478012X & MIDDLE-2051 & 1098 & Thallium & 1.1 & & B & U & UG/L & $05 / 30 / 2006$ & & DNT-237-06 \\
\hline GWM478012X & MIDDLE-2051 & 1098 & Uranium & 1.8 & & & & UG/L & 05/30/2006 & & DNT-237-06 \\
\hline GWM478012X & MIDDLE-2051 & 1098 & Vanadium & 4.5 & & B & & UG/L & 05/30/2006 & & DNT-237-06 \\
\hline GWM478012X & MIDDLE-2051 & 1098 & Zinc & 24.1 & & & & UG/L & 05/30/2006 & & DNT-237-06 \\
\hline GWM478012X & MIDDLE-2051 & 1098 & Mercury & 0.093 & & U & & UG/L & $05 / 30 / 2006$ & & DNT-237-06 \\
\hline GWM47801A1 & MIDDLE-2051 & 1098 & Total Alkalinity & 152 & & & & MG/L & 05/30/2006 & & DNT-285-06 \\
\hline GWM47801AN & MIDDLE-2051 & 1098 & Chloride & 13.1 & & & & $\mathrm{MG} / \mathrm{L}$ & 05/30/2006 & & DNT-285-06 \\
\hline GWM47801AN & MIDDLE-2051 & 1098 & Fluoride & 0.16 & & & $\mathrm{~J}$ & MG/L & 05/30/2006 & & DNT-285-06 \\
\hline GWM47801AN & MIDDLE-2051 & 1098 & Sulfate & 22.1 & & & & $\mathrm{MG} / \mathrm{L}$ & 05/30/2006 & & DNT-285-06 \\
\hline GWM47801N2 & MIDDLE-2051 & 1098 & Nitrate/Nitrite as $\mathrm{N}$ & 951 & & & & UG/L & $05 / 30 / 2006$ & & DNT-285-06 \\
\hline GWM47801VL & MIDDLE-2051 & 1098 & Acetone & 2 & & u & $\mathrm{R}$ & UG/L & 05/30/2006 & & JGJ-084-06 \\
\hline GWM47801VL & MIDDLE-2051 & 1098 & Benzene & 1 & & u & & UG/L & 05/30/2006 & & JGJ-084-06 \\
\hline GWM47801VL & MIDDLE-2051 & 1098 & Bromodichloromethane & 1 & & $u$ & & UG/L & 05/30/2006 & & JGJ-084-06 \\
\hline GWM47801VL & MIDDLE-2051 & 1098 & Bromoform & 1 & & u & & UG/L & 05/30/2006 & & JGJ-084-06 \\
\hline GWM47801VL & MIDDLE-2051 & 1098 & Bromomethane & 2 & & $u$ & & UG/L & 05/30/2006 & & JGJ-084-06 \\
\hline GWM47801VL & MIDDLE-2051 & 1098 & 2-Butanone & 5 & & $u$ & $\mathrm{R}$ & UG/L & 05/30/2006 & & JGJ-084-06 \\
\hline GWM47801VL & MIDDLE-2051 & 1098 & Carbon disulfide & 1 & & $u$ & & UG/L & 05/30/2006 & & JGJ-084-06 \\
\hline GWM47801VL & MIDDLE-2051 & 1098 & Carbon tetrachloride & 1 & & u & & UG/L & $05 / 30 / 2006$ & & JGJ-084-06 \\
\hline GWM47801VL & MIDDLE-2051 & 1098 & Chlorobenzene & 1 & & $u$ & & UG/L & 05/30/2006 & & JGJ-084-06 \\
\hline GWM47801VL & MIDDLE-2051 & 1098 & Dibromochloromethane & 1 & & $u$ & & UG/L & 05/30/2006 & & JGJ-084-06 \\
\hline GWM47801VL & MIDDLE-2051 & 1098 & 1,2-Dibromo-3-chloropropane & 1 & & $\mathrm{u}$ & $\mathrm{R}$ & UG/L & 05/30/2006 & & JGJ-084-06 \\
\hline GWM47801VL & MIDDLE-2051 & 1098 & Chloroethane & 2 & & $\mathrm{u}$ & & UG/L & 05/30/2006 & & JGJ-084-06 \\
\hline GWM47801VL & MIDDLE-2051 & 1098 & Chloroform & 1 & & $\mathrm{u}$ & & UG/L & 05/30/2006 & & JGJ-084-06 \\
\hline GWM47801VL & MIDDLE-2051 & 1098 & Chloromethane & 2 & & $u$ & & UG/L & 05/30/2006 & & JGJ-084-06 \\
\hline GWM47801VL & MIDDLE-2051 & 1098 & Cyclohexane & 1 & & $\mathrm{u}$ & & UG/L & 05/30/2006 & & JGJ-084-06 \\
\hline GWM47801VL & MIDDLE-2051 & 1098 & 1,2-Dibromoethane & 1 & & $u$ & & UG/L & 05/30/2006 & & JGJ-084-06 \\
\hline GWM47801VL & MIDDLE-2051 & 1098 & 1,2-Dichlorobenzene & 1 & & $\mathrm{u}$ & & UG/L & 05/30/2006 & & JGJ-084-06 \\
\hline GWM47801VL & MIDDLE-2051 & 1098 & 1,3-Dichlorobenzene & 1 & & $u$ & & UG/L & 05/30/2006 & & JGJ-084-06 \\
\hline GWM47801VL & MIDDLE-2051 & 1098 & 1,4-Dichlorobenzene & 1 & & $\mathrm{u}$ & & UG/L & 05/30/2006 & & JGJ-084-06 \\
\hline GWM47801VL & MIDDLE-2051 & 1098 & Dichlorodifluoromethane & 2 & & $\mathrm{u}$ & & UG/L & 05/30/2006 & & JGJ-084-06 \\
\hline GWM47801VL & MIDDLE-2051 & 1098 & 1,1-Dichloroethane & 1 & & u & & UG/L & 05/30/2006 & & JGJ-084-06 \\
\hline GWM47801VL & MIDDLE-2051 & 1098 & 1,2-Dichloroethane & 1 & & $\mathrm{u}$ & & UG/L & 05/30/2006 & & JGJ-084-06 \\
\hline GWM47801VL & MIDDLE-2051 & 1098 & 1,1-Dichloroethene & 1 & & $\mathrm{u}$ & & UG/L & 05/30/2006 & & JGJ-084-06 \\
\hline GWM47801VL & MIDDLE-2051 & 1098 & cis-1,2-Dichloroethene & 1 & & $\mathrm{u}$ & & UG/L & 05/30/2006 & & JGJ-084-06 \\
\hline GWM47801VL & MIDDLE-2051 & 1098 & trans-1,2-Dichloroethene & 1 & & $u$ & & UG/L & 05/30/2006 & & JGJ-084-06 \\
\hline GWM47801VL & MIDDLE-2051 & 1098 & 1,2-Dichloropropane & 1 & & $\mathrm{u}$ & & UG/L & 05/30/2006 & & JGJ-084-06 \\
\hline GWM47801VL & MIDDLE-2051 & 1098 & cis-1,3-Dichloropropene & 1 & & $\mathrm{u}$ & & UG/L & 05/30/2006 & & JGJ-084-06 \\
\hline GWM47801VL & MIDDLE-2051 & 1098 & trans-1,3-Dichloropropene & 1 & & $\mathrm{u}$ & & UG/L & 05/30/2006 & & JGJ-084-06 \\
\hline GWM47801VL & MIDDLE-2051 & 1098 & Ethylbenzene & 1 & & $u$ & & UG/L & 05/30/2006 & & JGJ-084-06 \\
\hline GWM47801VL & MIDDLE-2051 & 1098 & Trichlorofluoromethane & 1 & & $\mathrm{u}$ & & UG/L & 05/30/2006 & & JGJ-084-06 \\
\hline GWM47801VL & MIDDLE-2051 & 1098 & 2-Hexanone & 5 & & $u$ & $\mathrm{R}$ & UG/L & 05/30/2006 & & JGJ-084-06 \\
\hline GWM47801VL & MIDDLE-2051 & 1098 & Isopropylbenzene & 1 & & U & & UG/L & 05/30/2006 & & JGJ-084-06 \\
\hline GWM47801VL & MIDDLE-2051 & 1098 & Methyl acetate & 5 & & $u$ & $\mathrm{R}$ & UG/L & 05/30/2006 & & JGJ-084-06 \\
\hline GWM47801VL & MIDDLE-2051 & 1098 & Methyl cyclohexane & 4 & & $\mathrm{u}$ & & UG/L & 05/30/2006 & & JGJ-084-06 \\
\hline GWM47801VL & MIDDLE-2051 & 1098 & Methylene Chloride & 1 & & $u$ & & UG/L & 05/30/2006 & & JGJ-084-06 \\
\hline GWM47801VL & MIDDLE-2051 & 1098 & 4-Methyl-2-pentanone & 5 & & $\mathrm{u}$ & & UG/L & 05/30/2006 & & JGJ-084-06 \\
\hline GWM47801VL & MIDDLE-2051 & 1098 & Styrene & 1 & & $\mathrm{u}$ & & UG/L & 05/30/2006 & & JGJ-084-06 \\
\hline GWM47801VL & MIDDLE-2051 & 1098 & 1,1,2,2-Tetrachloroethane & 1 & & u & & UG/L & 05/30/2006 & & JGJ-084-06 \\
\hline GWM47801VL & MIDDLE-2051 & 1098 & Tetrachloroethene & 1 & & $u$ & & UG/L & 05/30/2006 & & JGJ-084-06 \\
\hline GWM47801VL & MIDDLE-2051 & 1098 & Toluene & 1 & & $\mathrm{u}$ & & UG/L & 05/30/2006 & & JGJ-084-06 \\
\hline GWM47801VL & MIDDLE-2051 & 1098 & 1,2,4-Trichlorobenzene & 1 & & $u$ & & UG/L & 05/30/2006 & & JGJ-084-06 \\
\hline GWM47801VL & MIDDLE-2051 & 1098 & $1,1,1$-Trichloroethane & 1 & & $u$ & & UG/L & 05/30/2006 & & JGJ-084-06 \\
\hline GWM47801VL & MIDDLE-2051 & 1098 & 1,1,2-Trichloroethane & 1 & & $\mathrm{u}$ & & UG/L & 05/30/2006 & & JGJ-084-06 \\
\hline GWM47801VL & MIDDLE-2051 & 1098 & Trichloroethene & 1 & & $\mathrm{u}$ & & UG/L & 05/30/2006 & & JGJ-084-06 \\
\hline GWM47801VL & MIDDLE-2051 & 1098 & 1,1,2-Trichloro-1,2,2-trifluoroethane & 1 & & $\mathrm{u}$ & & UG/L & 05/30/2006 & & JGJ-084-06 \\
\hline GWM47801VL & MIDDLE-2051 & 1098 & Vinyl Chloride & 2 & & u & & UG/L & 05/30/2006 & & JGJ-084-06 \\
\hline GWM47801VL & MIDDLE-2051 & 1098 & Xylenes & 3 & & $\mathrm{u}$ & & UG/L & 05/30/2006 & & JGJ-084-06 \\
\hline GWM47801VL & MIDDLE-2051 & 1098 & Methyl t-butyl ether & 2 & & u & & UG/L & 05/30/2006 & & JGJ-084-06 \\
\hline GWM47801CW & MIDDLE-2051 & 1098 & Carbon-14 & $-4.95 E+00$ & $1.53 \mathrm{E}+00$ & & $\mathrm{u}$ & $\mathrm{PCl} / \mathrm{L}$ & 05/30/2006 & $5.20 E+00$ & SOS-TL175-06 \\
\hline GWM47801R8 & MIDDLE-2051 & 1098 & Tritium & $4.28 \mathrm{E}+02$ & $1.25 \mathrm{E}+02$ & & & $\mathrm{PCl} / \mathrm{L}$ & $05 / 30 / 2006$ & $3.97 \mathrm{E}+02$ & SOS-TL176-06 \\
\hline GWM47801RH & MIDDLE-2051 & 1098 & Gross Alpha & $1.33 \mathrm{E}+00$ & $6.52 \mathrm{E}-01$ & & UJ & $\mathrm{PCl} / \mathrm{L}$ & $05 / 30 / 2006$ & $1.96 \mathrm{E}+00$ & SOS-TL177-06 \\
\hline GWM47801RH & MIDDLE-2051 & 1098 & Gross Beta & $3.04 \mathrm{E}+00$ & 8.29E-01 & & & $\mathrm{PCl} / \mathrm{L}$ & 05/30/2006 & $3.04 \mathrm{E}+00$ & SOS-TL177-06 \\
\hline GWM47801RH & MIDDLE-2051 & 1098 & Strontium-90 & $1.49 \mathrm{E}-01$ & $9.80 \mathrm{E}-02$ & & $U$ & $\mathrm{PCl} / \mathrm{L}$ & $05 / 30 / 2006$ & 4.17E-01 & SOS-TL177-06 \\
\hline GWM47801RH & MIDDLE-2051 & 1098 & Technetium-99 & $1.64 \mathrm{E}+00$ & $1.65 \mathrm{E}+00$ & & $\mathrm{u}$ & $\mathrm{PCl} / \mathrm{L}$ & 05/30/2006 & $5.51 \mathrm{E}+00$ & SOS-TL177-06 \\
\hline GWM47801UX & MIDDLE-2051 & 1098 & lodine-129 & 2.69E-01 & 7.07E-02 & & UJ & $\mathrm{PCl} / \mathrm{L}$ & $05 / 30 / 2006$ & $1.40 \mathrm{E}-01$ & SOS-TL174-06 \\
\hline GWM479012X & MIDDLE-2051 & 1148 & Aluminum & 8.9 & & B & & UG/L & 05/26/2006 & & DNT-284-06 \\
\hline GWM479012X & MIDDLE-2051 & 1148 & Antimony & 0.5 & & $u$ & & UG/L & $05 / 26 / 2006$ & & DNT-284-06 \\
\hline GWM479012X & MIDDLE-2051 & 1148 & Arsenic & 2 & & $u$ & & UG/L & 05/26/2006 & & DNT-284-06 \\
\hline GWM479012X & MIDDLE-2051 & 1148 & Barium & 35.3 & & & & UG/L & $05 / 26 / 2006$ & & DNT-284-06 \\
\hline GWM479012X & MIDDLE-2051 & 1148 & Beryllium & 0.088 & & U & & UG/L & 05/26/2006 & & DNT-284-06 \\
\hline GWM479012X & MIDDLE-2051 & 1148 & Cadmium & 0.057 & & u & & UG/L & $05 / 26 / 2006$ & & DNT-284-06 \\
\hline GWM479012X & MIDDLE-2051 & 1148 & Calcium & 35400 & & E & $\mathrm{J}$ & UG/L & 05/26/2006 & & DNT-284-06 \\
\hline GWM479012X & MIDDLE-2051 & 1148 & Chromium & 6 & & & & UG/L & $05 / 26 / 2006$ & & DNT-284-06 \\
\hline GWM479012X & MIDDLE-2051 & 1148 & Cobalt & 0.37 & & B & & UG/L & $05 / 26 / 2006$ & & DNT-284-06 \\
\hline GWM479012X & MIDDLE-2051 & 1148 & Copper & 1.7 & & & & UG/L & $05 / 26 / 2006$ & & DNT-284-06 \\
\hline GWM479012X & MIDDLE-2051 & 1148 & Iron & 9.4 & & $u$ & & UG/L & 05/26/2006 & & DNT-284-06 \\
\hline GWM479012X & MIDDLE-2051 & 1148 & Lead & 0.49 & & U & & UG/L & $05 / 26 / 2006$ & & DNT-284-06 \\
\hline GWM479012X & MIDDLE-2051 & 1148 & Magnesium & 17400 & & E & $\mathrm{J}$ & UG/L & $05 / 26 / 2006$ & & DNT-284-06 \\
\hline GWM479012X & MIDDLE-2051 & 1148 & Manganese & 9.9 & & & & UG/L & $05 / 26 / 2006$ & & DNT-284-06 \\
\hline GWM479012X & MIDDLE-2051 & 1148 & Nickel & 2.6 & & B & & UG/L & $05 / 26 / 2006$ & & DNT-284-06 \\
\hline
\end{tabular}


Table A-5. (continued).

\begin{tabular}{|c|c|c|c|c|c|c|c|c|c|c|c|}
\hline $\begin{array}{l}\text { Field Sample } \\
\text { Number }\end{array}$ & Location & Depth & Compound & $\begin{array}{l}\text { Sample } \\
\text { Result }\end{array}$ & $\begin{array}{l}\text { Sample } \\
\text { Error }\end{array}$ & $\begin{array}{c}\text { Result } \\
\text { Qualifier }\end{array}$ & $\begin{array}{l}\text { Validation } \\
\text { Flag }\end{array}$ & $\begin{array}{l}\text { Sample } \\
\text { Units }\end{array}$ & $\begin{array}{c}\text { Date Sample } \\
\text { Collected }\end{array}$ & MDA & $\begin{array}{l}\text { L\&V Report } \\
\text { Number }\end{array}$ \\
\hline GWM479012X & MIDDLE-2051 & 1148 & Potassium & 2380 & & & & UG/L & $05 / 26 / 2006$ & & DNT-284-06 \\
\hline GWM479012X & MIDDLE-2051 & 1148 & Selenium & 1.5 & & B & & UG/L & $05 / 26 / 2006$ & & DNT-284-06 \\
\hline GWM479012X & MIDDLE-2051 & 1148 & Silver & 0.2 & & U & & UG/L & $05 / 26 / 2006$ & & DNT-284-06 \\
\hline GWM479012X & MIDDLE-2051 & 1148 & Sodium & 7580 & & E & $\mathrm{J}$ & UG/L & 05/26/2006 & & DNT-284-06 \\
\hline GWM479012X & MIDDLE-2051 & 1148 & Strontium & 228 & & E & $\mathrm{J}$ & UG/L & 05/26/2006 & & DNT-284-06 \\
\hline GWM479012X & MIDDLE-2051 & 1148 & Thallium & 0.32 & & U & & UG/L & 05/26/2006 & & DNT-284-06 \\
\hline GWM479012X & MIDDLE-2051 & 1148 & Uranium & 1.6 & & & & UG/L & 05/26/2006 & & DNT-284-06 \\
\hline GWM479012X & MIDDLE-2051 & 1148 & Vanadium & 4.2 & & B & & UG/L & 05/26/2006 & & DNT-284-06 \\
\hline GWM479012X & MIDDLE-2051 & 1148 & Zinc & 106 & & & & UG/L & 05/26/2006 & & DNT-284-06 \\
\hline GWM479012X & MIDDLE-2051 & 1148 & Mercury & 0.093 & & U & & UG/L & 05/26/2006 & & DNT-284-06 \\
\hline GWM47901A1 & MIDDLE-2051 & 1148 & Total Alkalinity & 146 & & & $\mathrm{~J}$ & $\mathrm{MG} / \mathrm{L}$ & $05 / 26 / 2006$ & & DNT-286-06 \\
\hline GWM47901AN & MIDDLE-2051 & 1148 & Chloride & 13.3 & & $\mathrm{~J}$ & & MG/L & 05/26/2006 & & DNT-286-06 \\
\hline GWM47901AN & MIDDLE-2051 & 1148 & Fluoride & 0.14 & & & & $\mathrm{MG} / \mathrm{L}$ & $05 / 26 / 2006$ & & DNT-286-06 \\
\hline GWM47901AN & MIDDLE-2051 & 1148 & Sulfate & 20.9 & & & $\mathrm{~J}$ & $\mathrm{MG} / \mathrm{L}$ & $05 / 26 / 2006$ & & DNT-286-06 \\
\hline GWM47901N2 & MIDDLE-2051 & 1148 & Nitrate/Nitrite as $\mathrm{N}$ & 692 & & & $\mathrm{~J}$ & UG/L & $05 / 26 / 2006$ & & DNT-286-06 \\
\hline GWM47901VL & MIDDLE-2051 & 1148 & Acetone & 2 & & $\mathrm{u}$ & $\mathrm{R}$ & UG/L & $05 / 26 / 2006$ & & JGJ-083-06 \\
\hline GWM47901VL & MIDDLE-2051 & 1148 & Benzene & 1 & & $\mathrm{U}$ & & UG/L & 05/26/2006 & & JGJ-083-06 \\
\hline GWM47901VL & MIDDLE-2051 & 1148 & Bromodichloromethane & 1 & & u & & UG/L & $05 / 26 / 2006$ & & JGJ-083-06 \\
\hline GWM47901VL & MIDDLE-2051 & 1148 & Bromoform & 1 & & $u$ & & UG/L & $05 / 26 / 2006$ & & JGJ-083-06 \\
\hline GWM47901VL & MIDDLE-2051 & 1148 & Bromomethane & 2 & & u & & UG/L & $05 / 26 / 2006$ & & JGJ-083-06 \\
\hline GWM47901VL & MIDDLE-2051 & 1148 & 2-Butanone & 5 & & $\mathrm{u}$ & $\mathrm{R}$ & UG/L & 05/26/2006 & & JGJ-083-06 \\
\hline GWM47901VL & MIDDLE-2051 & 1148 & Carbon disulfide & 1 & & u & & UG/L & $05 / 26 / 2006$ & & JGJ-083-06 \\
\hline GWM47901VL & MIDDLE-2051 & 1148 & Carbon tetrachloride & 1 & & $u$ & & UG/L & $05 / 26 / 2006$ & & JGJ-083-06 \\
\hline GWM47901VL & MIDDLE-2051 & 1148 & Chlorobenzene & 1 & & U & & UG/L & $05 / 26 / 2006$ & & JGJ-083-06 \\
\hline GWM47901VL & MIDDLE-2051 & 1148 & Dibromochloromethane & 1 & & $\mathrm{U}$ & & UG/L & $05 / 26 / 2006$ & & JGJ-083-06 \\
\hline GWM47901VL & MIDDLE-2051 & 1148 & 1,2-Dibromo-3-chloropropane & 1 & & $\mathrm{u}$ & $\mathrm{R}$ & UG/L & $05 / 26 / 2006$ & & JGJ-083-06 \\
\hline GWM47901VL & MIDDLE-2051 & 1148 & Chloroethane & 2 & & $\mathrm{u}$ & & UG/L & $05 / 26 / 2006$ & & JGJ-083-06 \\
\hline GWM47901VL & MIDDLE-2051 & 1148 & Chloroform & 1 & & $\mathrm{u}$ & & UG/L & $05 / 26 / 2006$ & & JGJ-083-06 \\
\hline GWM47901VL & MIDDLE-2051 & 1148 & Chloromethane & 2 & & $\mathrm{U}$ & & UG/L & $05 / 26 / 2006$ & & JGJ-083-06 \\
\hline GWM47901VL & MIDDLE-2051 & 1148 & Cyclohexane & 1 & & $\mathrm{u}$ & & UG/L & $05 / 26 / 2006$ & & JGJ-083-06 \\
\hline GWM47901VL & MIDDLE-2051 & 1148 & 1,2-Dibromoethane & 1 & & u & & UG/L & $05 / 26 / 2006$ & & JGJ-083-06 \\
\hline GWM47901VL & MIDDLE-2051 & 1148 & 1,2-Dichlorobenzene & 1 & & $\mathrm{u}$ & & UG/L & $05 / 26 / 2006$ & & JGJ-083-06 \\
\hline GWM47901VL & MIDDLE-2051 & 1148 & 1,3-Dichlorobenzene & 1 & & $\mathrm{u}$ & & UG/L & $05 / 26 / 2006$ & & JGJ-083-06 \\
\hline GWM47901VL & MIDDLE-2051 & 1148 & 1,4-Dichlorobenzene & 1 & & $\mathrm{u}$ & & UG/L & $05 / 26 / 2006$ & & JGJ-083-06 \\
\hline GWM47901VL & MIDDLE-2051 & 1148 & Dichlorodifluoromethane & 2 & & u & & UG/L & $05 / 26 / 2006$ & & JGJ-083-06 \\
\hline GWM47901VL & MIDDLE-2051 & 1148 & 1,1-Dichloroethane & 1 & & $\mathrm{u}$ & & UG/L & 05/26/2006 & & JGJ-083-06 \\
\hline GWM47901VL & MIDDLE-2051 & 1148 & 1,2-Dichloroethane & 1 & & u & & UG/L & $05 / 26 / 2006$ & & JGJ-083-06 \\
\hline GWM47901VL & MIDDLE-2051 & 1148 & 1,1-Dichloroethene & 1 & & $\mathrm{u}$ & & UG/L & 05/26/2006 & & JGJ-083-06 \\
\hline GWM47901VL & MIDDLE-2051 & 1148 & cis-1,2-Dichloroethene & 1 & & $\mathrm{u}$ & & UG/L & 05/26/2006 & & JGJ-083-06 \\
\hline GWM47901VL & MIDDLE-2051 & 1148 & trans-1,2-Dichloroethene & 1 & & $\mathrm{u}$ & & UG/L & 05/26/2006 & & JGJ-083-06 \\
\hline GWM47901VL & MIDDLE-2051 & 1148 & 1,2-Dichloropropane & 1 & & $\mathrm{u}$ & & UG/L & 05/26/2006 & & JGJ-083-06 \\
\hline GWM47901VL & MIDDLE-2051 & 1148 & cis-1,3-Dichloropropene & 1 & & $\mathrm{U}$ & & UG/L & $05 / 26 / 2006$ & & JGJ-083-06 \\
\hline GWM47901VL & MIDDLE-2051 & 1148 & trans-1,3-Dichloropropene & 1 & & u & & UG/L & $05 / 26 / 2006$ & & JGJ-083-06 \\
\hline GWM47901VL & MIDDLE-2051 & 1148 & Ethylbenzene & 1 & & $\mathrm{u}$ & & UG/L & $05 / 26 / 2006$ & & JGJ-083-06 \\
\hline GWM47901VL & MIDDLE-2051 & 1148 & Trichlorofluoromethane & 1 & & $\mathrm{U}$ & & UG/L & $05 / 26 / 2006$ & & JGJ-083-06 \\
\hline GWM47901VL & MIDDLE-2051 & 1148 & 2-Hexanone & 5 & & $\mathrm{U}$ & & UG/L & $05 / 26 / 2006$ & & JGJ-083-06 \\
\hline GWM47901VL & MIDDLE-2051 & 1148 & Isopropylbenzene & 1 & & $\mathrm{U}$ & & UG/L & $05 / 26 / 2006$ & & JGJ-083-06 \\
\hline GWM47901VL & MIDDLE-2051 & 1148 & Methyl acetate & 5 & & $\mathrm{u}$ & $\mathrm{R}$ & UG/L & $05 / 26 / 2006$ & & JGJ-083-06 \\
\hline GWM47901VL & MIDDLE-2051 & 1148 & Methyl cyclohexane & 4 & & $\mathrm{u}$ & & UG/L & $05 / 26 / 2006$ & & JGJ-083-06 \\
\hline GWM47901VL & MIDDLE-2051 & 1148 & Methylene Chloride & 1 & & u & & UG/L & $05 / 26 / 2006$ & & JGJ-083-06 \\
\hline GWM47901VL & MIDDLE-2051 & 1148 & 4-Methyl-2-pentanone & 5 & & $\mathrm{U}$ & & UG/L & $05 / 26 / 2006$ & & JGJ-083-06 \\
\hline GWM47901VL & MIDDLE-2051 & 1148 & Styrene & 1 & & u & & UG/L & $05 / 26 / 2006$ & & JGJ-083-06 \\
\hline GWM47901VL & MIDDLE-2051 & 1148 & 1,1,2,2-Tetrachloroethane & 1 & & $\mathrm{u}$ & & UG/L & 05/26/2006 & & JGJ-083-06 \\
\hline GWM47901VL & MIDDLE-2051 & 1148 & Tetrachloroethene & 1 & & u & & UG/L & $05 / 26 / 2006$ & & JGJ-083-06 \\
\hline GWM47901VL & MIDDLE-2051 & 1148 & Toluene & 1 & & $\mathrm{U}$ & & UG/L & $05 / 26 / 2006$ & & JGJ-083-06 \\
\hline GWM47901VL & MIDDLE-2051 & 1148 & 1,2,4-Trichlorobenzene & 1 & & u & & UG/L & $05 / 26 / 2006$ & & JGJ-083-06 \\
\hline GWM47901VL & MIDDLE-2051 & 1148 & 1,1,1-Trichloroethane & 1 & & $\mathrm{u}$ & & UG/L & $05 / 26 / 2006$ & & JGJ-083-06 \\
\hline GWM47901VL & MIDDLE-2051 & 1148 & 1,1,2-Trichloroethane & 1 & & $\mathrm{U}$ & & UG/L & $05 / 26 / 2006$ & & JGJ-083-06 \\
\hline GWM47901VL & MIDDLE-2051 & 1148 & Trichloroethene & 1 & & $\mathrm{U}$ & & UG/L & $05 / 26 / 2006$ & & JGJ-083-06 \\
\hline GWM47901VL & MIDDLE-2051 & 1148 & 1,1,2-Trichloro-1,2,2-trifluoroethane & 1 & & u & & UG/L & $05 / 26 / 2006$ & & JGJ-083-06 \\
\hline GWM47901VL & MIDDLE-2051 & 1148 & Vinyl Chloride & 2 & & $\mathrm{u}$ & & UG/L & 05/26/2006 & & JGJ-083-06 \\
\hline GWM47901VL & MIDDLE-2051 & 1148 & Xylenes & 3 & & $\mathrm{u}$ & & UG/L & 05/26/2006 & & JGJ-083-06 \\
\hline GWM47901VL & MIDDLE-2051 & 1148 & Methyl t-butyl ether & 2 & & $\mathrm{U}$ & & UG/L & $05 / 26 / 2006$ & & JGJ-083-06 \\
\hline GWM47901CW & MIDDLE-2051 & 1148 & Carbon-14 & $-7.01 \mathrm{E}+00$ & $5.27 \mathrm{E}+00$ & & $\mathrm{u}$ & $\mathrm{PCl} / \mathrm{L}$ & 05/26/2006 & $1.77 \mathrm{E}+01$ & SOS-TL175-06 \\
\hline GWM47901R8 & MIDDLE-2051 & 1148 & Tritium & $5.87 \mathrm{E}+02$ & $1.23 \mathrm{E}+02$ & & & $\mathrm{PCI} / \mathrm{L}$ & $05 / 26 / 2006$ & $3.81 \mathrm{E}+02$ & SOS-TL176-06 \\
\hline GWM47901RH & MIDDLE-2051 & 1148 & Gross Alpha & $1.56 \mathrm{E}+00$ & 6.40E-01 & & UJ & $\mathrm{PCl} / \mathrm{L}$ & $05 / 26 / 2006$ & $1.80 \mathrm{E}+00$ & SOS-TL177-06 \\
\hline GWM47901RH & MIDDLE-2051 & 1148 & Gross Beta & 4.19E+00 & $8.88 \mathrm{E}-01$ & & & $\mathrm{PCI} / \mathrm{L}$ & $05 / 26 / 2006$ & $3.11 \mathrm{E}+00$ & SOS-TL177-06 \\
\hline GWM47901RH & MIDDLE-2051 & 1148 & Strontium-90 & $1.22 \mathrm{E}-01$ & 6.33E-02 & & $\mathrm{u}$ & $\mathrm{PCI} / \mathrm{L}$ & 05/26/2006 & $2.60 \mathrm{E}-01$ & SOS-TL177-06 \\
\hline GWM47901RH & MIDDLE-2051 & 1148 & Technetium-99 & $2.39 \mathrm{E}+00$ & $1.69 \mathrm{E}+00$ & & u & $\mathrm{PCI} / \mathrm{L}$ & 05/26/2006 & $5.58 \mathrm{E}+00$ & SOS-TL177-06 \\
\hline GWM47901UX & MIDDLE-2051 & 1148 & lodine-129 & $1.50 \mathrm{E}-02$ & $2.59 \mathrm{E}-02$ & & $\mathrm{u}$ & $\mathrm{PCI} / \mathrm{L}$ & 05/26/2006 & $1.02 \mathrm{E}-01$ & SOS-TL174-06 \\
\hline GWM46902VL & TRIP BLANK & NA & Acetone & 2 & & $\mathrm{u}$ & $\mathrm{R}$ & UG/L & 05/30/2006 & & JGJ-084-06 \\
\hline GWM46902VL & TRIP BLANK & NA & Benzene & 1 & & $\mathrm{U}$ & & UG/L & 05/30/2006 & & JGJ-084-06 \\
\hline GWM46902VL & TRIP BLANK & NA & Bromodichloromethane & 1 & & $\mathrm{u}$ & & UG/L & 05/30/2006 & & JGJ-084-06 \\
\hline GWM46902VL & TRIP BLANK & NA & Bromoform & 1 & & $\mathrm{u}$ & & UG/L & 05/30/2006 & & JGJ-084-06 \\
\hline GWM46902VL & TRIP BLANK & NA & Bromomethane & 2 & & u & & UG/L & 05/30/2006 & & JGJ-084-06 \\
\hline GWM46902VL & TRIP BLANK & NA & 2-Butanone & 5 & & u & $\mathrm{R}$ & UG/L & $05 / 30 / 2006$ & & JGJ-084-06 \\
\hline GWM46902VL & TRIP BLANK & NA & Carbon disulfide & 1 & & $\mathrm{u}$ & & UG/L & 05/30/2006 & & JGJ-084-06 \\
\hline GWM46902VL & TRIP BLANK & NA & Carbon tetrachloride & 1 & & $\mathrm{U}$ & & UG/L & 05/30/2006 & & JGJ-084-06 \\
\hline GWM46902VL & TRIP BLANK & NA & Chlorobenzene & 1 & & $\mathrm{U}$ & & UG/L & 05/30/2006 & & JGJ-084-06 \\
\hline GWM46902VL & TRIP BLANK & NA & Dibromochloromethane & 1 & & u & & UG/L & $05 / 30 / 2006$ & & JGJ-084-06 \\
\hline GWM46902VL & TRIP BLANK & NA & 1,2-Dibromo-3-chloropropane & 1 & & $\mathrm{u}$ & $\mathrm{R}$ & UG/L & $05 / 30 / 2006$ & & JGJ-084-06 \\
\hline GWM46902VL & TRIP BLANK & NA & Chloroethane & 2 & & $\mathrm{U}$ & & UG/L & 05/30/2006 & & JGJ-084-06 \\
\hline GWM46902VL & TRIP BLANK & NA & Chloroform & 1 & & $\mathrm{u}$ & & UG/L & $05 / 30 / 2006$ & & JGJ-084-06 \\
\hline GWM46902VL & TRIP BLANK & NA & Chloromethane & 2 & & u & & UG/L & $05 / 30 / 2006$ & & JGJ-084-06 \\
\hline GWM46902VL & TRIP BLANK & NA & Cyclohexane & 1 & & $\mathrm{U}$ & & UG/L & 05/30/2006 & & JGJ-084-06 \\
\hline GWM46902VL & TRIP BLANK & NA & 1,2-Dibromoethane & 1 & & u & & UG/L & 05/30/2006 & & JGJ-084-06 \\
\hline
\end{tabular}


Table A-5. (continued).

\begin{tabular}{|c|c|c|c|c|c|c|c|c|c|c|c|}
\hline $\begin{array}{c}\text { Field Sample } \\
\text { Number }\end{array}$ & Location & Depth & Compound & $\begin{array}{c}\text { Sample } \\
\text { Result }\end{array}$ & $\begin{array}{l}\text { Sample } \\
\text { Error }\end{array}$ & $\begin{array}{c}\text { Result } \\
\text { Qualifier }\end{array}$ & $\begin{array}{c}\text { Validation } \\
\text { Flag }\end{array}$ & $\begin{array}{l}\text { Sample } \\
\text { Units }\end{array}$ & $\begin{array}{c}\text { Date Sample } \\
\text { Collected }\end{array}$ & MDA & $\begin{array}{c}\text { L\&V Report } \\
\text { Number }\end{array}$ \\
\hline GWM46902VL & TRIP BLANK & NA & 1,2-Dichlorobenzene & 1 & & $\mathrm{U}$ & & UG/L & 05/30/2006 & & JGJ-084-06 \\
\hline GWM46902VL & TRIP BLANK & NA & 1,3-Dichlorobenzene & 1 & & $\mathrm{U}$ & & UG/L & 05/30/2006 & & JGJ-084-06 \\
\hline GWM46902VL & TRIP BLANK & NA & 1,4-Dichlorobenzene & 1 & & $\mathrm{u}$ & & UG/L & 05/30/2006 & & JGJ-084-06 \\
\hline GWM46902VL & TRIP BLANK & NA & Dichlorodifluoromethane & 2 & & $\mathrm{U}$ & & UG/L & 05/30/2006 & & JGJ-084-06 \\
\hline GWM46902VL & TRIP BLANK & NA & 1,1-Dichloroethane & 1 & & $\mathrm{U}$ & & UG/L & 05/30/2006 & & JGJ-084-06 \\
\hline GWM46902VL & TRIP BLANK & NA & 1,2-Dichloroethane & 1 & & $\mathrm{U}$ & & UG/L & 05/30/2006 & & JGJ-084-06 \\
\hline GWM46902VL & TRIP BLANK & NA & 1,1-Dichloroethene & 1 & & $\mathrm{U}$ & & UG/L & 05/30/2006 & & JGJ-084-06 \\
\hline GWM46902VL & TRIP BLANK & NA & cis-1,2-Dichloroethene & 1 & & $\mathrm{U}$ & & UG/L & 05/30/2006 & & JGJ-084-06 \\
\hline GWM46902VL & TRIP BLANK & NA & trans-1,2-Dichloroethene & 1 & & $\mathrm{U}$ & & UG/L & 05/30/2006 & & JGJ-084-06 \\
\hline GWM46902VL & TRIP BLANK & NA & 1,2-Dichloropropane & 1 & & $\mathrm{U}$ & & UG/L & 05/30/2006 & & JGJ-084-06 \\
\hline GWM46902VL & TRIP BLANK & NA & cis-1,3-Dichloropropene & 1 & & $\mathrm{U}$ & & UG/L & 05/30/2006 & & JGJ-084-06 \\
\hline GWM46902VL & TRIP BLANK & NA & trans-1,3-Dichloropropene & 1 & & $\mathrm{U}$ & & UG/L & $05 / 30 / 2006$ & & JGJ-084-06 \\
\hline GWM46902VL & TRIP BLANK & NA & Ethylbenzene & 1 & & $\mathrm{U}$ & & UG/L & 05/30/2006 & & JGJ-084-06 \\
\hline GWM46902VL & TRIP BLANK & NA & Trichlorofluoromethane & 1 & & $\mathrm{U}$ & & UG/L & 05/30/2006 & & JGJ-084-06 \\
\hline GWM46902VL & TRIP BLANK & NA & 2-Hexanone & 5 & & $\mathrm{U}$ & $\mathrm{R}$ & UG/L & $05 / 30 / 2006$ & & JGJ-084-06 \\
\hline GWM46902VL & TRIP BLANK & NA & Isopropylbenzene & 1 & & $\mathrm{U}$ & & UG/L & 05/30/2006 & & JGJ-084-06 \\
\hline GWM46902VL & TRIP BLANK & NA & Methyl acetate & 5 & & $\mathrm{U}$ & $\mathrm{R}$ & UG/L & 05/30/2006 & & JGJ-084-06 \\
\hline GWM46902VL & TRIP BLANK & NA & Methyl cyclohexane & 4 & & $\mathrm{U}$ & & UG/L & 05/30/2006 & & JGJ-084-06 \\
\hline GWM46902VL & TRIP BLANK & NA & Methylene Chloride & 1 & & $\mathrm{u}$ & & UG/L & 05/30/2006 & & JGJ-084-06 \\
\hline GWM46902VL & TRIP BLANK & NA & 4-Methyl-2-pentanone & 5 & & $\mathrm{U}$ & & UG/L & 05/30/2006 & & JGJ-084-06 \\
\hline GWM46902VL & TRIP BLANK & NA & Styrene & 1 & & $\mathrm{U}$ & & UG/L & 05/30/2006 & & JGJ-084-06 \\
\hline GWM46902VL & TRIP BLANK & NA & 1,1,2,2-Tetrachloroethane & 1 & & $\mathrm{U}$ & & UG/L & 05/30/2006 & & JGJ-084-06 \\
\hline GWM46902VL & TRIP BLANK & NA & Tetrachloroethene & 1 & & $\mathrm{U}$ & & UG/L & 05/30/2006 & & JGJ-084-06 \\
\hline GWM46902VL & TRIP BLANK & NA & Toluene & 1 & & $\mathrm{u}$ & & UG/L & 05/30/2006 & & JGJ-084-06 \\
\hline GWM46902VL & TRIP BLANK & NA & 1,2,4-Trichlorobenzene & 1 & & $\mathrm{U}$ & & UG/L & 05/30/2006 & & JGJ-084-06 \\
\hline GWM46902VL & TRIP BLANK & NA & 1,1,1-Trichloroethane & 1 & & $\mathrm{u}$ & & UG/L & 05/30/2006 & & JGJ-084-06 \\
\hline GWM46902VL & TRIP BLANK & NA & 1,1,2-Trichloroethane & 1 & & $\mathrm{U}$ & & UG/L & $05 / 30 / 2006$ & & JGJ-084-06 \\
\hline GWM46902VL & TRIP BLANK & NA & Trichloroethene & 1 & & $\mathrm{U}$ & & UG/L & 05/30/2006 & & JGJ-084-06 \\
\hline GWM46902VL & TRIP BLANK & NA & 1,1,2-Trichloro-1,2,2-trifluoroethane & 1 & & $\mathrm{U}$ & & UG/L & 05/30/2006 & & JGJ-084-06 \\
\hline GWM46902VL & TRIP BLANK & NA & Vinyl Chloride & 2 & & $\mathrm{u}$ & & UG/L & 05/30/2006 & & JGJ-084-06 \\
\hline GWM46902VL & TRIP BLANK & NA & Xylenes & 3 & & $\mathrm{u}$ & & UG/L & 05/30/2006 & & JGJ-084-06 \\
\hline GWM46902VL & TRIP BLANK & NA & Methyl t-butyl ether & 2 & & $\mathrm{u}$ & & UG/L & 05/30/2006 & & JGJ-084-06 \\
\hline GWM46901VL & TRIP BLANK & NA & Acetone & 2 & & $\mathrm{U}$ & $\mathrm{R}$ & UG/L & 05/23/2006 & & JGJ-083-06 \\
\hline GWM46901VL & TRIP BLANK & NA & Benzene & 1 & & $\mathrm{U}$ & & UG/L & $05 / 23 / 2006$ & & JGJ-083-06 \\
\hline GWM46901VL & TRIP BLANK & NA & Bromodichloromethane & 1 & & $\mathrm{U}$ & & UG/L & 05/23/2006 & & JGJ-083-06 \\
\hline GWM46901VL & TRIP BLANK & NA & Bromoform & 1 & & $\mathrm{u}$ & & UG/L & 05/23/2006 & & JGJ-083-06 \\
\hline GWM46901VL & TRIP BLANK & NA & Bromomethane & 2 & & $\mathrm{U}$ & & UG/L & 05/23/2006 & & JGJ-083-06 \\
\hline GWM46901VL & TRIP BLANK & NA & 2-Butanone & 5 & & $\mathrm{u}$ & $\mathrm{R}$ & UG/L & $05 / 23 / 2006$ & & JGJ-083-06 \\
\hline GWM46901VL & TRIP BLANK & NA & Carbon disulfide & 1 & & $\mathrm{U}$ & & UG/L & 05/23/2006 & & JGJ-083-06 \\
\hline GWM46901VL & TRIP BLANK & NA & Carbon tetrachloride & 1 & & $\mathrm{U}$ & & UG/L & 05/23/2006 & & JGJ-083-06 \\
\hline GWM46901VL & TRIP BLANK & NA & Chlorobenzene & 1 & & $\mathrm{U}$ & & UG/L & $05 / 23 / 2006$ & & JGJ-083-06 \\
\hline GWM46901VL & TRIP BLANK & NA & Dibromochloromethane & 1 & & $\mathrm{U}$ & & UG/L & 05/23/2006 & & JGJ-083-06 \\
\hline GWM46901VL & TRIP BLANK & NA & 1,2-Dibromo-3-chloropropane & 1 & & $\mathrm{U}$ & $\mathrm{R}$ & UG/L & 05/23/2006 & & JGJ-083-06 \\
\hline GWM46901VL & TRIP BLANK & NA & Chloroethane & 2 & & $\mathrm{U}$ & & UG/L & 05/23/2006 & & JGJ-083-06 \\
\hline GWM46901VL & TRIP BLANK & NA & Chloroform & 1 & & $\mathrm{u}$ & & UG/L & 05/23/2006 & & JGJ-083-06 \\
\hline GWM46901VL & TRIP BLANK & NA & Chloromethane & 2 & & $\mathrm{U}$ & & UG/L & 05/23/2006 & & JGJ-083-06 \\
\hline GWM46901VL & TRIP BLANK & NA & Cyclohexane & 1 & & $\mathrm{u}$ & & UG/L & 05/23/2006 & & JGJ-083-06 \\
\hline GWM46901VL & TRIP BLANK & NA & 1,2-Dibromoethane & 1 & & $\mathrm{U}$ & & UG/L & 05/23/2006 & & JGJ-083-06 \\
\hline GWM46901VL & TRIP BLANK & NA & 1,2-Dichlorobenzene & 1 & & $\mathrm{U}$ & & UG/L & 05/23/2006 & & JGJ-083-06 \\
\hline GWM46901VL & TRIP BLANK & NA & 1,3-Dichlorobenzene & 1 & & $\mathrm{U}$ & & UG/L & 05/23/2006 & & JGJ-083-06 \\
\hline GWM46901VL & TRIP BLANK & NA & 1,4-Dichlorobenzene & 1 & & $\mathrm{U}$ & & UG/L & 05/23/2006 & & JGJ-083-06 \\
\hline GWM46901VL & TRIP BLANK & NA & Dichlorodifluoromethane & 2 & & $\mathrm{U}$ & & UG/L & 05/23/2006 & & JGJ-083-06 \\
\hline GWM46901VL & TRIP BLANK & NA & 1,1-Dichloroethane & 1 & & $\mathrm{U}$ & & UG/L & $05 / 23 / 2006$ & & JGJ-083-06 \\
\hline GWM46901VL & TRIP BLANK & NA & 1,2-Dichloroethane & 1 & & $\mathrm{U}$ & & UG/L & 05/23/2006 & & JGJ-083-06 \\
\hline GWM46901VL & TRIP BLANK & NA & 1,1-Dichloroethene & 1 & & $\mathrm{U}$ & & UG/L & 05/23/2006 & & JGJ-083-06 \\
\hline GWM46901VL & TRIP BLANK & NA & cis-1,2-Dichloroethene & 1 & & $\mathrm{U}$ & & UG/L & 05/23/2006 & & JGJ-083-06 \\
\hline GWM46901VL & TRIP BLANK & NA & trans-1,2-Dichloroethene & 1 & & $\mathrm{U}$ & & UG/L & 05/23/2006 & & JGJ-083-06 \\
\hline GWM46901VL & TRIP BLANK & NA & 1,2-Dichloropropane & 1 & & $\mathrm{U}$ & & UG/L & 05/23/2006 & & JGJ-083-06 \\
\hline GWM46901VL & TRIP BLANK & NA & cis-1,3-Dichloropropene & 1 & & $\mathrm{U}$ & & UG/L & 05/23/2006 & & JGJ-083-06 \\
\hline GWM46901VL & TRIP BLANK & NA & trans-1,3-Dichloropropene & 1 & & $\mathrm{U}$ & & UG/L & 05/23/2006 & & JGJ-083-06 \\
\hline GWM46901VL & TRIP BLANK & NA & Ethylbenzene & 1 & & $\mathrm{U}$ & & UG/L & 05/23/2006 & & JGJ-083-06 \\
\hline GWM46901VL & TRIP BLANK & NA & Trichlorofluoromethane & 1 & & $\mathrm{U}$ & & UG/L & 05/23/2006 & & JGJ-083-06 \\
\hline GWM46901VL & TRIP BLANK & NA & 2-Hexanone & 5 & & $\mathrm{U}$ & & UG/L & 05/23/2006 & & JGJ-083-06 \\
\hline GWM46901VL & TRIP BLANK & NA & Isopropylbenzene & 1 & & $\mathrm{U}$ & & UG/L & 05/23/2006 & & JGJ-083-06 \\
\hline GWM46901VL & TRIP BLANK & NA & Methyl acetate & 5 & & $\mathrm{U}$ & $\mathrm{R}$ & UG/L & 05/23/2006 & & JGJ-083-06 \\
\hline GWM46901VL & TRIP BLANK & NA & Methyl cyclohexane & 4 & & $\mathrm{u}$ & & UG/L & 05/23/2006 & & JGJ-083-06 \\
\hline GWM46901VL & TRIP BLANK & NA & Methylene Chloride & 1 & & $\mathrm{U}$ & & UG/L & 05/23/2006 & & JGJ-083-06 \\
\hline GWM46901VL & TRIP BLANK & NA & 4-Methyl-2-pentanone & 5 & & $\mathrm{U}$ & & UG/L & 05/23/2006 & & JGJ-083-06 \\
\hline GWM46901VL & TRIP BLANK & NA & Styrene & 1 & & $\mathrm{U}$ & & UG/L & 05/23/2006 & & JGJ-083-06 \\
\hline GWM46901VL & TRIP BLANK & NA & 1,1,2,2-Tetrachloroethane & 1 & & $\mathrm{U}$ & & UG/L & 05/23/2006 & & JGJ-083-06 \\
\hline GWM46901VL & TRIP BLANK & NA & Tetrachloroethene & 1 & & $\mathrm{U}$ & & UG/L & 05/23/2006 & & JGJ-083-06 \\
\hline GWM46901VL & TRIP BLANK & NA & Toluene & 1 & & $\mathrm{U}$ & & UG/L & 05/23/2006 & & JGJ-083-06 \\
\hline GWM46901VL & TRIP BLANK & NA & 1,2,4-Trichlorobenzene & 1 & & $\mathrm{U}$ & & UG/L & 05/23/2006 & & JGJ-083-06 \\
\hline GWM46901VL & TRIP BLANK & NA & 1,1,1-Trichloroethane & 1 & & $\mathrm{U}$ & & UG/L & 05/23/2006 & & JGJ-083-06 \\
\hline GWM46901VL & TRIP BLANK & NA & 1,1,2-Trichloroethane & 1 & & $\mathrm{U}$ & & UG/L & 05/23/2006 & & JGJ-083-06 \\
\hline GWM46901VL & TRIP BLANK & NA & Trichloroethene & 1 & & $\mathrm{U}$ & & UG/L & 05/23/2006 & & JGJ-083-06 \\
\hline GWM46901VL & TRIP BLANK & NA & 1,1,2-Trichloro-1,2,2-trifluoroethane & 1 & & $\mathrm{U}$ & & UG/L & 05/23/2006 & & JGJ-083-06 \\
\hline GWM46901VL & TRIP BLANK & NA & Vinyl Chloride & 2 & & $\mathrm{U}$ & & UG/L & $05 / 23 / 2006$ & & JGJ-083-06 \\
\hline GWM46901VL & TRIP BLANK & NA & Xylenes & 3 & & $\mathrm{u}$ & & UG/L & 05/23/2006 & & JGJ-083-06 \\
\hline GWM46901VL & TRIP BLANK & NA & Methyl t-butyl ether & 2 & & $\mathrm{U}$ & & UG/L & $05 / 23 / 2006$ & & JGJ-083-06 \\
\hline
\end{tabular}




\section{A-2. DATA QUALIFIER FLAGS}

The data qualifier flags used in this appendix are a consolidation of laboratory- and validation-assigned flags. The data qualifier flags for organic, inorganic, and radiological results are defined as follows:

\section{Organic Qualifier Flags:}

- $\quad$ B-The analyte was detected in the associated laboratory method blank as well as in the sample.

- $\quad \mathrm{U}-$ The analyte was analyzed for, but it was not detected.

- UJ-The analyte was analyzed for, but it was not detected. The associated value is an estimate and might be inaccurate or imprecise.

- $\quad \mathrm{J}$ - The analyte was detected, but the associated values are an estimate and might be inaccurate or imprecise.

- $\quad \mathrm{N}$-There is presumptive evidence that a compound is present.

- $\quad \mathrm{NJ}$ or $\mathrm{JN}$-There is presumptive evidence that a compound is present, and the associated values are an estimate.

- $\quad \mathrm{R}$ - The accuracy of the data is so questionable that it is recommended that the data not be used. The "R" flag overrides all other applicable flags.

\section{Inorganic Qualifier Flags:}

- $\quad \mathrm{B}$-The result is less than the contract-required reporting limit but greater than or equal to the instrument detection limit.

- $\quad$ E-The reported value was estimated because of the presence of interference.

- $\quad \mathrm{N}$-The spiked sample recovery was outside control limits.

- $\quad \mathrm{U}-$ The analyte was not detected.

- UJ-The analyte was analyzed for, but it was not detected. The associated value is an estimate and might be inaccurate or imprecise.

- $\quad \mathrm{R}$ - The accuracy of the data is so questionable that it is recommended that the data not be used. The "R" flag overrides all other applicable flags.

\section{Radiological Qualifier Flags:}

- $\quad \mathrm{J}-$ The associated value is estimated. The result might not be an accurate representation of the amount of activity present in the sample.

- $\quad \mathrm{R}$ - The accuracy of the data is so questionable that it is recommended that the data not be used. The " $R$ " flag overrides all other applicable flags. 
- $\quad \mathrm{U}$-The radionuclide is not considered present in the sample (i.e., nondetect).

- $\quad$ UJ-The radionuclide might or might not be present, and the result is considered highly questionable. The associated value is an estimate and might be inaccurate or imprecise. The result is considered a nondetect for project data interpretation purposes.

\section{A-3. ACCELERATOR MASS SPECTROMETRY ANALYSES}

The I-129 and Cl-36 AMS method analyses were performed by the Purdue Rare Isotope Measurement laboratory (PRIME Lab) in Lafayette, Indiana.

\section{A-3.1 General Method Description}

AMS uses a particle accelerator in conjunction with negative ion sources, electric and magnetic analyzers, and nuclear detectors to separate interferences ( $\mathrm{S}$ for $\mathrm{Cl}-36$ ) and measure the isotope abundance to the level of $10^{-15}$.

Sample "targets" were prepared by precipitating $\mathrm{AgCl}$ for $\mathrm{Cl}-36$ analysis. To make sure a sufficient target was created, a "carrier" was added.

The measurements begin with negatively ionizing samples $(\mathrm{AgCl})$ by ion sputtering and charge exchange processes. The ion yield $(\mathrm{Cl}, \mathrm{I})$ is proportional to $\exp \left(A-\phi_{S}\right) / k T$ where $A, \phi_{s}, T$ and $k$ are the electron affinity of the sample, the effective work function, the temperature of the ionizer, and the Boltzmann constant.

This is followed by isotope selection through the injector magnet $\left(2 \times \frac{m}{q} \times \frac{E}{q}=B \times R\right.$ where $m, E, q$, $B$, and $R$ are atomic weight, energy, charge state, magnetic field strength, and radius), and the negative ions of the interest $\left({ }^{36} \mathrm{Cl}^{-},{ }^{129} \mathrm{I}^{-}\right)$are accelerated through the first half of the accelerator, acquiring kinetic energy up to $3-7 \mathrm{MeV}$, then converted to multiply charged positive ions $\left({ }^{36} \mathrm{Cl}^{7+},{ }^{127} \mathrm{I}^{5+}\right)$ by charge stripper (gas or a very thin carbon foil) at the center of the accelerator. The positive ions are accelerated through the second half of the accelerator; the final velocity is approximately $1.39 \times 10^{7} \sqrt{\frac{(q+1) V_{T}}{m}} \mathrm{~m} / \mathrm{second}$, where $V_{T}$ is accelerator terminal voltage in $\mathrm{MV}$, about 40 million mph for $\mathrm{Cl}^{7+}$.

Through further mass-to-charge $(\mathrm{m} / \mathrm{q})$ and energy-to-charge $(\mathrm{E} / \mathrm{q})$ analysis by the magnetic and the electrostatic analyzers, ions are directed to the gas ionization detector. The detector detects the ion total

energy $\mathrm{E}$ and energy loss per unit distance 0 through the gas, $-\frac{d E}{d x} \cong \frac{k z^{2}}{E}$ where $\mathrm{k}, \mathrm{z}$ are a constant and effective nuclear charge, which uniquely identify the isotope of interest.

\section{A-3.2 lodine-129}

A sample aliquot is selected so that when mixed with $2 \mathrm{mg}$ of stable carrier, the I-129 R/S ratio will not exceed 10,000. If this is not a concern, a sample aliquot of $800 \mathrm{~mL}$ is used.

The sample aliquot is filtered if necessary to remove any particulates, and a small amount of sodium thiosulfate solution is added to reduce any iodate ion to iodide. The sample is mixed and transferred to a 1L separatory funnel, acidified with nitric acid, and the iodide oxidized to free iodine with 
sodium nitrite solution. The sample is immediately extracted with dichloromethane, allowed to separate, and the dichloromethane layer drained off into a collection tube. The iodine in dichloromethane is immediately back extracted into silver nitrate solution. The extraction and back extraction is repeated two more times.

The silver iodide containing silver nitrate solution is separated from the dichloromethane, centrifuged, washed, and dried.

The ratios reported by the PRIME lab are for the "target" or the sample plus a carrier. The target values then need to be converted to insitu values or the value for water from the well. The raw or AMS target values for I-129/total iodine were converted to insitu values using the following formula:

$$
\text { Insitu }^{129} I / I=\frac{\text { carrier } w t+\text { sample } w t_{\text {iodine }}}{\text { Sample } w t_{\text {iodine }}} \times\left({ }^{129} I / I\right)^{t \arg e t}
$$

Where sample weight of iodine is calculated as:

Sample $W t_{\text {iodine }}=\frac{\text { Sample Wt }(\text { grams })}{1000 \mathrm{~g} / \mathrm{L}} \times I(\mathrm{mg} / \mathrm{L})$

The concentration of total iodine in the samples was assumed to be $0.001 \mathrm{mg} / \mathrm{L}$ to be consistent with previous studies performed by the USGS (Mann and Beasley 1994; Cecil et al. 2003).

The number of atoms of I-129/L was calculated by the following formula:

$$
\text { Atoms }^{129} \mathrm{I} / \mathrm{L}=\frac{\mathrm{I}(\mathrm{mg} / \mathrm{L}) \times\left({ }^{129} \mathrm{I} / \mathrm{I}\right)^{\text {insitu }} \times 6.022 \times 10^{23} \text { atoms } / \mathrm{mol}}{\text { Molecular weight }(126.9) \mathrm{g} / \mathrm{mol} \times 1000 \mathrm{mg} / \mathrm{g}}
$$

The insitu atoms of I-129/L were converted to $\mathrm{pCi} / \mathrm{L}$ using the following equation:

Atoms I-129/L $\times 3.78 \mathrm{E}-14=\mathrm{pCi} / \mathrm{L}$.

\section{A-3.3 Chlorine-36}

A sample aliquot is selected that would produce about 25 to $35 \mathrm{mg}$ of silver chloride for an AMS target, if possible. If the chloride concentration is low, the sample will be preconcentrated on an anion exchange column, eluted with dilute nitric acid before proceeding as directed below.

If the Cl-36 concentration is very high, stable chloride will be added to the sample aliquot to lower the radioactive/stable ratio to about 2,000.

A small amount of barium nitrate is added to the sample to precipitate sulfate. The sample is filtered, if necessary, to remove any particulates and acidified with nitric acid, and the chloride ion is precipitated with silver nitrate. The precipitate is allowed to settle and is separated from the acid solution by centrifugation. The supernatant is discarded. 
The silver chloride is dissolved in ammonium hydroxide solution, transferred to an anion exchange column, and generated in the nitrate form to remove possible interference from any remaining sulfur. The chloride is eluted from the column with dilute nitric acid solution, collected, and mixed with silver nitrate to reprecipitate the silver chloride.

The sample is centrifuged to collect the silver chloride precipitate, washed with water, recentrifuged, and dried.

The ratios reported by the PRIME lab are for the "target" or the sample plus a carrier. The target values then need to be converted to insitu values or the value for water from the well. The raw or AMS target values for Cl-36/total chloride were converted to insitu values using the following formula:

$$
\text { Insitu }{ }^{36} \mathrm{Cl} / \text { total } \mathrm{Cl}=\frac{\text { carrier } w t+\text { sample } w t_{\text {chloride }}}{\text { Sample } w t_{\text {chloride }}} \times\left({ }^{36} \mathrm{Cl} / \text { total } \mathrm{Cl}\right)^{\mathrm{targ} e t}
$$

Where sample weight of chloride is calculated as:

Sample $W t_{\text {chloride }}=\frac{\text { Sample } W t(\text { grams })}{1000 \mathrm{~g} / \mathrm{L}} \times C l(\mathrm{mg} / \mathrm{L})$

The number of atoms of Cl-36 was calculated by the following formula:

$$
\text { Atoms }{ }^{36} \mathrm{Cl}=\frac{\mathrm{Cl}(\mathrm{mg} / \mathrm{L}) \times\left({ }^{36} \mathrm{Cl} / \text { total }-\mathrm{Cl}\right)^{t \arg e t} \times 6.022 \times 10^{23} \text { atoms } / \mathrm{mol}}{\text { Molecular weight }(35.453) \times 1000 \mathrm{mg} / \mathrm{mol}}
$$

The value for insitu atoms of $\mathrm{Cl}-36 / \mathrm{L}$ was converted to $\mathrm{pCi} / \mathrm{L}$ using the following equation:

Atoms Cl-36/L $\left(10^{10}\right) \times 0.02=\mathrm{pCi} / \mathrm{L}$.

\section{A-4. REFERENCES}

Cecil, L. D, L. F. Hall, and J. R. Green, 2003, Reevaluation of Background Iodine-129 Concentrations in Water from the Snake River Plain Aquifer, Idaho, USGS Water Resources Investigations Report 03-4106.

Mann, Larry J., and T. M. Beasley, 1994, I-129 in the Snake River Plain Aquifer at and near the Idaho National Engineering Laboratory, Idaho, 1990-91, U.S. Geological Survey Water Resources Report 94-4053. 
A-74 
Appendix B

Geochemical Study 
B-2 


\section{Appendix B}

\section{Geochemical Study}

\section{B-1. INTRODUCTION}

This appendix presents the I-129 data that were collected as part of the geochemical study described in the OU 10-08 Groundwater Monitoring and Field Sampling Plan (DOE-ID 2005). Although the I-129 sampling was performed in 2005, the data were not available in time to be included into the 2005 annual report. Also, implications on groundwater flow paths from the vertical distribution of contaminants and major ions from the two Westbay well sampling events and the 2006 packer sampling results are discussed. In addition, two wells were sampled for I-129 in 2006 to address data gaps (DOE-ID 2006a).

The goals of the geochemical study were to resolve the source of the tritium in the aquifer at the RWMC, identify flow paths of contaminants from INTEC and RTC, determine the source of the anion anomaly south of the RWMC, and identify flow paths and evaluate contaminant influence south of the southern INL site boundary (Figure B-1). Identification of groundwater flow paths is essential for development and calibration of the OU 10-08 (i.e., Sitewide) groundwater model. In addition, data from the geochemical study are used to evaluate the potential for commingled plumes, which might elevate the cumulative risk above levels calculated for each plume individually.

The geochemical study used four tracers that are currently in the SRPA as a result of INL operations: C1-36, I-129, sulfur, and oxygen isotope ratios in sulfate, and nitrogen and oxygen isotope ratios in nitrate. The results for Cl-36, sulfur, and oxygen isotope ratios in sulfate, and nitrogen and oxygen isotope ratios in nitrate were previously presented in the FY 2005 Annual Report (DOE-ID 2006b).

Select plumes in the RTC-CFA-RWMC area are shown on Figure B-2 along with groundwater contours to illustrate the potential for commingling plumes and the possibility of upgradient influence on the RWMC. In general, the contaminant distributions in the SRPA agree with groundwater flow paths indicated by the water-level contours. However, the upgradient influences from RTC and INTEC on the RWMC is uncertain because of the possibility of preferential flow paths. If there is an upgradient influence at the RWMC, then it is within the scope of OU 10-08 to account for the effects of commingling plumes in the OU 10-08 RI/FS.

\section{B-2. I-129 RESULTS AND DISCUSSION}

The data goals for the I-129 sampling in the geochemical study are to define groundwater flow paths from INTEC and RTC, to determine the potential for upgradient influences at the RWMC, to evaluate the potential for commingling plumes, and to evaluate the extent of contaminant migration south of the INL Site boundary. 


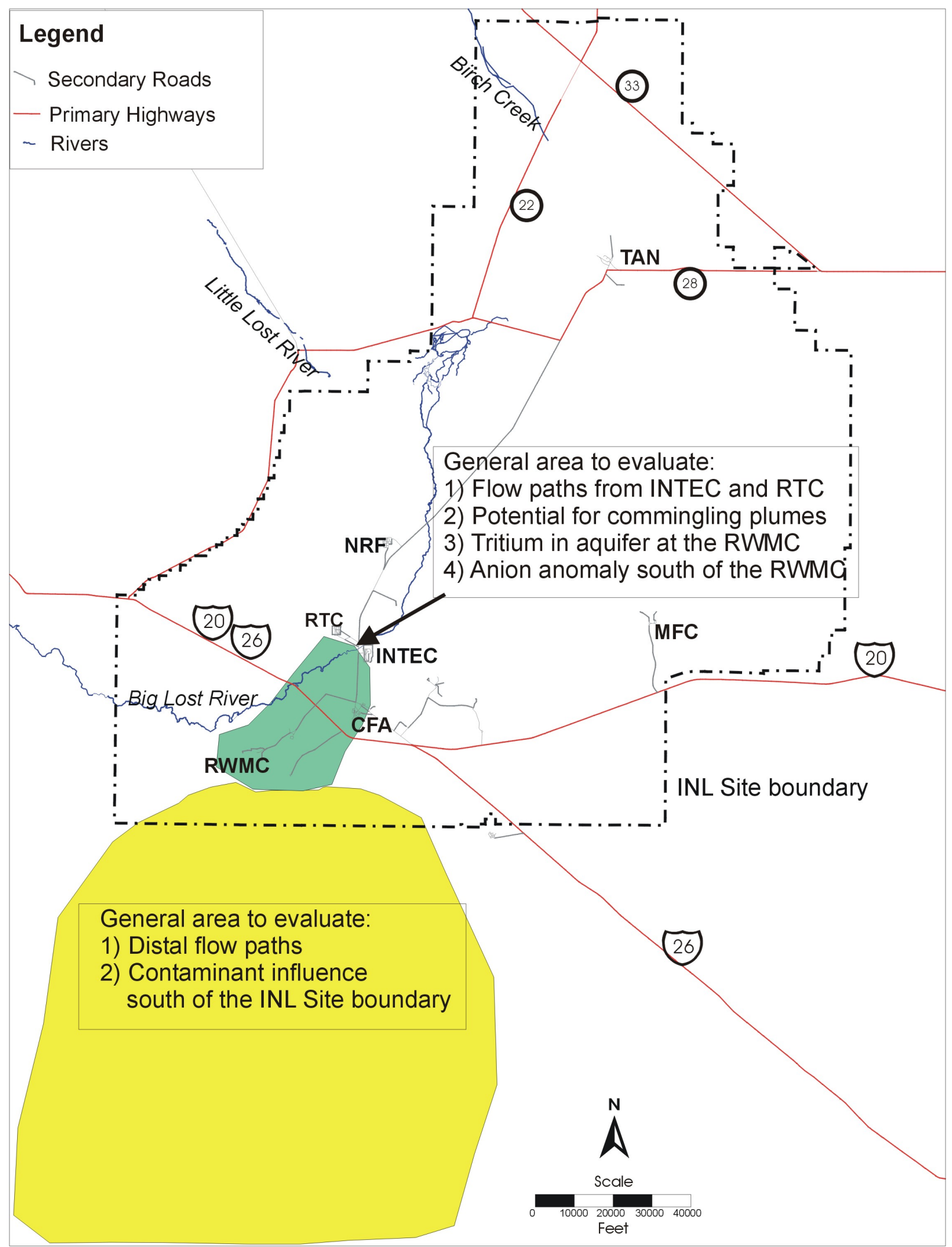

Figure B-1. Geochemical study areas. 


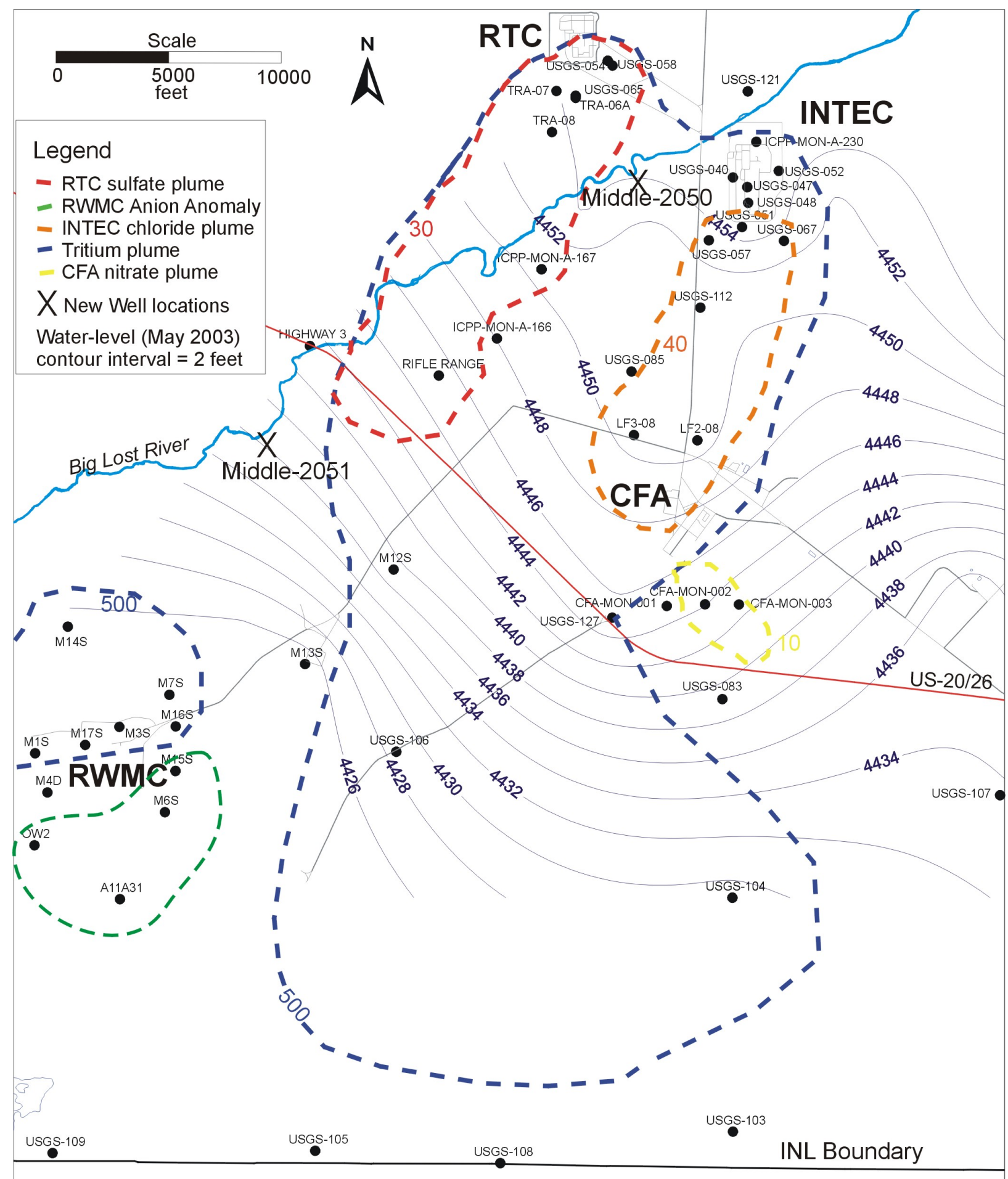

Figure B-2. Approximate boundaries of select plumes based on concentrations in 2003 for the RTC, INTEC, CFA, and RWMC areas. 
The I-129 results from this study are reported in Table B-1 along with USGS data from September 2003 (Gordon Rattray personal communication, 2004 ${ }^{\mathrm{a}}$ ). The calculation of the I-129 concentrations is described in Appendix A.

The difference in I-129 concentrations downgradient of INTEC and RTC can be used to evaluate groundwater flow paths from INTEC and the RTC and to evaluate the upgradient influences at the RWMC. Previous data indicated that I-129 concentrations downgradient of the RTC are much lower than I-129 concentrations downgradient of INTEC (Mann and Beasley 1994). This is consistent with the data presented in this report that show that the I-129 concentration downgradient of the RTC at Middle-1823 is more than an order of magnitude less than a similar distance downgradient of INTEC, at USGS-085 (Figure B-3).

Previous studies by the USGS indicated the possibility of upgradient influences for the RWMC (Mann and Beasley 1994; Beasley, Dixon, and Mann 1998; and Busenberg, Plummer, and Bartholomay 2001). In addition, water-level contour maps also indicated that the RWMC might be downgradient from RTC and INTEC (Figure B-2).

Although I-129 is present in plumes from INTEC, RTC, and in the wastes emplaced in the RWMC, the I-129 data are consistent with previously presented data that supported INTEC as the source of the I-129 in the SRPA on the north and east sides of the RWMC (DOE-ID 2006b). The I-129 concentrations in Wells M14S, M7S, and the RWMC production on the northern side of the RWMC are similar to Wells M12S and USGS-106 in the INTEC plume to the east (Figure B-3). The I-129 concentrations in wells on the north and northeastern side of the RWMC are similar to wells affected by the INTEC plume, suggesting the same source. The I-129 concentrations in M12S, M7S, M14S, RWMC production, and USGS-106 are three to six times higher than the concentration in Middle-1823 downgradient of RTC. The I-129 concentrations on the north side of the RWMC are higher than at Middle-1823 located downgradient of RTC, suggesting that RTC is not the source of the contaminants on the north side of the RWMC. Although the I-129 concentration is lower in Middle-1823 than in wells on the north side of the RWMC, the RTC cannot be completely ruled out as an influence on the RWMC, because Middle-1823 is an intermediate-depth well and the I-129 peak from RTC may have passed this location. The similar I-129 concentrations in M12S, M7S, M14S, RWMC production well, and USGS-106 make it more difficult to accept RWMC as the I-129 source because it requires waste from the Subsurface Disposal Area to impact the aquifer at approximately the same concentration as the INTEC plume.

In contrast to wells on the north and eastern sides of the RWMC that show influence from the I-129 plume from INTEC, wells to the south of the RWMC have I-129 concentrations that are similar to background or are slightly elevated above background (Figure B-3). The apparent groundwater divide that is observed with the I-129 data at the RWMC is also apparent with the nitrogen and sulfur isotope data along with the Cl-36 data (DOE-ID 2006b).

The Westbay wells were sampled and analyzed for I-129 by the AMS method in SeptemberOctober 2005. The highest I-129 concentrations were observed in the 748- and 834-ft depth samples from Westbay Well MIDDLE-2051, and tritium was also detected in these two samples. The shallow sample from MIDDLE-2051, at 608-ft depth, had a background I-129 concentration. In contrast to MIDDLE-2051, I-129 concentrations in MIDDLE-2050A were only slightly above background.

a. Rattray, G., United States Geological Survey, personal communication to M. Roddy, INL, October 2004. 
Table B-1. I-129 data for wells sampled in 2005, and USGS I-129 results from 2003.

\begin{tabular}{|c|c|c|c|c|c|}
\hline \multirow{2}{*}{ Location } & & \multicolumn{2}{|c|}{${ }^{129} \mathrm{I}$} & \multicolumn{2}{|c|}{${ }^{129} \mathrm{I}$} \\
\hline & & $\mathrm{pCi} / \mathrm{L}$ & $+/-$ & $\mathrm{aCi} / \mathrm{L}$ & $+/-$ \\
\hline \multicolumn{6}{|l|}{ Wells Sampled in 2005} \\
\hline M1S & & 0.000042 & 0.0000079 & 42 & 8 \\
\hline M6S & & 0.000027 & 0.0000092 & 27 & 9 \\
\hline M11S & & 0.000076 & 0.000015 & 76 & 15 \\
\hline M12S & & 0.011 & 0.00031 & 10505 & 308 \\
\hline M13S & & 0.000041 & 0.000011 & 41 & 11 \\
\hline M14S & & 0.019 & 0.00073 & 19354 & 733 \\
\hline A11A31 & & 0.00048 & 0.000032 & 485 & 32 \\
\hline A11A31 Dup & & 0.00045 & 0.000032 & 445 & 32 \\
\hline Crossroads & & 0.00000072 & 0.000000091 & 0.72 & 0.09 \\
\hline Crossroads Dup & & 0.00000074 & 0.000000061 & 0.74 & 0.06 \\
\hline Fingers butte & & 0.00000014 & 0.000000036 & 0.14 & 0.04 \\
\hline Graz Serv CCC3 & & 0.00000043 & 0.000000057 & 0.43 & 0.06 \\
\hline Grazing 2 & & 0.00000014 & 0.000000054 & 0.14 & 0.05 \\
\hline Houghland & & 0.000012 & 0.00000054 & 12 & 0.54 \\
\hline Middle-1823 & & 0.0020 & 0.000099 & 1982 & 99 \\
\hline USGS-009 & & 0.0000084 & 0.00000050 & 8.4 & 0.50 \\
\hline USGS-103 & & 0.0000080 & 0.00000037 & 8.0 & 0.37 \\
\hline USGS-105 & & 0.0027 & 0.000084 & 2740 & 84 \\
\hline USGS-108 & & 0.00097 & 0.000031 & 971 & 31 \\
\hline USGS-109 & & 0.00040 & 0.000015 & 396 & 15 \\
\hline USGS-106 & & 0.031 & 0.00090 & 31100 & 900 \\
\hline USGS-125 & & 0.00012 & 0.0000042 & 124 & 4.2 \\
\hline USGS-124 & & 0.0019 & 0.000062 & 1902 & 62 \\
\hline USGS-011 & & 0.000016 & 0.00000093 & 16 & 0.93 \\
\hline USGS-014 & & 0.000028 & 0.0000015 & 28 & 1.5 \\
\hline MIDDLE-2050A & 474 & 0.000018 & 0.00000076 & 18 & 0.76 \\
\hline MIDDLE-2050A & 647 & 0.000018 & 0.00000063 & 18 & 0.63 \\
\hline MIDDLE-2050A Dup & 647 & 0.000017 & 0.00000075 & 17 & 0.75 \\
\hline Field Blank QC & & 0.000000063 & 0.000000018 & 0.06 & 0.02 \\
\hline MIDDLE-2050A & 790 & 0.000019 & 0.00000058 & 19 & 0.58 \\
\hline MIDDLE-2050A & 1005 & 0.000046 & 0.0000017 & 46 & 1.7 \\
\hline MIDDLE-2050A & 1212 & 0.00014 & 0.0000047 & 138 & 4.7 \\
\hline MIDDLE-2050A & 1005 & 0.0000034 & 0.00000014 & 3.4 & 0.14 \\
\hline MIDDLE-2051 & 608 & 0.0000087 & 0.00000033 & 8.7 & 0.33 \\
\hline MIDDLE-2051 & 748 & 0.00054 & 0.000024 & 539 & 24 \\
\hline MIDDLE-2051 & 834 & 0.00091 & 0.000028 & 914 & 28 \\
\hline MIDDLE-2051 & 1098 & 0.00018 & 0.0000088 & 183 & 8.8 \\
\hline MIDDLE-2051 & 1148 & 0.000084 & 0.0000031 & 84 & 3.1 \\
\hline \multicolumn{6}{|l|}{ Wells Sampled in 2006} \\
\hline RWMC Production & & 0.019 & 0.001 & 19189 & 1042 \\
\hline M7S & & 0.021 & 0.00077 & 20797 & 779 \\
\hline M7S Dup & & 0.020 & 0.00038 & 20056 & 383 \\
\hline
\end{tabular}


Table B-1. (continued).

\begin{tabular}{|c|c|c|c|c|}
\hline \multirow[b]{2}{*}{ Location } & \multicolumn{2}{|c|}{${ }^{129} \mathrm{I}$} & \multicolumn{2}{|c|}{${ }^{129} \mathrm{I}$} \\
\hline & $\mathrm{pCi} / \mathrm{L}$ & $+/-$ & $\mathrm{aCi} / \mathrm{L}$ & $+/-$ \\
\hline \multicolumn{5}{|c|}{ Aquifer Wells Sampled in the Fall of 2003 (USGS Data) } \\
\hline CFA LF 2-10 & 0.079 & 0.006 & 78584 & 6164 \\
\hline USGS-009 & 0.0000088 & 0.0000007 & 8.8 & 0.70 \\
\hline USGS-011 & 0.000018 & 0.000001 & 18 & 1.0 \\
\hline USGS-014 & 0.000040 & 0.000004 & 40 & 4 \\
\hline USGS-014 & 0.000040 & 0.000004 & 40 & 4.4 \\
\hline USGS-020 & 0.026 & 0.002 & 26010 & 2134 \\
\hline USGS-035 & 0.0018 & 0.0000 & 1782 & 44 \\
\hline USGS-036 & 0.16 & 0.02 & 162398.9 & 16381 \\
\hline USGS-037 & 0.45 & 0.05 & 452138 & 50074 \\
\hline USGS-038 & 0.56 & 0.04 & 555773 & 37102 \\
\hline USGS-039 & 0.00028 & 0.00001 & 282 & 6.6 \\
\hline USGS-042 & 0.22 & 0.01 & 215936 & 12815 \\
\hline USGS-044 & 0.12 & 0.01 & 119752 & 6948 \\
\hline USGS-047 & 0.62 & 0.04 & 621058 & 43773 \\
\hline USGS-051 & 0.16 & 0.01 & 163547 & 14203 \\
\hline USGS-057 & 0.64 & 0.05 & 639573 & 45623 \\
\hline USGS-077 & 0.59 & 0.04 & 585787 & 38630 \\
\hline USGS-082 & 0.011 & 0.001 & 11243.77 & 823 \\
\hline USGS-085 & 0.28 & 0.02 & 282639 & 18041 \\
\hline USGS-103 & 0.000010 & 0.000002 & 10.0 & 2 \\
\hline USGS-103 & 0.0000087 & 0.0000005 & 8.7 & 0.53 \\
\hline USGS-103 & 0.0000085 & 0.0000005 & 8.5 & 0.53 \\
\hline USGS-104 & 0.0049 & 0.0004 & 4935 & 363 \\
\hline USGS-105 & 0.0017 & 0.0001 & 1652 & 127 \\
\hline USGS-106 & 0.034 & 0.002 & 33871 & 2077 \\
\hline USGS-108 & 0.0015 & 0.0001 & 1496 & 93 \\
\hline USGS-109 & 0.00040 & 0.00002 & 398 & 23 \\
\hline USGS-111 & 0.14 & 0.01 & 137903 & 13947 \\
\hline USGS-112 & 0.62 & 0.13 & 616543 & 133143 \\
\hline USGS-113 & 0.72 & 0.10 & 719638 & 102630 \\
\hline USGS-114 & 0.15 & 0.01 & 153026 & 12584 \\
\hline USGS-115 & 0.017 & 0.001 & 17158 & 1082 \\
\hline USGS-116 & 0.069 & 0.005 & 69428 & 5268 \\
\hline USGS-121 & 0.0000066 & 0.0000004 & 6.6 & 0.44 \\
\hline USGS-123 & 0.68 & 0.07 & 677810 & 68459.81 \\
\hline USGS-124 & 0.0023 & 0.0002 & 2266 & 157 \\
\hline USGS-125 & 0.00013 & 0.00000 & 128 & 1.7 \\
\hline USGS-127 & 0.0010 & 0.0001 & 1045 & 100 \\
\hline USGS-128 & 0.26 & 0.02 & 263384 & 17031 \\
\hline
\end{tabular}




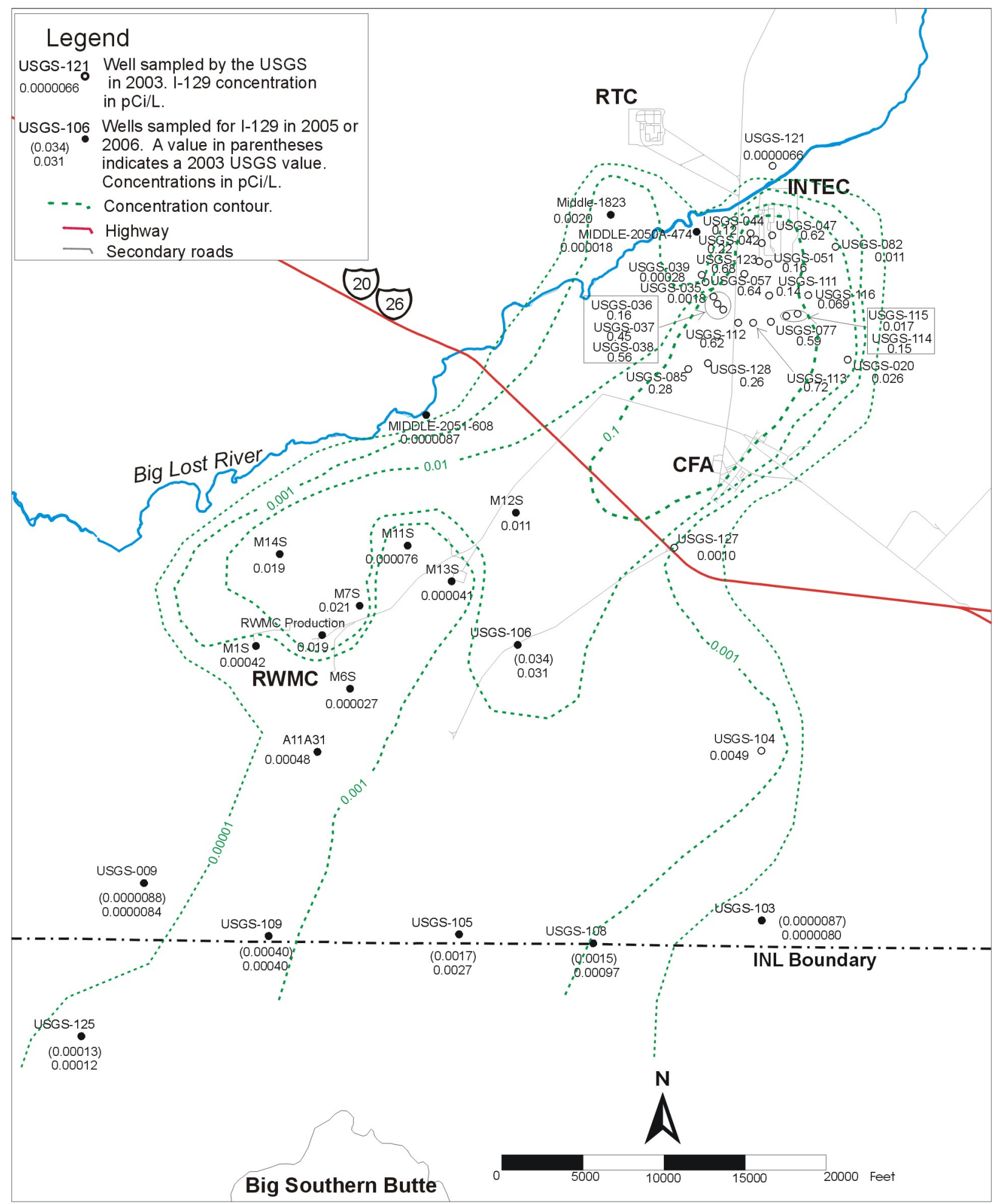

Figure B-3. Distribution of I-129 in the Snake River Plain Aquifer in the RTC/INTEC/RWMC area. 
The 2005 I-129 concentrations from the 748- and 834-ft depth samples from MIDDLE-2051 appear to be too low to be in the INTEC plume, but the I-129 concentrations are consistent with the RTC plume. The tritium and I-129 concentrations in the 834-ft depth sample from MIDDLE-2051 are both approximately one-half the tritium and I-129 concentrations in Middle-1823 downgradient of RTC, and this is consistent with dilution along a flow path. However, tritium concentrations in the 748- and 834-ft depth samples from MIDDLE-2051 are approximately one-half those in M14S and M12S, and the I-129 concentrations are more than an order of magnitude lower in the 834-ft depth sample from MIDDLE-2051. Thus, MIDDLE-2051 appears to be affected by RTC, implying that the RTC and INTEC plumes may be commingling on the north side of the RWMC.

Like the Cl-36 AMS data previously presented (DOE-ID 2006b), the I-129 plume from INTEC can be traced beyond the southern boundary of the INL. Wells USGS-105, -108 , and -109 on the southern INL boundary have I-129 concentrations above background (Figure B-4). The background I-129 concentration was re-evaluated from sampling results in 1993-94 and a background concentration of 5.4 attocuries per liter ( $\mathrm{aCi} / \mathrm{L} ; 1 \mathrm{aCi}=10^{-18}$ curies) was established (Cecil, Hall, and Green 2003). Out of the five stock wells sampled south of the INL boundary, only the Houghland well had an I-129 concentration above the background of $5.4 \mathrm{aCi} / \mathrm{L}$ (Figure B-4). The concentration of I-129 in the five stock wells sampled south of the INL boundary, Fingers Butte, Grazing-2, CCC-3 (Coffee Point), Houghland, and Crossroads had I-129 concentrations similar to their reported concentrations for 1993 (Cecil, Hall, and Green 2003). Sampling of Magic Valley wells and springs south of the INL Site from 1992 to 1994 indicated background I-129 concentrations (Cecil, Hall, and Green 2003). It is important to note that I-129 concentrations in wells on the southern INL boundary and south of the INL boundary are at least four orders of magnitude below the MCL.

\section{B-3. DISCUSSION OF CONTAMINANT AND MAJOR ION DATA FOR THE WESTBAY WELLS}

The vertical distributions of contaminants and major ions for the two Westbay wells, MIDDLE-2050A and MIDDLE-2051, are evaluated with respect to groundwater flow paths and the extent of contamination. Sampling data from September-October 2005 and May-June 2006 are presented and interpreted. The major ion data from the Westbay wells are compared to major ion data from nearby wells to give an indication of the groundwater flow paths and possible influences at each well for both the 2005 and 2006 sampling events. In order to evaluate groundwater flow paths, the major ion data from each sampling interval along with some nearby wells are plotted on a Schoeller diagram, which displays the log of the major ion concentrations expressed as milliequivilents per liter.

The vertical distributions of tritium, chloride, and sulfate at MIDDLE-2051 for 2005 are shown on Figure B-5. Although not shown on Figure B-5, the vertical distribution of I-129 and tritium are very similar. Both I-129 and tritium have near background concentrations in the first sampling port with a spike in concentration at the next two sampling ports, 748 and $834 \mathrm{ft}$, then a decrease with depth. Chloride concentrations show a gradual increase in concentrations with depth while sulfate concentrations show a modest upward spike in the 748- and 834-ft depth samples. The increase in sulfate concentrations corresponds with tritium detections and suggests a similar source for both.

A notable difference between the 2006 and 2005 data for MIDDLE-2051 is that tritium was detected in the two deepest sampling ports in addition to the 748- and 834-ft (depth) ports (Figure B-6). The occurrence of tritium in the two deepest ports in 2006 may be due to equilibration after installation of the Westbay system. Additional sampling in the future will help refine this interpretation. Although the vertical distribution of sulfate was similar in both sampling events, chloride concentrations level off at 834-ft sampling depth in 2006, instead of gradually increasing with depth as in 2005. 


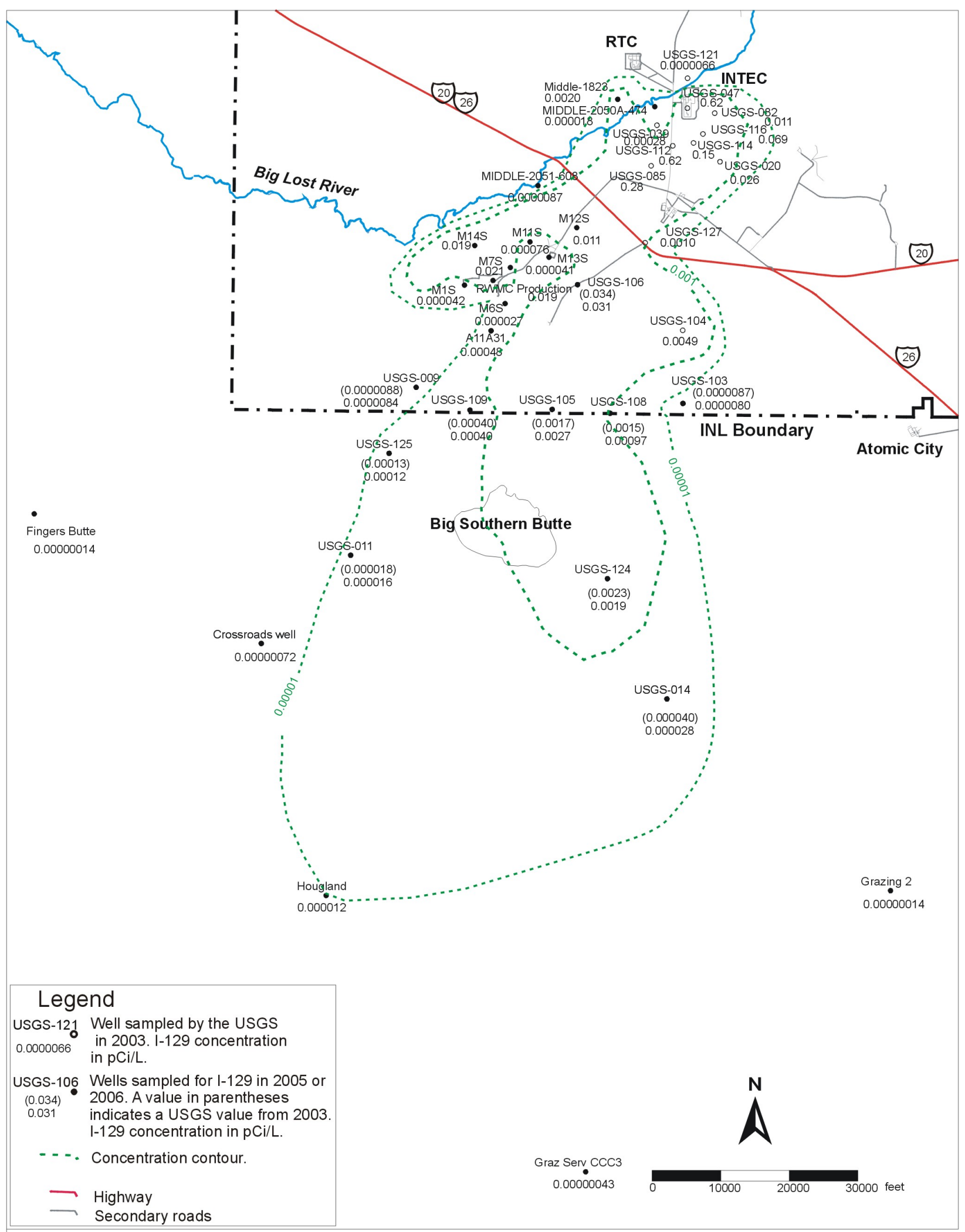

Figure B-4. Distribution of I-129 in the Snake River Plain Aquifer south of the INL boundary. 


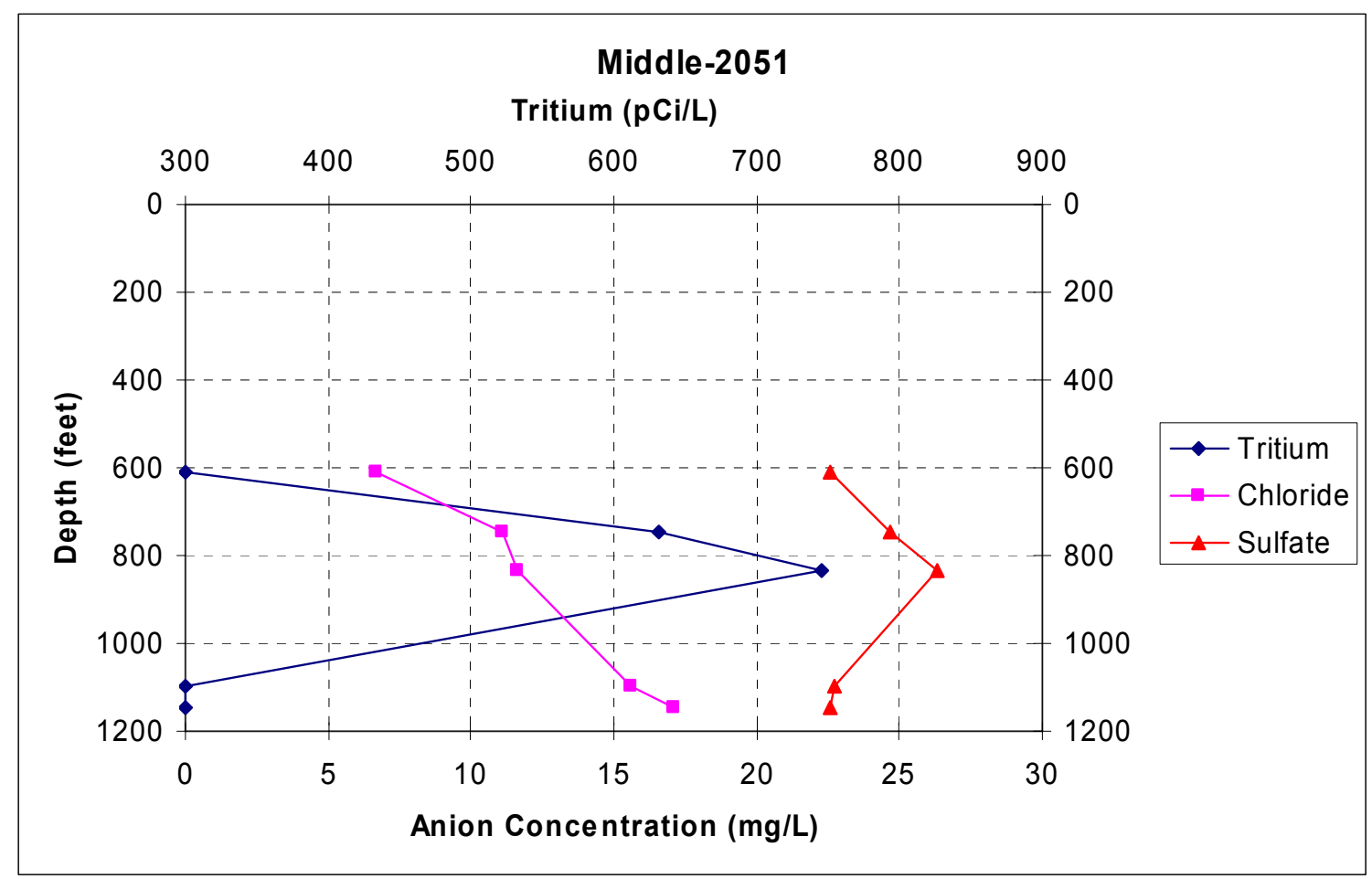

Figure B-5. Vertical distribution of tritium, chloride, and sulfate in MIDDLE-2051 in September 2005.

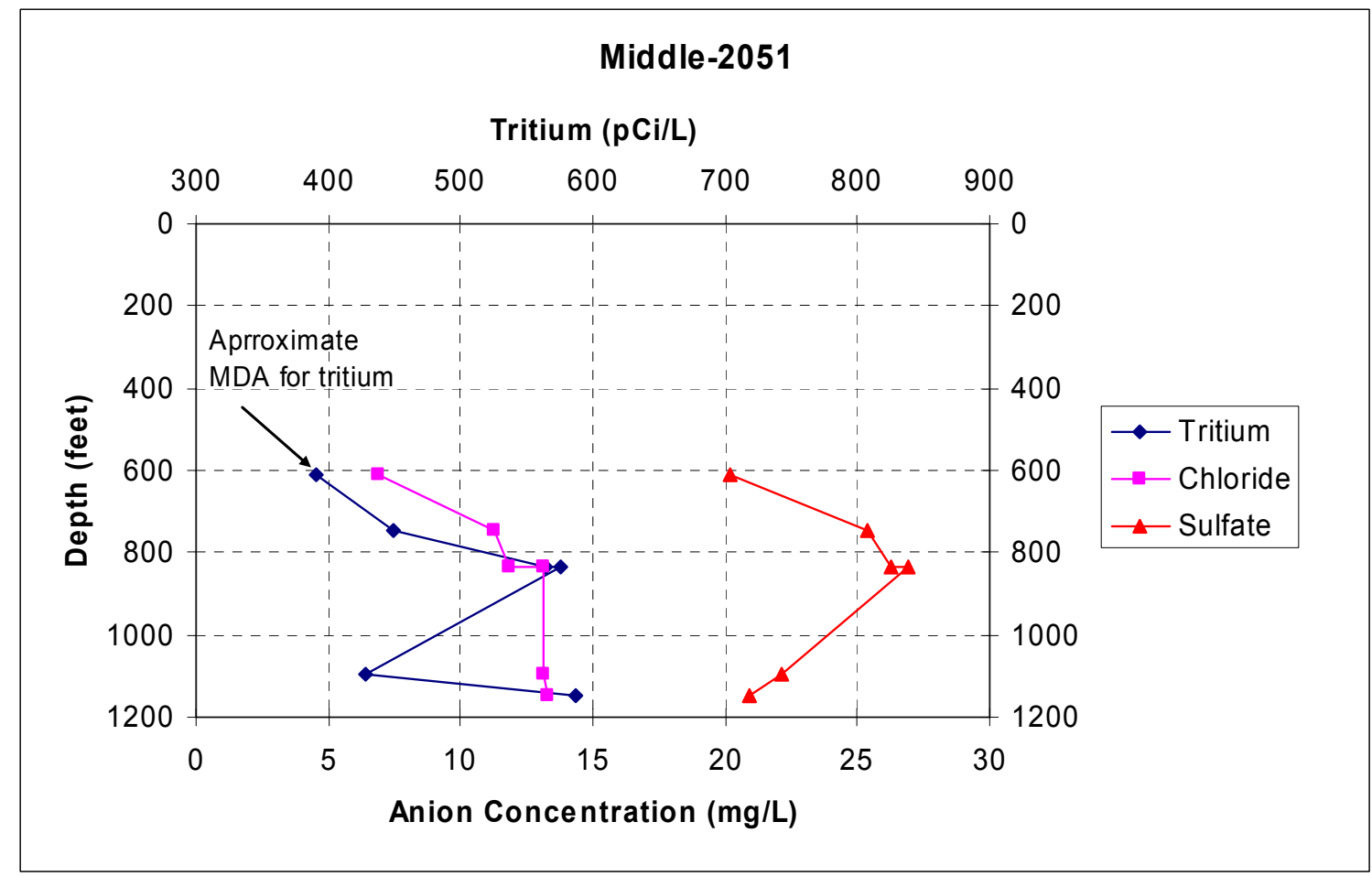

Figure B-6. Vertical distribution of tritium, chloride, and sulfate in MIDDLE-2051 in June 2006. 
A Schoeller diagram for the 2005 data for MIDDLE-2051 shows three different patterns or groupings for the five sampling intervals. The upper interval from MIDDLE-2051, 608-ft depth sampling port, is very similar to the Highway 3 well and suggests a similar water source for both wells. However, the Highway 3 well appears to be too deep to draw from the upper part of the aquifer since it is cased to $680 \mathrm{ft}$ bgs and open from 680 to $750 \mathrm{ft}$ bgs. If the Highway 3 well is capturing water primarily from the upper part of the aquifer, then this could explain why tritium concentrations are low in the Highway 3 well. The samples from 748 and $834 \mathrm{ft}$ have a major ion pattern that is more similar to the Rifle Range well (downgradient RTC plume) than wells affected by the INTEC plume (Figure B-7). The major ion data support the interpretation from the I-129 data that the 748- and 834-ft depth intervals from MIDDLE-2051 are influenced by the RTC plumes. The two deep sampling ports from MIDDLE-2051 have a major ion pattern that is similar to the deepest port from MIDDLE-2050A.

The major ion pattern for Well M14S could indicate mixing of the RTC and INTEC plumes north of the RWMC since it plots similar to M12S and USGS-106; however, Well M14S has a higher sulfate concentration that could indicate influence from RTC, since a sulfate plume originates at RTC. The slightly higher sulfate concentration in M14S, however, is not definitive evidence of mixing of the INTEC and RTC plumes since it is only one line of evidence for mixing.

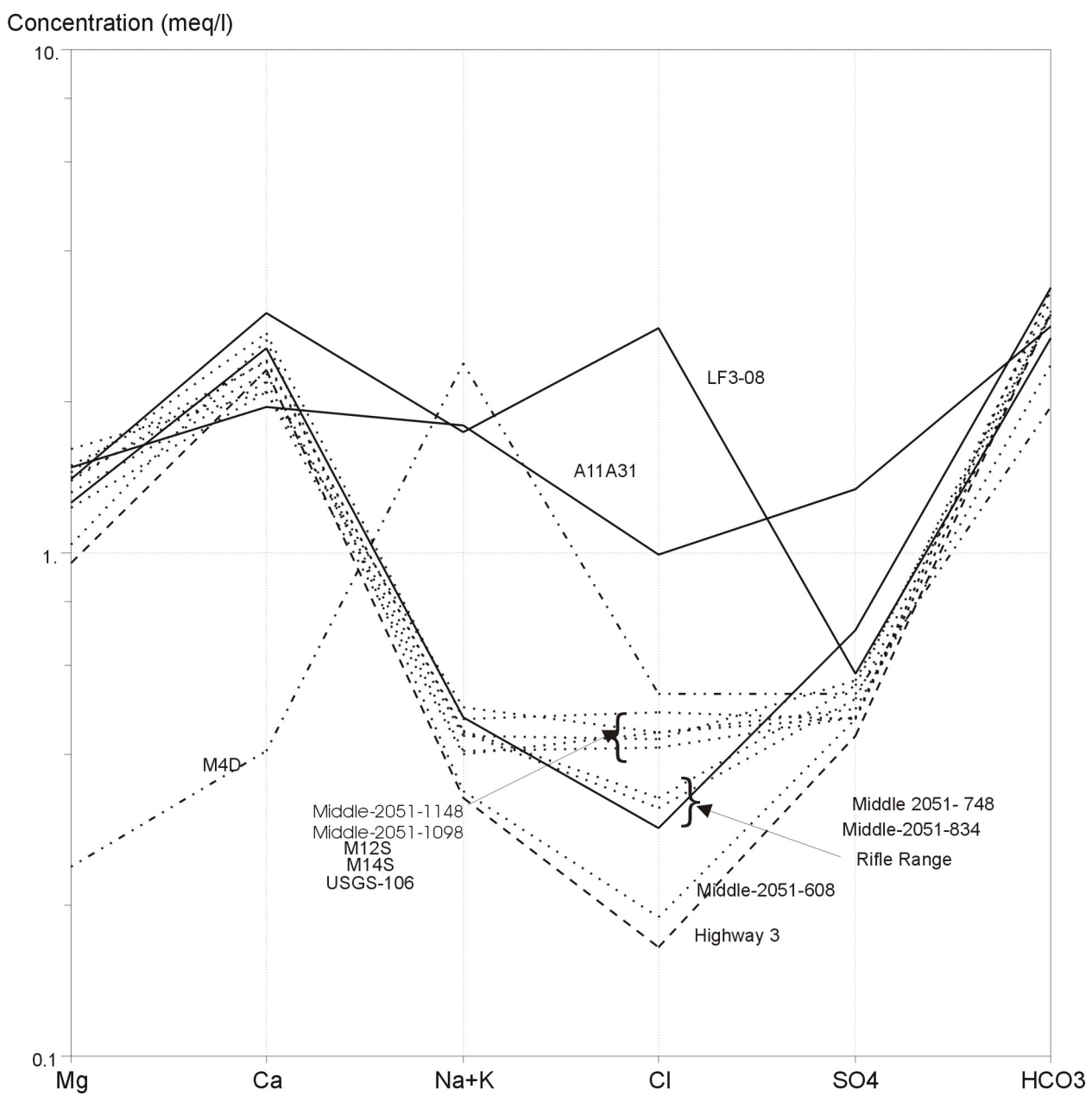

Figure B-7. Schoeller diagram for MIDDLE-2051 for September 2005. NOTE: Data for the Rifle Range well are from 2003, prior to influence from the new INTEC percolation ponds. 
None of the ports from MIDDLE-2051 have a major ion pattern similar to Well M4D located south of the RWMC (Figure B-7). The different chemistry of M4D may be key to understanding the apparent groundwater contaminant divide at the RWMC. Additionally, the Cl-36 from M7s and the RWMC production well may help address the issue of the groundwater contaminant divide. The major ion pattern for M4D is different from all other wells and will be compared in the future to the data for the newly installed Westbay system in USGS-132.

The 2006 Schoeller diagram for MIDDLE-2051 shows the same patterns as the 2005 diagram (Figure B-8). Although tritium was detected in the two deepest intervals of MIDDLE-2051 in 2006, the major ion pattern on the 2005 and 2006 Schoeller diagrams are the same.

The vertical distribution of tritium, chloride, and sulfate for MIDDLE-2050A shows that all three analytes are at background concentrations or below detection limits at all five sampling ports for the 2005 data (Figure B-9). I-129 concentrations are only slightly above background, indicating that the well site is not directly downgradient of either INTEC or RTC (Table B-1). In 2005, chloride and sulfate concentrations are relatively consistent except for a small drop in concentrations at a depth of 1,008 ft.

Except for sulfate in the 474-ft depth sample, the vertical distribution of tritium, chloride, and sulfate for MIDDLE-2050A shows that all three analytes are at background concentrations at all five sampling ports for the 2006 data (Figure B-10). The elevated sulfate concentration in the 474-ft sample could indicate influence from the RTC sulfate plume. Infiltration from the BLR would not be expected to produce this result based on previous analytical results of BLR water (DOE-ID 2003). A BLR water sample was not collected in 2006. The drop in chloride and sulfate concentrations at 1,008 ft also appears in the June 2006 data.

The 2005 Schoeller diagram for MIDDLE-2050A shows that all depths have a very similar pattern and are similar to USGS-121 located upgradient of INTEC (Figure B-11). This pattern is in sharp contrast to the wells in the INTEC plume, such as USGS-112 and LF3-08. The major ion pattern is also different for the Rifle Range well and ICPP-MON-A-167, contaminated with tritium from the RTC to the south and southwest of MIDDLE-2050A (Figure B-2). This suggests that MIDDLE-2050A is not strongly affected by INTEC and probably not affected by RTC. Note that the data for the Rifle Range well, ICPP-MON-A-166, and ICPP-MON-A-167 are from 2003, because by 2005 these wells began to show influence from the new INTEC percolation ponds. The impact on the Rifle Range well from the new INTEC percolation ponds is consistent with NE-SW flow in the aquifer. Two other wells, the Highway 3 well and ICPP-MON-A-166 located south and west of the MIDDLE-2050A, show much lower chloride concentration than the sampling intervals in MIDDLE-2050A and USGS-121, and probably indicate a water source different from that upgradient of INTEC.

The 2006 Schoeller diagram for MIDDLE-2050A shows differences for the 474- and 1,212-ft sampling depths compared to the 2005 samples (Figure B-12). As previously discussed, there is a noticeably higher sulfate concentration in the sample from $474 \mathrm{ft}$, which could indicate influence from the RTC sulfate plume. The 1,212-ft sample shows much higher sodium, potassium, and chloride concentrations. Given that the well was sampled shortly after installation, the difference in chemistry could reflect equilibration. Additional samples at 1,212-ft depth are needed to determine if this is true. 


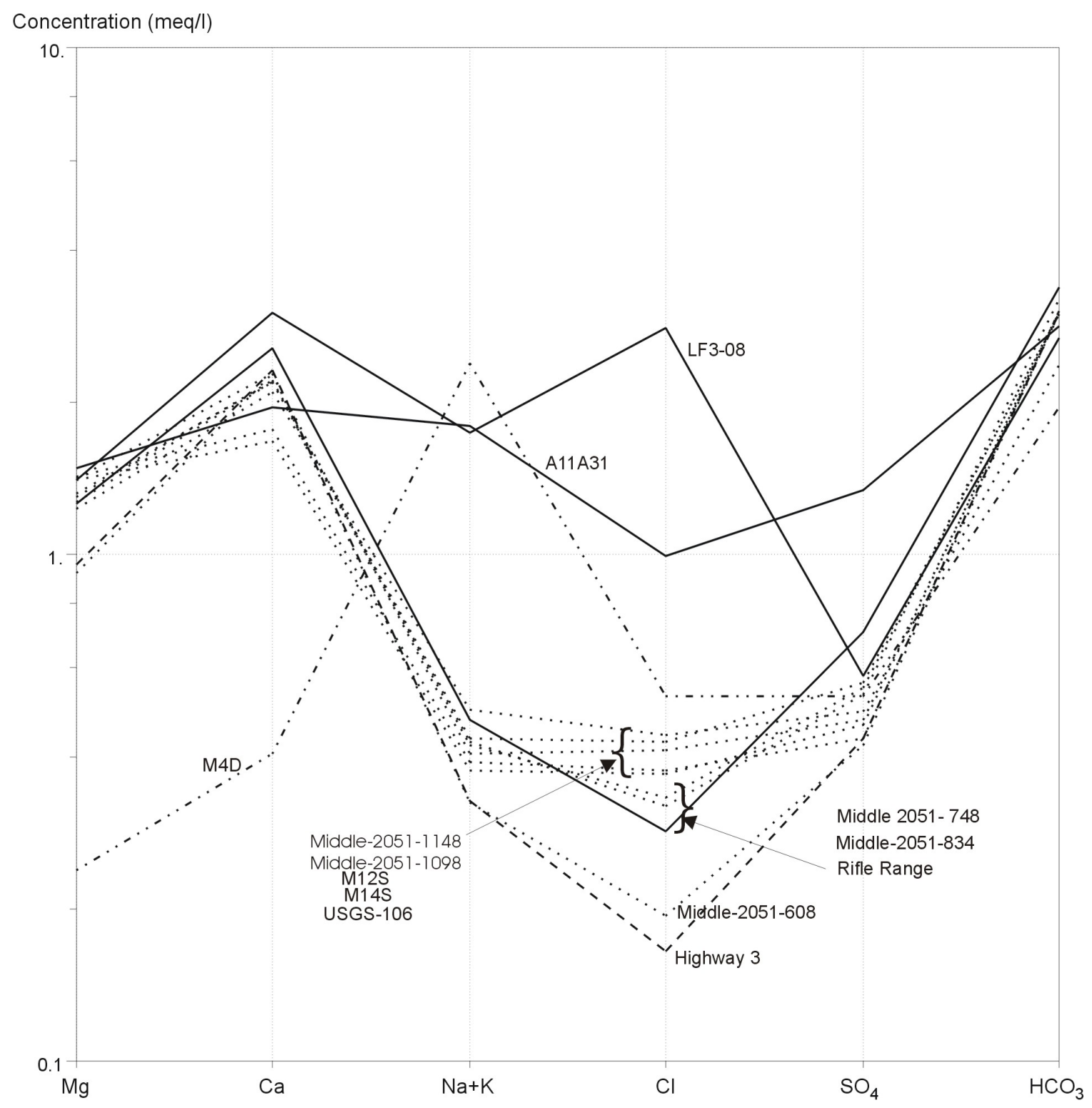

Figure B-8. Schoeller diagram for MIDDLE-2051 for June 2006. 


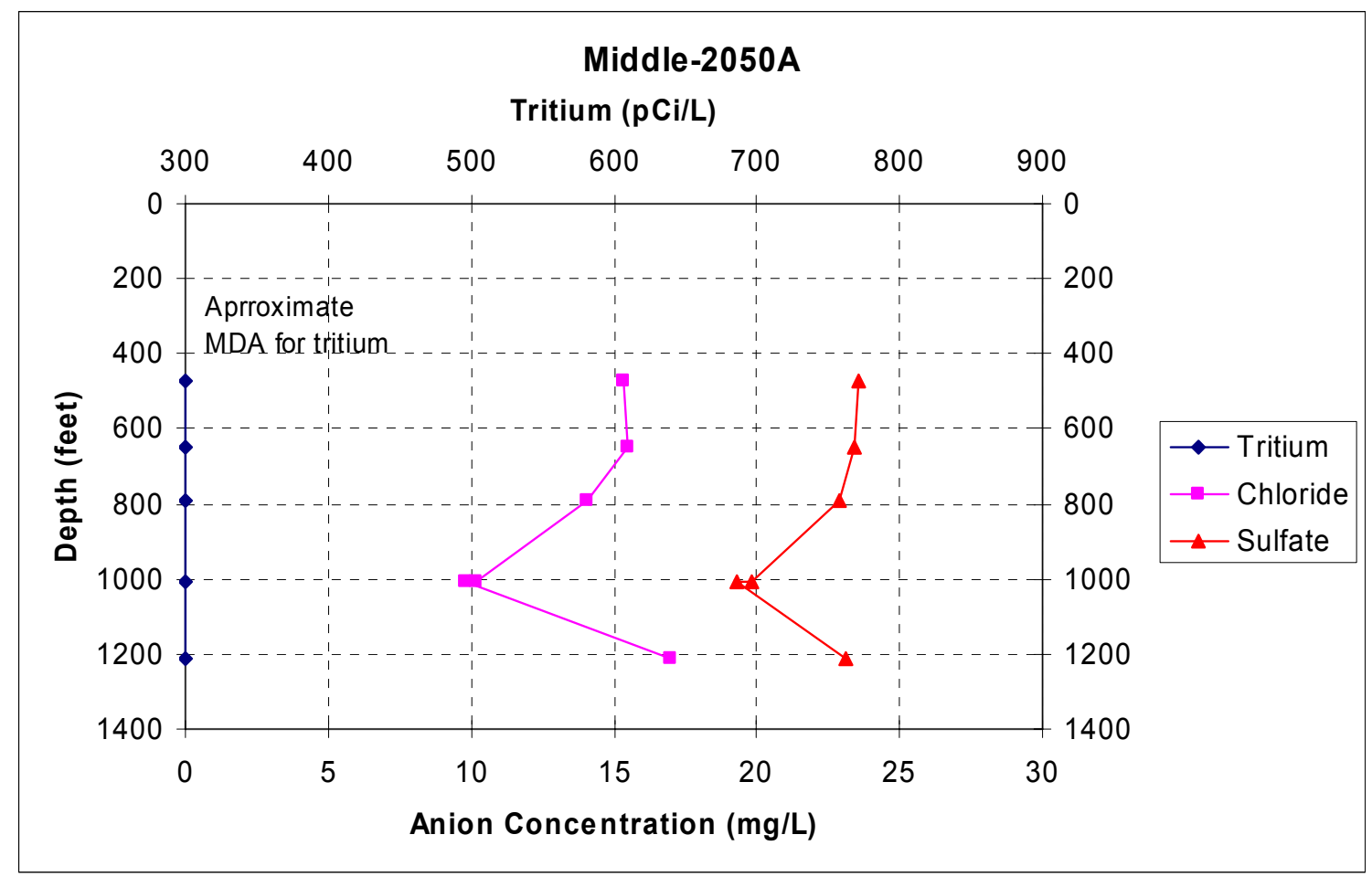

Figure B-9. Vertical distribution of tritium, chloride, and sulfate in MIDDLE-2050A in September 2005.

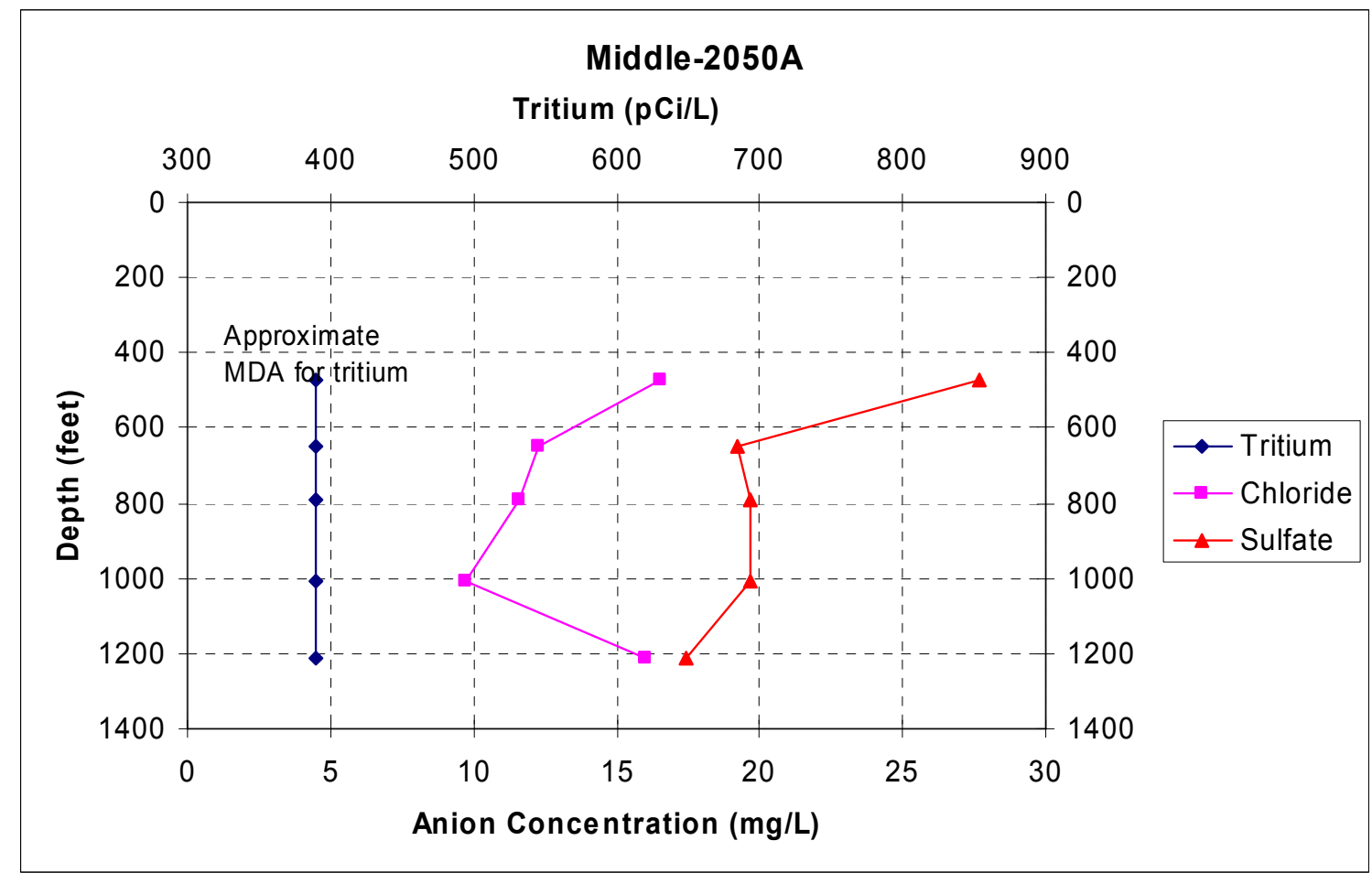

Figure B-10. Vertical distribution of tritium, chloride, and sulfate in MIDDLE-2050A in June 2006. 


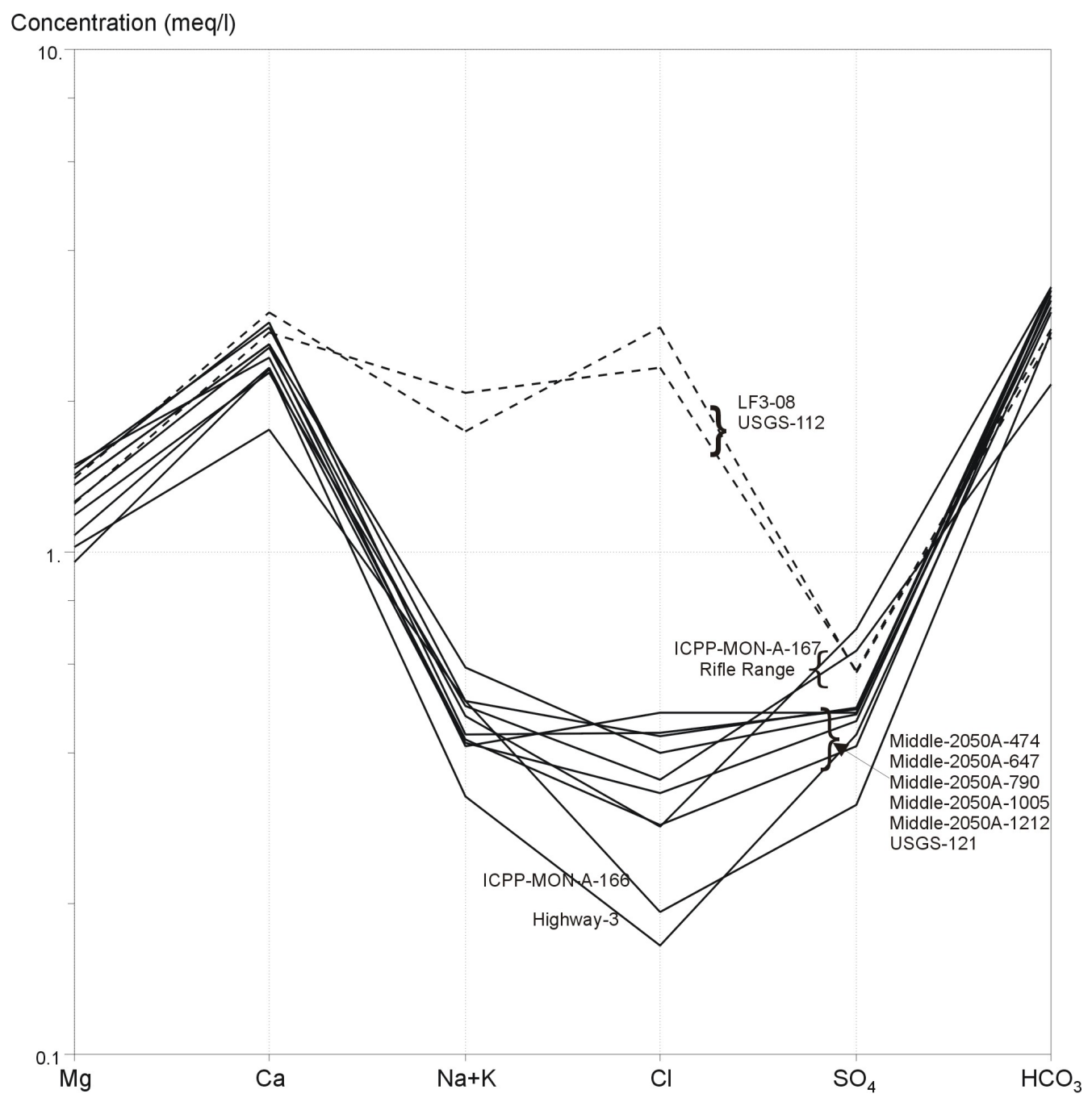

Figure B-11. Schoeller diagram for MIDDLE-2050A for September 2005. 


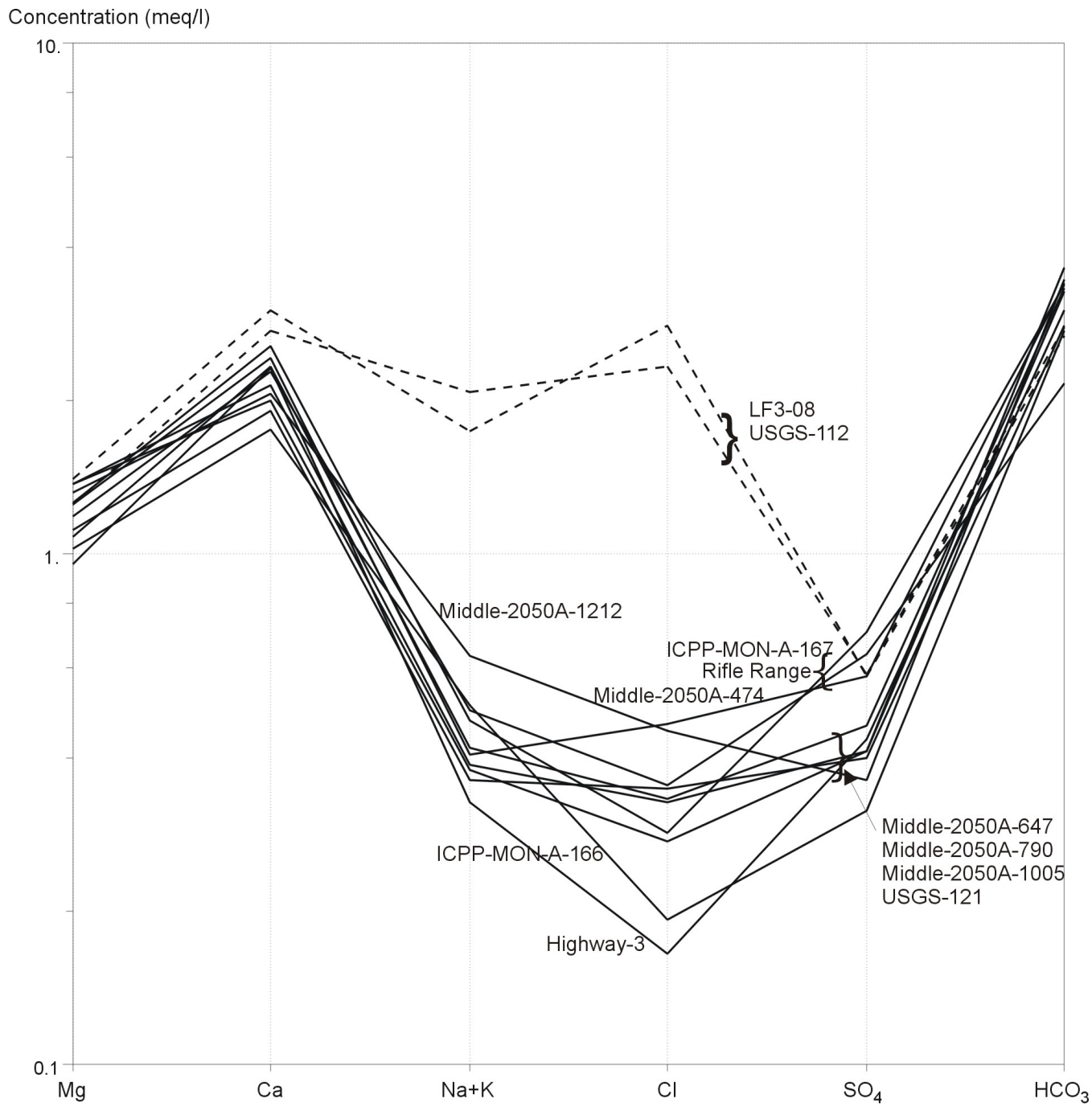

Figure B-12. Schoeller diagram for MIDDLE-2050A for June 2006. 


\section{B-4. DISCUSSION OF PACKER SAMPLING DATA FOR WELLS USGS-105 AND USGS-108}

Two discreet interval packer samples were collected from each USGS-105 and USGS-108 in addition to the regular fixed pump sample. Contaminant data are addressed in the main text of this report with the low detection limit I-129 data addressed previously in this appendix. The major ion data from the packer sampling are evaluated for implications regarding groundwater flow paths.

The concentrations of the major ions in the packer and regular samples from USGS-105 and USGS-108 are shown on Table B-2. Chloride and sulfate concentrations are elevated in the shallow sample from USGS-105 and could indicate migration from the anion anomaly south of the RWMC. However, VOCs, such as carbon tetrachloride or tricholorethene, were not detected in the 2006 samples from USGS-105, which would have corroborated migration from the RWMC area. In support of VOC migration to this location from the RWMC, VOCs were detected at very low levels in samples from USGS-105 in 2002 and 2004. Relatively elevated levels of F-113 were noted, however, by Busenberg, Plummer, and Bartholomay (2001) in USGS-105 using a very-low detection limit analytical method. In addition, carbon tetrachloride was detected in USGS-105 in 2004 at $0.24 \mu \mathrm{g} / \mathrm{L}$. The reason that VOCs are not detected on a consistent basis could be that the VOC concentrations are diluted to concentrations that are difficult to determine using the standard gas chromatography/mass spectrometry (GC/MS) analytical method. Also, the elevated I-129 concentration in the regular fixed pump sample from USGS-105 suggests influence from INTEC since the I-129 concentrations in the anion anomaly south of the RWMC are much lower than at USGS-105 (Table B-1). The elevated I-129 from INTEC with elevated anions (and potentially VOCs in the future) from the RWMC could indicate an area of potentially commingling plumes. The deep sample from USGS-105 has major ion concentrations similar to background.

In contrast to USGS-105, the packer samples from USGS-108 did not show significant differences in common ion chemistry (Table B-2 and Figure B-13). In addition, the major ion data from the two packer samples from USGS-108 were similar to the regular fixed pump depth sample from USGS-108. The similar common ion chemistry could be due to the smaller vertical separation between the samples in USGS-108 compared to USGS-105. A deeper sample could not be collected from USGS-108 due to the presence of polyvinyl chloride pipe in the well.

\section{B-5. DISCUSSION OF COMMINGLING PLUMES}

One of the goals of the geochemical study and of the packer and Westbay sampling was to evaluate commingling plumes. The I-129 data presented in this appendix and the previously presented Cl-36 data (DOE-ID 2006b) from the Purdue PRIME laboratory were used to define flow paths from INTEC and RTC and to determine potential areas of commingling plumes. Data from the geochemical study, the Westbay well sampling, and packer sampling indicated two potential areas where plumes from multiple facilities could overlap or commingle. The two areas that have a potential for plumes to commingle are on the north side of the RWMC and south of the RWMC near and along the INL boundary (Figure B-14).

The commingling of plumes on the southern boundary can only be seen using the ultra-low-detection limits of the Purdue PRIME laboratory. Using standard analytical methods, this area would not show commingling. Although carbon tetrachloride from the RWMC could increase in two INL boundary wells in the future, declining or steady tritium concentrations at the Guard wells suggest that influence from INTEC would be steady or decreasing (see Figure 4 in Section 2.1). Both I-129 and Cl-36 are several orders of magnitude below MCLs at both locations on the INL boundary. WAG 7 is addressing the carbon tetrachloride issue. 
The north side of the RWMC has carbon tetrachloride from the RWMC and tritium at detectable concentrations. Tritium is the only upgradient contaminant on the north side of the RWMC that is detectable using standard analytical methods because I-129 and Cl-36 are well below standard method detection limits. Tritium concentrations are declining or holding steady in wells monitored around INTEC (DOE-ID 2007) and RTC (ICP 2006), suggesting that tritium concentrations are unlikely to increase significantly in the future on the north side of the RWMC.

Table B-2. Major ion data for USGS-105 and USGS-108 packer and regular samples.

\begin{tabular}{|c|c|c|c|c|c|c|c|c|c|c|c|c|c|}
\hline \multirow[b]{2}{*}{ Location } & \multirow{2}{*}{$\begin{array}{c}\text { Depth } \\
\text { (feet bgs) }\end{array}$} & \multicolumn{3}{|c|}{ Sodium } & \multicolumn{3}{|c|}{ Magnesium } & \multicolumn{3}{|c|}{ Potassium } & \multicolumn{3}{|c|}{ Calcium } \\
\hline & & $\mathrm{ug} / \mathrm{L}$ & LF & VF & $\mathrm{ug} / \mathrm{L}$ & LF & $\mathrm{VF}$ & $\mathrm{ug} / \mathrm{L}$ & LF & $\mathrm{VF}$ & $\mathrm{ug} / \mathrm{L}$ & LF & $\mathrm{VF}$ \\
\hline USGS-105 & 700 & 12200 & & & 15000 & & & 2900 & & & 37500 & $\mathrm{E}$ & $\mathrm{J}$ \\
\hline USGS-105 & $676-704$ & 11200 & & & 12700 & & & 2530 & & & 36300 & & \\
\hline USGS-105 & $769-790$ & 11200 & & & 12600 & & & 2600 & & & 36300 & & \\
\hline USGS-105 & $769-790$ & 11200 & & & 12700 & & & 2590 & & & 37500 & & \\
\hline USGS-108 & 637 & 10000 & & & 12500 & & & 2300 & & & 32900 & & \\
\hline USGS-108 & $613-627$ & 11300 & & & 13900 & & & 2660 & & & 33700 & $\mathrm{E}$ & $\mathrm{J}$ \\
\hline \multirow[t]{2}{*}{ USGS-108 } & $657-760$ & 10100 & & & 12700 & & & 2310 & & & 33200 & & \\
\hline & \multirow{2}{*}{$\begin{array}{c}\text { Depth } \\
\text { (feet bgs) }\end{array}$} & \multicolumn{3}{|c|}{ Sulfate } & \multicolumn{3}{|c|}{ Nitrate/Nitrite as N } & \multicolumn{3}{|c|}{ Fluoride } & \multicolumn{3}{|c|}{ Chloride } \\
\hline Location & & $\mathrm{mg} / \mathrm{L}$ & LF & $\mathrm{VF}$ & $\mathrm{ug} / \mathrm{L}$ & $\mathrm{LF}$ & $\mathrm{VF}$ & $\mathrm{mg} / \mathrm{L}$ & LF & $\mathrm{VF}$ & $\mathrm{mg} / \mathrm{L}$ & LF & $\mathrm{VF}$ \\
\hline USGS-105 & 700 & 24.1 & & & 826 & & & 0.2 & & & 14.1 & & \\
\hline USGS-105 & $676-704$ & 28 & & & 1030 & & & 0.19 & & & 19.2 & & \\
\hline USGS-105 & $769-790$ & 23.4 & & & 691 & & & 0.18 & & & 13.1 & & \\
\hline USGS-105 & $769-790$ & 22.6 & & & 776 & & & 0.2 & & & 11.4 & & \\
\hline USGS-108 & 637 & 21.3 & & & 765 & & & 0.24 & & & 15.7 & & $\mathrm{~J}$ \\
\hline USGS-108 & $613-627$ & 22.2 & & & 752 & & & 0.26 & & & 16.3 & & $\mathrm{~J}$ \\
\hline USGS-108 & $657-760$ & 21.1 & & & 776 & & & 0.26 & & & 15.4 & & $\mathrm{~J}$ \\
\hline
\end{tabular}




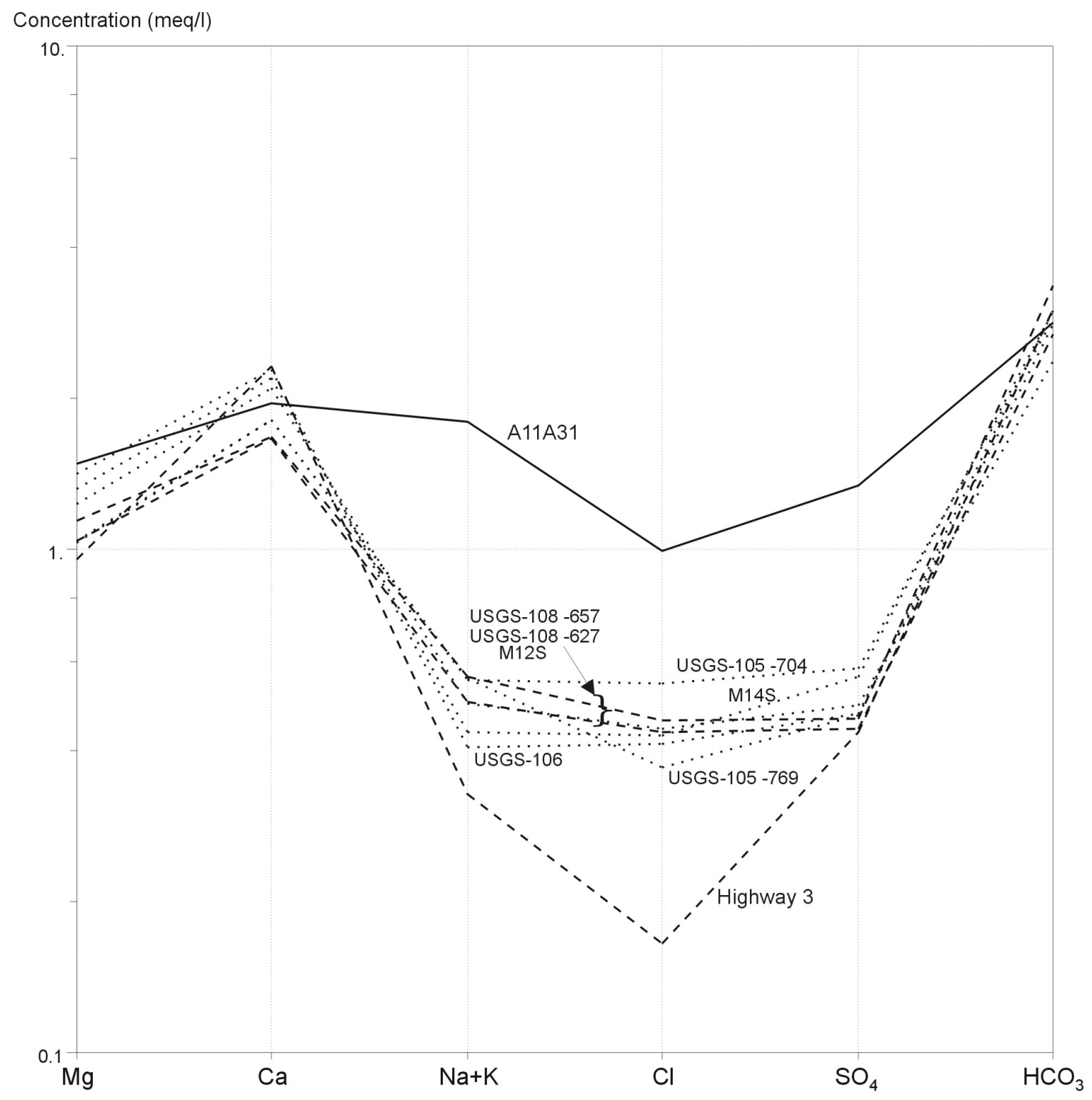

Figure B-13. Schoeller diagram for packer samples from USGS-105 and USGS-108. 


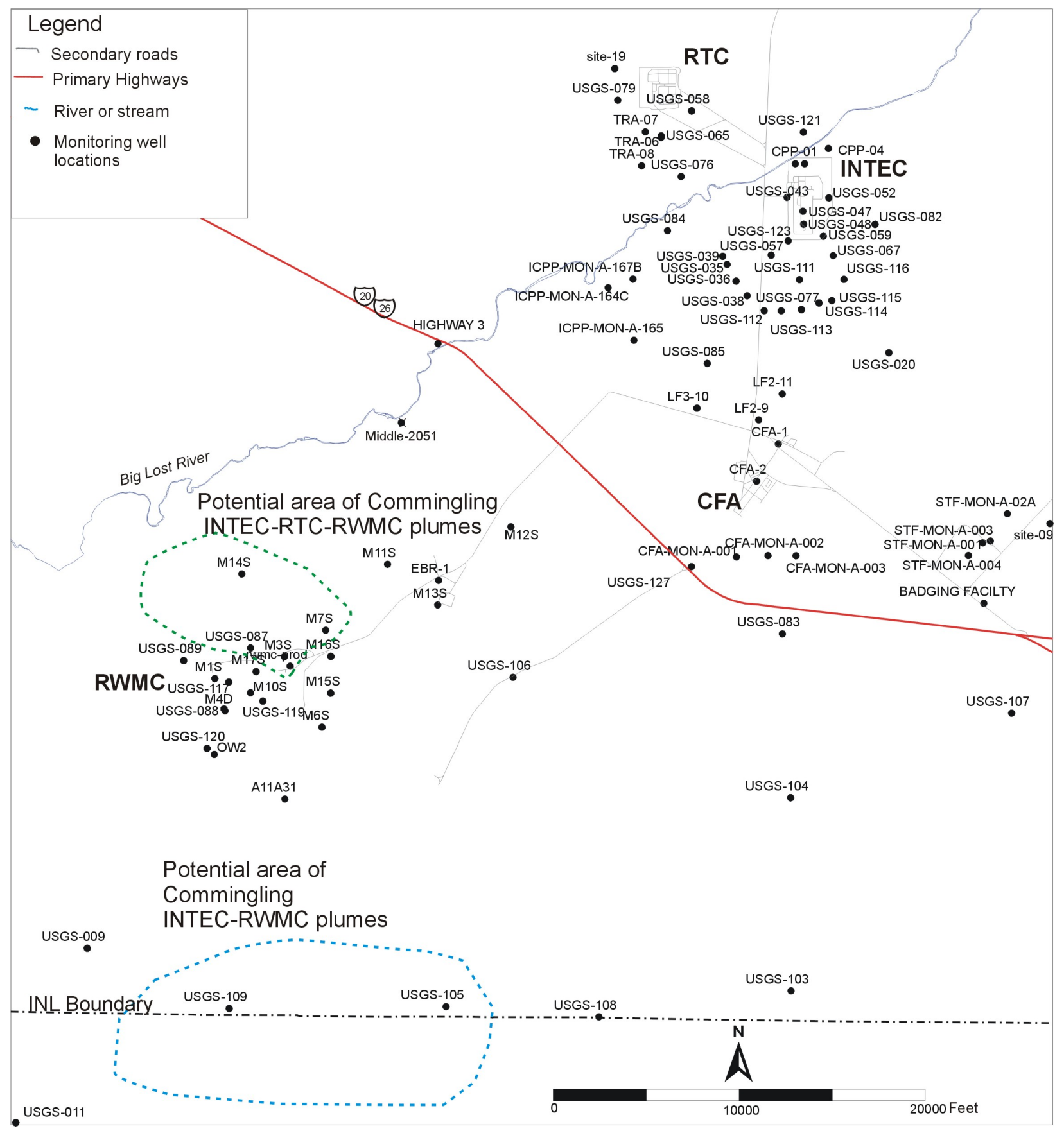

Figure B-14. Areas of potential commingling plumes. 


\section{B-6. SUMMARY AND CONCLUSIONS}

The following conclusions are drawn from the I-129 data collected for the geochemical study, the packer sampling data, and the Westbay sampling data presented in this appendix:

- The I-129 data support the interpretation that contaminant migration from INTEC is the source of the tritium on the north side of the RWMC. Previous USGS studies using I-129, Tc-99, and chlorofluorocarbons (freons) also support this conclusion.

- The source of contaminants at MIDDLE-2051 appears to be the RTC, based on I-129 and sulfate concentrations. Migration beyond MIDDLE-2051 of the RTC plume could lead to flow paths from INTEC and RTC overlapping at locations like Well M14S on the north side of the RWMC. In addition, carbon tetrachloride vapor migration from the RWMC impacts these wells. Thus, the north side of the RWMC shows evidence of commingling plumes. However, the concentrations of the upgradient contaminants are very low, with only tritium detectable using standard analytical methods.

- $\quad$ The similarity of water from the upper interval of MIDDLE-2051 with water from the Highway 3 well suggests that the Highway 3 well may draw water from the upper noncontaminated interval rather than from deeper intervals that may be contaminated with tritium. However, this interpretation is inconsistent with the well construction diagram.

- $\quad$ Overlap of the flow paths from the RWMC (anion plume and possibly the carbon tetrachloride plume, based on previous results) and INTEC was suggested by analysis on water samples collected from both the uppermost packer interval and the regular fixed-pump depth in Well USGS-105. The concentrations of the commingled contaminants are currently very low and concentrations of INTEC-related contaminants are not expected to increase in the future.

- $\quad$ Like the previously presented Cl-36 data, the I-129 data indicate that concentrations on the INL boundary are more than four orders of magnitude below the MCL and dilute even more south of the INL boundary. Using the AMS method, it is possible to trace groundwater flow paths as far south as the Houghland well or approximately $13 \mathrm{mi}$ south of the INL boundary. At this well, the I-129 concentration is approximately two times the background value of $5.4 \mathrm{aCi} / \mathrm{L}$ (Cecil, Hall, and Green 2003), or more than five orders of magnitude below the I-129 MCL. The I-129 data collected for this report support the conclusion that contaminants from the INL should not have an impact on wells in the Magic Valley.

\section{B-7. REFERENCES}

Beasley, T. M., P. R. Dixon, and L. J. Mann, 1998, “" ${ }^{99} \mathrm{Tc},{ }^{236} \mathrm{U}$, and ${ }^{237} \mathrm{~Np}$ in the Snake River Aquifer at the Idaho National Engineering and Environmental Laboratory, Idaho Falls, Idaho," Environmental Science and Technology, Vol. 32, No. 24, pp. 3875-3881.

Busenberg, E., L. N. Plummer, and R. C. Bartholomay, 2001, Estimated Age and Source of the Young Fraction of Ground Water at the Idaho National Engineering and Environmental Laboratory, USGS Water Resources Investigations Report 01-4265.

Cecil, L. D, L. F. Hall, and J. R. Green, 2003, Reevaluation of Background Iodine-129 Concentrations in Water from the Snake River Plain Aquifer, Idaho, USGS Water Resources Investigations Report 03-4106. 
DOE-ID, 2003, Phase I Monitoring Well and Tracer Study Report for Operable Unit 3-13, Group 4, Perched Water, DOE/ID-10967, Rev. 1, U.S. Department of Energy Idaho Operations Office, June 2003.

DOE-ID, 2005, Groundwater Monitoring and Field Sampling Plan of Operable Unit 10-08, DOE/NE-ID-11210, Rev. 0, U.S. Department of Energy Idaho Operations Office, March 2005.

DOE-ID, 2006a, Groundwater Monitoring and Field Sampling Plan of Operable Unit 10-08, DOE/NE-ID-11210, Rev. 1, U.S. Department of Energy Idaho Operations Office, May 2006.

DOE-ID, 2006b, Waste Area Group 10, Operable Unit 10-08, Remedial Investigation/Feasibility Study Annual Status Report for Fiscal Year 2005, DOE/NE-ID-11274, Rev. 1, U.S. Department of Energy Idaho Operations Office, May 2006.

DOE-ID, 2007, INTEC Groundwater Monitoring Report (2006), DOE/ID-11301, Rev. 0, U.S. Department of Energy Idaho Operations Office, February 2007.

ICP, 2006, Annual Groundwater Monitoring Status Report for Waste Area Group 2 for Fiscal Year 2006, RPT-237, Rev. 1, Idaho National Laboratory, Idaho Cleanup Project, October 2006.

Mann, Larry J., and T. M. Beasley, 1994, I-129 in the Snake River Plain Aquifer at and near the Idaho National Engineering Laboratory, Idaho, 1990-91, U.S. Geological Survey Water Resources Report 94-4053. 
Appendix C

\section{Summary of Operable Unit 10-08 Two-Dimensional} Transport Model 


$$
\text { C-2 }
$$




\section{CONTENTS}

Appendix C-Summary of Operable Unit 10-08 Two-Dimensional Transport Model ......................... C-1

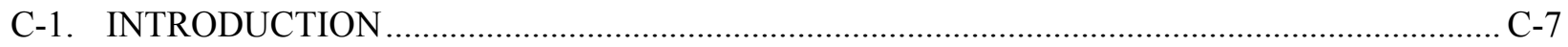

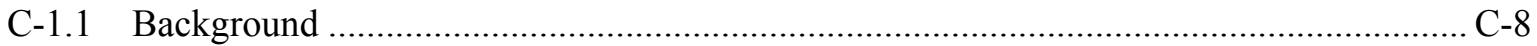

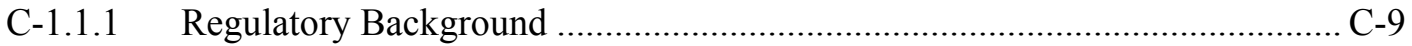

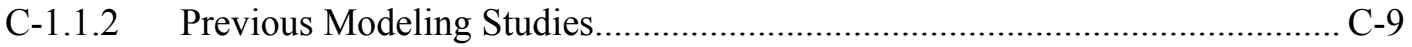

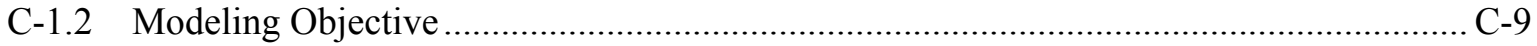

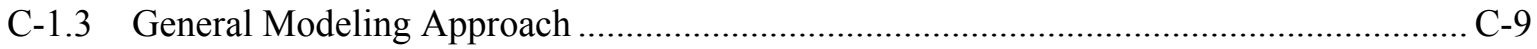

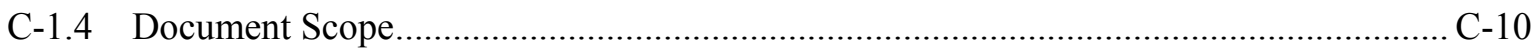

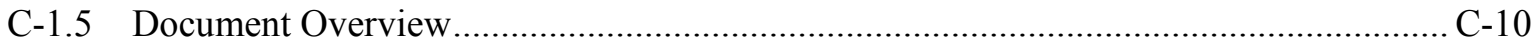

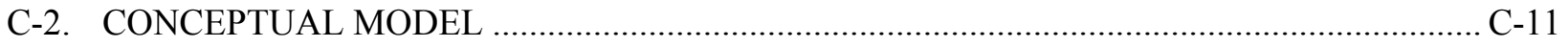

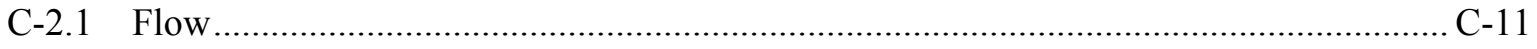

C-2.1.1 Operable Unit 10-08 Model Domain ......................................................... -11

C-2.1.2 Major Geologic Units and Hydrologic Subdomains .................................. C-12

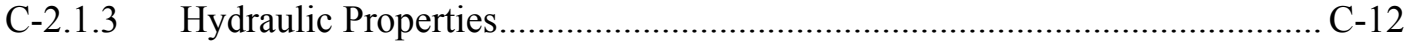

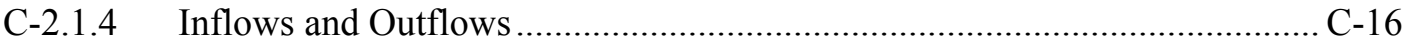

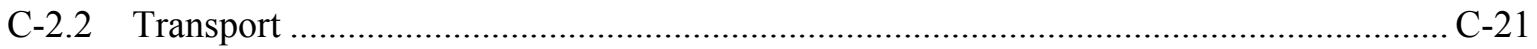

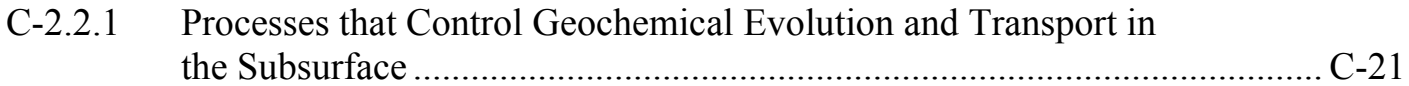

C-2.2.2 Velocities Inferred from Geochemical Studies ......................................... C-23

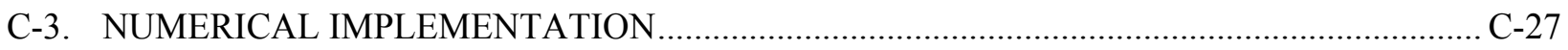

C-3.1 Two-Dimensional Flow Model Updates …...............................................................

C-3.1.1 Northeast Boundary Specified Flux …................................................... C-28

C-3.1.2 Constraints on Hydraulic Conductivity for Selected Pilot Points ................. C-28

C-3.1.3 Revisions to Southwest Specified Head Boundary ….................................. C-30

C-3.1.4 Revised Flow Model Results........................................................................... C-31

C-3.2 Two-Dimensional Transport Model Development.................................................... C-36

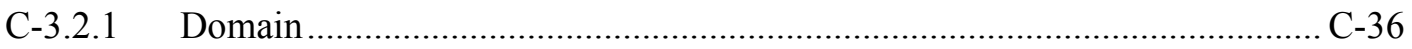

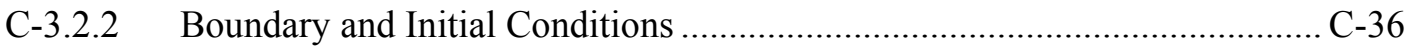

C-3.2.3 Contaminants and Contaminant Loading .................................................

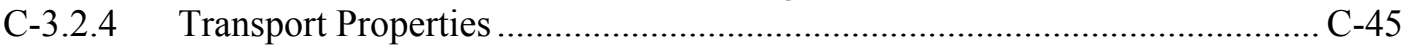

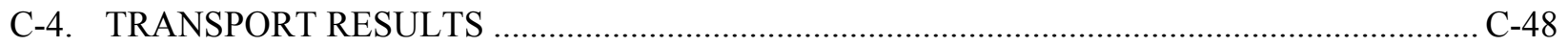


Appendix C-A - Three-Dimensional Characterization of Inflows to and Outflows from the Operable Unit 10-08 Model Domain.

\section{FIGURES}

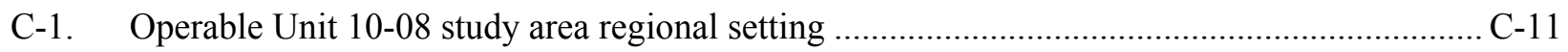

C-2. Aquifer bottom elevation for thick aquifer (top) and thin aquifer (bottom) scenarios ............. C-13

C-3. Distribution of geologic units and hydrologic subdomains in the Operable Unit 10-08

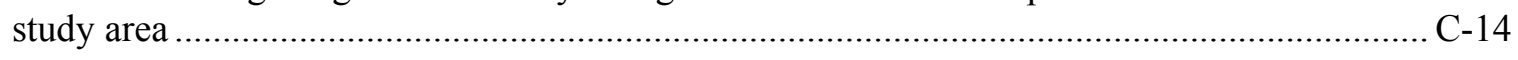

C-4. Hydraulic conductivity distribution of the study area ........................................................ C-16

C-5. Approximate distribution in 2003 of selected chemical and radiochemical contaminants in groundwater at Idaho National Laboratory ................................................................... C-24

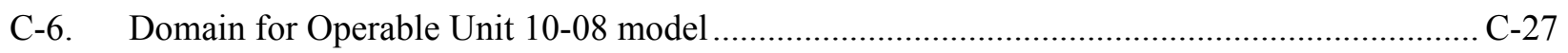

C-7. Example of head escalation in the thin scenario model resulting from using specified flux boundary conditions along the northeastern boundary ......................................................... C-29

C-8. Location of constrained pilot points near Big Lost River and Birch Creek model interface..... C-30

C-9. Comparison of hydraulic head contours and flux vectors near southwest specified-head

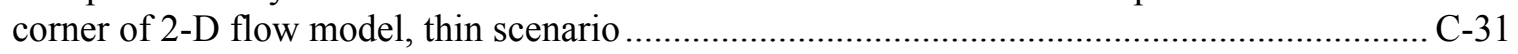

C-10. Final June 2004 simulated heads resulting from constrained pilot point approach during inverse modeling.

C-11. Final June 2004 simulated heads inside INL resulting from constrained pilot point approach inverse modeling for (a) thin aquifer scenario, (b) thick aquifer scenario

C-12. Final June 2004 heads for southern INL facilities resulting from constrained pilot point approach inverse modeling for the thick aquifer scenario...

C-13. Estimated hydraulic conductivity fields $(\mathrm{m} / \mathrm{d})$ that result from constrained pilot point inverse modeling for (a) thin aquifer scenario and (b) thick aquifer scenario

C-14. Residual versus head values for (a) the thin aquifer scenario (top figure) and (b) the thick aquifer scenario (bottom). 
C-15. Comparisons of estimated conductivity between Operable Unit 10-08 and individual WAG models at (a) OU 3-14 model and (b) OU 7-13/14 saturated model scales....

C-16. Location of contaminant sources simulated in the Operable Unit 10-08 2-D transport model.. C-39

C-17. Example time histories of wastewater volumes and contaminant concentrations disposed of in WAG 1 and WAG 3 injection wells....

C-18. Local-scale depiction of individual source cells represented in WAG 3 and WAG 7 vadose zone transport models

C-19. 140 recharge polygons (blue line) representing OU 7-13/14 vadose zone footprint and the underlying 2-D OU 10-08 transport model grid (black line)

C-20. Cumulative mass flux into the aquifer specified as input to GMS (output from WAG 7 model) and GMS output used in the aquifer model for $\mathrm{CCl}_{4}$, chromium, and nitrate (top to bottom figures, respectively)

C-21. The comparison between cumulative contaminant masses in the aquifer specified by individual WAG models and simulated by the 2-D OU 10-08 transport model

C-22. (a) The domains of individual WAG models relative to the OU 10-08 model domain and (b) the transport property values assigned in the individual WAG models (effective porosity and, in parentheses, longitudinal dispersivity in meters) for the variable-properties transport scenario

C-23. Plots of scaled tritium concentration at maximum simulation time (20,075 days, by year of 2005) for two aquifer thickness and two transport property scenarios

C-24. Plots of scaled I-129 concentration at maximum simulation time (20,075 days) for two aquifer thickness and two contaminant property scenarios.

C-25. Plots of scaled Sr-90 concentration at maximum simulation time (20,075 days) for two aquifer thickness and two contaminant property scenarios.

C-26. Plots of scaled Tc-99 concentration at maximum simulation time (20,075 days) for two aquifer thickness and two contaminant property scenarios.

C-27. Plots of nitrate concentration at maximum simulation time (20,075 days) for two aquifer thickness and two contaminant property scenarios.

C-28. Plots of chromium concentration at maximum simulation time (20,075 days) for two aquifer thickness and two contaminant property scenarios.

C-29. Plots of $\mathrm{CCl}_{4}$ concentration at maximum simulation time (20,075 days) for two aquifer thickness and two contaminant property scenarios....

C-30. Plots of TCE concentration at maximum simulation time (20,075 days) for two aquifer thickness and two contaminant property scenarios.

C-31. Comparison of the simulated plumes with individual WAG models (tritium plume by WAG 3 model and nitrate plume by WAG 7) for variable transport property scenario C-58 
C-32. Comparison of the simulated plumes with individual WAG models (tritium plume by WAG 3 model and nitrate plume by WAG 7) for uniform transport property scenario

C-33. Linear pore water velocity $(\mathrm{m} / \mathrm{d})$ distribution for the entire domain (uniform effective porosity of 0.10 ): left-thick scenario; right-thin scenario

C-34. Linear pore water velocity (m/d) distribution near vicinity of WAG 3 and WAG 7 (uniform effective porosity of 0.10 ): left-thick scenario; right-thin scenario

C-35. Linear pore water velocity $(\mathrm{m} / \mathrm{d})$ overlying the conductivity fields near vicinity of WAG 3 and WAG 7 (uniform effective porosity of 0.10 ): left-thick scenario; right-thin scenario

\section{TABLES}

C-1. Geologic units and hydrologic subdomains within the OU 10-08 model domain, including a summary of lithologic and stratigraphic characteristics controlling groundwater flow and contaminant transport.

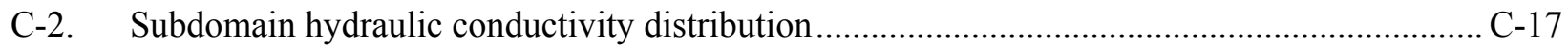

C-3. Inflows to the area represented by the Operable Unit 10-08 model domain.............................. C-18

C-4. Estimated underflow across the northeast model-domain boundary apportioned by segments to represent increasing aquifer thickness to the southeast.

C-5. Water balance components with specified flux and specified head along the northeastern boundary

C-6. Summary of sources and contaminants included in Operable Unit 10-08 2-D transport model

C-7. Radioactive decay half-lives and specific activities for radiological contaminants included in the Operable Unit 10-08 2-D transport model

C-8. Porosity and dispersivities from WAG-specific models that were used in the variable-property Operable Unit 10-08 2-D transport simulation. 


\section{Appendix C}

\section{Summary of Operable Unit 10-08 Two-Dimensional Transport Model}

\section{C-1. INTRODUCTION}

The Waste Area Group (WAG) 10, Operable Unit (OU) 10-08, groundwater modeling study is intended to provide a comprehensive evaluation of environmental impacts from operations at the Idaho National Laboratory (INL) Site to the underlying Snake River Plain Aquifer (SRPA). In particular, OU 10-08 groundwater studies address areas outside the boundaries of the other individual INL WAGs and consider the potential for risk created by the commingling of residual plumes left by those WAGs. The cumulative impacts on the SRPA are being evaluated during the OU 10-08 RI/FS.

The OU 10-08 groundwater modeling studies are guided by the Idaho National Engineering and Environmental Laboratory Operable Unit 10-08 Sitewide Groundwater Model Work Plan

(DOE-ID 2004). That plan was developed in collaboration with and review by the U.S. Department of Energy Idaho Operations Office (DOE-ID), the Idaho Department of Environmental Quality (DEQ), and the U.S. Environmental Protection Agency (EPA) to ensure that the products of the modeling studies match those needed for the OU 10-08 RI/FS process. This approach is intended to significantly expand the regulatory agencies' involvement in the development of the model by engaging them early and frequently over the course of the project. The overall modeling objectives statement and issues resulting from the collaborative planning effort are documented in the Groundwater Model Work Plan (DOE-ID 2004).

To enhance integration with the numerous parties involved in modeling aquifer flow and transport in the region, the project is using a tiered approach to the model design. Previously, the first step was completed, which was development of a steady-state two-dimensional flow model (Wood et al. 2005). This report presents the second step in our numerical simulations, i.e., a two-dimensional (2-D) transport model. The tiered approach is cost effective and will help to identify technical and administrative areas of conflict early enough in the process to solve issues in a timely manner during the RI/FS.

This appendix is presented for informational purpose only. The appendix contains material that was summarized in a presentation at the OU 10-08 Remedial Investigation/Feasibility Study (RI/FS) 2006 Annual Agency Summit Meeting held in Idaho Falls, Idaho, June 14th and 15th, 2006. The two-dimensional transport model was primarily developed to work out the methodology for including various contaminant sources in the MT3DMS simulator, which is linked via the Groundwater Modeling System shell (U.S. Army Corps of Engineers 2005) to the MODFLOW simulator. The two-dimensional transport simulations represent an interim step toward the final comprehensive three-dimensional flow and transport simulations. Concentration values reported in the model results are not calibrated and are not intended to be predictive.

Including this two-dimensional flow and transport model appendix meets the program milestone for a two-dimensional transport report. 


\section{C-1.1 Background}

A key component of the RI/FS effort (DOE-ID 2002) and long-term stewardship of the groundwater resources at the INL Site is the development of an INL Sitewide groundwater-flow and contaminant-transport numerical model (SWGM). The model will support decisions and serve as a tool for managing, compiling, and synthesizing data regarding the SRPA beneath the INL Site. Currently, several different aquifer models are used at the INL Site to satisfy specific program needs. These models are not consistent in some cases and are sometimes redundant in the regimes they represent. Preparation of the SWGM provides the opportunity to promote consistency and eliminate redundancies in INL aquifer models. In the short term, the SWGM will be used to satisfy requirements for preparation of the OU 10-08 Record of Decision (ROD) and will supplement and support existing aquifer models. However, the design of the SWGM will eventually allow incorporation of smaller individual aquifer models in a seamless, consistent manner. Although vadose zone transport modeling is the responsibility of individual WAGs, the assumptions and implementation used in the individual WAG vadose zone models will be reviewed as the contaminant fluxes are implemented into the SWGM.

The need for the SWGM also is driven by advancements in the understanding of the INL Site subsurface and greatly improved computational capabilities. During the past decade, INL Site contractors, the United States Geological Survey (USGS), and numerous academic institutions have obtained information that significantly changes the conceptual model of the subsurface beneath the eastern Snake River Plain (ESRP). In order to use these new data in determining the risk posed by contaminants from the INL Site, the data must be compiled and used to update conceptual and numerical models of flow and transport.

To the extent possible, the SWGM will be structured to integrate with and complement existing groundwater-flow and contaminant-transport models developed by individual WAGs and the USGS. This approach will enhance consistency across the INL Site and help resolve differences raised by different interpretations of subsurface data. Communication, staff integration, and data sharing are the foremost components in the strategy for integrating the SWGM with existing models. Meetings are held at regular intervals for technical and management staff involved with the active development or application of numerical simulations of the subsurface at the INL Site. Members of senior technical staff have been recruited from the major facility-scale groundwater projects to act as technical consultants on the design and construction of the SWGM. Additionally, use of the Environmental Data Warehouse to share and store data will ensure that the SWGM is developed and based on a common and consistent set of data.

The underlying strategy for the SWGM is a departure from the strategies for other models that have sought a single, unique solution to groundwater flow and transport. The SWGM strategy assumes that different and sometimes competing interpretations of groundwater flow and transport will develop because of the relatively sparse subsurface data set for the complicated INL Site subsurface and the many programs utilizing these data for varied purposes. Consensus on a single conceptual model will be difficult to achieve and will evolve as more data become available. The SWGM strategy for integration includes the capability to test interpretations generated by various projects (solving specific problems). Cross comparison between interpretations will define the bounds of flow and transport in the SRPA at the

scale of the INL Site. Thus, unique solutions derived by individual projects can be included in the SWGM as long as the solutions are consistent within a range that is reasonable for possible interpretations described by the subregional understanding of aquifer flow and transport. 


\section{C-1.1.1 Regulatory Background}

The WAG 10 OU 10-08 RI/FS Work Plan (DOE-ID 2002) describes the enforceable milestone schedule for OU 10-08, and the reader is referred to that plan for a detailed summary of the events controlling the final deliverable date for the OU 10-08 ROD.

The importance of the OU 10-08 SWGM is demonstrated by two important facts: (1) the SRPA, a sole-source aquifer, is the number one INL-related concern for the population of eastern Idaho, and (2) predicted contaminant levels in the SRPA drive the selection of most remedies for individual WAGs. For the aforementioned reasons, the INL Site management team (including the Agencies) has taken a proactive, technically robust approach for developing the SWGM.

\section{C-1.1.2 Previous Modeling Studies}

Numerical modeling of groundwater flow beneath the INL Site has been ongoing for many years, both at the INL Sitewide scale and for much larger areas of interest. Numerical models for INL groundwater problems were utilized as early as the mid-1970s (Robertson 1974). The USGS Regional Aquifer-System Analysis program produced several SRPA models at various scales for use as characterization tools dealing with regional water-resource issues (Lindholm 1981; Garabedian 1992; Spinazola 1994). Recent numerical modeling efforts have included the State of Idaho Regional Water Resource Model (Cosgrove et al. 2006) and the ongoing USGS Subregional Model. At the INL Site, remedial investigations mandated under the Comprehensive Environmental Response, Compensation, and Liability Act (CERCLA) (42 USC $\S 9601$ et seq.) have resulted in several flow and transport models; these include three numerical flow and transport models currently in preparation for WAGs 1, 3, and 7.

Historical modeling efforts are important, because they identify documented successes that can be incorporated into the SWGM and because they help to identify issues and problems that can be avoided. Several historical models provide input to the WAG 10 conceptual model and provide useful summaries of data to be used in the SWGM. Wood et al. (2005) summarized the basic features and applicable results for several regional ESRP and subregional INL Site models and three active individual WAG aquifer models.

\section{C-1.2 Modeling Objective}

From DOE-ID (2004), the overall goal for the SWGM project is to:

Develop a Sitewide flow and transport model of the active flow portion of the Snake River Plain Aquifer that can be used to evaluate OU 10-08 remedial action alternatives and to ensure all remedies remain protective of the aquifer. The model will provide credible estimates of contaminant concentrations from sources at the INL over relevant future timeframes.

\section{C-1.3 General Modeling Approach}

The approach taken by the OU 10-08 groundwater modeling project to attain this goal is to use multiple sources of information and perform a multi-objective calibration. These multiple sources of information include hydrologic data, stratigraphic data, contaminant data indicative of transport from facilities, isotope data to infer groundwater velocities away from facilities, and thermal data to corroborate the groundwater transport modeling. 
This approach results in a comprehensive model that addresses all impacts to the aquifer within the area defined by the model, which is termed sub-regional because it encompasses a region slightly larger than the INL, but not the entire ESRP aquifer. The modeling approach also emphasizes flexibility to be able to address different scenarios that result from uncertainty in particular aspects of the model, such as effective aquifer thickness. Credibility results from being both comprehensive in evaluating all features that might influence flow and transport as well as being flexible in evaluating aspects that are uncertain.

The modeling approach is tiered to allow phased development of both 2-D and 3-D models. This phased approach generally allows the methodology for simulating flow and transport to be developed and tested in simpler and less computationally intensive 2-D models, and then lessons learned may be applied to the more complex and representative 3-D models. Another aspect of the OU 10-08 approach is that, ultimately, a model will be abstracted from the final 3-D transport model that will be simpler to run but will provide consistent results and will allow easy extension of the 3-D model to include additional sources that may be identified over time. This abstracted model is called a Response Surface Model and was previously demonstrated for a proof-of-principle test (Rood 2005).

The specific steps of the approach are to develop the following:

- 2-D flow model

- $\quad$ 2-D transport model

- 3-D flow model

- 3-D transport model

- $\quad$ Response surface model (to mimic 3-D transport model).

\section{C-1.4 Document Scope}

This appendix presents the development of the 2-D transport model including the boundary conditions, transport properties, and the contaminant sources. The appendix also discusses revisions to the 2-D flow model that occurred as a result of switching to a prescribed flux along the northeast side of the model.

\section{C-1.5 Document Overview}

This appendix contains updated conceptual models for flow and transport (Section C-2). These are followed with updates to the numerical implementation of the 2-D flow model (Section C-3) and development of a 2-D transport model (Section C-4). Included in this transport model development are discussions of how contaminant sources are modeled and the problems that arose in implementing these sources to the numerical transport model. Initial transport simulation results are presented and compared to contour maps of simulated plumes from individual WAG transport models (Section C-5). 


\section{C-2. CONCEPTUAL MODEL}

The conceptual models of flow and transport provide the basis for numerical modeling of two-dimensional transport of contaminants within the area represented by the OU 10-08 model domain. These conceptual models are described in detail in Wood et al. (2005). Subsequent discussions summarize those conceptual models and identify revisions to specific concepts that are based on results from the two-dimensional flow model.

\section{C-2.1 Flow}

The two-dimensional contaminant transport model is derived from an understanding of the field of flow as derived from the numerical flow model. This flow model is based on the conceptual model of flow, including descriptions of the model domain, major geologic units and hydrologic subdomains, distribution of hydraulic properties, and inflows to the system.

\section{C-2.1.1 Operable Unit 10-08 Model Domain}

The study area represented by the OU 10-08 model domain originally encompassed approximately $7,770 \mathrm{~km}^{2}\left(3,000 \mathrm{mi}^{2}\right)$ of the ESRP extending from the Mud Lake area in the northeast to the Great Rift in the southwest. This study area was bounded on the northwest by the mountains and valleys of the Basin and Range Province and on the southeast by a generalized groundwater flowpath defined by regional flow models (Figure C-1). The study area was reduced in size as described in Wood et al. (2005) to eliminate complex hydrologic conditions in the Mud Lake area and to accommodate more precisely defined flow path along the southeast boundary that was based on recent water-level measurements. The area presently represented by the OU 10-08 model domain encompasses approximately $6,480 \mathrm{~km}^{2}\left(2,500 \mathrm{mi}^{2}\right)$.

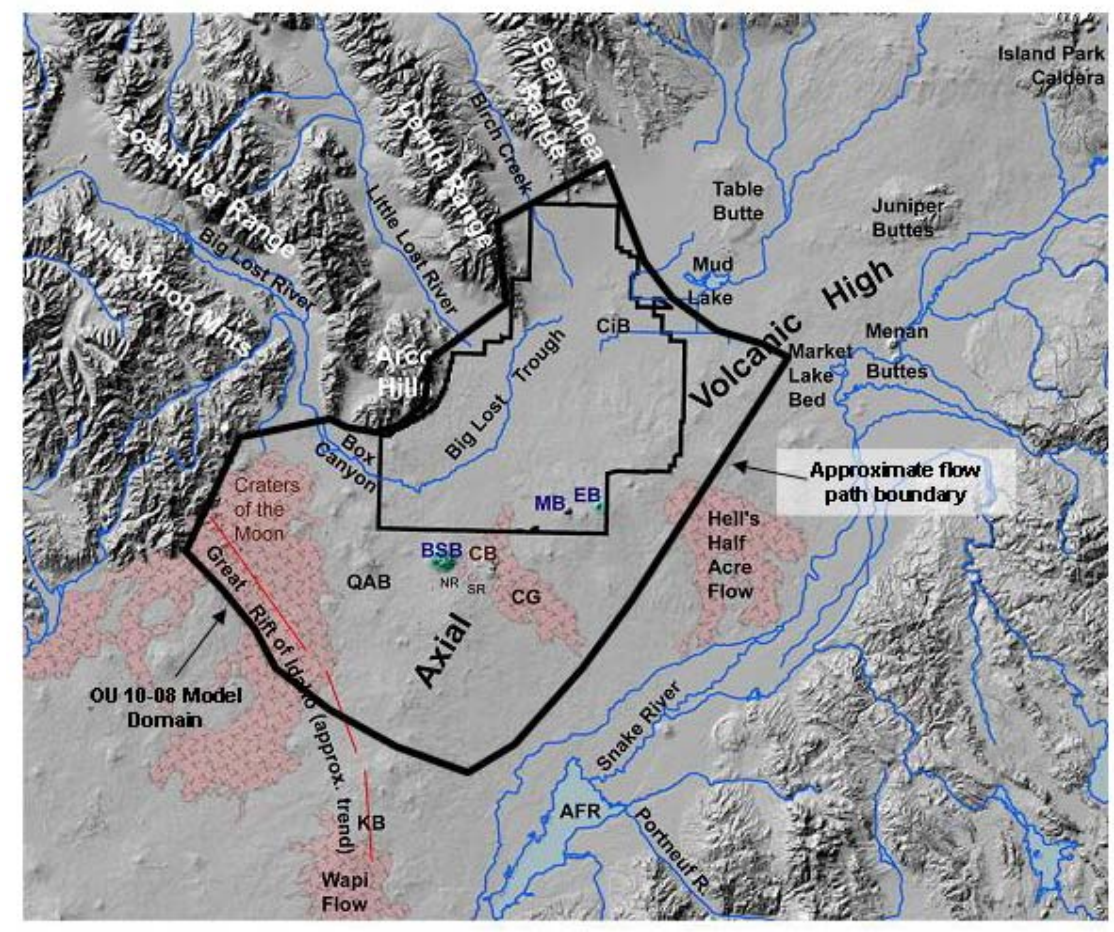

Figure C-1. Operable Unit 10-08 study area regional setting. 
Pertinent hydrologic features of the conceptual model relative to Figure $\mathrm{C}-1$ are inflow of regional groundwater into the study domain across the northeast boundary and outflow across the southwestern boundary. Also, tributary river drainages along the northwest boundary contribute inflow to the domain as underflow. Areal recharge from infiltrating precipitation that falls directly on the plain and the infiltration of seepage losses from surface water in the ephemeral Big Lost River are two additional sources of water entering the model domain.

Geologic and hydrologic data, including well geophysical and temperature logs and surface geophysical data, were used to develop two aquifer thickness scenarios (Figure C-2) for the OU 10-08 model domain. Both utilize limited direct evidence of the aquifer base from eight wells that fully penetrate the aquifer in the south-central part of the study area. The thick scenario also utilizes electrical resistivity and water-temperature at the top of the aquifer to extrapolate thicknesses to the northeast and southwest. Colder water temperatures are correlated with assumed thicker aquifer sections. The thin scenario does not use water temperature data away from areas of direct evidence, but assumes a general tendency for an increased aquifer thickness toward the center of the plain. Additional wells that extend deep into the aquifer have been drilled in recent years; logs of these are being analyzed as to whether they support or counter either of the two thickness scenarios.

\section{C-2.1.2 Major Geologic Units and Hydrologic Subdomains}

The area represented by the OU 10-08 model domain can be divided into five general geologic units. These units consist of active volcanic rift zones, inactive volcanic rift zones, floodplains, sedimentation areas, and volcanic tablelands. Each of these geologic units is further divided into hydrologic subdomains. The lithologic and stratigraphic properties of each of these geologic units and hydrologic subdomains control groundwater flow and contaminant transport. The distribution of these geologic units and hydrologic subdomains in the OU 10-08 study area is shown on Figure C-3, with the OU 10-08 model domain superimposed. For a detailed description of the distribution of these geologic units, refer to Wood et al. (2005). Table C-1 summarizes the five geologic units and 15 hydrologic subdomains identified within the entire study area.

\section{C-2.1.3 Hydraulic Properties}

Aquifer test data from 146 wells were used to estimate the hydraulic conductivity distribution for the SRPA within the area encompassed by the OU 10-08 model domain. The calculated hydraulic conductivity ranged over seven orders of magnitude, with a mean value of $3.1 \times 10^{3} \mathrm{ft} /$ day and a range from $1.6 \times 10^{-2}$ to $1.1 \times 10^{5} \mathrm{ft} / \mathrm{day}$. This range equates to values representative of dense basalts and loess to highly fractured basalts, interbeds, and coarse gravels (Freeze and Cherry 1979). The hydraulic conductivity distribution over the study area is also shown graphically in Figure C-4. For a more detailed description of the distribution of hydraulic properties, see Wood et al. (2005).

Because the tested wells only partially penetrate the active portion of the aquifer, the hydraulic conductivity was calculated assuming that the open interval of the well was the aquifer thickness and that flow in the vicinity of the well is laminar. While these assumptions likely result in conservatively high estimates of the hydraulic conductivity, they allow for testing the various aquifer thickness scenarios using the numerical model. 

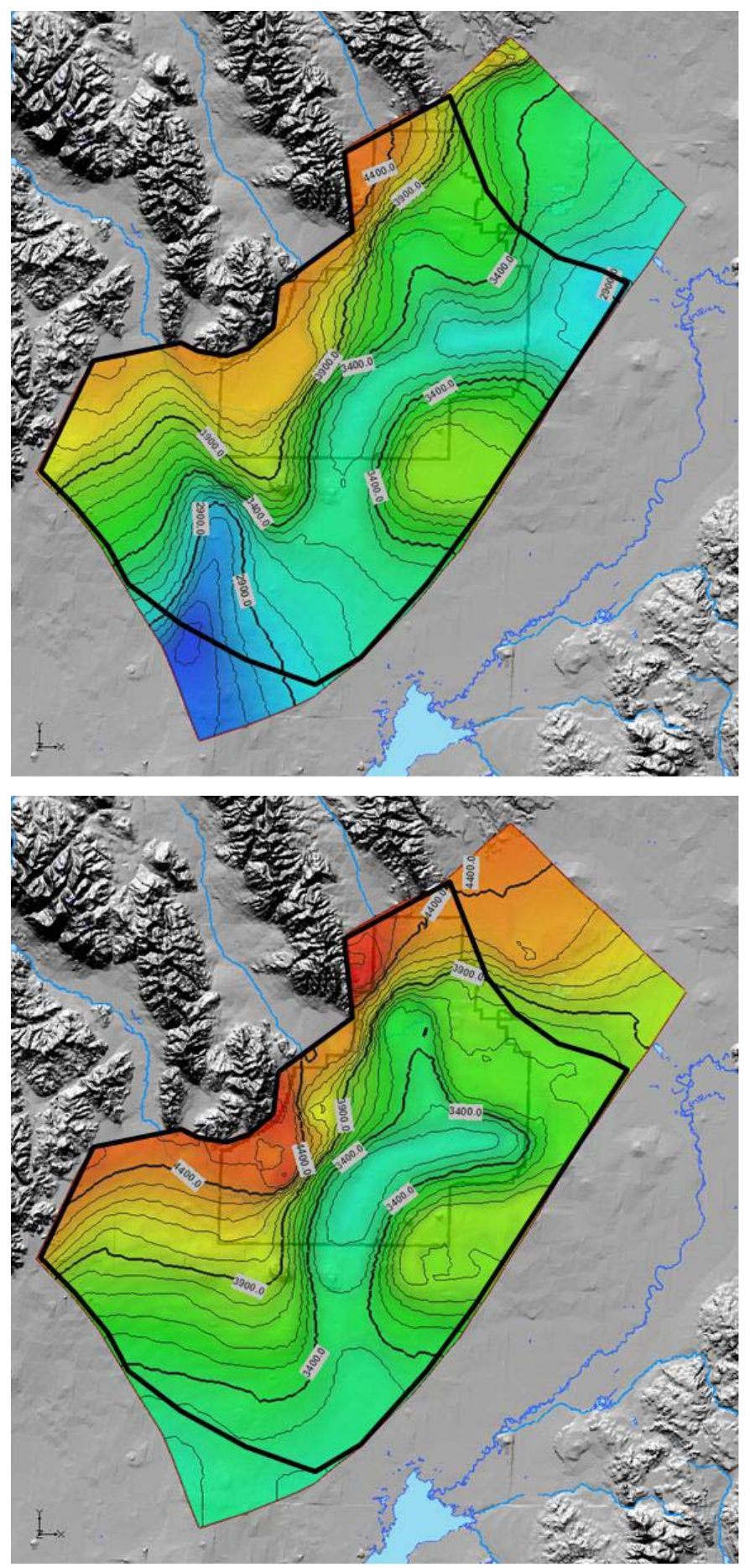

Figure C-2. Aquifer bottom elevation for thick aquifer (top) and thin aquifer (bottom) scenarios. 


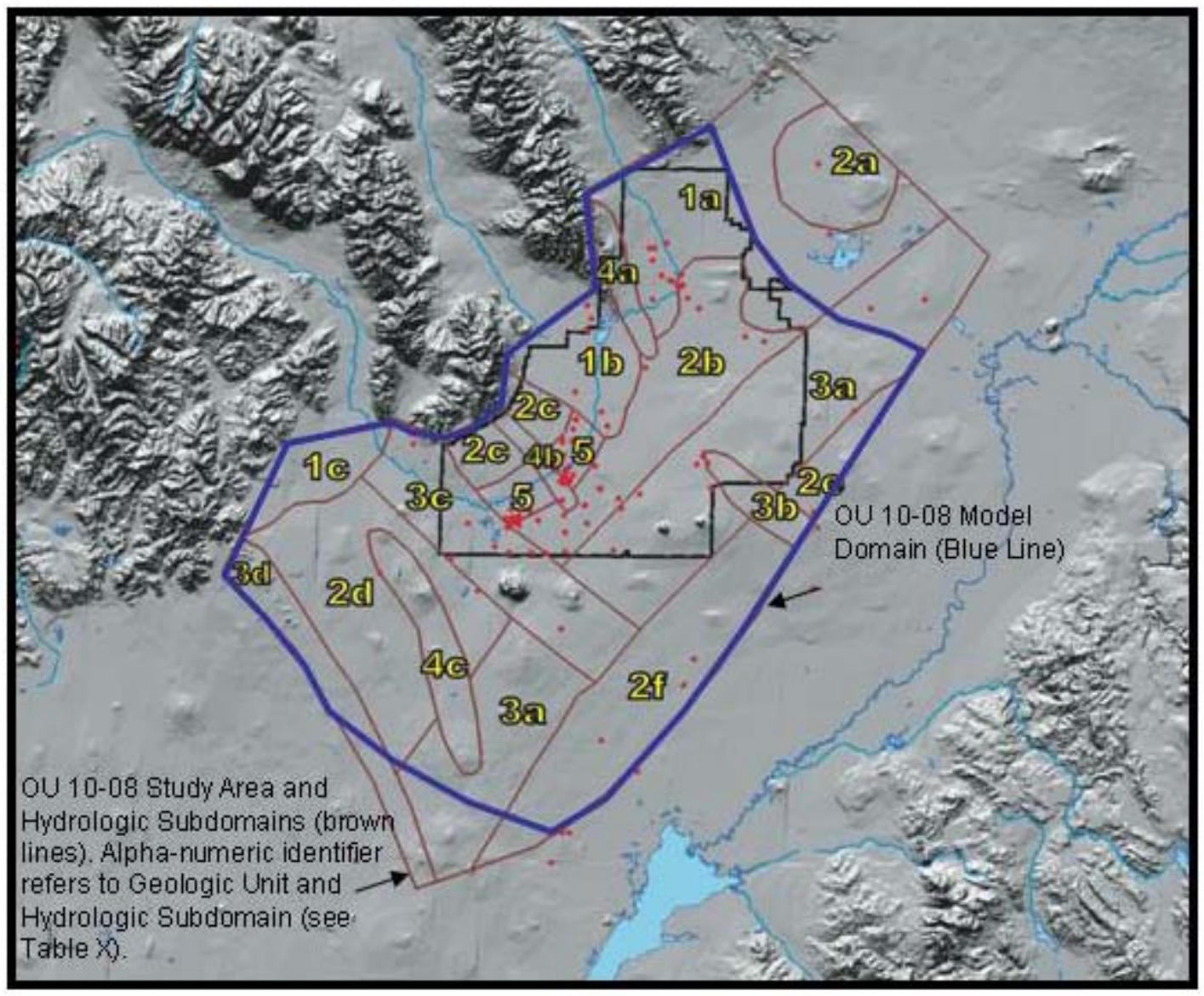

Figure C-3. Distribution of geologic units and hydrologic subdomains in the Operable Unit 10-08 study area. (Revised OU 10-08 model domain superimposed.) 
Table C-1. Geologic units and hydrologic subdomains within the OU 10-08 model domain, including a summary of lithologic and stratigraphic characteristics controlling groundwater flow and contaminant transport.

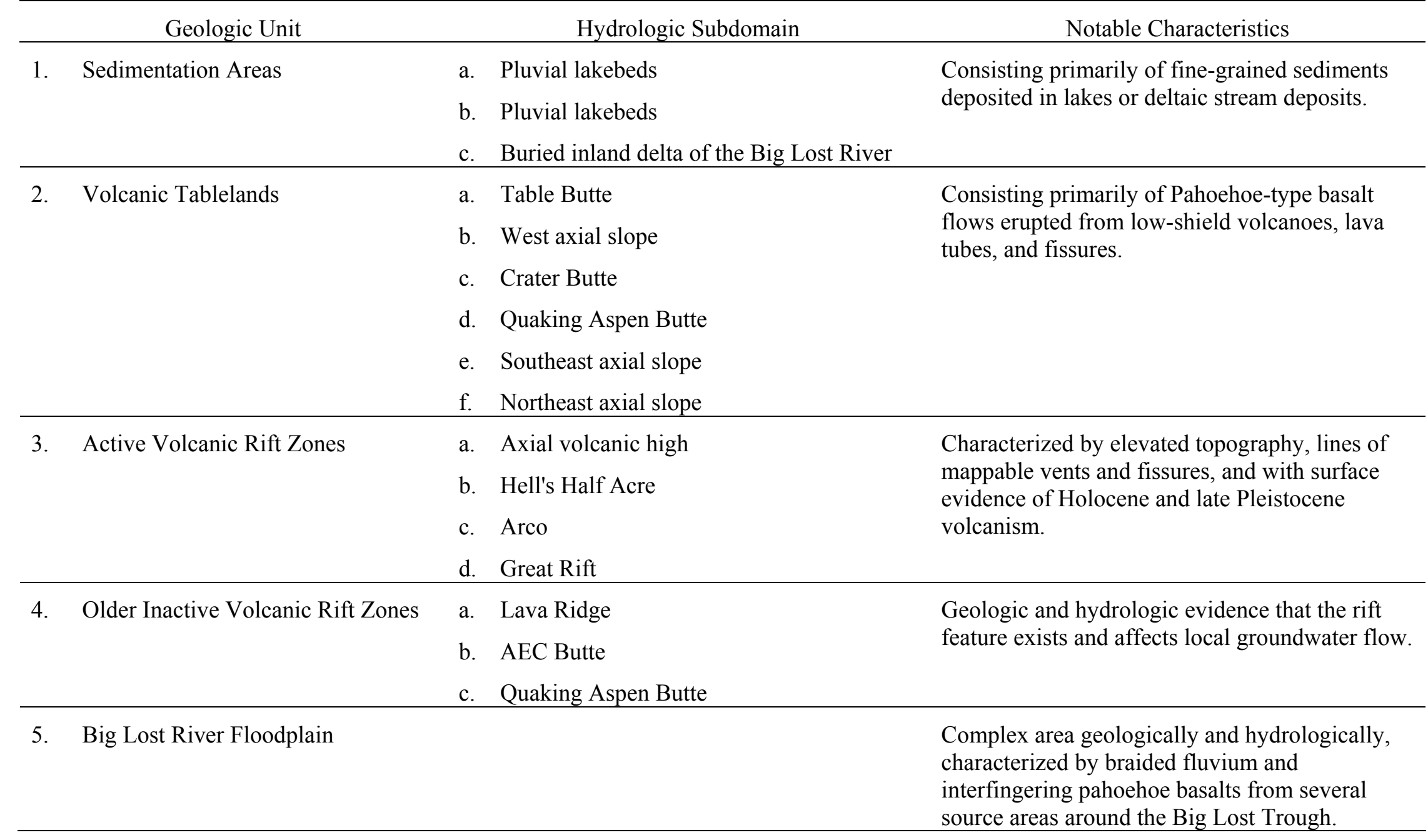




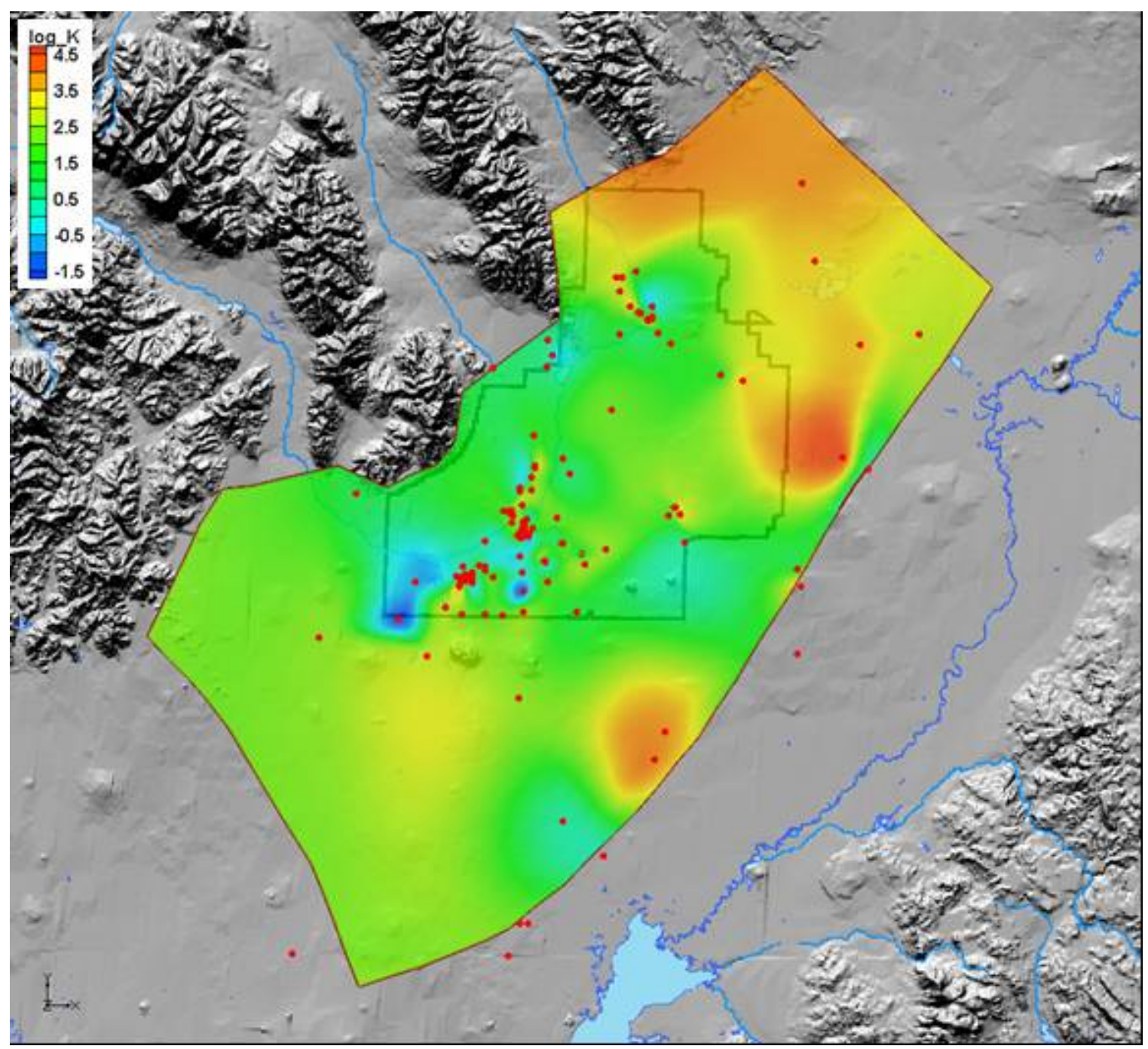

Figure C-4. Hydraulic conductivity distribution of the study area. Red dots indicate well locations with aquifer test data. Shown is the log-transformed hydraulic conductivity in $\mathrm{m} /$ day.

The aquifer test data were also evaluated based on the geology identified in the preceding section. No aquifer test data were available for a number of subdomains, including all of the inactive rift zones and the subdomain at the mouth of the Big Lost River drainage. Most of the aquifer tests were completed in the floodplain of the Big Lost River (Zone 5) and the volcanic tablelands in the central portion of INL (Zone 2b). These zones encompass the major facilities on the INL Site and have been studied in the greatest detail. Table C-2 summarizes the number of aquifer tests conducted in each subdomain and the range in hydraulic conductivity.

\section{C-2.1.4 Inflows and Outflows}

Major inflows to the area represented by the OU 10-08 model domain consist of (a) regional underflow across the northeast model-domain boundary, (b) tributary basin inflows as tributary aquifer underflow and as infiltrating streamflow, and (c) recharge from areal precipitation. These inflows are summarized in Table C-3. Expanded discussion about the characterization of the magnitude and point of recharge to the SRPA is presented in Appendix C-A. 
Table C-2. Subdomain hydraulic conductivity distribution.

\begin{tabular}{|c|c|c|c|c|}
\hline $\begin{array}{c}\text { Subdomain } \\
\text { Number }\end{array}$ & Description & $\begin{array}{c}\text { Number of } \\
\text { Aquifer Tests }\end{array}$ & $\begin{array}{c}\text { Hydraulic Conductivity } \\
\text { Range } \\
\text { (ft/day) }\end{array}$ & $\begin{array}{c}\text { Hydraulic Conductivity } \\
\text { Range } \\
\text { (m/day) }\end{array}$ \\
\hline \multicolumn{5}{|c|}{ Sedimentation Areas } \\
\hline $1 \mathrm{a}$ & Pluvial Lake Bed & 10 & $4.3 \mathrm{E}+00$ to $8.2 \mathrm{E}+02$ & $1.4 \mathrm{E}+01$ to $2.7 \mathrm{E}+03$ \\
\hline $1 b$ & Pluvial Lake Bed & 7 & $1.5 \mathrm{E}-01$ to $1.5 \mathrm{E}+01$ & $5.0 \mathrm{E}-01$ to $4.9 \mathrm{E}+01$ \\
\hline $1 \mathrm{c}$ & Buried Inland Delta of the Big Lost River & 0 & Not applicable & Not applicable \\
\hline \multicolumn{5}{|c|}{ Volcanic Tablelands } \\
\hline $2 \mathrm{a}$ & Table Butte Volcanic Tableland & 1 & $2.7 \mathrm{E}+03$ to $2.7 \mathrm{E}+03$ & $8.8 \mathrm{E}+03$ to $8.8 \mathrm{E}+03$ \\
\hline $2 b$ & West Axial Slope & 37 & $9.1 \mathrm{E}-02$ to $8.9 \mathrm{E}+02$ & $3.0 \mathrm{E}-01$ to $2.9 \mathrm{E}+03$ \\
\hline $2 \mathrm{c}$ & Crater Butte Volcanic Tableland & 1 & $3.3 \mathrm{E}+00$ to $3.3 \mathrm{E}+00$ & $1.1 \mathrm{E}+01$ to $1.1 \mathrm{E}+01$ \\
\hline $2 d$ & Quaking Aspen Butte Volcanic Tableland & 2 & $7.5 \mathrm{E}+01$ to $2.1 \mathrm{E}+02$ & $2.5 \mathrm{E}+02$ to $6.8 \mathrm{E}+02$ \\
\hline $2 \mathrm{e}$ & Northeast Axial Slope & 4 & $7.9 \mathrm{E}-01$ to $1.1 \mathrm{E}+04$ & $2.6 \mathrm{E}+00$ to $3.5 \mathrm{E}+04$ \\
\hline $2 \mathrm{f}$ & Southeast Axial Slope & 8 & $5.2 \mathrm{E}-01$ to $3.9 \mathrm{E}+00$ & $1.7 \mathrm{E}+00$ to $1.3 \mathrm{E}+01$ \\
\hline \multicolumn{5}{|c|}{ Active Rift Zones } \\
\hline $3 a$ & Axial Volcanic High & 9 & $9.8 \mathrm{E}-01$ to $1.1 \mathrm{E}+03$ & $3.2 \mathrm{E}+00$ to $3.6 \mathrm{E}+03$ \\
\hline $3 b$ & Hell's Half Acre Rift & 1 & $5.2 \mathrm{E}+02$ to $5.2 \mathrm{E}+02$ & $1.7 \mathrm{E}+03$ to $1.7 \mathrm{E}+03$ \\
\hline $3 \mathrm{c}$ & Arco Rift & 10 & $7.3 \mathrm{E}-03$ to $1.8 \mathrm{E}+03$ & $2.4 \mathrm{E}-02$ to $5.9 \mathrm{E}+03$ \\
\hline $3 d$ & Great Rift of Idaho & 1 & $4.8 \mathrm{E}+01$ to $4.8 \mathrm{E}+01$ & $1.6 \mathrm{E}+02$ to $1.6 \mathrm{E}+02$ \\
\hline \multicolumn{5}{|c|}{ Inactive Rift Zones } \\
\hline $4 a$ & Lava Ridge & 0 & Not applicable & Not applicable \\
\hline $4 \mathrm{~b}$ & AEC Butte Rift & 0 & Not applicable & Not applicable \\
\hline $4 c$ & Quaking Aspen Butte Rift & 0 & Not applicable & Not applicable \\
\hline \multicolumn{5}{|c|}{ Big Lost River Floodplain } \\
\hline 5 & Big Lost River Floodplain & 55 & $1.5 \mathrm{E}-02$ to $2.7 \mathrm{E}+03$ & $5.0 \mathrm{E}-02$ to $8.7 \mathrm{E}+03$ \\
\hline
\end{tabular}


Table C-3. Inflows to the area represented by the Operable Unit 10-08 model domain.

\begin{tabular}{|c|c|c|c|c|c|}
\hline \multicolumn{3}{|c|}{ Inflow } & Point of Recharge & Rate of Inflow & Source of Information \\
\hline \multicolumn{3}{|c|}{ Regional underflow across the northeast boundary } & Flow distributed horizontally across & $833,000 \mathrm{acre}-\mathrm{ft} /$ year & Spinazola five-layer \\
\hline \multirow[t]{4}{*}{$\begin{array}{l}\text { Tributary } \\
\text { underflow }\end{array}$} & Birch Creek & Underflow & $\begin{array}{l}\text { Top of aquifer in transition zone at } \\
\text { mouth of Birch Creek Valley }\end{array}$ & $\begin{array}{l}78,200 \text { acre-ft/year } \\
\left(264,300 \mathrm{~m}^{3} / \text { day }\right)\end{array}$ & Kjelstrom (1986) \\
\hline & Little Lost River & Underflow & $\begin{array}{l}\text { Top of aquifer in transition zone at } \\
\text { mouth of Little Lost River Valley }\end{array}$ & $\begin{array}{l}155,000 \mathrm{acre}-\mathrm{ft} / \mathrm{year} \\
\left(523,900 \mathrm{~m}^{3} / \text { day }\right)\end{array}$ & Kjelstrom (1986) \\
\hline & \multirow[t]{2}{*}{ Big Lost River } & Underflow & $\begin{array}{l}\text { Top of SRPA in transition zone at } \\
\text { mouth of Big Lost River Valley }\end{array}$ & $\begin{array}{l}295,400 \text { acre-ft/year } \\
\left(998,400 \mathrm{~m}^{3} / \text { day }\right)\end{array}$ & Kjelstrom (1986) \\
\hline & & Streamflow & $\begin{array}{l}\text { Top of SRPA in stream reach from } \\
\text { Arco to the Big Lost River playa }\end{array}$ & $\begin{array}{l}70,200 \text { acre-ft/year } \\
\left(237,200 \mathrm{~m}^{3} / \text { day }\right)\end{array}$ & $\begin{array}{l}\text { USGS stream-gaging } \\
\text { data, 1985-2003 } \\
\text { (USGS 2007) }\end{array}$ \\
\hline \multicolumn{3}{|c|}{ Direct precipitation } & $\begin{array}{l}\text { Distributed uniformly over the model } \\
\text { domain and applied to the top of the } \\
\text { aquifer }\end{array}$ & $\begin{array}{l}25,600 \text { to } 64,000 \text { acre-ft/year } \\
\left(86,500 \text { to } 216,300 \mathrm{~m}^{3} / \text { day }\right)- \\
\text { average is } 44,800 \text { acre-ft/year } \\
\left(151,400 \mathrm{~m}^{3} / \text { day }\right)\end{array}$ & Cecil et al. (1992) \\
\hline
\end{tabular}




\section{C-2.1.4.1 Regional Underflow Across the Northeastern Model Domain Boundary.}

Regional underflow in the Mud Lake area was estimated from a numerical modeling study of the Mud Lake area (Spinazola 1994) to be approximately 800,000 acre-ft/year (Wood et al. 2005). The magnitude of this regional underflow estimate was consistent with underflow estimates derived from the USGS Regional Aquifer System Analysis model (Garabedian 1992) and the Idaho Water Resources Research Institute model (Cosgrove et al. 2006).

The two-dimensional flow model constructed in 2005 (Wood et al. 2005) represented regional underflow from the northeast using a specified-head boundary. However, the flows simulated across this specified-head boundary were significantly smaller than those simulated by the Spinazola, Garabedian, and Idaho Water Resources Research Institute models. Further analysis of the Spinazola model results provided a refined underflow estimate of 833,000 acre-ft/year across the northeast boundary of the OU 10-08 model domain (Table C-3). This refined underflow estimate was required to support revision of the modeled boundary as a specified flux boundary to provide a better representation of transmissivity, groundwater flow, and contaminant transport throughout the model domain.

The two OU 10-08 aquifer thickness scenarios indicate that the SRPA thickens to the southeast in the vicinity of the northeast model-domain boundary. The rate of underflow is considered to increase with increasing thickness. This increase in underflow was estimated by apportioning the total estimated underflow along the northeast boundary with respect to thickness and percentage of flow with depth, as determined from the Spinazola numerical model (Table C-4). For a detailed discussion of the method by which flow was apportioned, refer to Appendix C-A. The estimated distribution of flow across the northeast boundary of the area represented by the OU 10-08 model domain is being used in the two-dimensional transport model.

The Spinazola numerical model represented the SRPA with five layers and overlapped the OU 10-08 model domain. Based on Spinazola's modeling results, approximately $70 \%$ of the groundwater flow may occur in the upper $150 \mathrm{ft}$ of the aquifer in the area of the northeast boundary of the OU 10-08 model domain. These flows likely do not fluctuate greatly and are considered to be reasonably stable over time.

C-2.1.4.2 Tributary Inflows. Tributary inflows to the area represented by the OU 10-08 model domain and used in the two-dimensional transport model were derived from the Birch Creek, Little Lost River, and Big Lost River drainage basins. These inflows occur in the form of underflow from tributary basin aquifers and from infiltration of streamflows.

Underflows into the SRPA from the Birch Creek and Little Lost River basins are estimated from Kjelstrom (1986) to be 78,200 acre-ft/year $\left(264,300 \mathrm{~m}^{3} /\right.$ day) and 155,000 acre-ft/year $\left(523,900 \mathrm{~m}^{3} /\right.$ day), respectively. These underflows represent the basin contributions to tributary basin inflow, and include infiltrating surface-water flows near the mouths of the drainage basins. Underflow from the Big Lost River basin is estimated to be 295,400 acre-ft/year $\left(998,400 \mathrm{~m}^{3} /\right.$ day). Underflows from all three tributary basins are considered to be reasonably stable over time, based on long-term stream-gaging data.

In contrast, streamflows from the Big Lost River are highly variable. These flows are transported along the Big Lost River, infiltrating along the river channel, in the INL spreading areas, and the Big Lost River Sinks. These episodic flows provide an average recharge to the top of the SRPA of 70,200 acre-ft/year $\left(237,200 \mathrm{~m}^{3} /\right.$ day $)$. For an expanded discussion of this episodic source of recharge, see Appendix C-A. 
Table C-4. Estimated underflow across the northeast model-domain boundary apportioned by segments to represent increasing aquifer thickness to the southeast.

\begin{tabular}{cccc}
\hline $\begin{array}{c}\text { Northeast Model } \\
\text { Domain Boundary } \\
\text { Segment }\end{array}$ & $\begin{array}{c}\text { Segment Start Distance } \\
\text { from the North Corner of } \\
\text { the Model Domain } \\
(\mathrm{m})\end{array}$ & $\begin{array}{c}\text { Segment End Distance } \\
\text { from the North Corner of } \\
\text { the Model Domain } \\
(\mathrm{m})\end{array}$ & $\begin{array}{c}\text { Estimated Aquifer } \\
\text { Underflow for } \\
\text { the Segment } \\
\text { (acre-ft/year) }\end{array}$ \\
\hline 1 & 0 & 3000 & 20,500 \\
2 & 3000 & 8000 & 37,700 \\
3 & 8000 & 12000 & 45,700 \\
4 & 12000 & 15000 & 39,300 \\
5 & 15000 & 17000 & 29,100 \\
6 & 17000 & 20000 & 44,600 \\
7 & 20000 & 20500 & 7,100 \\
8 & 20500 & 23000 & 36,700 \\
9 & 23000 & 28000 & 69,900 \\
10 & 28000 & 29000 & 15,400 \\
11 & 29000 & 30000 & 15,900 \\
12 & 30000 & 34000 & 66,400 \\
13 & 34000 & 35000 & 17,300 \\
14 & 35000 & 37500 & 45,000 \\
15 & 37500 & 38000 & 9,200 \\
16 & 38000 & 46000 & 163,400 \\
17 & 46000 & 47000 & 20,900 \\
18 & 47000 & 54373 & 148,900 \\
& & & 833,000 \\
\hline
\end{tabular}

C-2.1.4.3 Recharge from Precipitation. The average precipitation over the OU 10-08 model domain is 8 in. $(20 \mathrm{~cm})$ per year. Recharge from precipitation within this area has been estimated to range from 2 to $5 \%$ of precipitation. Recharge over the $2,500 \mathrm{mi}^{2}$ model domain is estimated to be 44,800 acre-ft/year $\left(151,400 \mathrm{~m}^{3} /\right.$ day $)$ (Table C-3).

Regional groundwater studies (Garabedian 1992; Contor 2004) developed regional distributions of recharge from precipitation based on the amount of precipitation, soil thickness, and infiltration capacity of the soil cover. Based on sensitivity tests using the OU 10-08 two-dimensional flow model, variable recharge with respect to these factors did not significantly affect groundwater flow (Wood et al. 2005).

C-2.1.4.4 Regional Underflow Out of the OU 10-08 Model Domain. No direct measurement is available of underflow out the area represented by the OU 10-08 model domain. However, this outflow can be estimated from the accumulated inflows to the area. The estimated outflow across the southwestern boundary of the model domain is estimated to be approximately 1,476,600 acre- $\mathrm{ft}$ /year $\left(4,990,600 \mathrm{~m}^{3} /\right.$ day $)$.

\section{C-2.1.4.5 Discussion of Steady-State Flow Versus Transient Flow in Context of}

Transport. Based on the conceptual model of groundwater flow within the OU 10-08 model domain, inflows from regional underflow, tributary underflow, and recharge from precipitation are stable and can 
be represented using long-term averages. However, recharge from the Big Lost River is highly episodic, with long periods of no recharge interspersed with periods of rapid local recharge along the stream channel, to the INL spreading areas, and to the Big Lost River Sinks. This episodic recharge, while only a small percentage of the total water budget, occurs in the vicinity of known INL contaminant sources and has been considered as causing transient changes in flow directions in these regions (Goode and Konikow 1990). These transient flow directions likely affect contaminant transport and therefore should be considered when simulating transport.

\section{C-2.2 Transport}

\section{C-2.2.1 Processes that Control Geochemical Evolution and Transport in the Subsurface}

This section presents a general discussion of the major processes that control the transport and fate of contaminants in the subsurface at the INL Site. Many of these processes are also important in the natural geochemical evolution of groundwater. These processes include advection and dispersion; dissolution, precipitation, and co-precipitation; sorption; matrix diffusion; transformation; and facilitated transport.

C-2.2.1.1 Advection and Dispersion. Advection and dispersion are primary transport mechanisms for aqueous wastes that were discharged directly into the SRPA. Advection is the movement of contaminants dissolved in water caused by the bulk movement of that water. Dispersion is the spreading of contaminants in water and is caused by differences in the length of the flow path traversed by different parcels of water (and the contaminants dissolved in that water) and differences in flow velocity in different flow paths. Dispersion mixes a plume of contaminated groundwater or vadose zone water with surrounding uncontaminated water. Additionally, it acts to reduce maximum contaminant concentrations, while concurrently increasing the volume of contaminated water.

C-2.2.1.2 Dissolution and Precipitation. Dissolution refers to the conversion of a solid phase into dissolved constituents, while precipitation is the formation of a solid phase from dissolved constituents. Dissolution is important in natural geochemical evolution because it is a major mechanism in water-rock interaction. Dissolution of mineral or amorphous phases by groundwater and vadose zone water is the source of most solutes in these waters.

With regard to waste disposal and contaminant transport, dissolution is probably most important in disposal facilities as a process that converts buried waste into dissolved contaminants. It could also be important if soluble solids were disposed via injection wells or if aggressive solutions were injected into the subsurface. Situations in which dissolution would be important include leaching of soluble salts in buried waste or corrosion of metals in buried waste. Dissolution may also be important if contaminants are precipitated, co-precipitated, or sorbed to minerals that are stable under one set of geochemical conditions and are subsequently released due to changes in geochemical conditions, such as change in $\mathrm{pH}$ or redox state. An example of this is dissolution of pre-existing minerals following disposal of acidic waste in the subsurface.

C-2.2.1.3 Sorption/Desorption. Sorption is the partitioning of dissolved constituents to solids. Conversely, desorption is the release of materials from solids to the aqueous phase. Sorption includes adsorption, in which the partitioning is a surface phenomenon, and absorption in which partitioning occurs in the interior of the solid. A common example of adsorption is cation exchange, in which positively charged ions are attracted to negatively charged mineral surfaces by electrostatic forces. An example of absorption is the incorporation of potassium ions in interlayer positions of illite, a clay mineral. In this discussion, sorption is used as a global term that includes both adsorption and absorption. 
Sorption is important primarily because it can drastically retard the movement of ionic contaminants. Cations are generally retarded much more than anions because most common minerals have negatively charged surfaces, which attract positively charged ions. Ions that are sorbed move more slowly than water is moving through the medium. The ratio of the rate of water velocity to contaminant velocity is the retardation coefficient, $R$. An unretarded solute, which moves at the same velocity as water, has a retardation coefficient of 1 . Retarded solutes, which move slower than water, have retardation coefficients greater than 1 . Some strongly sorbed contaminants have retardation coefficients on the order of 10,000 and are essentially immobile. Most contaminants have much lower retardation coefficients, and although they move more slowly than water, they are not immobile.

Sorption of contaminants has several effects. First, as previously discussed, it retards the migration rate. Second, it reduces concentrations in water. Third, it increases concentrations in the sorbed phase.

Desorption may also be important in the generation of dissolved contaminants from buried waste, sediments in percolation ponds, or from contaminated soils. If uncontaminated water comes in contact with these materials, desorption transfers contaminant mass from the waste into water.

Sorption is particularly important for attenuating radioactive contaminants. Retardation due to sorption slows the velocity of contaminant migration and increases the length of time needed for a mass of contaminant to migrate a given distance along a flowpath. The longer travel time provides additional time in which radioactive decay reduces concentrations more than it would if the contaminant were not retarded. This effect is more significant as travel times of a retarded radionuclide become longer, relative to the radioactive decay half-life. Hence, longer-lived nuclides must be more strongly sorbed than shorter-lived nuclides for the increased decay due to retarded transport to be significant.

C-2.2.1.4 Matrix Diffusion. Matrix diffusion is a physical-chemical process that substantially affects solute transport and reaction in some environments, which are referred to as dual porosity media. Dual porosity media are systems in which zones with strongly contrasting permeability are adjacent to one another and both zones have significant porosity. Examples of dual porosity media include fluvial aquifers with interbedded sand and silt or clay, fractured clay, and fractured sandstone or shale. In these media, the sand portions (or fractures) are the more permeable zones and the silt, clay, or rock matrices are the porous, less permeable zones. At the INL Site, fractured vesicular basalt and interflow zones have these characteristics. In dual porosity media, water moves much faster in the more permeable zones than in the less permeable zones. Therefore, solute concentrations can change much more rapidly in the more permeable zones than in the less permeable zones. Differences in solute concentrations cause diffusion to transport mass from high-concentration zones to low-concentration zones. This process attenuates the migration of sharp solute breakthrough fronts and causes extensive tailing during elution curves. This phenomenon is a major transport process in some environments, especially porous rocks, but has received little attention at INL. The term matrix diffusion refers to the process of diffusion between more permeable zones and the less permeable matrix, and is synonymous with transport in dual porosity media.

C-2.2.1.5 Transformation. Transformation refers to the change in chemical species. It includes both abiotic and biologically mediated processes.

Transformations can be very important relative to the mobility, toxicity, and solubility of contaminants. Common transformations change in speciation due to redox reactions (i.e., Fe-II $\Leftrightarrow \mathrm{Fe}-\mathrm{III}$ or $\mathrm{Cr}-\mathrm{VI} \Leftrightarrow \mathrm{Cr}-\mathrm{III}$ ), and due to change in $\mathrm{pH}$ (i.e., $\mathrm{Pb}$ ). Hexavalent chromium forms oxyanions that are soluble, not significantly retarded, and toxic. Trivalent chromium forms cationic species that are much less mobile, less soluble, and less toxic than hexavalent chromium species. The characteristics of many metals vary with $\mathrm{pH}$ and redox state. Hull and Pace (2000) present activity diagrams for contaminants of potential concern at the Radioactive Waste Management Complex (RWMC). 
Biologically-mediated transformations also are important factors that affect the release of contaminants from waste and subsequently the transport and fate of contaminants in the subsurface. For example, biodegradation of organic matter (i.e., paper, cardboard, and wood containers) in landfills such as the RWMC can generate strongly reducing conditions that are favorable to release of some metals, and the inorganic and organic acids produced by organic matter biodegradation may accelerate corrosion of waste metals and complexation of metals. Biotransformation is important in converting nitrate into innocuous nitrogen. Biotransformation is very important in the fate of organic contaminants in the subsurface. For example, tetrachloroethene (PCE) and trichloroethene (TCE) in the groundwater plume emanating from Technical Support Facility (TSF) - 05 at Test Area North (TAN) are being biotransformed into less chlorinated ethenes and ultimately into innocuous products, such as ethene, chloride, bicarbonate, and water. Biodegradation also commonly degrades contaminants derived from petroleum into innocuous products.

Another transformation that affects contaminants is radioactive decay which reduces the mass and activity of a parent nuclide, at a rate depending on the half-life. Daughter products resulting from this decay may have different half-lives and the relative change in activity likewise depends on those half-lives.

C-2.2.1.6 Facilitated Transport. Facilitated transport can be defined as the movement of a contaminant in the subsurface at a rate that is significantly faster than expected as a result of the contaminant being associated with a mobile phase. In other words, if a contaminant is expected to be substantially retarded by sorption to mineral surfaces in the vadose zone or aquifer, but in fact moves more quickly than predicted, the faster transport is attributed to facilitated transport. The mechanisms responsible for facilitated transport include complex formation and colloids. At INL, actinides form complexes with carbonate species that are common constituents of groundwater in the SRPA, and this complexation can substantially enhance their mobility in the subsurface. Evidence exists that colloid facilitated transport may be important in actinide transport in the subsurface beneath INL but this evidence is not conclusive.

\section{C-2.2.2 Velocities Inferred from Geochemical Studies}

Geochemical studies have provided information about groundwater flow velocities within the area of the OU 10-08 model domain. These studies have included contaminant and environmental tracer monitoring, chlorofluorocarbon (CFC) field studies, Cl-36 arrival time studies, and chemical dis-equilibrium studies. Each is discussed in turn.

\section{C-2.2.2.1 Summary of Selected Contaminant Distributions in INL Groundwater.}

Figure C-5 presents the approximate distribution in 2003 of tritium, sulfate, and nitrate in groundwater at INL. The distribution of these contaminants in groundwater did not change significantly during 2005.

The tritium plume that is above the instrument detection limit of $500 \mathrm{pCi} / \mathrm{L}$ originates from Idaho Nuclear Technology and Engineering Center (INTEC) and Reactor Technology Complex (RTC) and extends south within a mile of INL's southern boundary. This plume included tritium that had been injected to deeper parts of the aquifer through the INTEC injection well and tritium transported to the top of the SRPA with infiltration pond wastewater at both facilities. A separate tritium plume was shown to originate from RWMC. Recent tritium data from the newly constructed Westbay well MIDDLE-2051 indicate that the tritium plume may be extended to the west and that the two separate plumes between INTEC/RTC and RWMC may commingle. 


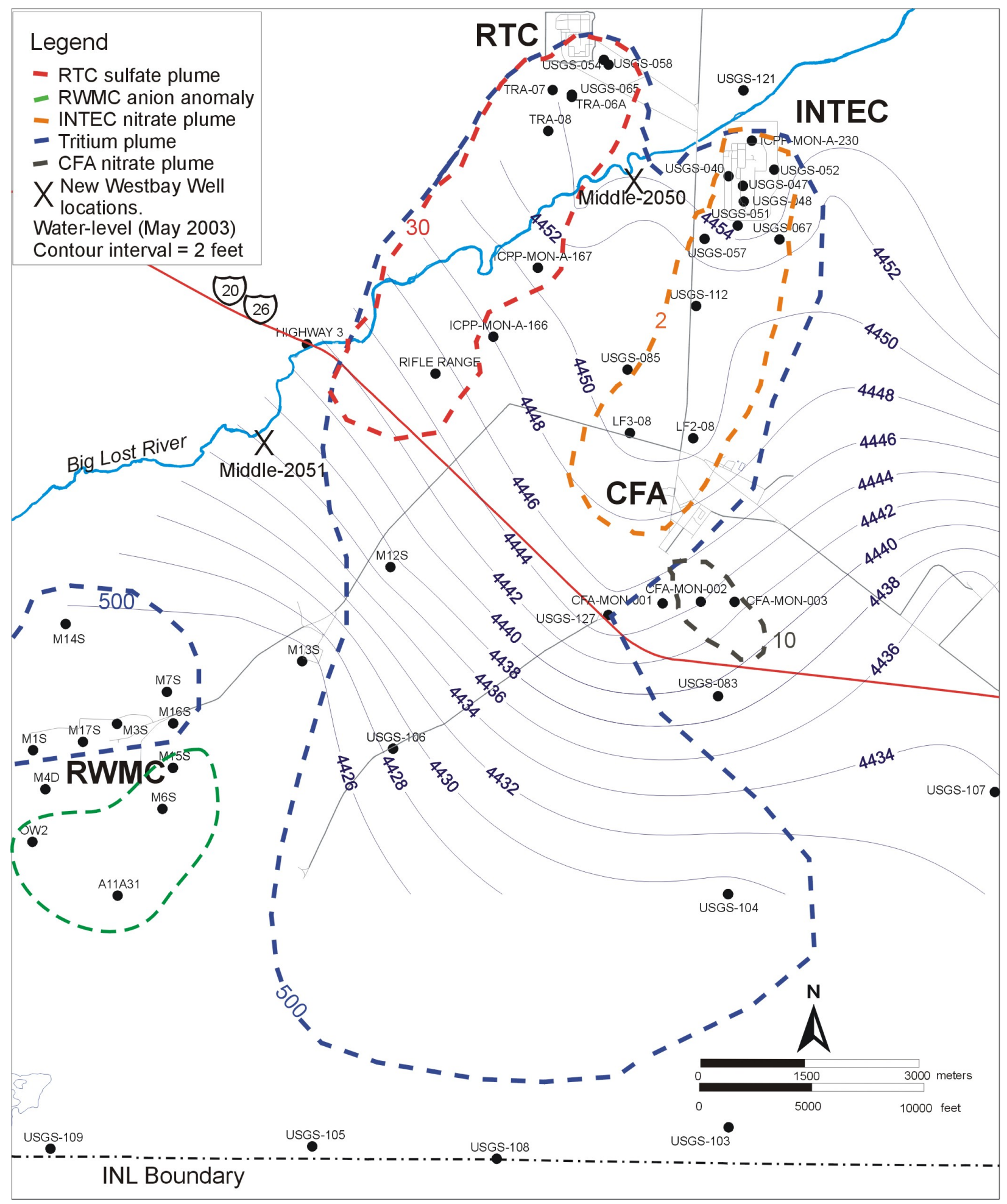

Figure C-5. Approximate distribution in 2003 of selected chemical and radiochemical contaminants in groundwater at Idaho National Laboratory.

A traceable sulfate plume in groundwater with concentrations exceeding $20 \mathrm{mg} / \mathrm{L}$ originated from RTC infiltration ponds and extends south past Highway 20/26. Similarly, a nitrate plume with 
concentrations exceeding $2 \mathrm{mg} / \mathrm{L}$ originated at INTEC and extends south to the Central Facilities Area (CFA).

A plume of dissolved I-129 has been identified in INL groundwater. Preliminary 2005 water-chemistry data indicate that I-129 with concentrations exceeding $10^{-5} \mathrm{pCi} / \mathrm{L}$ extends as far as 12 miles south of the INL boundary. Largest concentrations are in the area south and southwest of INTEC.

C-2.2.2.2 Velocity Estimates from CFC and Other Environmental Tracer Data. The USGS collected and analyzed environmental tracer data to estimate the age and source of the young fraction of groundwater at INL (Busenberg, Plummer, and Bartholomay 2001). Tracers utilized as part of this study consisted of chlorofluorocarbons (CFCs), sulfur hexafluoride, helium, and tritium. Based on this study, USGS concluded that the SRPA is stratified with increasing age with depth. The environmental tracers were present only at or near the water table, except where they were introduced at depth through injection wells.

This environmental tracer study determined that a preferential flowpath extends from the Big Lost Sinks through the central part of INL and east of Big Southern Butte. Flow velocities within this flowpath were estimated from environmental tracer data to be approximately $3 \mathrm{~m} /$ day. Flow velocities across the rest of the INL Site were estimated to be approximately $1 \mathrm{~m} /$ day.

C-2.2.2.3 Chlorine-36. Chlorine-36 was released to the SRPA from the deep injection well at INTEC, with disposals peaking in 1958. Water samples from Wells USGS-11 and -14, both located south of the INL boundary, were analyzed for Cl-36. Peak Cl-36 concentrations were measured in water from these wells in 1983 and 1987, respectively. Based on these peak arrivals, travel times may have been approximately 25 to 29 years, respectively, resulting in a calculated flow velocity of approximately $3 \mathrm{~m} /$ day. However, the first arrival times for $\mathrm{Cl}-36$ were much earlier, indicating a maximum flow velocity of up to $6 \mathrm{~m} /$ day (Cecil et al. 2000).

C-2.2.2.4 Chemical Dis-equilibrium Studies. The earliest estimates of groundwater velocities (average linear velocity) in the INTEC-CFA region were 11 to $25 \mathrm{ft} /$ day (3.4 to $7.6 \mathrm{~m} /$ day)

(Barraclough et al. 1967) and were determined from evaluation of tritium arrival at CFA wells. Wood (1989) estimated velocities for the larger area from INTEC to RWMC as $5 \mathrm{ft} /$ day $(1.5 \mathrm{~m} /$ day) assuming tritium in the aquifer at the RWMC was from INTEC. More recently, water samples showing chemical disequilibrium with host rock have been used to evaluate rates of water movement across INL (Luo et al. 2000; Johnson et al. 2000). These studies have provided a refined estimate of velocity distribution. Based on this recent work, groundwater flow path regions have been identified in which velocities appear to be faster compared to the surrounding areas.

One "fast path" region was inferred from these geochemical data as originating at the mouth of the Little Lost River and extending south across the southern boundary of the INL Site. Based on isotope and radioisotope data from Wells USGS-124, $-17,-83$, and -107 , the velocity of groundwater within this region may range from approximately 8 to $16 \mathrm{ft} /$ day ( 2.6 to $4.8 \mathrm{~m} /$ day).

Groundwater flow velocities in regions to the east and west of the "fast path" may be substantially slower. Based on isotope and radioisotope data in these regions, calculated ages of water are six to nine times older than ages of water within the central part of the fast path. The apparent flow velocities in these slower regions range from 1 to $1.3 \mathrm{ft} / \mathrm{day}(0.3$ to $0.4 \mathrm{~m} /$ day $)$. These calculated ages and velocities are based on the assumption that all of the equilibration of the water chemistry is a result of in situ digenetic activities, and that no significant upwelling of deep equilibrated waters impacts water chemistry. These 
assumptions are not well founded, and calculated flow velocities in these regions are severely hampered by lack of well control and head data.

Isotope and radioisotope data also have been used to infer groundwater flow velocity on the eastern side of INL. The estimated flow velocity in this region is calculated to be approximately $8 \mathrm{ft} /$ day $(2.6 \mathrm{~m} /$ day $)$. To the east of TAN, calculated velocities are larger, $28 \mathrm{ft} /$ day $(8.5 \mathrm{~m} /$ day $)$. However, this estimate is problematic because of likely influence of recent water from the Birch Creek drainage short-circuiting the system and impacting the geochemical signal. This is further exacerbated by local interbeds that intersect the water table in this area enhancing the impact of recent water.

While the recent estimates give more refined estimates spatially, they are still generally within the original estimates of Barraclough et al. (1967).

C-2.2.2.5 Limitations of Vertically Averaged Model. The basalts and sediments of the ESRP form an extremely complex aquifer that is characterized by large ranges in horizontal and vertical hydraulic properties, with three-dimensional groundwater flow and contaminant transport strongly controlled by the anisotropy of the layered system. Contaminant transport also is controlled by the point of contaminant entry into the aquifer. In some cases, contaminants were directly injected deep within the aquifer. In other cases, they were transported through a thick vadose zone to within the very upper parts of the aquifer.

Because of the anisotropy of flow and different points of contaminant entry, the flow and transport system is strongly three-dimensional. A two-dimensional model cannot adequately represent this highly layered, heterogeneous flow system for the following reasons:

- $\quad$ The two-dimensional model averages hydraulic properties across the entire active aquifer thickness and cannot represent anisotropy derived from the complex layered architecture.

- The two-dimensional model averages contaminant concentrations across the entire active aquifer thickness and cannot represent the observed vertical contaminant distribution or differentiate between contaminant distributions occurring in response to deep injection and those occurring in response to entry at the top of the SRPA.

The principle objective of the OU 10-08 two-dimensional transport model was to provide an intermediate step in construction of a fully three-dimensional model of flow and transport. This intermediate step was taken to develop a mechanism for incorporation of contaminant source terms from individual WAGs. 


\section{C-3. NUMERICAL IMPLEMENTATION}

The implementation of the conceptual model with numerical modeling codes includes both a flow model and a transport model. The flow model solves the flow field, and the velocity field output from the flow model is used as input to the transport model. This report only considers a two-dimensional representation of the system for flow and transport.

This section consists of two principle topics. The first topic is updates to the flow model that was originally presented in Wood et al. (2005). This is followed by the development of the two-dimensional transport model.

\section{C-3.1 Two-Dimensional Flow Model Updates}

The OU 10-08 model domain was modified and documented in the OU 10-08 Summary Report on the Subregional-scale Two-dimensional Aquifer Model (Wood et al. 2005). The summary report contains discussion of how the model domain was revised on the southeast and northeast boundaries. The southeastern boundary was slightly revised to reflect more recent analysis of flow lines in this portion of the aquifer. The northeast boundary was revised to shorten the extent of the domain to avoid the complex hydrologic nature of the highly irrigated Mud Lake area, which was originally included as part of the model. The OU 10-08 model domain is shown along with individual WAG locations in Figure C-6.

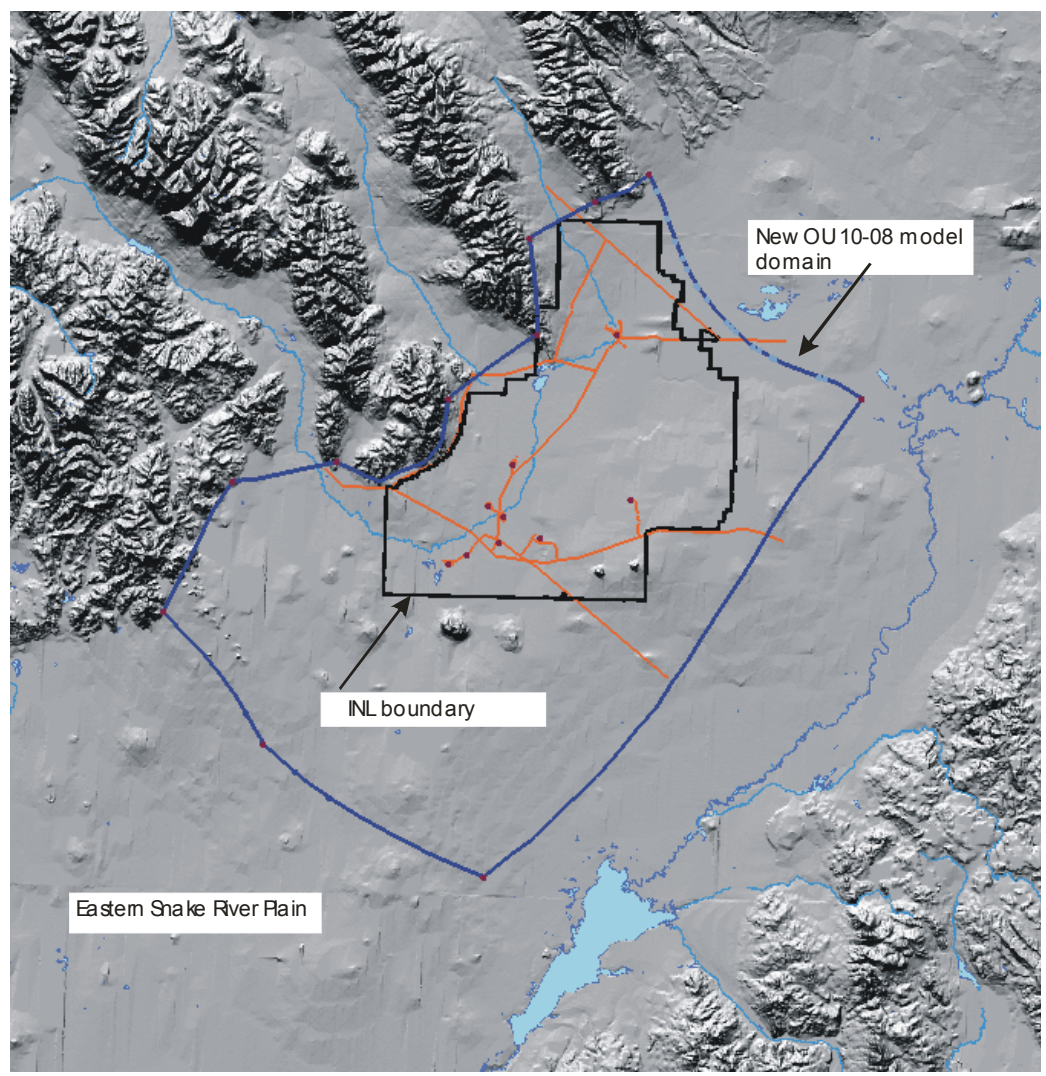

Figure C-6. Domain for Operable Unit 10-08 model. 
This section describes revisions to the 2-D flow model that result primarily from changing the boundary condition along the northeast side of the model domain from a prescribed head to a prescribed flux. This change was made to improve the agreement with the system water balance identified in the conceptual model. Other changes to the flow model caused changes to the simulated groundwater flow field and are also discussed. These changes include constraining hydraulic conductivity for selected pilot points during the automated calibration process and revising the specified heads for the southwestern boundary.

\section{C-3.1.1 Northeast Boundary Specified Flux}

The model presented in Wood et al. (2005) had an overall water budget that was determined to be deficient compared to the regularly accepted quantities as simulated by the USGS regional aquifer model (Garabedian 1992) and the State of Idaho's 2-D aquifer model (Cosgrove et al. 2006). The deficiency, resulting from specified heads at both the northeast and southwest boundaries, was that the water flux through the system was only one-third of the expected amount budget (see Table C-5). As discussed in Section C-2, the northeastern boundary, which had been modified in location and shape, was changed from a specified head boundary condition to a specified flux boundary condition. Water fluxes for the northeast boundary were implemented according to the 18 segments, defined in Table C-4.

Applying these specified fluxes to the northeastern boundary produced an overall water budget that showed better agreement with the conceptual model water budget. The components of both the previous and the revised water budget are listed in Table C-5 and are compared to the conceptual model water budget. With specified fluxes along the northeastern boundary, the simulation results were within $1.8 \%$ of the conceptual model values, and therefore show good agreement with the conceptual understanding of water fluxes through the system.

Table C-5. Water balance components with specified flux and specified head along the northeastern boundary.

\begin{tabular}{|l|c|c|c|}
\hline & $\begin{array}{c}\text { Conceptual Model Fluxes } \\
\left(\mathrm{m}^{3} / \text { day }\right)\end{array}$ & $\begin{array}{c}\text { Specified Flux Along } \\
\text { Northeast Boundary } \\
\left(\mathrm{m}^{3} / \text { day }\right)\end{array}$ & $\begin{array}{c}\text { Specified Head Along } \\
\text { Northeast Boundary } \\
\left(\mathrm{m}^{3} / \text { day }\right)\end{array}$ \\
\hline NE boundary & $2,815,300$ & $2,825,116$ & $1,241,360$ \\
\hline Tributary inflow & $1,786,600$ & $1,686,510$ & $1,686,510$ \\
\hline $\begin{array}{l}\text { Precipitation+ } \\
\text { BLR infiltration }\end{array}$ & 363,742 & 366,385 & 369,449 \\
\hline Total input & $4,965,642$ & $4,878,010$ & $3,297,319$ \\
\hline $\begin{array}{l}\text { Difference from } \\
\text { conceptual model }\end{array}$ & $-1.8 \%$ & $-33.6 \%$ \\
\hline BLR = Big Lost River. & & & \\
\hline
\end{tabular}

\section{C-3.1.2 Constraints on Hydraulic Conductivity for Selected Pilot Points}

Simulation results with the revised specified flux boundary along the northeast side of the domain produced heads that increase dramatically where the tributary underflow from the Big Lost River and Birch Creek entered the model. Previously, the use of specified head conditions at both the northeast and southeast model boundaries yielded heads in these areas that were consistent with field observations. With the additional and correct amount of water entering into the system, simulated heads near these interfaces rose to levels that exceeded field observations and sometimes even land surface in spite of the inverse calibration process. An example of these excessive heads is shown in Figure C-7. 

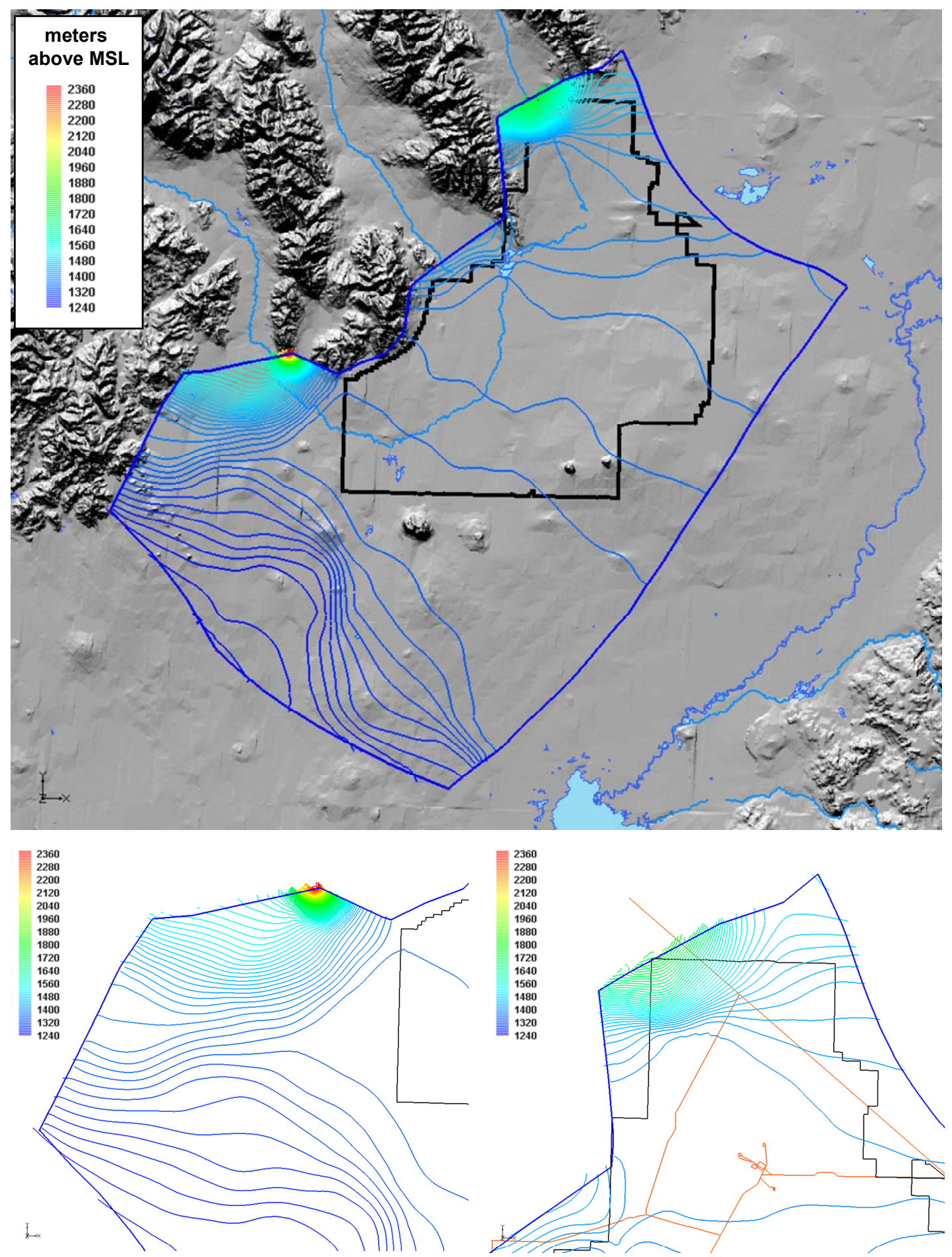

(a)

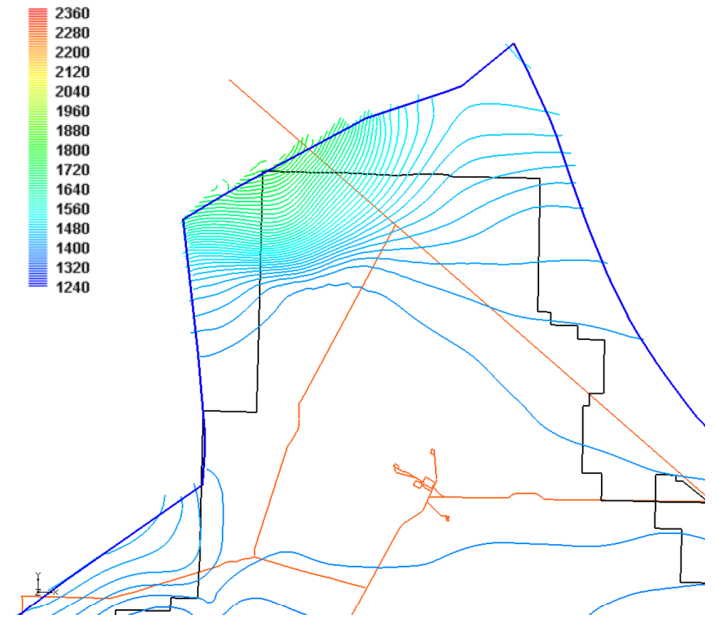

(b)

Figure C-7. Example of head escalation in the thin scenario model resulting from using specified flux boundary conditions along the northeastern boundary; shown are (a) near Big Lost River and (b) near Birch Creek (land surface is represented with color-filled contours and simulated head as colored contour lines with 10-m intervals; elevations are meters above sea level). 
To correct this problem, without modifying tributary underflow fluxes or interface thicknesses, the hydraulic conductivity values were fixed for eight pilot points out of the 275 pilot points used for the 2-D inverse model calibration. Five pilot points near the Big Lost River interface and three pilot points near the Birch Creek interface were constrained (see Figure C-8). These pilot points were effectively removed from the inverse calibration procedure for the 2-D model. By constraining the pilot point values of conductivity to sufficiently high values at these locations, the model was able to accommodate the same tributary underflows without producing excessive hydraulic heads.

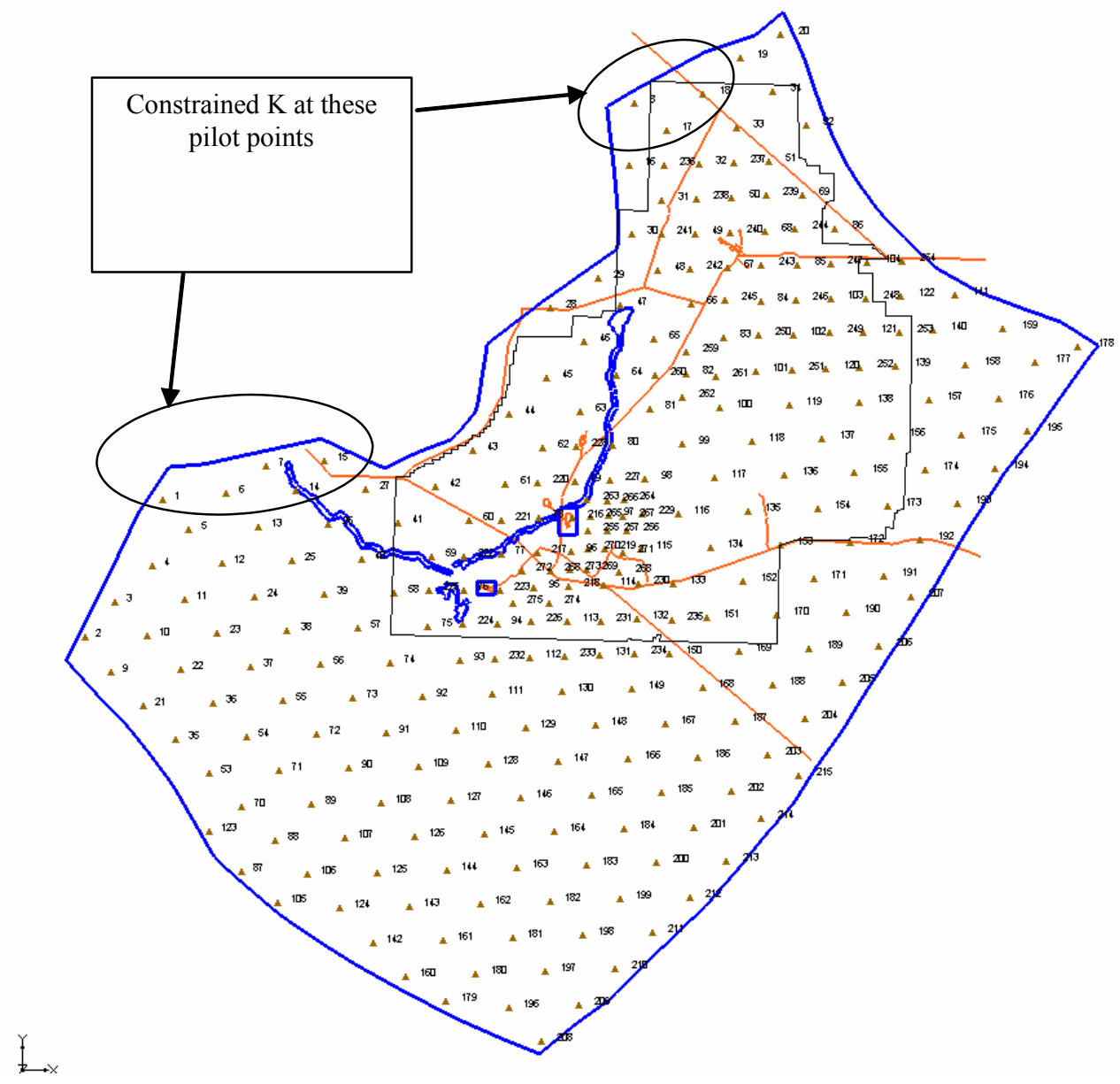

Figure C-8. Location of constrained pilot points near Big Lost River and Birch Creek model interface.

\section{C-3.1.3 Revisions to Southwest Specified Head Boundary}

An analysis of the flow of water across the southwest boundary revealed that flow was not entirely out of the model as indicated by the conceptual flow model. Very steep and concave water table contours at the southwest boundary were caused by a lone observation point (Well Site-2). Using high specified head values at the two endpoint nodes of this boundary arc produced inflow of water entering the model across this boundary (see Figure C-9). The specified head values for this boundary were selected based on interpolated June 2004 water table data. There are few actual observation points near this boundary and the resulting specified heads along this boundary have high uncertainty. By lowering the specified head values at the end points of this boundary, less concave contours were produced and inflow of water was prevented across this boundary (see Figure C-9). 


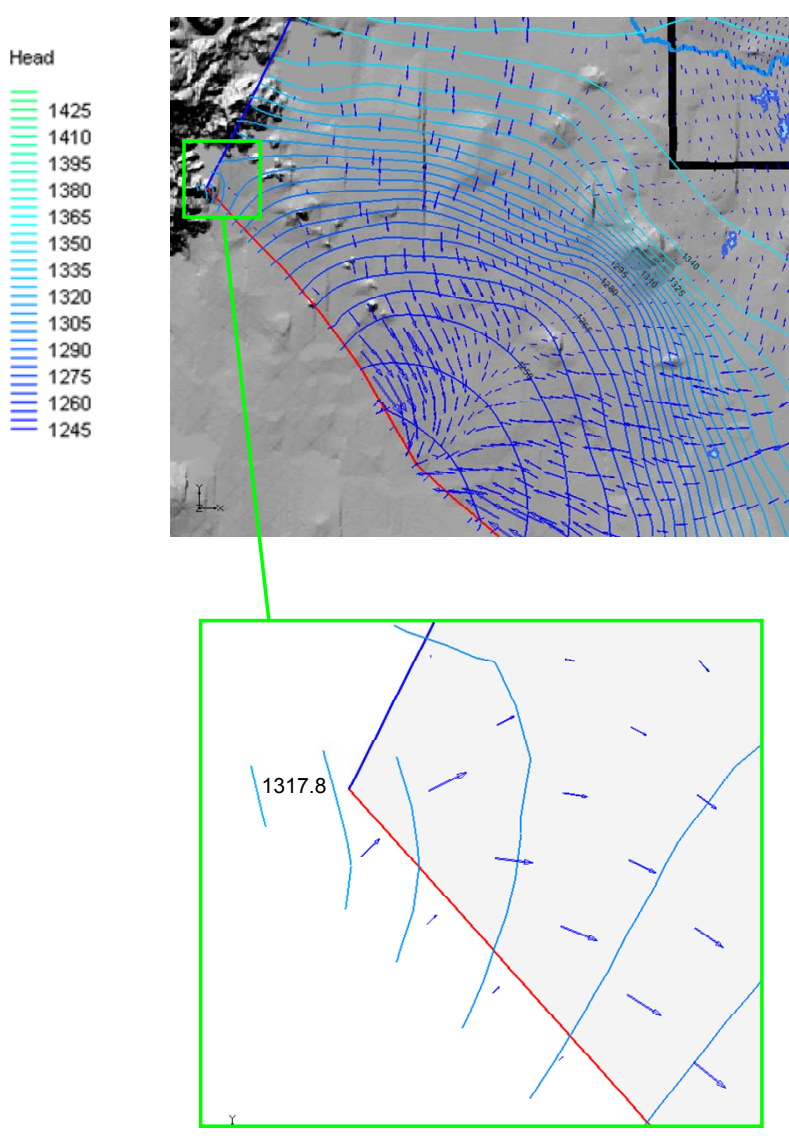

(a)

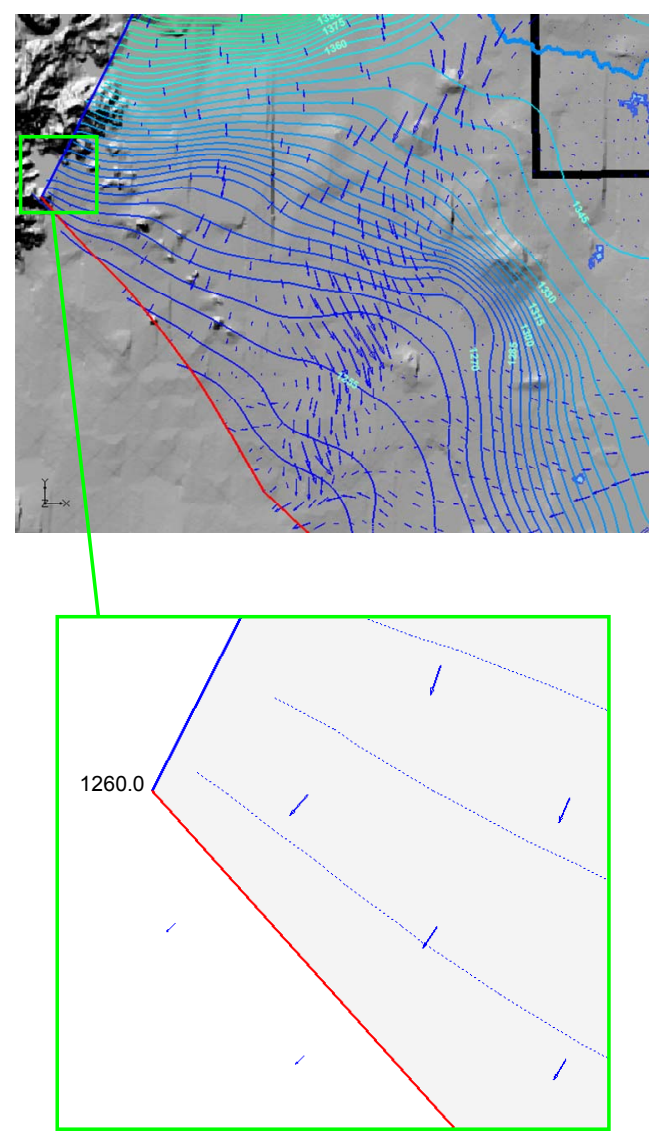

(b)

Figure C-9. Comparison of hydraulic head contours and flux vectors near southwest specified-head corner of 2-D flow model, thin scenario; (a) on left, shows interpolated specified head of $1317.8 \mathrm{~m} \mathrm{msl}$ and resulting flow reversal, (b) on right, shows lowered specified head of $1260.0 \mathrm{~m} \mathrm{msl}$ and resulting flow vectors that are now consistent with conceptual model.

\section{C-3.1.4 Revised Flow Model Results}

By fixing the conductivity values at a total of eight pilot points at the Big Lost River and Birch Creek interfaces, acceptable head matches were obtained. The final simulated hydraulic heads, for both the thin aquifer and thick aquifer scenarios, are shown in Figure C-10. Well Site-2 in the southwest portion of the domain still exerts a large influence on the model results and causes focusing of flow. The lack of well coverage in this region is, in part, the reason for the large influence of the Site- 2 well. The agreement within the primary region of interest, meaning from the RTC and INTEC facilities down to the southern INL boundary, shows good agreement with measured water levels and with generally understood flow directions. This can be seen well in Figure C-11 which shows the same results for the region inside the INL boundaries. Figure $\mathrm{C}-12$ shows the results for the thick scenario for an even smaller region covering the southern INL facilities.

Figure C-13 shows, for both thickness scenarios, estimated hydraulic conductivity distributions that result from constrained pilot point inverse modeling to fit observed heads. The conductivity distributions generally agree with the conceptual model, especially the conductivity contrasts seen in areas of known geologic nonconformities such as the axial volcanic high and Arco volcanic rift zones. 


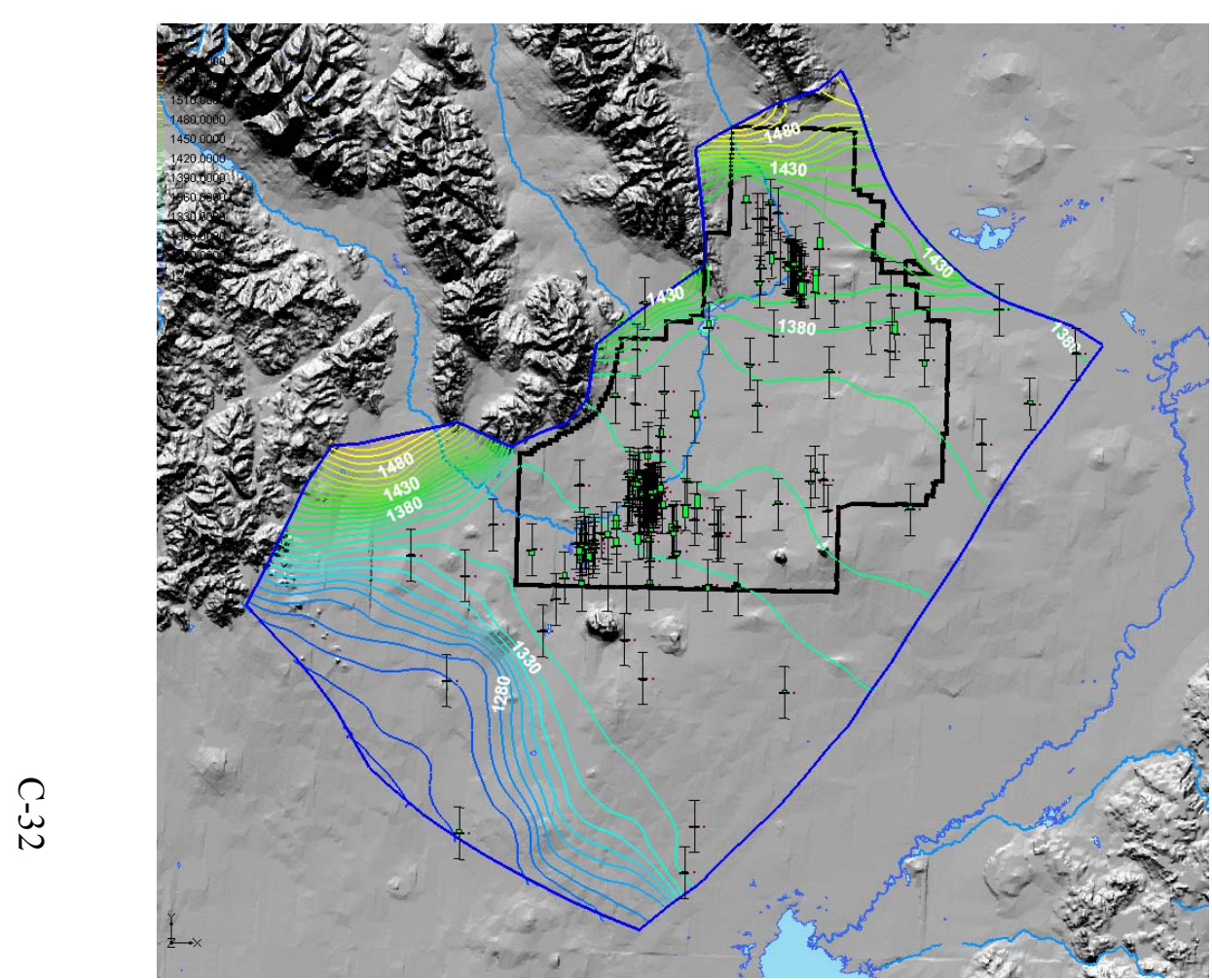

(a)

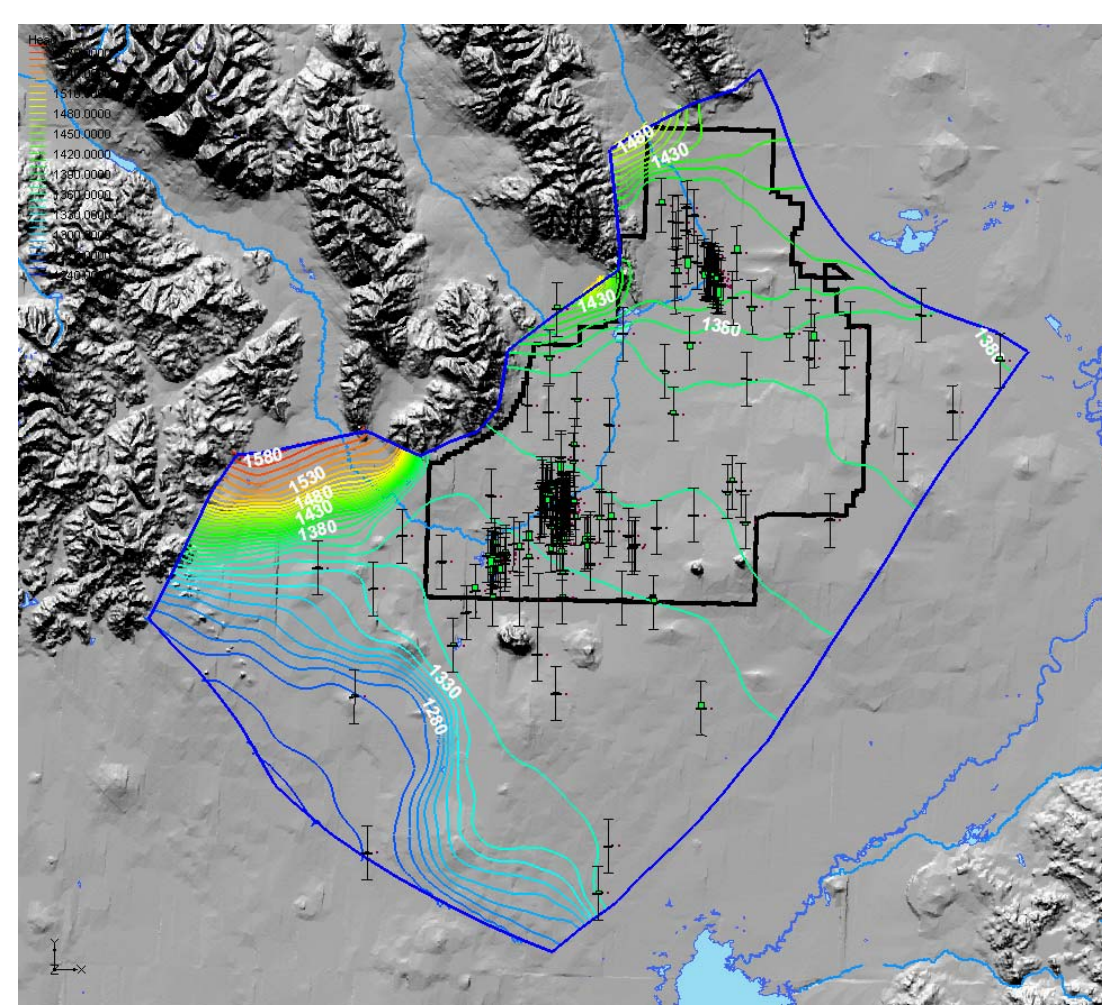

(b)

Figure C-10. Final June 2004 simulated heads resulting from constrained pilot point approach during inverse modeling; shown are (a) thin aquifer scenario, and (b) thick aquifer scenario (contour interval is $10 \mathrm{~m}$, elevations are meters above mean sea level; error bars are at observation well locations). 


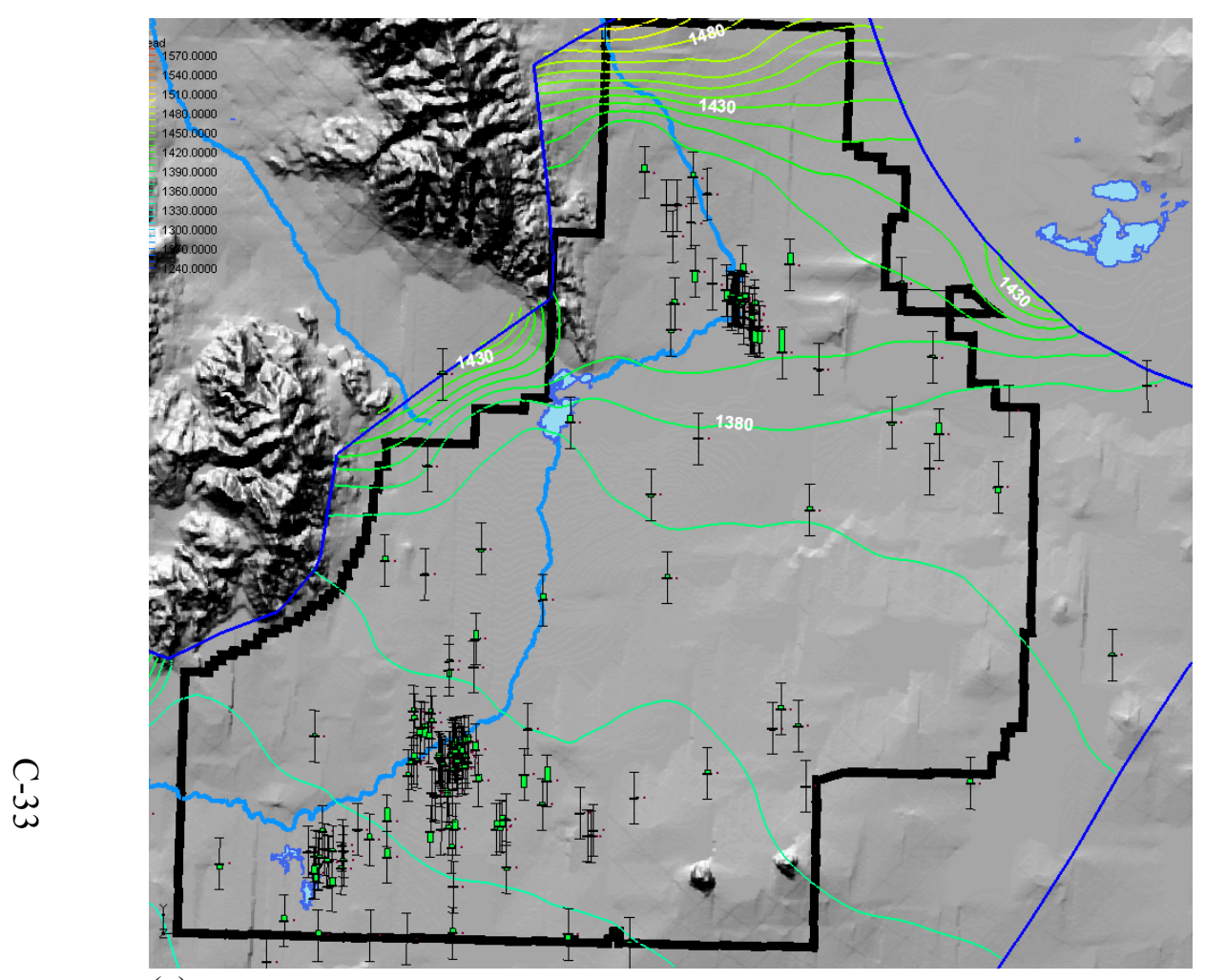

(a)

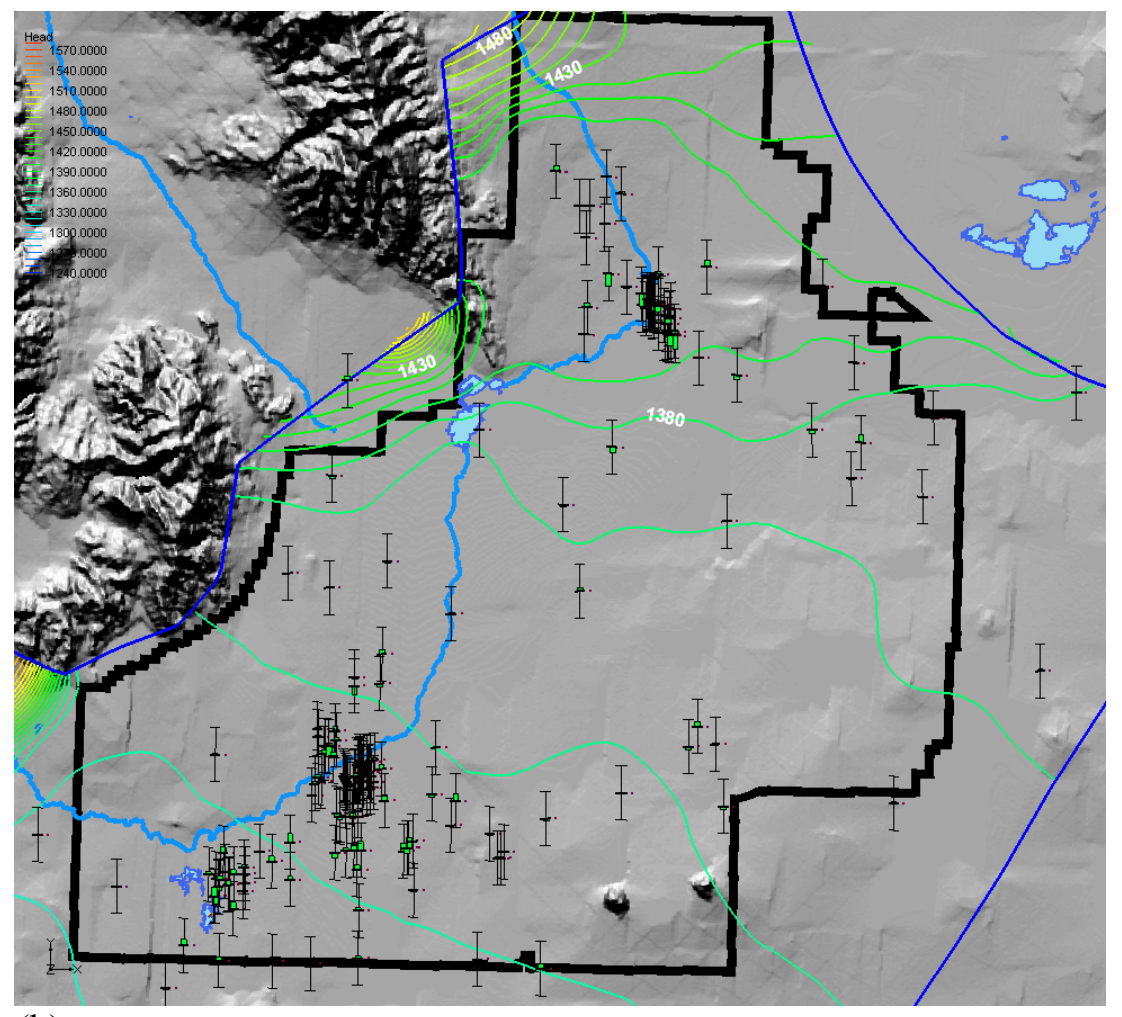

(b)

Figure C-11. Final June 2004 simulated heads inside INL resulting from constrained pilot point approach inverse modeling for (a) thin aquifer scenario, (b) thick aquifer scenario (contour interval is 10 meters, elevations are meters above mean sea level; error bars are at observation well locations). 


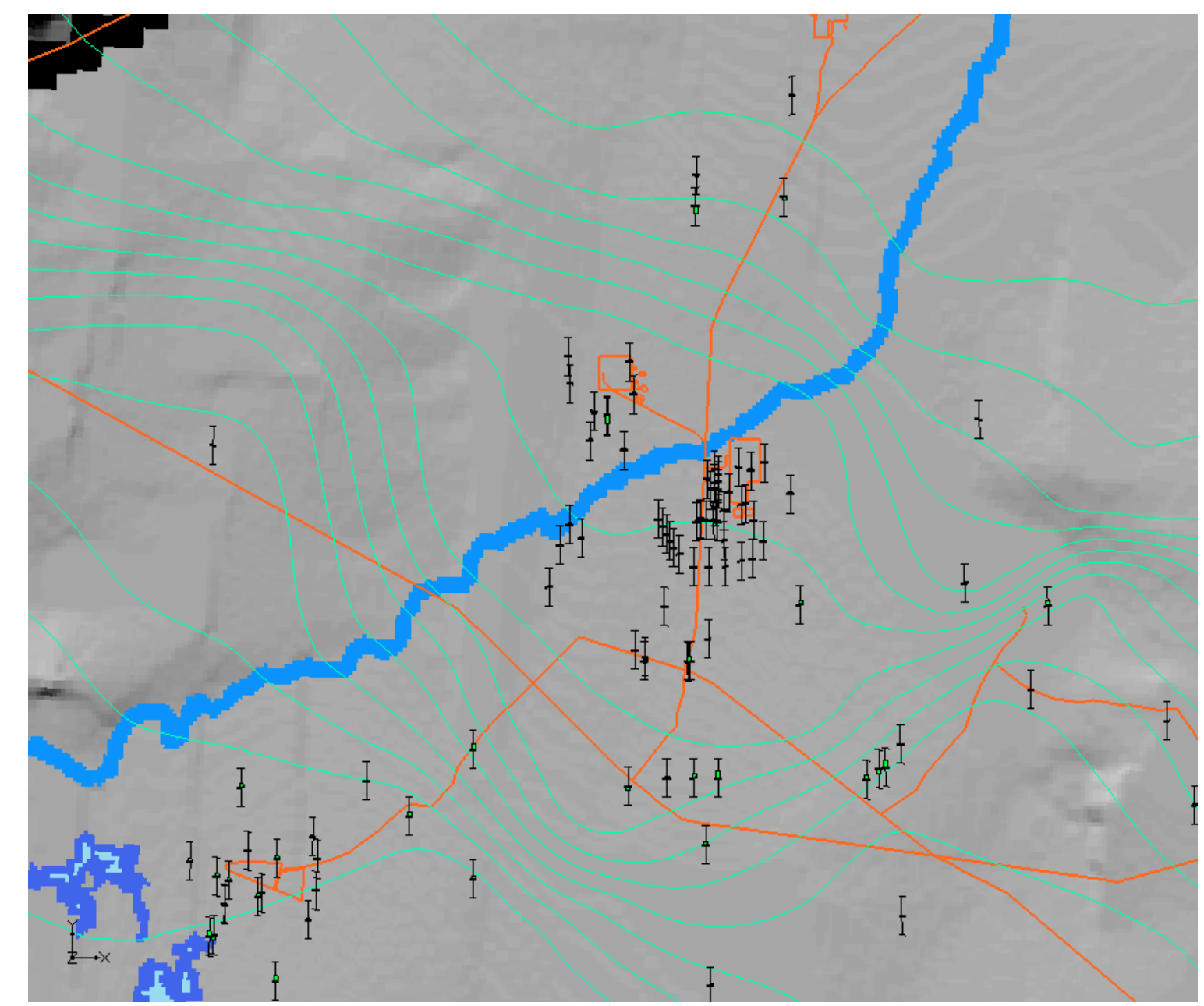

Figure C-12. Final June 2004 heads for southern INL facilities resulting from constrained pilot point approach inverse modeling for the thick aquifer scenario (contour interval is 1 meter, error bars are at observation well locations). 


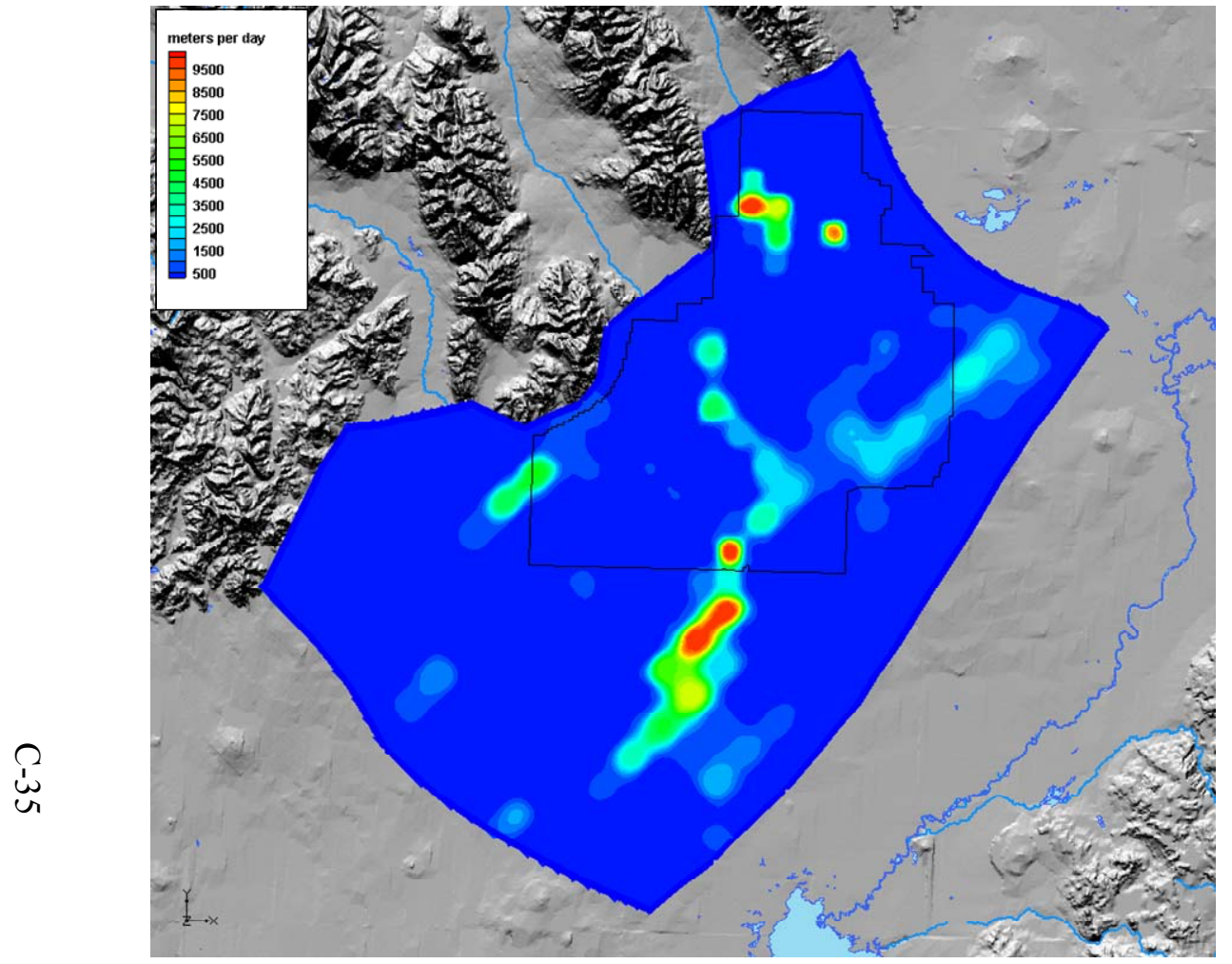

(a)

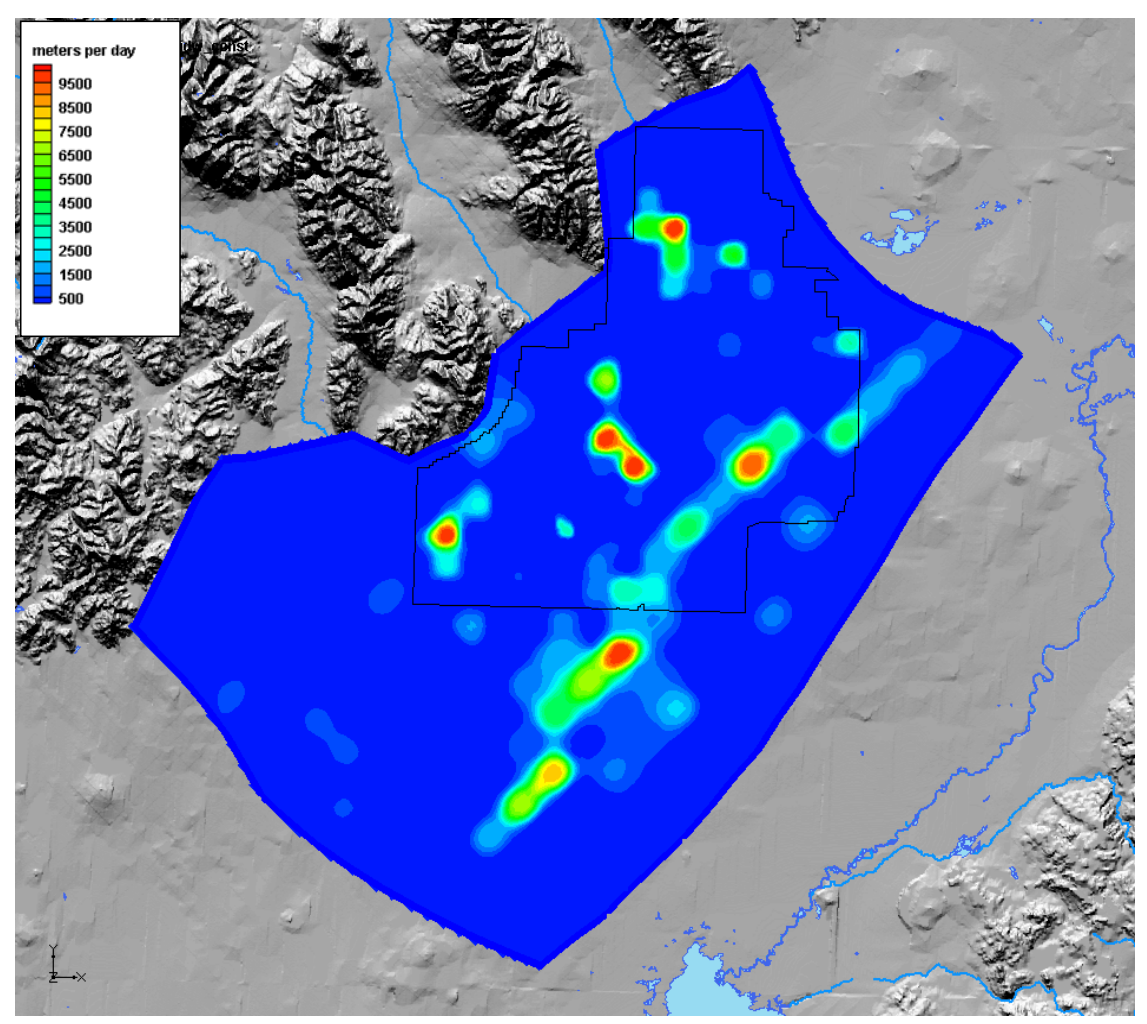

(b)

Figure C-13. Estimated hydraulic conductivity fields $(\mathrm{m} / \mathrm{d})$ that result from constrained pilot point inverse modeling for (a) thin aquifer scenario and (b) thick aquifer scenario. 
Figure $\mathrm{C}-14$ shows the distributions of head residuals obtained for both aquifer thickness scenarios. For both thickness scenarios, there is no systematic bias observed in the residual distributions after the specified flux condition was applied to the northeastern boundary. Secondly, both scenarios achieved satisfactory fittings to the measured heads. Particularly, inside INL, most head residuals are less than $0.5 \mathrm{~m}$, with only a few wells having residuals greater than $0.5 \mathrm{~m}$, but still less than $1 \mathrm{~m}$, as shown in Figure C-11. Finally, the overall residuals of the thick aquifer scenario are slightly less than those of the thin aquifer scenario. However, as can be seen in the following transport section, the small difference in the simulated heads between the thick and thin scenarios does impact simulated plume shapes.

On a local scale, there is some agreement between the OU 10-08 conductivity distribution and those obtained from the individual WAG modeling efforts at WAGs 1, 3, and 7. However, there is also considerable disagreement likely resulting from the particular density of pilot points and their locations that were used in the OU 10-08 model. Additionally, the two OU 10-08 aquifer thickness scenarios do not identically match the individual WAG-modeled aquifer thicknesses. Examples of this are shown in Figure C-15. This conductivity comparison is for demonstration purposes only; it is expected that the OU 10-08 3-D flow model will produce a better conductivity match to those localized conductivities estimated by the individual-WAG 3-D flow models.

\section{C-3.2 Two-Dimensional Transport Model Development}

The OU 10-08 implementation of the conceptual model into a numerical simulation requires two parts: flow, as discussed above, and contaminant transport. This section explains how the transport simulation was accomplished. The use of MT3DMS to simulate contaminant transport requires an existing flow simulation from MODFLOW; in this case, the simulated heads as shown in Figure C-10 represent the input flow fields to the transport simulations.

\section{C-3.2.1 Domain}

MT3DMS is linked to MODFLOW using the GMS user interface. The domain for the transport simulation is, therefore, the same as the domain for the flow simulation. Heads and cell-by-cell flux terms computed by MODFLOW during the flow simulation are written to a specially formatted file read by MT3DMS and used as the water fluxes for the transport simulation.

\section{C-3.2.2 Boundary and Initial Conditions}

The northwestern, northeastern, and southeastern boundaries were assigned as no contaminant flux boundaries. The southwestern boundary was modeled and assigned a zero gradient concentration condition, which implies free advection when contaminants reach this boundary.

Initial conditions for all transport simulations consisted of zero concentrations everywhere in the simulation domain. 

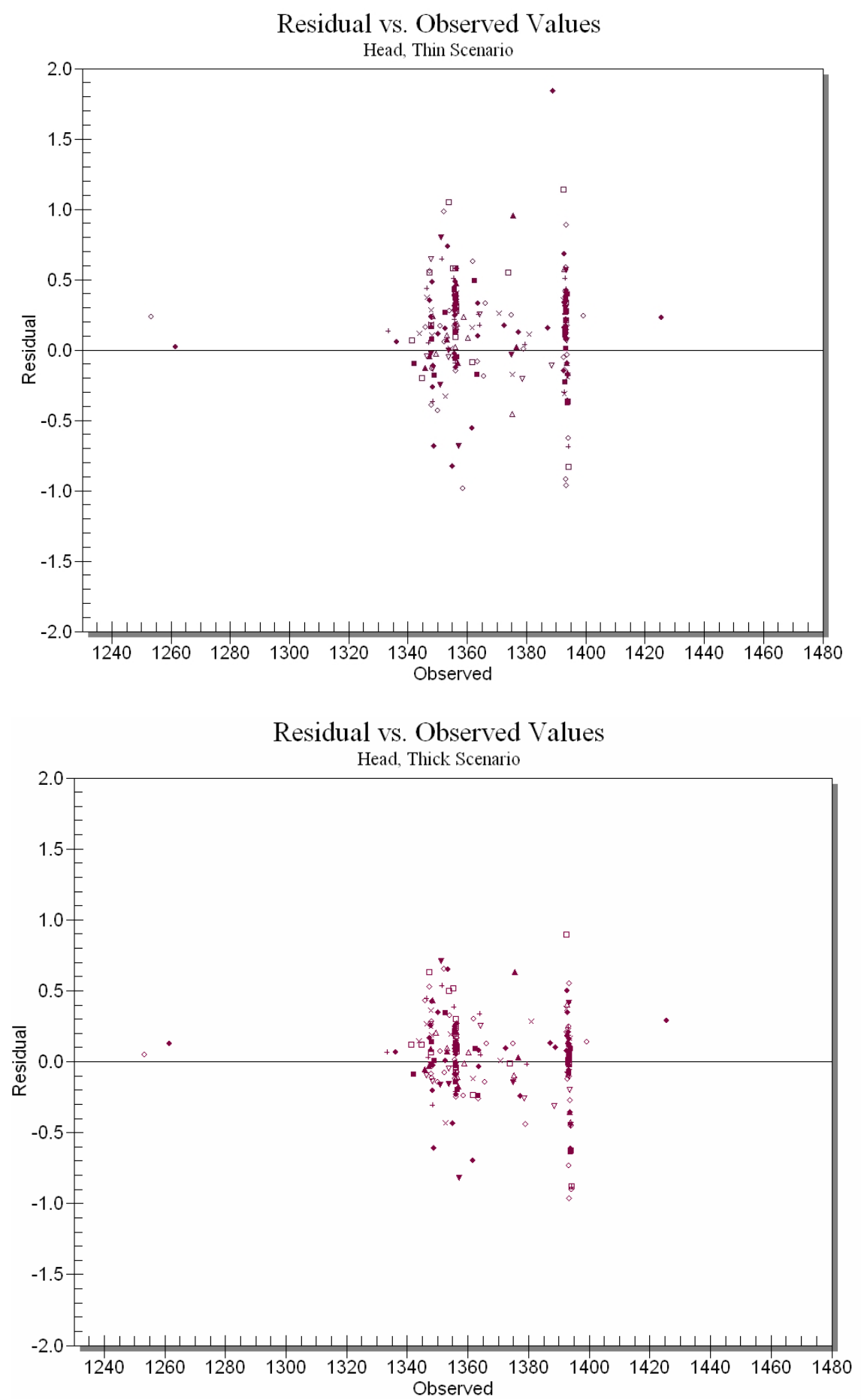

Figure C-14. Residual versus head values for (a) the thin aquifer scenario (top figure) and (b) the thick aquifer scenario (bottom). 


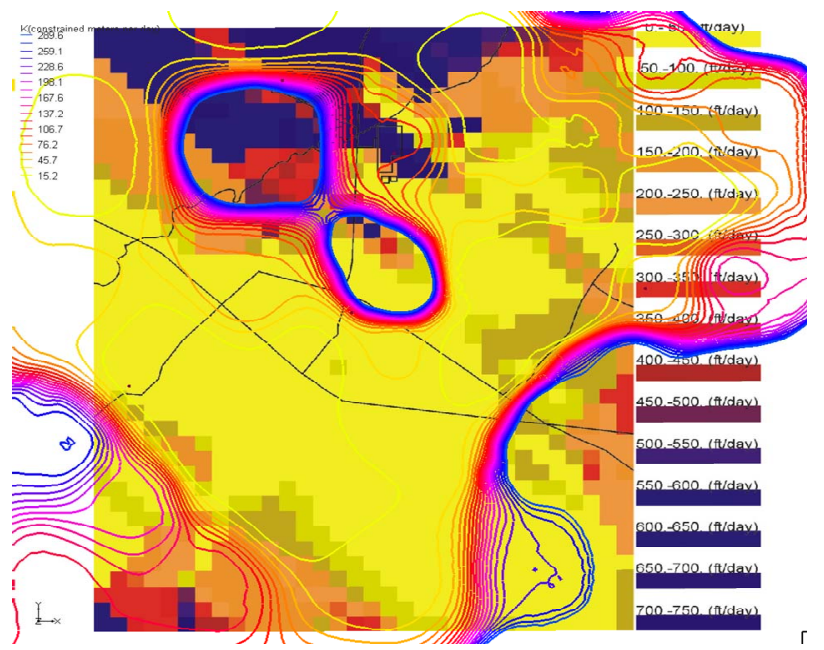

(a)

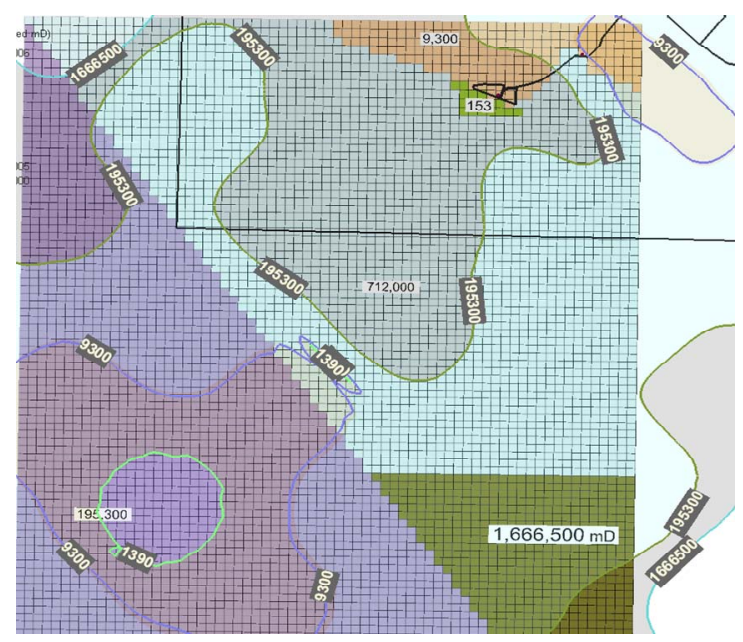

(b)

Figure C-15. Comparisons of estimated conductivity between Operable Unit 10-08 and individual WAG models at (a) OU 3-14 model and (b) OU 7-13/14 saturated model scales. The WAG 3 comparison shows OU 3-14 estimated conductivity (ft/d) (as solid contours) with OU 10-08 estimated conductivity over a similar range (as lines), with similar colors and contour interval. The WAG 7 comparison shows OU 7-13/14 permeability distribution in millidarcies $(\mathrm{mD})$ (solid contours with labels of black text on white background) compared to permeability converted from OU 10-08 estimated conductivity (labels are white text on black background) for similar range, intervals, and colors.

\section{C-3.2.3 Contaminants and Contaminant Loading}

This section identifies the contaminants that were simulated with the two-dimensional transport model and describes the implementation of contaminant sources into the model. There were two primary contaminant sources considered: migration through the vadose zone and direct aquifer injection. Direct aquifer injection is no longer practiced at INL, so only historical injection is considered. Remedial actions under consideration for OU 3-14 and OU 7-13/14 will impact future migration of contaminants in the vadose zone. The two-dimensional transport model uses base case vadose zone model flux results from both the OU 3-14 and OU 7-13/14 remedial investigation/baseline risk assessment (RI/BRA) for the individual facilities that assume no remedial actions are implemented.

C-3.2.3.1 Contaminants. The OU 10-08 2-D transport model was developed to demonstrate how to incorporate the various outputs of contaminant fluxes simulated by individual WAG transport models into our subregional scale model. As a demonstration model, the OU 10-08 2-D transport model was not intended to include simulation of all contaminants from all known INL sources. The demonstration used selected results from individual facilities to focus on contaminants with the most widespread observed plumes in the aquifer.

For the OU 10-08 2-D transport model, contaminants from three INL facilities were simulated: Test Area North (WAG 1), Idaho Nuclear Technology and Engineering Center (WAG 3), and the Radioactive Waste Management Complex (WAG 7). These sources include injection wells at WAG 1 and WAG 3 and vadose zone sources at WAG 3 and WAG 7. Locations of these sources relative to other features of the INL Site are depicted in Figure C-16. 


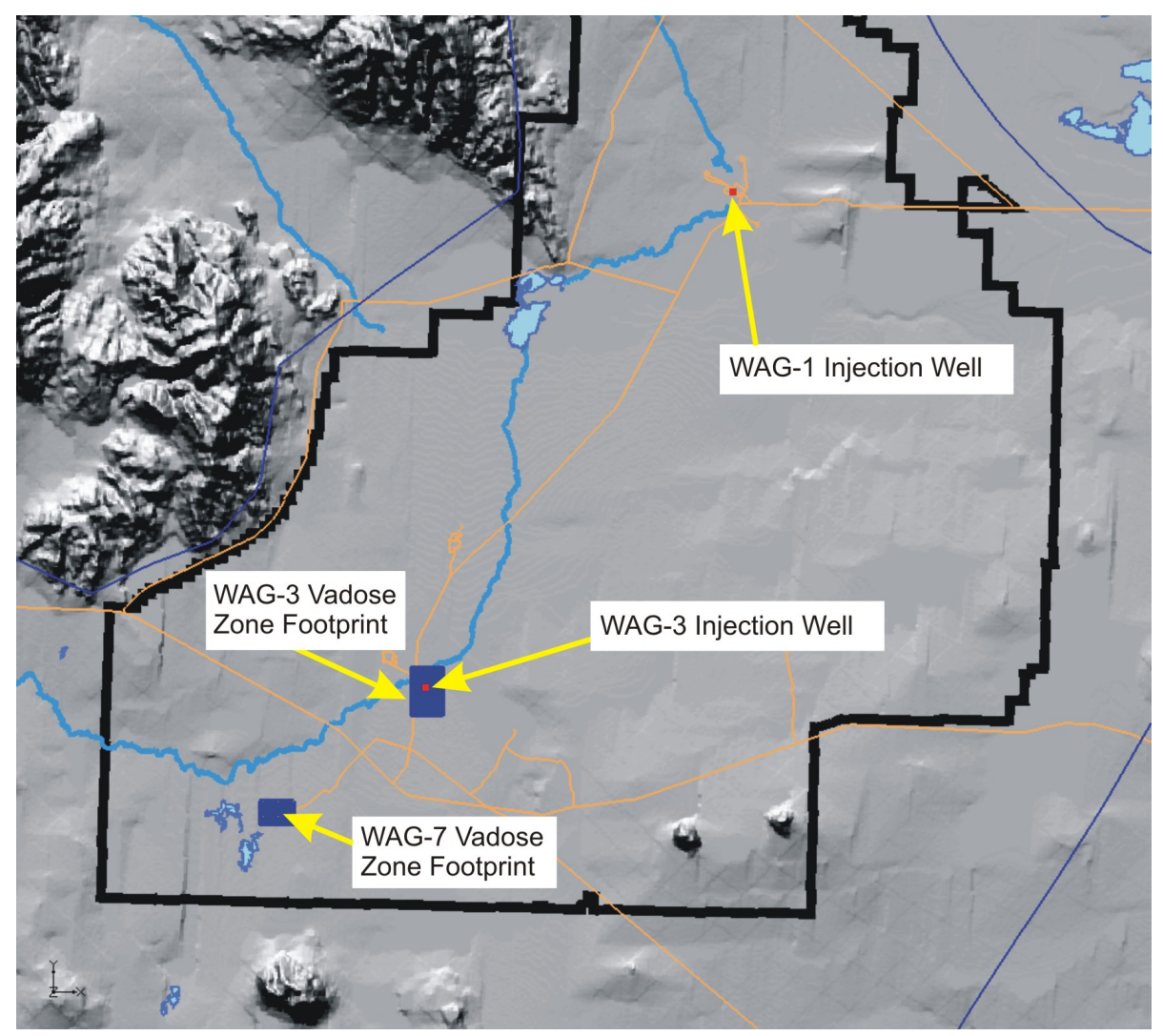

Figure C-16. Location of contaminant sources simulated in the Operable Unit 10-08 2-D transport model.

Eight contaminants were simulated and are listed in Table C-6. They include two organics, two inorganics, and four radionuclides. Although a vadose zone tritium source was available from an ongoing low-level waste performance assessment at the RWMC, tritium from this vadose zone source was not included in this version of the OU 10-08 transport model as it was integrated for the entire footprint and therefore is different than the individual cell fluxes for the rest of the RWMC sources. The starting time for the OU 10-08 two-dimensional transport model is at the beginning of 1950. Source terms from the individual WAG models start at 1955.0 for WAG 1, 1953.92 for WAG 3, and 1952.0 for WAG 7.

Table C-6. Summary of sources and contaminants included in Operable Unit 10-08 2-D transport model.

\begin{tabular}{|c|c|c|c|}
\hline $\begin{array}{l}\text { Waste Area } \\
\text { Group }\end{array}$ & Facility & Source to OU 10-08 Model & Contaminants \\
\hline WAG 1 & TAN & Injection well (TSF-05) & TCE \\
\hline WAG 3 & INTEC & Injection well (CPP-03) & Tritium, I-129, Sr-90, Tc-99 \\
\hline WAG 3 & INTEC & OU 3-14 vadose zone model & Tritium, I-129, Sr-90, Tc-99 \\
\hline WAG 7 & RWMC & OU 7-13/14 vadose zone model & $\begin{array}{l}\text { Carbon tetrachloride, chromium, nitrate, I-129, } \\
\text { Tc-99 }\end{array}$ \\
\hline \multicolumn{4}{|c|}{$\begin{array}{l}\text { OU = operable unit. } \\
\text { INTEC = Idaho Nuclear Technology and Engineering Center. } \\
\text { RWMC = Radioactive Waste Management Complex. } \\
\text { TAN = Test Area North. } \\
\text { TCE = trichloroethene. } \\
\text { WAG = waste area group. }\end{array}$} \\
\hline
\end{tabular}


Scaling was used in this modeling effort because of the extremely low concentration of some contaminants provided as input to the 2-D transport model. The low input concentrations are made even smaller since they are averaged across the entire aquifer thickness in the 2-D transport model. The extremely small concentrations pose challenges to obtaining an accurate solution in the numerical model due to truncation errors. The input sources were scaled up in order to minimize the impact of discretization truncation errors on the simulated contaminant plumes. The simulation results were likewise scaled back down by dividing by the scaling factors before the results were used.

C-3.2.3.2 Aquifer Injection Wells at TAN and INTEC. For the OU 10-08 model, the point source application method was used to simulate the CPP-03 injection well at INTEC and the TSF-05 injection well at TAN. Both injection wells had long service lives. At TAN, liquid waste was injected via the TSF-05 well to the aquifer from 1953 through 1972. At INTEC, the CPP-03 well began receiving approximately 1 million gallons per day of service wastewater from 1952 to 1984.

For the WAG 1 transport model, two source term scenarios were considered: (a) a biodegrading TCE source, in which an initial 1,100 gal of TCE were injected followed by a residual term of 0.0026 gal/day; and (b) a non-degrading source of an initial 66 gal injected followed by 0.012 gal/day (Martian 1999). These quantities were prepared for the WAG 1 model by assuming the TCE injection pattern followed that of tritium injection, the history of which is better documented.

Quantities of injected waste water, and concentrations of TCE in the waste water, as a function of time were obtained from the WAG 1 model and input to the OU 10-08 model as a point source.

Biodegradation of TCE was not included in the OU 10-08 simulation. The source term from the TAN model that was applied to the OU 10-08 simulation included a degrading source term. MT3DMS requires this injection well contaminant input as concentration; therefore, TCE volumes were converted to masses using a constant density of $1.465 \mathrm{~g} / \mathrm{cm}^{3}$. The waste water injection was simulated as a pulse of a constant $123.5 \mathrm{~m}^{3} / \mathrm{d}$ occurring from 1955-1972 which is the average injected rate for the period. Additional details are presented in Appendix C-B.

The CPP-03 injection well at INTEC received a total volume of approximately 12 billion gallons of service wastewater. Quantities of injected wastewater and concentrations of H-3, Sr-90, and I-129, as functions of time, were obtained from the OU 3-14 RI/BRA model and input to the OU 10-08 model as a point source. The injected wastewater was modeled as a pulse of constant $3,175 \mathrm{~m}^{3} / \mathrm{d}$ occurring from 1952-1984, which is the average injected rate for that period. More detail for this source is provided in Appendix C-B. Figure C-17 provides examples of the time-series of injected wastes originating from these two wells.

C-3.2.3.3 Vadose Zone Sources. Simulation of the WAG 3 and WAG 7 vadose zone sources using the GMS interface was not as straightforward as the injection well method. The locations of the OU 3-14 and OU 7-13/14 vadose zone transport model "footprints" on the aquifer are shown in Figure C-16 with their local discretization shown in Figure C-18. The OU 7-13/14 vadose zone model has 140 individual model grid blocks or cells that have a fluxes of water and contaminants exiting the bottom of the model domain. In a manner similar to the OU 7-13/14 model, the OU 3-13/14 vadose zone model has 600 individual grid blocks at the vadose zone/aquifer interface that result in 600 individual source terms to the aquifer. These facility fluxes had to be implemented spatially in the OU 10-08 aquifer model. 


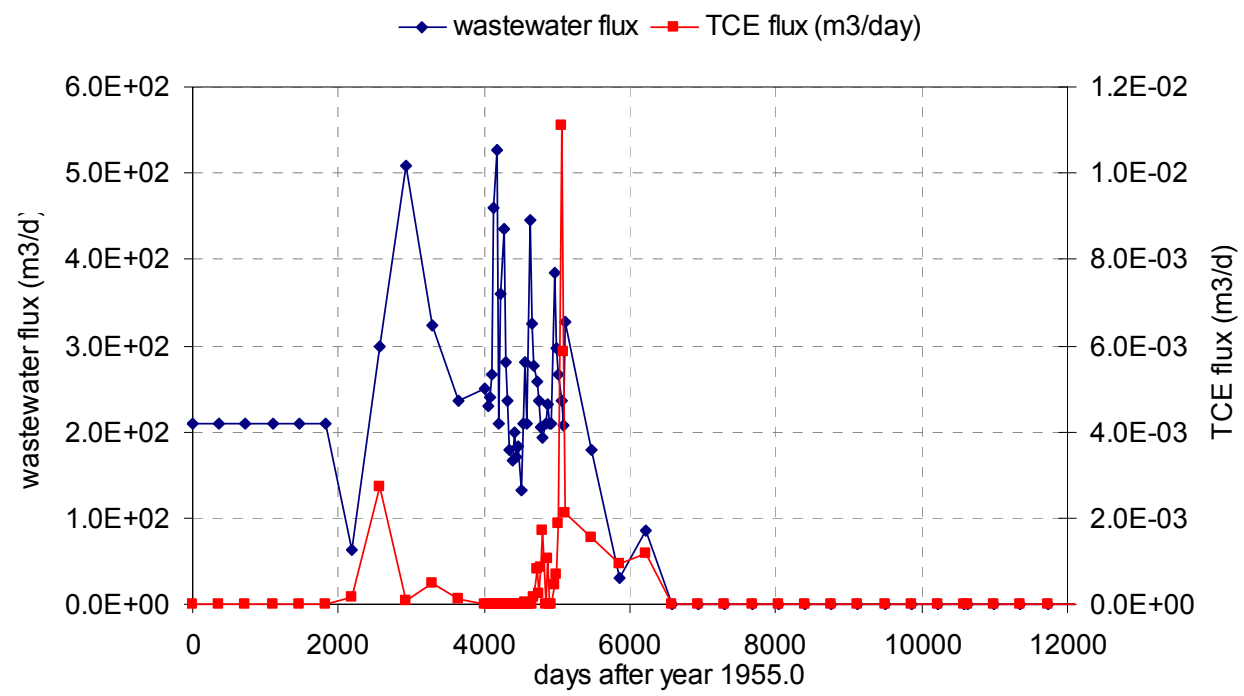

(a) TSF-05

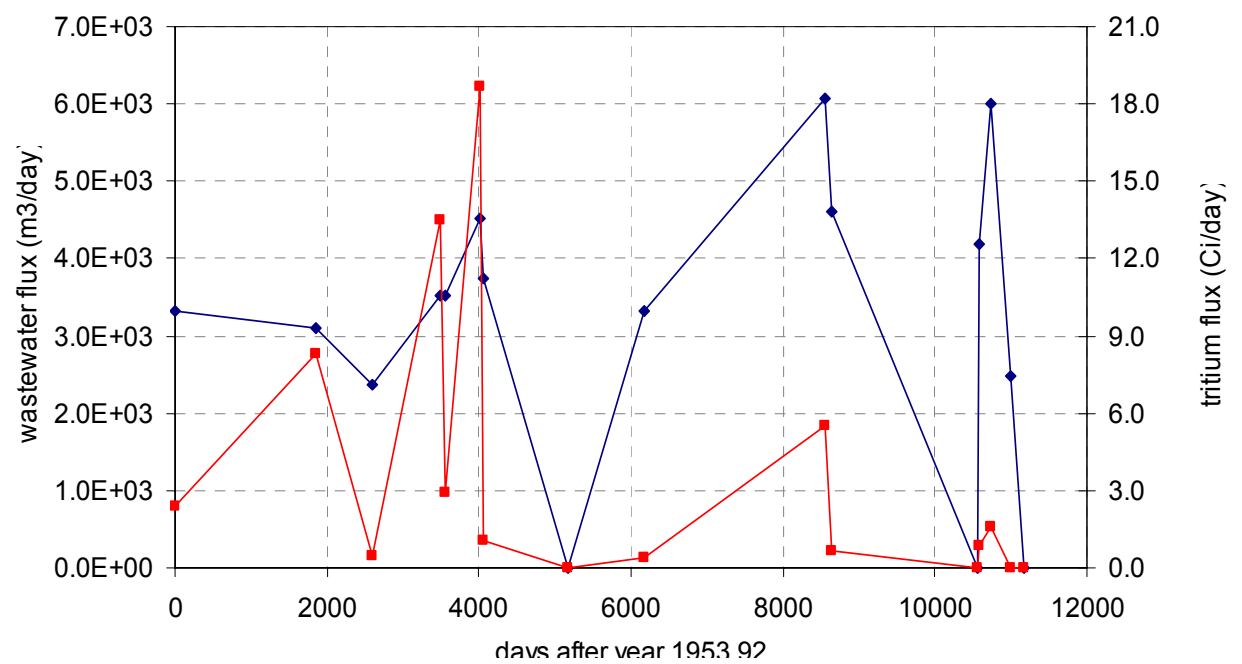

(b) CPP-03

Figure C-17. Example time histories of wastewater volumes and contaminant concentrations disposed of in WAG 1 and WAG 3 injection wells. 


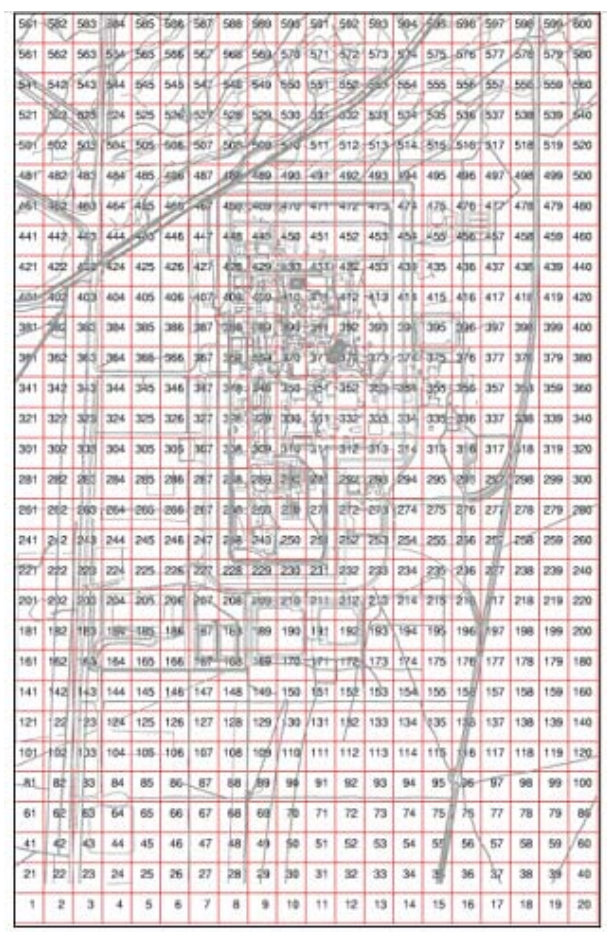

(a) OU 3-13/14 modeled vadose zone.

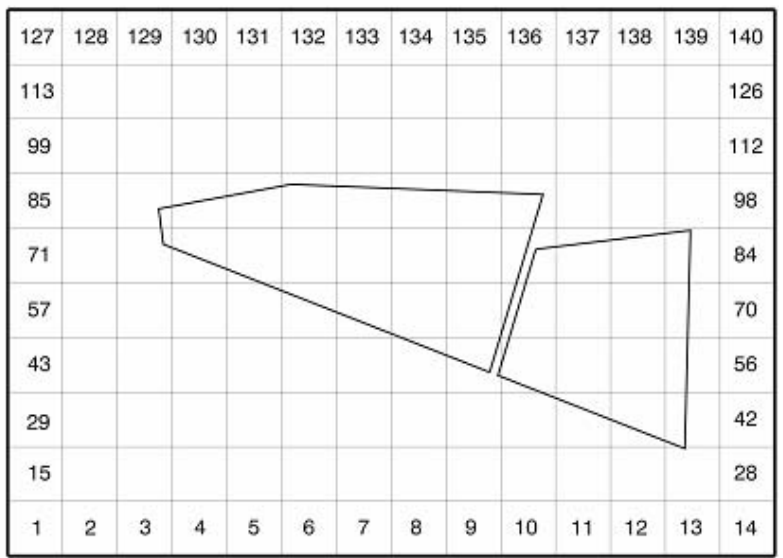

(b) OU 7-13/14 modeled vadose zone.

Figure C-18. Local-scale depiction of individual source cells represented in WAG 3 and WAG 7 vadose zone transport models (not to scale).

For each cell there was a unique time-series of water fluxes and contaminant mass loading fluxes. The OU 7-13/14 vadose zone source terms included in the OU 10-08 two-dimensional transport model were I-129, Tc-99, carbon tetrachloride, chromium, and nitrate. OU 3-14 vadose zone source terms included in the OU 10-08 two-dimensional model were H-3, I-129, Sr-90, and Tc-99. There are five unique time-series for each of 140 source cells for the OU 7-13/14 model and four unique time-series for each of 600 source cells in the OU 3-14 model. For more detail regarding the source terms for the OU 3-14 and OU 7-13/14, see Appendix C-C. Implementing these unique time-series proved to be a significant challenge in constructing the 2-D transport model since the GMS user-interface only allows manual input of individual time-series.

For all contaminant flux terms, the data from the specific WAG models were provided as mass fluxes, i.e., $\mathrm{kg} /$ day or $\mathrm{Ci} /$ day. MT3DMS requires input specifying the contaminant concentration (i.e., $\mathrm{kg} / \mathrm{m}^{3}$ or $\mathrm{Ci} / \mathrm{m}^{3}$ ) of the influxing water. This requires dividing the provided contaminant mass flux by the supplied water flux to obtain concentration. To maintain a steady-state flow simulation, the input water flux terms, which are also input to the MODFLOW model, were treated as constant water fluxes. For each source, an average water flux was determined and input to MODFLOW. To simplify flow simulation, the following average water flux rates were simulated for these sources and applied uniformly across each footprint as follows: WAG 3 vadose zone footprint $\left(10.56 \mathrm{~m}^{3} / \mathrm{d}\right)$. WAG 7 vadose zone footprint $\left(0.894 \mathrm{~m}^{3} / \mathrm{d}\right)$. The lower WAG 7 water fluxes are related to background precipitation rates, while the WAG 3 fluxes include process water discharges to ponds and other anthropogenic sources.

To use the average water flux in MODFLOW and still include time-variant contaminant fluxes in the MT3DMS model, a modified contaminant concentration time-series was determined for each source term that is based on this average water flux, rather than the actual time-variant water flux; this ensured 
that the same amount of contaminant mass would enter the aquifer during simulation. The water fluxes entering the aquifer from the vadose zone footprints were too small to impact the groundwater flow field.

C-3.2.3.4 GMS Contaminant Loading Limitation and Solution. In both cases of injection wells and vadose zone sources, the GMS interface approach proved impractical for preparing the necessary MT3DMS input files. The most straightforward approach is to construct 740 coverage polygons (representing each individual vadose zone footprint cell) within GMS to represent the vadose zone footprints of WAGs 3 and 7, then assign each polygon a water flux time series and eight contaminant time series accordingly. During the 2-D OU 10-08 transport modeling efforts, these 740 recharge polygons were first constructed within GMS.

However, because the model grid is not aligned with individual WAG models as shown in Figure C-19, in order to assign the correct water flux and contaminant mass loading flux to a specific model grid within the source footprint, partitioning of the surrounding recharge polygons is necessary. The current version of GMS is unable to correctly perform this partitioning as shown in Figure C-20. There is a significant difference between the contaminant mass loading specified as input within GMS and the mass resulting from GMS output that is actually used as input by the MT3DMS model, as shown in Figure C-20.

Another important limitation of the GMS interface is that the temporal interpolation of individual WAG model flux table time steps were not adequately captured. Inaccurate temporal interpolation of contaminant mass loading also contributed to mass imbalances. In all cases, the use of recharge polygons to simulate the vadose zone source cells produced some mass imbalance.

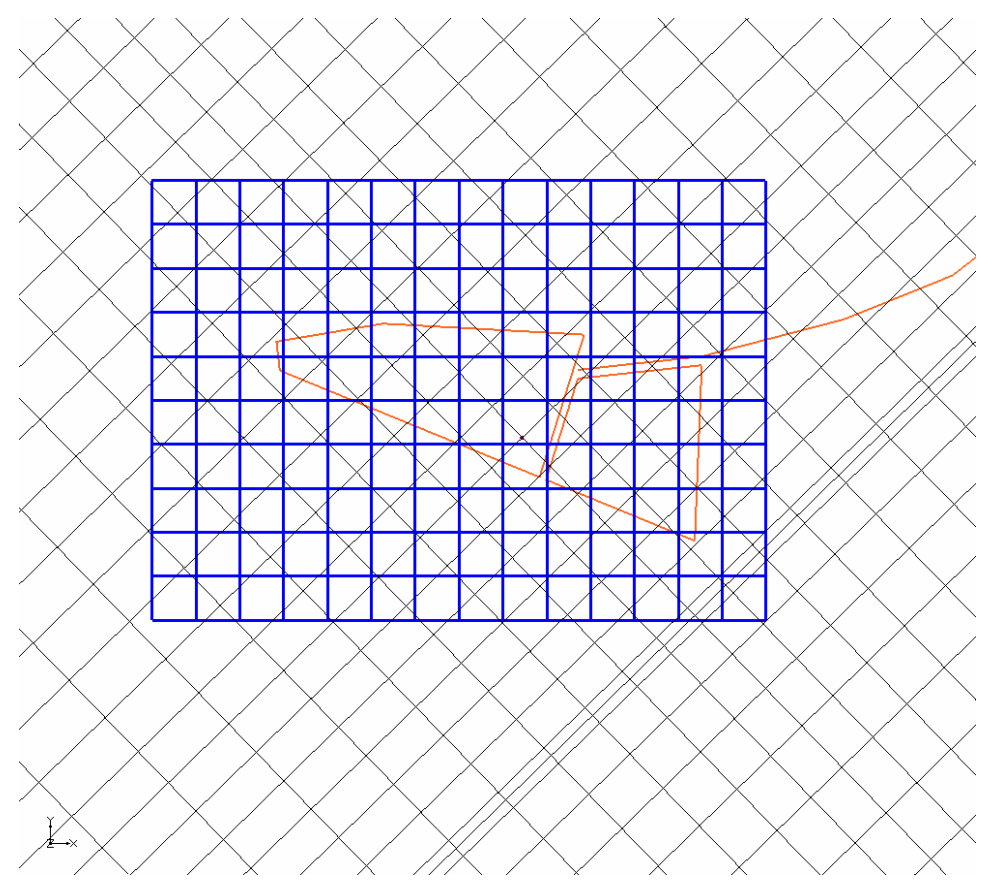

Figure C-19. 140 recharge polygons (blue line) representing OU 7-13/14 vadose zone footprint and the underlying 2-D OU 10-08 transport model grid (black line). 

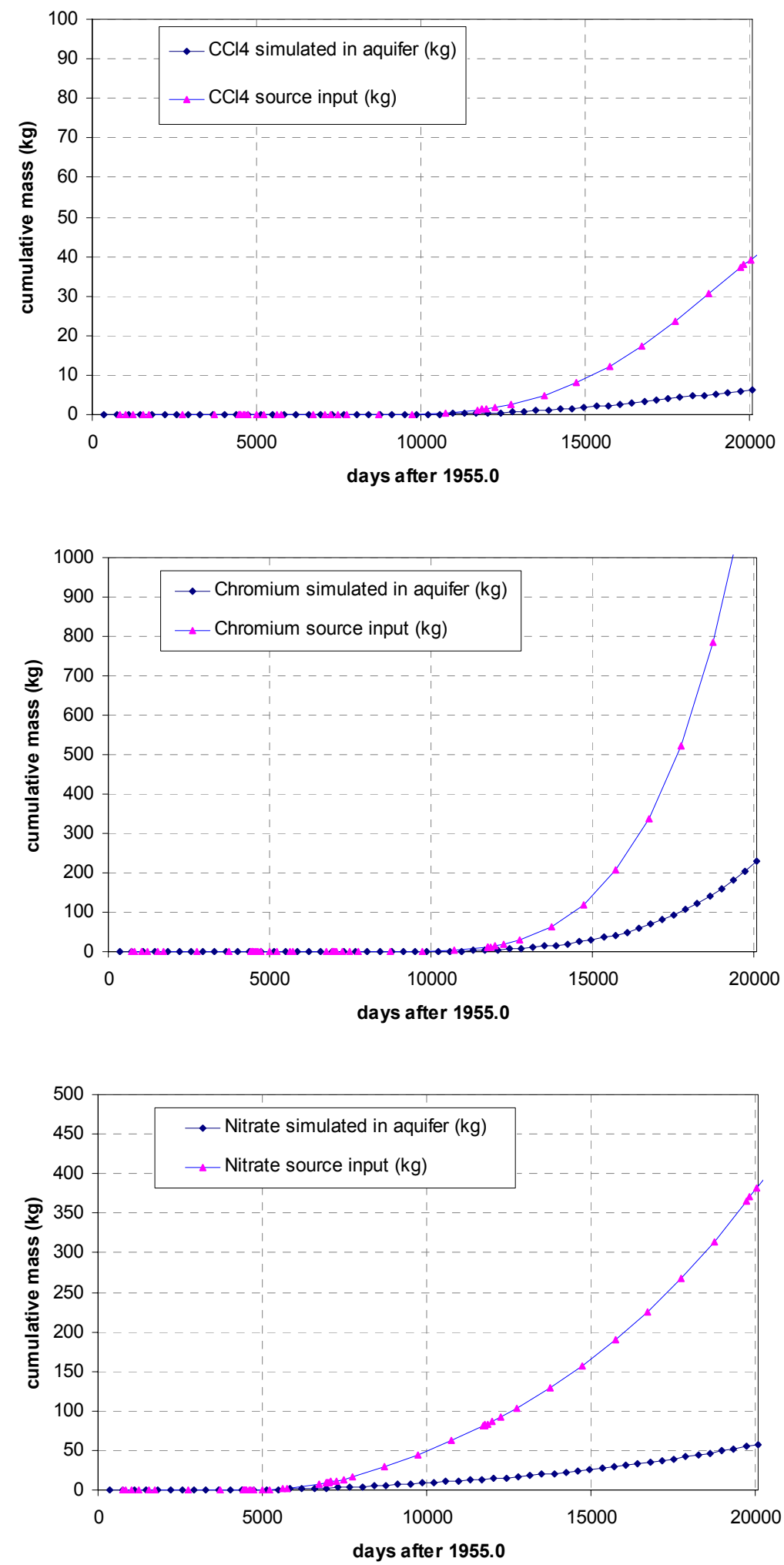

Figure C-20. Cumulative mass flux into the aquifer specified as input to GMS (output from WAG 7 model) and GMS output used in the aquifer model for $\mathrm{CCl}_{4}$, chromium, and nitrate (top to bottom figures, respectively). 
To preserve mass and overcome these limitations, an alternative method for preparing MT3DMS input files was used. Instead of using recharge polygons to represent source cells, an injection source well is used to represent each vadose zone source cell. The water "injection" rate is calculated by multiplying the water flux from a source cell by its area; then the mass loading fluxes are divided by this injection rate to obtain contaminant concentrations associated with the "injected" water. This "injection" well approximation to the vadose zone footprints is equivalent to the recharge polygon approach since these two approximations mathematically result in the same amount of contaminant mass loading rate into a specific numerical model grid. However, the injection well approach does not require any partitioning, which will exactly conserve the mass.

To facilitate this alternative approach, a FORTRAN program was created to manage the contaminant flux terms for 742 sources, i.e., 140 WAG 7 vadose zone sources, 600 WAG 3 vadose zone sources, one WAG 3 injection well, and the WAG 1 injection well. The FORTRAN program converts the source terms from area mass flux time-series to concentration time series, temporally interpolates them to match the OU 10-08 transport model stress periods, assigns the correct concentration terms to individual model cells, and writes this in the correct format for input to the MT3DMS input file. The use of this program significantly eased the burden of source term input and resolved all the mass-balance issues occurring with GMS-input terms. Figure C-21 shows the agreement between the cumulative contaminant mass entering into the aquifer from individual WAG sources and the simulated result of contaminant mass loading into the OU 10-08 model. Note that the simulation results for tritium and Sr-90 include decay.

\section{C-3.2.4 Transport Properties}

Table C-7 provides half-lives and specific activities for the four radiological contaminants simulated with the two-dimensional transport model. Biodegradation of TCE was not included in the simulations. Contaminant-specific sorption partition coefficients were not needed as sorption was not included anywhere in the 2-D transport model.

Porosities and dispersivities were required for each grid cell in the transport model domain. Two transport scenarios involving porosity and dispersivity were simulated. In the first scenario, the porosity and dispersivity were treated as variable and were assigned based on the values from the individual WAG-scale transport models as shown in Table C-8. This variable-properties transport scenario attempted to honor the parameters used in the individual WAG models. For the portions of the OU 10-08 model domain outside the individual WAG models, a porosity value of 0.05 and longitudinal and transverse dispersivities of $91 \mathrm{~m}$ and $45 \mathrm{~m}$ were assigned. The porosity value was selected from within the range of porosities (0.03-0.06) used in the individual WAG models. The 91-m longitudinal dispersivity is based on previous INL modeling (Robertson 1974; Arnett, Martineau, and Lehto 1990). Figure C-22 shows the domain of OU 10-08 and the domains of individual WAG models and the assigned porosity and dispersivity values.

For the second transport scenario, the entire modeled domain was represented more simplistically with a uniform porosity of 0.10 , a uniform longitudinal dispersivity of $91 \mathrm{~m}$, and a transverse dispersivity of $45 \mathrm{~m}$ assigned for the entire domain. The uniform porosity value was selected to result in more reasonable velocities. The change to prescribed fluxes on the northeast boundary resulted in more (and appropriately so) water moving through the domain. Previous individual WAG facility models used prescribed head boundaries and likely underestimated the water fluxes through the system and used smaller porosities to match the velocities responsible for contaminant transport. With a larger water flux, larger porosities likely allow a closer match to the velocities from the individual WAG facility models. In any event, the assigned values for porosity and dispersivity will be subject to change in the 3-D transport model calibration. 


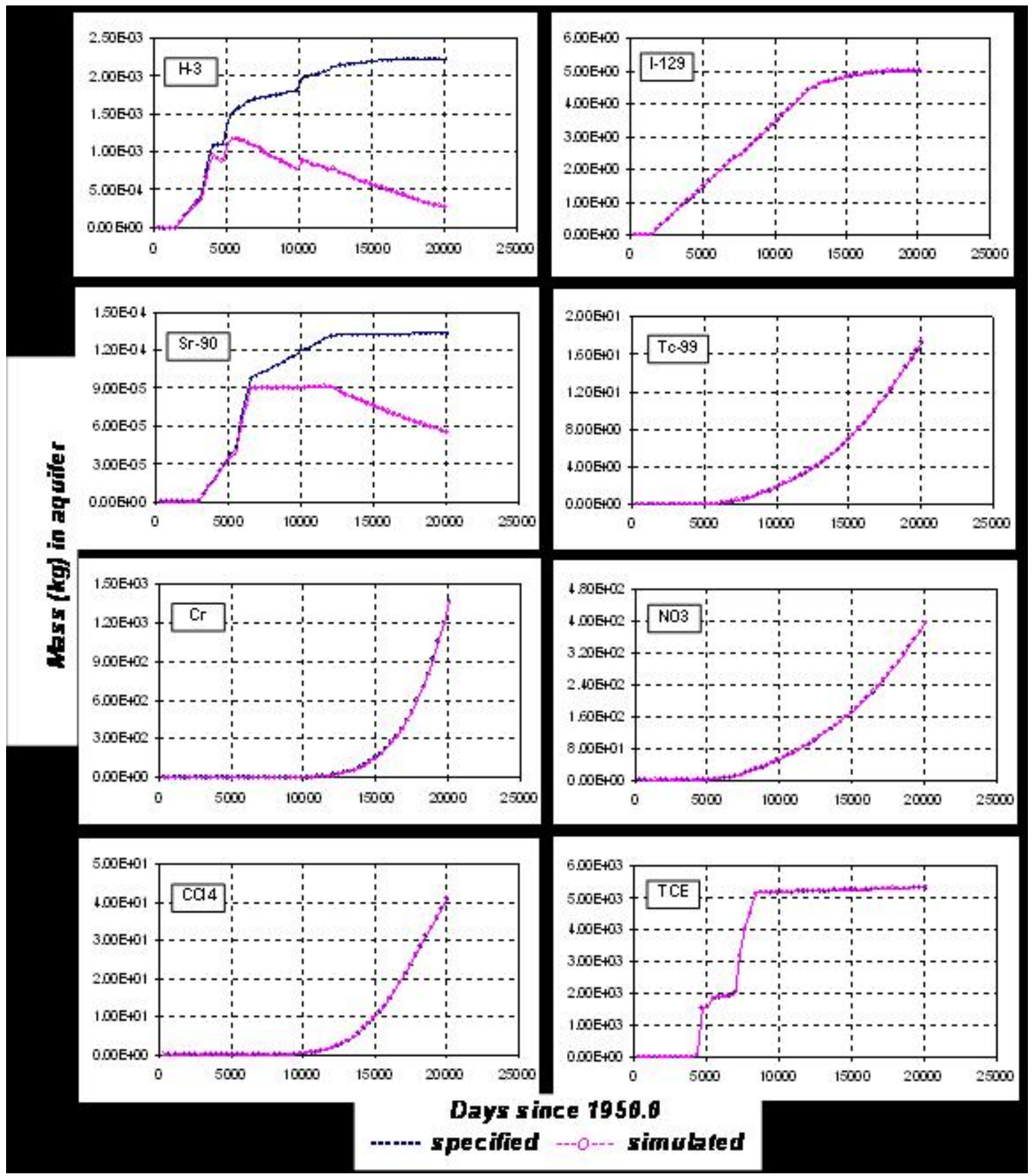

Figure C-21. The comparison between cumulative contaminant masses in the aquifer specified by individual WAG models and simulated by the 2-D OU 10-08 transport model. 
Table C-7. Radioactive decay half-lives and specific activities for radiological contaminants included in the Operable Unit 10-08 2-D transport model.

\begin{tabular}{ccc}
\hline Contaminant & $\begin{array}{c}\text { Half-life, t } 1 / 2 \\
(\mathrm{yr})\end{array}$ & $\begin{array}{c}\text { Specific Activity } \\
(\mathrm{Ci} / \mathrm{g})\end{array}$ \\
\hline H-3 & 12.33 & $9.685 \mathrm{E}+03$ \\
I-129 & $1.70 \mathrm{E}+07$ & $1.765 \mathrm{E}-04$ \\
Sr-90 & 29.1 & $1.365 \mathrm{E}+02$ \\
Tc-99 & 213,000 & $1.695 \mathrm{E}-02$ \\
\hline
\end{tabular}

Table C-8. Porosity and dispersivities from WAG-specific models that were used in the variable-property Operable Unit 10-08 2-D transport simulation.

\begin{tabular}{cccc}
\hline Area & Porosity & \multicolumn{1}{c}{ Dispersivity } & \multicolumn{1}{c}{ Reference } \\
\hline RWMC & 0.06 & $\mathrm{D}_{\mathrm{l}}=9 \mathrm{~m}, \mathrm{D}_{\mathrm{t}}=4 \mathrm{~m}$ & $\begin{array}{l}\text { DOE/ID-11241 } \\
\text { (DOE-ID 2006a) }\end{array}$ \\
\hline INTEC & 0.03 & $\begin{array}{l}\mathrm{D}_{\mathrm{l}}=\mathrm{D}_{\mathrm{t}}=0 \text { within vadose zone model footprint } \\
\text { and } \mathrm{D}_{\mathrm{l}}=40 \mathrm{~m}, \mathrm{D}_{\mathrm{t}}=60 \mathrm{~m} \text { outside the footprint }\end{array}$ & $\begin{array}{l}\text { DOE/NE-ID-11227 } \\
\text { (DOE-ID 2006b) }\end{array}$ \\
\hline TAN & 0.035 & $\mathrm{D}_{\mathrm{l}}=25 \mathrm{~m}, \mathrm{D}_{\mathrm{t}}=12.5 \mathrm{~m}^{1}$ & $\begin{array}{l}\text { INEEL/EXT-02-00632 } \\
\text { (INEEL 2002) }\end{array}$ \\
\hline
\end{tabular}

INTEC $=$ Idaho Nuclear Technology and Engineering Center.

RWMC $=$ Radioactive Waste Management Complex.

TAN $=$ Test Area North.
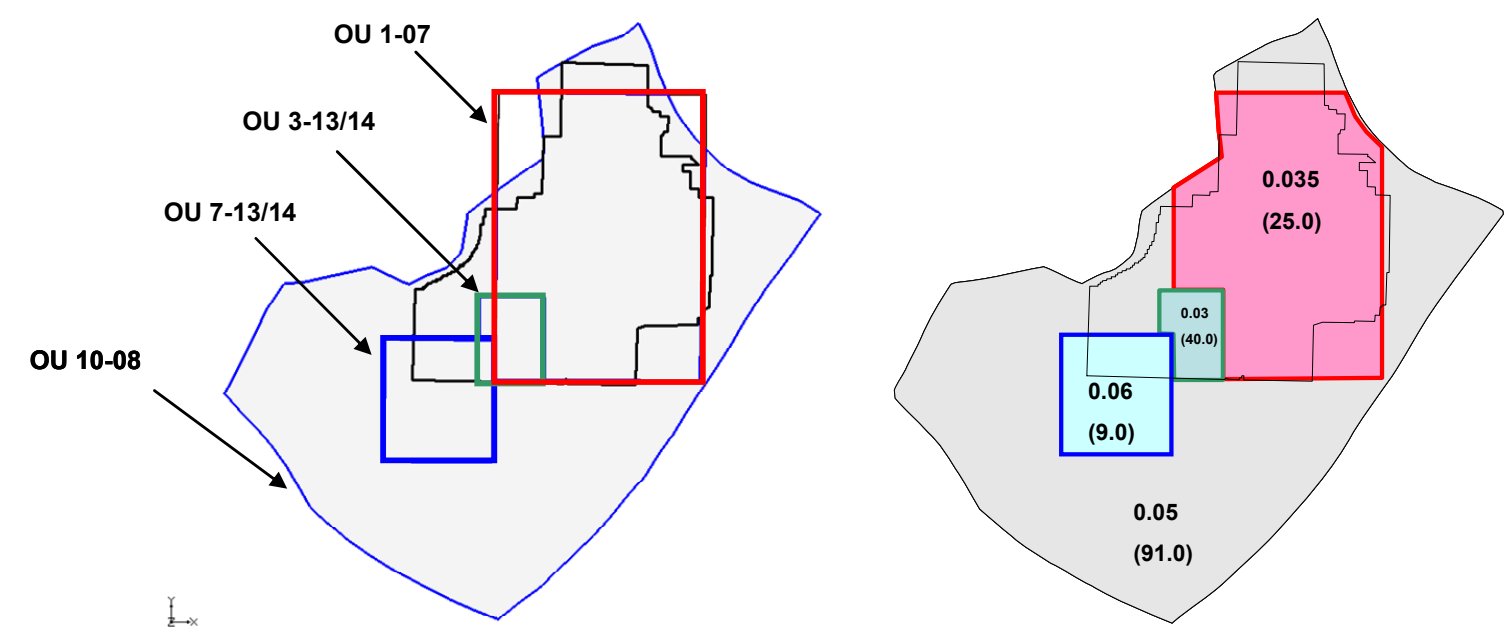

(a)

(b)

Figure C-22. (a) The domains of individual WAG models relative to the OU 10-08 model domain and (b) the transport property values assigned in the individual WAG models (effective porosity and, in parentheses, longitudinal dispersivity in meters) for the variable-properties transport scenario. 


\section{C-4. TRANSPORT RESULTS}

This section summarizes the results from the simulation of eight contaminants from four INL facilities (listed in Table C-6) using both the thick aquifer and thin aquifer scenarios and the uniform and variable transport property scenarios. Calibration of the two-dimensional transport model was not attempted. The two-dimensional model has uniform concentrations over the entire vertical aquifer thickness and does not represent three-dimensional transport. Concentration measurements sampled from a limited interval in aquifer monitoring wells are actually 3-D measurements, and represent average concentrations from the open interval in the well, whereas the 2-D OU 10-08 transport model assumes uniform profiles across the entire aquifer thickness. Defensible representation of the anisotropy resulting from the layered architecture of the SRPA requires construction of a fully three-dimensional transport model. The two-dimensional transport model described in this report was only constructed as an intermediate step to incorporate the complex addition of source terms in a simplified numerical tool.

This section shows visual comparisons of simulation results for contaminant concentrations at calendar year 2005 (simulation day 20,075 days) for the two aquifer thickness scenarios and two transport property scenarios. Simulated concentrations are vertically uniform over the entire simulated thickness of the aquifer and are therefore not representative of measured three-dimensional concentrations.

Tritium was released to the SRPA through an injection well and infiltration ponds at INTEC in the OU 10-08 model. Simulated distribution of tritium in groundwater in 2005 is shown in Figure C-23 for the two thickness scenarios and the two transport property scenarios. Plume distributions "a" and "b" were simulated using the variable transport properties. In both simulations for these variable transport properties, the tritium plume migrated beyond the southern INL boundary by 2005 with the thick scenario showing the furthest extent of the tritium plume at 2005. Plume distributions "c" and "d" were simulated using uniform transport properties, and show less extent to the plume distribution at 2005, albeit the contour levels are higher because the tritium mass is more concentrated in the simulations, which results in higher concentrations. There is a notable difference between the simulation results due to both the aquifer thickness scenarios and the method used to assign transport properties. Simulated plumes that extend southward are impacted by a high-velocity zone that further spreads the plumes. This high-velocity zone is discussed below.

Modeled I-129 and Sr-90 distributions (Figures C-24 and C-25) show results similar to the tritium simulations, with plumes extending south of the INL boundary for all except the thin aquifer, uniform transport properties scenario. These results also show the impact of a higher velocity region that the plumes contact southward from INTEC.

Contaminants released from the WAG 7 footprint that were simulated with the OU 10-08 two-dimensional transport model were I-129, Tc-99, nitrate, chromium, and carbon tetrachloride. The simulation results for I-129 do not show an impact from the WAG 7 source at calendar year 2005 (Figure C-24) because it is overshadowed by the much larger mass entering the model at INTEC. Modeled distributions of Tc-99, nitrate, chromium, and carbon tetrachloride originating from WAG 7 are shown in Figures C-26 through C-29. These simulation results show a different result from those with the INTEC sources. The farthest southward migration occurs with the thin aquifer and variable transport scenario. Although there is more similarity in these results, selecting between them would require calibration with comparison to observed concentrations. 


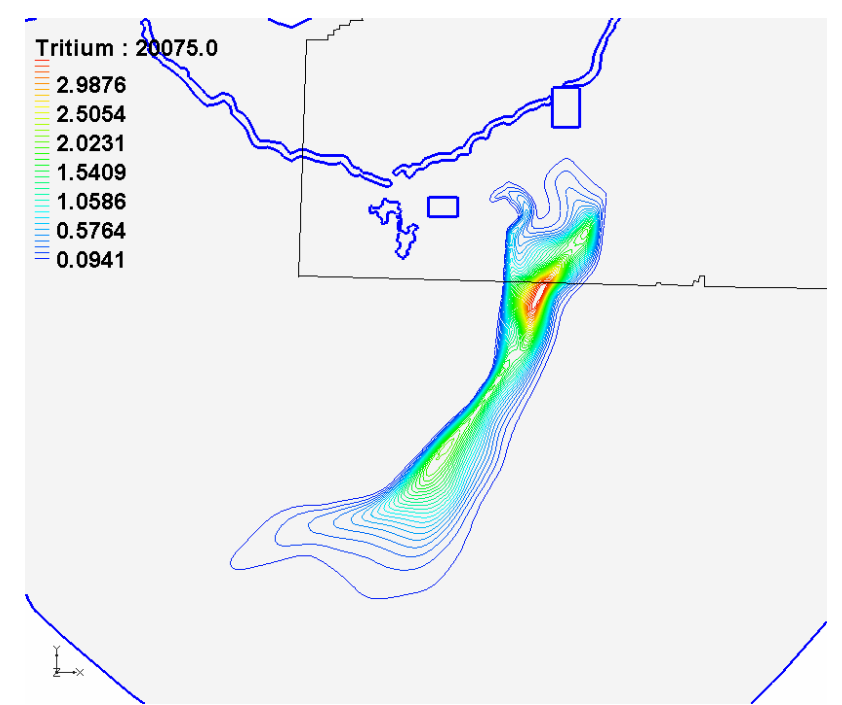

a. thick, variable properties

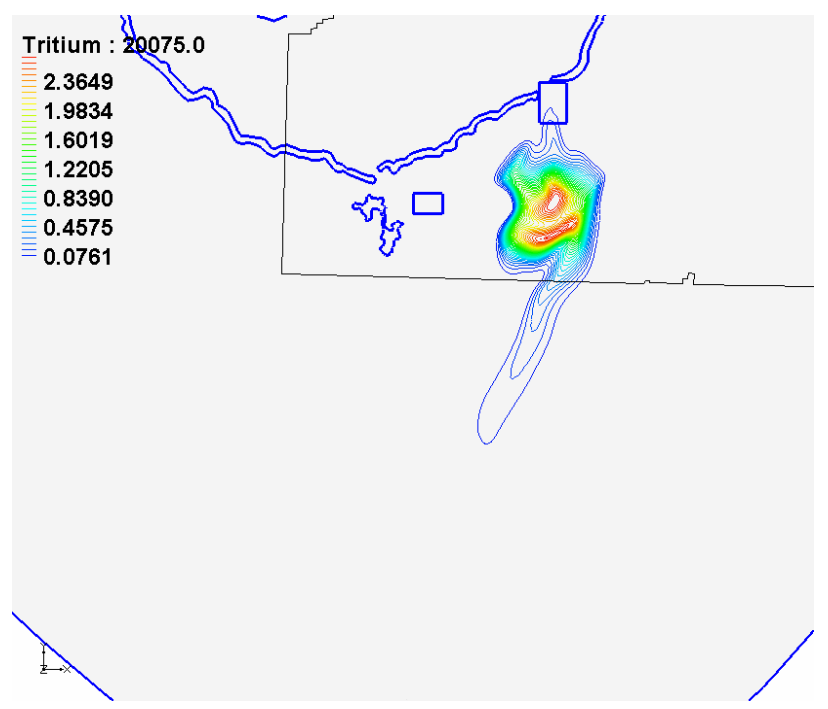

c. thick, uniform properties

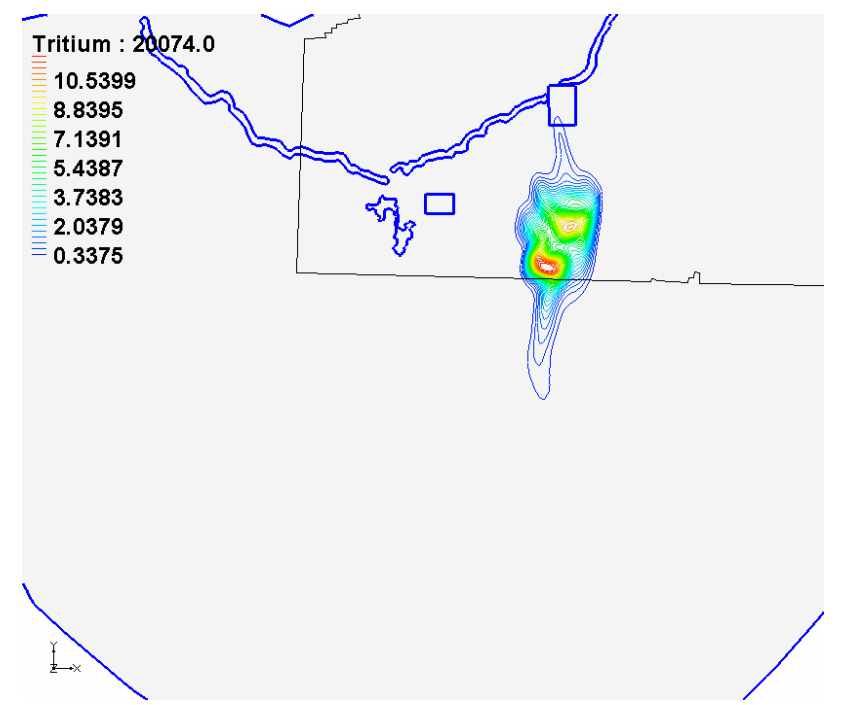

b. thin, variable properties

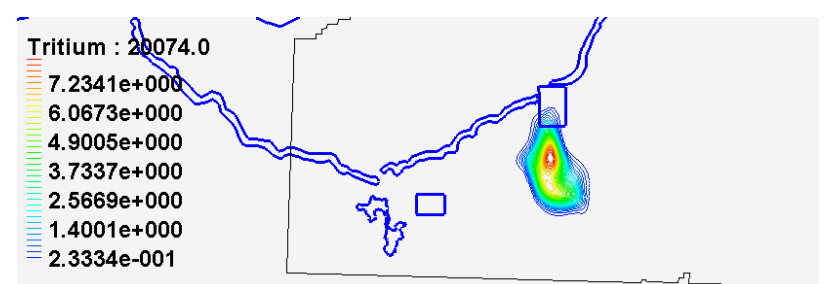

$2.3334 \mathrm{e}-001$

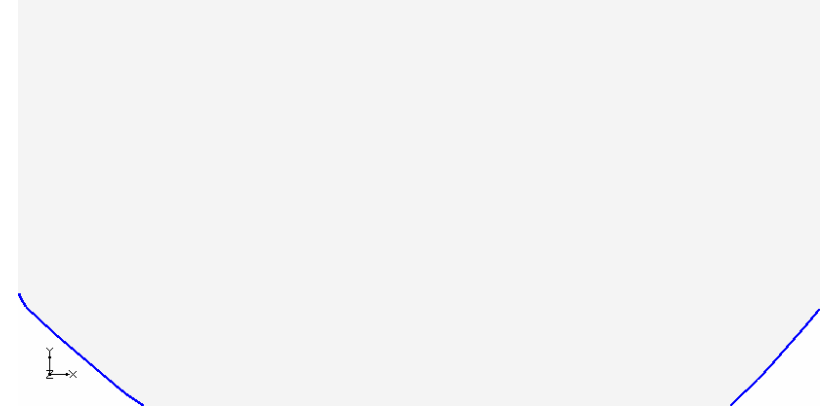

d. thin, uniform properties

Figure C-23. Plots of scaled tritium concentration at maximum simulation time (20,075 days, by year of 2005) for two aquifer thickness and two transport property scenarios. 


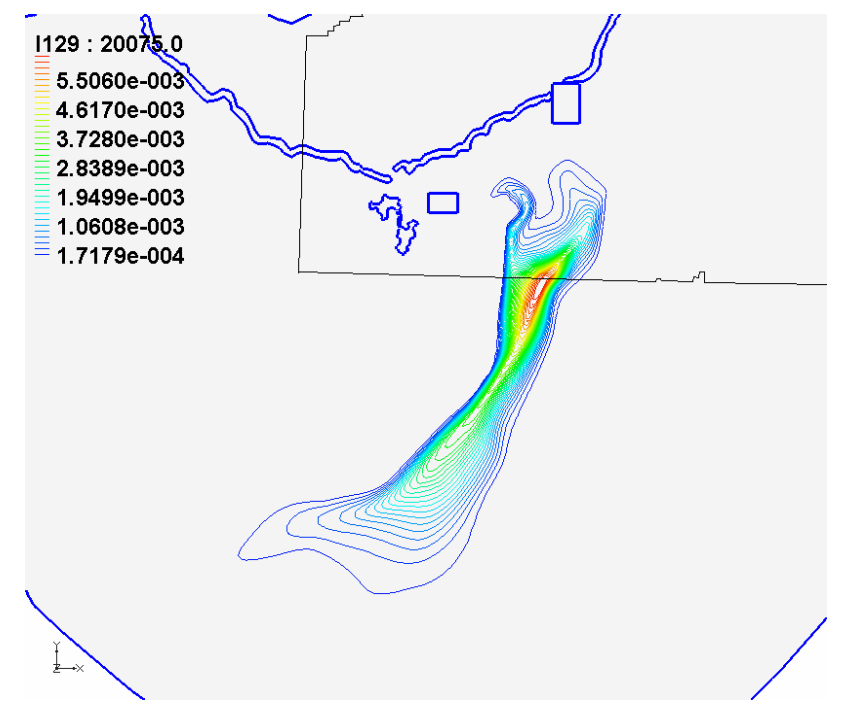

a. thick, variable properties

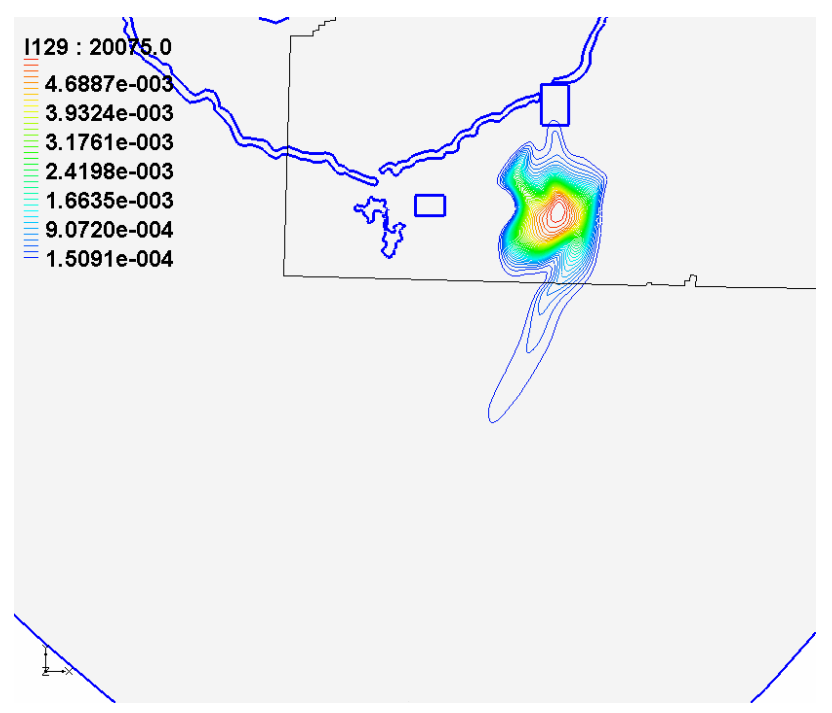

c. thick, uniform properties

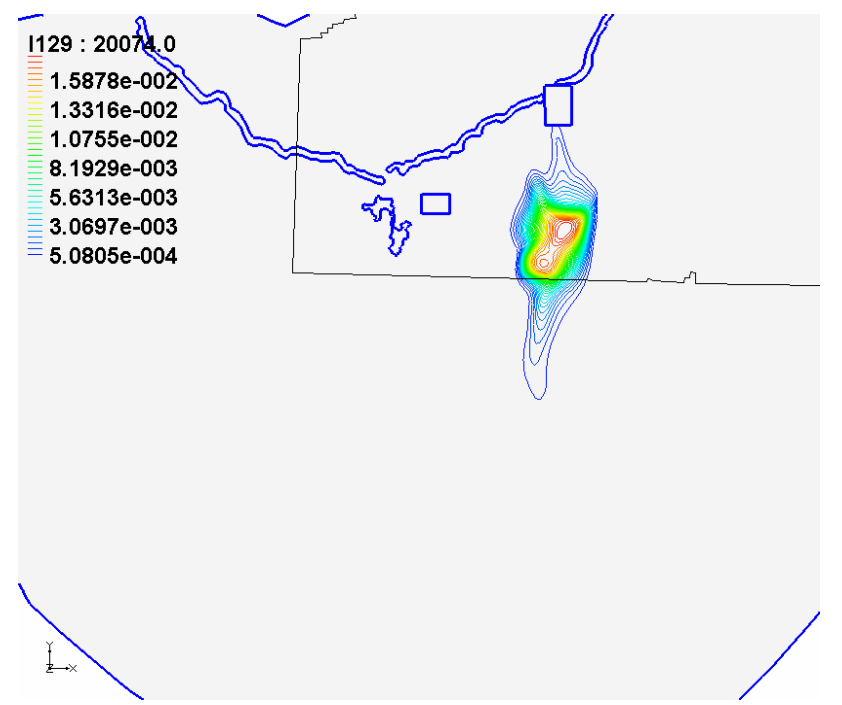

b. thin, variable properties

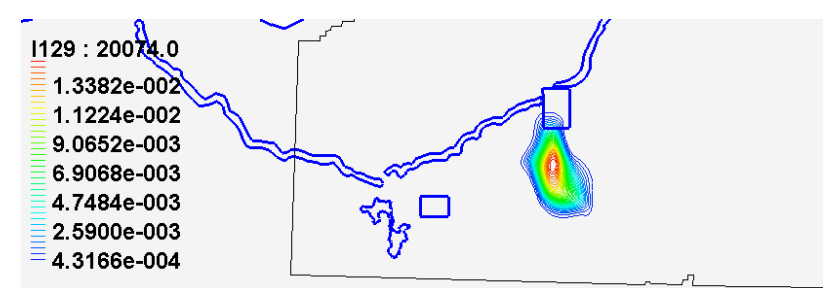

$3166 \mathrm{e}-004$

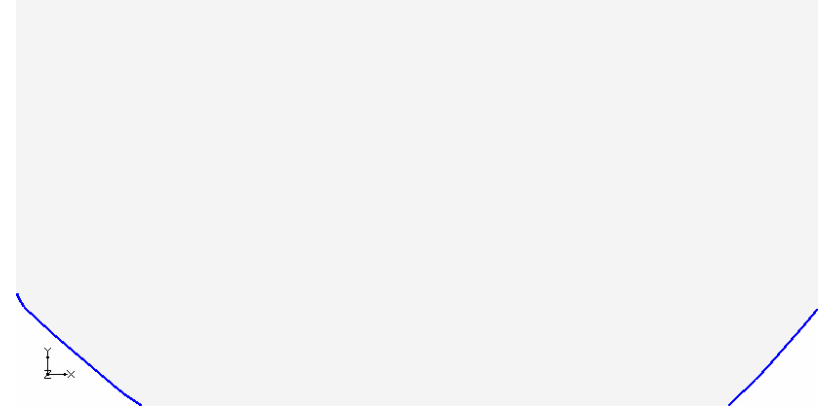

d. thin, uniform properties

Figure C-24. Plots of scaled I-129 concentration at maximum simulation time (20,075 days) for two aquifer thickness and two contaminant property scenarios. 


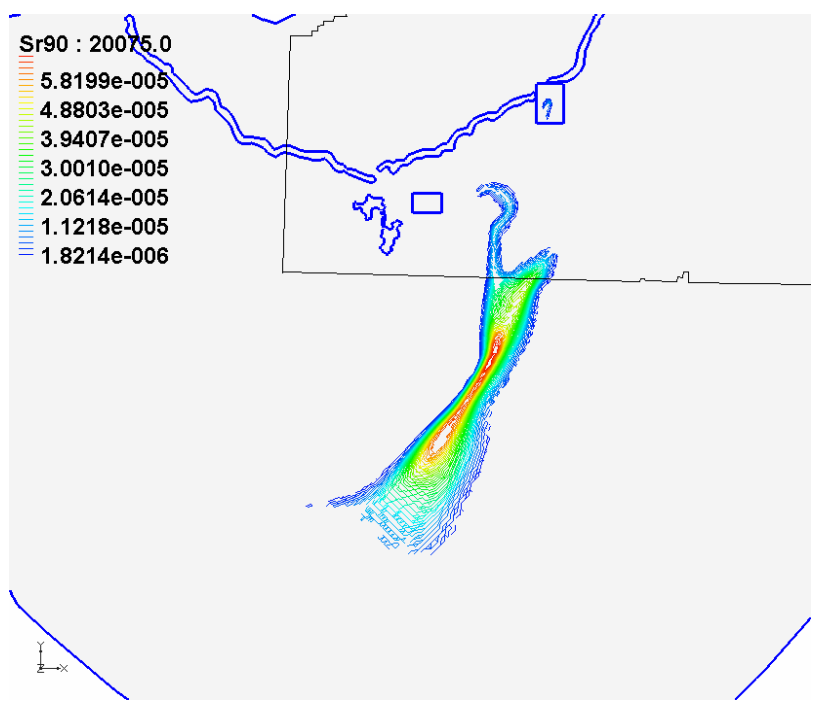

a. thick, variable properties

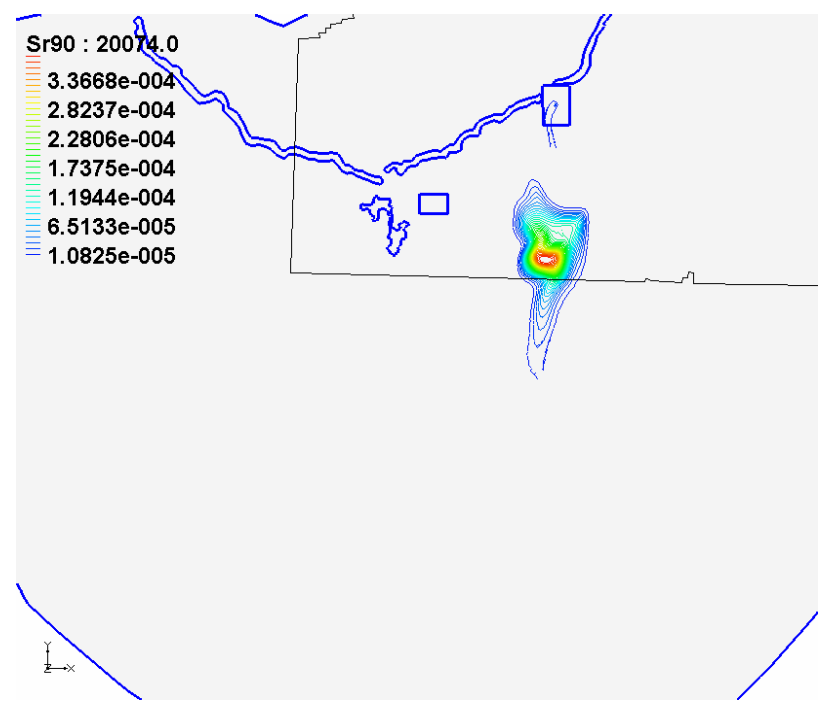

b. thin, variable properties

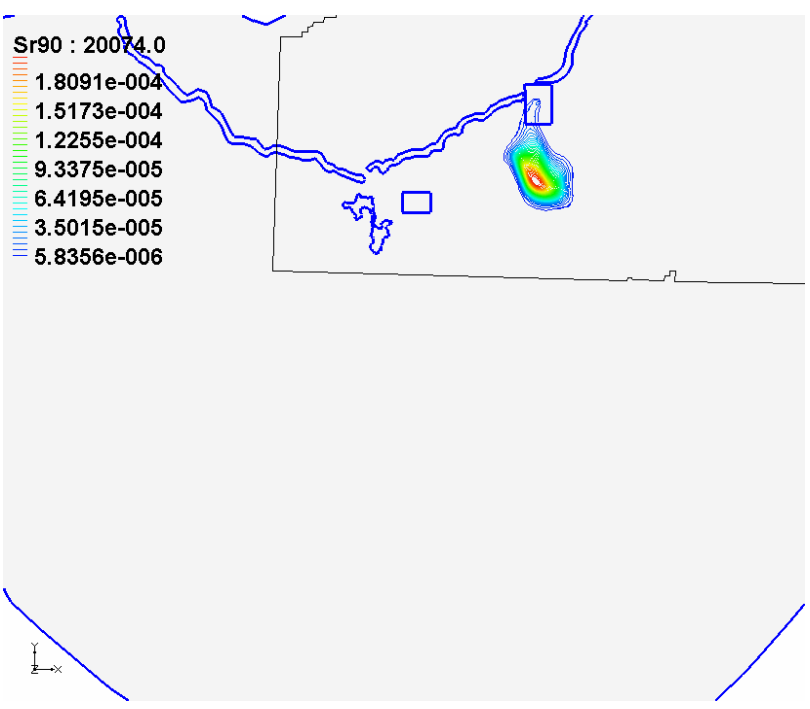

d. thin, uniform properties

c. thick, uniform properties

Figure C-25. Plots of scaled Sr-90 concentration at maximum simulation time (20,075 days) for two aquifer thickness and two contaminant property scenarios. 


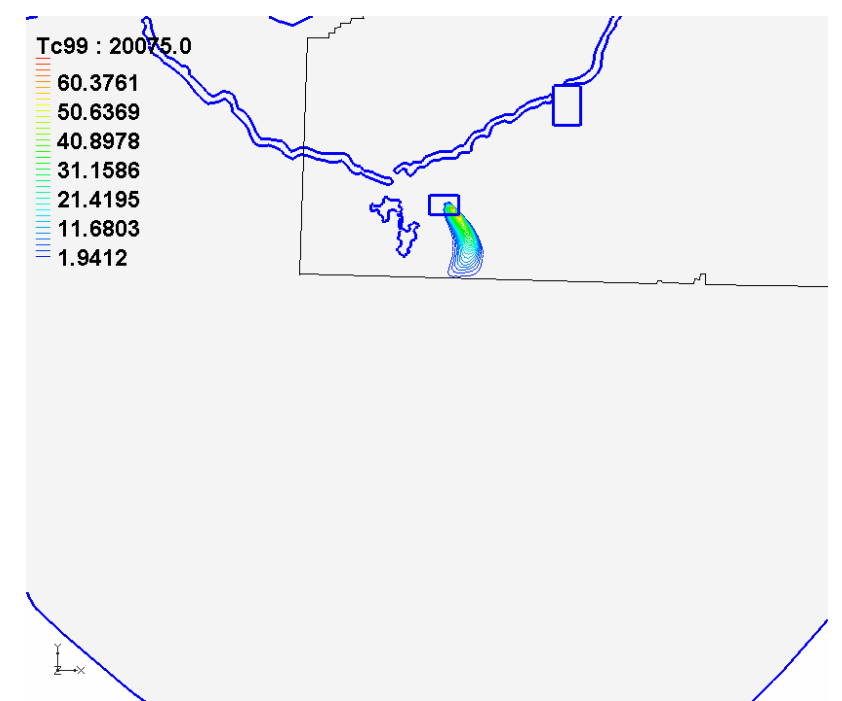

a. thick, variable properties
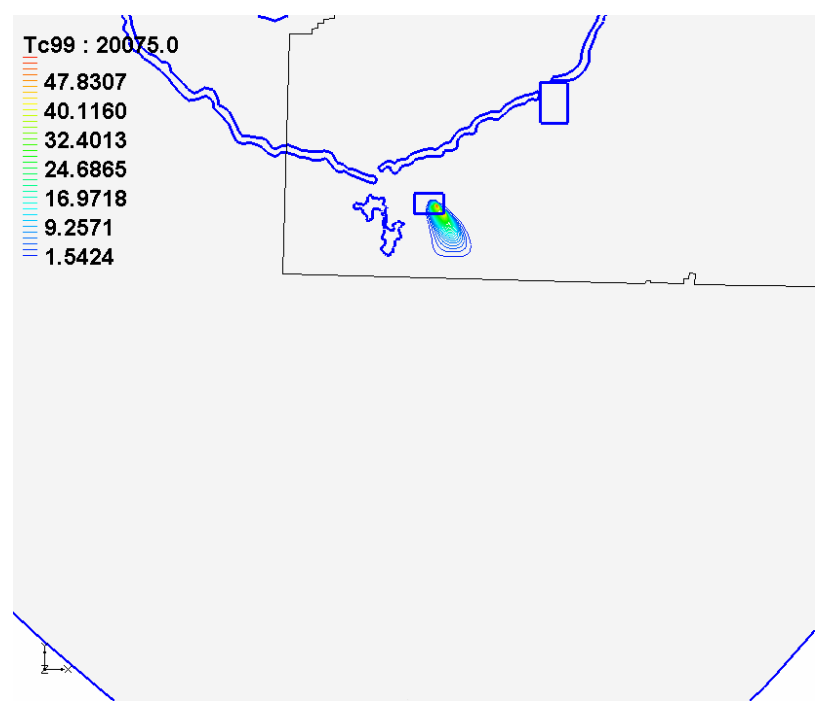

c. thick, uniform properties

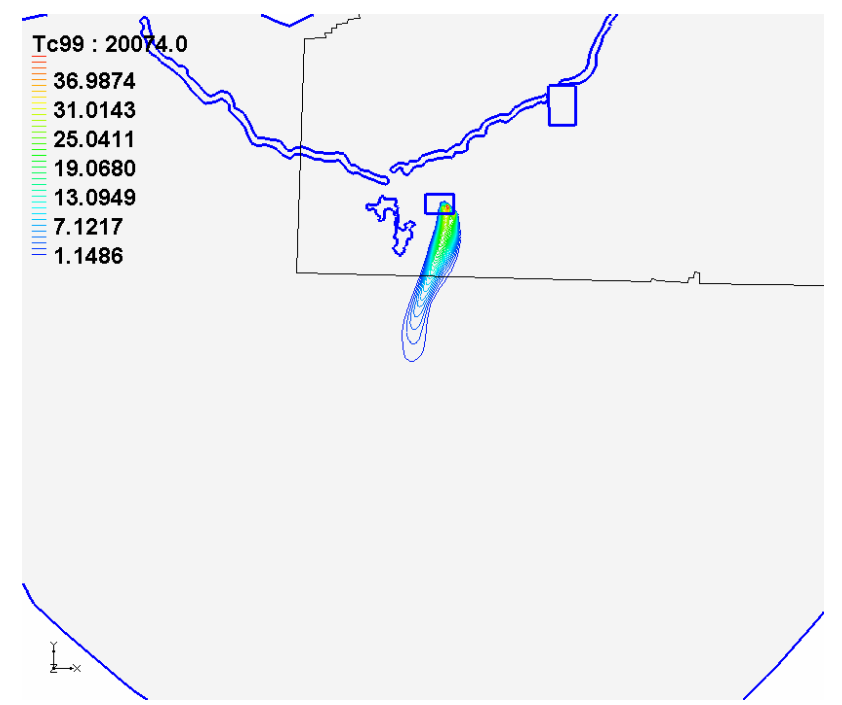

b. thin, variable properties
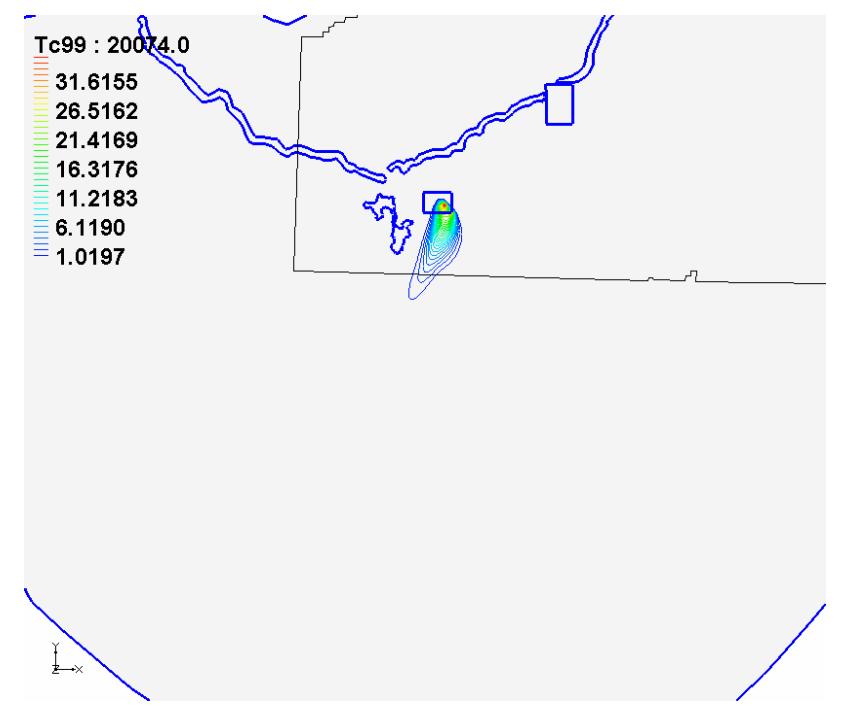

d. thin, uniform properties

Figure C-26. Plots of scaled Tc-99 concentration at maximum simulation time (20,075 days) for two aquifer thickness and two contaminant property scenarios. 


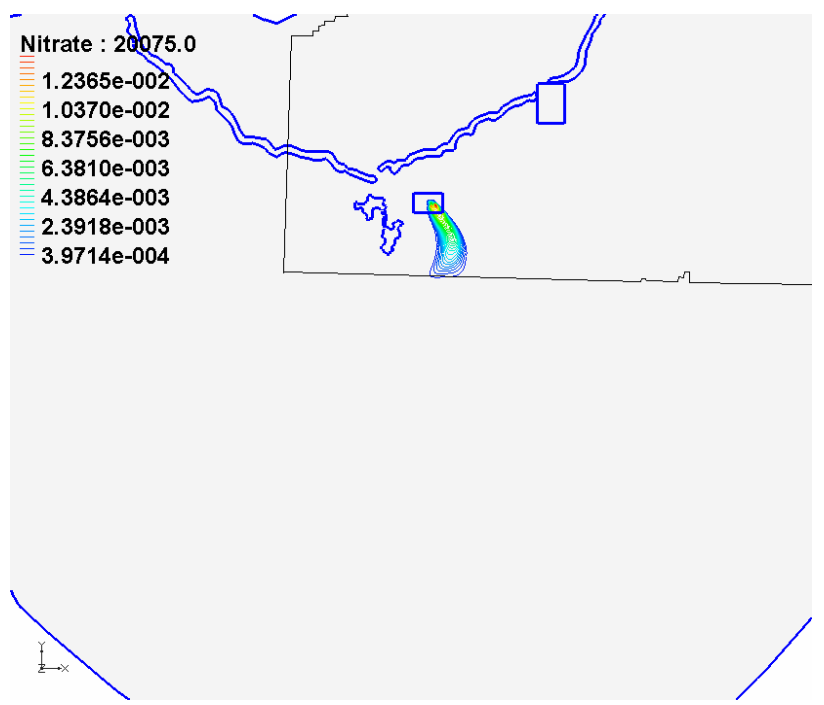

a. thick, variable properties
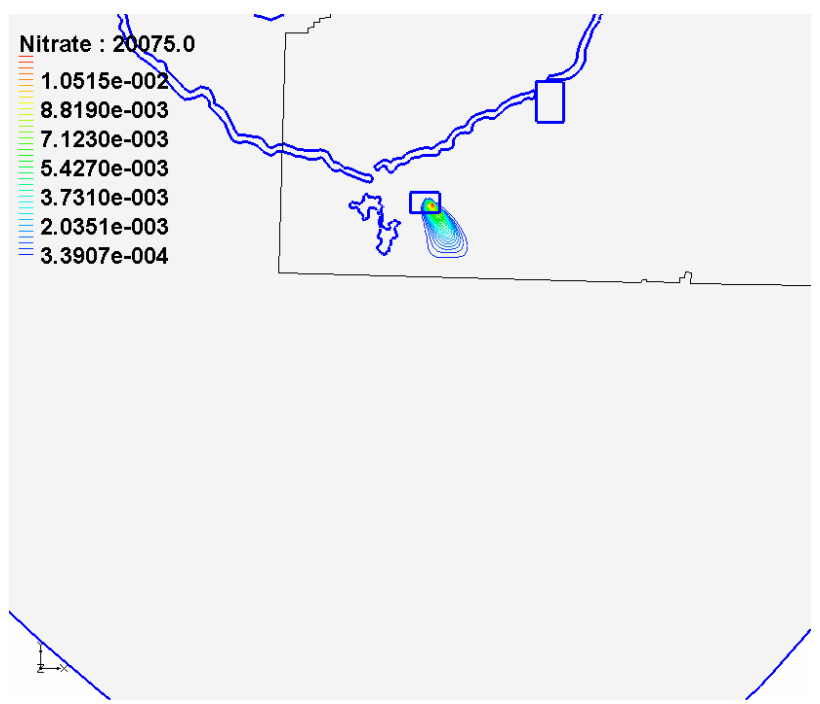

c. thick, uniform properties

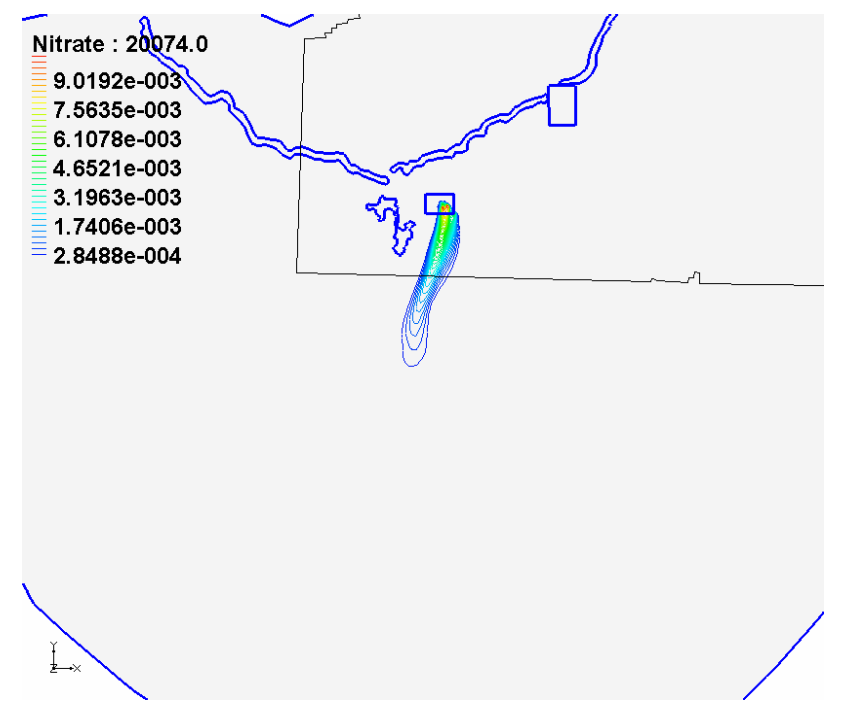

b. thin, variable properties

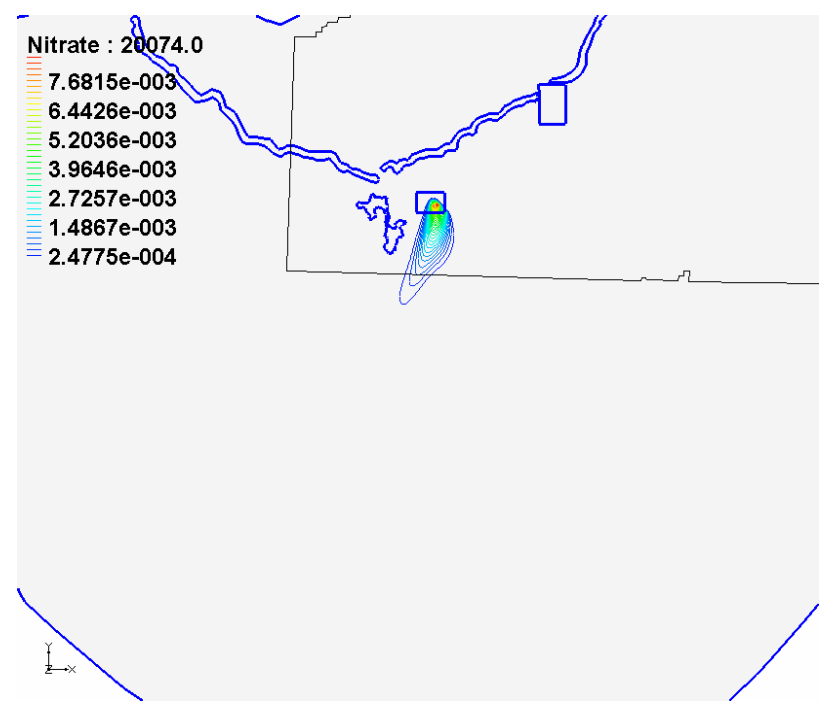

d. thin, uniform properties

Figure C-27. Plots of nitrate concentration at maximum simulation time (20,075 days) for two aquifer thickness and two contaminant property scenarios. 


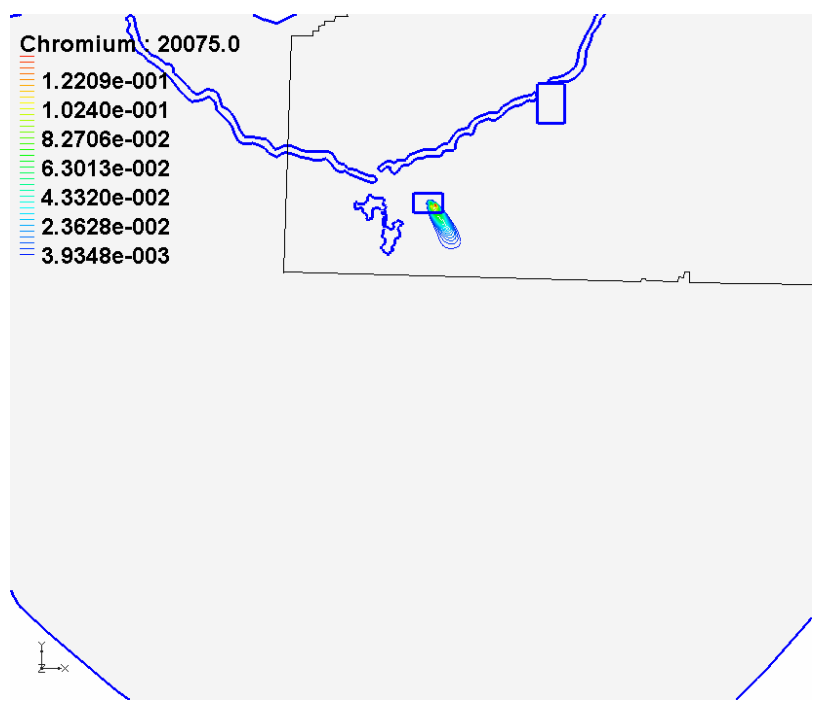

a. thick, variable properties
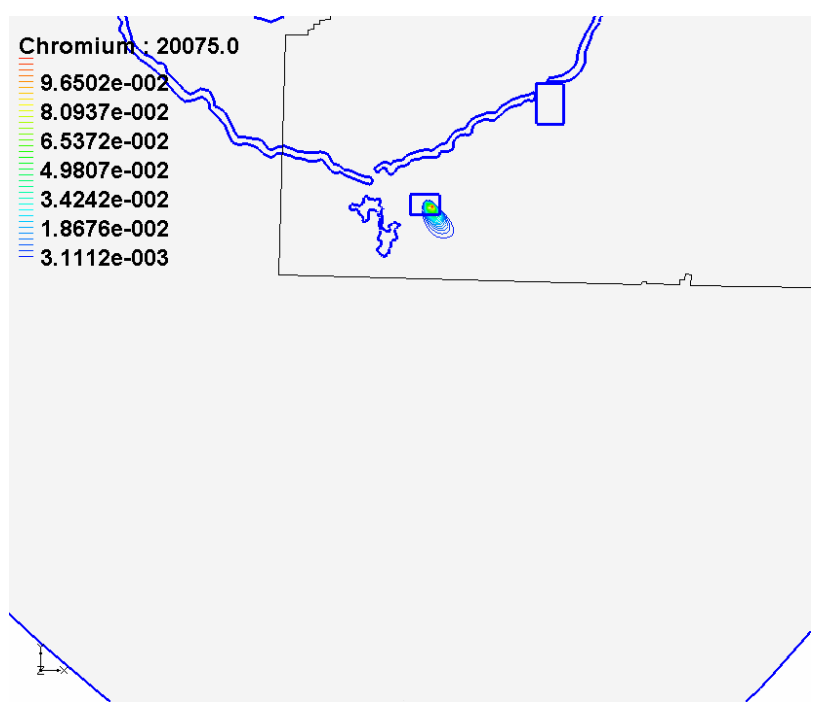

c. thick, uniform properties

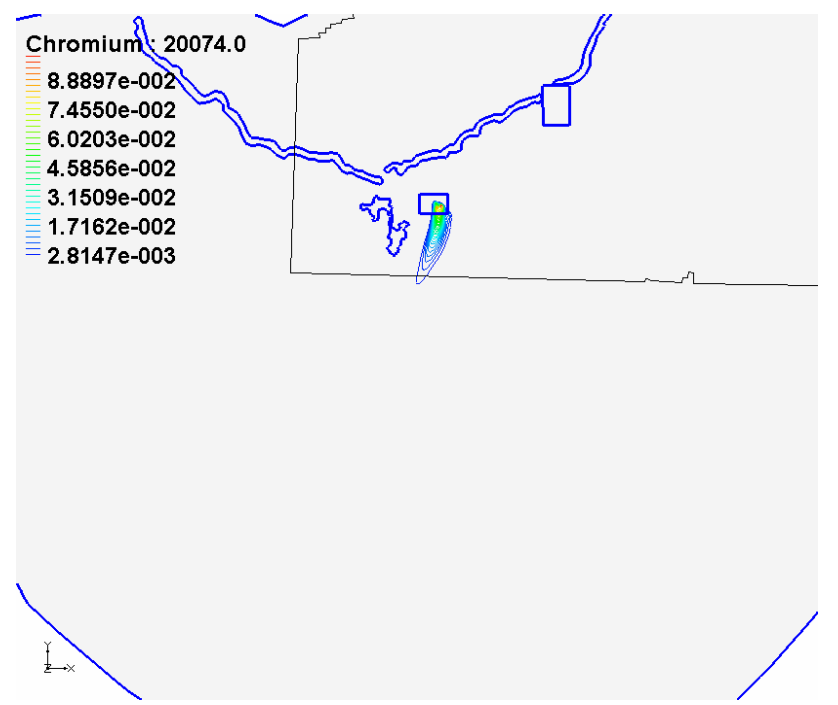

b. thin, variable properties

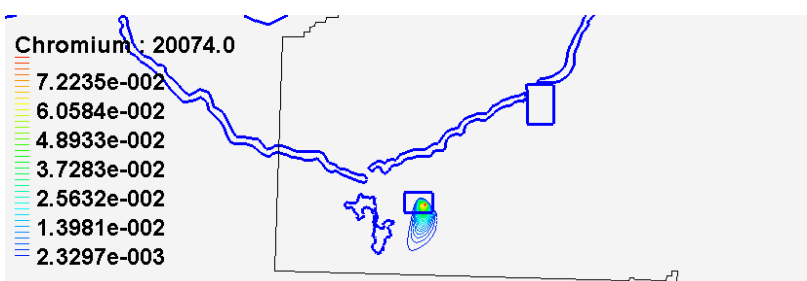

$1.3981 \mathrm{e}-002$
$=$

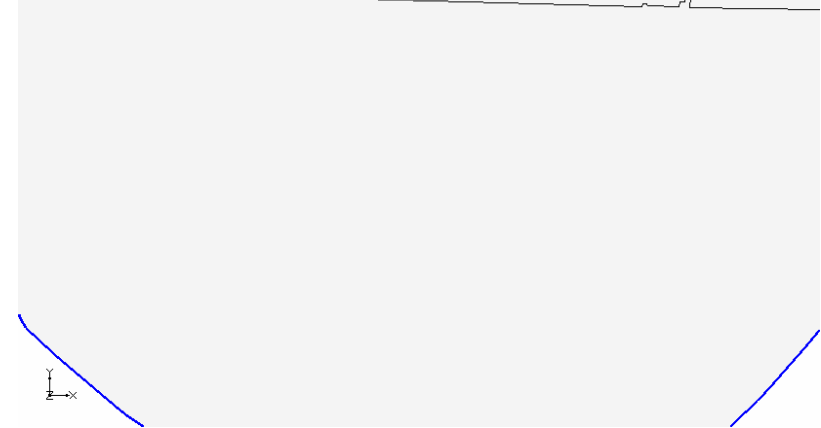

d. thin, uniform properties

Figure C-28. Plots of chromium concentration at maximum simulation time (20,075 days) for two aquifer thickness and two contaminant property scenarios. 


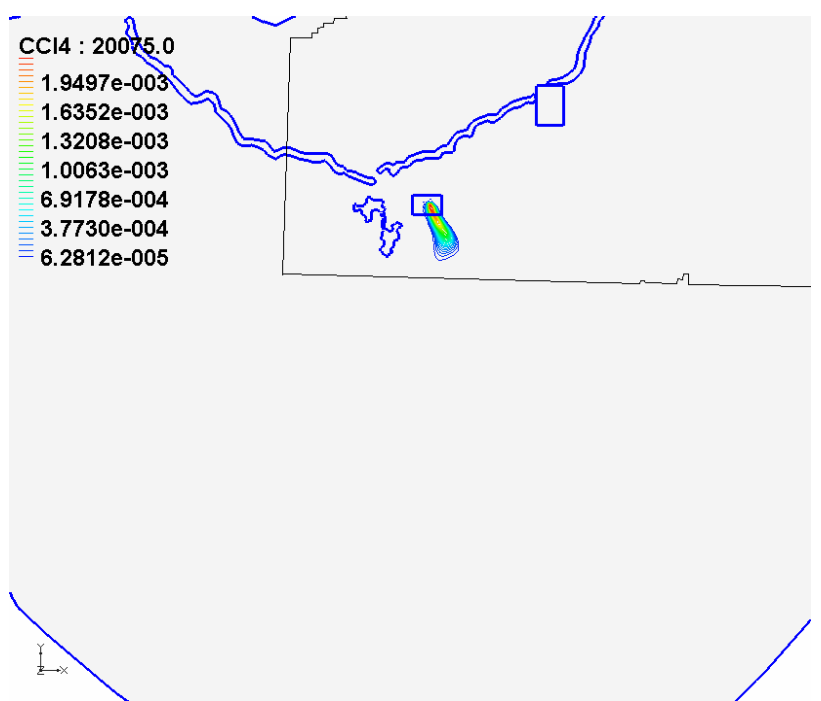

a. thick, variable properties
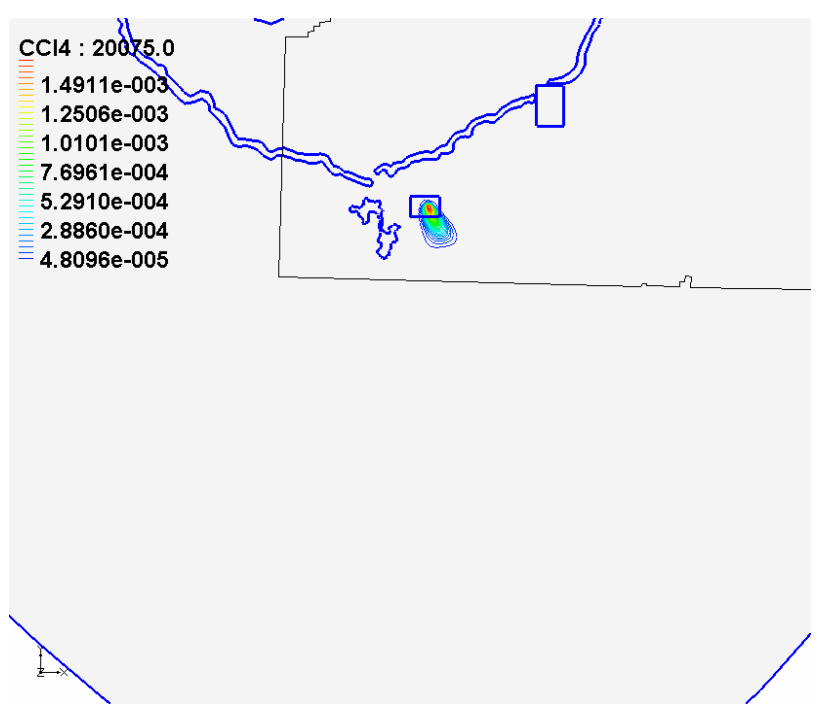

c. thick, uniform properties

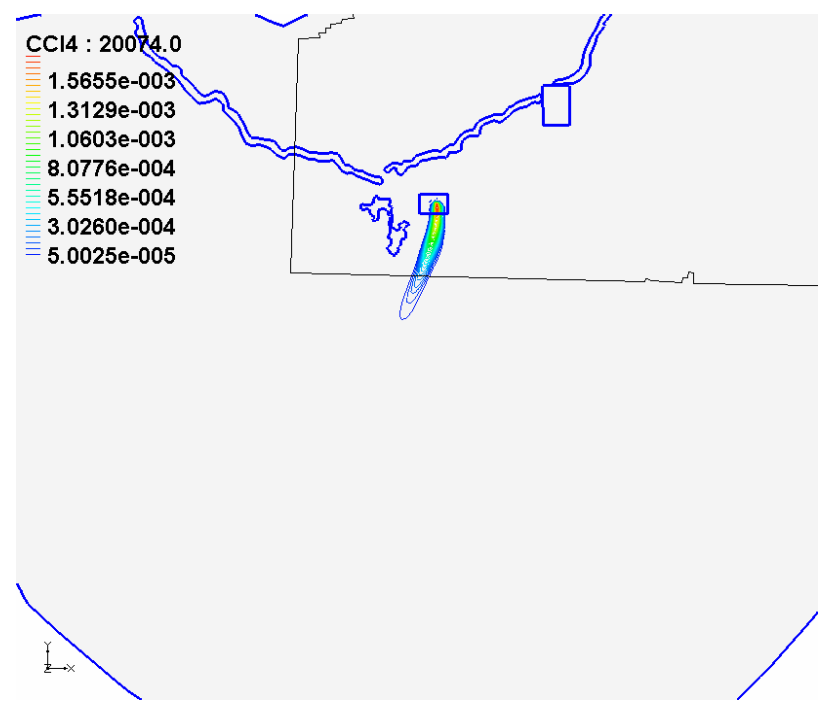

b. thin, variable properties

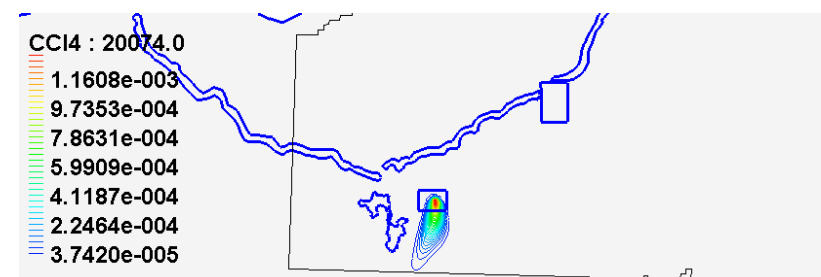

$.2464 \mathrm{e}-004$
$.7420 \mathrm{e}-005$

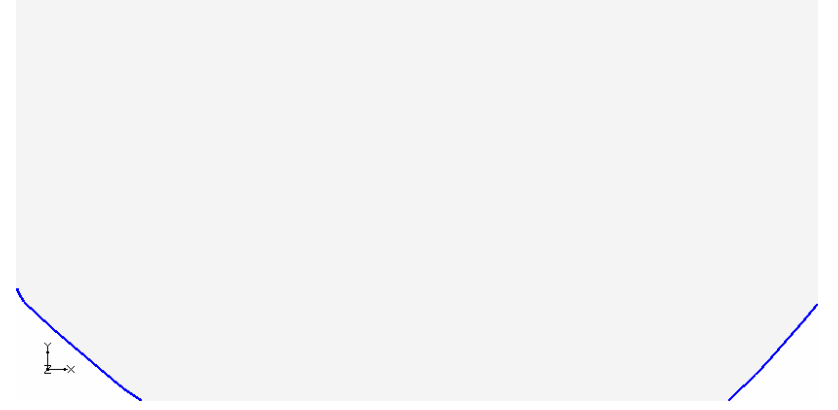

d. thin, uniform properties

Figure C-29. Plots of $\mathrm{CCl}_{4}$ concentration at maximum simulation time (20,075 days) for two aquifer thickness and two contaminant property scenarios. 
The only contaminant from the WAG 1 footprint that was simulated with the OU 10-08 two-dimensional transport model was TCE (Figure C-30). The simulated TCE plume for either aquifer thickness scenario with the variable transport properties extended along a very narrow pathway all the way southward across the INL by 2005 (Figure C-30). Obviously, there is no monitoring data to support this simulation result. The simulation result occurs primarily because of high velocities that are a result of the low porosities used in the variable transport property scenario. The plume simulated using the higher, uniform porosity of 0.10 did not migrate as far but moved much farther than the observed plume extent. In all four cases, the simulated plume is impacted by a high-velocity corridor in the two-dimensional flow model at the location of the axial volcanic ridge.

As expected, in all of these approximate plume distributions, the relative distribution of contaminants proved to be very sensitive to the porosity. Estimates of porosity that were derived from individual WAG models likely are representative of fluxes derived from constant head boundaries. The larger porosity required in the OU 10-08 two-dimensional transport model to more closely represent known contaminant distributions is likely attributed to use of the larger, and believed to be more correct, specified flux boundary along the northeastern OU 10-08 model domain boundary.

Graphical comparison of OU 10-08 simulation results with results for tritium and nitrate from individual WAG models is provided in Figures C-31 and C-32. Figure C-31 contains a comparison for the variable transport property scenario and Figure C-32 demonstrates the uniform property results. The region portrayed is smaller than those shown previously, in order to illustrate more detail in the comparison to individual WAG model results. While there are observable differences, overall the OU 10-08 simulated plume configurations are generally consistent with the individual WAG models' directions and extents. Simulated concentrations will be different because the OU 10-08 two-dimensional model are lower as a result of having uniform concentrations over the entire vertical thickness of the aquifer.

The plume configurations in these figures show that the velocity field resulting from the simulated water levels differs between the thick and thin aquifer scenarios near important contamination sources such as INTEC and the RWMC. In Figure C-32, the thick aquifer scenario shows a simulated nitrate plume that moves noticeably to the southeast, whereas the simulated nitrate plume for the thin scenario initially moves almost directly south before turning more southwest. Investigation of the water velocity field resulting from the two scenario's MODFLOW simulation explains the difference.

Figure C-33 shows the distribution of linear pore water velocity vectors for the entire model domain for each aquifer thickness scenario. Regions of higher velocity resulting from the high-conductivity region that was estimated in the inverse solution for the two-dimensional flow model are noteworthy. These regions correspond well with the estimated location of the axial volcanic high discussed in the conceptual model section. Simulated contaminant plumes that extend southward sufficiently far from facilities are impacted by this higher velocity zone.

Differences in velocities between the thick and thin aquifer scenarios can be discerned at the model domain scale but can be seen more easily in Figure C-34, which shows only the southern portion of the INL Site. The two rectangles in the figure show the location of the RWMC and INTEC vadose zone footprints. A wider range of velocities is observed in the thick aquifer scenario, in both direction and magnitude of velocity. Both aquifer thickness scenarios show an initial southerly flow direction from INTEC. Slightly farther south, a southwesterly velocity occurs, which bodes well for eventual calibration to monitoring data that also shows a southwesterly component to transport in this key region between RTC, INTEC, and RWMC. 


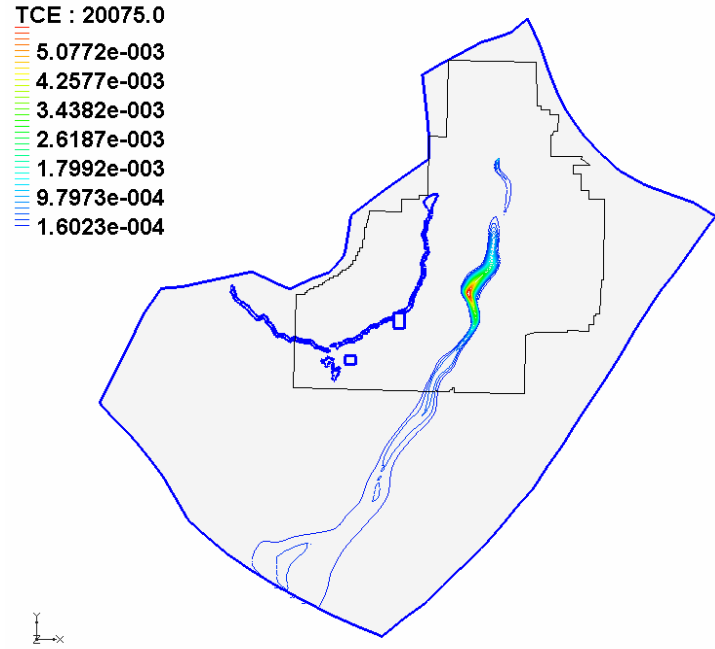

a. thick, variable properties

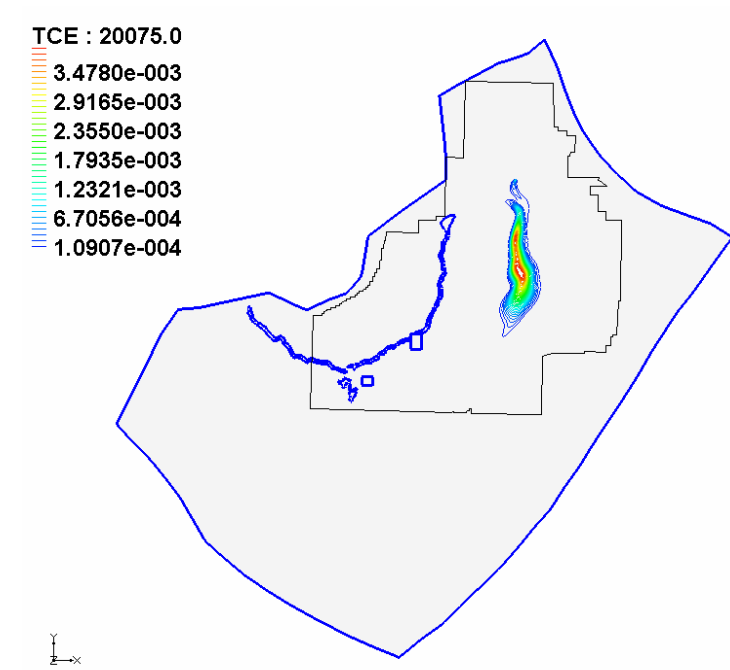

c. thick, uniform properties

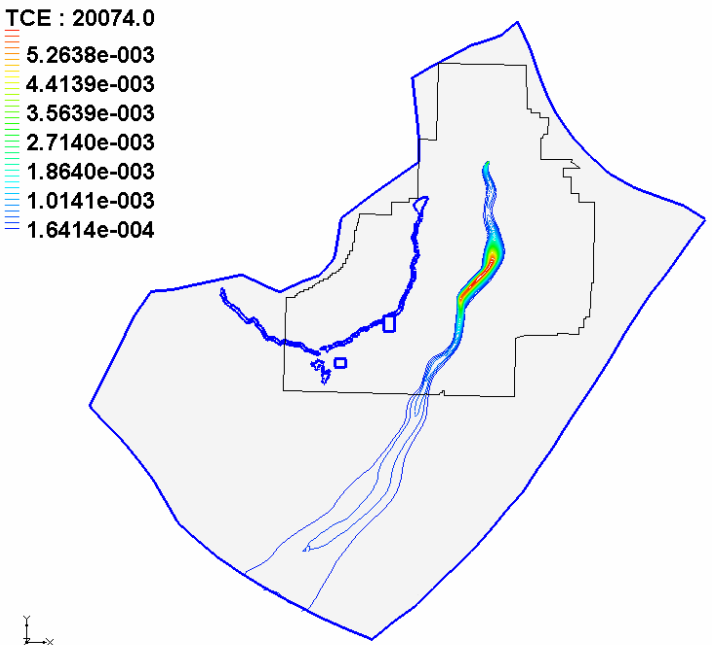

b. thin, variable properties

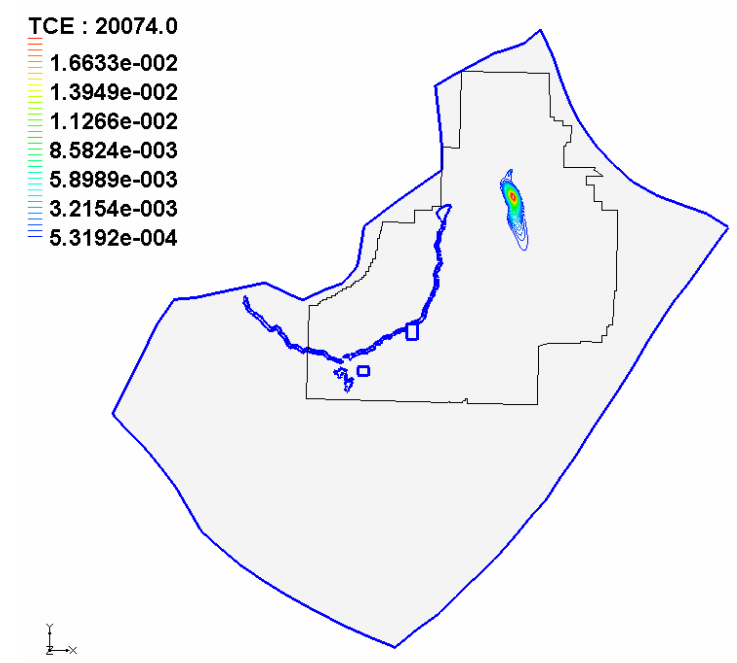

d. thin, uniform properties

Figure C-30. Plots of TCE concentration at maximum simulation time (20,075 days) for two aquifer thickness and two contaminant property scenarios. 


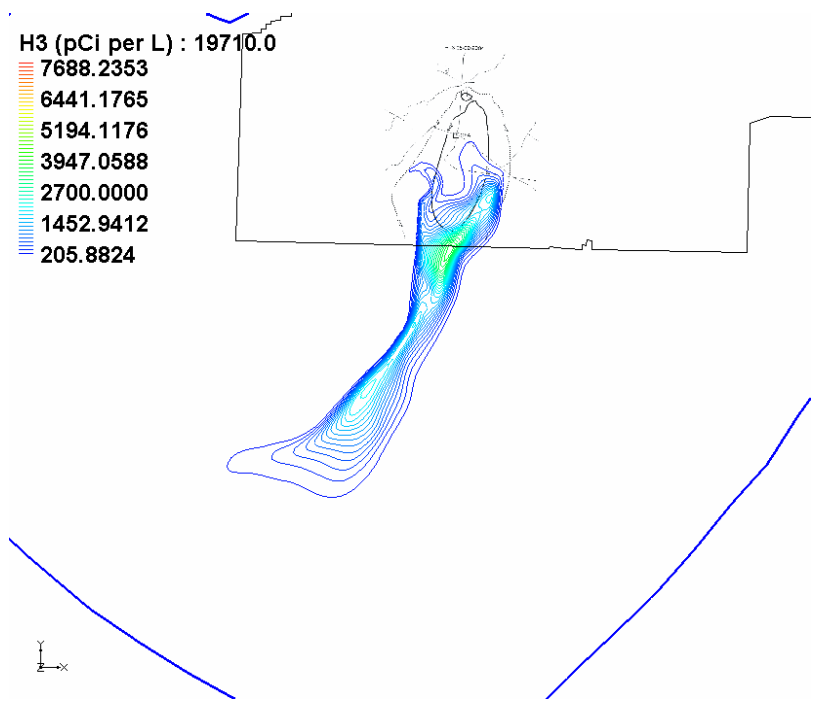

a. thick, variable properties

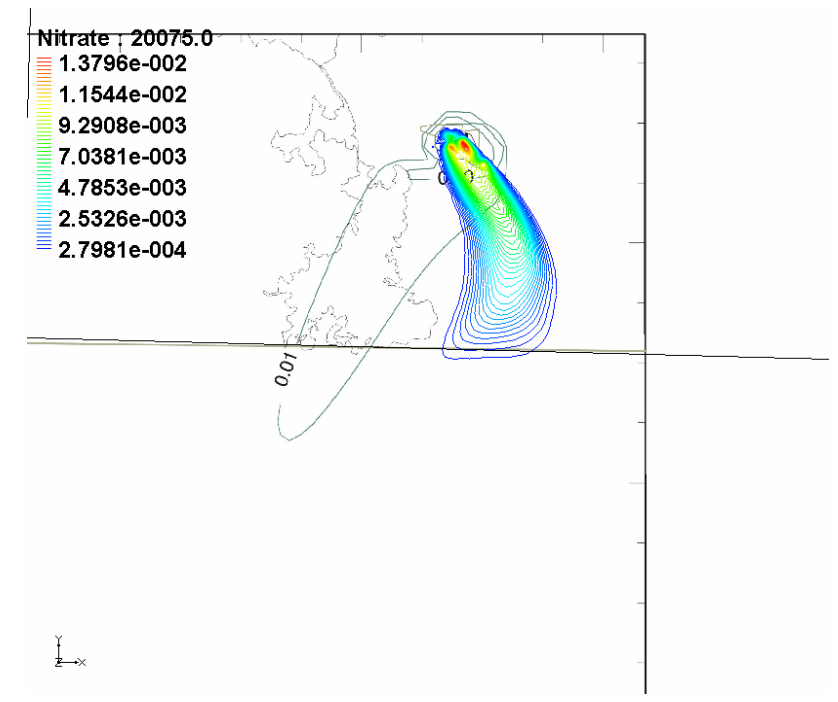

c. thick, uniform properties

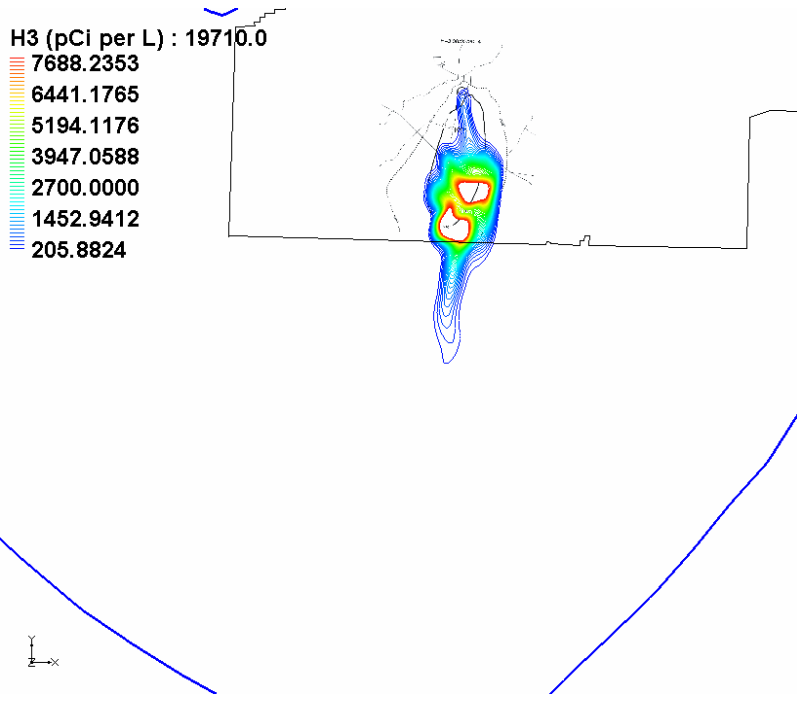

b. thin, variable properties

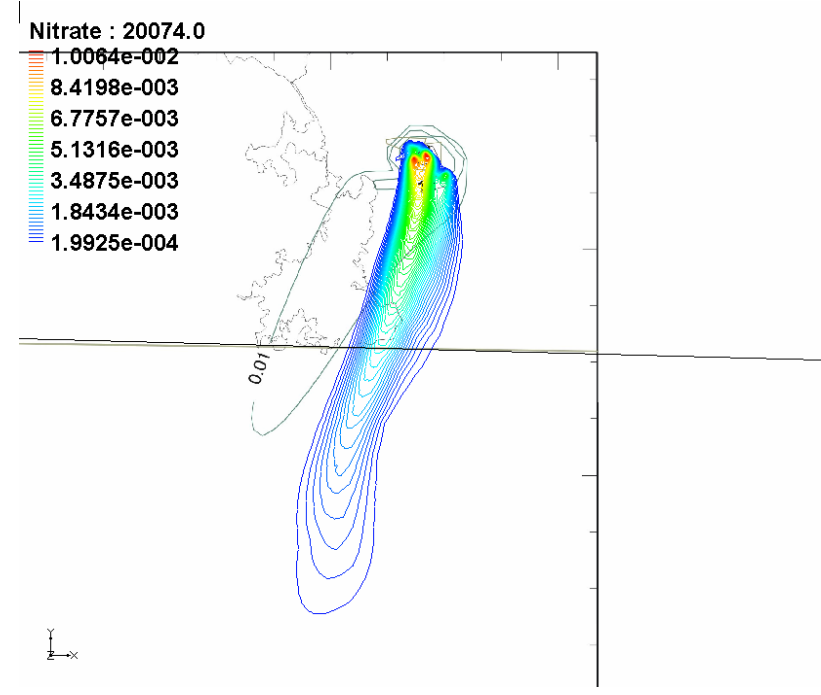

d. thin, uniform properties

Figure C-31. Comparison of the simulated plumes with individual WAG models (tritium plume by WAG 3 model and nitrate plume by WAG 7) for variable transport property scenario. 


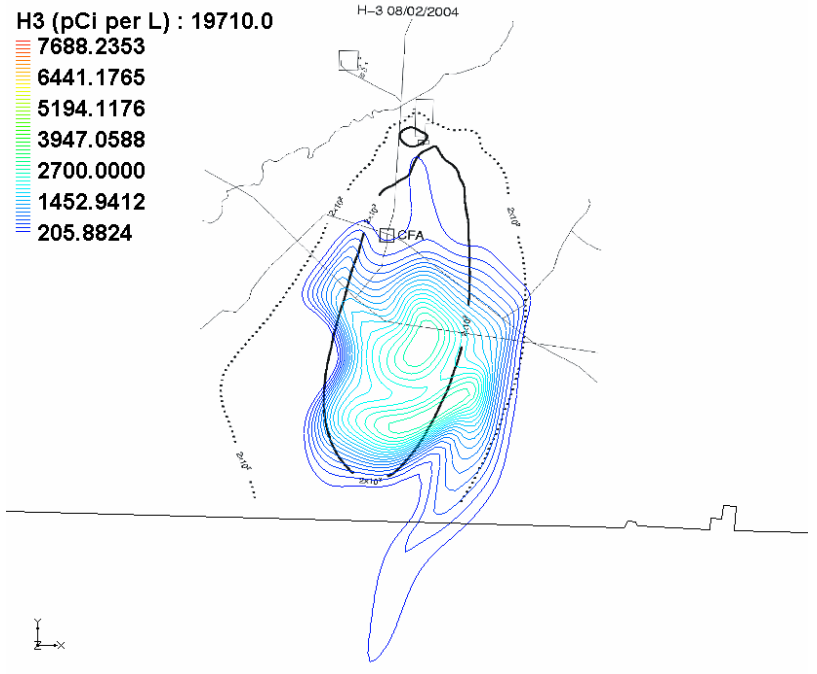

a. thick, uniform properties

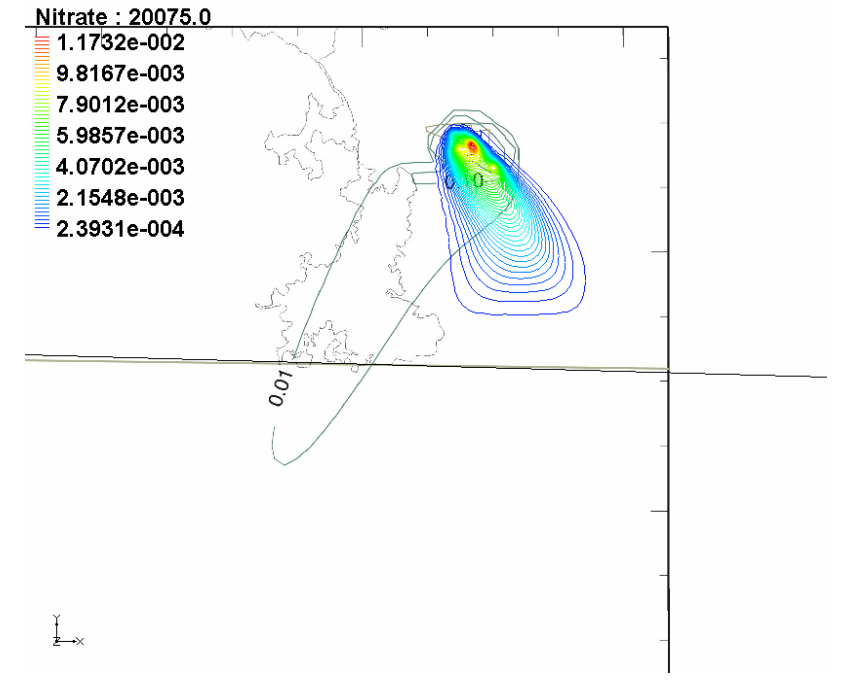

c. thick, variable properties

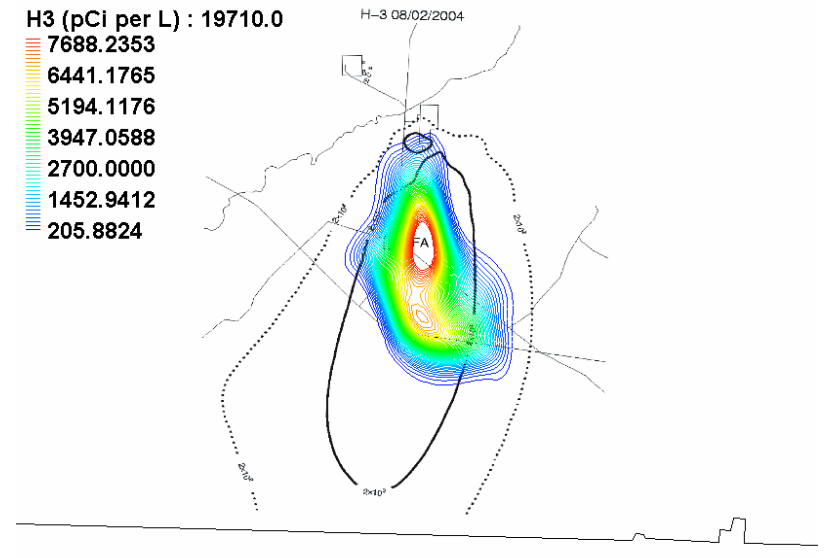

$L_{x}$

b. thin, uniform properties

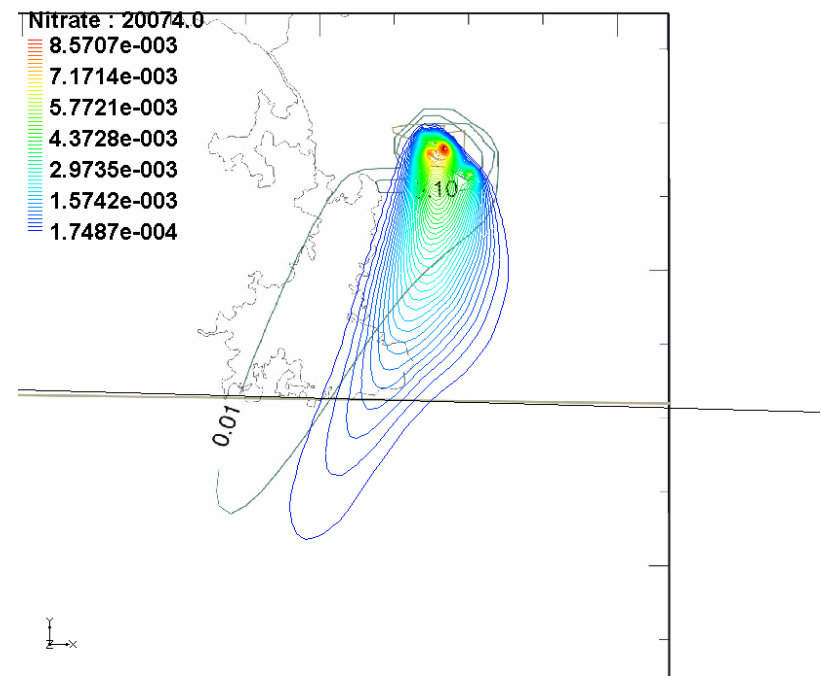

d. thin, variable properties

Figure C-32. Comparison of the simulated plumes with individual WAG models (tritium plume by WAG 3 model and nitrate plume by WAG 7) for uniform transport property scenario. 

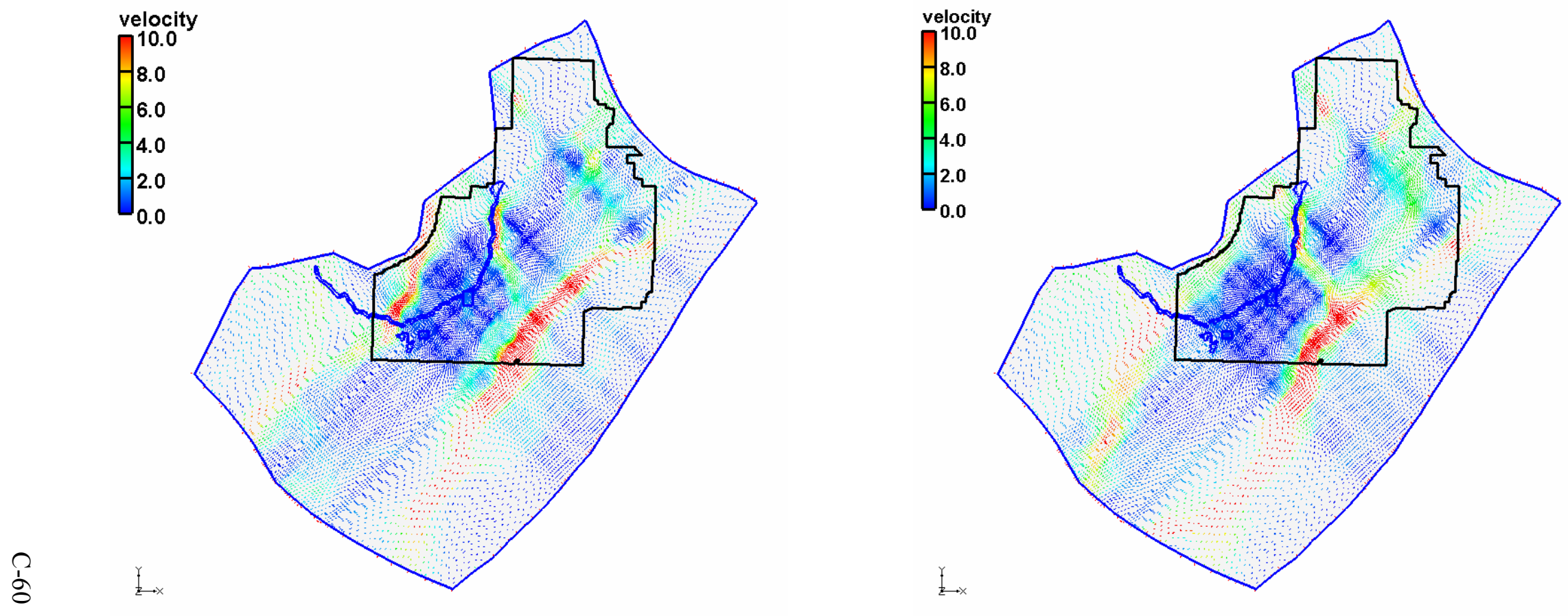

Figure C-33. Linear pore water velocity ( $\mathrm{m} / \mathrm{d}$ ) distribution for the entire domain (uniform effective porosity of 0.10 ): left-thick scenario; right-thin scenario. 

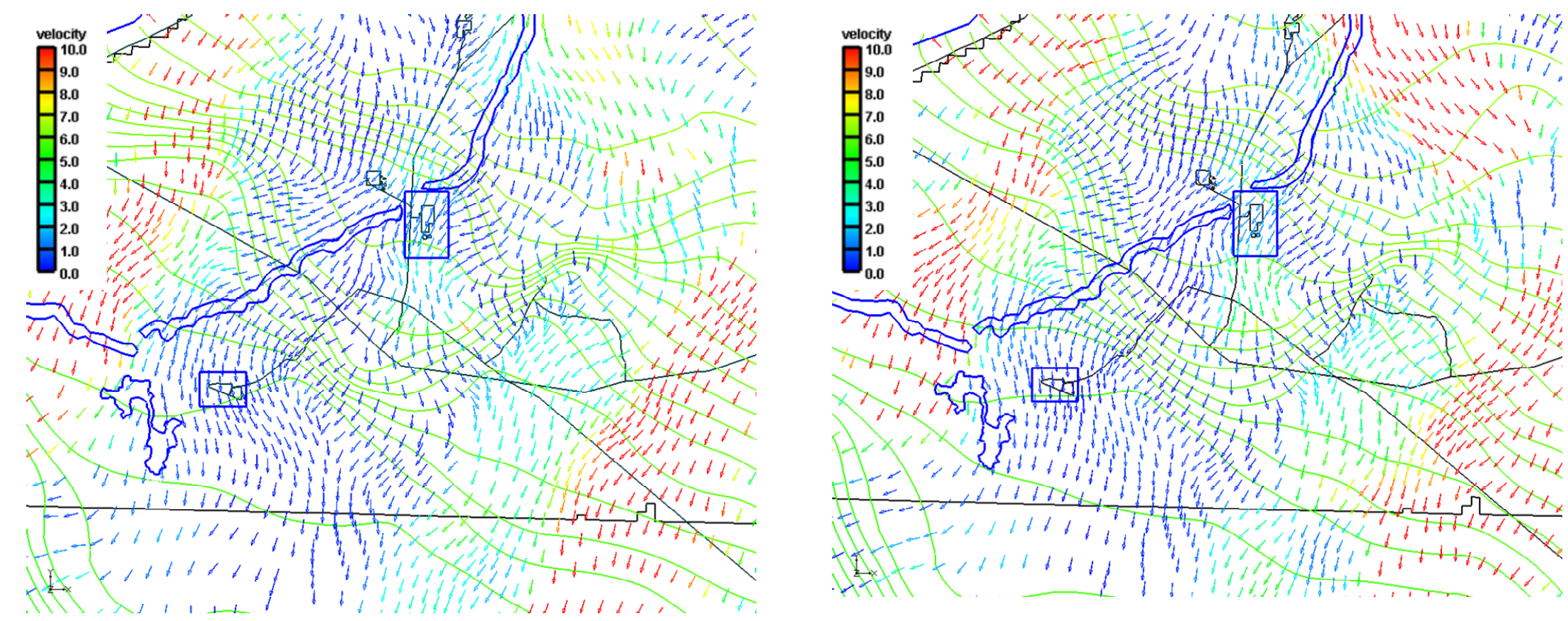

Figure C-34. Linear pore water velocity (m/d) distribution near vicinity of WAG 3 and WAG 7 (uniform effective porosity of 0.10 ): left-thick scenario; right-thin scenario. 
The simulated southeasterly velocities beneath the RWMC footprint for the thick aquifer scenario (that were previously seen to result in a southeasterly direction to the nitrate plume) can be seen in Figure C-34. The velocities beneath RWMC are more southerly in the thin aquifer scenario. Another difference that can be seen in Figure C-34 is a high-velocity "channel" to the northwest of RWMC that is more prevalent in the thick aquifer scenario than in the thin aquifer scenario. This "channel" does not necessarily correspond to anything in the conceptual model but was apparently necessary in the inverse solution to transmit sufficient water. This "channel" actually conflicts with interpretations of stagnant water at the USGS-22 location and thereby highlights the need to consider more than just water levels when calibrating a model of contaminant transport in the aquifer.

Figure C-35 shows the velocity distributions from Figure C-34 overlying the estimated conductivity distributions that resulted from solving the inverse problem for each scenario with a constrained set of pilot points and the updated boundary conditions. As this figure illustrates, areas of low conductivity between RWMC and INTEC for the thick scenario have a large effect on resulting contaminant plume configurations. The southeasterly direction of the nitrate plume (see the thick uniform property scenario in Figure C-32) results from a low conductivity area to the northeast of RWMC that serves as a flow barrier. This barrier forces water coming from the northeast (flowing in the more regional southwest direction) around the low conductivity area. Groundwater movement directly north of RWMC moves in behind this barrier and flows to the southeast. This southeast movement affects plume orientation of contaminants entering the aquifer from RWMC, as shown previously in Figure C-32.

In summary, this two-dimensional transport model was an intermediate step in development of a fully three-dimensional transport model. The two-dimensional model provided the opportunity to develop an approach for adding the contaminant source terms in a manner compatible with the individual WAG models. It should be reiterated that the simulated distributions of contaminants do not adequately represent actual distributions because the model treats them as uniformly distributed across the entire aquifer thickness. In actuality, WAG 1, WAG 3, and WAG 7 source terms enter the layered system at the top of the aquifer or are injected at depth. Because of the different points of entry and anisotropy of this layered system, a fully three-dimensional numerical model is required. 

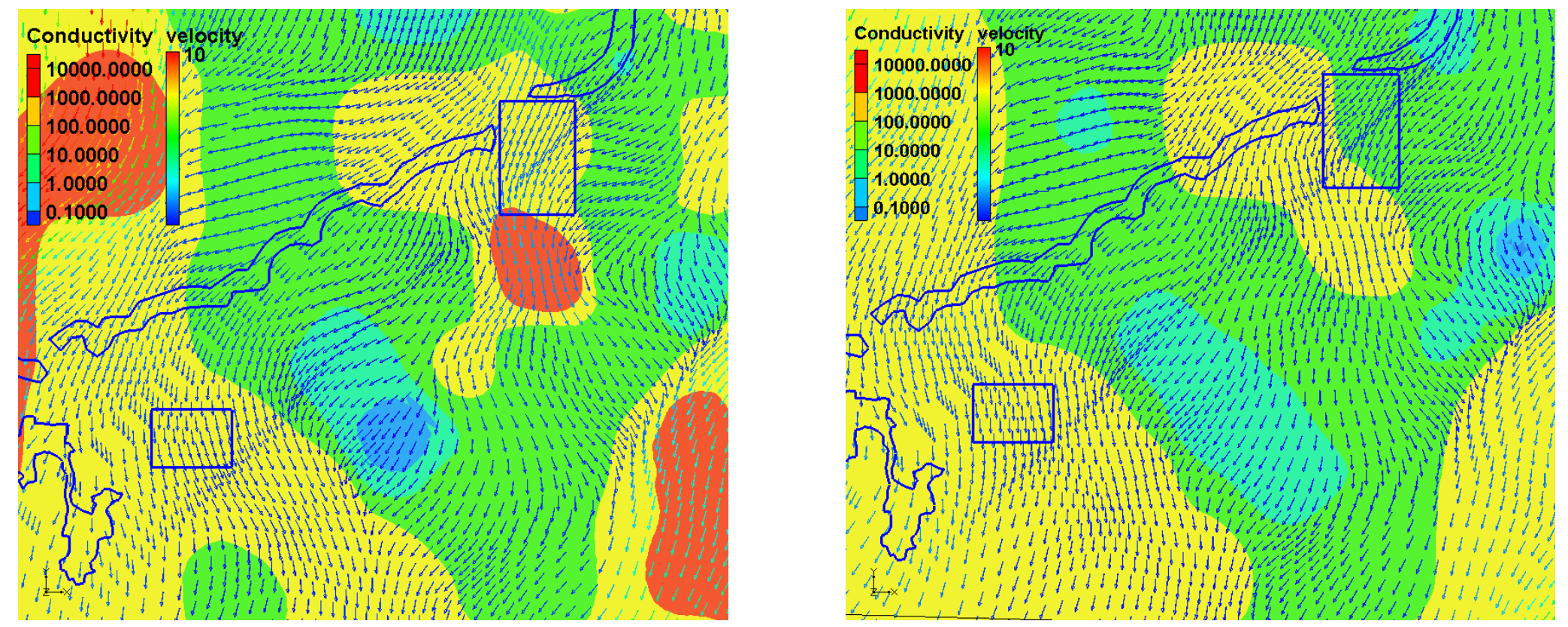

f Figure C-35. Linear pore water velocity $(\mathrm{m} / \mathrm{d})$ overlying the conductivity fields near vicinity of WAG 3 and WAG 7 (uniform effective porosity of 0.10 ): left-thick scenario; right-thin scenario. 


\section{C-5. SUMMARY}

Implementation of contaminant source terms for sources at WAGs 1, 3, and 7 was accomplished with the OU 10-08 two-dimensional transport model. Although calibration was not performed, initial transport simulation results were compared with results from individual WAG transport models from the OU 3-14 and OU 7-13/14 programs. Updates to the two-dimensional flow model were also accomplished. These include updating the model's northeastern boundary conditions to reflect specified-flux conditions and balance the model's water budget against more widely-accepted concepts of water flux through the aquifer.

Thick aquifer and thin aquifer scenarios were implemented in the 2-D transport model. The transport model examined both a uniformly-distributed transport property and a scenario reflecting transport properties assigned in the individual WAG transport models.

Relative distribution of contaminants proved to be extremely sensitive to the porosity. A larger porosity than those used in the individual WAG facility models was required in the OU 10-08 transport model to keep velocities small enough to resemble known contaminant distributions. This change in porosities likely is attributed to using specified flux boundaries for the northeastern OU 10-08 model domain boundary. This change increased the amount of water moving through the system by approximately a factor of three.

The two-dimensional transport model described in this report was constructed as an intermediate step to both gain insight into how the model performs and to work through the process of incorporating the complex addition of source terms into the model. Contaminant distributions in the ESRP aquifer cannot be accurately simulated with relatively simple 2-D systems assuming fully integrated, constant contaminant concentration across the depth of this dimension. Rather, transport is inherently threedimensional as contaminants enter the aquifer both at the top of the aquifer from the vadose zone and at depth in the aquifer from injection. 


\section{C-6. REFERENCES}

42 USC $\S 9601$ et seq., 1980, “Comprehensive Environmental Response, Compensation and Liability Act of 1980 (CERCLA/Superfund)," United States Code, December 11, 1980.

Arnett, R. C., R. C. Martineau, and Michael J. Lehto, 1990, Preliminary Numerical Model of Radionuclide Transport in the Snake River Plain Aquifer Near the Idaho National Engineering Laboratory, EGG-WM-8820, Idaho National Engineering Laboratory.

Busenberg, E., L. N. Plummer, and R. C. Bartholomay, 2001, Estimated Age and Source of the Young Fraction of Ground Water at the Idaho National Engineering and Environmental Laboratory, USGS Water Resources Investigations Report 01-4265.

Barraclough, J. T., W. E. Teasdale, J. B. Robertson, and R. G. Jensen, 1967, Hydrology of the National Reactor Testing Station, Idaho, 1966, U.S. Geological Survey Open-File Report, IDO-22049, October 1967.

Cecil, L. D., J. R. Pittman, T. M. Beasley, R. L. Michel, R. L. Kubik, P. Sharma, U. Fehn, and H. E. Gove, 1992, "Water Infiltration Rates in the Unsaturated Zone at the Idaho National Engineering Laboratory Estimated from Chlorine-36 and Tritium Profiles, and Neutron Logging," Kharaka Yousifk and Ann S. Maest (eds.), Proceedings of the $7^{\text {th }}$ International Symposium on Water-Rock Interaction, July 13-18, 1992, pp. 709-714.

Cecil, L. D., J. A. Welhan, J. R. Green, S. K. Frape, and E. R. Sudicky, 2000, Use of chlorine-36 to determine regional-scale aquifer dispersivity, eastern Snake River Plain aquifer, Idaho/USA. Nuclear Instruments and Methods in Physics Research B 172, p. 679-687.

Contor, B. A., 2004, Recharge on Non-irrigated Lands, Idaho Water Resource Research Institute Technical Report 04-006, Eastern Snake River Plain Aquifer Model Enhancement Project Scenario Document DDW-003, September 2004.

Cosgrove, D. M., B. A. Contor, and G. S. Johnson, 2006, Enhanced Snake Plain Aquifer Model Final Report, Idaho Water Resources Research Institute Technical Report 06-002, July 2006.

DOE-ID, 2002, Waste Area Group 10, Operable Unit 10-08, Remedial Investigation/Feasibility Study Work Plan (FINAL), DOE/ID-10902, Rev. 0, U.S. Department of Energy Idaho Operations Office, August 2002.

DOE-ID, 2004, Idaho National Engineering and Environmental Laboratory Operable Unit 10-08 Sitewide Groundwater Model Work Plan (FINAL), DOE/NE-ID-11188, Rev. 0, U.S. Department of Energy Idaho Operations Office, December 2004.

DOE-ID, 2006a, Remedial Investigation and Baseline Risk Assessment for Operable Unit 7-13/14, DOE/ID-11241, Rev. 0, U.S. Department of Energy Idaho Operations Office, May 2006.

DOE-ID, 2006b, Operable Unit 3-14 Tank Farm Soil and Groundwater Remedial Investigation/Baseline Risk Assessment, DOE/NE-ID-11227, Rev. 0, U.S. Department of Energy Idaho Operations Office, April 2006.

Freeze, R. A., and J. A. Cherry, 1979, Groundwater, Englewood Cliffs, New Jersey: Prentice-Hall. 
Garabedian, S. P., 1992, Hydrology and Digital Simulation of the Regional Aquifer System, Eastern Snake River Plain, Idaho, U.S. Geological Survey Professional Paper 1408-F.

Goode, D. J., and L. F. Konikow, 1990, "Reevaluation of Large-Scale Dispersivities for a Waste Chloride Plume: Effects of Transient Flow," International Conference on Calibration and Reliability in Groundwater Modeling, International Association of Hydrological Sciences, The Hague, The Netherlands, September 1990.

Hull, L., and M. N. Pace, 2000, Solubility Calculations for Contaminants of Potential Concern, OU 7-13/14, INEEL/EXT-2000-00465, Rev. 0, Idaho National Engineering and Environmental Laboratory, April 2000.

INEEL, 2002, TAN Large Scale Groundwater Model TETRAD to MODFLOW/MT3MDMS Conversion Task, INEEL/EXT-02-00632, Rev. 0, Idaho National Engineering and Environmental Laboratory, November 2002.

Johnson, T. M., R. C. Roback, T. L. McLing, T. D. Bullen, D. J. DePaolo, C. Doughty, R. J. Hunt, R. W. Smith, L. D. Cecil, and M. T. Murrell, 2000, 'Groundwater 'Fast Paths' in the Snake River Plain Aquifer-Radiogenic Isotope Ratios as Natural Groundwater Tracers," Geology, Vol. 28, No. 10, October 2000, pp. 871-874.

Kjelstrom, L. C., 1986, "Flow Characteristics of the Snake River and Water Budget for the Snake River Plain, Idaho and Eastern Oregon," U.S. Geological Survey Hydrologic Investigations Atlas, HA-680, scale 1:1,000,000.

Lindholm, G. F., 1981, Plan of Study for the Regional Aquifer-System Analysis of the Snake River Plain, Idaho and Eastern Oregon, U.S. Geological Survey Open-File Report 81-689.

Luo, S., T. Ku, R. Roback, M. Murrell, and T. L. McLing, 2000, "In-situ Radionuclide Transport and Preferential Groundwater Flows at the INEEL (Idaho)," Decay-series Disequilibrium Studies, Geochimica et Cosmochimica Acta, Vol. 64, No. 5, pp. 867-881.

Martian, P., 1999, Numerical Modeling Support of the Natural Attenuation Field Evaluation for Trichlorethene at the Test Area North, Operable Unit 1-07B, Idaho National Engineering and Environmental Laboratory, INEEL/EXT-97-01284, Lockheed Martin Idaho Technologies Company, January 1999.

Robertson, J. B., 1974, Digital Modeling of Radioactive and Chemical Waste Transport in the Snake River Plain Aquifer at the National Reactor Testing Station, Idaho - 1952-1970, U.S. Geological Survey Open-File Report IDO-22054.

Rood, A. S., 2005, Groundwater Transport Modeling Using Response Surface Modeling: Proof of Principle, ICP/EXT-05-00952, Rev. 0, Idaho Cleanup Project, Idaho National Laboratory, June 2005.

Spinazola, J. M., 1994, Geohydrology and Simulation of Flow and Water Levels in the Aquifer System in the Mud Lake Area of the Eastern Snake River Plain, Eastern Idaho, U.S. Geological Survey Water-Resources Investigations Report 93-4227. 
U.S. Army Corps of Engineers, Coastal and Hydraulics Laboratory, Department of Defense (DoD) Groundwater Modeling System (GMS), Version 6.0, Engineer Research and Development Center, Vicksburg, MS, 2005.

USGS, 2007, USGS Annual Statistics for Idaho, http://nwis.waterdata.usgs.gov/id/nwis/annual/calendar_year?search_criteria=station_nm\&submitte d form=introduction, U.S. Geological Survey, Wed page visited January 11, 2007.

Wood, T. R., 1989, Preliminary Assessment of the Hydrogeology at the Radioactive Waste Management Complex, Idaho National Engineering Laboratory, EGG-WM-8694, December 1989.

Wood, T. R., C. M. Helm-Clark, H. Huang, S. Magnuson, T. McLing, B. Orr, M. Roddy, M. J. Rohe, M. A. Plummer, and R. Podgorney, 2005, Operable Unit 10-08 Summary Report on the Subregional-scale Two-dimensional Aquifer Model, ICP/EXT-05-00979, Rev. 1, Idaho Cleanup Project, October 2005. 
C-68 


\section{Appendix C-A}

Three-Dimensional Characterization of Inflows to and Outflows from the Operable Unit 10-08 Model Domain 
C-70 


\section{Appendix C-A}

\section{Three-Dimensional Characterization of Inflows to and Outflows from the Operable Unit 10-08 Model Domain \\ C-A-1. INTRODUCTION}

Transient three-dimensional simulation of groundwater flow through the area represented by the OU 10-08 model domain (Figure C-A-1) requires an understanding of the areal and vertical character of inflows and outflows to the system. This appendix addresses the three-dimensional character and magnitude of inflows to the model domain. Those inflows, points of recharge, rates, and sources of information are summarized on Table C-A-1 and will be used in the development of OU 10-08 two-dimensional and three-dimensional numerical models. This appendix also summarizes net outflows in the context of accumulated inflows.

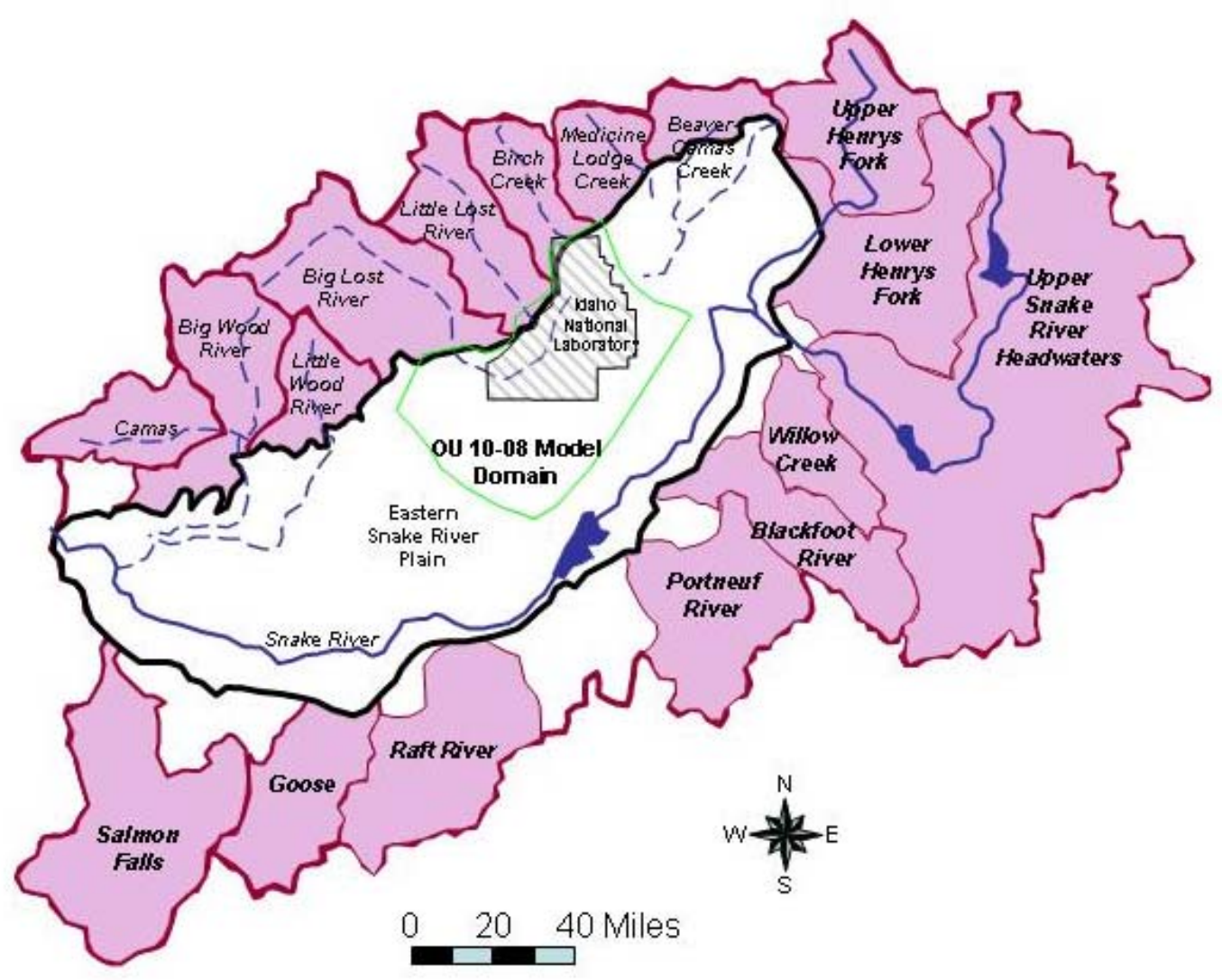

Figure C-A-1. Eastern Snake River Plain, tributary drainages, major surface-water features, Idaho National Laboratory, and area represented by the OU 10-08 model domain. 
Table C-A-1. Inflows to the area represented by the Operable Unit 10-08 model domain.

\begin{tabular}{|c|c|c|c|c|c|}
\hline \multicolumn{3}{|c|}{ Inflow } & Point of Recharge & Rate of Inflow & Source of Information \\
\hline \multicolumn{3}{|c|}{ Regional underflow across the northeast boundary } & Flow distributed horizontally across & $833,000 \mathrm{acre}-\mathrm{ft} /$ year & Spinazola five-layer \\
\hline \multirow[t]{4}{*}{$\begin{array}{l}\text { Tributary } \\
\text { underflow }\end{array}$} & Birch Creek & Underflow & $\begin{array}{l}\text { Top of aquifer in transition zone at } \\
\text { mouth of Birch Creek Valley }\end{array}$ & $\begin{array}{l}78,200 \text { acre-ft/year } \\
\left(264,300 \mathrm{~m}^{3} / \text { day }\right)\end{array}$ & Kjelstrom (1986) \\
\hline & Little Lost River & Underflow & $\begin{array}{l}\text { Top of aquifer in transition zone at } \\
\text { mouth of Little Lost River Valley }\end{array}$ & $\begin{array}{l}155,000 \mathrm{acre}-\mathrm{ft} / \mathrm{year} \\
\left(523,900 \mathrm{~m}^{3} / \text { day }\right)\end{array}$ & Kjelstrom (1986) \\
\hline & \multirow[t]{2}{*}{ Big Lost River } & Underflow & $\begin{array}{l}\text { Top of SRPA in transition zone at } \\
\text { mouth of Big Lost River Valley }\end{array}$ & $\begin{array}{l}295,400 \text { acre-ft/year } \\
\left(998,400 \mathrm{~m}^{3} / \text { day }\right)\end{array}$ & Kjelstrom (1986) \\
\hline & & Streamflow & $\begin{array}{l}\text { Top of SRPA in stream reach from } \\
\text { Arco to the Big Lost River playa }\end{array}$ & $\begin{array}{l}70,200 \text { acre-ft/year } \\
\left(237,200 \mathrm{~m}^{3} / \text { day }\right)\end{array}$ & $\begin{array}{l}\text { USGS stream-gaging } \\
\text { data, 1985-2003 } \\
\text { (USGS 2007) }\end{array}$ \\
\hline \multicolumn{3}{|c|}{ Direct precipitation } & $\begin{array}{l}\text { Distributed uniformly over the model } \\
\text { domain and applied to the top of the } \\
\text { aquifer }\end{array}$ & $\begin{array}{l}25,600 \text { to } 64,000 \text { acre-ft/year } \\
\left(86,500 \text { to } 216,300 \mathrm{~m}^{3} / \text { day }\right)- \\
\text { average is } 44,800 \text { acre-ft/year } \\
\left(151,400 \mathrm{~m}^{3} / \text { day }\right)\end{array}$ & Cecil et al. (1992) \\
\hline
\end{tabular}




\section{C-A-2. INFLOWS TO THE OPERABLE UNIT 10-08 MODEL DOMAIN}

Major inflows include regional underflow within the SRPA across the northeastern boundary of the model domain, recharge from groundwater and surface-water inflows from tributary basins, and recharge from areal precipitation.

\section{C-A-2.1 Regional Underflow into the Model Domain}

Regional underflow within the SRPA enters the area represented by the OU 10-08 model domain across the northeastern boundary (Figure C-1-2). This inflow is derived from recharge of runoff from the Yellowstone Plateau to the northeast and from tributary basin inflows (Camas, Beaver, Medicine Lodge Creeks and other minor tributaries) draining onto the ESRP northeast of INL.

The northeastern model-domain boundary is located downgradient from the Mud Lake area to avoid complexities associated with the transient hydrologic regime in that area (Wood et al. 2005). Underflow across this boundary is estimated from simulated outflows for 1980 across the southwestern boundary of a five-layer groundwater flow model of the Mud Lake area (Spinazola 1994). The year 1980 was selected by Spinazola as representing near-steady-state conditions for the SRPA. This simulated outflow, totaling 918,000 acre-ft/year was consistent with underflow estimates derived from Garabedian's regional flow model (1992) and with estimates derived from the Idaho Water Resources Research Institute model (Cosgrove et al. 2006).

The Spinazola model extended into the northeastern part of the OU 10-08 model domain (Figure C-A-2) and included tributary inflows from Birch Creek. Regional underflow across the northeastern boundary of the OU 10-08 model domain was estimated by subtracting inflows within the area of overlap (estimated to be approximately $400 \mathrm{mi}^{2}$ ) from Spinazola's simulated outflow. These inflows consisted of 78,200 acre-ft of surface water and groundwater inflows from Birch Creek and an estimated 3,400 to 8,500 acre-ft/year of areal precipitation infiltration (using a range of 2 to $5 \%$ of precipitation). Based on these estimated inflows, underflow across the northeastern model domain boundary may be approximately 833,000 acre-ft/year (Table C-A-1).

Because the vertical character of regional underflow across the northeastern boundary of the OU 10-08 model domain could not be directly observed, underflow from the five-layer Spinazola model was used to derive a three-dimensional approximation of this source of inflow. Table C-A-2 presents apportioned underflow out of the Spinazola model as a percentage of total underflow and presents depth-integrated estimates of inflow to the OU 10-08 model domain based on the inflow estimate of 833,000 acre-ft/year. Spinazola's layers one and two each were as much as $100 \mathrm{ft}$ thick. Layer three was as much as $300 \mathrm{ft}$ thick. Layers four and five were less than or equal to $500 \mathrm{ft}$ thick and $1,000 \mathrm{ft}$ thick respectively. In the OU 10-08 overlap area, thickness of the bottom two layers ranged from zero to $500 \mathrm{ft}$ thick. The approximate thickness of the Spinazola model layers in the vicinity of the northeastern boundary of the OU 10-08 model domain is shown in Figure C-A-3.

Based on Spinazola's layered estimates of outflow, approximately $32 \%$ of underflow (263,000 acre-ft/year) may occur within the upper $100 \mathrm{ft}$ of the SRPA across the northeastern boundary of the OU 10-08 model domain. Approximately 70\% of underflow (581,000 acre-ft/year) into the model domain may occur within the upper $150 \mathrm{ft}$ of the SRPA. 


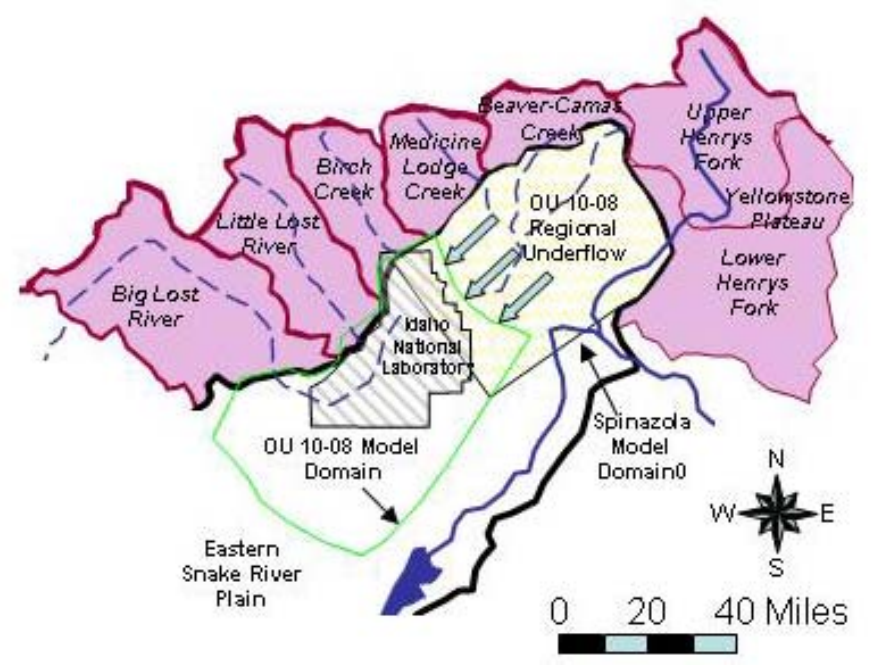

Figure C-A-2. Operable Unit 10-08 model domain and location of regional underflow across northeast boundary, tributary basins contributing recharge to regional underflow at the INL, and area of overlap between the OU 10-08 and Spinazola model domains.

Table C-A-2. Layer thickness, underflow out, and percentage of total underflow, Spinazola model (1994), and estimated underflow into the OU 10-08 model domain.

\begin{tabular}{ccccc}
\hline Layer & $\begin{array}{c}\text { Thickness of } \\
\text { Sinazola's Model } \\
\text { Layers, in Feet }\end{array}$ & $\begin{array}{c}\text { Estimated Outflow by Layer } \\
\text { Across the Southwest } \\
\text { Boundary of the Spinazola } \\
\text { Model, in Acre-ft/year }\end{array}$ & $\begin{array}{c}\text { Percentage of } \\
\text { Total Simulated } \\
\text { Underflow }\end{array}$ & $\begin{array}{c}\text { Estimated Underflow into the } \\
\text { OU 10-08 Model Domain, } \\
\text { Based on Spinazola Layered } \\
\text { Estimates, in Acre-ft/year }\end{array}$ \\
\hline 1 & $\leq 100$ & 150,000 & 16.3 & 135800 \\
2 & $\leq 100$ & 140,000 & 15.3 & 127400 \\
3 & $\leq 300$ & 350,000 & 38.1 & 317400 \\
4 & $\leq 500$ & 220,000 & 24.0 & 199900 \\
5 & $\leq 500$ & 58,000 & 6.3 & 52500 \\
\hline Total & $\leq 1,500$ & 918,000 & 100.0 & 833,000 \\
\hline
\end{tabular}




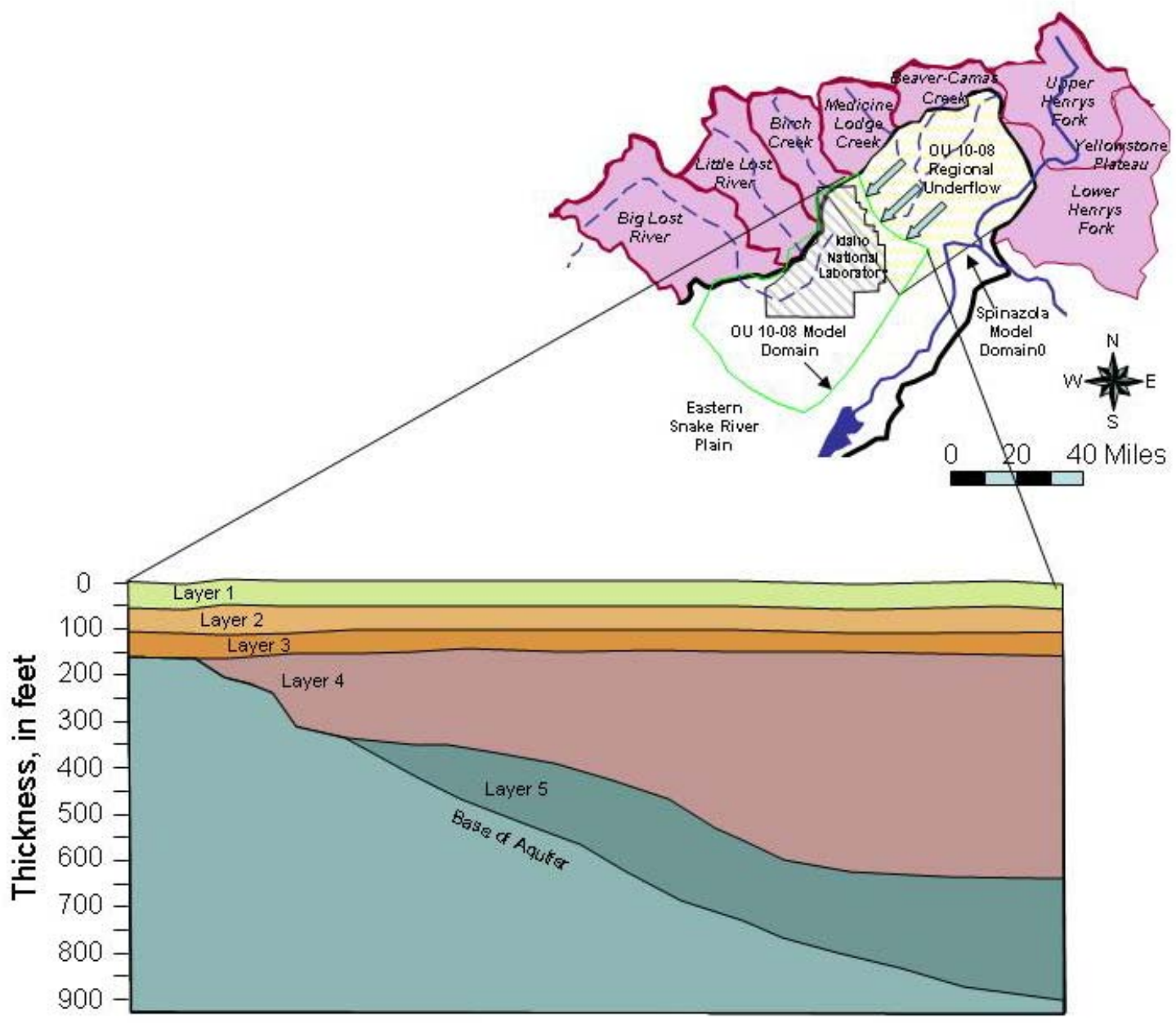

Figure C-A-3. Approximate distribution of layers in the Spinazola model along a line of section corresponding to the OU 10-08 model domain northeast boundary.

Two-dimensional distribution of regional underflow across the OU 10-08 northeast boundary was derived from simulated flows through the layered Spinazola model. The two-dimensional flow volume was estimated by dividing the northeast boundary into 18 segments to approximate changes in layer thickness and flow. Flows per unit width, as $\mathrm{m}^{3} / \mathrm{m} /$ day, were estimated in each layer for each segment. These layered unit flows were then summed and multiplied by the segment length to approximate flow across the aquifer thickness for that segment. Table C-A-3 presents the 18 segments that comprise the northeast boundary of the OU 10-08 model domain and two-dimensional flow totals for each segment. The summed flows were $2,815,300 \mathrm{~m}^{3} / \mathrm{m} /$ day or 833,000 acre- $\mathrm{ft} /$ year. 
Table C-A-3. Approximation of segment flows across the Operable Unit 10-08 model domain northeast boundary.

\begin{tabular}{|cccccc|}
\hline & $\begin{array}{c}\text { Segment Start } \\
\text { Distance from } \\
\text { North Model } \\
\text { Corner }\end{array}$ & $\begin{array}{c}\text { Segment End } \\
\text { Distance from } \\
\text { North Model } \\
\text { Corner }\end{array}$ & $\begin{array}{c}\text { Sum of Layered } \\
\text { Flows per Unit } \\
\text { Width of Segment } \\
(\mathrm{m})\end{array}$ & $\begin{array}{c}\text { Segment Flows } \\
(\mathrm{m})\end{array}$ & $\begin{array}{c}\text { Segment Flows } \\
\text { (acre-ft/year) }\end{array}$ \\
\hline 1 & 0 & 3000 & 23.1 & 69,200 & 20,500 \\
2 & 3000 & 8000 & 25.5 & 127,400 & 37,700 \\
3 & 8000 & 12000 & 38.6 & 154,400 & 45,700 \\
4 & 12000 & 15000 & 44.3 & 132,800 & 39,300 \\
5 & 15000 & 17000 & 49.1 & 98,200 & 29,100 \\
6 & 17000 & 20000 & 50.2 & 150,700 & 44,600 \\
7 & 20000 & 20500 & 47.9 & 24,000 & 7,100 \\
8 & 20500 & 23000 & 49.6 & 124,000 & 36,700 \\
9 & 23000 & 28000 & 47.3 & 236,400 & 69,900 \\
10 & 28000 & 29000 & 52.1 & 52,100 & 15,400 \\
11 & 29000 & 30000 & 53.8 & 53,800 & 15,900 \\
12 & 30000 & 34000 & 56.1 & 224,400 & 66,400 \\
13 & 34000 & 35000 & 58.5 & 58,500 & 17,300 \\
14 & 35000 & 37500 & 60.8 & 152,000 & 45,000 \\
15 & 37500 & 38000 & 62.5 & 31,200 & 9,200 \\
16 & 38000 & 46000 & 69.0 & 552,300 & 163,400 \\
17 & 46000 & 47000 & 70.7 & 70,700 & 20,900 \\
18 & 47000 & 54373 & 68.3 & 503,200 & 148,900 \\
\hline Sum & & & & $2,815,300$ & 833,000 \\
\hline
\end{tabular}

\section{C-A-2.2 Tributary Inflows}

Tributary inflows to the area represented by the OU 10-08 model domain were derived from the Birch Creek, Little Lost River, and Big Lost River drainage basins (Figure C-A-1). These inflows occur as underflow from tributary aquifers and from infiltration of streamflows.

\section{C-A-2.2.1 Birch Creek}

Birch Creek drains a tributary basin exceeding $400 \mathrm{mi}^{2}$ in the mountains of the Lemhi and Bitterroot ranges (Figure C-A-4). Kjelstrom (1986) estimated that an average volume of approximately 78,200 acre-ft/year $\left(264,300 \mathrm{~m}^{3} /\right.$ day $)$ of water is derived from the Birch Creek drainage area. This source of inflow to the SRPA occurs as infiltration of streamflow and as underflow through tributary aquifers.

Streamflow in Birch Creek has been monitored intermittently since the early 1900s at three streamgaging stations upstream from the ESRP. Annual flows at these stations have ranged from approximately 60 to $90 \mathrm{cfs}\left(146,800\right.$ to $220,200 \mathrm{~m}^{3} /$ day). These flows are measured in the vicinity of a bedrock high and likely represent most of the water derived from the drainage area. (USGS 2007) 


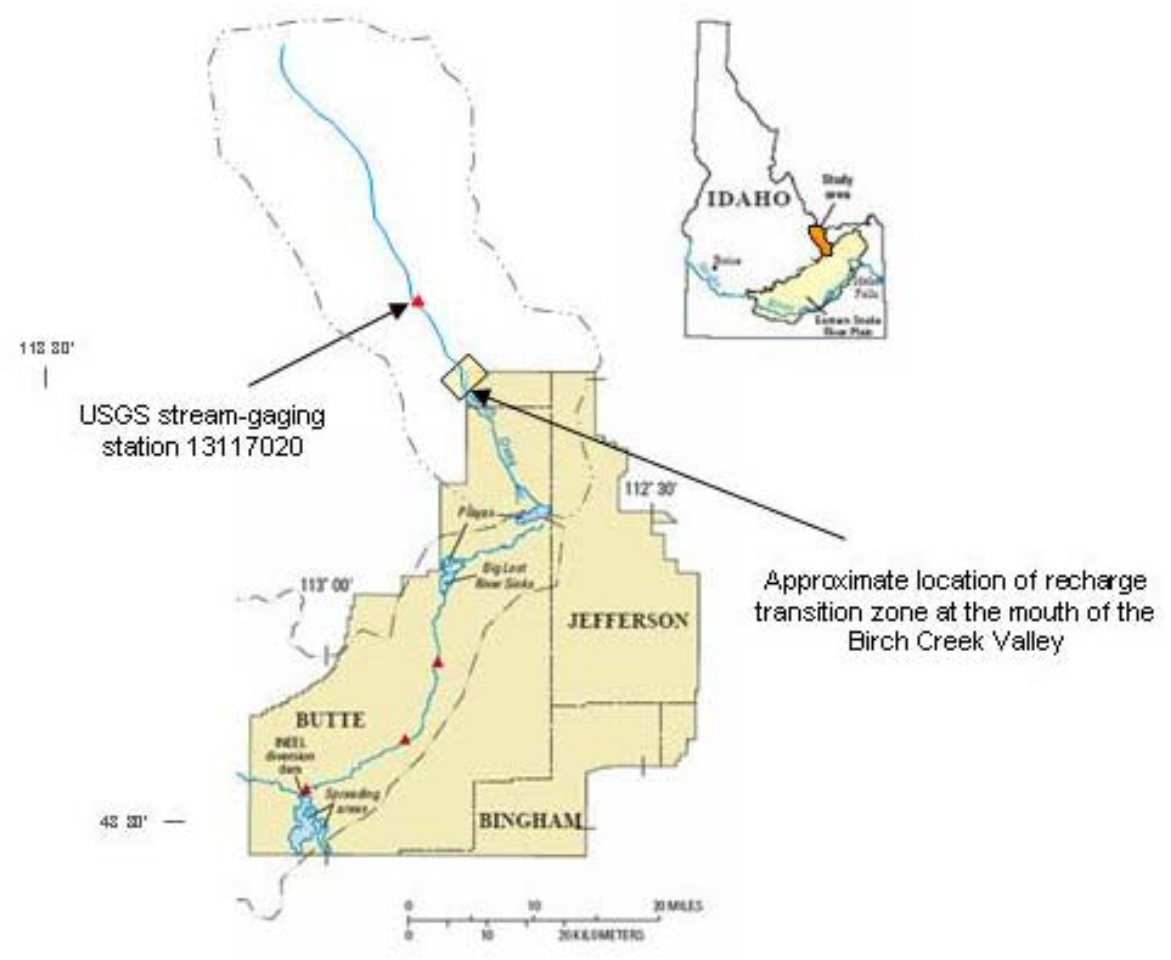

Figure C-A-4. Drainage area for Birch Creek, location of stream gaging stations, and selected surface-water features at the Idaho National Laboratory.

Based on stream-gaging station data, Birch Creek streamflows typically remain stable throughout the year. These stable baseflows are sustained primarily by spring discharge, with relatively small contributions occurring from snowmelt runoff. Streamflows infiltrate along the stream channel and diversions to recharge tributary basin aquifers or the SRPA near the mouth of the Birch Creek valley. The limited range in flow volumes indicates that recharge from this source does not vary significantly from year to year.

Recharge to the SRPA from Birch Creek tributary inflows is described in Wood et al. (2005) and probably is similar to that derived from other drainage basins in the area of the OU 10-08 model domain. This source of recharge likely takes place at the top of the aquifer and occurs through downward percolation of infiltrating streamflows through the Birch Creek and diversion channels or through vertical percolation of tributary aquifer underflow moving into the basalts and sediments of the ESRP, as shown in a generalized cross-sectional conceptual model (Figure C-A-5). In either case, interfingering sediments and basalts may spread these groundwater underflows to recharge the SRPA within a transitional zone (Figure C-A-4) at the edge of the plain. Information characterizing the location and extent of this transition zone is limited, but likely is similar to transition zones observed for the Little Lost River and Big Lost River (Wood et al. 2005). 


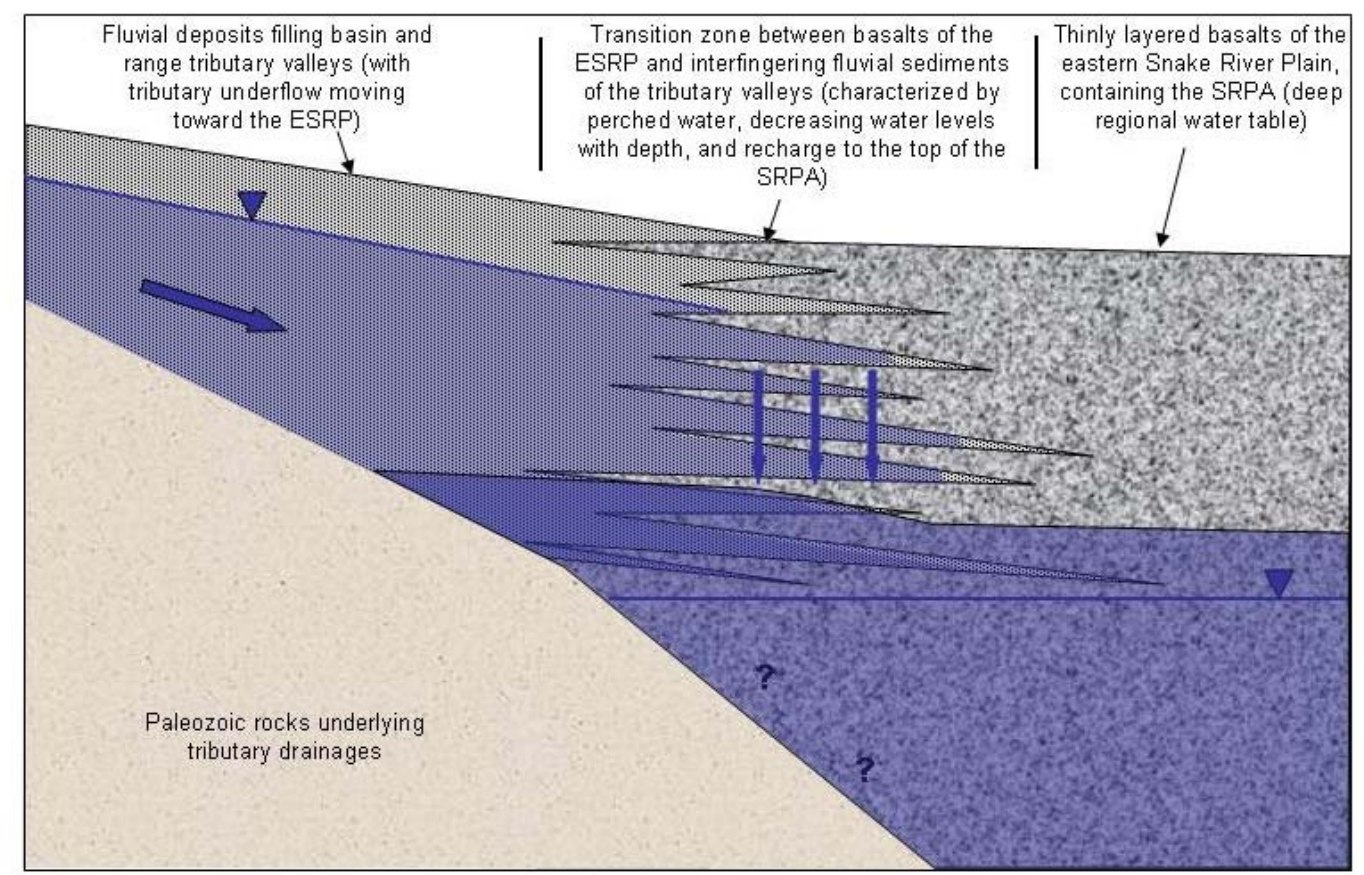

Figure C-A-5. Generalized cross-sectional conceptual model of recharge to the SRPA from tributary basin underflow.

\section{C-A-2.2.2 Little Lost River}

The Little Lost River drains a tributary basin of about $963 \mathrm{mi}^{2}$ of the northeastern slopes of the Lost River Range and the western slopes of the Lemhi Range (Figure C-A-6). Kjelstrom (1986) calculated a basin yield of approximately 155,000 acre-ft/year $\left(523,900 \mathrm{~m}^{3} /\right.$ day $)$. This yield represents an average annual inflow to the SRPA of $214 \mathrm{cfs}$ in the form of streamflow and tributary aquifer underflow.

Streamflows were monitored during 1941-89 at a gaging station on the Little Lost River approximately $7 \mathrm{mi}$ upstream from Howe. The average discharge for this period of record was $77 \mathrm{cfs}$ $\left(188,400 \mathrm{~m}^{3} /\right.$ day), and ranged annually from 49 to $107 \mathrm{cfs}\left(119,900\right.$ to $261,800 \mathrm{~m}^{3} /$ day) (USGS 2007). Most of these flows generally are diverted for irrigation downstream of the gaging station or infiltrate. The long-term volume of Little Lost River streamflows onto the ESRP is considered to be inconsequential.

The small range of annual stream discharge at the Little Lost River gaging station indicates that inflows to the SRPA from this tributary are relatively stable. These flows likely represent only a part of the inflows that are available from this basin, with a significant percentage of basin flows occurring as tributary aquifer underflow. The estimated tributary underflow of 155,000 acre-ft/year takes into account consumptive losses within the tributary basin from evaporation or plant transpiration.

Recharge to the SRPA from the Little Lost River tributary basin, similar to that from other basins in the region, occurs at the top of the aquifer from downward percolation of tributary-basin underflow in a transitional zone at the mouth of the basin (Figure C-A-5). Within that transitional zone, the stratigraphic sequence consists of fluvial sediments that interfinger with ESRP basalts and pluvial sediments. Within this transition zone, the interfingered basalt/sediment sequence is characterized by multiple shallow and perched basin-fill aquifers. The transition zone extends $3 \mathrm{mi}$ or less onto the ESRP from the mouth of the Little Lost River valley north from Howe (Figure C-A-6). 


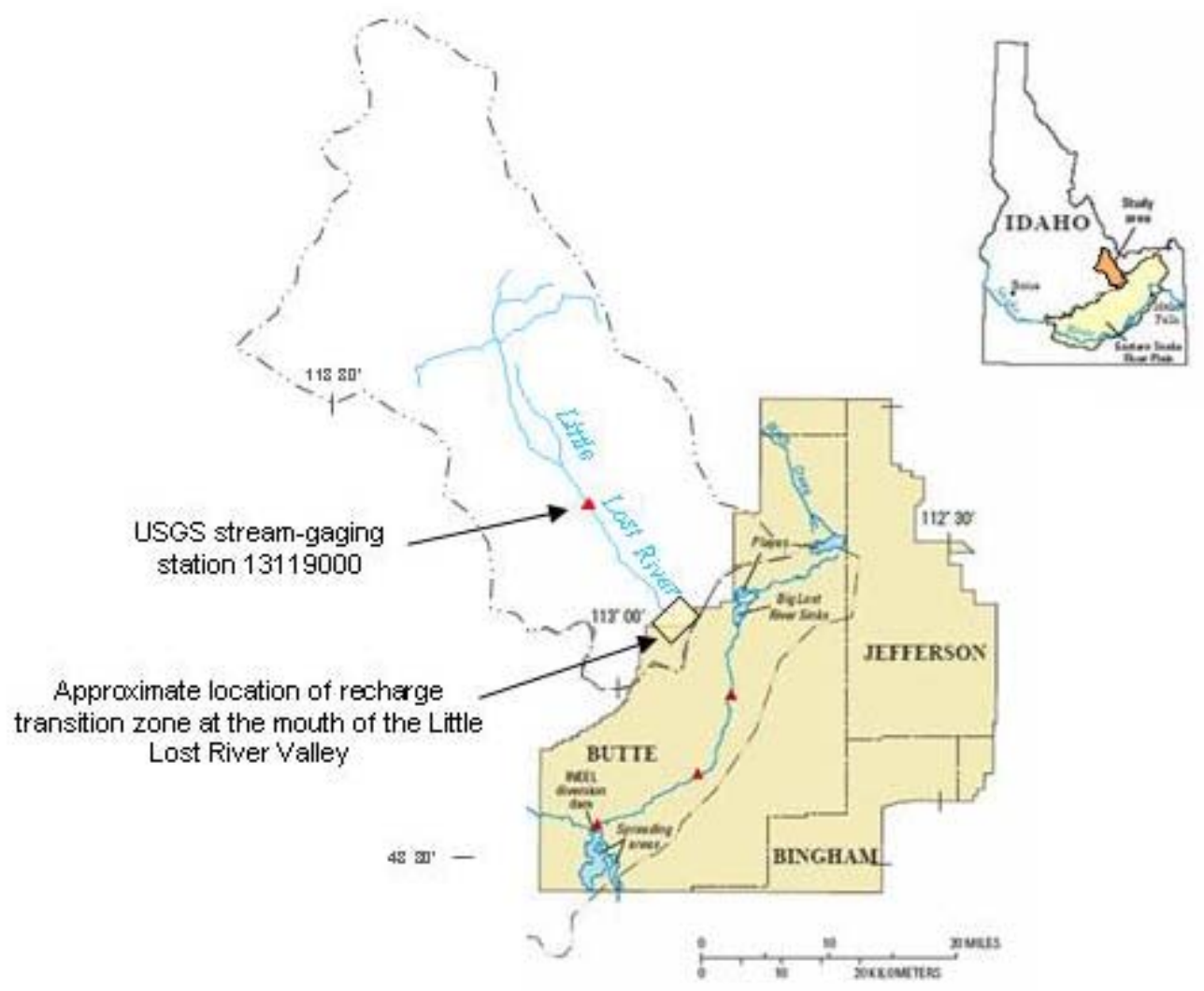

Figure C-A-6. Drainage area for the Little Lost River, location of stream gaging stations, and surfacewater features at Idaho National Laboratory.

\section{C-A-2.2.3 Big Lost River}

The Big Lost River drains a mountainous tributary basin encompassing approximately $1,410 \mathrm{mi}^{2}$ and including the Lost River Range and Pioneer Mountains to the west of the ESRP (Figure C-A-7). Inflows to the SRPA from the Big Lost River drainage basin are derived from tributary aquifer underflow and from infiltration of streamflow in the river channel and associated surface-water features on the ESRP. These two sources of inflow are distinctly different in location and variability of flow.

C-A-2.2.3.1 Underflow. Tributary basin deposits within the Big Lost River valley contain groundwater. The potentiometric surface indicates that the direction of groundwater flow within this tributary basin aquifer is toward the ESRP. Underflow was estimated by Kjelstrom from basin-yield equations to total 295,400 acre-ft/year $\left(998,400 \mathrm{~m}^{3} /\right.$ day). This source of recharge to the SRPA may vary minimally in response to climatologic changes. 


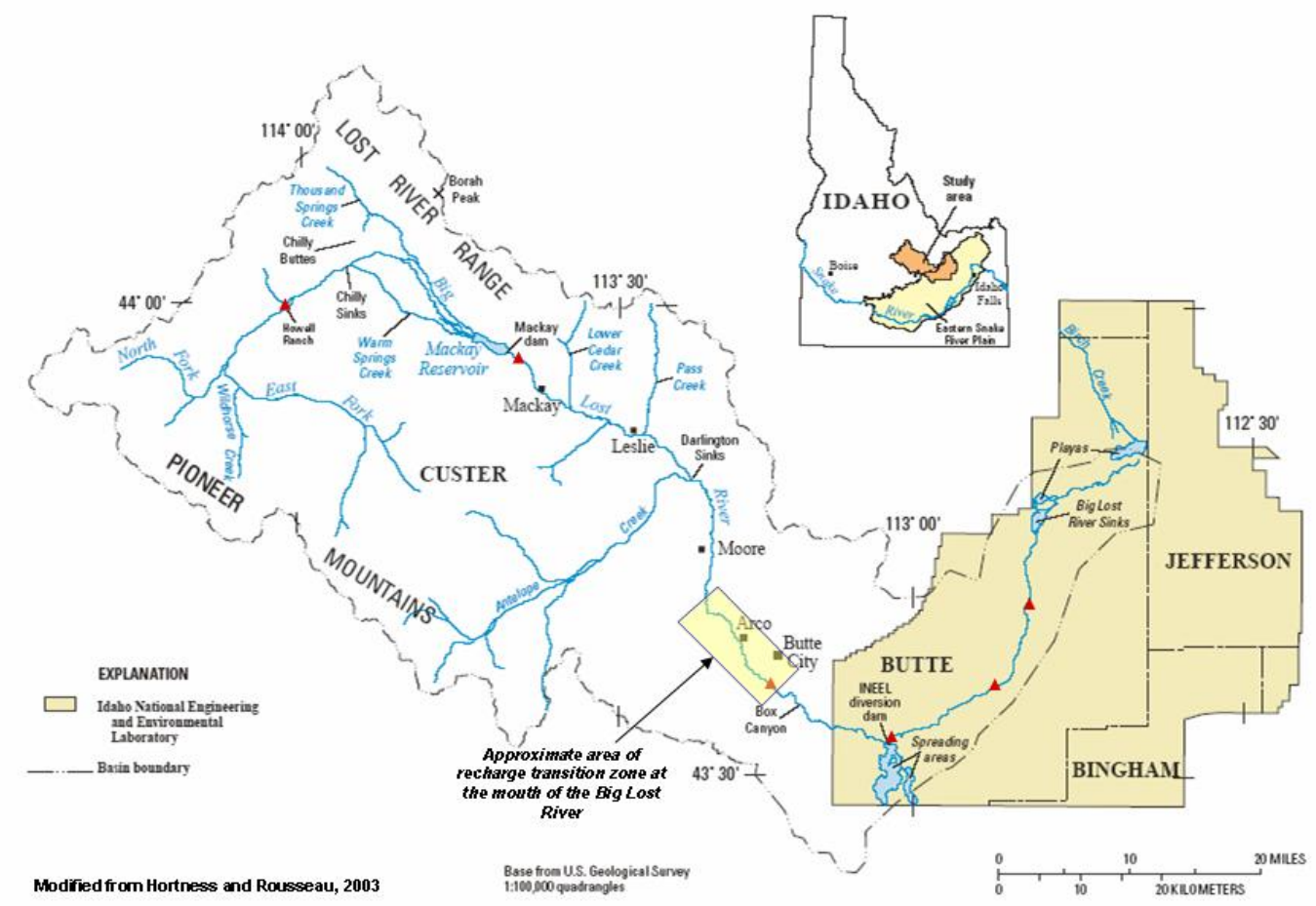

Figure C-A-7. Drainage area for the Big Lost River, location of stream gaging stations, and surface-water features at Idaho National Laboratory.

Groundwater levels in the tributary aquifer typically are hundreds of feet higher than those in the SRPA to the east. A transition zone in the vicinity of Arco (Figure C-A-7) consists of a stratigraphic sequence of fluvial sediments derived from the mountainous drainage basin to the west that interfinger with basalts of the ESRP (Wood et al. 2005). This zone is characterized by multiple bodies of perched water and decreasing water levels with depth (Figure C-A-5).

Within this transition zone, groundwater that flows out from the mouth of the Big Lost River valley is perched on interfingering sedimentary units, with recharge to the SRPA occurring through downward percolation through these interbeds and basalts to the top of the regional flow system. This source of recharge occurs downgradient from INL but constitutes an important source of subregional inflow.

C-A-2.2.3.2 Streamflow. Streamflow in the Big Lost River is primarily controlled by annual snowpack in the mountainous areas of the basin, water storage in Mackay Reservoir, and reservoir releases and diversions for downstream irrigation water use. Streamflows in the Big Lost River are monitored at a series of USGS stream-gaging stations (Figure C-A-7) (USGS 2007). Based on a total of 48 years of streamflow data for the gaging station near Arco (1947-1960, 1967-1979, and 1983-2003), annual mean discharge is variable, ranging from $488 \mathrm{cfs}$ in 1984 to zero during several years. The mean discharge for the period of record was $97.3 \mathrm{cfs}$ or approximately $70,200 \mathrm{acre}-\mathrm{ft} / \mathrm{year}\left(237,200 \mathrm{~m}^{3} /\right.$ day $)$. 
Aquifer recharge occurs from infiltration of episodic streamflow along the channel of the Big Lost River, in INL spreading areas constructed in the 1960s for floodwater protection of INL facilities, in the area described as the Big Lost River Sinks north of INTEC, Reactor Technology Complex (RTC), the Naval Reactors Facility, and in terminal playas. Water infiltrating the channel of the Big Lost River must move through a thick sequence of unsaturated basalts and interbedded sediments, with a vadose zone ranging from more than $600 \mathrm{ft}$ thick near the RWMC to approximately $200 \mathrm{ft}$ thick near the playas. Water levels in shallow, vadose-zone wells located as much as $0.5 \mathrm{mi}$ away from the river channel have been observed to change rapidly in response to streamflows, indicating that infiltrating water spreads away from the channel in the shallow subsurface, and that the effective area of recharge is wider than the narrow river channel (Barraclough et al. 1967).

Streamflow records measured during 1985-2003 at a series of five stream-gaging stations located on the river within the ESRP (Figure C-A-8) were used to estimate the percentage of Big Lost River streamflow near Arco that infiltrated along specified reaches downstream from Arco to the Big Lost River sinks during that time period (Wood et al. 2005). These estimates were derived with the assumption that recharge is rapid and they do not take into account losses that may occur from evapotranspiration in the channel, streambanks, diversions, and playas.

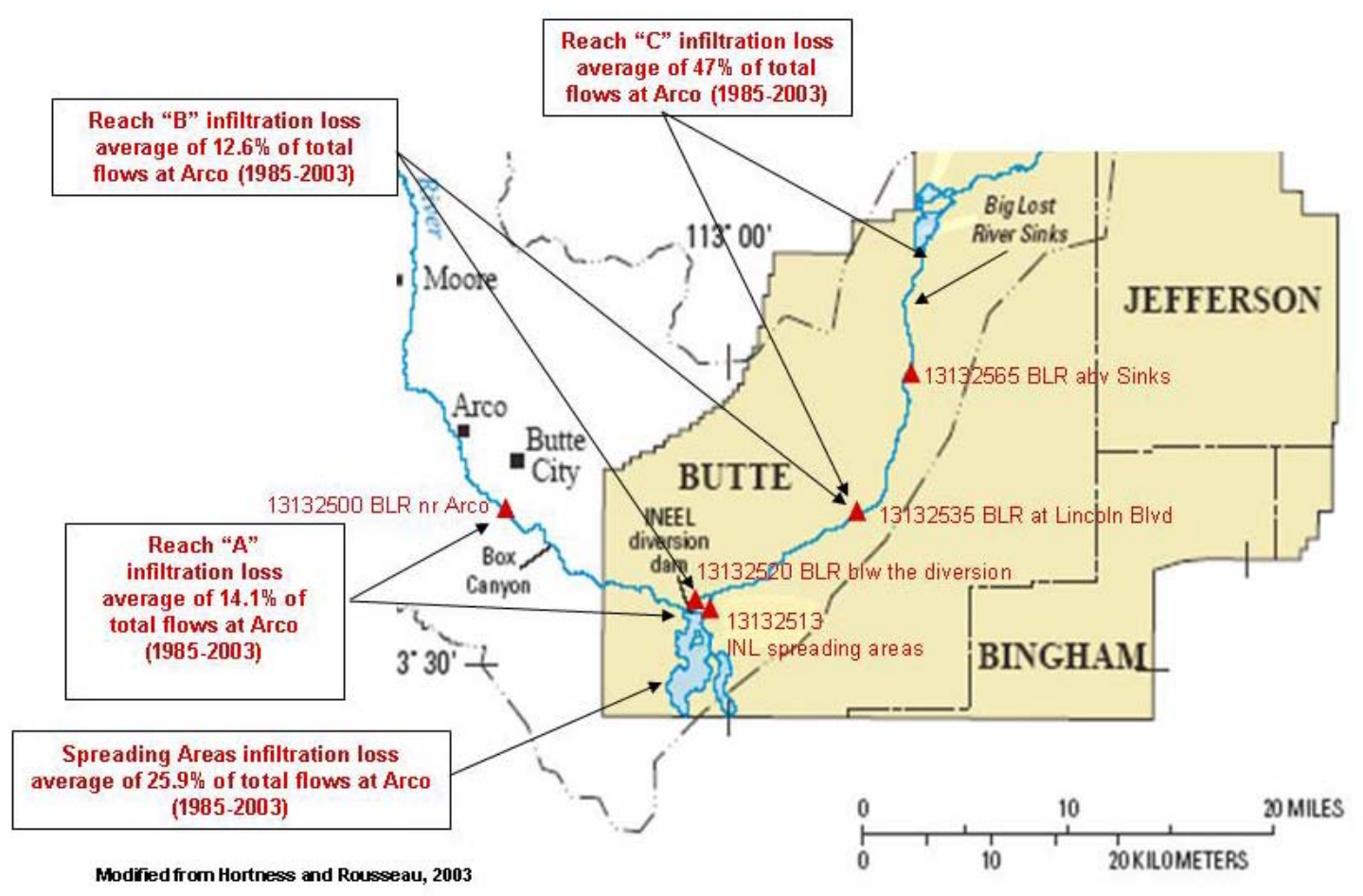

Figure C-A-8. Recharge reaches along the Big Lost River used to estimate channel losses and recharge rates from streamflow.

Based on these data, as much as $14.5 \%$ of the 1985-2003 Big Lost River streamflow near Arco infiltrated in the 14.1-mi reach extending from the gaging station near Arco to a gaging station at the spreading area diversion channel. This reach consists of a river channel incised into basalt flows and includes the Box Canyon area. The river channel is narrow, with flows moving directly over bedrock or a very thin alluvial cover. Based on a long-term average discharge of $97.3 \mathrm{cfs}$ at Arco, average infiltration losses within this reach may approach $1 \mathrm{cfs} /$ river mile. 
An estimated 25.9\% of the Big Lost River streamflow near Arco was diverted during 1985-2003 to the INL spreading areas. These spreading areas consist of low-lying, sediment-filled depressions that were developed to divert excess flood flows in the 1960s. Diverted flood flows rapidly infiltrate in the spreading areas through a thin alluvial covering. Diversions are episodic, with six major diversions occurring in 1984-86 and 1995-99 (Figure C-A-9) (USGS 2007).

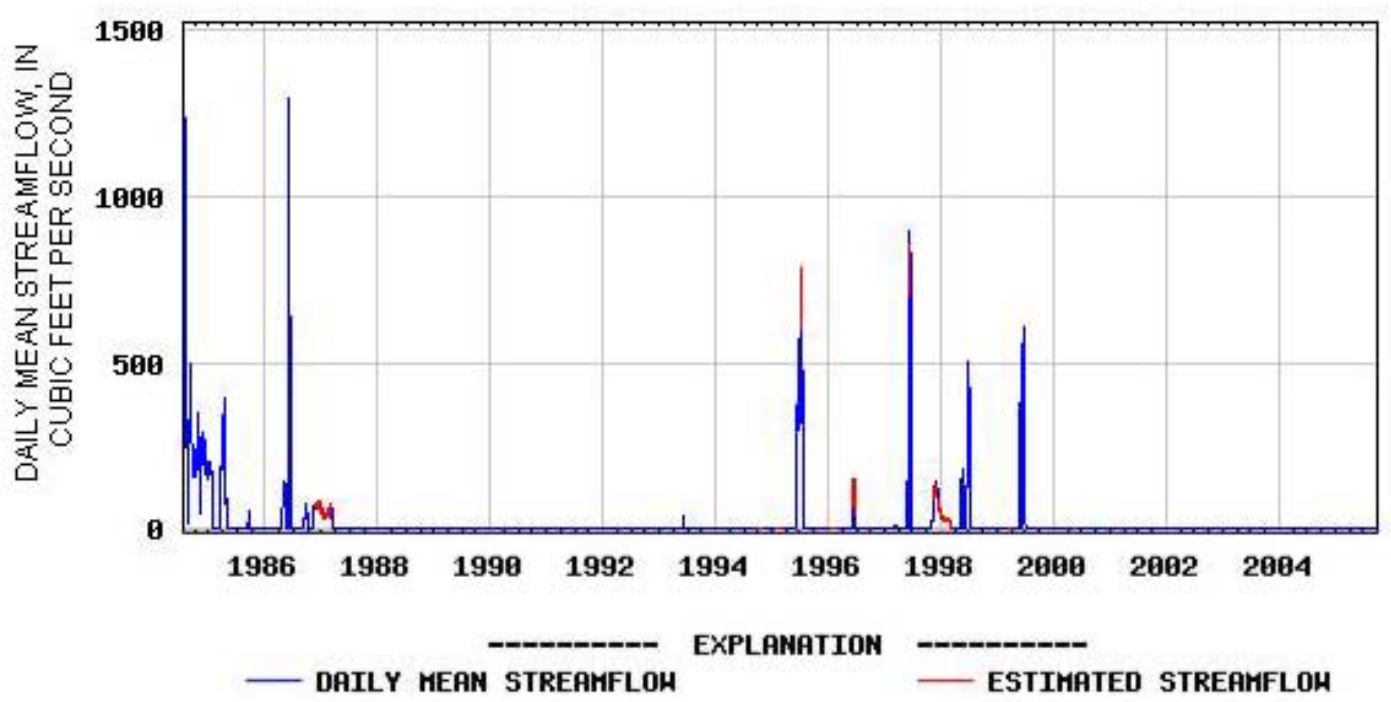

Figure C-A-9. Streamflow diversions to the INL spreading areas during 1984-2005 (from USGS streamflow records, station 13132513, INL Diversion at head near Arco).

A total of $12.6 \%$ of the Big Lost River streamflow near Arco episodically infiltrated in the 10.7-mi stream reach from the spreading-area diversion to Lincoln Boulevard. The upper part of this reach (approximately $3 \mathrm{mi}$ in length) includes sections where the channel is incised into basalt flows and sections crossing thin alluvial deposits over basalt. The lower part of this reach (approximately $10 \mathrm{mi}$ ) consists of a channel cut into alluvial deposits as thick as $50 \mathrm{ft}$. Infiltration losses along this reach may average $1.1 \mathrm{cfs} /$ river mile.

A total of $47 \%$ of the Big Lost River streamflow near Arco episodically infiltrated in the 21-mi stream reach extending from Lincoln Boulevard to the playas terminating the Big Lost River. At the Big Lost River Sinks, the channel bifurcates and stream losses characteristically increase. Based on a limited set of discharge data for four years, most of the flows likely infiltrate downstream from USGS gaging station 13132565, recently installed at the upstream end of the sinks. Infiltration losses for the entire reach may average more than $4 \mathrm{cfs} /$ river mile, but may be much larger in the area of the sinks, based on recent discharge data from this new gaging station. Again, this assumption is based on rapid recharge and minimal losses to evapotranspiration.

These estimated recharge rates from infiltration along the Big Lost River channel on the ESRP represent an average for the period of record and do not reflect the large range in episodic discharge, nor do they reflect annual variations in flow over the period of record. This range is evident from monthly discharges measured in the Big Lost River near Arco during 1995-99 (Figure C-A-10). This period, typical of normal periods of streamflow, demonstrates that recharge is variable during the year with higher rates of recharge typically occurring during June in response to runoff of melting snow in the upper drainage basin. 


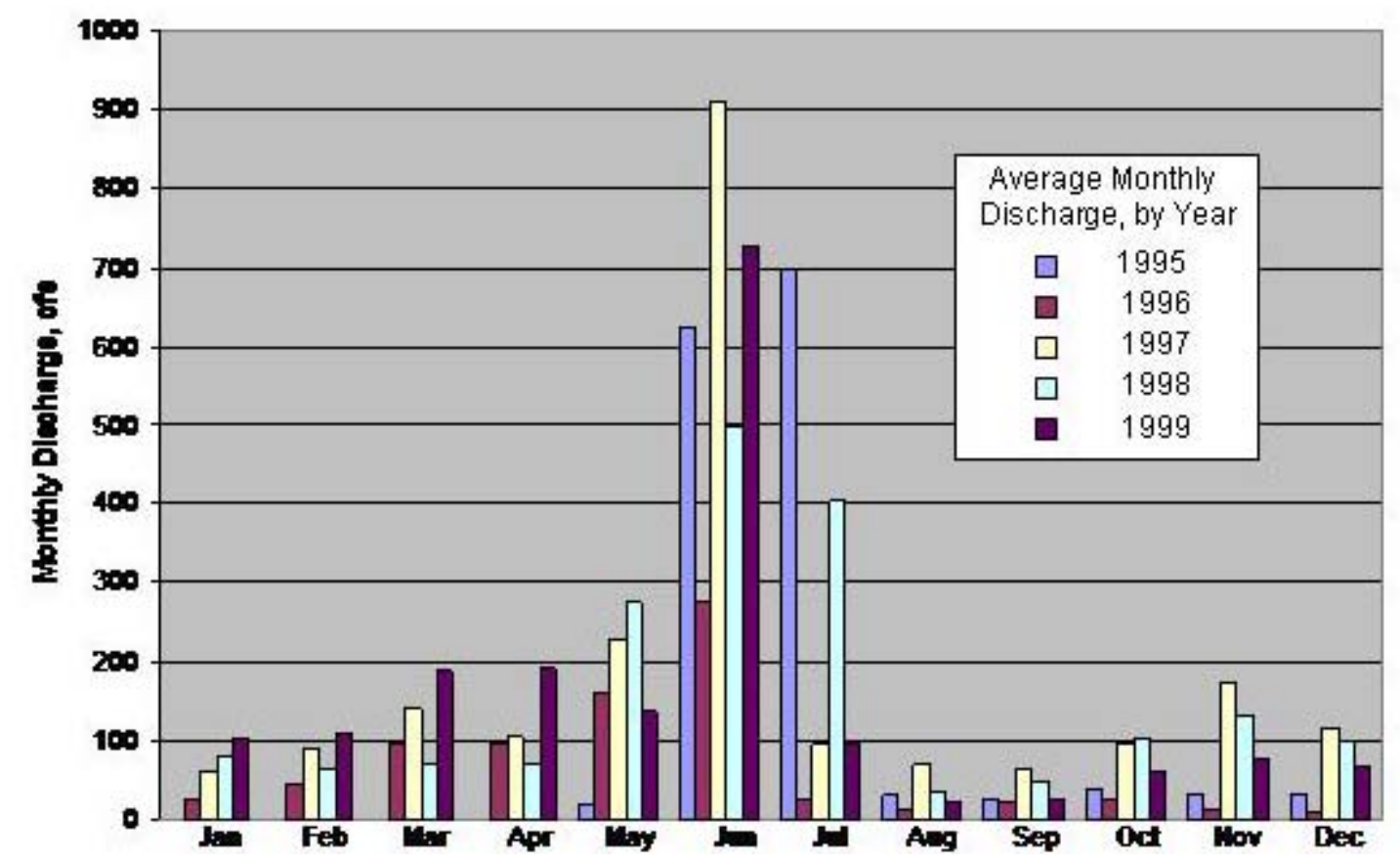

Figure C-A-10. Monthly discharge, in cubic feet per second, for the Big Lost River near Arco, 1995-1999.

In summary, recharge from the infiltration of streamflow in the Big Lost River occurs episodically, with long periods of no flow or recharge interspersed with periods of high flows and high recharge. During periods of sustained flows, some water retained in the near surface as bank storage will be lost to evaporation or plant transpiration. However, most of the infiltrating water will move past the root zone in sediments and will penetrate fractures in the underlying basalt flows. Recharge from this inflow source takes place at the top of the SRPA, with much of the total recharge (approximately 73\%) focused beneath the INL spreading areas and in the Big Lost River Sinks. Prior to construction of the spreading areas in the 1960s, all of this water was diverted north to the Big Lost River Sinks. This focused recharge to the spreading areas and sinks may be reflected in aquifer water temperatures and in stable isotope data from the top of the aquifer. The remainder is distributed as linear recharge beneath and adjacent to the channel, with some linear recharge occurring episodically in proximity to known contaminant sources at INTEC and RTC.

\section{C-A-2.3 Direct Precipitation}

Some recharge to the SRPA is derived from infiltration of rainfall and melting winter snows. Precipitation at the INL Site averages approximately 8 in./year, with largest precipitation rates occurring in May and June. Most of this potential source of recharge is returned to the atmosphere through evaporation and transpiration.

Drainages are poorly developed on the undulating surface of the ESRP. Topographically higher surfaces are subjected to wind and commonly have thin or no loess deposits on top of basalt flows. Runoff from rainfall and snowmelt accumulates locally in low-lying sediment-filled areas. Accumulated water in these areas is susceptible to evaporation from free water surfaces and from saturated soils. Infiltration of this accumulated runoff may occur as locally focused recharge. 
The amount of water that infiltrates past the root zone and through the thick vadose zone probably represents only a small percentage of direct precipitation. Cecil et al. (1992) estimated that actual recharge is from 2 to $5 \%$ of direct precipitation. Based on this estimate and the average rainfall on the plain, from 25,600 to 64,000 acre-ft/year $\left(86,500\right.$ to $216,300 \mathrm{~m}^{3} /$ day) of recharge may be derived from direct precipitation over the area represented by the OU 10-08 model domain (Table C-A-1). For this OU 10-08 study, recharge is assumed to be the average of this range or 44,800 acre- $\mathrm{ft} / \mathrm{year}$ $\left(151,400 \mathrm{~m}^{3} /\right.$ day). This source of recharge occurs at the top of the SRPA over the area represented by the OU 10-08 model domain.

The distribution of direct precipitation recharge likely is variable, depending on the amount of precipitation, soil thickness and type, rock type, and soil cover infiltration capacity. The distribution of recharge from direct precipitation, derived from Garabedian (1992) and from the State of Idaho Regional Water Resource Model (Contor 2004) is shown in Figure C-A-11. Based on this distribution, recharge from direct precipitation is less than or equal to $0.5 \mathrm{in}$./year over most of the OU 10-08 model domain, which is reasonably consistent with the estimated 2 to $5 \%$ of precipitation. A sensitivity test based on the Garabedian/Contor distribution of direct-precipitation recharge was conducted using the OU 10-08 two-dimensional flow model. Test results indicated that variably distributed direct-precipitation recharge did not significantly change results from those based on a uniformly distributed recharge.

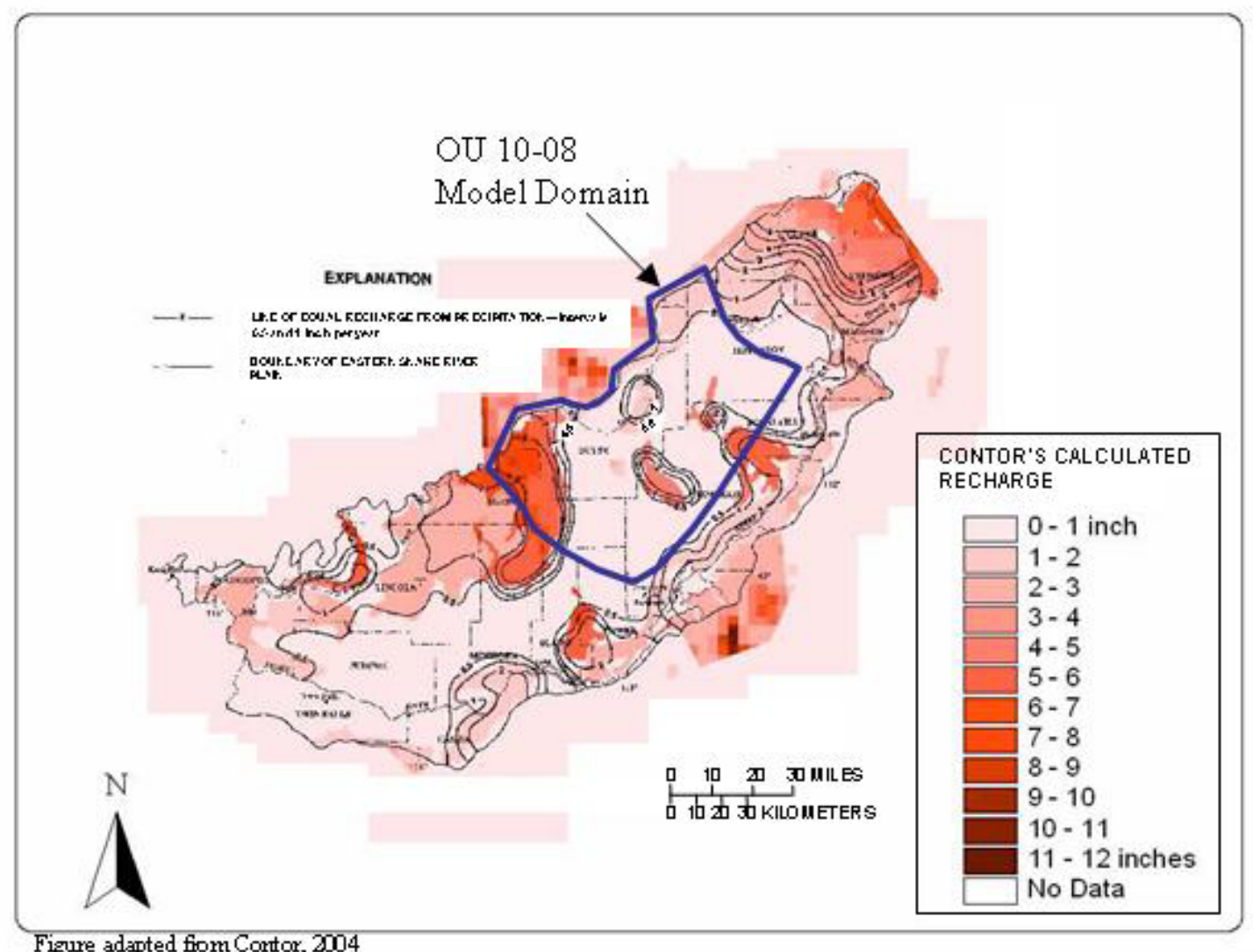

Figure C-A-11. Estimates of recharge from infiltration of precipitation over the Eastern Snake River Plain (Garabedian 1992; Contor 2004). 


\section{C-A-3. SUMMARY}

Major inflows to the OU 10-08 model domain consist of regional groundwater underflow from the Mud Lake area; tributary inflows as groundwater underflow and infiltrating streamflow from the Birch Creek, Little Lost River, and Big Lost River basins; and from infiltration of direct precipitation over the model domain.

Regional underflow across the northeast boundary is approximately 833,000 acre-ft/year, with perhaps $70 \%$ of this inflow occurring in the upper $150 \mathrm{ft}$ of active aquifer.

Inflows from Birch Creek and the Little Lost River are largely in the form of groundwater underflow. Birch Creek underflow is 78,200 acre-ft/year and Little Lost River underflow is approximately 155,000 acre-ft/year. These inflows occur at the top of the SRPA in transition zones at the mouths of the two valleys.

Inflow from the Big Lost River is in the form of groundwater underflow and infiltration of streamflow on the ESRP. Underflow is estimated to be 295,400 acre-ft/year, again occurring at the top of the SRPA in a transitional zone at the mouth of the valley.

Long-term streamflow infiltration is estimated to average 70,200 acre-ft/year, with largest contributions to the INL spreading areas (25.9\% of streamflow near Arco) and to the area north of INTEC and including the Big Lost River Sinks (47\%). The remainder is distributed along the stream channel. Again, this source of recharge is to the top of the aquifer. Aquifer inflow from infiltrating Big Lost River flows is episodic, with long periods of no flow and short cycles of high flows. Recharge also varies annually with highest flows in June. Approximately $73 \%$ of streamflow probably infiltrates as focused recharge to the INL spreading areas and to the Big Lost Sinks. Prior to the 1960s, all of this water likely recharged in the vicinity of the sinks.

Inflow from direct precipitation over the OU 10-08 model domain is estimated to be $3.5 \%$ of average precipitation. This inflow may vary areally, based on topography, rate of precipitation, and infiltration capacity of surficial materials, but sensitivity tests indicate that direct precipitation recharge can be considered to be uniformly distributed over the model domain without significant effect on groundwater flow and contaminant transport. Direct precipitation recharge is estimated to average 44,800 acre-ft/year.

The accumulation of inflows to the area represented by the OU 10-08 model domain moves to the southwest as outflow across the southwest model-domain boundary. The sum of these inflows is approximately 1,476,600 acre-ft/year (4,990,600 $\mathrm{m}^{3} /$ day) and likely represents underflow across the southwest boundary. Outflow is distributed vertically across the active section of flow; however, information is not available to evaluate the vertical distribution of flow, largely because of the limited number of wells and large depths to the aquifer. 


\section{C-A-4. REFERENCES}

Barraclough, J. T., W. E. Teasdale, J. B. Robertson, and R. G. Jensen, 1967, Hydrology of the National Reactor Testing Station, Idaho 1966, USGS Open-File Report TID-4500, October 1967.

Cecil, L. D., J. R. Pittman, T. M. Beasley, R. L. Michel, R. L. Kubik, P. Sharma, U. Fehn, and H. E. Gove, 1992, "Water Infiltration Rates in the Unsaturated Zone at the Idaho National Engineering Laboratory Estimated from Chlorine-36 and Tritium Profiles, and Neutron Logging," Kharaka Yousifk and Ann S. Maest (eds.), Proceedings of the $7^{\text {th }}$ International Symposium on Water-Rock Interaction, July 13-18, 1992, pp. 709-714.

Contor, B. A., 2004, Recharge on Non-irrigated Lands, Idaho Water Resource Research Institute Technical Report 04-006, Eastern Snake River Plain Aquifer Model Enhancement Project Scenario Document DDW-003, September 2004.

Cosgrove, D. M., B. A. Contor, and G. S. Johnson, 2006, Enhanced Snake Plain Aquifer Model Final Report, Idaho Water Resources Research Institute Technical Report, in publication, April 2006.

Garabedian, S. P., 1992, Hydrology and Digital Simulation of the Regional Aquifer System, Eastern Snake River Plain, Idaho, U.S. Geological Survey Professional Paper 1408-F.

Kjelstrom, L. C., 1986, "Flow Characteristics of the Snake River and Water Budget for the Snake River Plain, Idaho and Eastern Oregon," U.S. Geolgoical Survey Hydrologic Investigations Atlas, HA-680, scale 1:1,000,000.

Spinazola, J. M., 1994, Geohydrology and Simulation of Flow and Water Levels in the Aquifer System in the Mud Lake Area of the Eastern Snake River Plain, Eastern Idaho, U.S. Geological Survey Water-Resources Investigations Report 93-4227.

USGS, 2007, USGS Annual Statistics for Idaho, http://nwis.waterdata.usgs.gov/id/nwis/annual/calendar year?search criteria=station nm\&submitte d form=introduction, U.S. Geological Survey, Wed page visited January 11, 2007.

Wood, T. R., C. M. Helm-Clark, H. Huang, S. Magnuson, T. McLing, B. Orr, M. Roddy, M. J. Rohe, M. A. Plummer, and R. Podgorney, 2005, Operable Unit 10-08 Summary Report on the Subregional-scale Two-dimensional Aquifer Model, ICP/EXT-05-00979, Rev. 1, Idaho Cleanup Project, October 2005. 


\section{Appendix C-B}

\section{Injection Well Histories}


C-88 


\section{Appendix C-B}

\section{Injection Well Histories}

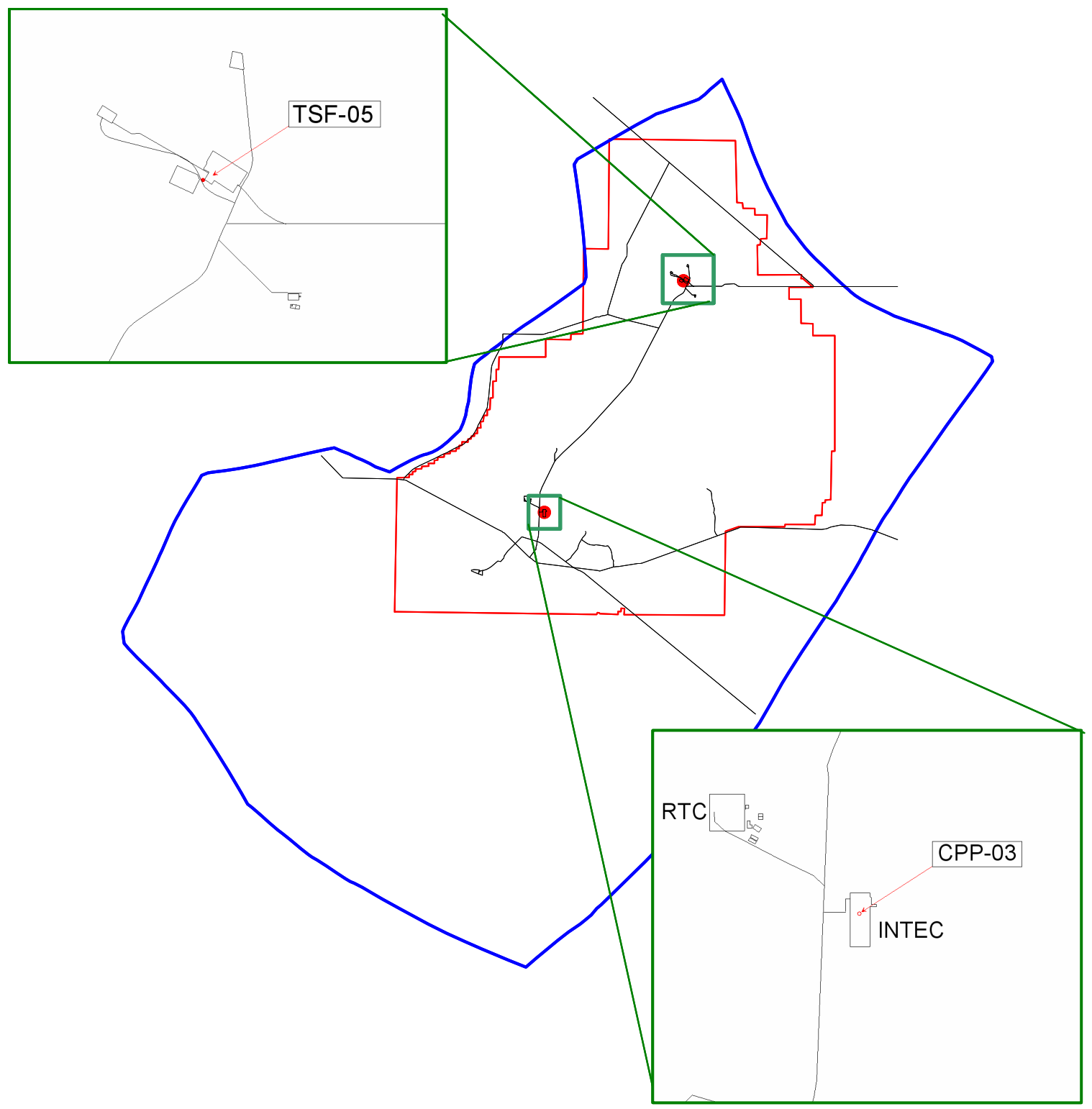

Figure C-B-1. Location of injection wells at TAN (TSF-05) and INTEC (CPP-03). 

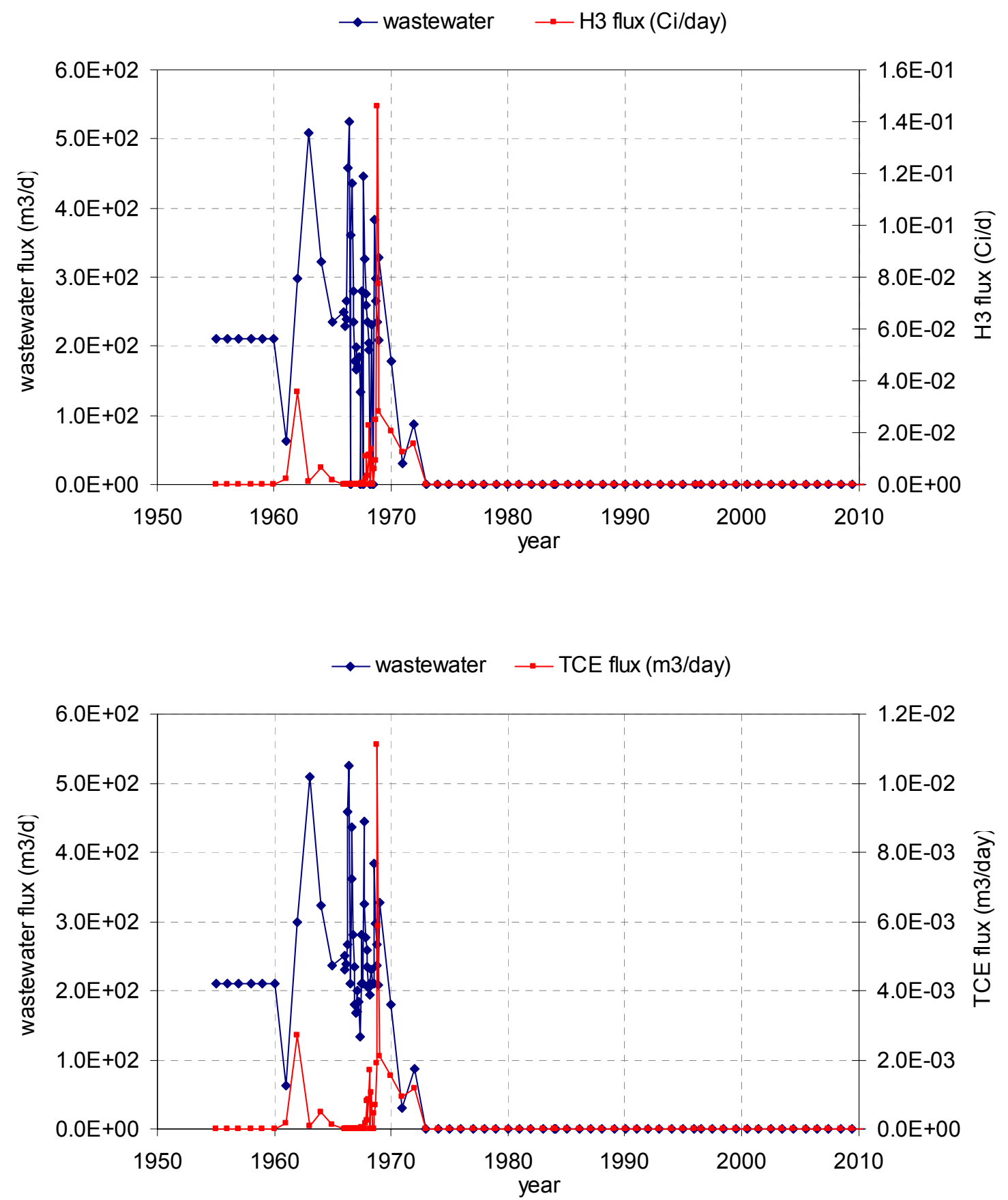

Figure C-B-2. Injection well history for TAN TSF-05. 


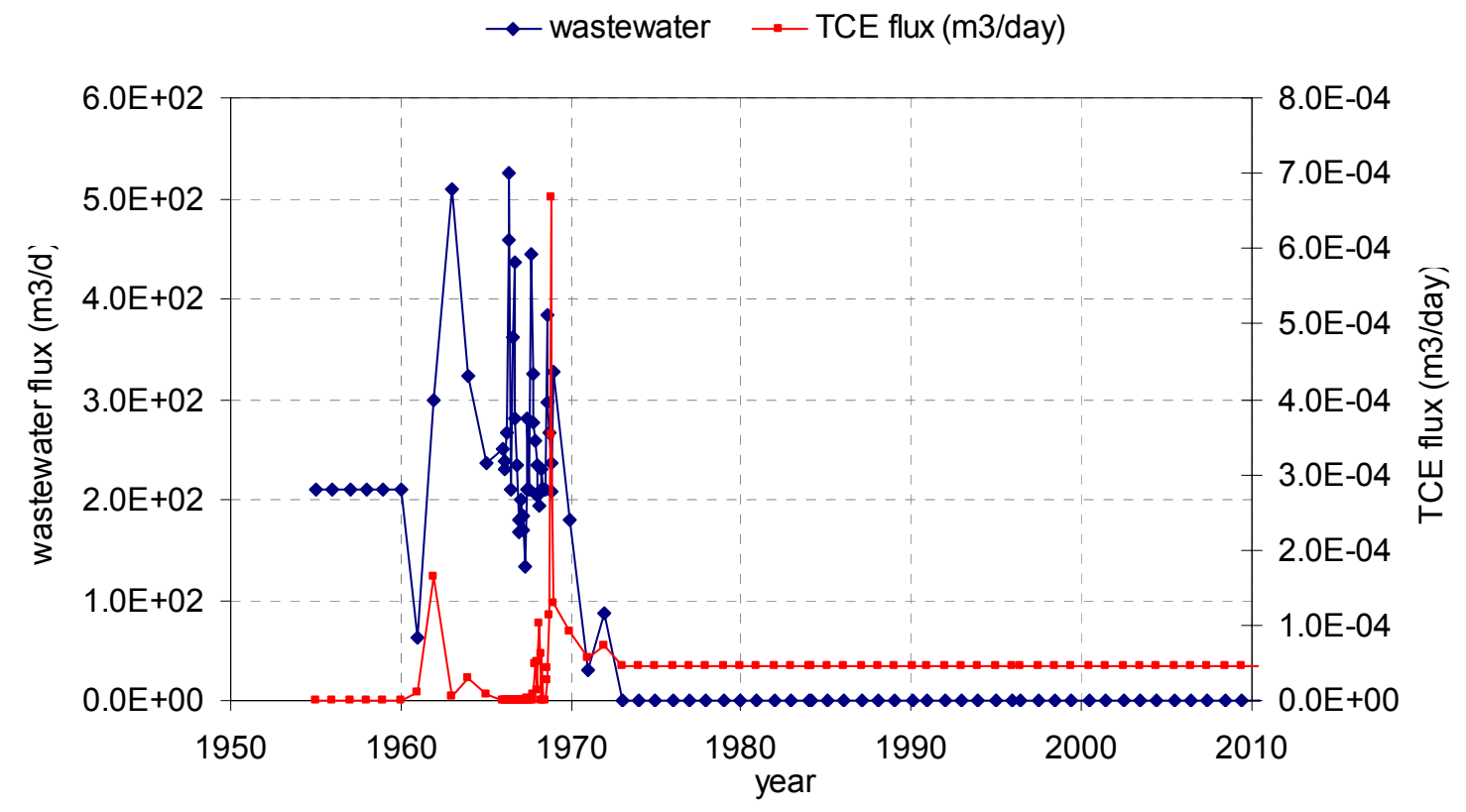

Figure C-B-3. Alternate TCE injection well history for TAN TSF-05 (non-degrading scenario). 

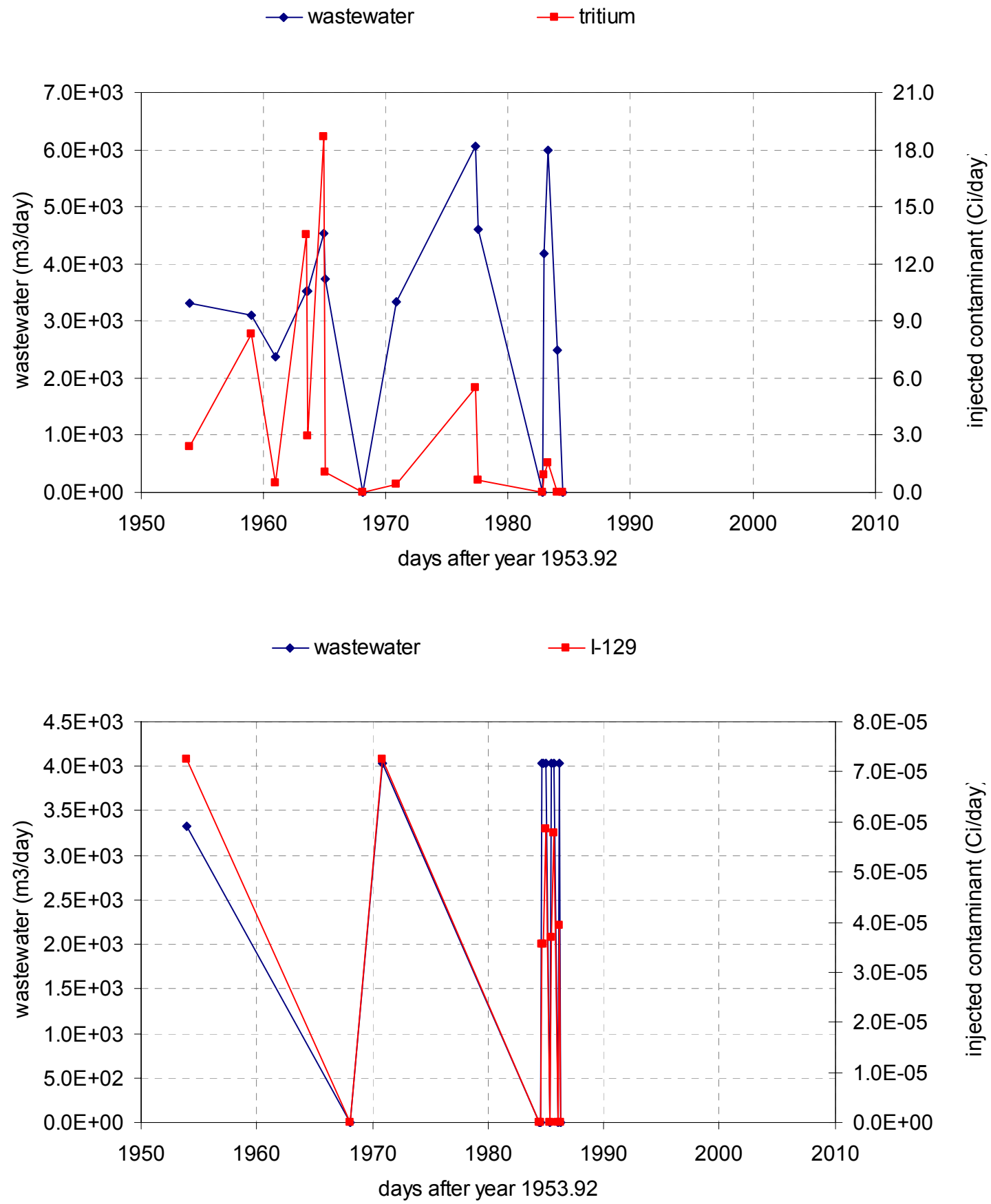

Figure C-B-4. Injection well history for INTEC CPP-03. 

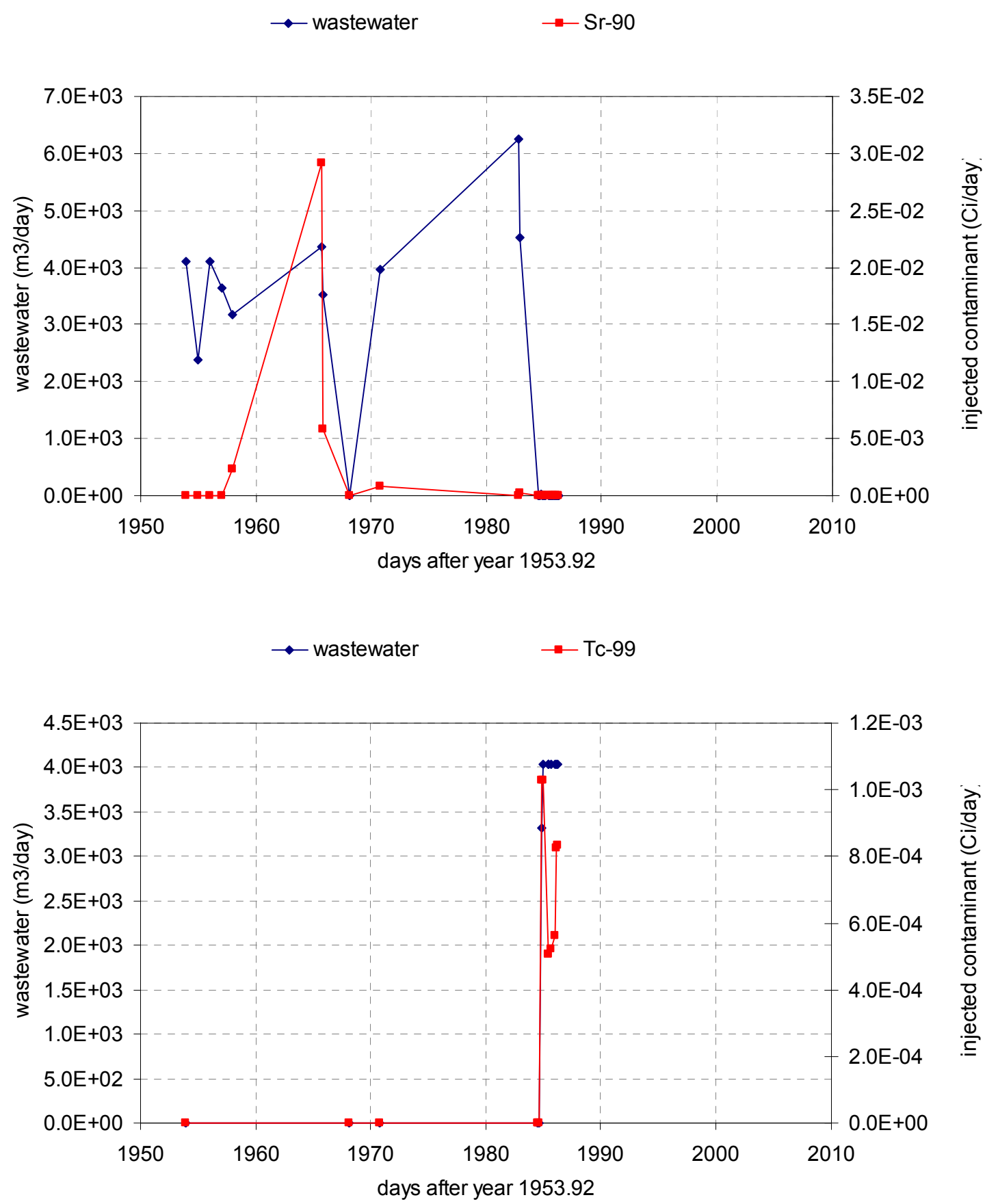

Figure C-B-4 (continued). Injection well history for INTEC CPP-03. 
C-94 


\section{Appendix C-C}

\section{Vadose Zone Flux Histories from Individual WAG Models}


C-96 


\section{Appendix C-C}

\section{Vadose Zone Flux Histories from Individual WAG Models}

This appendix shows the vadose zone source terms provided from the two WAG 3 and WAG 7 facility-specific models. The terms provided by the individual WAG models were unique for each of 600 source cells (WAG 3) and 140 cells (WAG 7) (see Figure C-18).

However, only integrated source terms are provided graphically in this appendix. These are the sum of individual source cell flux terms that have been integrated over the entire vadose zone footprint for WAG 3 and WAG 7.

The terms presented here are in units of $\mathrm{kg}$ /day for non-radiological contaminants or $\mathrm{Ci} /$ day for radiological contaminants. For comparison purposes, the only WAG 7 tritium sources presented in this appendix are derived from the ongoing RWMC low-level waste performance assessment and composite analysis.

Since the OU 10-08 2-D transport model was studied for a historical period of 1950 to present, and not for simulations of distant future time periods, the data presented here (Figures C-C-1 through C-C-3) are shown only for the time period from 1950 to present. 

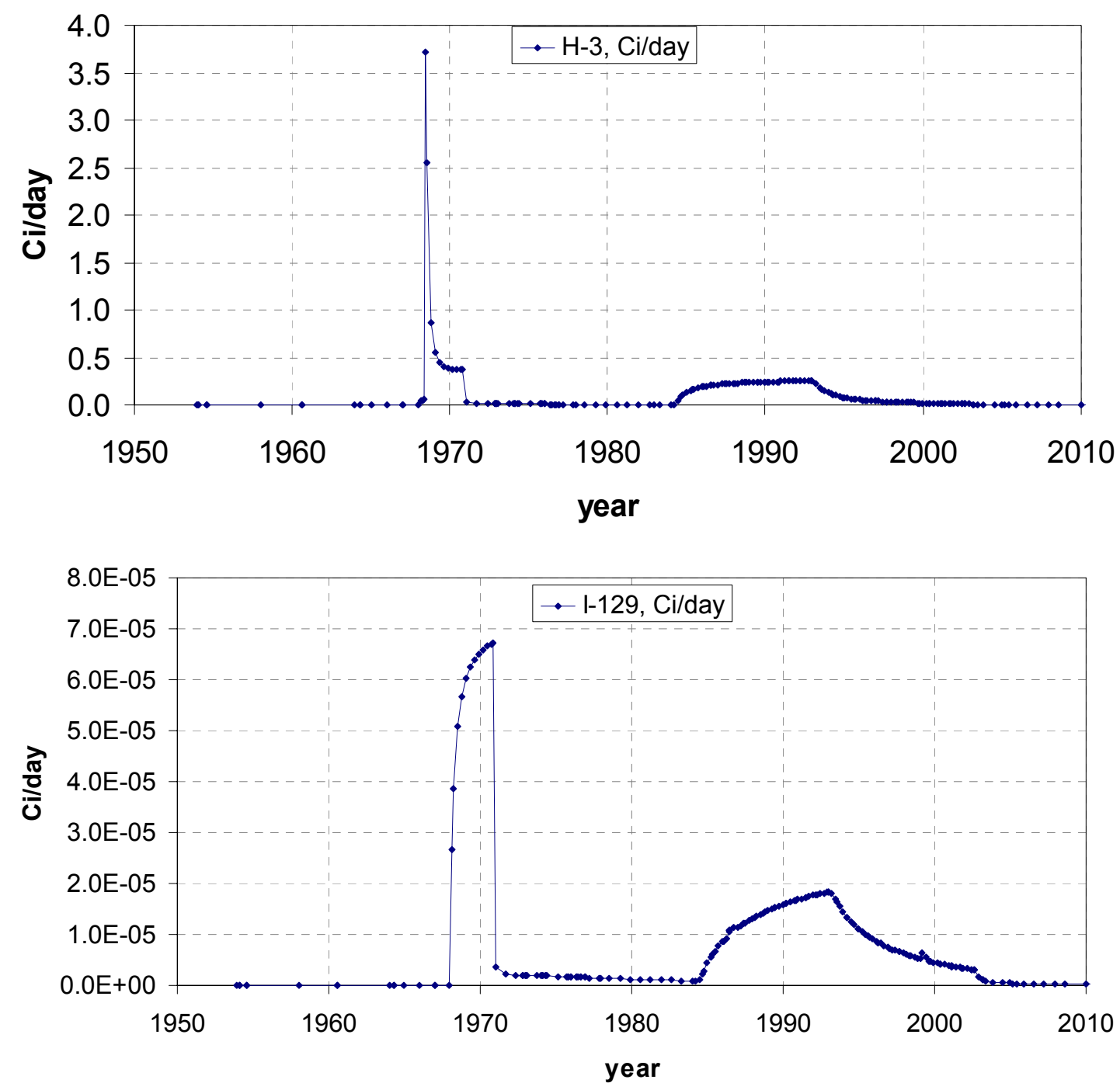

Figure C-C-1. Integrated flux terms from OU 3-14 vadose zone model. 

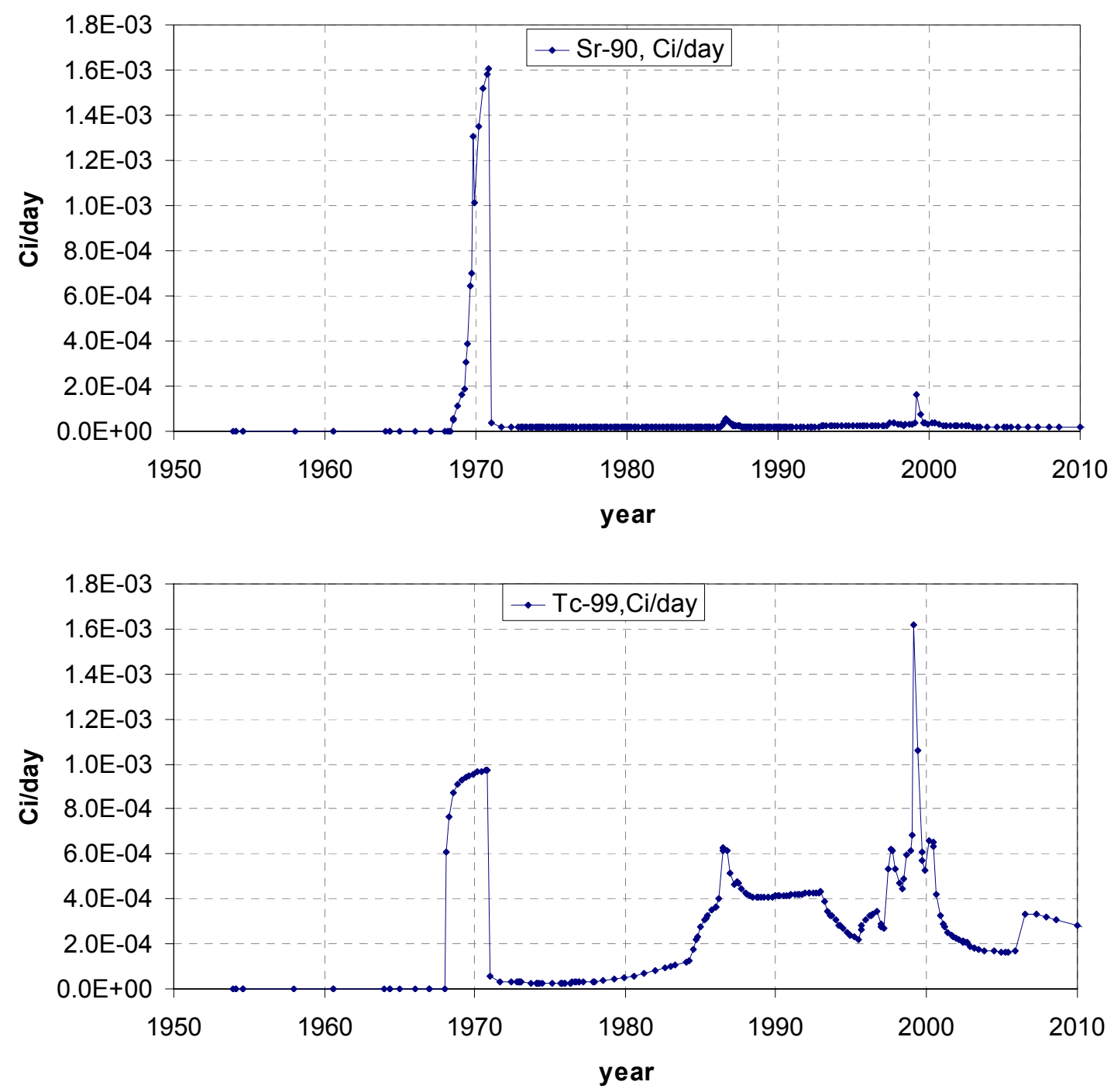

Figure C-C-1 (continued). Integrated flux terms from OU 3-14 vadose zone model. 

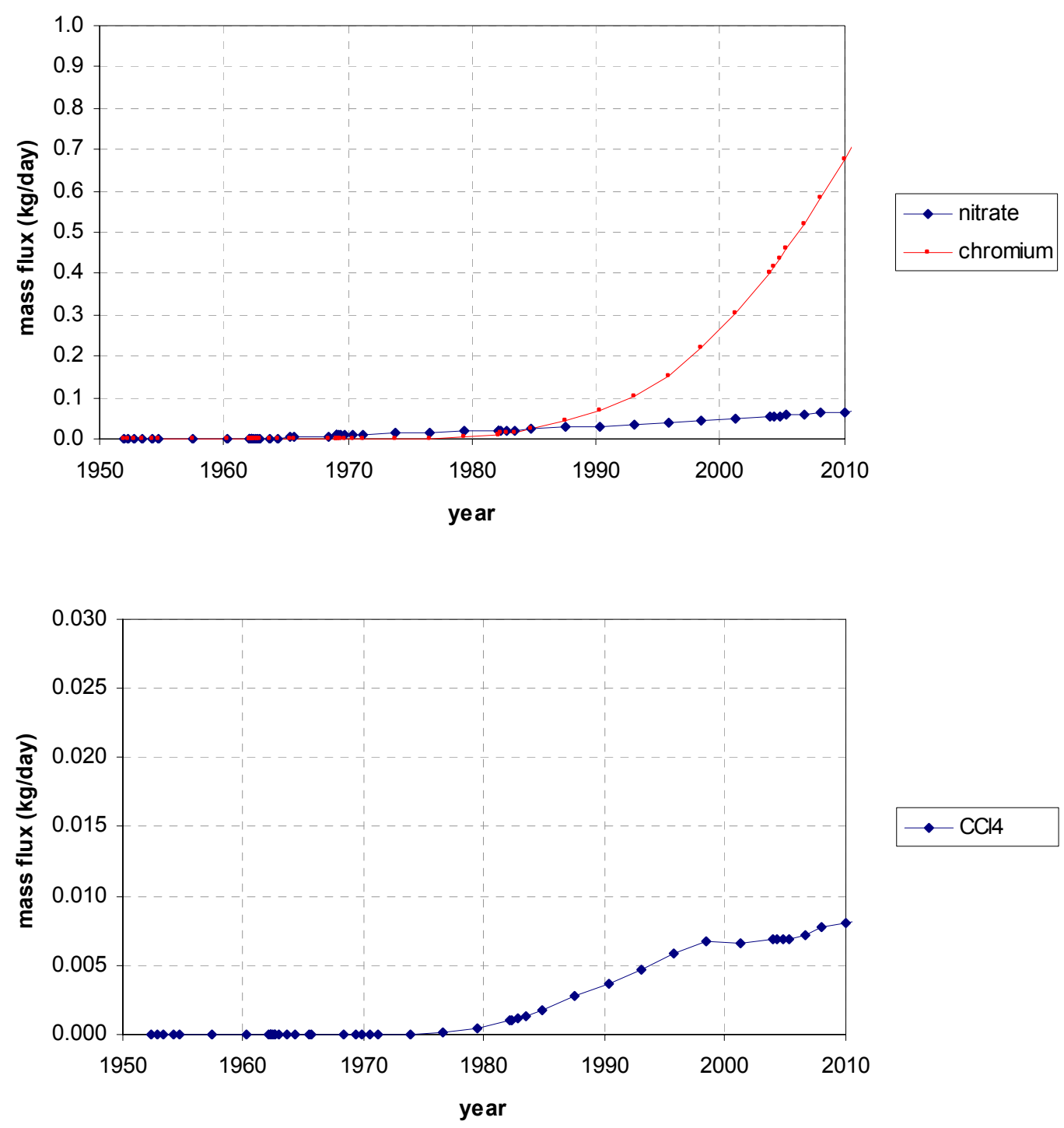

Figure C-C-2. Integrated flux terms from OU 7-13/14 vadose zone model. 

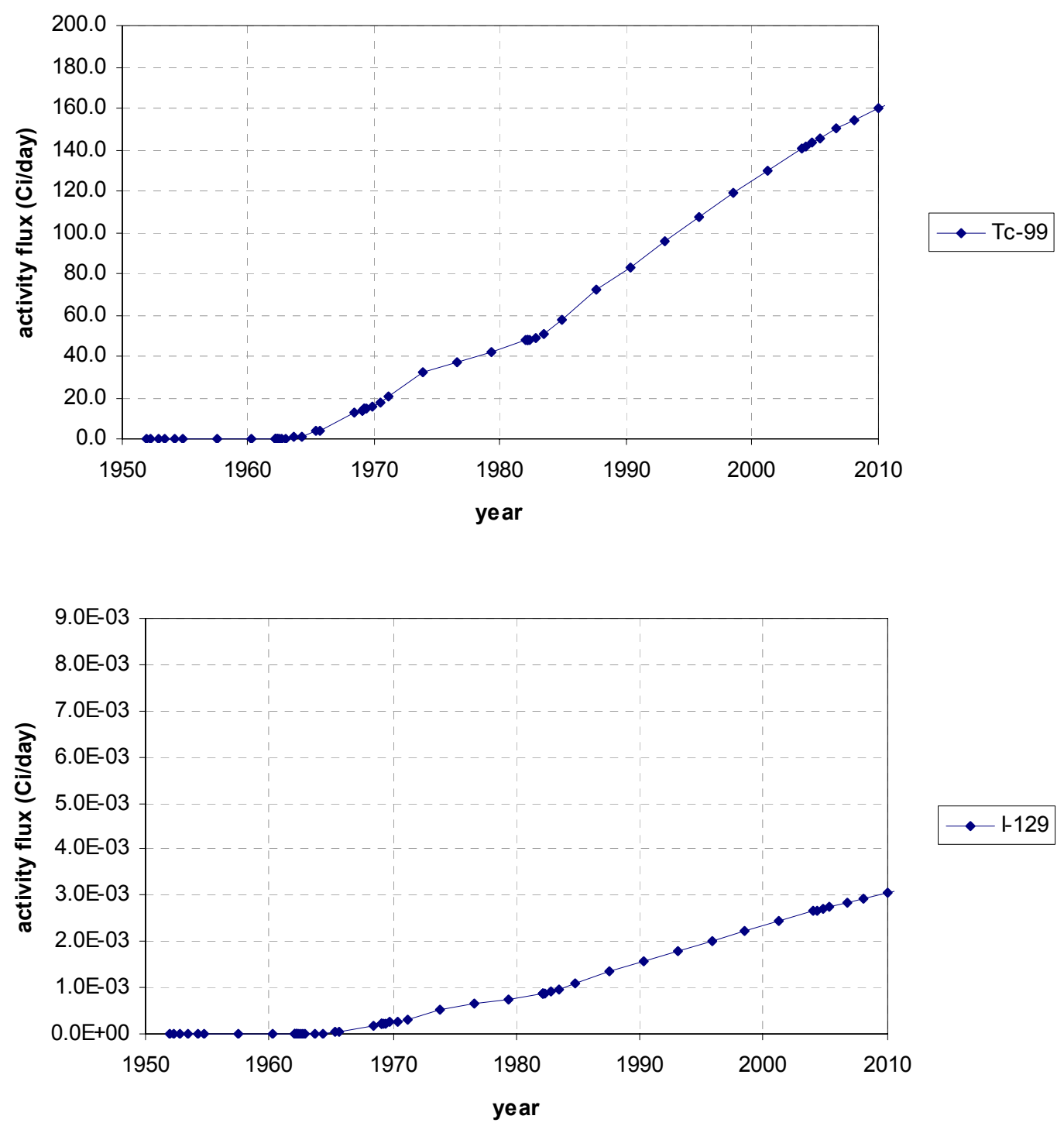

Figure C-C-2 (continued). Integrated flux terms from OU 7-13/14 vadose zone model. 


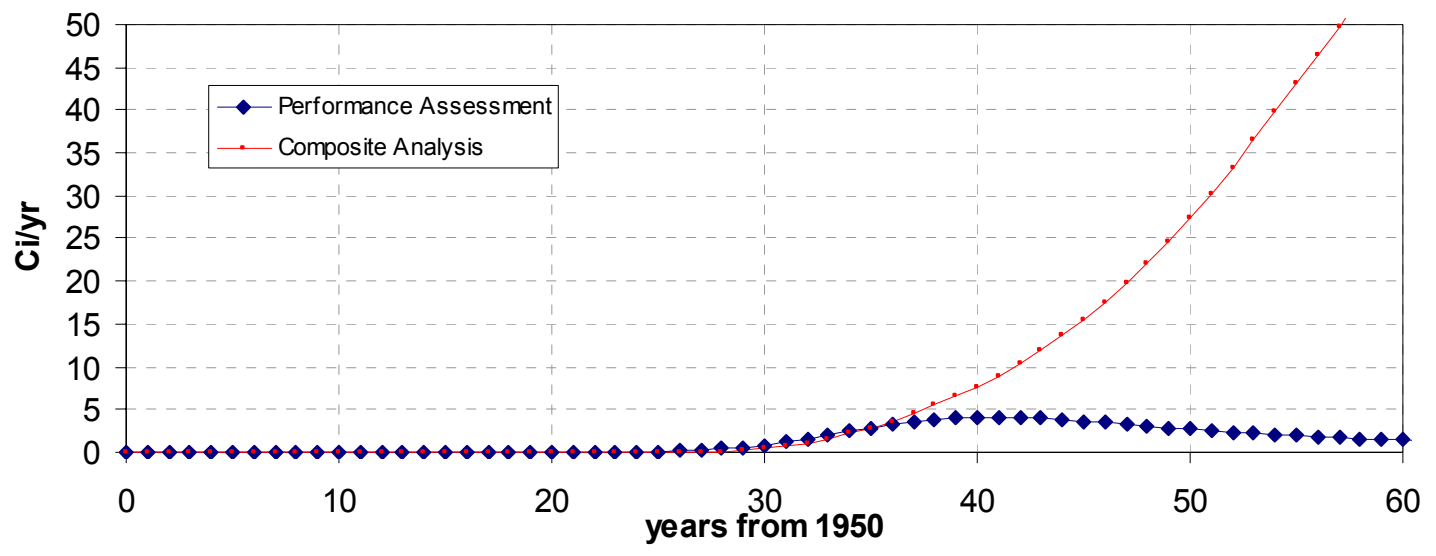

Figure C-C-3. Integrated tritium flux terms from ongoing RWMC low-level waste performance assessment and composite analysis. 